NIST Special Publication 973

\title{
Eleventh International Conference on Computer Technology in Welding
}

T.A. Siewert and C. Pollock, Editors
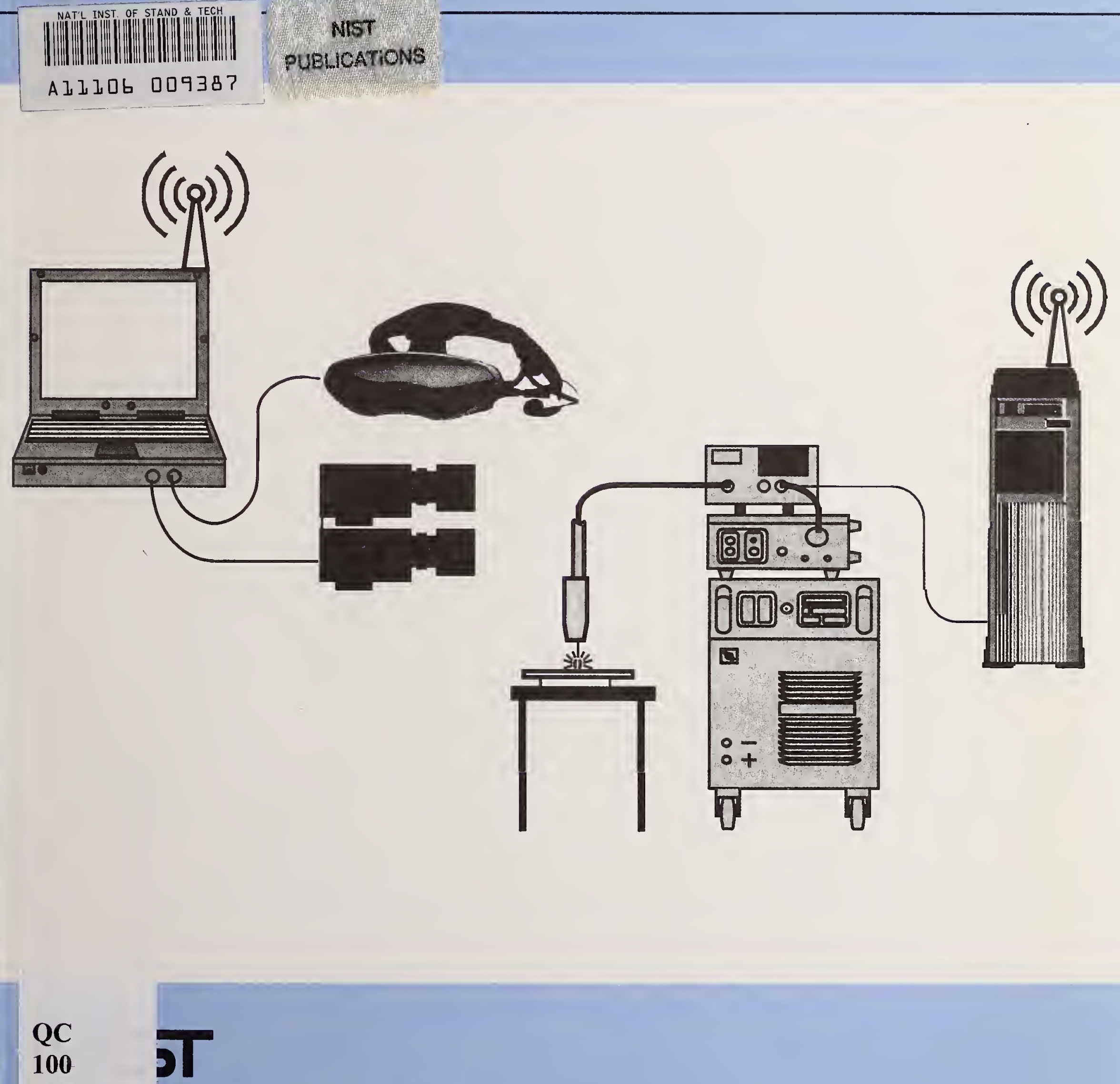

.U57 nstitute of Standards and Technology

no. 973 Administration, U.S. Department of Commerce

2002 c. 2 

processes, to ensure product reliability . . . and to facilitate rapid commercialization ...of products based on new scientific discoveries."

NIST, originally founded as the National Bureau of Standards in 1901, works to strengthen U.S. industry's competitiveness; advance science and engineering; and improve public health, safety, and the environment. One of the agency's basic functions is to develop, maintain, and retain custody of the national standards of measurement, and provide the means and methods for comparing standards used in science, engineering, manufacturing, commerce, industry, and education with the standards adopted or recognized by the Federal Government.

As an agency of the U.S. Commerce Department's Technology Administration, NIST conducts basic and applied research in the physical sciences and engineering, and develops measurement techniques, test methods, standards, and related services. The Institute does generic and precompetitive work on new and advanced technologies. NIST's research facilities are located at Gaithersburg, MD 20899, and at Boulder, CO 80303. Major technical operating units and their principal activities are listed below. For more information contact the Publications and Program Inquiries Desk, 30I-975-3058.

\section{Office of the Director}

- National Quality Program

- International and Academic Affairs

Technology Services

- Standards Services

- Technology Partnerships

- Measurement Services

- Information Services

\section{Advanced Technology Program}

- Economic Assessment

- Information Technology and Applications

- Chemistry and Life Sciences

- Materials and Manufacturing Technology

- Electronics and Photonics Technology

\section{Manufacturing Extension Partnership}

Program

- Regional Programs

- National Programs

- Program Development

\section{Electronics and Electrical Engineering}

Laboratory

- Microelectronics

- Law Enforcement Standards

- Electricity

- Semiconductor Electronics

- Radio-Frequency Technology

- Electromagnetic Technology

- Optoelectronics ${ }^{1}$

\section{Materials Science and Engineering}

\section{Laboratory}

- Theoretical and Computational Materials Science

- Materials Reliability

- Ceramics

- Polymers

- Metallurgy

- NIST Center for Neutron Research

\section{Chemical Science and Technology}

\section{Laboratory}

- Biotechnology

- Physical and Chemical Properties ${ }^{2}$

- Analytical Chemistry

- Process Measurements

- Surface and Microanalysis Science

Physics Laboratory

- Electron and Optical Physics

- Atomic Physics

- Optical Technology

- Ionizing Radiation

- Time and Frequency'

- Quantum Physics

\section{Manufacturing Engineering}

Laboratory

- Precision Engineering

- Automated Production Technology

- Intelligent Systems

- Fabrication Technology

- Manufacturing Systems Integration

Building and Fire Research Laboratory

- Applied Economics

- Structures

- Building Materials

- Building Environment

- Fire Safety Engineering

- Fire Science

\section{Information Technology Laboratory}

- Mathematical and Computational Sciences ${ }^{2}$

- Advanced Network Technologies

- Computer Security

- Information Access and User Interfaces

- High Performance Systems and Services

- Distributed Computing and Information Services

- Software Diagnostics and Conformance Testing

- Statistical Engineering

'At Boulder, CO 80303.

Some elements at Boulder, $\mathrm{CO}$. 


\title{
Eleventh International Conference on Computer Technology in Welding
}

\author{
T. A. Siewert \\ and \\ C. Pollock, \\ Editors
}

Materials Reliability Division

Materials Science and Engineering Laboratory

325 Broadway

Boulder, Colorado 80305

January 2002

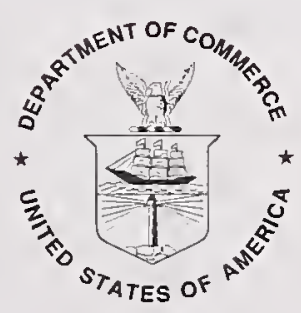

U.S. Department of Commerce Donald L. Evans, Secretary

Technology Administration

Phillip J. Bond, Under Secretary for Technology

National Institute of Standards and Technology Arden L. Bement, Jr., Director 
Certain commercial entities, equipment, or materials may be identified in this document in order to describe an experimental procedure or concept adequately. Such identification is not intended to imply recommendation or endorsement by the National Institute of Standards and Technology, nor is it intended to imply that the entities, materials, or equipment are necessarily the best available for the purpose.

National Institute of Standards and Technology Special Publication 973

Natl. Inst. Stand. Technol. Spec. Publ. 973, 319 pages (January 2002)

CODEN: NSPUE2

U.S. GOVERNMENT PRINTING OFFICE

WASHINGTON: 2002

For sale by the Superintendent of Documents, U.S. Government Printing Office Internet: bookstore.gpo.gov Phone: (202) 512-1800 Fax: (202) 512-2250 Mail: Stop SSOP, Washington, DC 20402-000 1 


\section{CONTENTS}

\section{TRACK A: SENSING}

Chair: Dr. J. E. Jones, N. A. Technologies, Golden, Colorado

\section{Session A1: Sensing and Control I: GMAW}

A1-1 The Experimental Studies on the Process-Integrated Quality Assurance in

Pulsed Current GMAW of Aluminum

Dr. S. Rajasekaran, Tamilnadu, India.

A1-2 Droplet Oscillation and Weld Pool Imaging Using Computer-Controlled

Composite Pulse Current

Dr. B. Zheng, EWI, Columbus, Ohio

A1-3 Optimization of PGMAW Using Online Observation and Statistical Data

S. Nordbruch, University of Bremen, Germany

A1-4 Through the Arc Tracking of 5G Narrow Gap Pipe Welds

T. S. Rajagopalan, CRC-Evans Automatic Welding, Houston, Texas

A1-5 Development of Rotating GMA Welding System and its Application to

Arc Sensors

C. H. Kim, Korean Adv. Inst. of Science and Tech., Taejeon, Korea

\section{Keynote}

"Lights Out" Welding: Progress towards Complete Welding Automation

T. P. Quinn, Past Chairman, AWS A9 Committee on Computerization,

NIST, Boulder, Colorado

Chair: Dr. D. M. Barborak, WeldWare Inc., Columbus, Ohio

Session A2: Sensing and Control II: GMAW and GTAW

A2-1 A System for Real-Time Control of Gas Metal Arc Weld Profile

Dr. D. M. Barborak, WeldWare Inc., Columbus, Ohio

A2-2 Weld Penetration Control During GTA Welding Using Weld Pool

Oscillation Sensing

Dr. M. J. M. Hermans, Delft University of Technology, The Netherlands

A2-3 Real-Time Molten Pool Area Extraction for Control of Gas Tungsten

Arc Welding

Prof. R. Kovacevic, SMU, Richardson, Texas.

Chair: W. Rippey, NIST, Gaithersburg, Maryland

\section{Session A3: Sensing and Control III: VPPAW}

A3-1 Monitoring Stability of Keyhole Formation in VPPAW

Prof. R. Kovacevic, SMU, Richardson, Texas

A3-2 Systems Technology for Variable Polarity Plasma Arc Welding of

Aluminum Alloys

Dr. B. Zheng, EWI, Columbus, Ohio 
Chair: Dr. A. C. Hall, Sandia National Laboratories, Albuquerque, New Mexico

\section{Session A4: Imaging}

A4-1 Characterizing the Microstructure of A GTA Weld In-Process Using

High-Speed, High Magnification, Digital Imaging

Dr. A. C. Hall, Sandia National Laboratories, Albuquerque, New Mexico

A4-2 Vision-based Weld Defect Measurement and Depiction

C. Reichert, EWI, Columbus, Ohio

A4-3 Computer Micro-image Analysis in the Heat Affected Zone of HQ130

High Strength Steel

Dr. Y. Li, Shandong University, Peoples Republic of China

Chair: Dr. T. P. Quinn, NIST, Boulder, Colorado

Session A5: Fabrication Quality and Standards

A5-1 Virtual and Augmented Reality for Quality Improvement of Manual Welds

P. Tschirner, University Bremen, Germany

A5-2 A9 Mission, Current Activities, and What Should We Do Next

W. Rippey, NIST, Gaithersburg, Maryland

Chair: Dr. K. Matsuyama, MIT, Cambridge, Massachusetts

Session A6: Sensing and Control Systems IV

A6-1 On-Line Weld Quality Diagnostics in Friction Stir Welding

Prof. R. Kovacevic, SMU, Richardson, Texas

A6-2 A Real-Time Monitoring and Control System for Resistance Spot Welding

Dr. K. Matsuyama, Massachusetts Institute of Technology (MIT),

Cambridge, Massachusetts.

\section{TRACK B: MODELING}

Chair: Dr. R. D. Everhart, EWI, Columbus, Ohio

\section{Session B1: Modeling I: Distortion and Stress}

B1-1 An Integrated FEA Based Procedure for Weld Fixture Design

Dr. Z. Yang, Caterpillar Inc., Peoria, Illinois 203

B1-2. Welding Induced Distortions Modeling of Large Plate Structures

Dr. R. D. Everhart, EWI, Columbus, Ohio

B1-3 Computational Modeling of Residual Stress in Welds

Dr. K. Koppenhoefer, EWI, Columbus, Ohio

B1-4 A Study on the Prediction of Welding Distortions of the Hull in Shipbuilding

S. B. Shin, Hyundai Heavy Ind., Co., Korea

B1-5 Numerical Simulation of Shear Stress Distribution in the Fillet of an $\mathrm{Al}^{-\mathrm{Al}_{2} \mathrm{O}_{3}}$

Soldering Assembly

Dr. X. Ma, National Key Laboratory of Reliability Physics of

Electronic Product, Peoples Republic of China 
Chair: Dr. M. V. Li, EWI, Columbus, Ohio

Session B2: Modeling II: Predicting Microstructure and Performance

B2-1 Advances in Aluminium Weld Simulations Applying WELDSIM

H. G. Fjær, Institute for Energy Technology, Norway

B2-2 A Computational Model for Heat Affected Zone of Reactor Pressure Vessel Steel

Dr. J.-S. Kim, Korea Power Engineering Co., Korea ……........................................265

B2-3 Novel Computer-aided Method of Welding Materials Design

V. Mazurovsky, CWC, Ltd, Ariel, Israel....

B2-4 Finite Element Analysis of Creep Strength Mis-matched Dissimilar

Welded Joints

H. Chen, Southwest Jiaotong University, Chengdu, China

Chair: Dr. D. F. Farson, The Ohio State University, Columbus, Ohio

\section{Session B3: Modeling III}

B3-1 Laser Weld Keyhole Dynamics: Simulation and Experiment

Dr. D. F. Farson, OSU, Columbus, Ohio

B3-2 Finite Element Modeling on Welding Processes and Welding Phenomena

Dr. B. Palotas, Budapest University of Technology and

Economics, Hungary....

Chair: Dr. M. Williams, Los Alamos National Laboratory, Los Alamos, New Mexico

\section{Session B4: Modeling IV}

B4-1 Three-Dimensional Simulation of Robot Path and Heat Transfer of a TIG-Welded Part with Complex Geometry

M. Ericsson, University of Trollhattan/Uddevalla, Sweden

B4-2 Dynamic Modeling of GTAW for Rapid Manufacturing

Prof. R. Kovacevic, SMU, Richardson, Texas

B4-3 Three Dimensional Transient Heat Transfer Analysis For GTA Welding Process M. Jou, National Taiwan Normal University, Taipei City, Taiwan. 



\section{Preface}

These are the proceedings of the eleventh conference in this series. The conference was held December 5 and 6, 2001, in Columbus, Ohio, under the sponsorship of the American Welding Society, NIST, and The Welding Institute. These proceedings include 32 presentations grouped into sessions on: sensing and control of GMAW, sensing and control of GTAW, sensing and control of VPPAW, imaging, fabrication quality and standards, sensing and control systems, modeling of distortion and stress, predicting microstructure and performance, and general modeling topics. The large number of submissions required the papers to be divided into two parallel tracks: a sensing and control track, and a modeling track. In addition, there was a keynote talk on our progress toward total automation. These proceedings include all the manuscripts that were submitted (including viewgraphs). Additional copies are available from NTIS (National Technical Information Service) or GPO (Government Printing Office) as NIST Special Publication 973 (See below).

The first U.S. workshop on computerization of welding data was held in 1986, under the sponsorship of the American Welding Institute (AWI) and the then National Bureau of Standards (now, National Institute of Standards and Technology, NIST). The workshop produced a list of national needs in welding data, a list designed to guide database developers. The proceedings of that first workshop are available from NTIS as NIST Special Publication 742.

There had been sufficient advances in database activity by 1988 to justify a second meeting, this time as a joint workshop-conference under the sponsorship of AWI, NIST, and the American Welding Society (AWS). The scope was expanded to include a conference on the latest developments and a preconference tutorial to provide novices with a background in common computer applications. The conference was attended by 61 managers, welding engineers, and computer professionals. The proceedings of the workshop and conference are available from NTIS as NIST Special Publication 781.

By 1990, the welding database activity had grown large enough to justify a third conference on this topic, again sponsored by AWI, AWS, and NIST. It consisted of a series of tutorials, a keynote presentation, and technical sessions on the topics: off-line planning, real-time welding information, data systems and standards, and industrial applications. The conference included demonstrations of welding software, and was followed by a meeting of the AWS Committee on Computerization of Welding Data.

The fourth conference, held during November 1992 in Orlando, Florida, was truly an international conference; speakers representing 10 countries presented papers on the topics of standards, applications, quality and NDE, sensing, control, and databases. Once again, a preconference tutorial was organized and taught by AWI personnel covering PC networks, expert systems, neural networks, Windows and the Excel Spreadsheet, and databases. The conference also included a keynote presentation, tabletop exhibits, and hands-on demonstrations of welding software. The AWS Committee on Computerization of Welding Data met following the Conference. These proceedings are available from AWS (Code: CP-1192). 
The fifth conference, held August 1994 in Golden, Colorado, continued the trend of growth in size and scope. It consisted of 31 papers (by 69 authors) on the topics of quality control, off-line planning and simulation, commercial software systems, control and automation, welding optimization, data acquisition and sensors, application case studies, welder and procedure qualification systems, weld prediction and control, and large-scale systems. This year the preconference tutorials were on the topics of computing platforms, sensing and data acquisition, and line planning for welding automation. These tutorials were taught by experts from ABB Robotics, AMET, CSM, Ford Research Laboratory, and NIST. The conference was cosponsored by AWI, AWS, the Colorado School of Mines, and NIST. The conference proceedings are available from AWS (code: CP-794).

The sixth conference was held June 9-12, 1996, in the "Heart of Europe," where Belgium, Germany, and the Netherlands converge in the Limberg region. The Technical Program was aimed to embrace all known facets of computing practice as applied to welded fabrication and manufacturing. Expert advice was provided in process selection, consumable selection, and welding procedure generation and interpretation of standards. Proceedings are available through Woodhead Publishing Ltd. (see below).

The seventh conference was held July 8-12, 1997, in San Francisco, California, and was presented in conjunction with the American Welding Society, the National Institute of Standards and Technology, and The Welding Institute. Attendees were able to observe how computers can be used for more than just databases. In addition to tutorials, the following were covered: case studies, controls and controllers, sensing, process automation, modeling heat and fluid flow, modeling thermomechanical effects, and modeling residual stress or mechanical effects. Also covered were the Internet and networked systems in the welding industry. Additional copies of the proceedings are available from NTIS as NIST Special Publication 923.

The eighth conference was held June 22 to 24,1998 , in Liverpool, U.K., and was sponsored by The Welding Institute. The significant advances in technologies such as expert systems, neural networks, and the internet, were highlighted in tutorial sessions. The 35 papers were grouped into sessions on: sensors/vision, modeling and control, modeling of process and joint properties, modeling and simulation, education and training, and quality control and quality assurance. Proceedings are available through Woodhead Publishing Ltd.

The ninth conference was held September 28 to 30, 1999, in Detroit, Michigan, under the sponsorship of the American Welding Society, NIST, and The Welding Institute. There were 57 papers grouped into sessions on resistance weld simulation, simulation of gas metal arc welding, modeling of weld shape and distortion, modeling of solidification, general modeling, welding documentation, sensing and control of arc quality, droplet control and process automation, automation communication and interfaces, and documentation and database applications. The large number of papers required the papers to be divided into two parallel tracks: a modeling track, and a sensing and control track. In addition, there were tutorials on weld cell communication issues and on web page design. The printed proceedings were supplemented with a CDROM disk, which included electronic versions of most of the papers and some electronic files of the presentations. Additional copies are available from NTIS or GPO as NIST Special Publication 949. 
The tenth conference was held June 6 and 7, 2000, in Copenhagen, Denmark, under the leadership of The Welding Institute and the Force Institute. The papers were divided into sessions on sensor and vision systems, equipment control, manufacturing and production, education and training, and modeling and simulation. The proceedings are available in both paper and electronic forms from Woodhead Publishing Ltd.

To order the NIST Special Publications, contact:

Superintendent of Documents

Government Printing Office

Washington, DC 20402

(202) 512-1800

http://www.access.gpo.gov/ or National Technical Information Service

Springfield, VA 22161

(703) 487-4650

http://www.ntis.gov/

To order the 1992 and 1994 proceedings, contact:

Order Department

American Welding Society

550 N.W. LeJeune Road

Miami, Florida 33126

(800) 334-9353 (or (305) 443-9353, Ext. 280)

To order the 1996, 1998, or 2000 proceedings, contact:

Woodhead Publishing Ltd.

Abington Hall, Abington

Cambridge CB1 6AH

England

$+44(0) 1223891358$

Except where attributed to NIST authors, the content of individual sections of this volume has neither been reviewed nor edited by the National Institute of Standards and Technology. NIST therefore accepts no responsibility for comments or recommendations therein. The mention of trade names in this volume neither constitutes nor implies any endorsement or recommendation by the National Institute of Standards and Technology.

Acknowledgment: The editors wish to express their appreciation to those who helped run the conference, chair the sessions, and prepare these proceedings, especially to Gladys Santana of the American Welding Society and Vonnie Ciaranello of NIST. 

Session A1: Sensing and Control I: GMAW 



\title{
THE EXPERIMENTAL STUDIES ON THE PROCESS-INTEGRATED QUALITY ASSURANCE IN PULSED CURRENT GMA WELDING OF ALUMINUM
}

\author{
Dr. S. Rajasekaran
}

\begin{abstract}
Al-Mg alloys are widely used in the production of new breed lightweight cars. The pulsed current gas metal arc welding (GMAW-P) is widely used to weld these alloys. However, quality of the weld mainly depends upon the parameters of the pulsed current such as peak current $\left(I_{P}\right)$, peak duration $\left(T_{P}\right)$, background current $\left(I_{B}\right)$, background duration $\left(T_{B}\right)$, welding speed $\left(W_{S}\right)$, and wire feed speed $\left(\mathrm{W}_{\mathrm{F}}\right)$. Selecting the most suitable GMAW-P parameter is very complex due to interdependence of parameters.

Therefore effects of the GMAW-P parameters on different types of droplet detachments, namely one droplet detachment during peak duration, one droplet detachment during background duration, two droplets detachment during peak duration, and three droplets detachment during peak duration have been studied. The best type of droplet detachment for GMAW-P has been selected on the basis of process-integrated quality assurance. This process-integrated quality assurance enables online monitoring of welding voltage, welding current as well as the reproduction of welding data and complete documentation of all parameters and welding machine adjustments. Therefore, this method was used to select the suitable pulse parameter to welding.

The $1.2 \mathrm{~mm}$ diameter of ER 5356 filler metal and 6mm thick AA 5083 Al-Mg alloy base metal were used throughout welding experiments. Electrical signal especially pulsed current waveform as time function, contains essential information about physical phenomena in arc. Therefore, the average current per pulse cycle time was measured from the pulsed current waveforms using AutoCAD 2000 and a self-developed computer software EL-JS. The statistical analysis was carried out to determine uniformity of average current. Results show that one droplet detachment during peak duration can provide stable weld compared to other types of droplet detachments. This experimental study helped to achieve high-quality cost-effective welds with high production rate.
\end{abstract}

\section{KEY WORDS}

Pulsed Current GMAW, Pulse Parameters, Waveform, Process-Integrated Quality Assurance, Al-Mg Alloys Welding, Droplet Detachment, Data Analysis, Computer Data, Arc Sensing.

At the time of this work, Dr. S. Rajasekaran was Research Scholar in the Department of Mechanical Engineering, Indian Institute of Technology-Bombay, Mumbai 400076, Maharastra, India. He is now Assistant Professor in the Department of Mechanical Engineering, Sri Krishna College of Engineering and Technology, Kuniamuthur Post, Coimbatore 641008, Tamilnadu, India. 


\section{INTRODUCTION}

Aluminum has become increasingly important to manufacturing products. Aluminum has replaced many more traditional steel materials. The most conspicuous product of aluminum and its alloys is the new breed lightweight cars. The reason is its higher resistance to climatic conditions and higher corrosion resistance over steel cars. However, the most recognized benefit is its greater strength combined with lower weight. The pulsed current gas metal arc welding (GMAW-P) is the most advanced and promising one among various GMA welding processes, for the welding of aluminum and its alloys, because it can greatly improve the weld quality and promote the automation of all-position welding. It offers the possibility of welding thin metal with spray transfer or using thick wire diameter for low average welding current.

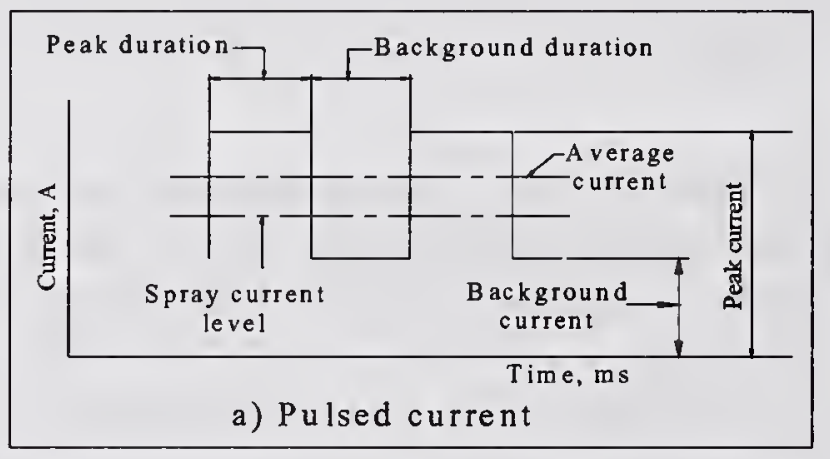

a) Pulsed current

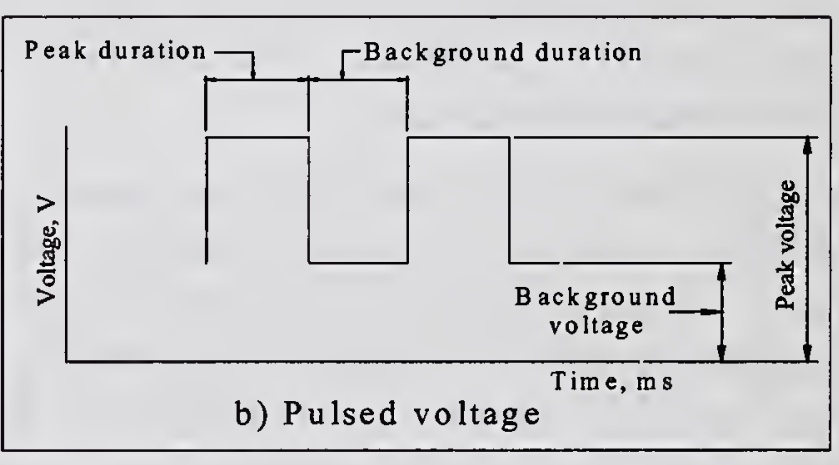

$\mathrm{I}_{\mathrm{B}}$-Background current (A)

$\mathrm{V}_{\mathrm{P}}$-Peak voltage $(\mathrm{V})$;

$\mathrm{V}_{\mathrm{B}}$-Background voltage $(\mathrm{V})$

$\mathrm{T}_{\mathrm{P}}$-Peak duration (ms);

$\mathrm{T}_{\mathrm{B}}$-Background duration (ms)

Figure 1: Pulsed current and pulsed voltage waveform for GMAW-P

In GMAW-P, the current fluctuates between a high peak current for the pulses and a low background current in a regular cycle (Fig. 1). The molten metal is detached either as a single droplet or multiple droplets depending upon the pulse parameters, namely peak current $\left(\mathrm{I}_{\mathrm{P}}\right)$, peak duration $\left(T_{P}\right)$, background current $\left(I_{B}\right)$, and background duration $\left(T_{B}\right)$ (Refs. 1-3). If it is a single droplet, this detachment may occur either in peak duration or background duration, and if it is multiple droplet detachment, the detachment may occur in peak duration (Refs. 4-5). This difference in the timing of droplet detachment affects the arc stability and the overall quality of the weld. There are also shortcomings in the conventional GMAW-P process. First, setting of GMAW-P variables can be very complicated. Secondly, it has a poor self-adjust ability in response to accidental arc length disturbance. Studies on the arc characteristics are very important to understand the stability of arc under various pulsing conditions. Therefore, studies are carried out by focusing efforts on solving these problems. 
All manufacturing industries and welding engineers are considering quality control to be an important factor. Recently, there has been a lot of pressure to achieve the welding benchmark standards. Process-integrated quality assurance enables online monitoring of welding voltage, welding current as well as the reproduction of welding data and complete documentation of all parameters and welding machine adjustments.

In this research paper, a optimum GMAW-P parameter is selected on the basis of process integrated quality assurance: For this, first, the effects of GMAW-P parameters on the various types of droplet detachments were studied and classified on the basis of type of droplet detachment. Secondly, the average current per pulse cycle time was measured for the various types of droplet detachments and the values of mean, standard deviation, and coefficient of variation were calculated. The optimum GMAW-P was selected by choosing the pulse parameter which resulted in the least standard deviation and the coefficient of variation, to produce costeffective, spatter free, high quality welds.

\section{OBJECTIVE}

The objective of this work was to study how GMAW-P parameters influence droplet detachment, and to determine to what extent pulsed current can be used to produce stable Al-Mg alloy welds.

\section{EXPERIMENTAL WORK}

\section{Filler Metal and Base Metal}

The welding was carried out using solid filler metal ER $5356 \mathrm{Al}-\mathrm{Mg}$ alloy having a diameter of $1.2 \mathrm{~mm}$ and with the compatible AA $5083 \mathrm{Al}-\mathrm{Mg}$ alloy of $6.0 \mathrm{~mm}$ thick base metal. Argon gas having a commercial purity of $99.97 \%$ and a flow rate of $3.333 \times 10^{-4} \mathrm{~m}^{3} / \mathrm{s}$ was used throughout the welding as a shielding gas.

\section{Welding Power Supply}

A transistorized welding power supply was used for carrying out GMAW-P operations (Fig. 2) in a pulsed current mode, operating with constant voltage output characteristics. In the pulsed current mode, peak voltage, background voltage, peak duration, and cycle time can be vary over the ranges 10 to $40 \mathrm{~V}, 10$ to $30 \mathrm{~V}, 1$ to $15 \mathrm{~ms}$ and 3.3 to $50 \mathrm{~ms}$, respectively. Direct setting of levels at peak and background current is not possible with the welding power supply used in the present study. Hence, levels of peak and background voltage were adjusted by trial and error to obtain the desired peak and background current levels, which were measured using high speed, double-channel, digital storage oscilloscope. The peak duration, wire feed speed, and cycle time could be independently adjusted using the welding power supply control. 


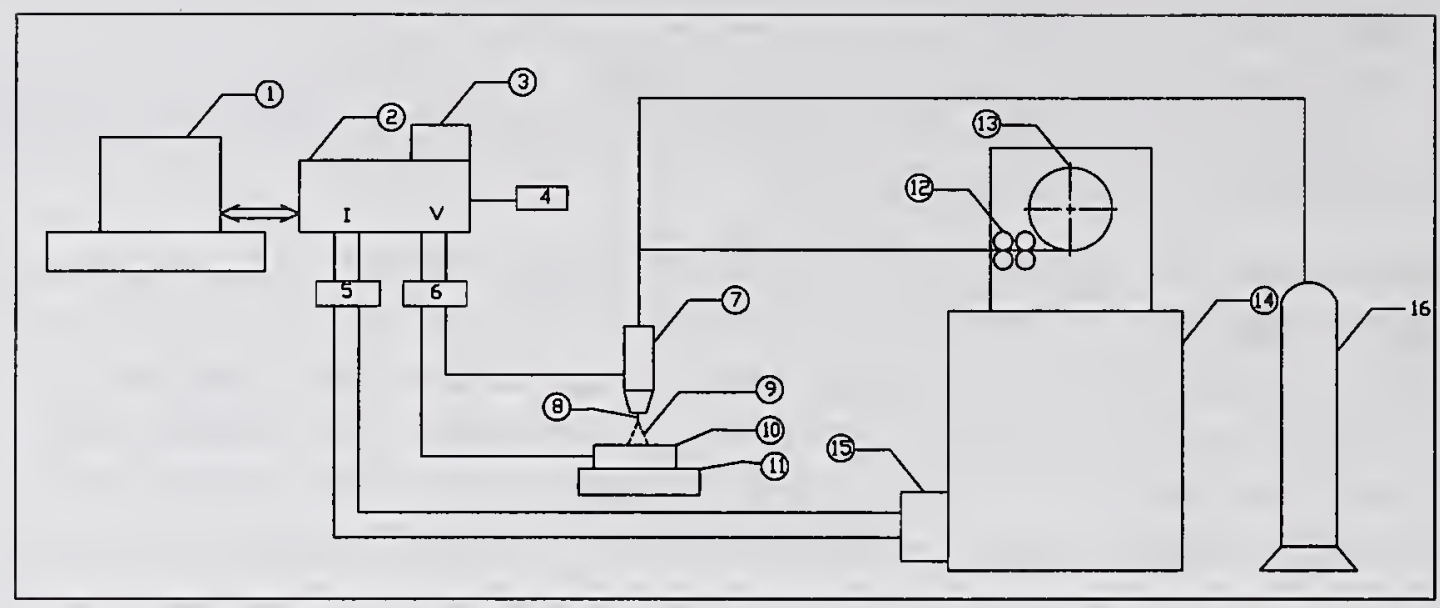

1. Personal computer

2. Double channel digital storage oscilloscope

3. Internal color digital plotter

4. Waveform processor

5. $5 \mathrm{kHz}$ low pass passive filter for current signal

6. $5 \mathrm{kHz}$ low pass passive filter for voltage signal

7. Torch

8. Filler metal
9. Electric arc

10. Base metal

11. Carriage

12. Filler metal feed rollers

13. Filler metal reel

14.Transistorized welding power supply

15. Control plug

16. Argon gas cylinder

Figure 2: The schematic of the pulsed current gas metal arc welding

\section{Low Pass Passive Filters}

The waveforms, which were captured by a high speed, double channel, digital storage oscilloscope (Gould 4072) were not clear. Moreover, carrying out any measurements on the waveform was difficult because of the poor appearance of the waveforms. Therefore the problem of high frequency noise and process noise in the signals of the voltage and current was discussed with the manufacturer and eliminated from the signals using two $5 \mathrm{kHz}$ low pass passive filters as shown in Fig. 2. Totally, two filters were designed and fabricated. The first one was used for the pulsed voltage signal and the second one for the pulsed current signal. These filters permit frequencies below $5 \mathrm{kHz}$ to pass but reject all other frequencies, which are above $5 \mathrm{kHz}$. Since the expected molten droplet frequency is well within $5 \mathrm{kHz}$, the droplet detachment phenomena could be clearly studied from the voltage spikes on the waveforms of pulsed voltage.

\section{Data Acquisition}

The pulsed current was sensed from the power supply and the pulsed voltage was sensed between the base metal and the contact tip. Sufficient time was allowed during welding to reach a stable welding condition. For this, the delay time was set in the oscilloscope and then instantaneous current and voltage were monitored and recorded simultaneously with a high speed, double channel, digital storage oscilloscope (Gould 4072) through $5 \mathrm{kHz}$ low-pass passive filters (Fig. 2).

Droplet detachment was observed from the voltage spikes on the waveform of the pulsed voltage. There were variations in the peak voltage values along the peak duration apart from those owing to the intentional pulsing. The reason for these variations is that when the droplet was transferred, the instantaneous arc voltage momentarily increased and formed voltage spikes 
on the peak voltage levels along the peak duration. Droplet detachment time and the number of droplet detachments were obtained from the pulsed voltage traces.

Stored waveforms of pulsed current and voltage were transferred from the oscilloscope to a personal computer and saved for later analysis. The personal computer was equipped with the General purpose Instrumentation Bus (GB-IB) for current and voltage data acquisition. Totally 1000 data samples were saved during each welding experiments. Analysis of the fluctuations of these values as a function of various pulsing parameters was useful to understand arc's behavior and this could be correlated to the various types of molten metal droplet detachments.

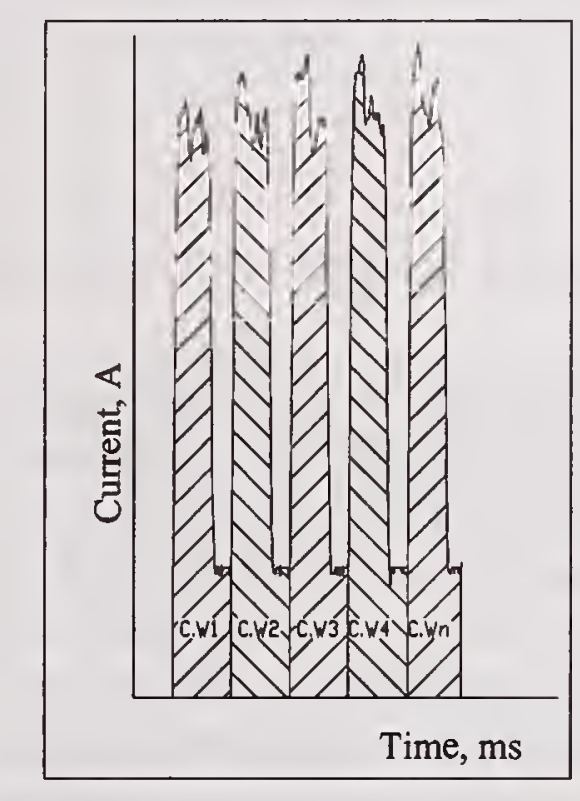

C. $W_{A V}=\left(C \cdot W_{1}+C . W_{2}+C \cdot W_{3} \ldots \ldots \ldots+C . W_{n}\right) / n$

Standard deviation, $\sigma=\sqrt{ }\left(\Sigma\left[\mathrm{C} . \mathrm{W}_{\mathrm{AV}}\right]^{2} / \mathrm{n}\right)-\left(\Sigma \mathrm{C} . \mathrm{W}_{\mathrm{AV}} / \mathrm{n}\right)^{2}$

Coefficient of variance, $\mathrm{C} . \mathrm{V}=\left(\sigma / \mathrm{C} . \mathrm{W}_{\mathrm{AV}}\right) \times 100$

Figure 3: Measurement of average current for each pulse cycle time, and calculation of the standard deviation and coefficient of variation.

\section{Pulsed Current Signal}

A typical pulsed current waveform is shown in Fig. 3. Area under every pulsed current waveform (A. ms) per cycle time was calculated using AutoCAD 2000 and a self-developed computer package EL-JS. The average current per cycle time was calculated by dividing area of waveform under one pulse by pulse cycle time. Statistical analysis was carried out on the basis of mean average current, standard deviation, and coefficient of variance, to determine the suitable pulse parameter.

The level of arc stability was assessed from the regularity of average current per cycle time. The standard deviation and coefficient of variation of average current were taken as the best measure of the regularity of filler metal melting, during welding operation. The pulsed current waveform with lesser standard deviation and coefficient of variation of average current was considered to be good from the standpoint of good arc stability. 


\section{Welding Procedure}

The flat position bead-on-plate welds were prepared under mechanized conditions at a fixed welding speed of $10 \mathrm{~mm} / \mathrm{s}$ and fixed wire speed of $100 \mathrm{~mm} / \mathrm{s}$. The welding torch was positioned normal to the base metal surface. Filler metal was connected to the direct-current-electrodepositive (DCEP) polarity. Prior to welding, the oxide film was removed from the base metal by mechanical means. The welding torch was mounted on four-wheeled variable speed tractor (Esab A2 Mini trac). It was moved above the base metal and welding was carried out (down hand type) in the rolling direction of base metal. The torch-to-work-angle and contact tip-to-work distance were maintained at $90^{\circ}$ and $15 \mathrm{~mm}$, respectively. The most suitable parameters of the pulsed current and the type of droplet detachment to obtain a good quality weld were selected on the basis of uniformity of average current.

\section{RESULTS AND DISCUSSION}

The improvement of arc stability in GMAW-P through optimization of the control parameters can extend the operational range of filler metal. This is because the level of peak current exceeds the level of spray current for axial droplet detachment, even at low average currents. The high peak current exerts a pinch effect on the molten droplet at the tip of the filler metal sufficient to propel a single droplet axially through the arc to the base metal. This pinch effect gives good control over droplet detachment during welding. The generation of excessive spatter mainly depends on whether the critical duration is too long or too short. The background current is used to just maintain the arc between each pulse without extinguishing.

\section{Optimum Waveform of Pulsed Current}

Experiments were conducted at fixed values of $W_{S}=10 \mathrm{~mm} / \mathrm{s}, W_{F}=100 \mathrm{~mm} / \mathrm{s}, I_{B}=20 \mathrm{~A}$, and $T_{B}$ $=6 \mathrm{~ms}$ to establish the relationship between $\mathrm{I}_{\mathrm{P}}$ and $\mathrm{T}_{\mathrm{P}}$ for different types of droplet detachments, namely, one droplet detachment during peak duration, one droplet detachment during background duration, two droplets detachment during peak duration, and three droplets detachment during peak duration, respectively. For this, levels of $I_{P}$ were kept above the spray current level, and $I_{P}$ and $T_{P}$ were allowed to vary. In order to obtain the above types of droplet detachments, pulse cycle time was allowed to vary. For this, it was not assumed in this study that molten droplet diameter would be equal to the diameter of weld metal.

Various types of droplet detachments were observed for different GMAW-P parameters by means of high speed, double channel, digital storage oscilloscope and classified them in terms of $I_{P}$ and $T_{P}$. Such a result is shown in Fig. 4.

\section{Zone 'A'}

In the zone ' $\mathrm{A}$ ' a large droplet is formed and arc behavior becomes unstable accompanied by short circuits (Fig. 5), erratic droplet detachment, and much spatter, because the electromagnetic force applied to the molten filler metal tip is not directed to the base metal. Note that in this zone the value of $\mathrm{I}_{\mathrm{P}}$ is smaller than $168 \mathrm{~A}$, which is $18 \mathrm{~A}$ higher than spray current level of $150 \mathrm{~A}$ with $1.2 \mathrm{~mm}$ diameter ER $5356 \mathrm{Al}-\mathrm{Mg}$ filler metal used in this research work. It shows that higher currents are necessary to realize the spray type of molten droplet detachment. The pendant 
droplet is stationary at the end of the background period; therefore the additional current is required to overcome the drop inertia. But in the steady D.C. GMAW process the pendant droplet is in constant streaming motion once it is initiated.

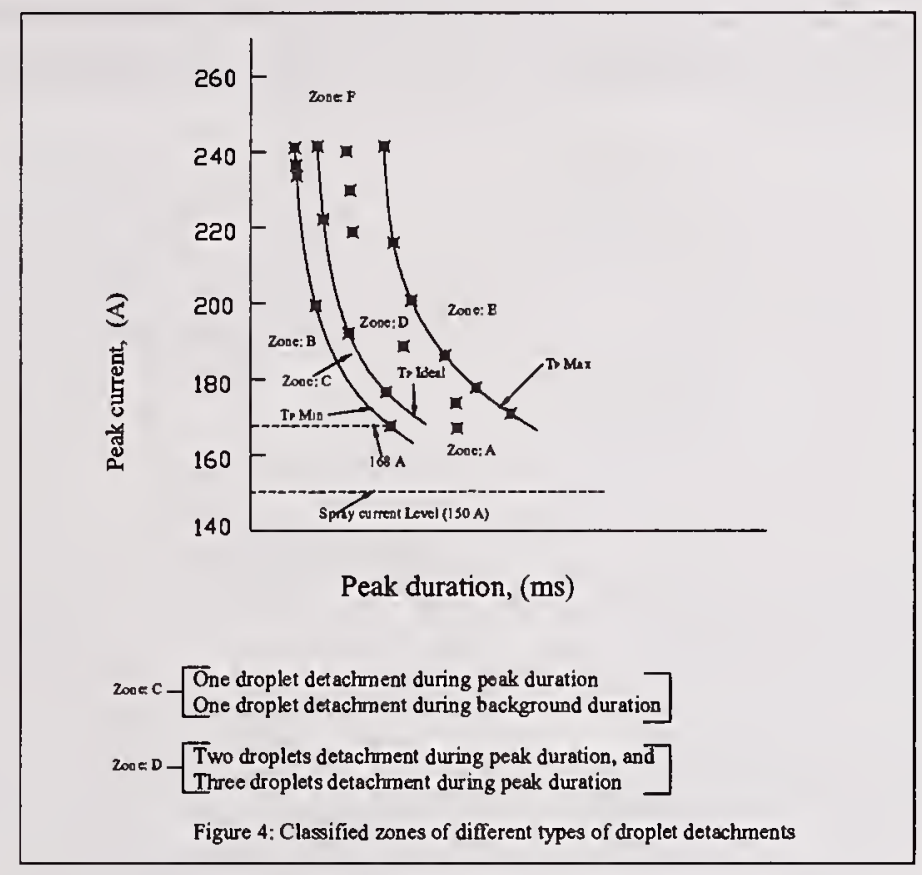

\section{Zone 'B'}

In the zone ' $B$ ', where $I_{P}>168 A$ and $T_{P}<T_{P}$ min, the peak energy is too small to make a droplet detachment in one pulse and the pendant droplet swells into a large globe during several pulses. Therefore this zone is undesirable.

\section{Zone 'C'}

One droplet with a diameter close to that of the filler metal detaches regularly and reliably per every pulse cycle time either during background period (Fig.6) or during peak duration (Fig.7) in the single droplet detachment zone ' $C$ ' where $I_{P}>168 A$ and $T_{P} \min \leqq T_{P} \leqq T_{P}$ ideal. The waveforms with less variations in the average current for the one droplet detachment during background duration and for the one droplet detachment during peak duration are shown in Fig. 8 and Fig. 9, respectively.

\section{Zone 'D'}

The droplet detachment was apparently the spray type in the zone ' $D$ '. Where $I_{P}>168 \mathrm{~A}$ and $T_{P}>$ $\mathrm{T}_{\mathrm{P}}$ max. In this multiple droplet detachment zone, either two droplets (Fig. 10) or three droplets (Fig. 11) were detached during peak duration, depending upon the combination of GMAW-P within this zone ' $\mathrm{D}$ '.

There was a considerable spatter during experiments with multiple droplet detachments. This is because when the peak duration is too long, beyond the optimum value, the molten filler metal tip is prolonged. This resulted in much spatter due to contact with the base metal, and the subsequent explosion of droplets. The peak energy was also high because of the application of large peak duration in multiple detachments as shown in Table 1. As can be seen from the Table1, one droplet detaches during background period for average peak energy of $18.5 \mathrm{~J}$, and 
one droplet detaches during peak duration for average peak energy of $21.2 \mathrm{~J}$. At the same time, average peak energy of $28.7 \mathrm{~J}$ was required to obtain two droplets detachment during peak, and average peak energy of $30.7 \mathrm{~J}$ was required in the case of three droplets detachment during peak. Fig. 12 shows a typical pulsed current waveform with less variation in the mean average current for the two droplets detachment during peak duration. The pulsed current waveform with less variation in the mean average current for the three droplets detachment during peak duration is presented in Fig. 13.

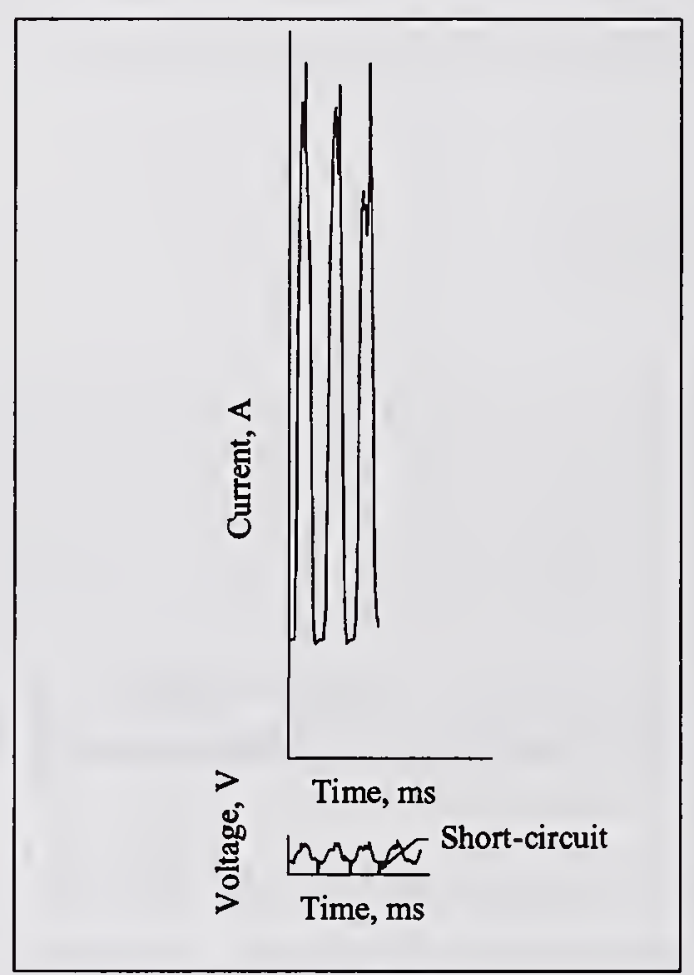

$\mathrm{I}_{\mathrm{P}}=160 \mathrm{~A} ; \mathrm{T}_{\mathrm{P}}=5 \mathrm{~ms} ; \mathrm{I}_{\mathrm{B}}=20 \mathrm{~A} ; \mathrm{T}_{\mathrm{B}}=11 \mathrm{~ms} ;$

$\mathrm{V}_{\mathrm{P}}=20.8 \mathrm{~V} ; \mathrm{V}_{\mathrm{B}}=13.8 \mathrm{~V}$

Figure 5: Typical pulsed voltage and current waveform with short-circuit

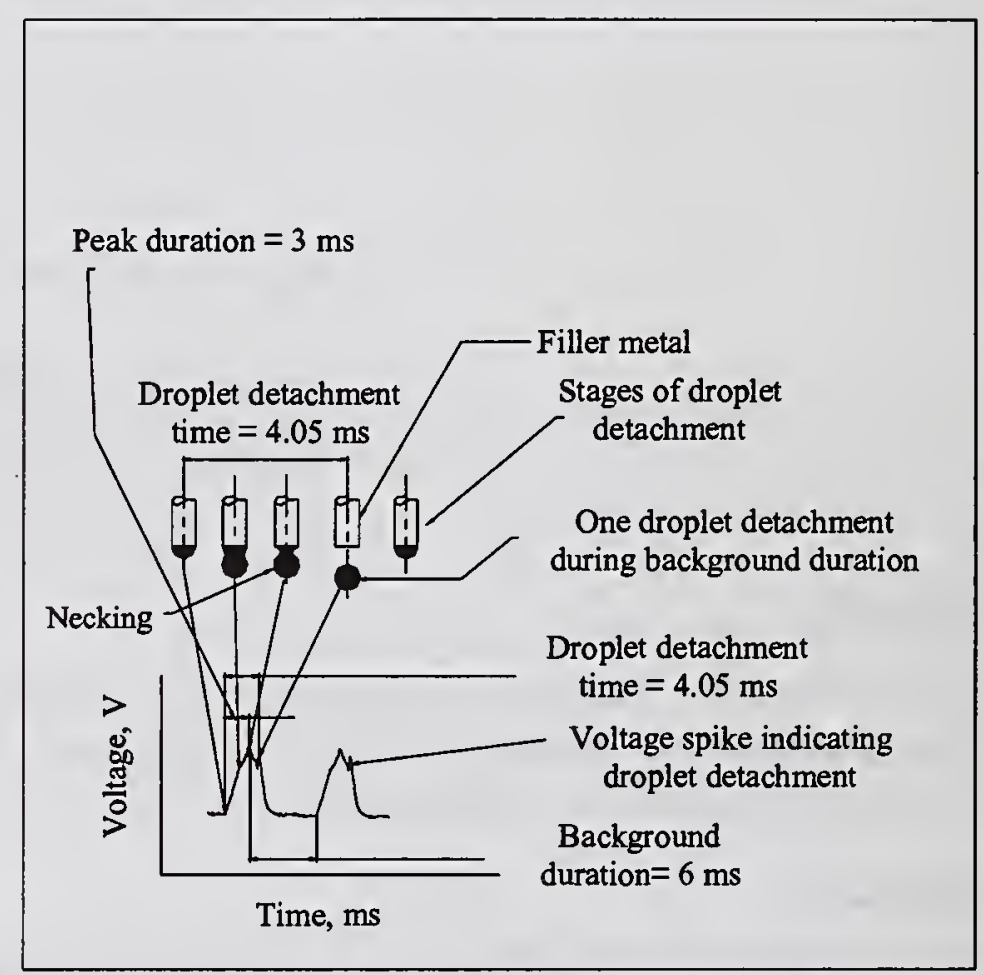

$I_{P}=260.8 \mathrm{~A} ; T_{P}=3 \mathrm{~ms} ; I_{B}=20 \mathrm{~A} ; T_{B}=6 \mathrm{~ms} ; T_{D}=4.05 \mathrm{~ms} ;$
$V_{P}=25.32 \mathrm{~V} ; V_{B}=15.98 \mathrm{~V} ; I_{A V}=108.4 \mathrm{~A} ; V_{A V}=19.08 \mathrm{~V} ;$
$W_{F}=100 \mathrm{~mm} / \mathrm{s} ;$ and $W_{S}=10.0 \mathrm{~mm} / \mathrm{s}$

Figure 6: Voltage signal showing one droplet detachment during background duration

\section{Zone 'E'}

The droplet detachment was apparently the spray type in the zone 'E', where $I_{P}>168 A$ and $T_{P}>T_{P}$ max. But, the molten filler metal tip was prolonged and touches the base metal causing much spatter. Moreover, as $T_{P}$ increases in this zone ' $E$ ', frequency of droplet detachment decreases at a constant feeding rate of filler metal, resulting in continual increased fluctuations of the arc length.

\section{Zone 'F'}

In the zone ' $F$ ', arc breaks because of the excessive arc length. Therefore, this zone is highly undesirable. 


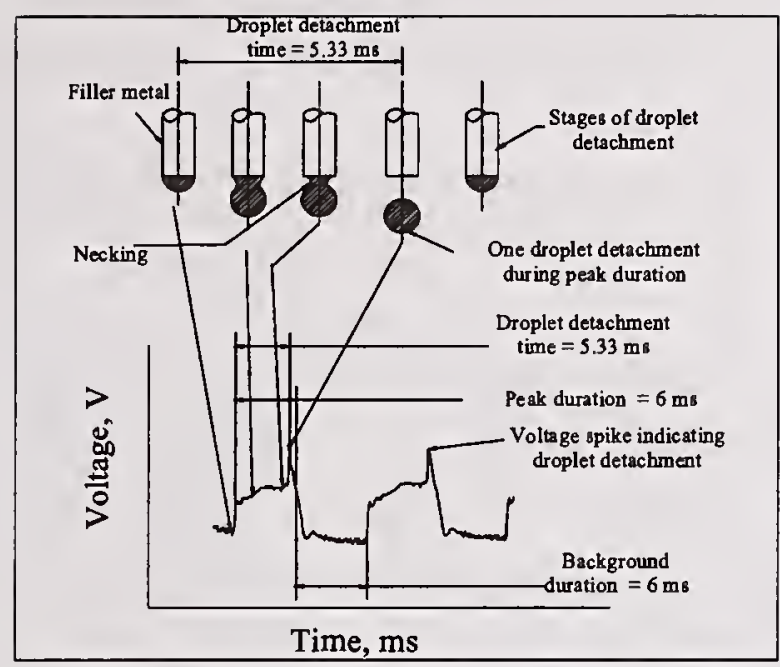

$$
\begin{aligned}
& I_{P}=176.5 \mathrm{~A} ; T_{P}=6 \mathrm{~ms} ; I_{B}=20 \mathrm{~A} ; T_{B}=6 \mathrm{~ms} ; T_{D}=5.33 \mathrm{~ms} ; V_{P}=23.3 \mathrm{~V} ; V_{B}=16.6 \mathrm{~V} \\
& I_{A V}=95.1 \mathrm{~A} ; V_{A V}=19.6 \mathrm{~V} ; W_{F}=100 \mathrm{~mm} / \mathrm{s} ; \text { and } W_{S}=10.0 \mathrm{~mm} / \mathrm{s}(\text { Weld no. EL-3) }
\end{aligned}
$$

Figure 7: Voltage signal showing one droplet detachment during peak duration

\section{Selection of Suitable GMAW-P Parameter and Type of Droplet Detachment}

Average pulse current has been one of the criteria considered for selection of the most suitable pulse parameter combinations in order to obtain good quality weld. Also, on the basis of the above criterion, a suitable type of droplet detachment was recommended from among the four different types of droplet detachments, namely, one droplet detachment during peak duration, one droplet detachment during background duration, two droplets detachment during peak duration, and three droplets detachment during peak duration.

The standard deviation and coefficient of variation of the average current were calculated (Table 1) for four types droplet detachments, namely, one droplet detachment during peak duration (Region-1), one droplet detachment during background duration (Region-2), two droplets detachment during peak duration (Region-3), and three droplets detachment during peak duration (Region-4) and represented in Figs. 14 and 15, respectively. Different types of droplet detachments were obtained at various pulsing conditions and grouped in Region-1, Region-2, Region-3, and Region-4.

Good correlation was obtained between the standard deviation of average current and peak energy, and between the coefficient of variation of the average current and peak energy. Region1 consists of the lowest range of standard deviation among all four regions. This shows good regularity of average current in Region-1, during filler metal melting. Region- 4 consists of the highest range of standard deviation among all four regions. Region-2 and Region-3 are in between Region-1 and Region-4 as shown in Figs. 14 and 15.

The lowest standard deviation and the lowest coefficient of variation for the average current were observed for the welds EL-1, EL-8, EL-10, EL-18, which were obtained at different pulsing conditions, namely, one droplet detachment during peak duration, one droplet detachment during background duration, two droplets detachment during peak duration, and three droplets 
detachment during peak duration, respectively. Among these four types of droplet detachments, the lowest standard deviation $(0.9228)$ and the lowest coefficient of variation $(0.8361)$ were found for the weld no. EL-1, which was made with one droplet detachment during peak duration.

\begin{tabular}{|l|l|l|l|l|l|l|l|l|l|l|}
\hline $\begin{array}{l}\text { Weld } \\
\text { No. }\end{array}$ & $\mathrm{I}_{\mathrm{P}}$ & $\mathrm{T}_{\mathrm{P}}$ & $\mathrm{V}_{\mathrm{P}}$ & $\mathrm{T}$ & $\mathrm{T}_{\mathrm{D}}{ }^{*}$ & $\begin{array}{l}\text { Deta } \\
\text {-ch } \\
\text { ment } \\
\text { Type }\end{array}$ & $\begin{array}{l}\text { Peak } \\
\text { Energy } \\
(\mathrm{J})\end{array}$ & $\mathrm{C} . \mathrm{W}_{\mathrm{AV}}$ & $\mathrm{C} . \mathrm{W}_{\mathrm{S} . \mathrm{D}}$ & $\mathrm{C} . \mathrm{W}_{\mathrm{C} . \mathrm{V}}$ \\
\hline EL-1 & 196.8 & 5 & 22.9 & 11.0 & 5.00 & $\mathrm{~A}$ & 22.53 & 110.3718 & $\mathbf{0 . 9 2 2 8}$ & $\mathbf{0 . 8 3 6 1}$ \\
\hline EL-2 & 181.8 & 6 & 23.1 & 12.0 & 5.14 & $\mathrm{~A}$ & 25.20 & 103.5011 & 0.9597 & 0.9272 \\
\hline EL-3 & 176.5 & 6 & 23.3 & 12.0 & 5.33 & $\mathrm{~A}$ & 24.67 & 95.0075 & 1.2426 & 1.3079 \\
\hline EL-4 & 231.9 & 3 & 24.8 & 9.0 & 2.85 & $\mathrm{~A}$ & 17.25 & 108.9990 & 1.1516 & 1.0565 \\
\hline EL-5 & 240.5 & 3 & 23.2 & 9.0 & 4.38 & $\mathrm{~B}$ & 16.74 & 109.9400 & 1.2834 & 1.1674 \\
\hline EL-6 & 211.5 & 4 & 23.1 & 10.0 & 5.90 & $\mathrm{~B}$ & 19.54 & 110.3000 & 1.3218 & 1.1984 \\
\hline EL-7 & 244.2 & 3.5 & 22.1 & 9.5 & 4.20 & $\mathrm{~B}$ & 18.89 & 120.2500 & 1.2627 & 1.0499 \\
\hline EL-8 & 229.0 & 4 & 22.1 & 10.0 & 4.38 & $\mathrm{~B}$ & 20.24 & 120.4600 & $\mathbf{1 . 2 5 0 4}$ & $\mathbf{1 . 0 3 8 0}$ \\
\hline EL-9 & 243.6 & 3.5 & 22.0 & 9.5 & 4.20 & $\mathrm{~B}$ & 18.76 & 122.2900 & 1.3006 & 1.0635 \\
\hline EL-10 & 217.5 & 4 & 23.1 & 10 & 3.60 & $\mathrm{C}$ & 20.10 & 109.6437 & $\mathbf{1 . 4 2 2 7}$ & 1.2976 \\
\hline EL-11 & 226.0 & 4 & 23.6 & 10 & 3.72 & $\mathrm{C}$ & 21.33 & 112.8266 & 1.5072 & 1.3359 \\
\hline EL-12 & 224.0 & 5 & 23.1 & 11 & 3.54 & $\mathrm{C}$ & 25.87 & 118.2110 & 1.5921 & 1.3468 \\
\hline EL-13 & 180.5 & 6 & 22.8 & 12 & 4.71 & $\mathrm{C}$ & 24.69 & 103.3983 & 1.5579 & 1.5067 \\
\hline EL-14 & 187.1 & 8 & 24.9 & 14 & 3.87 & $\mathrm{C}$ & 37.27 & 112.7396 & 1.7526 & 1.5546 \\
\hline EL-15 & 185.6 & 7 & 23.0 & 13 & 3.86 & $\mathrm{D}$ & 29.88 & 111.7484 & 2.1722 & 1.9439 \\
\hline EL-16 & 192.9 & 8 & 23.9 & 14 & 3.74 & $\mathrm{D}$ & 36.88 & 120.6306 & 2.0297 & 1.6826 \\
\hline EL-17 & 180.2 & 9 & 23.6 & 15 & 4.02 & $\mathrm{D}$ & 38.27 & 118.0505 & 2.0210 & 1.7120 \\
\hline EL-18 & 168.3 & 9 & 22.6 & 15 & 4.42 & $\mathrm{D}$ & 34.23 & 114.1309 & $\mathbf{1 . 9 0 6 6}$ & $\mathbf{1 . 6 7 0 6}$ \\
\hline EL-19 & 177.1 & 9 & 23.8 & 15 & 4.51 & $\mathrm{D}$ & 37.93 & 115.6615 & 2.0776 & 1.7963 \\
\hline EL-20 & 172.7 & 10 & 23.6 & 16 & 4.8 & $\mathrm{D}$ & 40.76 & 111.3447 & 2.3147 & 2.0789 \\
\hline
\end{tabular}

I $\quad$ Peak current

$\mathrm{T}_{\mathrm{P}} \quad$ Peak duration

$\mathrm{V}_{\mathrm{P}} \quad$ Peak voltage

T Pulse cycle time

$\mathrm{T}_{\mathrm{D}} \quad$ Droplet detachment time

* For multiple droplet detachments, $\mathrm{T}_{\mathrm{D}}=$ Time between the beginning of the pulse and the first droplet detachment
A: One droplet detachment during peak duration

B: One droplet detachment during background duration

C: Two droplets detachment during peak duration, and

D: Three droplets detachment during peak duration

Peak Energy $=I_{P} \times T_{P} \times V_{P}(J)$

C. $W_{\text {A.V }}$ Mean value of average current

C.W S.D Standard deviation of average current

C.W C.V Coefficient of variation of average current

Table 1. Type of droplet detachment, and statistical analysis of average current 


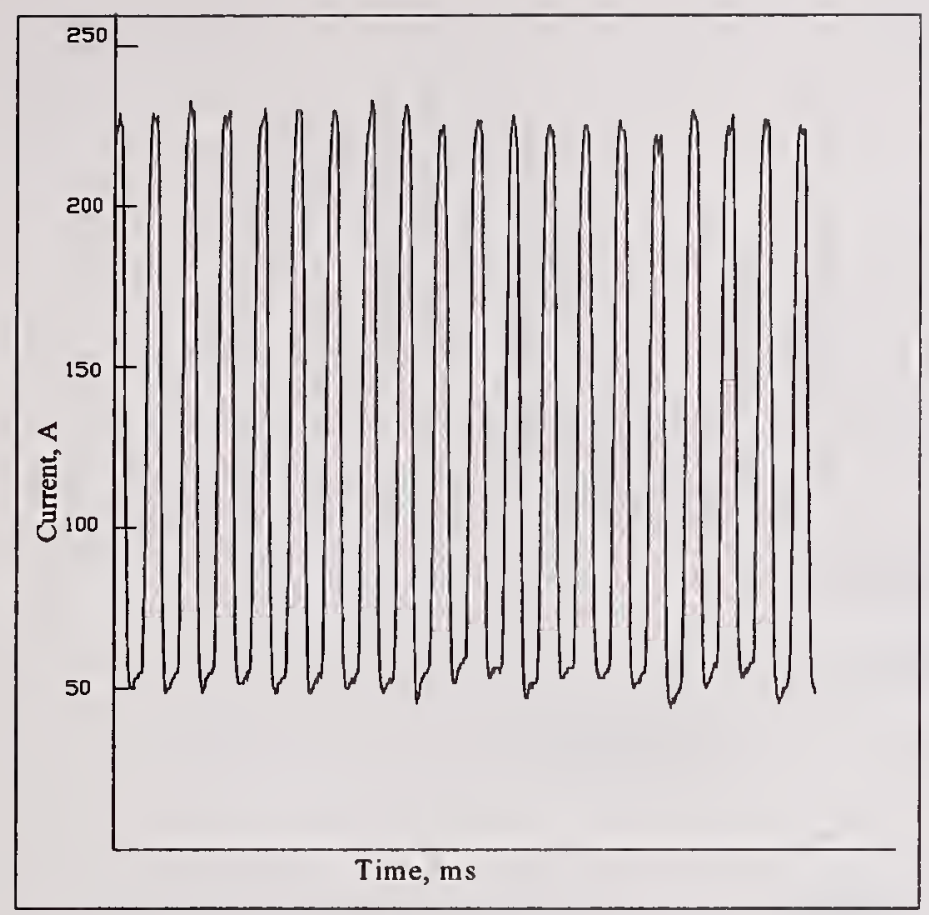

$I_{P}=229.0 \mathrm{~A} ; T_{P}=4 \mathrm{~ms} ; I_{B}=20 A ; T_{B}=6 \mathrm{~ms}$;

$\mathrm{T}_{\mathrm{D}}=4.38 \mathrm{~ms} ; \mathrm{V}_{\mathrm{P}}=22.1 \mathrm{~V} ; \mathrm{V}_{\mathrm{B}}=14.8 \mathrm{~V}$;

$\mathrm{I}_{\mathrm{AV}}=120.46 \mathrm{~A} ; \mathrm{V}_{\mathrm{AV}}=17.4 \mathrm{~V} ; \mathrm{W}_{\mathrm{F}}=100 \mathrm{~mm} / \mathrm{s}$;

$\mathrm{W}_{\mathrm{S}}=10.0 \mathrm{~mm} / \mathrm{s} ; \mathrm{C} . \mathrm{W}_{\mathrm{AV}}=120.46 \mathrm{~A}$;

C. $W_{\text {S.D }}=1.2504 \mathrm{~A}$; and C. $\mathrm{W}_{\mathrm{C} . \mathrm{V}}=1.0380$.

Figure 8: The waveform with less variation in the average current for the one droplet detachment during background duration (Weld no. EL-8).

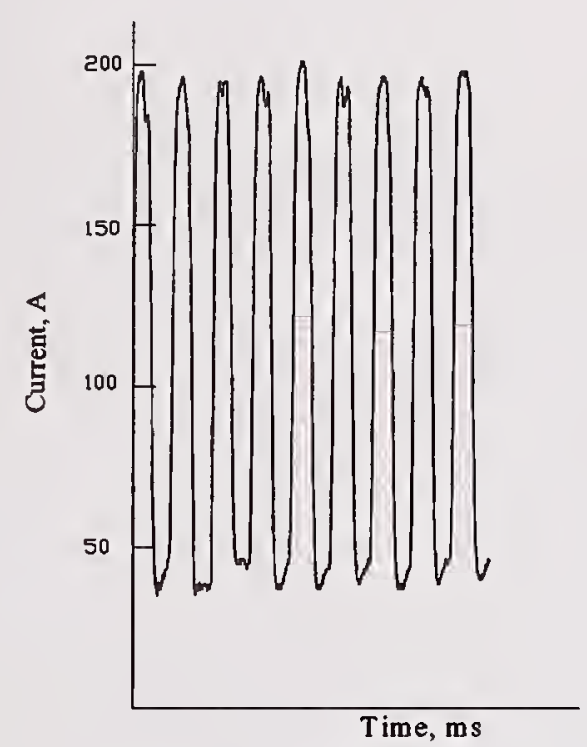

$\mathrm{I}_{\mathrm{P}}=196.8 \mathrm{~A} ; \mathrm{T}_{\mathrm{P}}=5 \mathrm{~ms} ; \mathrm{I}_{\mathrm{B}}=20 \mathrm{~A} ; \mathrm{T}_{\mathrm{B}}=6 \mathrm{~ms}$;

$\mathrm{T}_{\mathrm{D}}=5 \mathrm{~ms} ; \mathrm{V}_{\mathrm{P}}=22.9 \mathrm{~V} ; \mathrm{V}_{\mathrm{B}}=15.03 \mathrm{~V}$;

$\mathrm{I}_{\mathrm{AV}}=110.3718 \mathrm{~A} ; \mathrm{V}_{\mathrm{AV}}=18.1 \mathrm{~V} ; \mathrm{W}_{\mathrm{F}}=100 \mathrm{~mm} / \mathrm{s}$;

$\mathrm{W}_{\mathrm{S}}=10.0 \mathrm{~mm} / \mathrm{s} ; \mathrm{C} . \mathrm{W}_{\mathrm{AV}}=110.3718 \mathrm{~A}$;

C. $W_{\text {S.D }}=0.9228 \mathrm{~A}$; and C. $\mathrm{W}_{\mathrm{C} . \mathrm{V}}=0.8361$

Figure 9: The waveform with less variation in the average current for the one droplet detachment during peak duration (Weld no. EL-1)

The smaller the standard deviation and the smaller the coefficient of variation, the more uniform the average current is. That means this type of droplet detachment provides a droplet from the filler metal tip regularly during peak duration in order to produce spatter free, high quality, and stable weld. Hence, one droplet detachment during peak duration was considered to be the best for providing a more uniform average current than the other types of droplet detachments. Based on this, the following parameters of the pulsed current were found to be more suitable than the other combinations of pulsed current: $\mathrm{I}_{\mathrm{P}}=196.8 \mathrm{~A} ; \mathrm{T}_{\mathrm{P}}=5.0 \mathrm{~ms} ; \mathrm{I}_{\mathrm{B}}=20 \mathrm{~A} ; \mathrm{T}_{\mathrm{B}}=6 \mathrm{~ms} ; \mathrm{W}_{\mathrm{F}}=100$ $\mathrm{mm} / \mathrm{s} ; \mathrm{I}_{\mathrm{AV}}=110.3718 \mathrm{~A}$; and $\mathrm{W}_{\mathrm{S}}=10.0 \mathrm{~mm} / \mathrm{s}$.

Therefore, only the GMAW-P combinations within the optimum zone ' $\mathrm{C}$ ' (Fig. 4), which provides one droplet detachment during peak duration, are suitable for carrying out good quality weld. The above parameter combination can be programmed to the computer so that whenever operator wants to use the wire feed rate, $W_{F}=100 \mathrm{~mm} / \mathrm{s}$, automatically this particular pulsed waveform is selected, and the operator gets good quality weld. Thus process-integrated quality assurance of the weld can be possible on the basis of more uniform average current. This above experimental work can be suitably extended to the other wire feed rates in order to achieve stable weld.

Furthermore, the standard deviation (1.2504) and coefficient of variation (1.0380) of the weld no. EL-8, which was welded with one droplet detachment during background duration, was found to be the second best in terms of the uniform average current. 


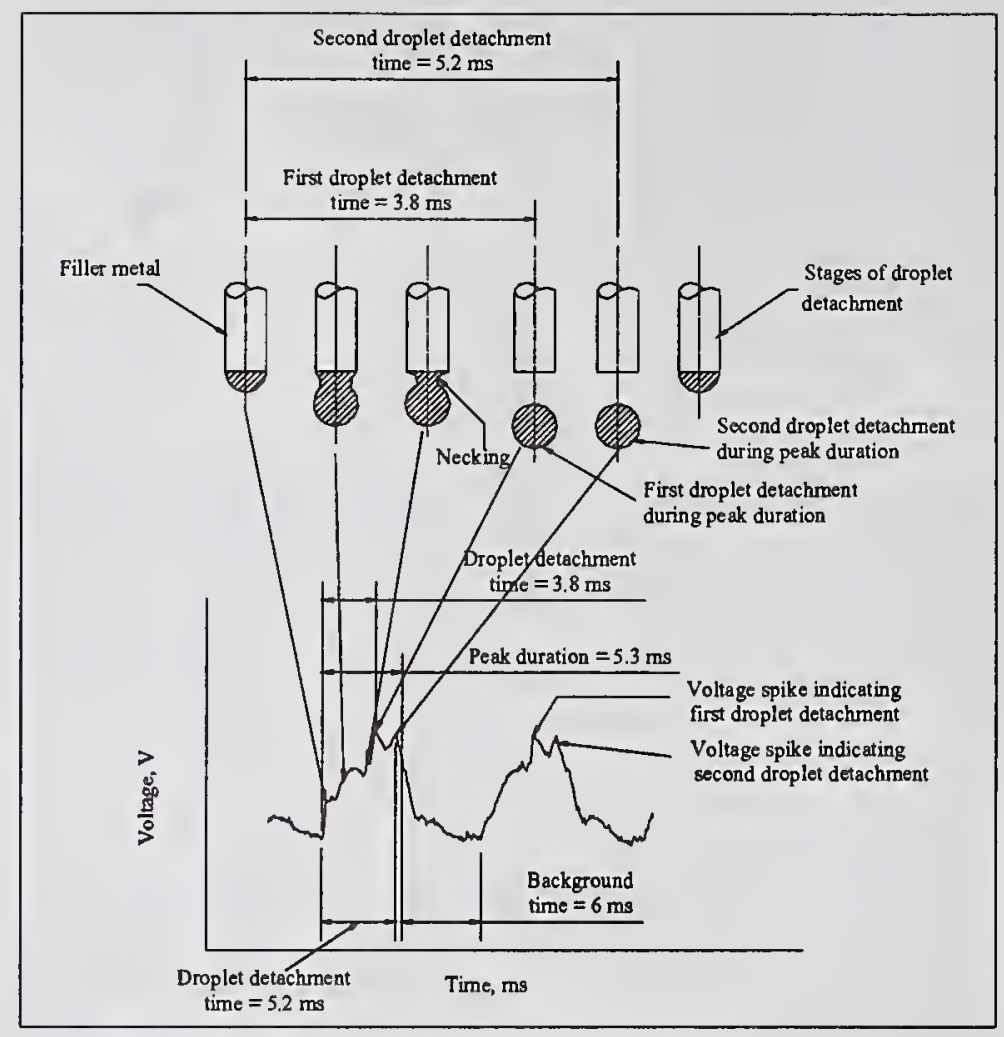

$\mathrm{I}_{\mathrm{P}}=230.3 \mathrm{~A} ; \mathrm{T}_{\mathrm{P}}=5 \mathrm{~ms} ; \mathrm{I}_{\mathrm{B}}=20 \mathrm{~A} ; \mathrm{T}_{\mathrm{B}}=6 \mathrm{~ms} ; \mathrm{T}_{\mathrm{D} 1}=3.8 \mathrm{~ms} ; \mathrm{T}_{\mathrm{D} 2}=5.2 \mathrm{~ms} ; \mathrm{V}_{\mathrm{P}}=23.02 \mathrm{~V} ;$ $\mathrm{V}_{\mathrm{B}}=15.2 \mathrm{~V} ; \mathrm{I}_{\mathrm{AV}}=109.4 \mathrm{~A} ; \mathrm{V}_{\mathrm{AV}}=18.3 \mathrm{~V} ; \mathrm{W}_{\mathrm{F}}=100 \mathrm{~mm} / \mathrm{s} ;$ and $\mathrm{W}_{\mathrm{S}}=10.0 \mathrm{~mm} / \mathrm{s}$

Figure 10: Voltage signal showing two droplets detachment during peak duration.

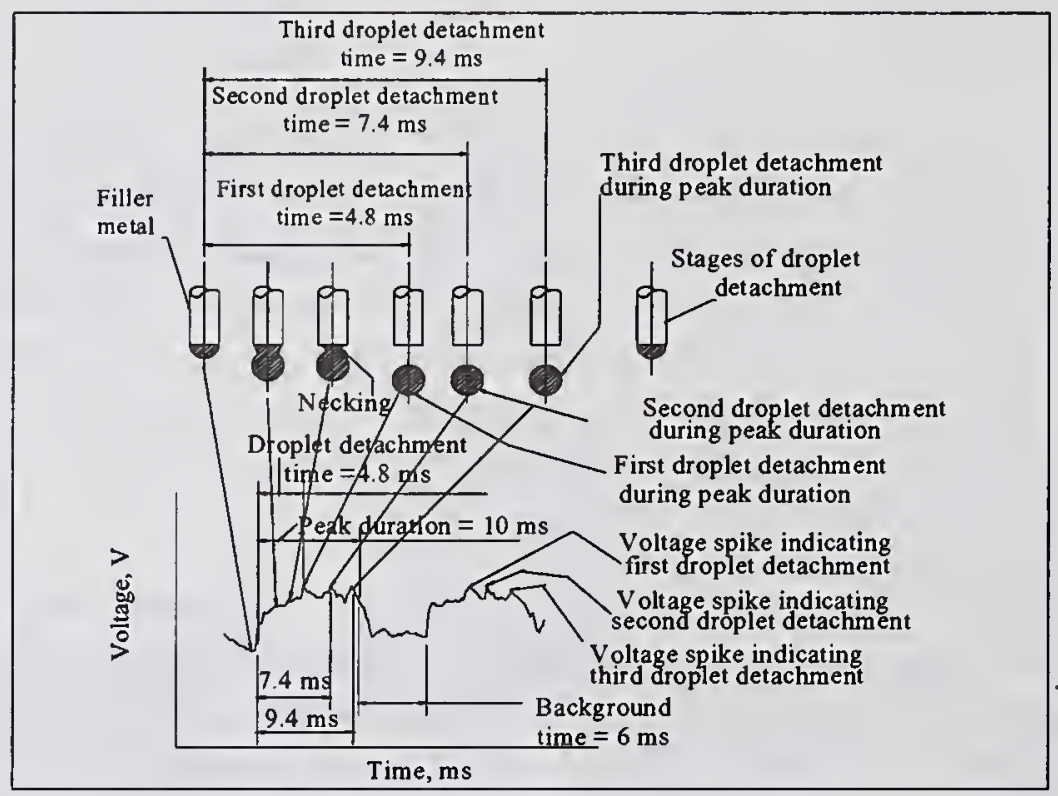

$I_{P}=172.7 \mathrm{~A} ; \mathrm{T}_{\mathrm{P}}=10 \mathrm{~ms} ; \mathrm{I}_{\mathrm{B}}=20 \mathrm{~A} ; \mathrm{T}_{\mathrm{B}}=6 \mathrm{~ms} ; \mathrm{T}_{\mathrm{D} 1}=4.8 \mathrm{~ms} ; \mathrm{T}_{\mathrm{D} 2}=7.4 \mathrm{~ms} ; \mathrm{T}_{\mathrm{D} 3}=9.4 \mathrm{~ms} ;$ $\mathrm{V}_{\mathrm{P}}=23.6 \mathrm{~V} ; \mathrm{V}_{\mathrm{B}}=16.5 \mathrm{~V} ; \mathrm{I}_{\mathrm{AV}}=111.3 \mathrm{~A} ; \mathrm{V}_{\mathrm{AV}}=20.2 \mathrm{~V} ; \mathrm{W}_{\mathrm{F}}=100 \mathrm{~mm} / \mathrm{s} ;$ and $\mathrm{W}_{\mathrm{S}}=10.0 \mathrm{~mm} / \mathrm{s}$.

Figure 11: Voltage signal showing three droplets detachment during peak duration. 


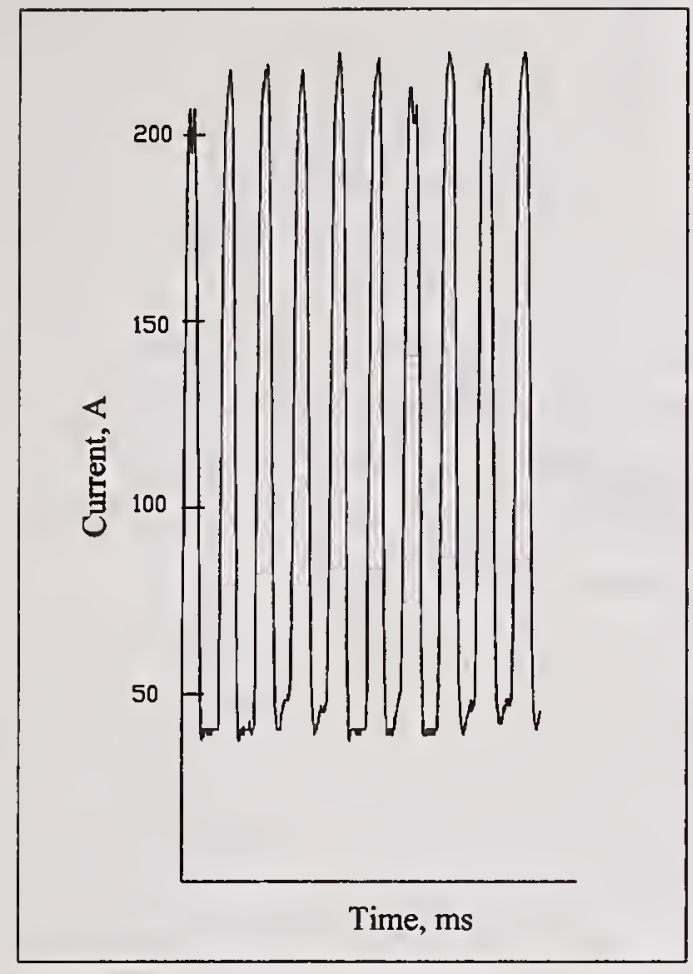

$I_{P}=217.5 \mathrm{~A} ; T_{P}=4 \mathrm{~ms} ; I_{B}=20 \mathrm{~A} ; T_{B}=6 \mathrm{~ms} ;$

$\mathrm{T}_{\mathrm{D}}=5 \mathrm{~ms} ; \mathrm{V}_{\mathrm{P}}=23.1 \mathrm{~V} ; \mathrm{V}_{\mathrm{B}}=14.7 \mathrm{~V}$;

$\mathrm{I}_{\mathrm{AV}}=109.6437 \mathrm{~A} ; \mathrm{V}_{\mathrm{AV}}=17.98 \mathrm{~V}$;

$W_{\mathrm{F}}=100 \mathrm{~mm} / \mathrm{s} ; W_{\mathrm{S}}=10.0 \mathrm{~mm} / \mathrm{s}$;

C. $\mathrm{W}_{\mathrm{AV}}=109.6437 \mathrm{~A} ; \mathrm{C} . \mathrm{W}_{\mathrm{S} . \mathrm{D}}=1.4227 \mathrm{~A}$;

and C. $\mathrm{W}_{\mathrm{C} . \mathrm{V}}=1.2976$.

Figure 12: The waveform with less variation in the average current for the two droplets detachment during peak duration (Weld no. EL-10).

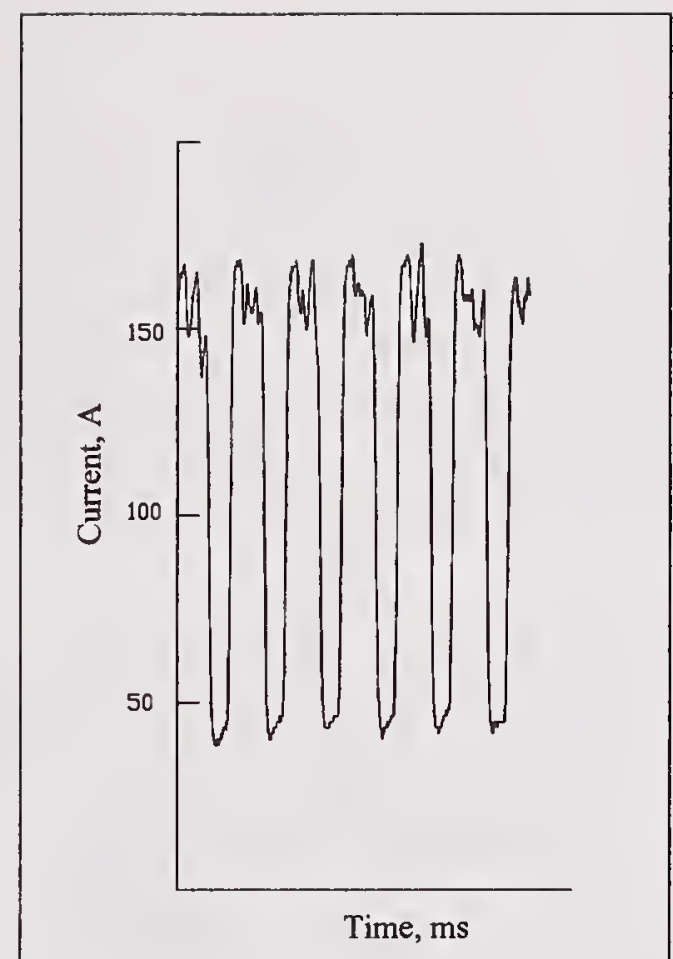

$I_{P}=168.3 \mathrm{~A} ; T_{P}=9 \mathrm{~ms} ; I_{B}=20 \mathrm{~A} ; T_{B}=6 \mathrm{~ms}$;

$\mathrm{T}_{\mathrm{D}}=4.42 \mathrm{~ms} ; \mathrm{V}_{\mathrm{P}}=22.6 \mathrm{~V} ; \mathrm{V}_{\mathrm{B}}=15.32 \mathrm{~V}$;

$\mathrm{I}_{\mathrm{AV}}=114.1309 \mathrm{~A} ; \mathrm{V}_{\mathrm{AV}}=18.77 \mathrm{~V}$;

$\mathrm{W}_{\mathrm{F}}=100 \mathrm{~mm} / \mathrm{s} ; \mathrm{W}_{\mathrm{S}}=10.0 \mathrm{~mm} / \mathrm{s}$;

C. $W_{\text {AV }}=114.1309$ A; C. $W_{\text {S.D }}=1.9066 \mathrm{~A}$;

and C. $\mathrm{W}_{\mathrm{C} . \mathrm{V}}=1.6706$

Figure 13: The waveform with less variation in the average current for the three droplets detachment during peak duration (Weld no. EL-18).

\section{SUMMARY}

The automotive industry is interested to furthering the use of Al-Mg alloys in the production of new breed lightweight cars. The pulsed current gas metal arc welding (GMAW-P) is widely used to weld these alloys. However, quality of the weld mainly depends upon the parameters of the pulsed current. Selecting the most suitable GMAW-P parameter is very complex due to interdependence of parameters. Therefore, in this research paper, an optimum GMAW-P parameter was selected on the basis of process-integrated quality assurance. Because, processintegrated quality assurance enables online monitoring of welding voltage, welding current as well as the reproduction of welding data and complete documentation of all parameters and welding machine adjustments. 

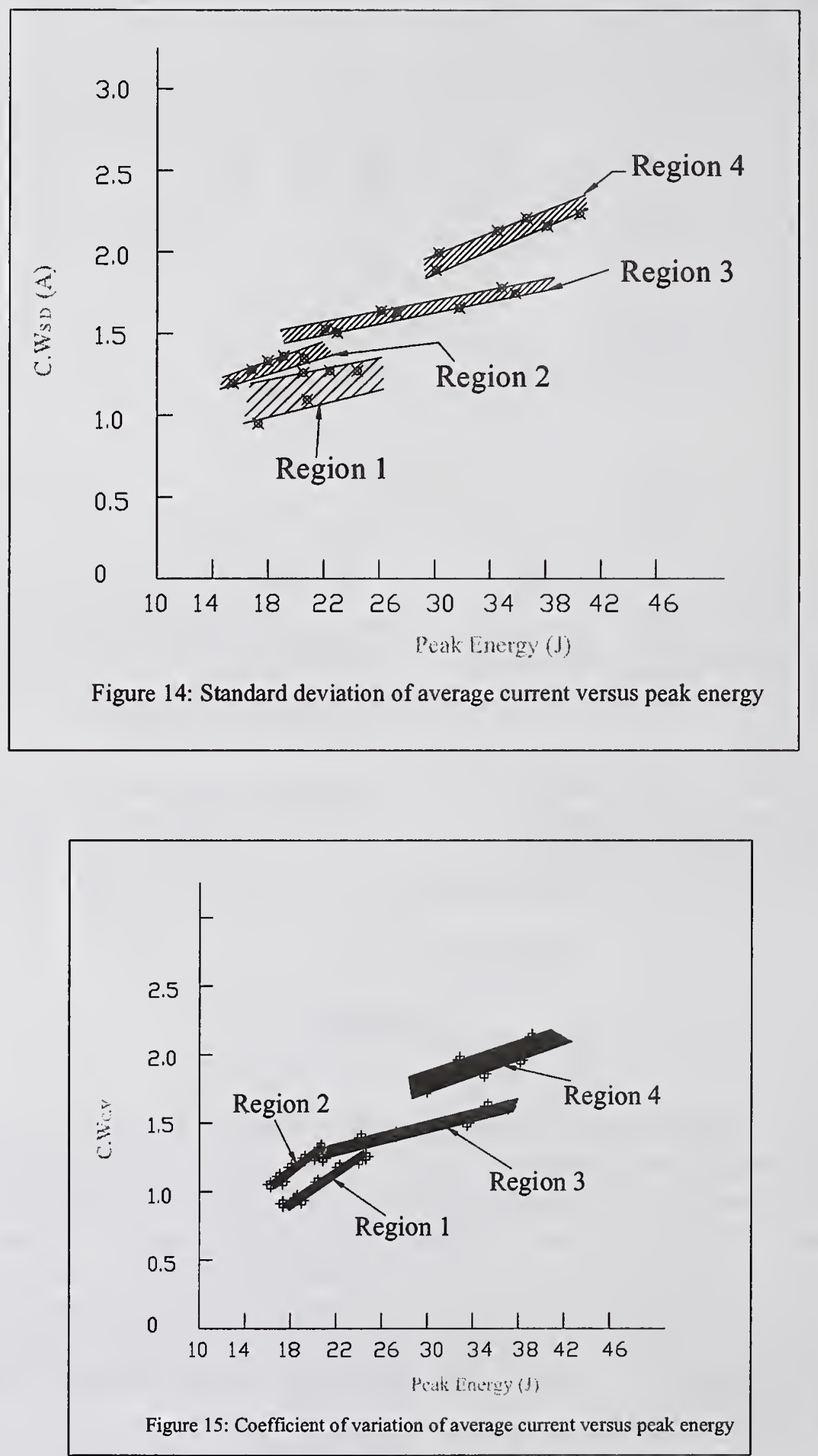
For this, first, the effects of GMAW-P parameters on the various types of droplet detachments were studied and classified in different zones on the basis of type of droplet detachment. Secondly, the average current per pulse cycle time was measured from the each waveform for the various types of droplet detachments and the values of mean, standard deviation, and coefficient of variation were calculated. The optimum GMAW-P was selected by choosing the pulse parameter which resulted in the least standard deviation and the coefficient of variation, to produce cost-effective, spatter free, high quality welds. Among four types of droplet detachments studied, one droplet detachment during peak duration was considered to be the best for providing a more uniform average current than the other types of droplet detachments. Thus processintegrated quality assurance of the weld was possible on the basis of more uniform average current.

Statistical analysis of fluctuations in average current per pulse cycle time using a personal computer was carried out for the quantitative assessment of welding arc stability. The computer analysis represents a powerful means of measuring the degree of arc stability and filler metal melting uniformity. This technique facilitates research into process-integrated quality assurance and to improve the design of GMAW-P equipment to ensure that preferred welding parameters are used and to research the cause of variable performance of GMAW-P equipment in actual practice. The study can also be extended to monitor the manual skill of welding machine operators during their training or actual work. Importantly during underwater welding, the instructor cannot find out the weld quality because of poor visibility of the prevailing or existing condition.

\section{CONCLUSION}

1. Statistical analyses of average current from the pulsed current waveform using a computer provide a powerful means of directly assessing the arc stability. This can be very helpful for monitoring and improving the GMAW-P process and for optimizing the parameters of the GMAW-P process, optimizing filler metal, welding equipment, and power sources.

2. Every variation in the melting and detachment of filler metal can be seen in the waveforms of pulsed voltage and pulsed current. This study helped to obtain optimum arc behavior in terms of arc stability, spatter level, and droplet detachment in order to ensure optimum weld quality.

3. The peak duration is critical, for if it is too long or too short it results in the generations of excessive spatter.

4. The zone ' $\mathrm{C}$ ' is preferable among all the zones to carryout welding. In this zone, only the one droplet detachment during peak duration was desirable because the standard deviation and coefficient of variation of average current were found to be the lowest, among from the four types of droplet detachments.

5. The peak energy required for one droplet detachment during background duration was the lowest. Furthermore, one droplet detachment during peak duration, two droplets detachment during peak duration, and three droplets detachment during peak duration 
require higher peak energies, increasing in the order given. The highest peak energy of $40.76 \mathrm{~J}$ was observed in the case of three droplets detachment during peak duration.

6. The most uniform average current was observed for the welds made with one droplet detachment during peak duration (weld no. EL-1) compared with the average currents for one droplet detachment during background duration, two droplets detachment during peak duration, and three droplets detachment during peak duration. Because, among from the four types of droplet detachments, the lowest standard deviation (0.9228) and the lowest coefficient of variation (0.8361) were found for the weld no. EL-1. Based on this, the following parameters of the pulsed current were found to be more suitable than the other combinations of pulsed current: $I_{P}=196.8 \mathrm{~A} ; T_{P}=5.0 \mathrm{~ms} ; I_{B}=20 \mathrm{~A} ; T_{B}=6 \mathrm{~ms} ; W_{F}$ $=100 \mathrm{~mm} / \mathrm{s} ; \mathrm{I}_{\mathrm{AV}}=110.3718 \mathrm{~A} ;$ and $\mathrm{W}_{\mathrm{S}}=10.0 \mathrm{~mm} / \mathrm{s}$. Thus process-integrated quality assurance of the weld was possible on the basis of more uniform average current. This study can be very much useful for fabricators to derive the fullest benefits from GMAW$P$ in high quality or automatic or robotic welding.

7. The value of $I_{P}$ should be set above the spray current level and $T_{P}$ should be so adjusted that the filler metal tip is melted to a proper size and one droplet is detached appropriately to the base metal during peak duration.

8. Control parameters that characterize the arc stability include average current, peak energy, and droplet detachment time. Monitoring and analyzing these parameters can make sure that the GMAW-P process will be carried out in a optimum droplet detachment type, thus ensuring the best welding conditions for the optimum droplet detachment.

\section{LIST OF SYMBOLS}

\begin{tabular}{|c|c|c|c|}
\hline $\begin{array}{l}\text { C.V } \\
\text { C. } W_{\text {A.V }} \\
\text { C. } W_{\text {C.V }}\end{array}$ & $\begin{array}{l}\text { Coefficient of variation } \\
\text { Mean value of average current } \\
\text { Coefficient of variation of }\end{array}$ & $\mathrm{T}_{\mathrm{D} 2}$ & $\begin{array}{l}\text { Time between the beginning } \\
\text { of the pulse and the second } \\
\text { droplet detachment }\end{array}$ \\
\hline & average current & $\mathrm{T}_{\mathrm{D} 3}$ & Time between the beginning \\
\hline C.W $W_{\text {S.D }}$ & Standard deviation of average current & & of the pulse and the third \\
\hline DCEP & Direct Current Electrode Positive & & droplet detachment \\
\hline$F_{P U}$ & Pulse frequency $(1 / T)$ & $T_{P}$ & Peak duration \\
\hline GMAW-P & Pulsed Current Gas Metal Arc Welding & $T_{D}$ & Droplet detachment time \\
\hline I & Welding current & $\mathrm{V}$ & Voltage \\
\hline $\mathrm{I}_{\mathrm{B}}$ & Background current & $\mathrm{V}_{\mathrm{B}}$ & Background voltage \\
\hline $\mathrm{I}_{\mathbf{P}}$ & Peak current & $V_{P}$ & Peak voltage \\
\hline $\mathrm{I}_{\mathrm{T}}$ & Spray current level & $\mathrm{V}_{\mathrm{AV}}$ & Average arc voltage \\
\hline $\mathrm{I}_{\mathrm{AV}}$ & Average current & $\mathrm{W}_{\mathrm{F}}$ & Wire feed speed \\
\hline 1 & Pulse cycle time & $\mathrm{W}_{\mathbf{S}}$ & Welding speed \\
\hline $\mathrm{T}_{\mathrm{B}}$ & Background duration & $\sigma$ & Standard deviation \\
\hline $\mathrm{T}_{\mathrm{D} 1}$ & $\begin{array}{l}\text { Time between the beginning of the } \\
\text { pulse and the first droplet detachment }\end{array}$ & & \\
\hline
\end{tabular}




\section{REFERENCES}

1. Rajasekaran, S.; Kulkarni, S.D.; Mallya, U.D.; Chaturvedi, R. C. 1998. Droplet detachment and plate fusion characteristics in pulsed current gas metal arc welding. Welding Journal 77 (6): 254-s to 269-s.

2. Rajasekaran, S. 1999. Weld bead characteristics in pulsed GMA welding of Al-Mg alloys. Welding Journal 78 (12): 397-s to 407-s.

3. Rajasekaran, S. 2000. Weld surface undulation characteristics in the pulsed GMA welding process. The Ninth International Conference on Computer Technology in Welding, September 28-30,1999, National Institute of Standards and Technology, United States Department of Commerce, Special Publication No. 949, May 2000, 349-360.

4. Rajasekaran, S.; Kulkarni, S.D.; Mallya, U.D.; Chaturvedi, R. C. 1995. Molten droplet detachment characteristics in steady and pulsed current GMA welding Al-Mg alloys. Proceedings of the Sixth International Conference on Aluminum Weldments (INALCO '95), April 3-5, 1995, Cleveland, Ohio, U.S.A. American Welding Society, Miami, Fla, 207-224.

5. Rajasekaran, S.; Kulkarni, S.D.; Mallya, U.D.; Chaturvedi, R. C. 1994. Droplet detachment and plate fusion characteristics in pulsed current gas metal arc welding. Proceedings of the Advanced Joining Technologies for New materials II, March 2-4, 1994, The Cocoa Beach Hilton, Florida, U.S.A, American Welding Society, Miami, Fla, 172-187. 


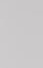




\title{
DROPLET OSCILLATION AND WELD POOL IMAGING USING COMPUTER-CONTROLLED COMPOSITE PULSE CURRENT
}

\author{
B. Zheng ${ }^{*}$
}

\begin{abstract}
This paper presents a unique solution to real-time monitoring of both droplet detachment and weld pool during a pulsed gas metal arc welding process: a composite pulse current consisting of a square wave form followed by a sine wave form was designed. The instant for initiating a constant base current adaptively started at the instant droplet detachment was sensed. During the period of the base current, a flag signal was generated to trigger the imaging of a weld pool. The approach makes droplet detachment and image acquisition be proceeded without outside intervention. This provides the possibility for real time quantifiable monitoring and control for both droplet transfer and weld pool penetration.
\end{abstract}

\section{INTRODUCTION}

The results of the previous researches showed that metal transfer and weld pool penetration both have a large influence on the generation of the defects such as undercut, burn through, insufficient melting, spatter, gas pore, and even weld cracks during a gas metal arc welding (GMAW) process $\{$ Ref. 1-3). Hence, in-process monitoring and control for metal transfer and weld pool penetration are crucial in order to minimize the cost of post-weld inspection and repair. Currently, a droplet/arc oriented control strategy is often used even though it is inefficient in some applications. Since maintaining a consistent weld pool has a dramatically direct impact on weld quality, a weld pool oriented control strategy has been largely demanded.

In a GMAW process with argon-rich shielding, the different metal transfer modes of shortcircuiting, globular, and spray can be observed in sequence as the welding current is increased (Ref. 2-5) when steel electrode wire is used. The critical welding currents at which the metal transfer mode changes are defined as transition currents, one of which is the spray transition current at which globular transfer becomes spray transfer (Ref. 2-3). The level of the spray transition current mainly depends on many factors such as wire diameter and composition of shielding gas. Quality welds can be achieved using the projected spray transfer mode of one droplet per pulse (ODPP). To ease the flexible selection of ODPP and simplify the adjustment of process parameters, pulsed current welding (GMAW-P) is a preferred process (Ref. 3 and 6-9). However, most of GMAW-P processes with ODPP mode use open loop control that regulates only the metal transfer mode and pay little attention to the weld pool (even though some researches have been pursued on sensing and closed-loop control of droplet transfer) (Ref. 4-21). The partial reason for this is the difficulty in measuring characteristic signals and control compatibility of droplet detachment with weld pool penetration.

Besides through-arc sensing of a weld pool (Ref. 22-28), machine vision systems have been used to sense the weld pool and control the full penetration during a PAW, or GTAW, or GMAW process (Ref. 29-45). These vision systems can be characterized into band-pass arc light filtering,

" Edison Welding Institute, 1250 Arthur E. Adams Drive, Columbus, OH 43221 
coaxial viewing, high-shutter-speed camera synchronizing with laser-assisted illumination, and infrared thermography. The instantaneous decrease of the welding current has also been used to weaken the intensity of the arc light to capture a clear weld pool image in GMAW-P (Ref. 44). However, the interference resulting from metal transfer while imaging a weld pool was not solved because of the inaccurate open-loop control that was used to determine the instant of the droplet detachment. Also, the camera had some limitations, such as the shutter speed and the luminance, which could not meet the needs of imaging and controlling a GMAW weld pool.

The objective of this paper is to explore a unique solution to the compatibility of sensing and control of a droplet detachment and imaging a weld pool during a GMAW-P process, which will set a basis for real time control of a fully penetrated weld pool (Ref. 44-46).

\section{EXPERIMENT PROCEDURE}

A PC-controlled, inverter welding power source and a wire feeder are used in this experiment. A high frame rate digital camera with adjustable frame rate and a frame grabber is used for monitoring the droplet with the back illuminating light from a He-Ne laser. Current and voltage sensors are integrated with a data acquisition system. A positioning system driven by stepper motors is also interfaced with the system. A CCD camera with high shutter speed is attached to the front of the torch to synchronously image the weld pool.

To explore a droplet detachment method associated with the imaging of a weld pool, a controlled signal generator was designed, the current wave form of which is shown in Fig. 1. The period of pulse consists of a square wave form and a sine wave form with a pre-selected frequency. The peak current for a square pulse is experimentally set at around $160 \mathrm{~A}$ that is below the spray transition current, the function of which is to initiate the droplet growth and minimize the energy input to a workpiece. The function of a sine wave pulse following the square pulse is to activate an externally forced oscillation of a droplet before it detaches from the tip of an electrode wire. The sine wave pulse with its peak level (260 A) being higher than transition current (220-240 A, in this case) adaptively terminates at the instant of the droplet detachment monitored by the arc voltage sensor. The imaging of a weld pool is initiated during the base current $(40$ A) period after a time delay that is experimentally set. The frequency of the sine wave pulse can be varied from 0 to 500 $\mathrm{Hz}$. The total cycle frequency is less than $30 \mathrm{~Hz}$ due to the limitation of the

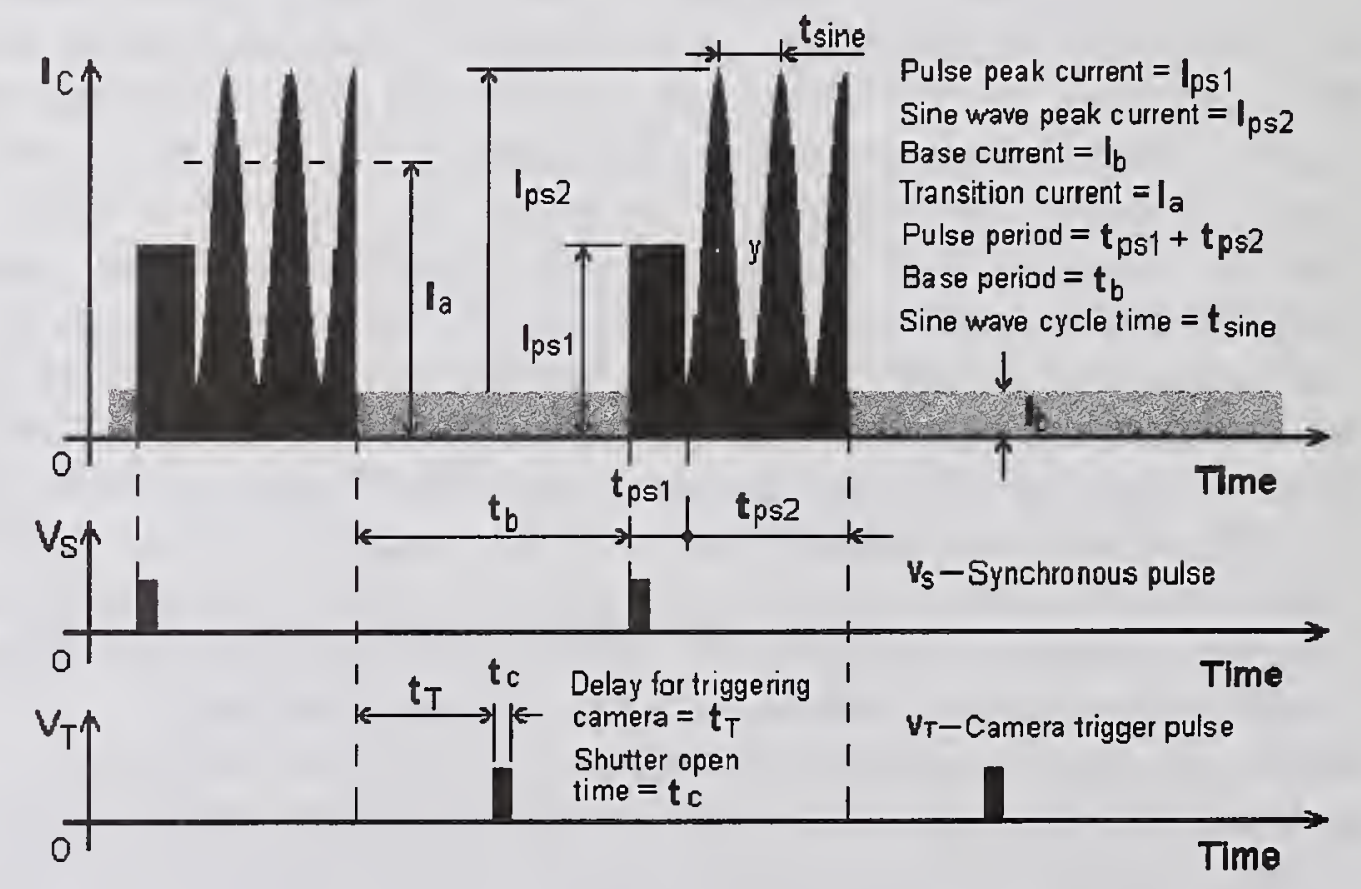

Fig. 1 Composite Pulse Current $\left(\mathrm{I}_{\mathrm{C}}\right)$, Synchronous Pulse $\left(\mathrm{V}_{\mathrm{S}}\right)$, and Trigger Pulse $\left(\mathbf{V}_{\mathrm{T}}\right)$ 
recording frequency by the high shutter speed camera. A droplet cannot detach during the period of a square pulse because of the low electromagnetic pinch-off force resulted from the acting current. The sine wave current pulse not only promotes the continuous growth of a droplet as it oscillates but also assists in droplet detachment and further decreases the energy input to a workpiece. At the end of the base period for maintaining an arc, a new cycle repeats the same process.

Bead-on-plate welds were made in a flat position on $6.0-\mathrm{mm}$ thick mild steel plates. The electrode wire is 1.2-mm diameter steel (AWS standard ER 70S) and the shielding gas is argonrich $\left(85 \% \mathrm{Ar}+15 \% \mathrm{CO}_{2}\right)$.

\section{RESULTS AND DISCUSSION}

According to the static force balance theory (Ref. 10-12), the forces promoting droplet detachment in a flat welding position are the electromagnetic force resulting from the welding current, gravity and the plasma gas drag force. The force resisting the droplet detachment is mainly the surface tension. The other resistant forces are anode spot pressure and metallic vapor force acting on the anode spot. When the forces promoting droplet detachment are equal to or greater than the resisting forces, the droplet will detach from the tip of an electrode wire. The electromagnetic force will be a promoting force for droplet detachment when the lower half of the droplet is covered with the spread arc anode spots and the welding current is above the transition current. Otherwise, it will become a resisting force for droplet detachment. The magnitude of the electromagnetic force depends on the level of welding current (Ref. 11-13). The control strategies for droplet detachment may be categorized into two types: electrical and mechanical (Ref. 6-19).

Since electrically controlling the electromagnetic force inside the droplet and arc is reliable and fast, most investigations on droplet detachment control focus on this approach. In an ODPP mode, the current during the pulse period of a cycle forms a droplet with a certain size, while the current during the base period of a cycle prevents the arc from extinguishing. The melting rate of the wire (the wire feed speed) is directly proportional to the pulse frequency: the higher the frequency, the higher both the average welding current and the melting rate. The pulse period and level also have the positive effect on the melting rate when the pulse frequency is constant. Therefore, the mass and heat transferred to the workpiece may be flexibly regulated by the adjustment of average welding current.

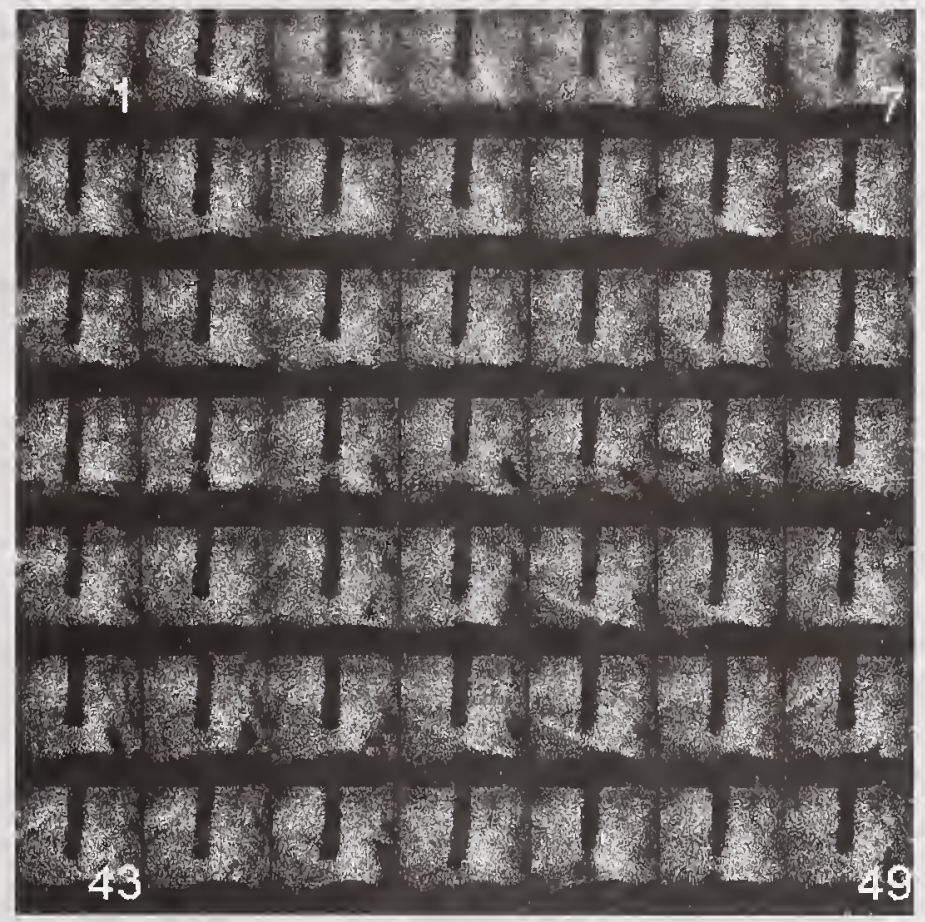

Fig. 2 Control for Droplet Detachment with Resonance Oscillation

Both the static force balance theory and the pinch instability theory reveal that the ODPP approach, characterized by approximately the same 
droplet diameter as that of the wire, can be electrically achieved only when the pulse current is above the transition current. The droplet may detach during the pulse period or the base period in a cycle, depending upon different combinations of the selected parameters.

During its growth, the droplet at the tip of an electrode wire oscillates vertically (sometimes with a horizontal component of oscillation) at random frequencies (multiresonant oscillation) due to the forces acting on it. The random oscillation of the droplet was observed and analyzed mostly in a globular

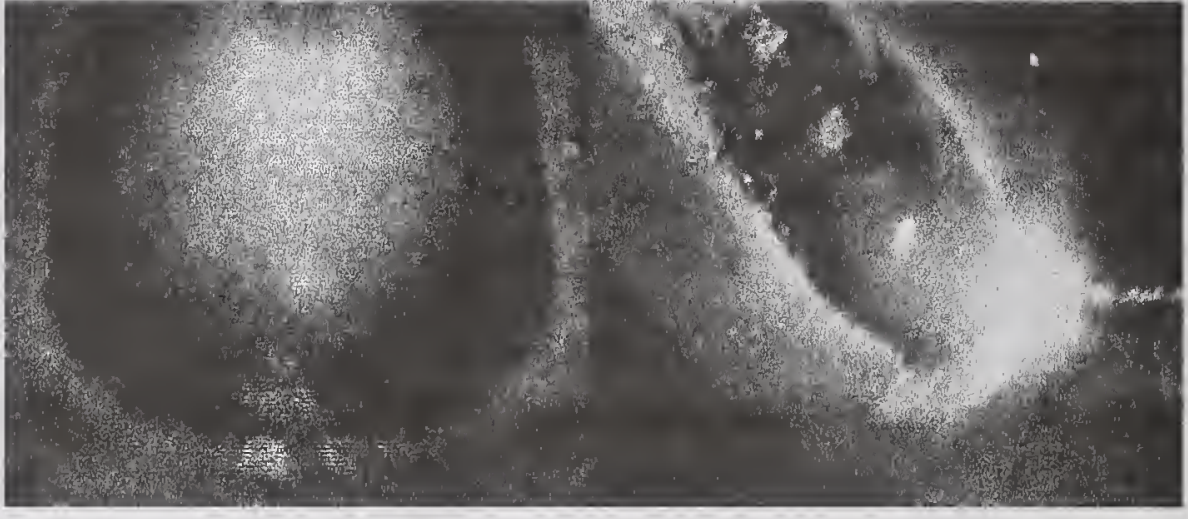

a

Fig. 3 Images of a Weld Pool in Pulsed Current Gas Metal Arc Welding of Steels

a. viewing over a weld pool from the front of a welding torch

b. side view over a weld pool from the side of a weld torch transfer mode where the droplet suspends at the tip of an electrode wire for a comparatively longer time because the electromagnetic force inside the droplet is not large enough to detach it (Ref. 11-13). This type of oscillation is not controllable and thus not a candidate for droplet detachment control. The active control of droplet detachment was developed using a high frame rate camera as a vision sensor to detect the vertical coordinate of the droplet along the axis of the wire (Ref. 13-14). However, there are some issues that still need to be addressed, such as the

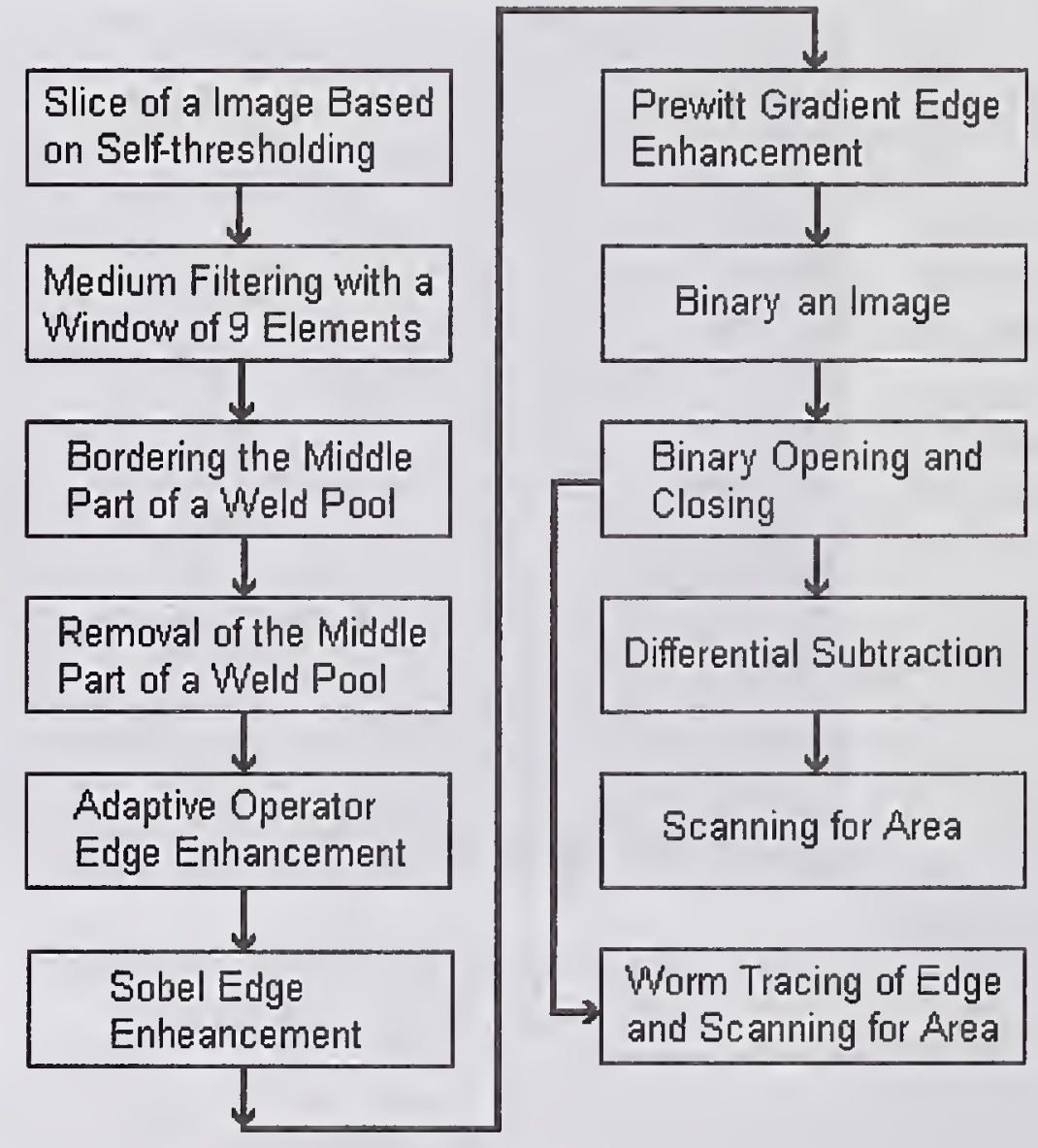
possibility to change the instant of the droplet detachment through changing the number of oscillations in a cycle and the compatibility with the imaging of a weld pool.

An active variation in the welding current between a level above the spray transition current and a level at the base current during the pulse period in a cycle will make the electromagnetic force inside the droplet change accordingly. Suppose the surface tension, the plasma drag force and other forces are constant, the droplet at the tip of an electrode wire may be forced to oscillate synchronously due to this varying and controllable electromagnetic force. A frequency-controlled variation of a current with a sine-wave form is an ideal approach (as shown in Fig. 1). The natural frequency of oscillation

Fig. 4 Procedure for Processing a Weld Pool Image 
for a given droplet depends on its size. When this frequency equals that of a periodically varied external composite force applied on the droplet, resonance will occur. In this case, the droplet may detach if the magnitude of the force due to resonance is high enough. The essence of this approach is to convert the electrical energy from a welding current into the mechanical energy of a droplet oscillation that can be used to control the timing of droplet detachment and to image the weld pool. During droplet oscillation, although the heat generated during the period when the sine wave-form current is acting might be less than the heat due to a square pulse with the same magnitude and duration, it still is high enough to continuously melts the wire.

Figure 2 shows sequential images of droplet oscillations and detachment created using the composite pulse wave form shown in Fig. 1. It can be seen that the arc is maintained and the electrode wire melts from frame 1 to frame 7 . From frame 8 to frame 12, the droplet finishes the first cycle of oscillation. From frame 12 to frame 15, the droplet makes the second oscillation. From frame 15 to frame 18 , the droplet oscillates the third time. The droplet also makes the fourth oscillation from frame 18 to frame 21 , and then detaches at the instant between frame 22 and frame 23 . The frequency of the droplet oscillation $\quad(496 \mathrm{~Hz}$, determined by counting the number of image frames and making a simple calculation) is nearly the same as that of a sine wave pulse $(500 \mathrm{~Hz})$. Compared with the droplet oscillation, the sine wave current has just two and a half cycles. Therefore, in this case, the droplet can oscillate only twice based on the sine wave frequency and one induced oscillation resulting from the reduction of the current after the square wave pulse. The fourth oscillation and the detachment of the droplet indicate that the droplet resonance occurs because of the effect of inertia produced by the forced multiple oscillations. An algorithm for controlling the welding current is developed based on the composite wave form using

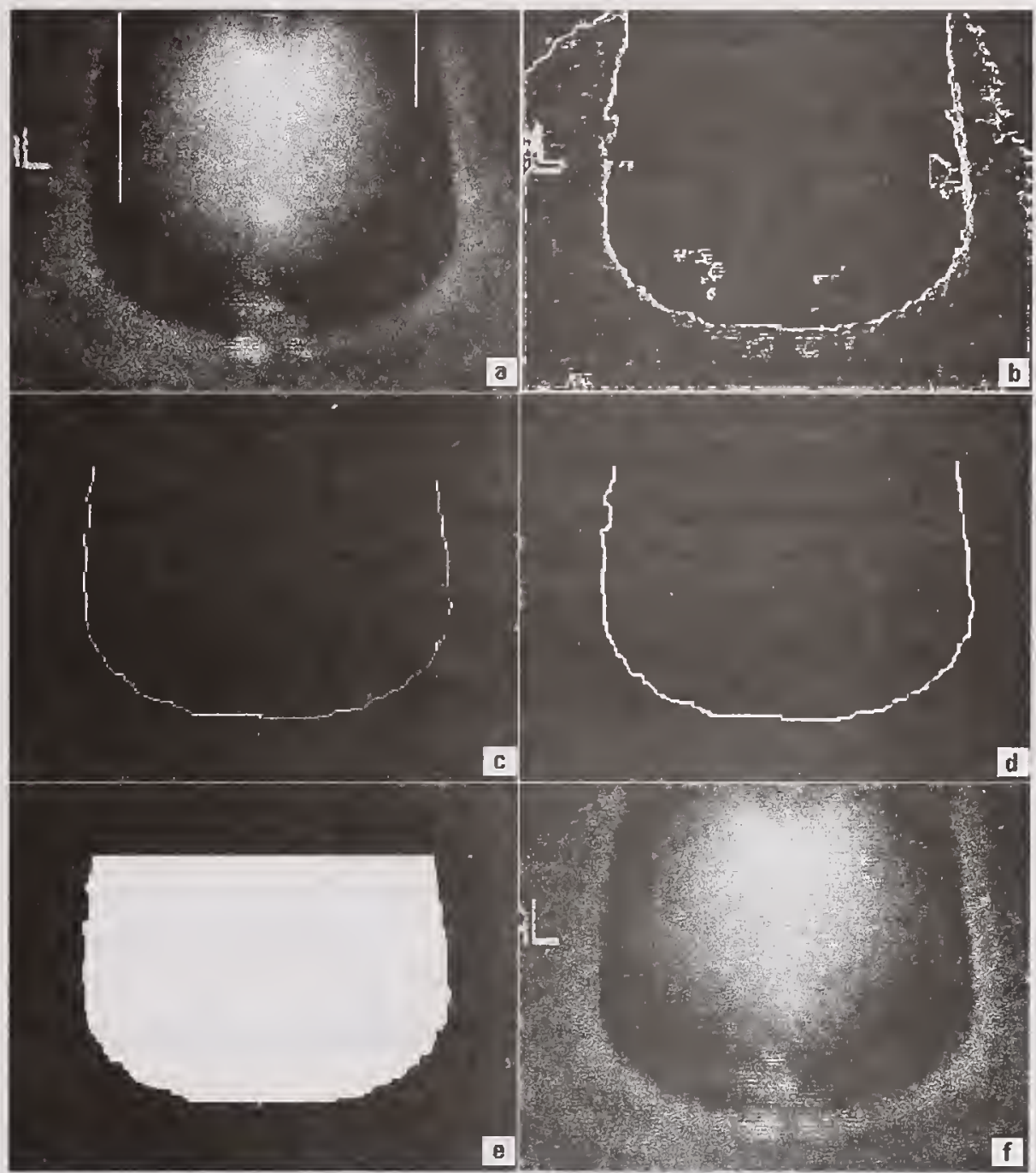

Fig. 5 Processed Images of a Weld Pool Using Different Algorithms

(a)Bordering the middle part of an image

(b)Prewitt gradient edge enhancement

(c) Scanning an image differentially subtracted

(d)Worm tracing of an edge for binary image

(e)Area caculation of weld pool in an image

(f) Sum of an edge and an original image of a weld pool 
arc voltage as a monitoring signal.

Since droplet detachment can be achieved at a controllable instant using the unique composite pulse designed in this paper, a high shutter speed camera is externally triggered to capture the image of a weld pool under the active projection of light from a laser source. This is done after a droplet detaches during the period of the base current. A band pass filter that allows $330 \mathrm{~nm} \mathrm{~N} \mathrm{~N}_{2}$ laser light to pass through is attached to the front of the camera. The light is guided through a fiber optics cable and projected on the weld pool. The time delay of $\mathrm{t}_{\mathrm{T}}$, shown in Fig. 1, can be adjusted. The process of droplet transfer also can be simultaneously imaged on the same frame as that of the image of the weld pool. In order to guarantee the image quality of a weld pool, $\mathrm{t}_{\mathrm{T}}$ was experimentally determined to match that instant immediately after a droplet is transferred. Because of the decrease of the welding current, the image of a weld pool illuminated by the laser light forms under a very low light radiation from an arc. This sequential timing approach uniquely makes a trade-off between the droplet transfer control and the weld pool imaging. The acquired images viewed at different camera positions are shown in Fig. 3. It can be seen that the metal transfer process is avoided while the image of a weld pool is captured. The side view of a weld pool shows that the weld pool possesses a convex shape that is much different from that of a concave weld pool which occurred in gas tungsten arc welding or plasma arc welding. The boundary located at the rear part of a weld pool can not be clearly identified because of its convex shape. The image of a weld pool from the front view satisfies the requirements needed for image processing, even though a brighter area resulting from an arc during the base current exists in the middle area.

The features of a weld pool image acquired in this paper lead to the determination of an off-line image processing procedure that is shown in Fig. 4. Also, scanning and worm tracing for edge extraction and area calculation are comparatively pursued. The procedure for image processing includes routine algorithms and core algorithms such as adaptive thresholding for image slicing, bordering the middle part of an image, and worm tracing of an edge. The boundary for a weld pool image by using worm tracing algorithm can be seen in Fig. 5(d). Some processed images corresponding to Fig. 4 are shown in Fig. 5. The variation of the area calculation between scanning and worm tracing for an edge are in the range of a 100-200 pixel difference for the defined weld pool area (the white area in Fig. 5 (e)) that has more than 30,000 pixels.

\section{CONCLUSIONS}

The following conclusions can be drawn based on the research of this paper:

1. In-process monitoring and control of weld quality is one of the crucial issues for gas metal arc welding (GMAW). The unique solution presented demonstrates real-time monitoring of both droplet detachment and the weld pool during a GMAW-P process.

2. A composite pulse current consisting of a square wave followed by a sine wave can initiate the growth and oscillation of a droplet before its detachment from the tip of an electrode wire. The instant for activating a constant base current can be adaptively started after the sensed instant of droplet detachment.

3. The cyclic number of droplet oscillations depends largely on pulse parameters. Careful selection of welding parameters, monitoring arc voltage, and applying appropriate algorithms 
to extract the information of droplet detachment will guarantee real time control for smooth metal transfer.

4. Imaging of a weld pool can be reliably triggered by a delayed flag signal generated during the period of a base current. In a cycle, droplet detachment and image acquisition can be compatibly accomplished.

5. In addition to other image processing routines, worm tracing and scanning are developed for calculating the area of a weld pool image. The procedure developed for processing a weld pool image includes its boundary extraction and area calculation and provides the possibility for real time quantifiable monitoring and control of the weld pool penetration state in the future.

\section{ACKNOWLEDGEMENT}

The author expresses his great acknowledgement to the supports of Mechanical Engineering Department of Southern Methodist University, Dallas, Texas and Edison Welding Institute, Columbus, Ohio.

\section{REFERENCES}

1. The Lincoln Electric Company. 1995. The Procedure Handbook of Arc Welding (Thirteen edition), The Lincoln Electric Company, Cleveland, $\mathrm{OH}$.

2. Lancaster, J. and Ed F.,. 1986. The Physics of Welding, 2nd ed. Edition. New York, Pergamon Press.

3. AWS. 1991. Gas Metal Arc Welding, Welding Handbook, American Welding Society (Eighth edition), Miami, FL, Vol. 2: 109-155.

4. Kim Y.S. and Eagar T. W.. 1993. Analysis of Metal Transfer in Gas Metal Arc Welding. Welding Journal 6: 269 to278.

5. Jones L. A.; Eagar T. W.; and Lang J H.. 1998. Images of a Steel Electrode in Ar-2\%O2 Shielding during Constant Gas Metal Arc Welding. Welding Journal 4: 269 to 278.

6. Wang Q. L. and Li P. J.. 1997. Arc Light Sensing of Metal transfer and Its Analysis in Pulsed GMAW Process. Welding Journal 11: 458s to 469s.

7. Johnson J. A.; Carlson N. M.; and Smartt H. B.. 1989. Detection of Metal Transfer Mode in GMAW. Proc. 2nd International Conf. on Trends in Welding Res., 5: 377-381, Gatlinburg, Tenn.

8. Madigan R. B.; Quinn T. P.; and Siewert T. A.. 1989. Sensing Droplet Detachment and Electrode Extension for Control of Gas Metal Arc Welding. Proc. 3rd International Conf. on Trends in Welding Res., Gatlinburg, Tenn, 11: 999-1002,

9. ESAB Group. 1994. Size and Shape of the Liquid Droplet at the Molten Tip of an Arc Electrode. Journal of Physics 4: 1433 to 1442.

10. Amson J. C.. 1962. An Analysis of the Gas-shielded Consumable Metal Arc Welding System. British Welding Journal 41(4): 232 to 249.

11. Greene J. W.. 1960. An Analysis of Transfer in Gas-shielded Welding Arcs. Trans. AIEE Part 2, 7:194 to 203. 
12. Waszink J. H. and Graat L. H. J.. 1983. Experiment Investigation of the Forces Acting on a Droplet of Weld Metal. Welding Journal 62(4): 109s to 116s.

13. Kovacevic R.; Er L. G.; and Zhang Y. M.. 1997. Achieving Projected Spray Based on Excited Droplet Oscillation. The Second World Congress on Intelligent Manufacturing Progresses and Systems, Budapest, Hungary, 6: 10-13.

14. Zhang Y. M.; Er L. G.; and Kovacevic R.. 1998. Active Metal Transfer Control by Monitoring Excited Droplet Oscillation. Welding Journal 77(9): $388 \mathrm{~s}$ to $395 \mathrm{~s}$.

15. Dubon Z.. 1965. A Device for Interrupted Electrode Wire Feed during Arc Welding. Czechoslovakian Priority Certificate No. 116243.

16. Paton B. E. et al.. 1977. Controlling the Arc Welding Process by Programming the Electrode Wire Feed Rate. Automatic Welding 30(1): 1 to 4.

17. Dmitrienko V. P.. 1977. Calculation of the Speed of Movement of the Electrode Tip during Welding with Mechanical Control of Metal Transfer. Automatic Welding 32(2): 1 to 4.

18. Jones L. A.; Eagar T. W.; and Lang J. H.. 1992. Investigation of Droplet Detachment Control in Gas Metal Arc Welding. Proceedings of the 3rd International Conference on Trends in Welding Research, Gatlinburg, TN. 6: 1009-1013.

19. Yang S. Y.. 1998. Projected Metal transfer Control with Additional Mechanical Forces (AMF) in MIG/MAG Welding Process. Ph. D. Dissertation, Harbin Institute of Technology.

20. Kovacevic R.; Beardsley H.; and Fan H. G.. 1998. Modeling, Sensing and Control of Droplet Based Solid Freeform Fabrication Process. The 75th Anniversary Energy Sources Technology Conference, ASME International Petroleum Division. Houston, TX. 2:

21. Stava E. K.. 1995. A New, Low-Spatter Arc Welding Machine. The Lincoln Electric Company, Cleveland, Ohio.

22. Yang C. L.. 1991. The Real Time Control of Weld Full-penetration in the Thin Steel Plate TIG Welding with Arc Sensor Based on the Weld Pool Resonance. Ph. D. Dissertation, Harbin Institute of Technology, Harbin, China: 1-116.

23. HU B. and Den G.. 1998. OUDEN Weld Penetration Sensing and Control during GTA [TIG] Welding Using Weld Pool Oscillation. Trends in Welding Research. Proceedings of the 5th International Conference, Pine Mountain, GA, June: 1125-1130.

24. Hu B. X.. 1980. The Study of Controlling System of Welding Quality at All-position by Using the Arc Sound in Pulsed Plasma Arc Welding of Steel. China, Welding 3: 17 to 20.

25. Zhang J.H. and Wang Q.L.. 1985. Study on Arc Sound in TIG and Plasma Processes. IIW Doc. $212-610-85$.

26. Martinez L. F.. 1992. Front Side Keyhole Detection in Aluminum Alloys. Welding Journal 5: 49 to $52 . J$.

27. Metcalfe C. and Quigley M. B. C.. 1975. Keyhole Stability in Plasma Arc Welding. Welding Journal 11: 401 to 404.

28. Steffens H. D.. 1972. Automatic Control for Plasma Arc Welding with Constant Keyhole. Welding Journal 6:40 to 45 .

29. Zheng B.; Wang Q. L.; and Kovacevic R.. 1999. Imaging the Keyhole Weld Pool in Variable Polarity Plasma Arc Welding of Aluminum Alloys. ASME Journal of Manufacturing Science and Engineering 9.

30. Zheng B.; Wang Q.L.; and Kovacevic R.. 2000. Closed Loop Control for Weld Formation Based on the Front Image Signal of the Keyhole in Variable Polarity Plasma Arc Welding of Aluminum Alloys. Welding Journal 12: 363 s to $371 \mathrm{~s}$ 
31. Agapakis J. E. and Bolstad J.. 1991. Vision Sensing and Processing System for Monitoring and Control of Welding and Other High Luminosity Processes. International Robots \& Vision Automation Conference: 23-29.

32. Nakata S.; Huang J.; Tsuruha Y.. 1988. Visual Sensing System for In-Process Control of Arc Welding Process. Welding International 12: 1086 to 1090.

33. Hoffman T.. 1991. Real-time Imaging for Process Control. Advanced Materials \& Processes. 9: 37 to 43

34. Guu C. and Rokhlin S. I.. 1989. Computerized Radiographic Weld Penetration Control with Feedback on Weld Pool Depression. Materials Evaluation (10): 1204 to 1210.

35. Guu C. and Rokhlin S. I.. 1992. Arc Weld Process Control Using Radiographic Sensing. Materials Evaluation (11): 1344-1348.

36. Rokhlin S. I. and Guu A. C.. 1990. Computerized Radiographic Sensing and Control of an Arc Welding Process. Welding Journal 69(3): 83 to 95.

37. Richardson R. W. and Gutow D. A.. 1984. Coaxial Arc Weld Pool Viewing for Process Monitoring and Control. Welding Journal 63(3): 43 to 50

38. Kovacevic R. and Zhang Y. M.. 1993. Three-dimensional Measurement of Weld Pool Surface. International Conference on Modeling and Control of Welding Processes, Florida,

39. Kovacevic R. and Zhang Y. M.. 1995. Vision Sensing of 3D Weld Pool Surface. The 4th International Conference on Trends in Welding Research, Gatlinburg,

40. Kovacevic R. and Zhang Y. M.. 1996. Monitoring of Weld Penetration Based on Weld Pool Geometrical Appearance. Welding Journal 10: 317 to 329.

41. Nagarajan S.; Chen W. H.; and Chin B. A.. 1989. Infrared sensing for adaptive arc welding. Welding Journal 68(11): 462 to 466.

42. Nagarajan S.; Banerjee P.; Chen W. H.; and Chin B. A.. 1990. Weld pool size and position control using IR sensors. Proceedings of NSF Design and Manufacturing Systems Conference, Arizona State University.

43. Chen W. and Chin B. A.. 1990. Monitoring Joint Penetration Using Infrared Sensing Techniques. Welding Journal 69(4): $181 \mathrm{~s}$ to $185 \mathrm{~s}$.

44. Oshima K. and Morita M.. 1992. Sensing and Digital Control of Weld Pool in Pulsed MIG Welding. Transactions of the Japan Welding Society 23(4): 36 to 42.

45. Eagar, T. W. 1989. Recent Trends in Welding Science and Technology. S. A. David, Ed. P. 341, ASM International, Materials Park, Ohio.

46. Zheng B. and Kovacevic R.. 1999. A Novel Control Approach for the Droplet Detachment in GMA Welding of steel. Computer Trend in Welding, Detroit, 8, 


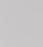




\title{
OPTIMIZATION OF PGMAW USING ONLINE OBSERVATION \\ AND STATISTICAL DATA
}

\author{
S. Nordbruch ${ }^{1,2}$, A. Gräser ${ }^{2}$
}

\begin{abstract}
In this paper a monitoring system that simplifies the finding of optimal welding parameters, the analysis and the optimization of pulsed gas metal arc welding (PGMAW) is described.

The system allows the visual online observation of all states of the welding process, including the droplet transfer, without an additional lighting unit. Additionally, the synchronized measurement of the welding current and welding voltage signals during image recording and the extraction of characteristic parameters of the signals is possible. Furthermore, the system allows the visual analysis of the material transition images.

For an analysis and optimization of the process the systems computes statistical data of all collected and calculated visual and electrical data of a recording sequence.
\end{abstract}

\section{KEYWORDS}

PGMAW, droplet transfer, online observation, visual data, electrical welding data, statistical data

\section{INTRODUCTION}

The pulsed gas metal arc welding process is an important component in many industrial and manufacturing operations. It is highly suited to a wide range of applications. Due to the complex processes, the extreme brightness of the welding arc, the high number of different welding tasks, etc., the finding of optimal parameters, the test of new welding parameter combinations or the analyses by process errors is difficult.

For the solution of the problems the visual observation of the droplet transfer in combination with the measurement of electrical welding parameters is an approach. Typically, the droplets should be even and in uniform size and the material transition should be splashless.

The visual observation of the material transition has been used extensively. Normally digital Charge-Coupled-Device (CCD) high-speed cameras in combination with an optical laser are used. Due to the extreme brightness these approaches are using the shadowgraphing technique, described by Allemand et. al. (Ref. 1).

For the mentioned problems the systems are unsuitable due to a set of disadvantages. The most important are:

» The necessity of the lighting unit and the limited possibilities of observation caused by this (shadowgraphing technique).

» The acquisition and maintenance costs are very high.

1 Friedrich-Wilhelm-Bessel-Institut Forschungsgesellschaft m.b.H, Postfach 106364, 28063 Bremen, Germany

2 University Bremen, Institute of Automation (IAT), Kufsteiner Str. NW1, 28359 Bremen, Germany 


\section{SYSTEM}

The monitoring system consists of a High-Dynamic-Range-CMOS camera (HDRC) with an external trigger input, an intelligent measuring board for the triggering of the image recording and the synchronized and simultaneous measurement of electrical welding parameters, a signal processing unit for the automatic calculation of characteristic welding process parameters, an image processing unit for the automatic visual analysis of the droplet transfer images and a statistical unit. Due to the properties of the HDRC camera the system requires no additional lighting unit. The basic set-up of the system is shown in figure 1 .

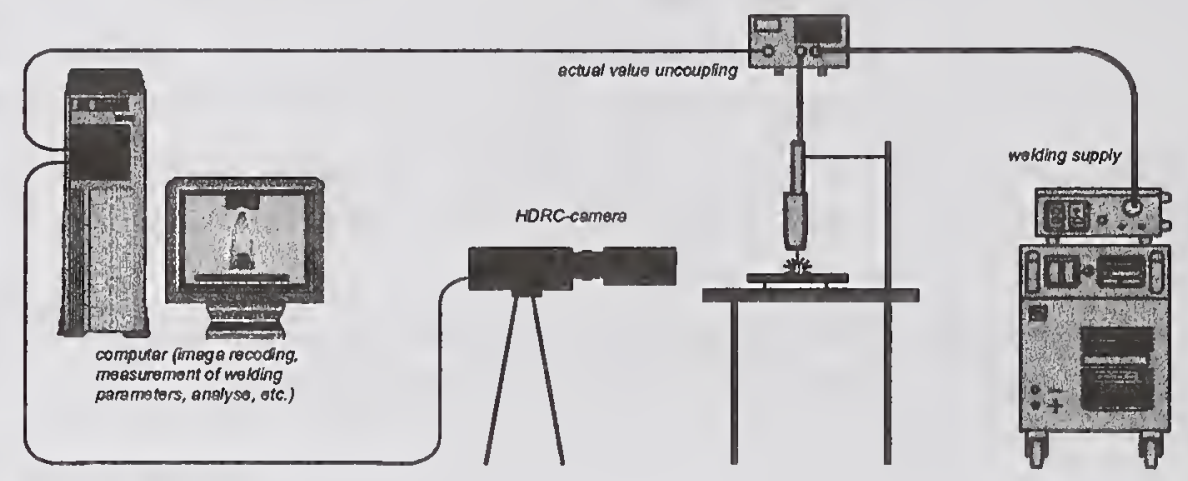

Figure 1: Experimental set-up

\section{High-Dymamic-Range-CMOS Camera (HDRC)}

HDRC cameras use an image sensor with a brightness dynamic of approximately $10^{6}: 1$. This corresponds approximately to the intensity difference of the welding process. HDRC cameras are therefore able to observe all states of the material transition in all welding positions without an additional lighting unit.

The principle of HDRC cameras can be described as follows. The level of intensity, i.e. the color/gray values of an object, are essentially dependent on the exposure rate and the material properties of the object with its reflection properties. The information of an object is essentially dependent on the contrast. A CCD camera maps the absolute color/gray values caused by respective irradiation in the image. As opposed to the CCD camera the HDRC camera maps the contrast, which is caused by different reflections. I. e., brightness conditions (color/gray values of the object) are independently represented by the intensity of illumination as constant number differences. Before further processing, this high intensity dynamic is compressed logarithmically in every cell of the image sensor just like in the human eye. Due to this compression, the information content of the image that is included in the contrast is not reduced.

A further advantage of HDRC cameras is, that at every time each sensor cell can be accessed independently of all others. The constraint of CCD cameras that must read out complete images does not apply for HDRC cameras. This property of HDRC cameras allows the selection of sub areas of the image sensor instead of the complete image. Considerably higher image recording frequencies become possible.

\section{Principle of Image Recording and Measurement of Electrical Welding Parameters}

The system uses the periodicity of the droplet transfer of the PGMAW process. For the observation of the regularity of such processes it is sufficient to take one picture per period of the same 
process phase. In order to observe the exactly same process phase in each case (also at period times varying), the image recording is synchronized on an electrical welding parameter and is not carried out in fixed time intervals. The image recording starts after a trigger criterion is detected and an additional variable delay time (see figure 2 ).

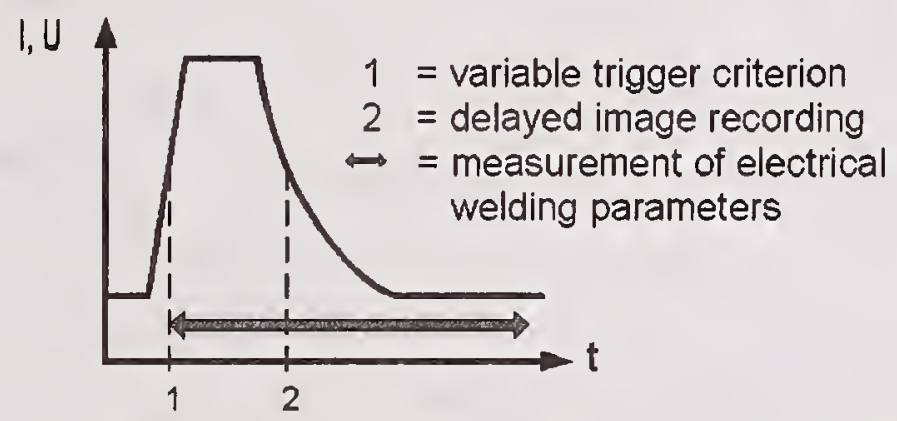

Figure 2: Procedure of image and electrical welding parameter recording

Due to the variable trigger criterion and the variable delay time, every state of the welding process (the melting of filler wire through the arc, the detachment of the drop and finally the immersion of the drop into the welding bath) can be observed in online mode, even if this state itself has no unambiguous trigger criterion.

With periodically recorded images, the virtual picture of a quasi-stationary process, i.e. of a stationary drop is generated. Irregularities within the welding process are immediately recognized as different images in a series. The influence of varying welding parameters on the process is immediately visible.

Furthermore the variable delay time between the trigger criterion and the trigger impulse for the camera in one recording series can be continuously enlarged. In this case a virtual picture of a continuous process, i.e. of a virtual droplet transfer through the sequence of the different consecutive drops, is recorded.

Simultaneous to the image recording the welding current and voltage are measured. The measurement starts at the trigger criterion and holds after a variable time within the period of the process (see figure 2).

\section{Signal Processing}

The signal processing unit allows the automatic calculation of the following typical characteristic PGMAW process parameters from the measured current and voltage signals (see figure 3 ).

" Pulse current $I_{p}$ and voltage $U_{p}$

» Background current $I_{g}$ and voltage $U_{g}$

» Mean value (of the period) of current $I_{m}$ and voltage $U_{m}$

" Pulse $t_{p}$ and background $t_{g}$ time

" Period time $t_{\text {ges }}$ and frequency $f_{\text {ges }}$

Additionally, the unit calculates specific parameters of the used welding supply (Ref. 2). The supply generates current and voltage signals that contain a so-called 'backpack' in the falling edge of the pulse (see figure 3 ). The calculated special parameters are:

" Backpack current $I_{r}$ and voltage $U_{r}$

» Backpack time $t_{r}$ 


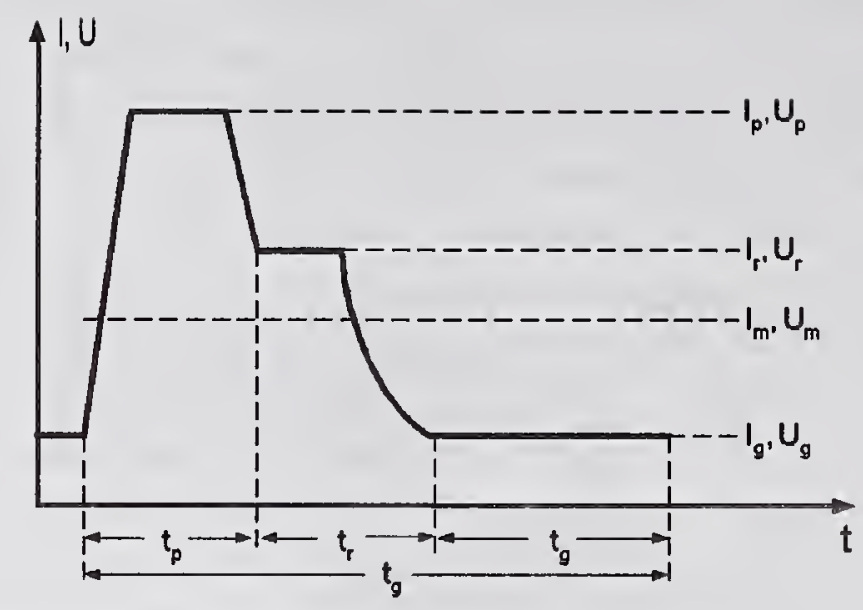

Figure 3: Calculated electrical welding parameters

\section{$\underline{\text { Image Analysis }}$}

For further conclusions onto the process the droplet transfer images are analyzed visually. The image processing unit allows the automatic detection and geometric measurement of all objects in the images and their classification. The unit is divided into the classical image processing parts preprocessing, segmentation, feature extraction and classification.

Due to the extreme brightness conditions and the characteristics of the HDRC camera, objects within the arc differ only by few gray levels from the background and objects outside of the arc clearly visible against the background (see figure 4). The images cannot be analyzed with standard image processing techniques. Pre-processing is essential. For pre-processing a procedure is necessary, which is robust against varying image contents (e.g. arc brightness). A procedure was developed, which enables the ,recognition" of the high-contrast areas that have to be maintained and low-contrast areas which have to be improved. Additionally pre-processing contains a compensation of the locally varying lighting influences, so that the result image of the pre-processing only shows the contrasts with the interesting information. The segmentation is carried out regionoriented by the calculation of an optimal gray level threshold for each image. The feature extraction part detects the objects and measures their geometry (volume, size, width, height, perimeter, among other things). With a statistical classification procedure, which characterizes the object classes by distribution densities, the objects are assigned to the given object classes (,drops", "splashes" or ,unknown") according to the criterion of the maximal probability. Figure 4 demonstrates the steps of the image analyze.
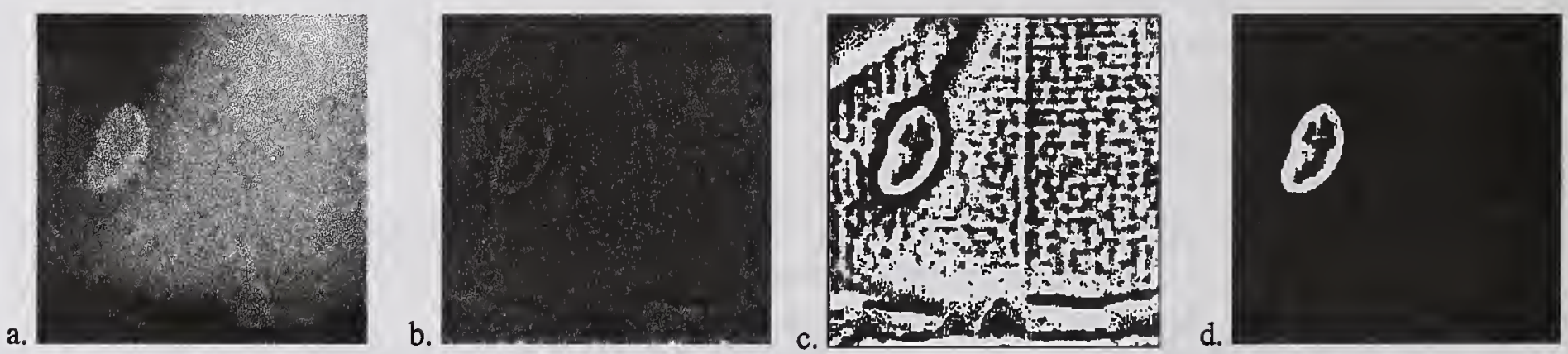

Figure 4: Image processing: a.) Original image b.) Image with contrast enhancement and shading corrections $\mathrm{c}$.) Image after threshold segmentation d.) Image with detected and classified object 


\section{Statistical Data}

The statistical unit computes the following data of all measured and calculated visual and electrical parameters of a recording sequence.

" Minimum, maximum and range

" Mean and median

" Standard deviation and variance

" Linear deviation dimension

"Coefficient of variation

" Histogram chart

" Modal value(s) of the histogram chart

"Skewness and kurtosis

" Regressions line (trend analysis)

The unit also performs sequential analysis, which allows the user to analysis and optimizes the process.

\section{$\underline{\text { Results }}$}

The efficiency of the system is illustrated by experimental data ${ }^{3}$. Figure 5 presents the quasistationary process through the recording of several different drops at the same states of the process. The first three images (figure $5 \mathrm{a}, 5 \mathrm{~b}$ and $5 \mathrm{c}$ ) of the sequence show an even droplet transfer. The drops have approximately the same size and shape and are nearly at the same position. Image $5 \mathrm{~d}$ shows the consequences of irregularities. The material transition splits up into several little drops.

a.

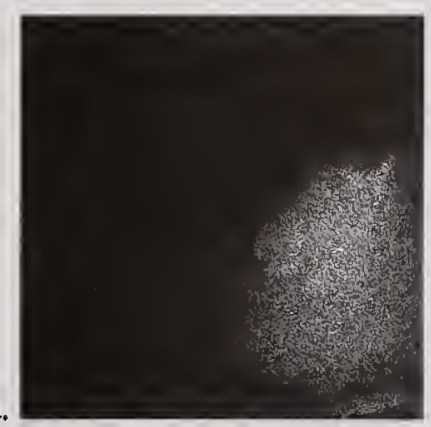

b.

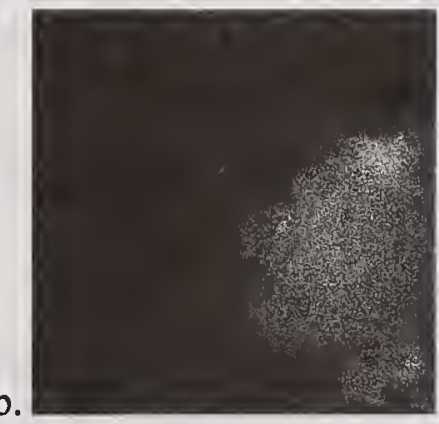

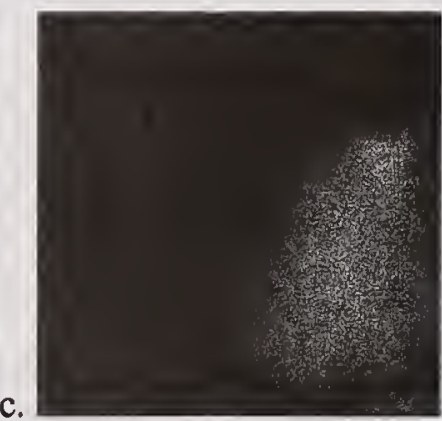

d.

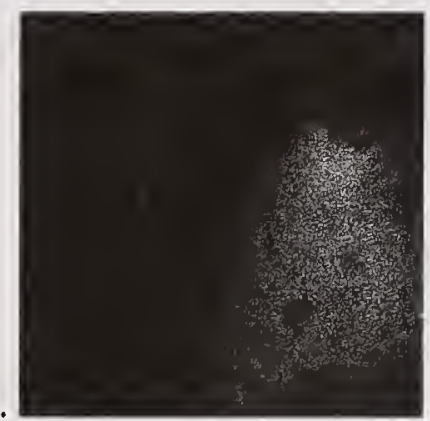

Figure 5: Quasi-stationary drop with irregularities

Figure 6 illustrates an example for all measured and calculated data of a material transition. It shows an image of the droplet transfer, results of the visual analysis, synchronized and simultaneously recorded electrical welding parameters current and voltage and calculated characteristic electrical welding process parameters. Figure 7, figure 8, table 1 and table 2 present examples for the statistical unit. The figures and tables show the mean values of the individual periods of the welding current and voltage of a sequence as well as its calculated statistical data.

${ }^{3}$ All images were recorded during weld-surfacing of unalloyed steel with a thickness of $4 \mathrm{~mm}$ and a diameter of the welding wire of $1,2 \mathrm{~mm}$. Welding was carried out using a EWM High-Tech Inverter power supply "Integral Inverter MIG 350 Pulse (Update 2.0)" (Ref. 2) and with a vertical position of the welding torch in tub position. The protective gas consists of $82 \%$ argon and $18 \%$ carbon dioxide. 


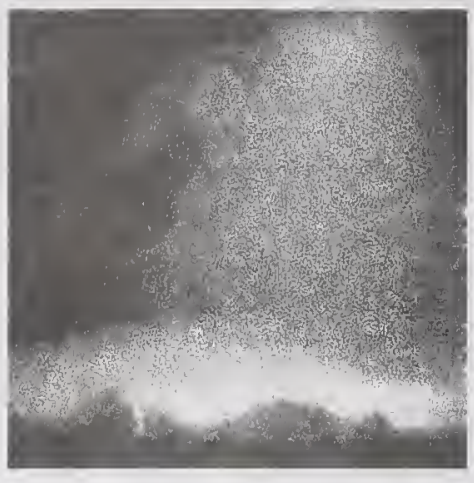

a.

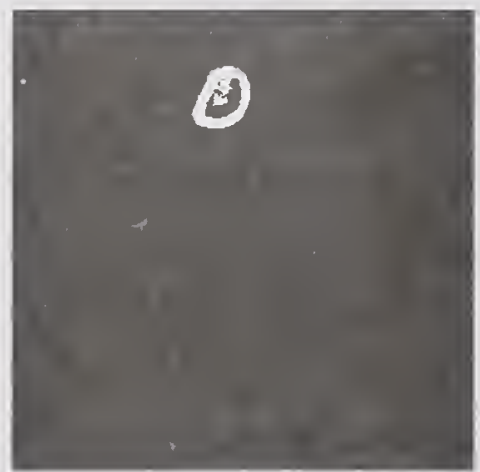

b.

\begin{tabular}{|l|l|}
\hline Object & Drop \\
\hline Width $[\mathrm{mm}]$ & 1,41 \\
\hline Height $[\mathrm{mm}]$ & 1,49 \\
\hline Size $\left[\mathrm{mm}^{2}\right]$ & 1,57 \\
\hline Perimeter $[\mathrm{mm}]$ & 4,77 \\
\hline
\end{tabular}

c.

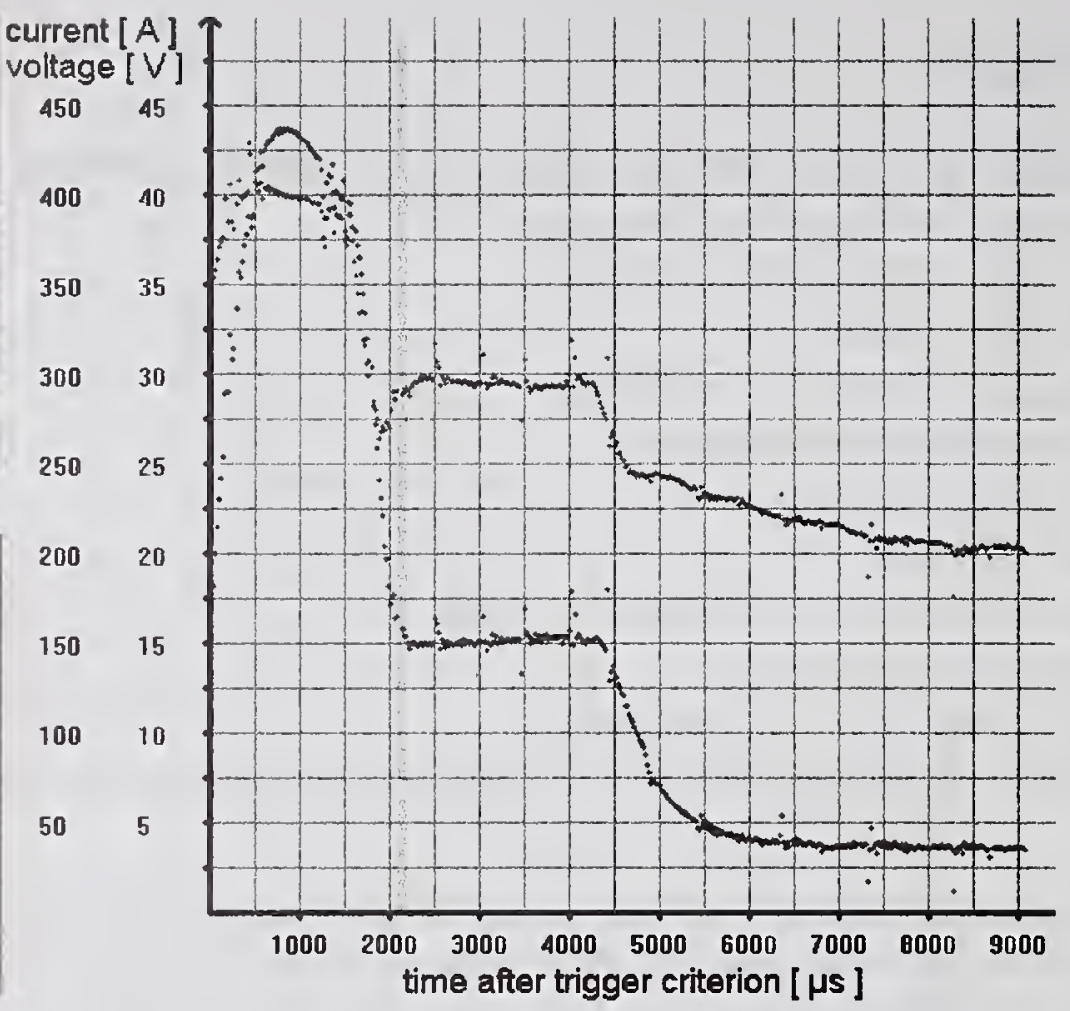

d.

\begin{tabular}{|l|l|l|l|}
\hline Current & A & Voltage & V \\
\hline Mean & 121,7 & Mean & 25,8 \\
\hline Pulse & 434,6 & Pulse & 40,0 \\
\hline Backpack & 152,7 & Backpack & 29,5 \\
\hline Background & 36,3 & Background & 20,3 \\
\hline
\end{tabular}

e.

Figure 6: Common representation of recorded and calculated data of one period a.) Image of the droplet transfer b.) Result image of the visual analysis c.) Synchronized and simultaneously measured electrical welding parameters current and voltage including the time of the image recording d.) Results of the calculation of characteristic process parameters

\section{$\underline{\text { Specifications }}$}

The capability of the system is dependent on the employed components (model of the processor, the main memory of the computer, etc.), the size of the image area and the monitoring modes (online/offline observation and display and online/offline analysis).

On a computer with two Pentium at III $850 \mathrm{MHz}$, an image area size of $256 \times 256$ pixel and the operating system Windows NT 4.0 for example

- a visual online observation and display of the droplet transfer with 160 pictures per second,

- a simultaneous and synchronized measurement of the welding current and voltage with offline display,

- offline analyses (visual, characteristic process parameters and statistical data)

- and the saving of all data in the main memory.

are possible. 
a.)

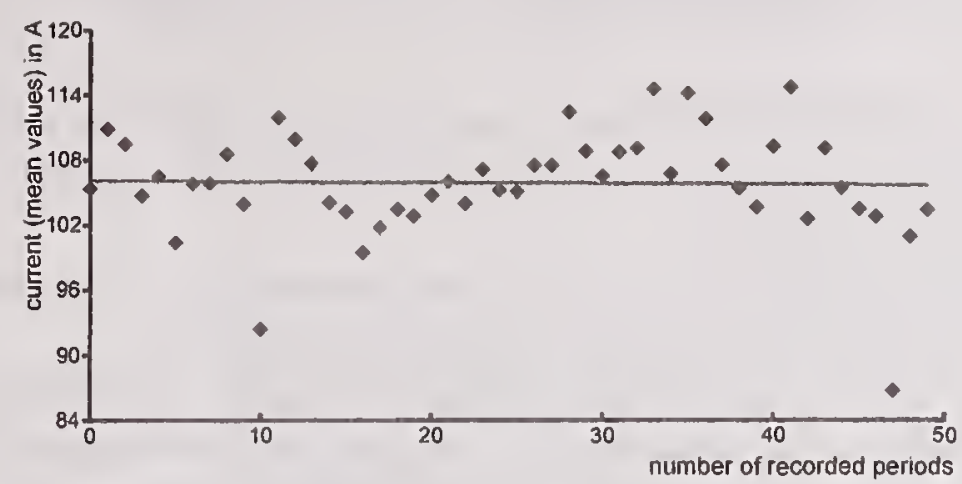

b.)

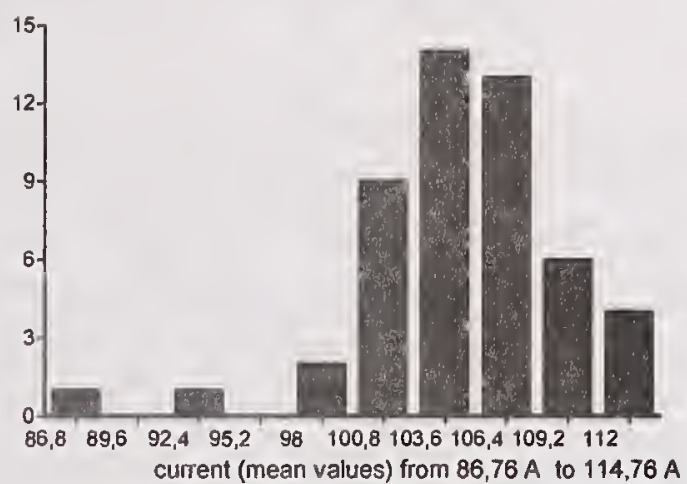

Figure 7: Mean values of the individual periods of the welding current of a recorded sequence and its calculated statistical data a.) Graph of the mean values including regressions line b.) Histogram chart c.) Statistical data (selection)

\begin{tabular}{|l|r|l|r|}
\hline Mean & $105,87 \mathrm{~A}$ & Minimum & $86,76 \mathrm{~A}$ \\
\hline Median & $105,88 \mathrm{~A}$ & Maximum & $114,76 \mathrm{~A}$ \\
\hline Modal & $104,96 \mathrm{~A}$ & Range & $28,0 \mathrm{~A}$ \\
\hline Variance & $24,42 \mathrm{~A}$ & Coefficient of variation & $0,04 \mathrm{~A}$ \\
\hline Standard deviation & $4,94 \mathrm{~A}$ & Linear deviation dimension & $3,43 \mathrm{~A}$ \\
\hline
\end{tabular}

Table 1: Statistical data (selection) of mean values of the individual periods of the welding current of a recorded sequence

a.)

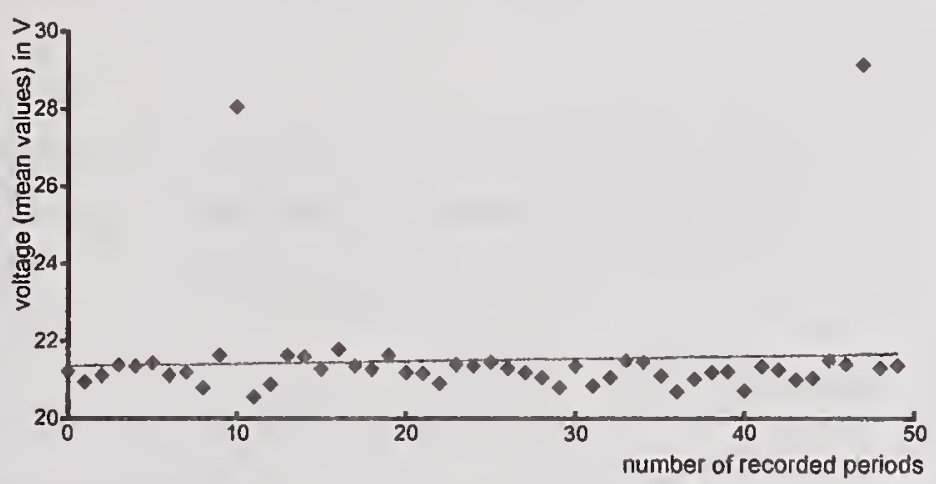

b.)

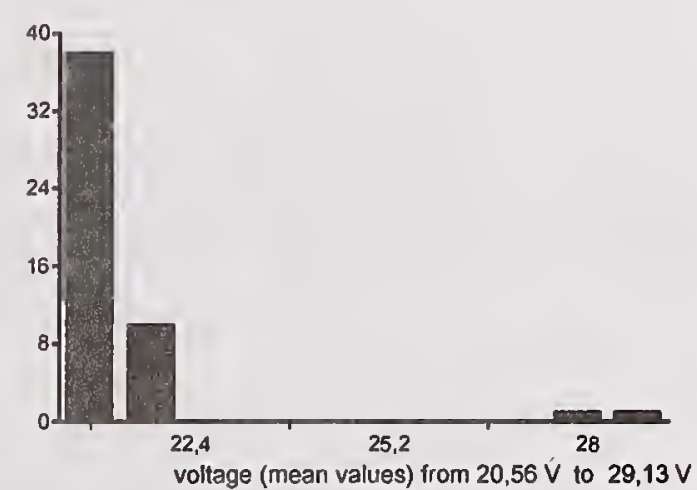

Figure 8: Mean values of the individual periods of the welding voltage of a recorded sequence and its calculated statistical data a.) Graph of the mean values including regressions line b.) Histogram chart c.) Statistical data (selection)

\begin{tabular}{|l|r|l|r|}
\hline Mean & $21,50 \mathrm{~V}$ & Minimum & $20,56 \mathrm{~V}$ \\
\hline Median & $21,25 \mathrm{~V}$ & Maximum & $29,13 \mathrm{~V}$ \\
\hline Modal & $20,98 \mathrm{~V}$ & Range & $8,57 \mathrm{~V}$ \\
\hline Variance & $2,22 \mathrm{~V}$ & Linear deviation dimension & $0,59 \mathrm{~V}$ \\
\hline Standard deviation & $1,49 \mathrm{~V}$ & Coefficient of variation & $0,06 \mathrm{~V}$ \\
\hline
\end{tabular}

Table 2: Statistical data (selection) of mean values of the individual periods of the welding voltage of a recorded sequence 


\section{SUMMARY AND CONCLUSIONS}

The sensor system described in this paper allows the online visual observation of all states of the PGMAW process, including the droplet transfer, without additional lighting. The image recording is synchronized to an electrical welding parameter.

Additionally, with the system the simultaneous and synchronized measurement of electrical welding parameters and the calculation of characteristic process parameters is possible.

Furthermore, the system is featured with an image processing unit for the automatic detection, geometry measurement and classification of the material transition. With this unit, visual 2-D features of the material transition can be directly assigned to the electrical welding parameters.

For an analysis and optimization of the process the systems computes statistical data (extreme values, mean values, standard deviation, etc.) of all measured and calculated visual and electrical data of a recording sequence.

Presently, the optimization is restricted to the online observation of the images of the droplet transfer and the offline analysis of all measured and calculated data.

The system can be used

" for the fast and economical adjustment of suitable welding parameters prior to manufacturing,

》 to test different combinations of parameter settings, filler metals, inert gases, etc.,

" and the development of welding devices.

\section{ACKNOWLEDGMENT}

This project is supported by the German Federal Ministry of Economics and Technology (BMWi) via the "Arbeitsgemeinschaft industrieller Forschungsvereinigungen (AiF)" (No. 12583 $\mathrm{N} / 1)$.

\section{REFERENCES}

1. Allemand, C.D.; Schoeder, R.; Ries, D.E.; and Eagar T.W. 1985. A Method of Filming Metal Transfer in Welding Arcs, Welding Journal (64) (1): 45-47.

2. EWM High-Tech Precision, Mündersbach, Germany. Welding supply type: Integral Inverter MIG 350 Pulse (Update 2.0).

3. Cheremisinoff, N. P. 1987. Practical Statistics for Engineers and Scientists, Technomic Publishing Company, Lancaster. 


\title{
THROUGH THE ARC TRACKING OF 5G NARROW GAP PIPE WELDS
}

\author{
T.S. Rajagopalan*, P.A. Tews*
}

\begin{abstract}
A through-the-arc joint tracking system was developed for use with mechanized orbital pipeline welding equipment designed to weld pipeline girth welds in the $5 \mathrm{G}$ position. Although through the arc joint tracking has been successfully applied to lap and fillet welds in the $1 \mathrm{G}$ and $2 \mathrm{G}$ positions, and is offered by many robotic manufacturers, through the arc tracking of pipeline girth welds in the $5 \mathrm{G}$ position is new. The system was successfully used to aid welders in welding over 8200 joints of 42 inch $(1.06 \mathrm{~m})$ diameter .417 ", .500 " and $.600 "(10.6 \mathrm{~mm}, 12.7 \mathrm{~mm}$, and $15.2 \mathrm{~mm}$ ) wall thickness grade $X-70$ line pipe during the summer of 2000 . The mechanized equipment utilized the solid wire pulsed gas metal arc process with Argon- $\mathrm{CO}_{2}$ shield gas. The joint design was a compound bevel narrow gap with 5-degree sidewalls. Voltage and current waveforms were recorded along with torch position and timing signals using a high-speed multichannel data acquisition system developed specifically for this research. Analysis of the recorded waveforms enabled the sample timing to be optimized. The recorded waveforms show the effect of joint depth and oscillation width on the arc voltage waveforms. Provisions were made to vary the torch to work target value as a function of position around the pipe, but it was found that this feature was unnecessary. Provisions were also made to vary the sensitivity and speed of correction for each weld pass, but this too was found to be unnecessary. Optimum sensitivity and maximum correction values were developed that worked for all fill passes. This reduced weld repairs on this project by enabling the use of an optimum oscillation width. Too wide an oscillation width caused excessive sidewall melting, which increased weld pool fluidity, creating an improper weld contour in the vertical position. Too narrow an oscillation width caused lack of sidewall fusion defects. Through the arc joint tracking eliminated these problems by centering the torch oscillation pattern. This enabled the use of an oscillation width wide enough to eliminate lack of sidewall fusion defects, but narrow enough to minimize dilution
\end{abstract}

\section{KEYWORDS}

Mechanized welding, Through-the-arc tracking, GMAW-P

\section{INTRODUCTION}

In mechanized welding of cross-country pipelines, guide bands are temporarily clamped around the outside of the pipe near the joint to be welded. Devices referred to as welding bugs are temporarily clamped onto the guide bands. The welding bugs travel around the pipe on the band. The bugs have a wire feeder mounted either on the bug or nearby. When the wire feeder is mounted on the bug, small (10 pound or less) spools of welding electrode are carried on the bug. When the wire feeder is mounted nearby, such as on the wall of a welding shelter, 30 pound

\footnotetext{
*CRC-Evans Automatic Welding, Houston, TX, 77086
} 
spools of welding electrode are used. In this case, the wire feeder feeds the welding electrode through a cable-hose to the welding torch on the bug. The bugs also provide a means for oscillating the torch across the joint, either using a shuttle type oscillator or a pendulum type oscillator. If a shuttle type oscillator is used, the center of oscillation is controlled by the shuttle. If a pendulum oscillator is used, an additional motorized horizontal slide must be provided to move the center of oscillation to the right or left. The pendulum oscillator itself cannot be used, because the angle between the torch and the two joint sidewalls must be equal. Welding bugs typically have a motorized axis for moving the torch toward or away from the surface of the pipe to maintain the desired contact-tip-to-work distance.

In pulsed arc welding with a constant wire feed speed, the welding power supply varies the voltage at the contact tip to maintain the desired pulsed current waveform. When the contact tip to work distance increases, the welding power supply increases the voltage applied to the contact tip in order to maintain the desired current waveform. Conversely, when the contact tip to work distance decreases, the welding power supply decreases the voltage applied to the contact tip so the welding current will not increase beyond the desired waveform. This relationship can be used to provide a measure of both the contact tip to work distance and the center of the oscillation pattern with respect to the sidewalls. By comparing the measured arc voltage as it sweeps across the center of the joint to a target arc voltage, the result of the comparison can be used to move the torch upward or downward to constantly balance the measured arc voltage against the target voltage. Similarly, the voltage measured when the torch is melting one sidewall can be compared to the voltage measured when the torch is melting the other sidewall, and this comparison used to maintain the center of oscillation equally between the joint sidewalls. The pulsed arc process was used in the work reported on in this paper.

In short arc welding with a constant wire feed speed and constant potential welding power supply, the welding current increases as the contact tip to work distance decreases. The same principals described above can be applied to measurements of welding current to maintain a desired contact tip to work distance and maintain the center of oscillation equally between the joint sidewalls.

\section{PROCEDURE}

Welding was carried out on 42 " $(1.06 \mathrm{~m})$ diameter 0.563 inch wall grade X70 pipe using $1.2 \mathrm{~mm}$ ER70S-6 welding electrode with $85 \%$ Argon- $15 \% \mathrm{CO}_{2}$ shielding gas. As stated above, the gas metal arc pulsed welding process was used. The electrode was fed at a constant rate for each fill pass. Travel speed was constant for all except the last pass. A manual travel speed override of $+/-25 \%$ was permitted for the last pass to accommodate variations in joint gap, which affect joint fill. Remote wire feeders were used with 12 foot cable hoses and water cooled welding torches. Welding electrode was supplied on 30 pound spools. Figure 1 shows the welding shelters used on this project. The shelters rest on the pipe, and contain the welding bugs, wire feeders, and control cabinet. The welding power supplies were mounted on the rear deck of the shelter support tractor, and powered by an alternator driven by the tractor power take-off.

Each of the welding bugs had two welding torches. Each torch was independently oscillated across the joint by means of a shuttle type oscillator driven by a brushless DC servo motor and 
ball-nut lead screw. The torches were moved towards or away from the joint by means hollow shaft stepper motors with brass nut lead screws.

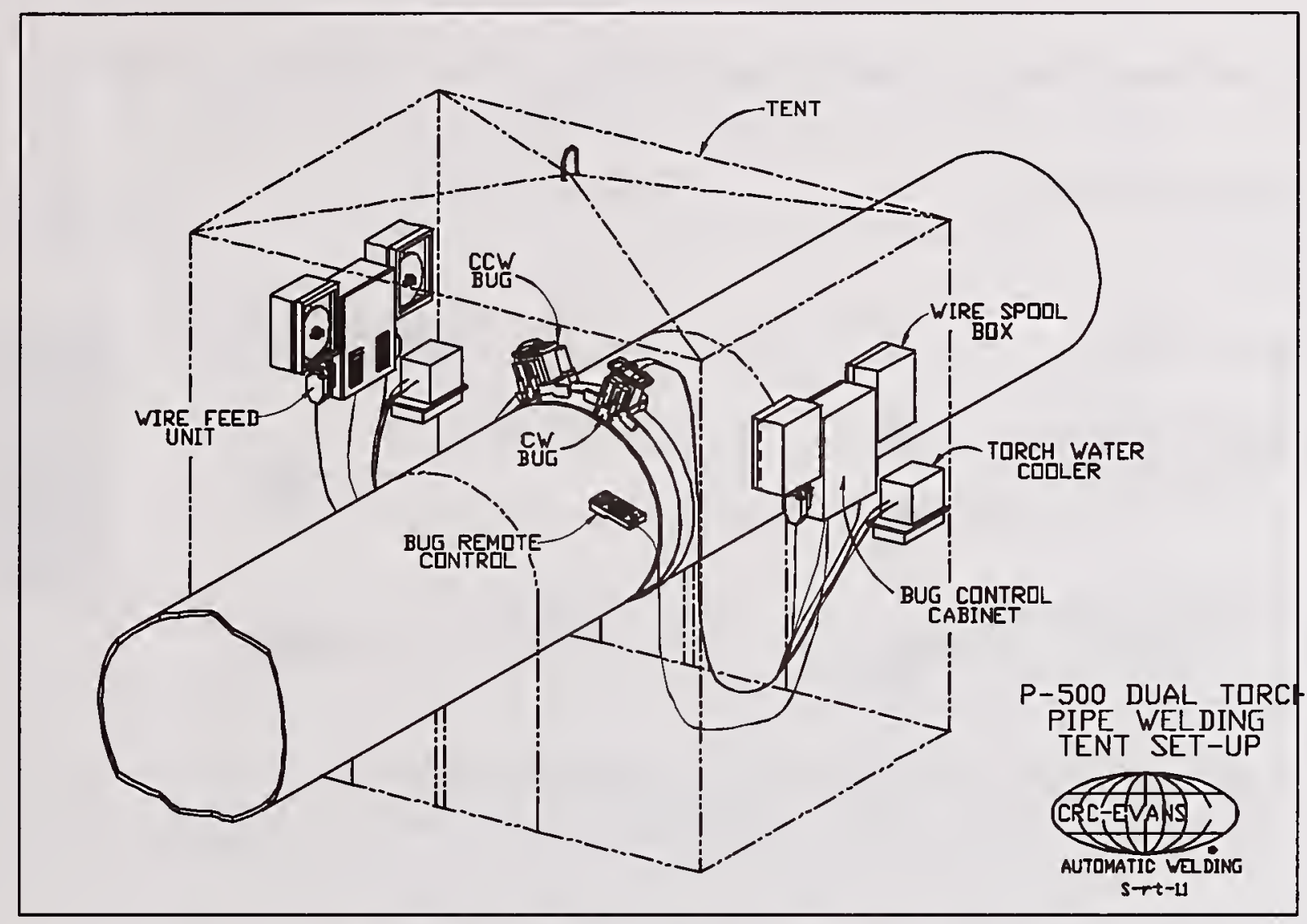

Figure 1 Typical Welding Shelter with Pipe, Bugs, and Control Cabinets

Figure 2 is a block diagram of the overall bug control system. Five host microprocessors with peer-to-peer communications were used in the system. The host microprocessor used was an Intel $80 \mathrm{C} 152$, a variety of the popular 8051 family of microprocessors, with an on board CSMACD serial communications channel. An RS-485 type twisted pair communications link operating at 1.3 megabaud connected the host microprocessors to one another. There were two stacks of boards in the control cabinet for controlling the wire feeders, travel motor, oscillating motors, and vertical adjust motors for the two torches. Each stack contained a power supply board, host CPU board, and four daughter boards. One daughter board controlled the encoder motors used for wire feed and travel. A second daughter board controlled the stepper motors used for vertical adjust. A third daughter board controlled the bushless DC motor used to oscillate the torch across the joint. The fourth daughter board digitized the arc voltage and arc amperage from the associated torch. A third host microprocessor was built into the hand held pendant used to operate the welding bug. Two more host microprocessors located on the support tractor had daughter boards connected to them for controlling the welding power supplies and monitoring the arc voltage and current for quality reporting.

Figure 3 shows a block diagram of the board used to control the brushless DC motor for torch oscillation. The board contained an Atmel AT-89S8252 microprocessor, a Precision Motion Devices MC-1231 motion control chipset, and Linear Devices LT-11581 FET drivers. The Atmel controlled the PMD chipset, overseeing the oscillation motion, recomputing the trajectory in response to host commands to change rate, width, torch center of oscillation, right dwell, or 


\section{BLOCK DIA GRAM OFP-50O CONTROL SYSTEM}

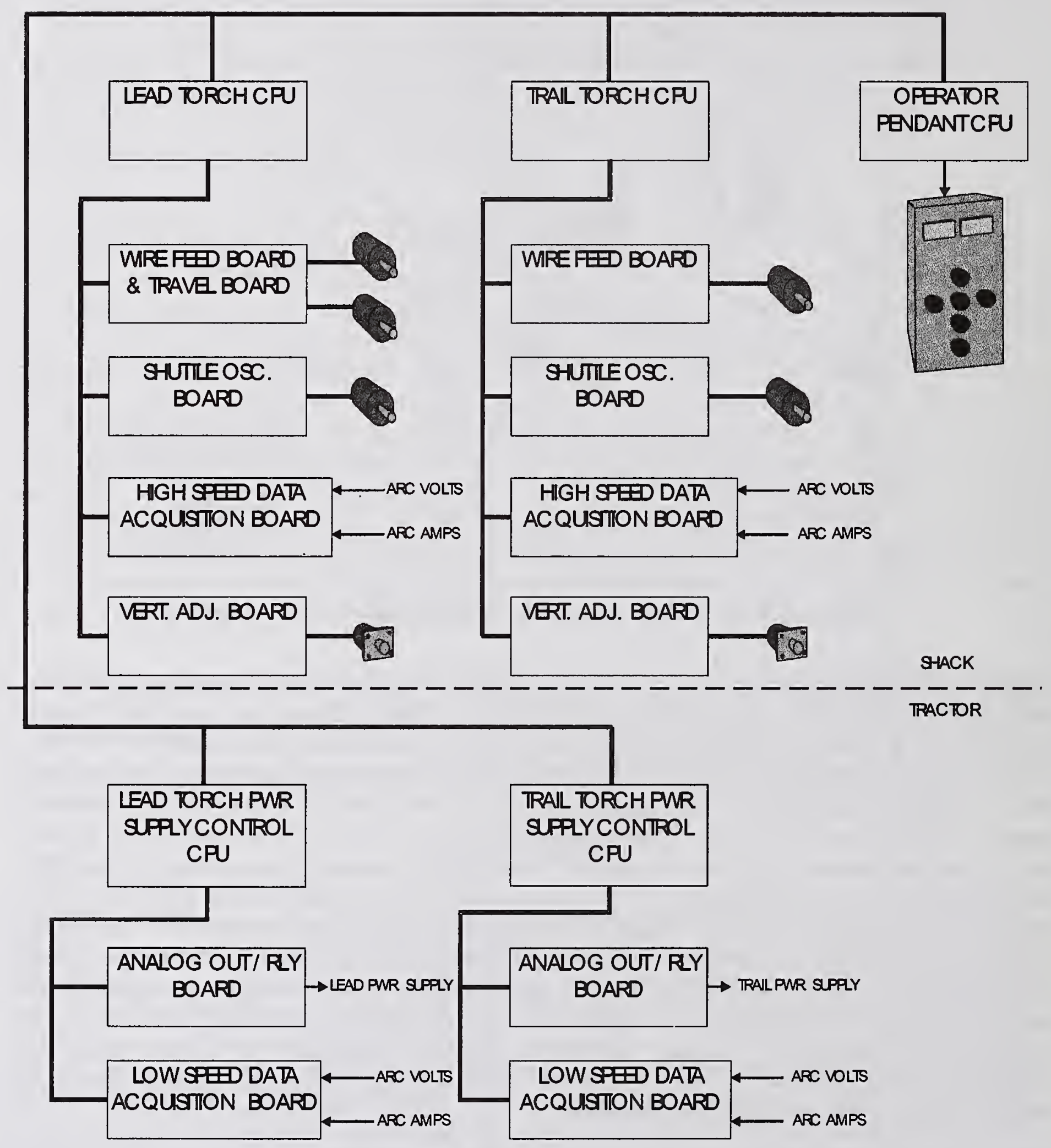

Figure 2 Block Diagram of Control System

left dwell. The Atmel microprocessor set bits in a status word when the oscillator reached programmable percentages of the cross seam distance. The host microprocessor interrogated the Atmel microprocessor 1000 times per second to detect these status word bit transitions. When 
detected, the host scheduled the appropriate tracking task, which would normally read the analog to digital converter data or start the analog to digital converter.

\section{BLOCK DIAGRAM OF SHUTTLE OSCILLATOR BRUSHLESS DC SERVO BOARD}

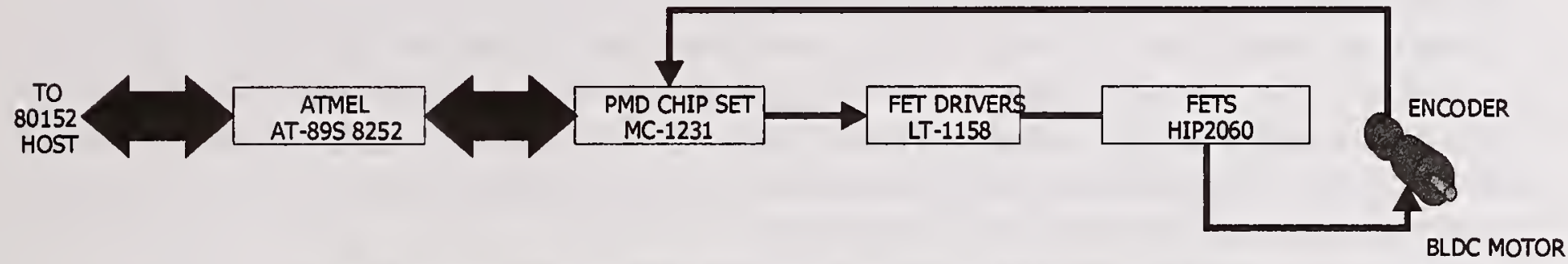

Figure 3 Block Diagram of Oscillator Daughter Board

Figure 4 is a block diagram of the arc voltage and current measurement daughter board. Analog values from sense wires and shunts were sampled 40,000 times per second and digitized using Hewlett Packard HP7800 isolation amplifiers and a 12 bit Analog Devices LM12454 converter. An Analog Devices ADSP2181 Digital Signal Processor processed the converted values. The board sampled the data for the commanded length of time, and returned the processed values of the sampled data when queried. One type of processing performed was excluding sampled arc voltage readings from the average when sampled arc current was below peak levels.

\section{BLOCK DIAGRAM OF HIGH SPEED DATA ACQUISITION BOARD}

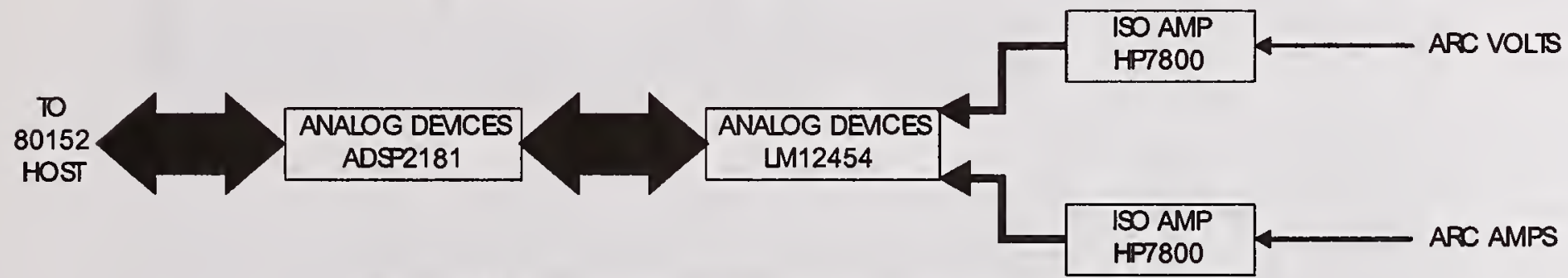

Figure 4 Block Diagram of Data Acquisition Board

The host microprocessor compared the center samples to the target voltage, and used the error times a scaling factor to command the torch to work motor to raise or lower the torch. The computed move limited to a programmable maximum value per sweep. The computed move command was based solely on the proportional error, without integral or derivative terms.

Studies were carried out to determine the sampling window that would optimize the comparison of the voltage drops that occur as the torch and welding electrode approach each sidewall of the joint. To carry out this research, an eight-channel analog to digital converter board from National Instruments was purchased. Isolation amplifiers were designed and built to protect the analog to digital converter board from the welding arc voltage, and to allow arc voltage, arc current, digital timing signals, and analog processes signals to be measured simultaneously. 
Timing signals were provided by the Atmel microprocessor on the brushless DC servo motor board that controlled the torch movement. Arc voltage was measured at the arc, and arc current was measured close to the arc by means of a clamp-on Hall Effect current probe. Processed analog values were obtained from a digital to analog converter connected to a serial output on the ADSP2181 digital signal processor. Waveforms were stored on disk, and were printed out on a laser printer.

Trials were also conducted to determine optimum attenuation factors and maximum move factors for vertical and horizontal tracking. Tracking was given time to stabilize and then a step change was made to either the cross seam or vertical adjust motor. The response time and overshoot of the cross seam and vertical adjust axis were measured by means of linear potentiometers attached for this purpose. The optimum values for attenuation and maximum correction per sweep were selected and used on the pipeline welding project.

\section{Results}

Figure 5 shows a typical waveform obtained using the national instruments data acquisition system. The top trace is the arc voltage, the second trace down is the arc current, the third trace down is the "late indicator", the bit set by the brushless DC servo motor board Atmel chip indicating the torch has reached a programmable percentage of its sweep. The bit goes low after the host CPU detects it has gone high and has sent a command back to the Atmel to clear the bit. The next trace down is the right wall indicator, another bit set by the Atmel when the torch has reached its extreme point of oscillation in one direction. The trace below the right wall indicator

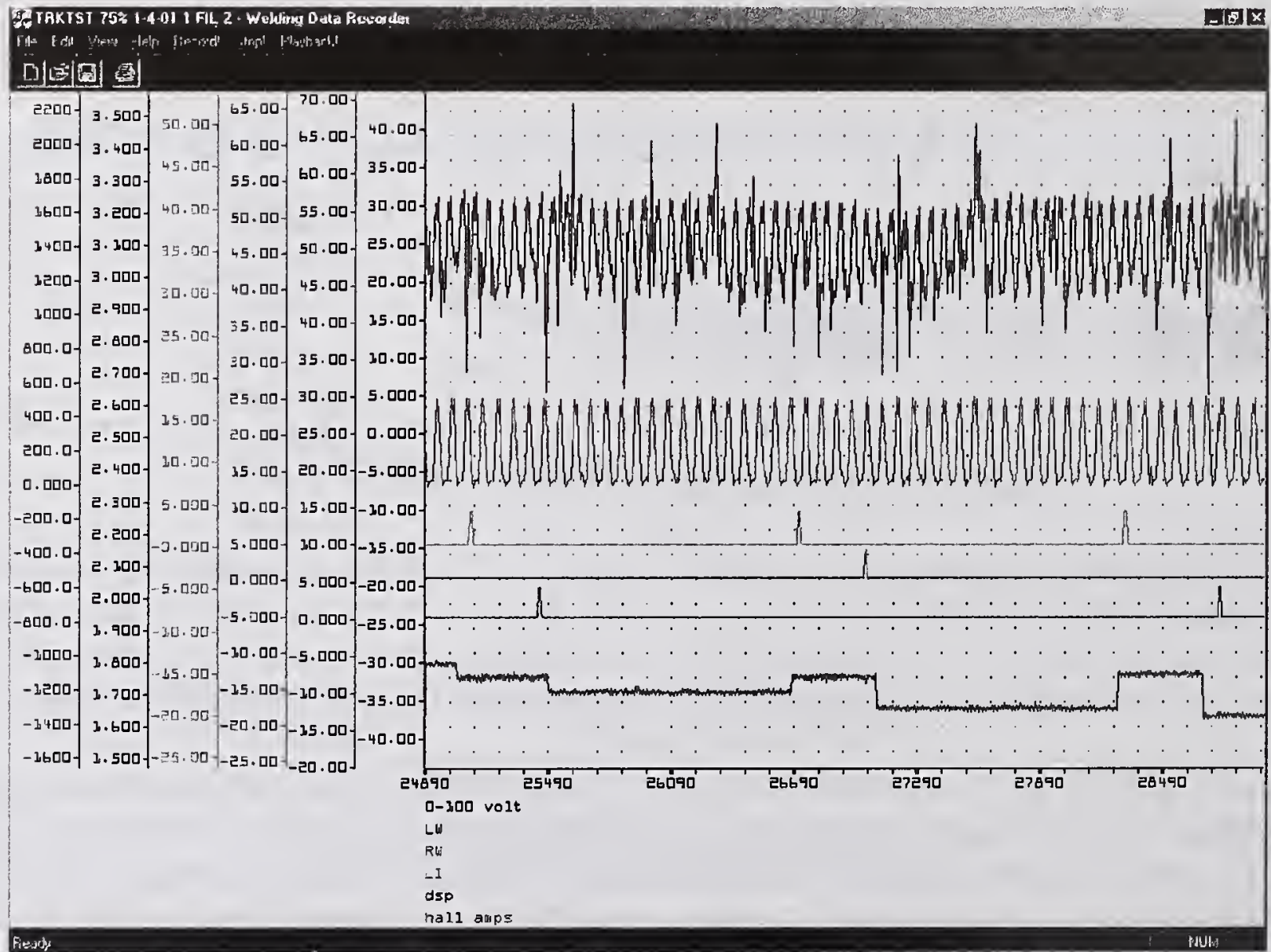

Figure 5 High Speed Data Acquisition Traces 
is the left wall indicator. The bottom trace is the analog value of the result of the processing done by the high speed data acquisition board. The data was taken at a rate of 180 oscillations per minute. The time period between the right and left wall timing signals was 333 milliseconds. The pulse frequency was about $135 \mathrm{~Hz}$. The short segments of the bottom trace represent the value of the processed arc voltage signal measured from the end of one sidewall to the start of the opposite sidewall measurement. Because these represent the torch to work measurement, they are nearly equal in value. The longer segments of the bottom trace represent the value of the processed arc voltage measured while the torch approached the sidewall. The values are lower because the contact tip to work distance decreases as the torch approaches the side wall.

Figure 6 shows the effect of a step change to the cross seam position. The top trace is arc voltage, the second, third and fourth traces are the late indicator, right wall, and left wall indicators respectively. The second trace from the bottom is the processed arc voltage measurement for the previous period. The bottom trace is oscillation motion measured by a linear potentiometer. The center of oscillation was intentionally shifted 0.010 inches after the tracking had stabilized. The time for the oscillation pattern to return is approximately seconds, or 18 oscillations. Figure 8 shows the result of excessive response to the difference in sidewall measurements. The system overcorrected in an under-damped fashion.

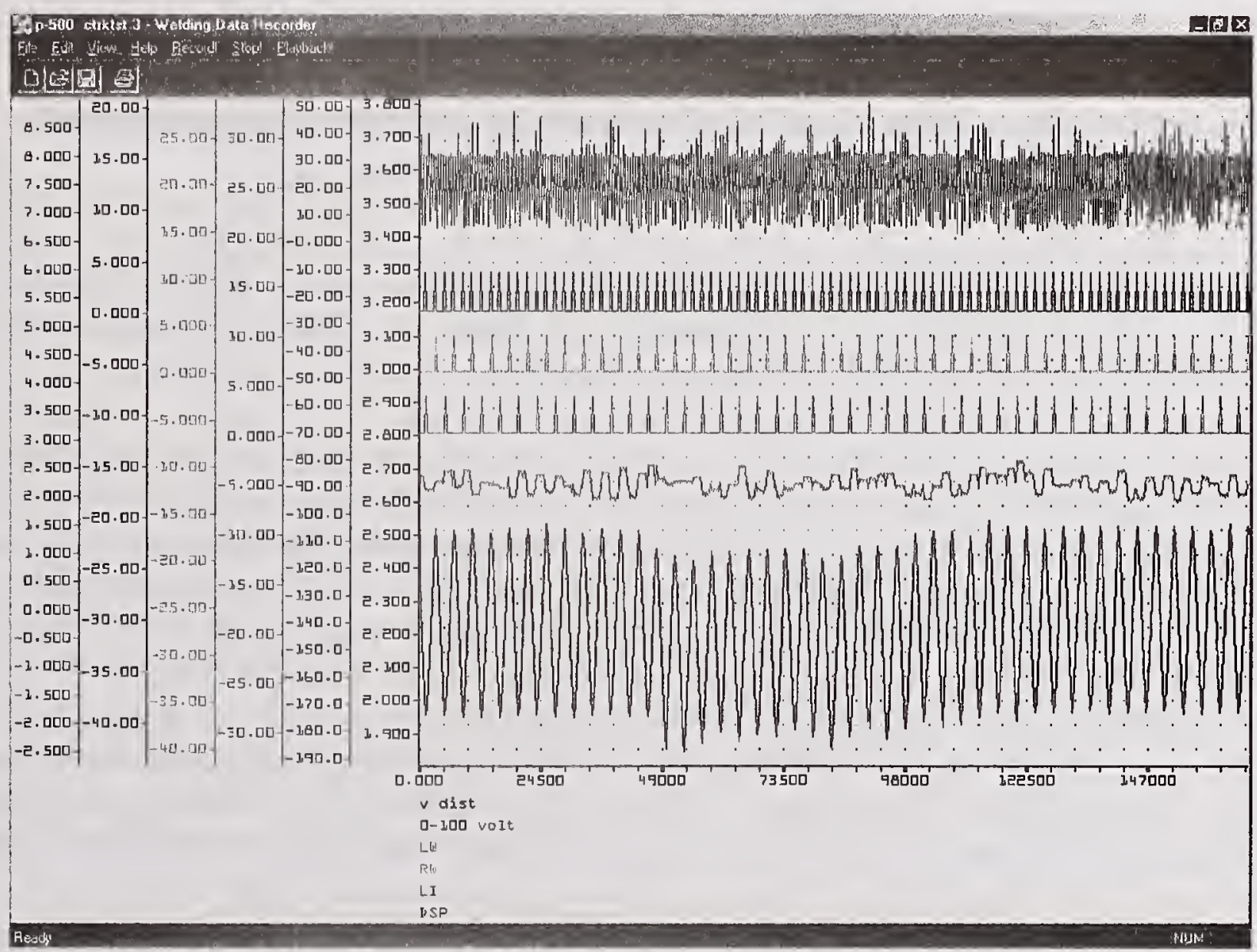

Figure 6 Critically Damped Cross Seam Tracking

\section{Discussion}

The 12 bit analog to digital converter provides a resolution of arc voltage of 0.024 volts per bit. The scale factor applied to the torch to work error was equivalent to 0.125 inch of movement per 


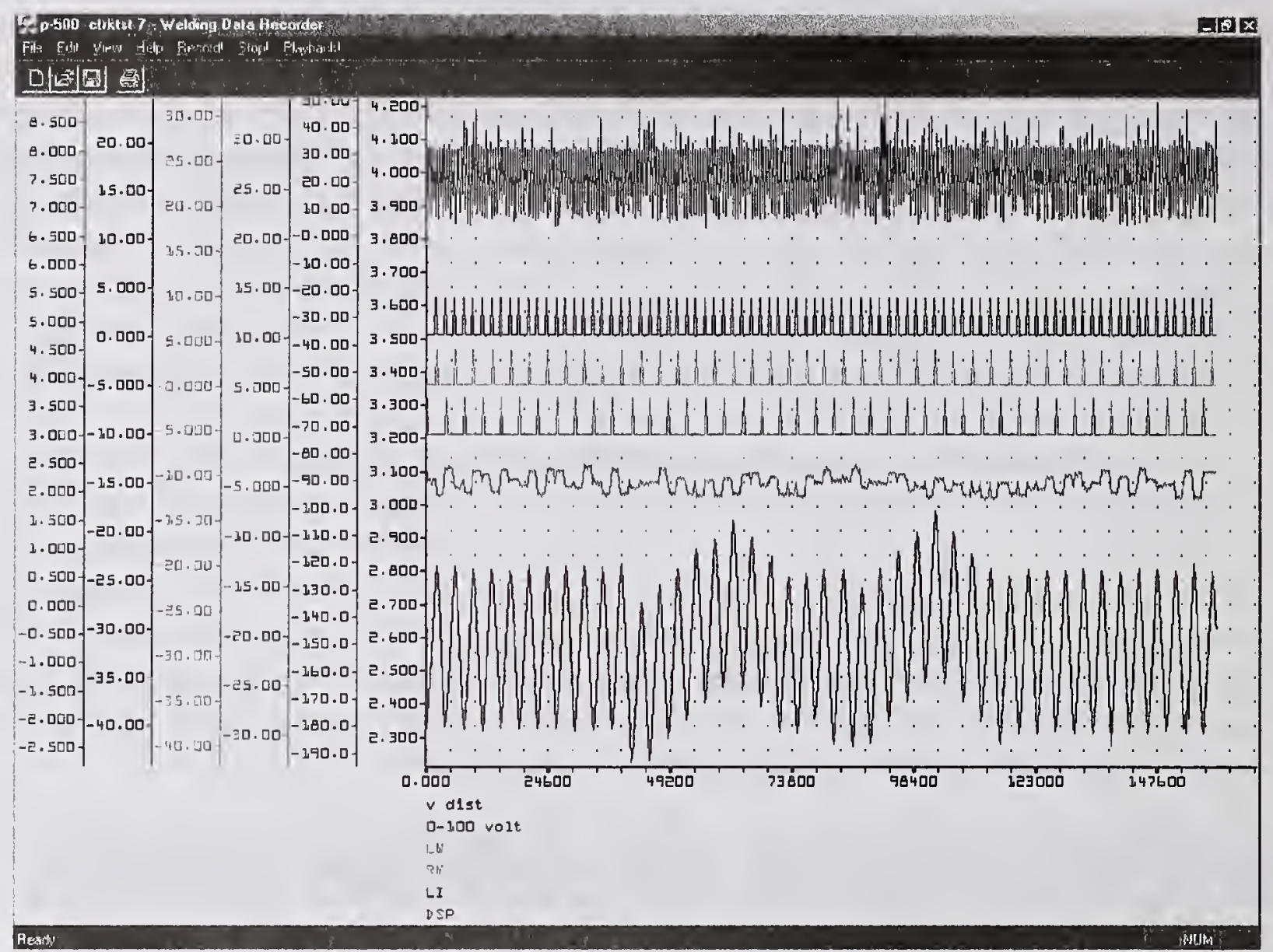

Figure 7 Under Damped Cross Seam Tracking

2 volt difference between measured arc voltage and target arc voltage. This scale factor was attenuated to achieve the damped response shown in figure 6 . The under damped response shown in Figure 7 occurred when no attenuation was used.

Prior to introducing through the arc tracking on the project site, manually guided dual torch bugs were welding 90 joints per day with a $9 \%$ repair rate. After introducing through the arc tracking, the repair rate dropped to less than $4 \%$. The most common weld defect that required repairs without through the arc tracking was lack of sidewall fusion in the second and third fill passes. The engineering critical assessment required welds with small stacked lack of sidewall fusion defects be repaired. The cause of this defect was excessive puddle fluidity caused by excessive weld metal dilution. The excessive weld metal dilution was in turn caused by setting oscillation width wide in an attempt to insure sidewall fusion regardless of the torch not being centered in the groove. Through the arc tracking allowed the use of a width that was just wide enough to touch both sidewalls by maintaining the torch oscillation pattern centered between the sidewalls.

\section{SUMMARY}

A through the arc seam tracking system has been developed and applied to a dual torch external welding bug used for cross country pipe line girth welding. The system was successfully used to weld 122 miles $(196 \mathrm{Km})$ of 42 inch $(1.06 \mathrm{~m})$ diameter grade X-70 pipe. Use of through the arc seam tracking reduced weld repair rate from the $9 \%$ to less than $4 \%$. 


\title{
DEVELOPMENT OF ROTATING GMA WELDING SYSTEM AND ITS APPLICATION TO ARC SENSORS
}

\author{
C.-H. Kim* and S.-J. Na*
}

\begin{abstract}
The sensitivity of rotating arc sensor is related with the dynamic wire melting due to the insufficient self-regulation of the arc length. This paper presents the dynamic simulation of wire melting by using the variable space network method and by modeling the heat flux from the molten end of the wire into the electrode. A new type of arc rotation mechanism with a hallowshaft motor was devised to implement a high-speed rotating arc and used to develop the arc sensor.
\end{abstract}

\section{KEYWORDS}

GMA welding, Arc Sensor, Dynamic Wire Melting, Rotating Arc

\section{INTRODUCTION}

Through-arc-sensing is widely used for automatic seam tracking because of its many advantages such as the possibilities for real time control, no auxiliary parts around the welding torch, no need for maintenance and low cost. The arc sensing method uses the electrical arc as a sensor and is based on signal variations as a function of the CTWD (contact-tip-to-workpiece distance). Therefore it is generally necessary to weave or rotate the welding torch to intentionally stimulate the differences in the CTWD. With the conventional torch weaving method the upper limit of oscillation frequency is about $4-5 \mathrm{~Hz}$ owing to the mechanical restraint. The arc rotation method enables a high-speed rotation of arc over several tens of Hz. In Japan and Germany, arc rotation mechanisms that rotate the electrode nozzle by an external motor have been developed (Ref. 1-2). However, accessibility to the joint location may be limited by the external motor system attached to the welding torch.

Self-regulation in GMAW occurs due to using a constant voltage power source. The arc length remains approximately constant for variations in the CTWD, because the time constant of the self-regulating process is shorter than the torch weaving rate in conventional GMA welding (Ref. 3 ). However, the self-regulation of the arc length is not fully performed in high-speed rotating GMAW owing to a rapid movement of the welding torch. Consequently the rotating arc sensor operates in a dynamic state and the sensitivity of the sensor is greater than that of the conventional weaving arc sensor.

In a static state, the electrode melting model was confirmed experimentally by Lesnewich in 1958 , and was proved theoretically by Halmoy in 1979 and experimentally by many researchers.

\footnotetext{
* Dept. of Mech. Eng., Korea Advanced Institute of Science and Technology (KAIST), 373-1 Kusung Dong, Yusong Gu, Taejeon, Korea (South)
} 
Recently, dynamic wire melting models have been developed using an energy balance approach which uses an 'action integral' and neglecting the heat conduction along the electrode wire (Ref. 4). However, Kim et al. addressed that the Peclet number is not high enough to neglect the thermal conduction (Ref. 5). They modeled the heat transferred to the melting tip of the solid electrode from two sources : the heat transferred from the molten end of the wire, and the heat directly delivered to the solid electrode by electron condensation.

In this study, a dynamic model of the GMA welding process and an arc rotation mechanism were developed for high-speed rotating arc welding. The mathematical model includes :

(1) dynamic model that predicts the electrode melting by considering the heat conduction in the electrode and the heat transfer by condensing the electrons directly from the welding arc to the electrode

(2) heat transfer model that describes the heat from the molten end of the electrode wire to its solid ends

(3) simplified weld pool model under assumption that the arc rotates very rapidly

The developed arc rotation mechanism was used to improve the weld quality and to develop an automatic seam tracking sensor. It could improve the accessibility to the joint and adaptability to the conventional welding torch system.

\section{ARC ROTATION MECHANISM}

A schematic diagram and a photograph of the developed arc rotating mechanism are shown in Fig. 1. The mechanism includes a hollow-shaft motor, an eccentric tip and 3 carbon brushes. The electrode wire is deflected circularly by an eccentric tip that is rotated by the hollow shaft of rotating motor and galvanized through the carbon brushes. This mechanism can be installed inside the electrode nozzle and connected directly to a conventional torch system. The weight and size of the nozzle can be reduced considerably by using an adequately small motor.

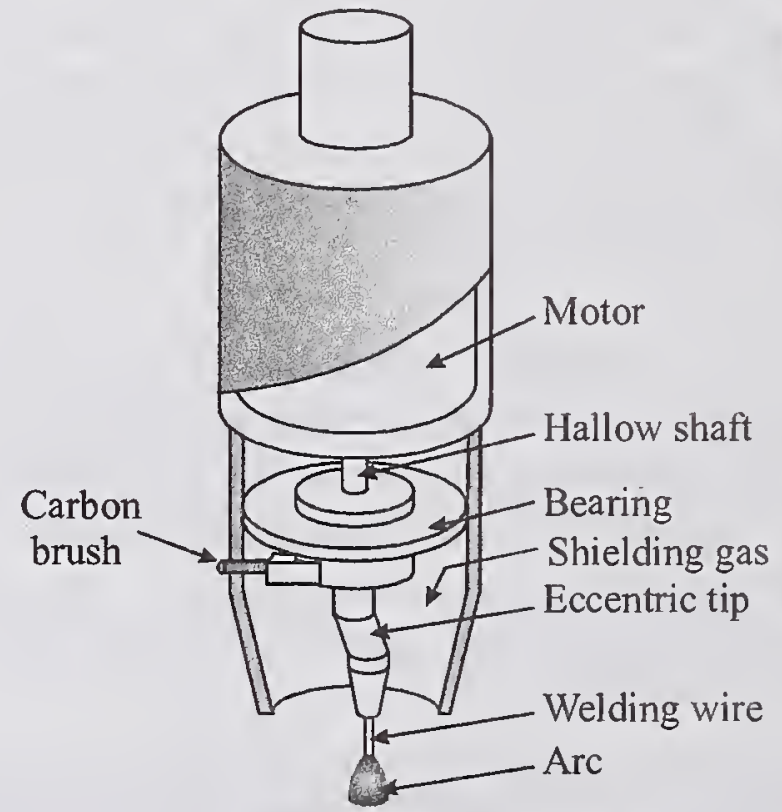

(a) Schematic diagram

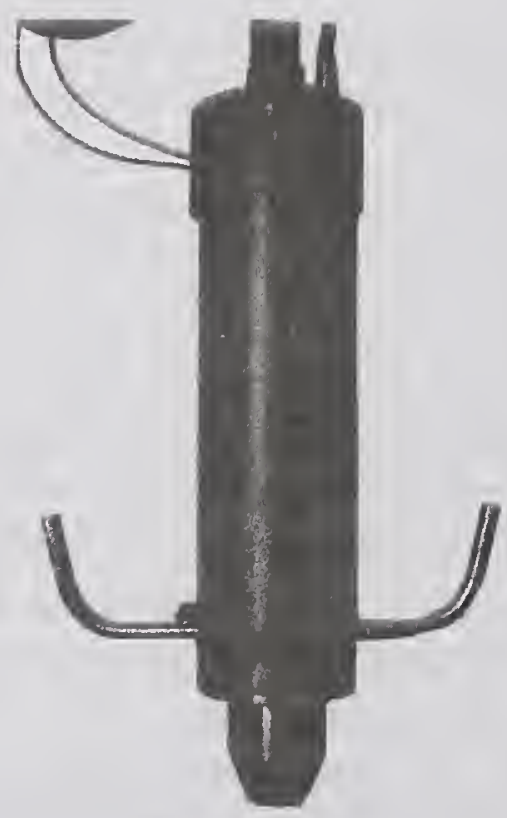

(b) Photograph

Figure 1: Devised rotation mechanism 
The arc positions are defined in Fig. 2(a) and the images of $20 \mathrm{~Hz}$ rotating arc taken by a highspeed camera at various arc positions are shown in Fig. 2(b). The arc length seems to be shorter at $\mathrm{C}_{\mathrm{r}}$ than at $\mathrm{C}_{\mathrm{f}}$, because the molten pool is formed at the position of $\mathrm{C}_{\mathrm{r}}$.

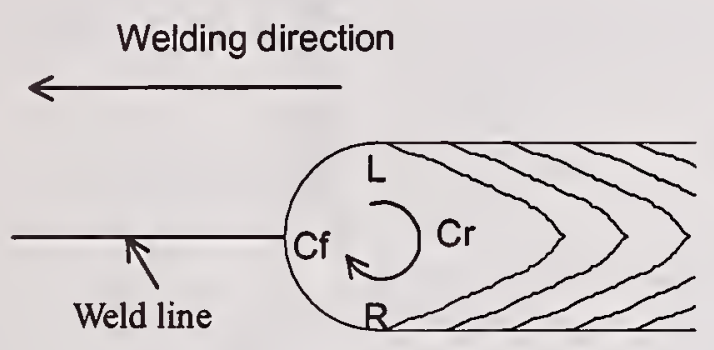

(a) Position of arc

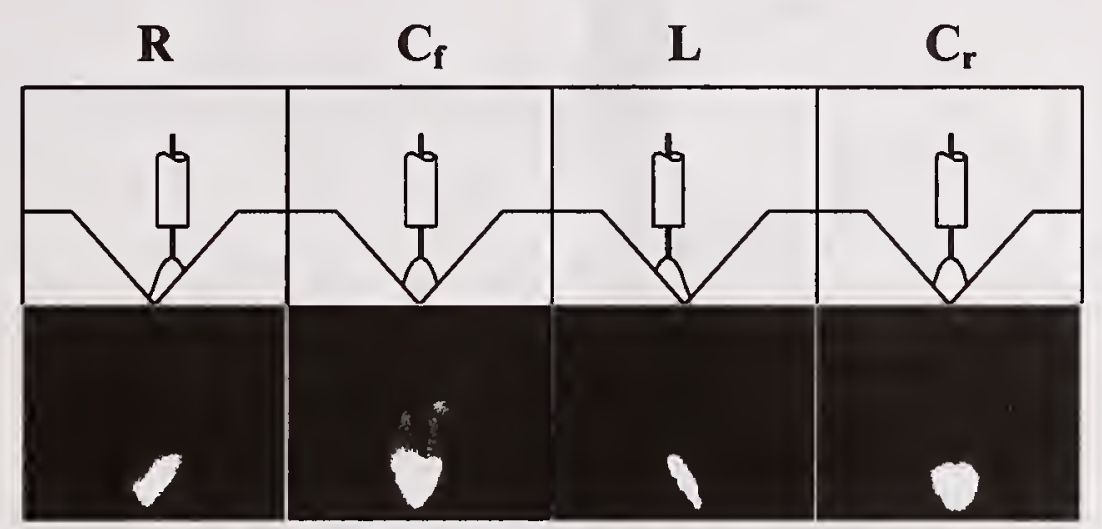

(b) Images taken by high-speed camera

Figure 2: Arc shapes at various arc positions

\section{MODEL OF GMA WELDING SYSTEM}

Figure 3 shows arc heating at the electrode and the equivalent electrical circuit of conventional GMA welding system having a power source with constant-voltage characteristics. For a dynamic state the one-dimensional conduction equation in electrode wire is given by

$$
\frac{\partial}{\partial z}\left(k(T) \frac{\partial T}{\partial z}\right)+\rho_{e}(T) j^{2}+F=C_{p}(T) \rho(T)\left(w \frac{\partial T}{\partial z}+\frac{\partial T}{\partial t}\right)
$$

and the dynamic wire melting model is given by the following expression.

$$
-k(T) \frac{\partial T}{\partial z}+\frac{Q_{m}(t)}{S}=\left(w-d L_{e} / d t\right) \Delta H \rho(T)
$$

In Eq. 2, $Q_{m}$ can be calculated from the temperature distribution of the electrode and Waszink's experimental results (Ref. 6). From Fig. $4, Q_{m} / I$ shows a linear relation with the Peclet number for spray and globular transfer mode. The charcteristic length of the Peclet number is $S / L_{e}$, where $S$ is the cross-sectional area of wire and $L_{e}$ is the wire extension length. $S$ is in proportion to the volumetric melting rate and $L_{e}$ to the Joule heating inside the electrode.

Equation 1 is a kind of one-phase Stefan problem, and for low Peclet number the conduction heat transfer along the electrode axis is not negligible. To analyze this one-phase melting phenomenon, the Murray-Landis method (the variable space network method) is used. For each iteration step, the electrode is divided into the same number of equally sized space increments, which expand or shrink in time as shown in Fig. 5 (Ref.7). 


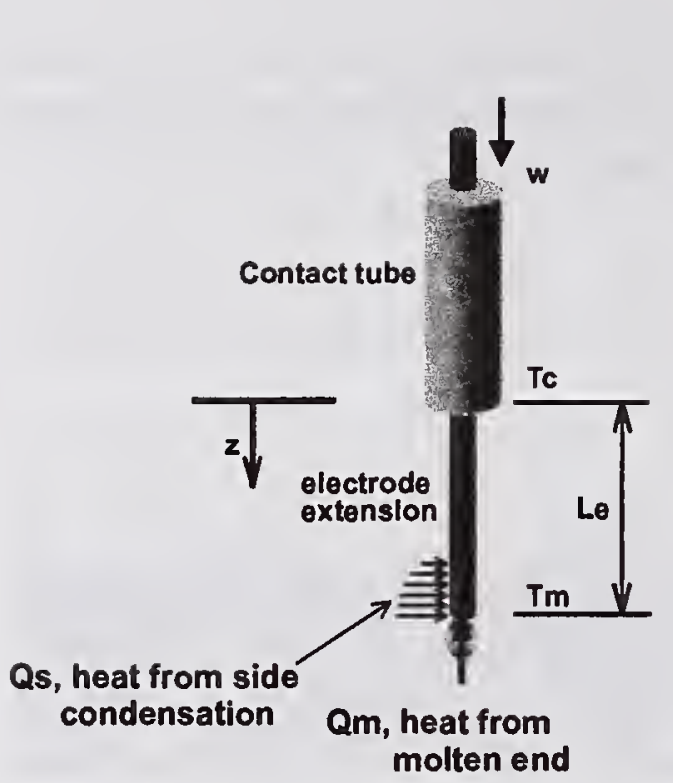

(a) Wire melting model

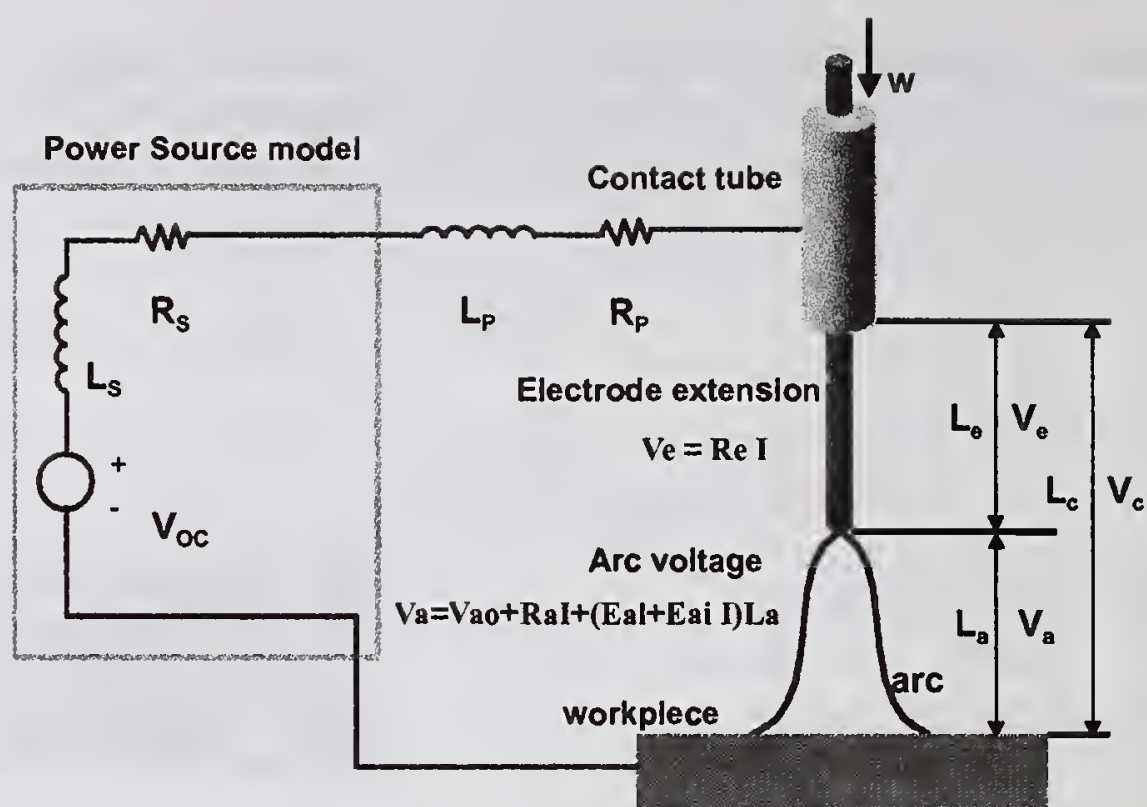

(b) Euqivalent circuit of GMA welding system

Figure 3: Mathematical modeling of GMA welding system

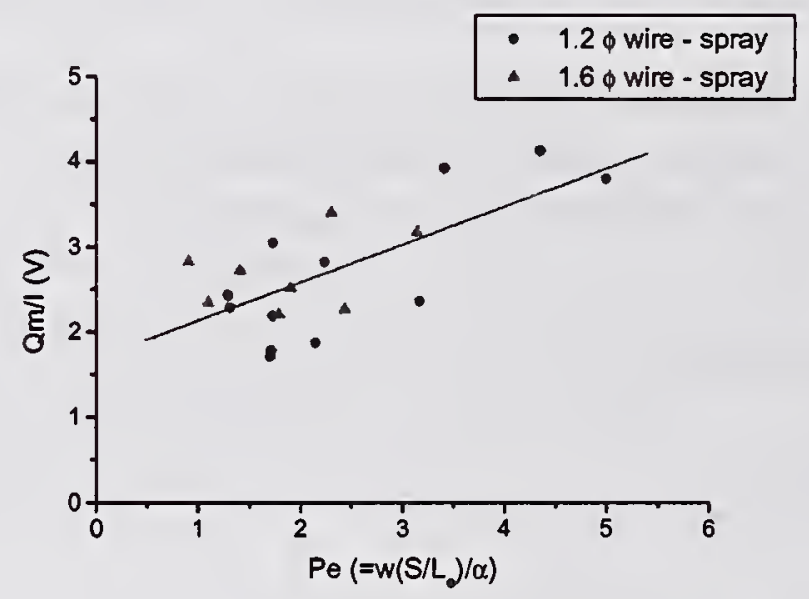

(a) Spray transfer mode

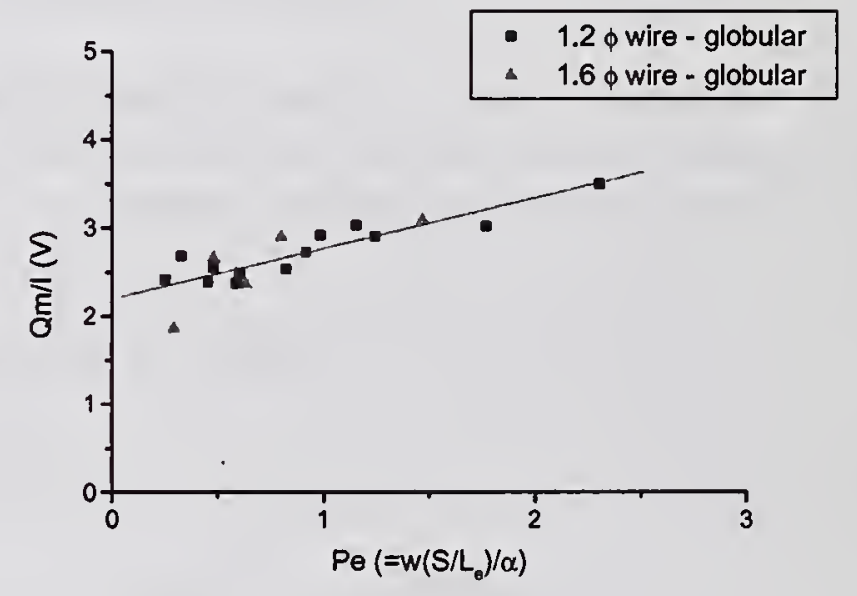

(b) Globular transfer mode

Figure 4: Heat flux entering the solid wire from the molten end

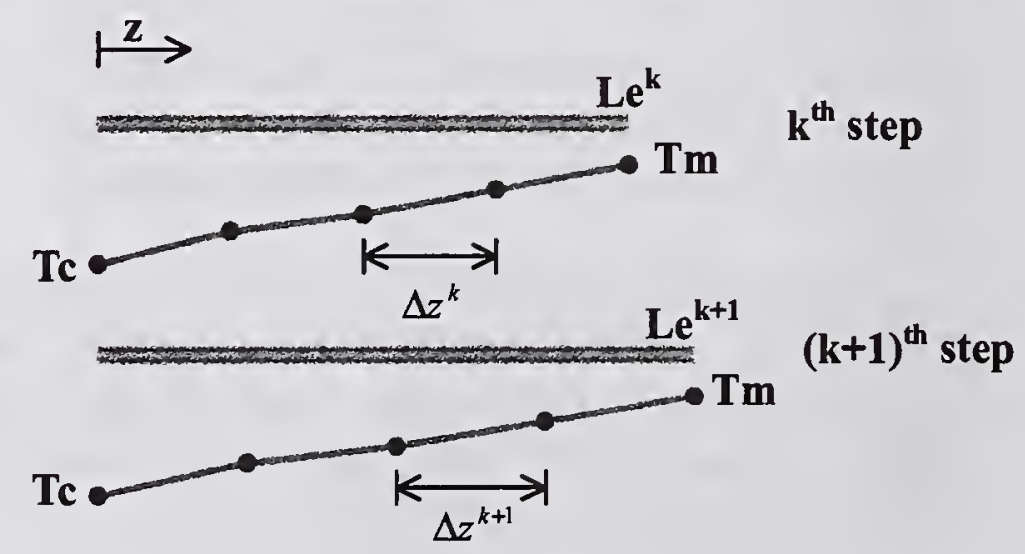

Figure 5: Variable space network 
The governing equation for the whole loop of the welding circuit can be written as

$$
\frac{d I}{d t}=\frac{V_{o C}-V_{a 0}}{L_{s}+L_{p}}-\frac{R_{s}+R_{p}+R_{a}+R_{e}}{L_{s}+L_{p}} I-\frac{E_{a l}+E_{a i} I}{L_{s}+L_{p}} L_{a},
$$

where the elecrical resistance in the electrode $(R e)$ is calculated as follows.

$$
R_{e}=\int_{b}^{L_{e}} \frac{\rho_{e}(T(z))}{S} d z
$$

The welding current signals were simulated by using eq. 1-4 and compared with experimental ones as shown in Fig. 6. To minimize the effect of weld pool shape, the amplitudes of welding current were measured for bead-on-plate welding with sinusoidal variation of torch height.

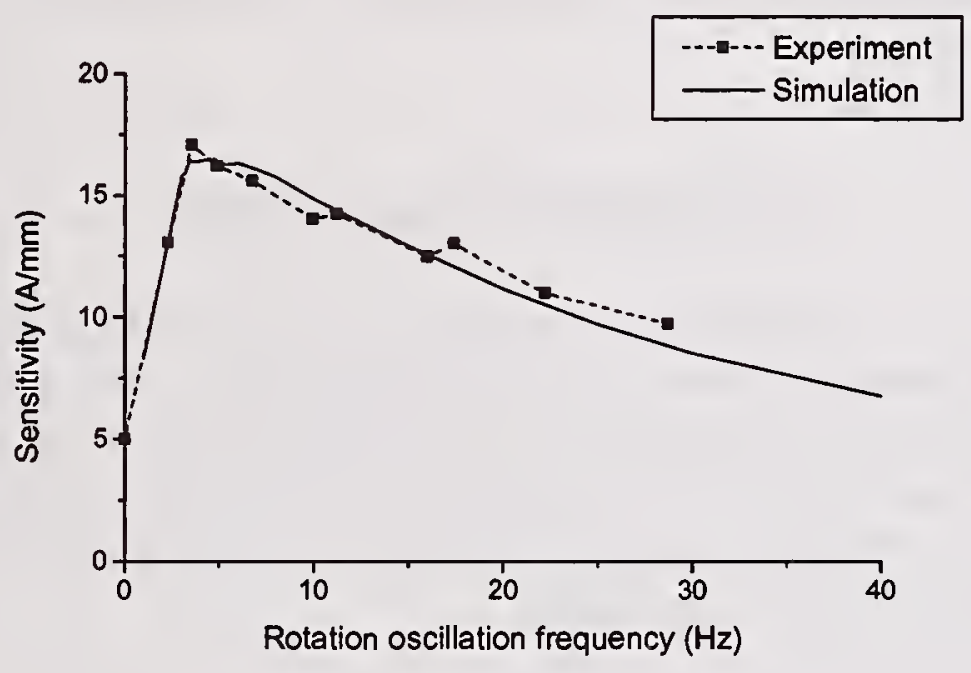

Figure 6: Sensitivity of arc sensor for bead-on-plate welding with torch height oscillation

In rotating arc welding, the CTWD is considerably affected by the weld pool shape. Especially for rotating along the rear half-circle, the arc moves above the previously formed pool and calculation of the CTWD considering the molten pool height at arc position is crucial. Analyses of dynamic 3-D GMA weld pool are regarded as of major importance but are so sophisticated and time-consuming that it is beyond the scope of this paper.

Under the assumption that the arc rotates very rapidly, a simplified 3-D quasi-steady model of molten pool was adopted in this study. The previous work has demonstrated that the conduction model, without consideration of fluid flow, can predict the shape of weld pool boundary in the vicinity of arc with reasonable accuracy (Ref. 8). A quasi-steady conduction model was used for calculation of the CTWD during high-speed arc rotation. Welding current waveforms were simulated by solving the governing equations (1-4) and the simplified weld pool model. For example, experimental and simulated results under $25 \mathrm{~Hz}$ rotation speed are shown in Fig. 7. Although the experimental welding signals contain high-frequency fluctuations, their basic waveforms can be predicted reasonably well by the simulations. 


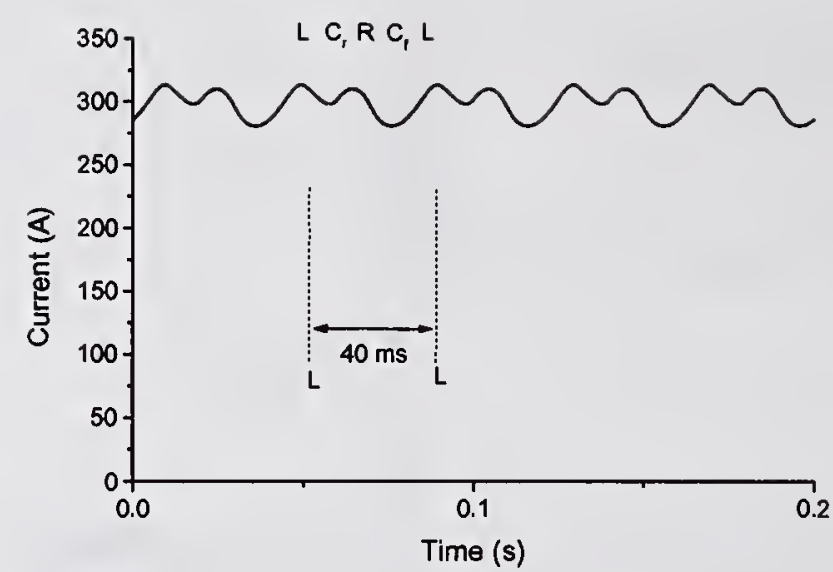

(a) Simulated results

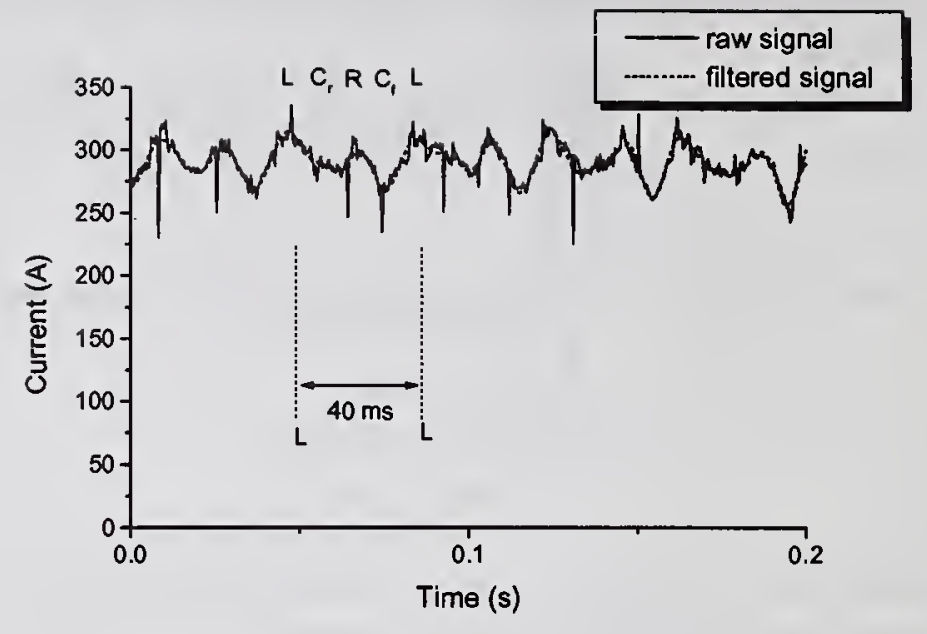

(b) Experimental results

Figure 7: Simulated and experimental welding current waveform for V-groove welding with $25 \mathrm{~Hz}$ arc rotation

\section{ROTATING ARC SENSOR}

By self-regulation of the arc length, the variation of the CTWD generates the change of the electrode extension in conventional torch weaving system. The weaving arc sensor has relatively poor resolution and limitation of application to the aluminum welding because the resistivity of $\mathrm{Al}$ electrode is much lower than that of the welding arc. But by the change of the arc length due to the variation of the CTWD, the rotating arc sensor has an improved sensitivity as shown in Fig. 6 , and can be applied to the aluminum welding.

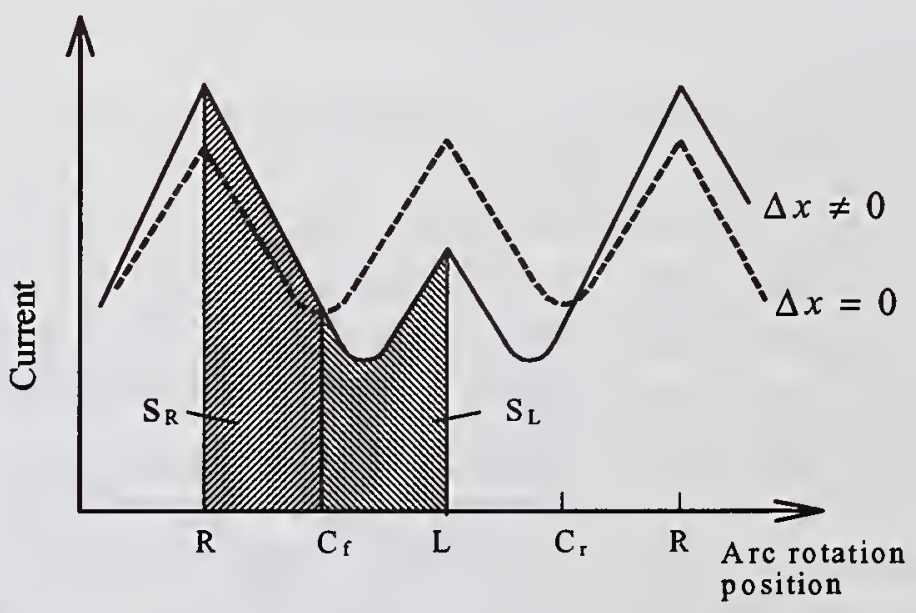

Figure 8: Arc sensing algorithm

When the welding torch is positioned at the center of groove, the current waveform becomes symmetric at $\mathrm{C}_{\mathrm{f}}$. If the welding torch is deviated from the center of groove, the current waveform changes to asymmetrical shape as shown in Fig. 8. The area difference $\left(\mathrm{S}_{\mathrm{L}}-\mathrm{S}_{\mathrm{R}}\right)$ shows a linear relationship with the offset distance as in Fig. 9 and is adopted for the error criterion. The rotating arc sensor can track the weld seam by using the correction data proportional to the area 
difference. Figure 10 shows examples of the automatic weld seam tracking for mild steel and Al 5052 respectively. The weld seam could be tracked with an accuracy of $0.07 \mathrm{~mm}$ maximum error and $0.035 \mathrm{~mm}$ standard deviation of error in the welding of mild steel. The rotating arc sensor showed a fairly good performance also in the aluminum welding in spite of the slightly increased error.

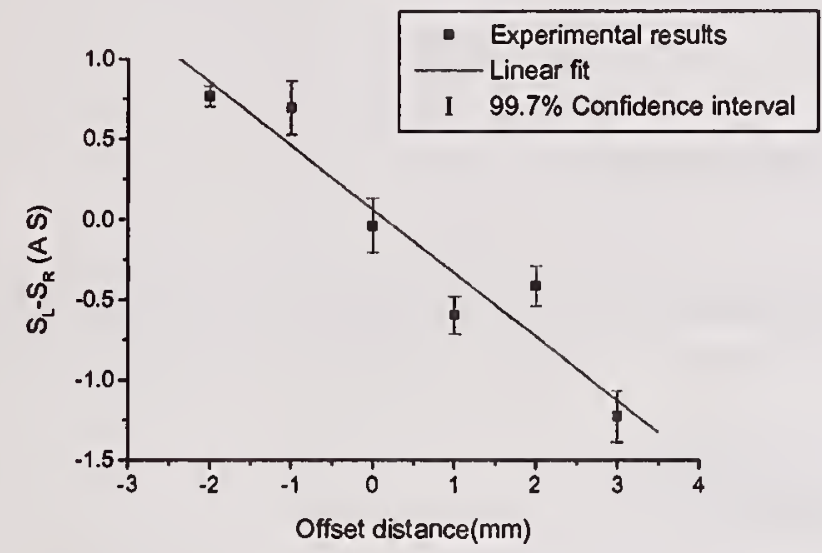

(a) Mild steel

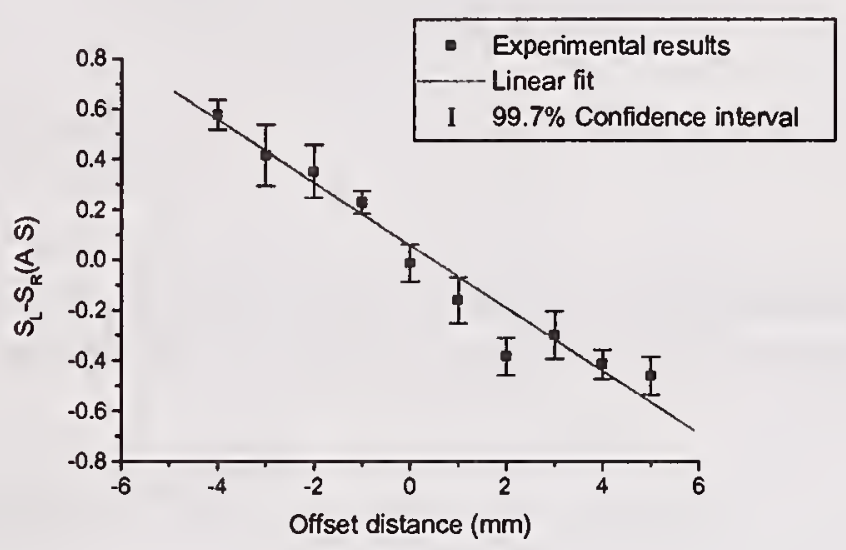

(b) Al 5052

Figure 9: Correlation between offset distance and area difference

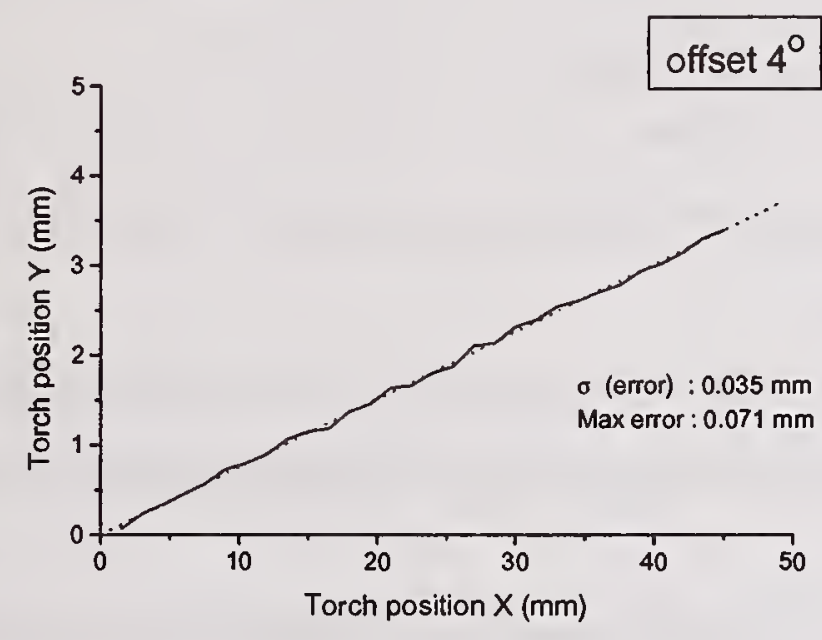

(a) Mild steel

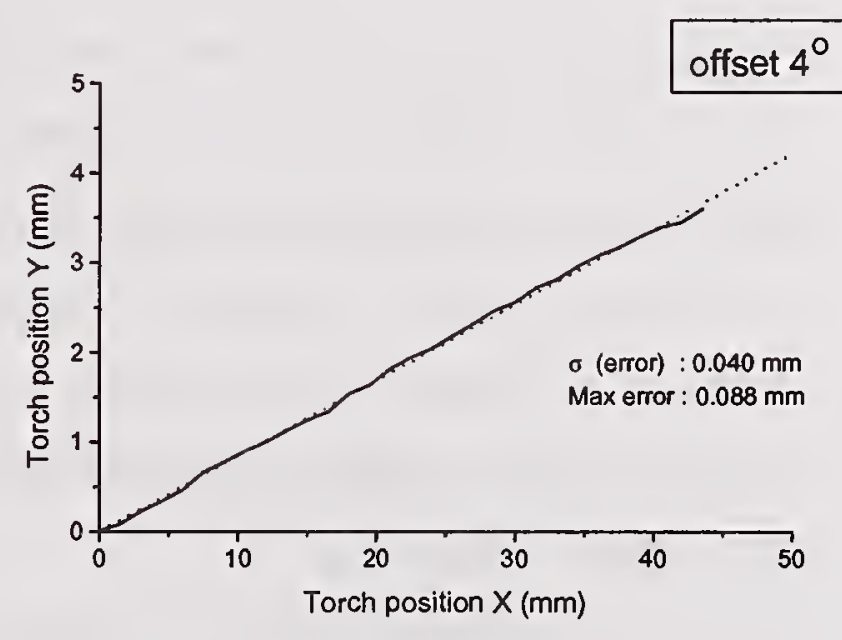

(b) Al 5052

Figure 10: Examples of automatic seam tracking by rotating arc sensor

\section{CONCLUSION}

(1) New-type arc rotation system was developed to implement a mechanically high-speed rotating arc. By using a hollow-shaft motor the rotating mechanism could improve the accessibility to the joint.

(2) Welding current was simulated by considering the dynamic characteristics of GMA welding system such as dynamic wire melting, transient temperature distribution of wire 
and internal resistance and inductance of welding power source. By time domain simulations the welding current was predicted and used for the development of the arc sensor.

(3) Rotating arc sensor was developed to track the weld seam with improved sensitivity, responsiveness and accuracy. Besides the rotating arc sensor could be applied to aluminum welding successfully.

\section{ACKNOWLEGEMENT}

The authors would like to thank the Ministry of Science and Technology, Korea, for the financial support by a grant from the Critical Technology 21 Project.

\section{REFERENCES}

1 Sugitiani, Y.; Kobayashi, Y.; and Murayama, M. 1991. Development and application of automatic high speed rotation arc welding. Welding International 7 (5): 577 to 583.

2 Dilthey, U.; Oester, M.; and Gollinick, J. 1996. Mechanized metal-gas-shielded arc welding with high-speed rotary wire electrode. Welding \& Cutting 48 (11): 866 to 875.

3 Cook, G.E.; Andersen, K.; Fernadez, K.R.; Sheperd, M.E.; and Wells, A.M.Jr. 1987. Electrical arc sensing for robot positiòning control. Robotic arc welding. Eds. J.D. Lane: 181 to 216: Springer-Verlag: IFS Publication.

4 Shepard, M.E.; and Cook, G.E. 1992. A frequency-domain model of self-regulation in gasmetal arc welding. Third International Conference on Trends in Welding Research: 899 to 903: ASM International.

5 Kim, Y.-S.; McEligot, D.M.; and Eagar, T.W. 1991. Analysis of electrode heat transfer in gas metal arc welding. Welding Journal 70 (1): 20-s to 31 -s.

6 Waszink, J.H.; and Van Den Heuvel, G.J.M. 1982. Heat generation and heat flow in the filler metal in GMA welding. Welding Journal 61 (8): 269-s to 282-s.

7 Murray, W.D.; and Landis, F. 1959. Numerical and machine solution of transient heatconduction problems involving melting or freezing. Journal of Heat Transfer 81: 106 to 112.

8 Kim, J.-W. 1991. A study on the analysis of weld pool convection and seam tracking by considering the arc length characteristics in GMA welding. Ph.D. dissertation. Taejeon, Korea: KAIST. 


\section{Keynote Lecture}




\section{"Lights Out" Welding:}

Where We Are and Where

We Need To Go

We Need To Go

Progress towards Complete Welding Automation

T.P. Quinn

National Institute of Standards and Technology

Boulder, CO 80305

\section{"Lights out" welding}

Welding automated to the point that no human intervention necessary 


\section{Challenges to Automation}

Reproduce the welder's hand motion $\checkmark$

- Automatically track joint $\checkmark$

- Adaptively fill large or uneven gaps $\checkmark$

- Sense weld defects - take corrective action

- From blueprint to assembly with no "training"

\section{Welder's hand motion}

Articulated arm robots, crawlers, rotation devices, ...

Faster

More accurate

Untiring 


\section{Automatically track joint}

Laser stripes, Vision systems, Touch Sensors, Through-the-arc sensors

$\rightarrow$ Integrated into the controller software can track the joint with two-pass algorithms and single-pass algorithms

\section{Adaptively fill}

- With the joint tracker signals, software exists that can effectively handle large or uneven gaps, while controlling heat input 


\section{Sense weld defects}

- Through-the-arc sensing (no feedback)

- Research-grade weld pool visualization

Through-the-torch (TIG available)

$\Rightarrow$ IR, laser stripe (etc.)

Weld-pool vibration

\section{From blueprint to assembly}

Least developed area

- Solutions usually unique, proprietary

- Standards necessary 


\section{A-9 Welding Activity Model}

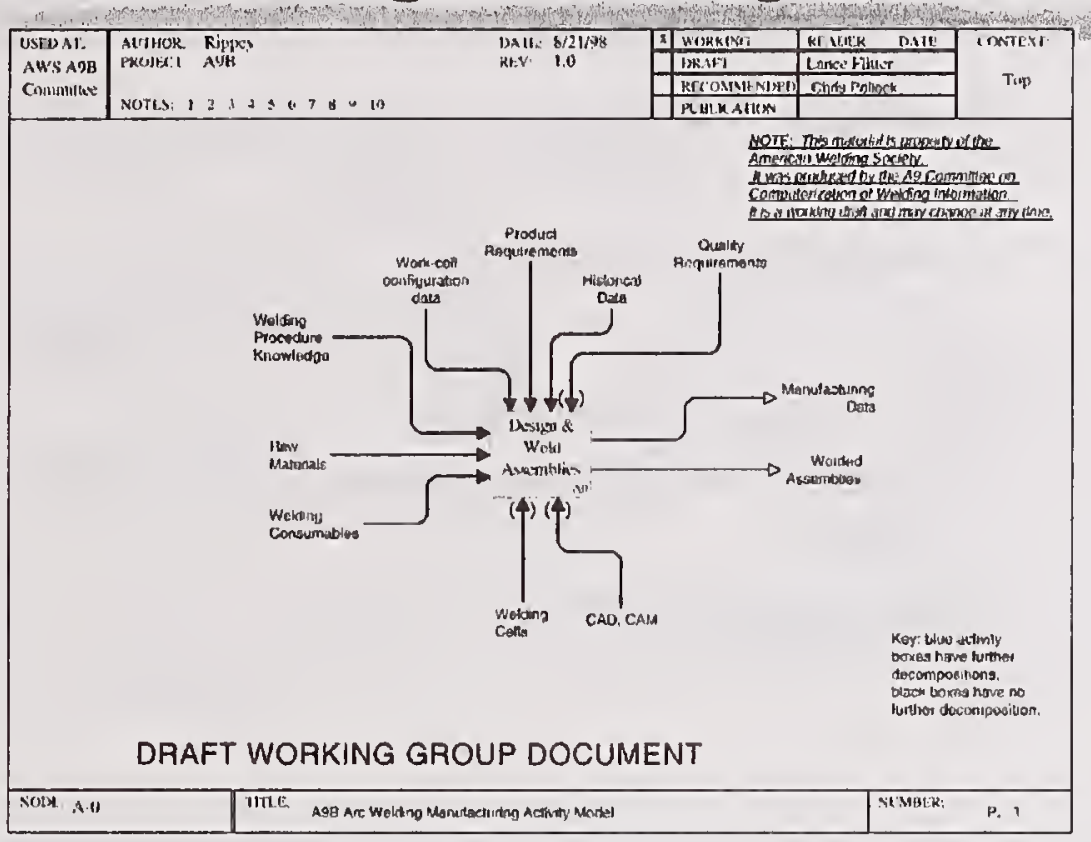

\section{Design}

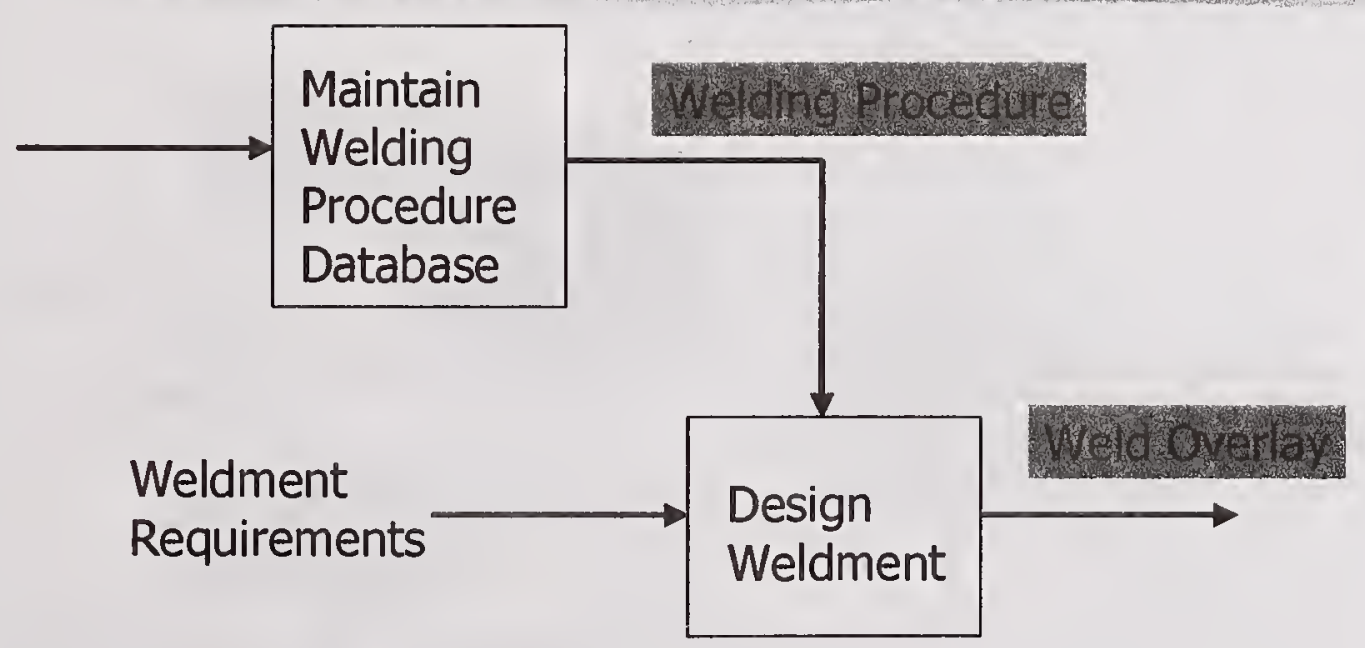




\section{Possible Standards}

Welding Procedure

*9.1 Early database standard

- Considering withdrawal

* Little interest in standard database interface

Weld Overlay Standards

- Interchange Welding Overlay between CAD systems

Interest unknown

\section{Manufacturing Planning}

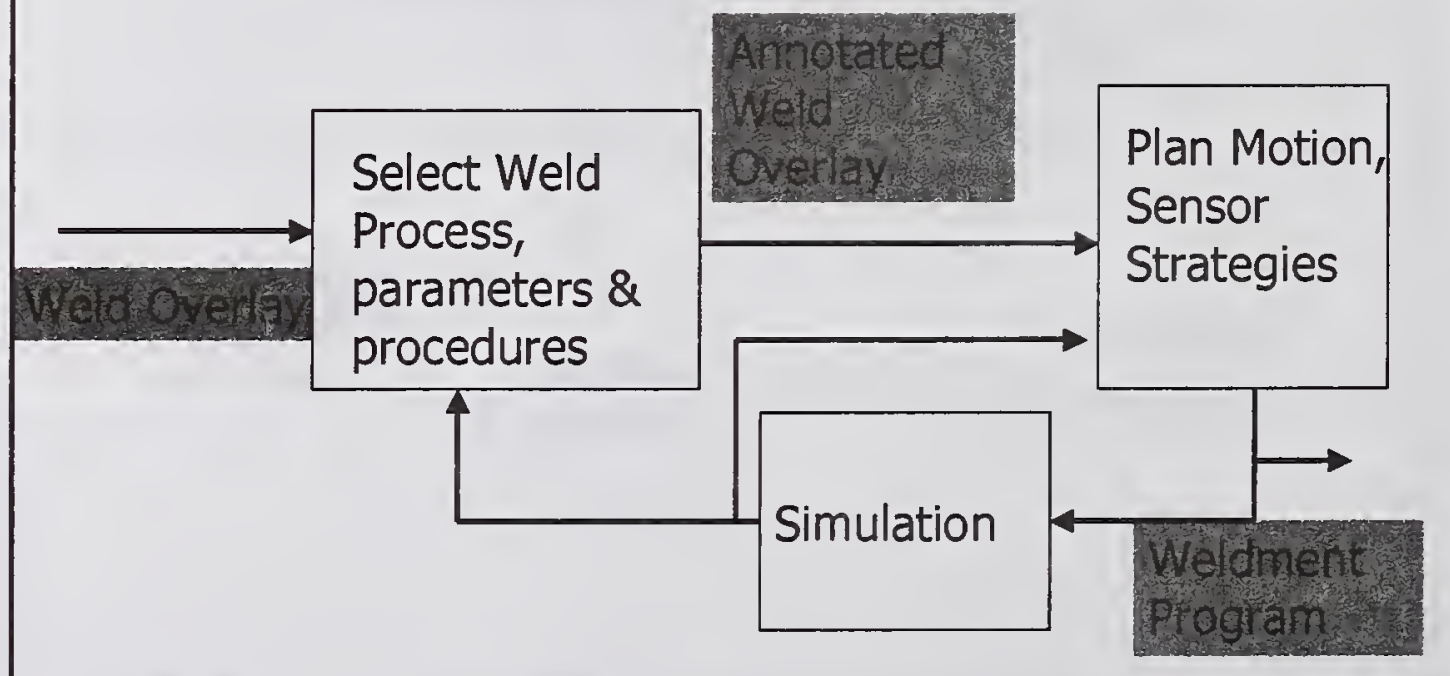




\section{Possible Standards}

- Annotated Weld Overlay

- CAD Description

$\rightarrow$ Interest unknown

- Weldment Program

Output of an offline programmer - to interface with welding cell

- Interest from shipyards

- Navy sponsored document available for standard

\section{Intra-cell Communication}

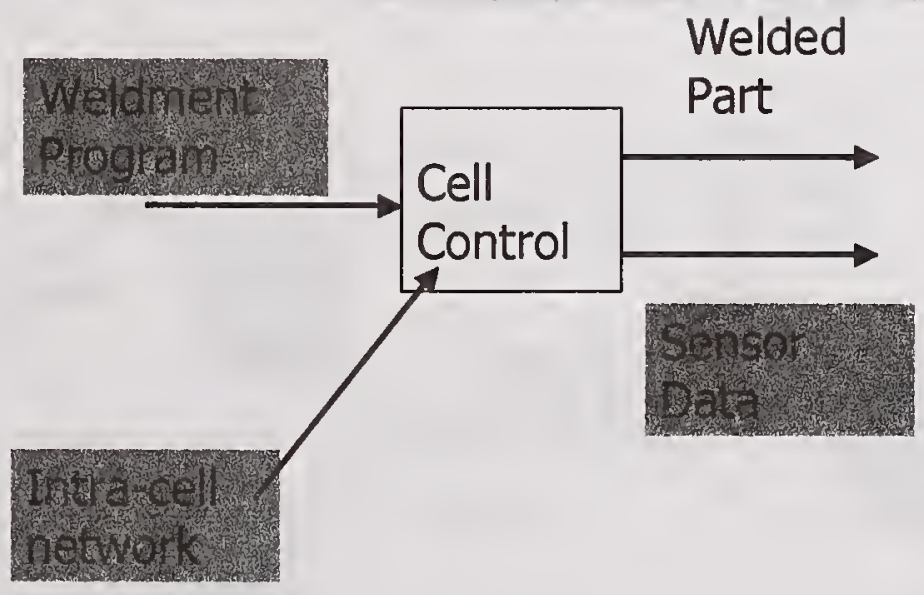




\section{Possible Standards}

Sensor Data

- Database interface for quality documentation and statistical process control

Scope could include inspection reports

Interest unknown

\section{Intra-cell Network}

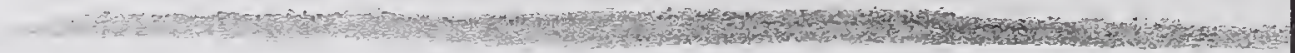

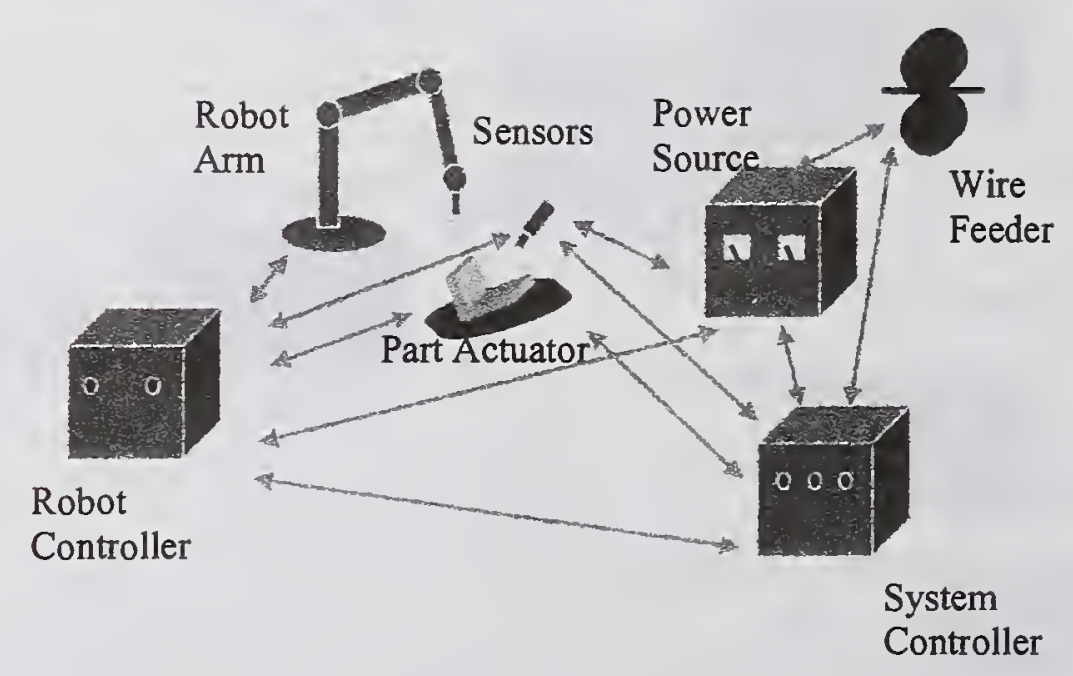




\section{Intra-cell network (cont.)}

Idea is to have a standard hardware and software connection along all communications paths

- Advantages:

Easier integration for complex systems

* Plug-and-play for simple systems

Network cabling simpler

- Not limited to a proprietary system or proprietary components

\section{Intra-cell network (cont.)}

Disadvantages

- Proprietary systems are well established

Which network protocol to use?

Interest

Large consumers of cells universally interested

- Power source manufacturers also interested

$\Rightarrow$ Robot producers lukewarm

Integrators ?? 


\section{Status}

A9.4 based on "ArcLink" in development

$\Rightarrow$ Ready for balloting

- DOJ review underway

Controversy over perception of AWS "endorsing" one network over another

A9.? (or ODVA) based on a DeviceNet standard under consideration

\section{The Pitches}

Standards development can lead to faster time-to-market

AWS needs input from those most affected

More people and energy required 


\section{Contact Information}

A9 Chair

Bill Rippey

Telephone 301.975.3417

NIST

100 Bureau Drive, MS 8230

Gaithersburg,MD 20899-8230

william.rippey@nist.gov 



\section{Session A2: Sensing and Control II: GMAW and GTAW}





\title{
A SYSTEM FOR REAL-TIME CONTROL OF GAS METAL ARC WELD PROFILE
}

\author{
D.M. Barborak ${ }^{*}$, H.W. Ludewig $^{\dagger}$, R.W. Richardson ${ }^{\ddagger}$, D.F. Farson ${ }^{\ddagger}$, and S. Yurkovich ${ }^{\ddagger}$
}

\begin{abstract}
The objective of this research was to investigate the use of a unique, hybrid robotic Gas Metal Arc Welding control system for robustly controlling weld shape of single-pass fillet welds in the presence of common production perturbations and disturbances. Such a system could improve the fatigue properties of weldments, increasing service life and decreasing design requirements.

A hybrid robotic control system was developed consisting of a 6-axis articulated arm welding robot, laser-based machine vision system, and weld profile controller. The control architecture consisted of integrated feedforward and feedback, multi-input/multi-output control loops that ran simultaneously to perform joint finding, joint tracking, modified fill, contact-tip-to-work (CTWD) regulation, and weld symmetry control. Complex weld profile features such as weld symmetry and weld profile were addressed as reference tracking and disturbance rejection control problems.
\end{abstract}

\section{KEYWORDS}

Robotic GMAW, Fillet Weld, Weld Geometry, Weld Profile, Feedback Control, Feedforward Control, Adaptive Fill, Joint Finding, Joint Tracking, Weld Symmetry Control.

\section{INTRODUCTION}

The surface profile of a weld deposit and presence of any discontinuities are very influential to its fatigue performance. Robotic arc welding relies on process set points derived during the development of an optimal welding procedure to produce a desired weld profile with minimal discontinuities. Classified as open-loop control, this technique assumes all the process inputs and disturbances remain fixed and produce a repeatable output in terms of weld quality. Any error, change in plant dynamics, or disturbance to the process is likely to cause deterioration in weld quality which may require expensive rework or cause premature failure.

The objective of this research has been to investigate the application of a hybrid GMAW control system for robustly controlling the weld profile of single-pass fillet welds in the presence of common production perturbations and disturbances. The purpose of this is to control fatigue properties of weldments to increase service life or decrease design requirements. It has been shown that fatigue properties are strongly affected by weld geometry and discontinuities ${ }^{1,2}$. For fillet-welds, this includes all geometrical surface aspects of the weld face and weld root including any discontinuities. This application of a hybrid weld profile control system requires integration of multi-input/multi-output, feedforward and feedback control loops to perform joint tracking, adaptive fill, contact-tip-to-work (CTWD) regulation, and weld symmetry control. Complex weld profile features such as weld symmetry and weld profile are addressed as reference tracking and disturbance rejection control problems. Robustness to disturbance

\footnotetext{
"WeldWare, Inc., Columbus, Oh.

${ }^{\dagger}$ Caterpillar, Inc., Peoria, Ill.

$\ddagger$ The Ohio State University, Columbus, Oh.
} 
rejection of common production perturbations such as tack welds, root gaps, variable heat sinking, joint deviations, and changes in CTWD are considered. This research promises to lead to better control of weld properties beyond what has been previously accomplished.

\section{WELD PROFILE CONTROL SYSTEM}

The control system developed for this research implemented a 6-axis welding robot interfaced with a laser-based, structured light machine vision sensor (Figure 1). The welding robot is a standard ABB IRB2400 6-axis articulated arm robot with S4 controller interfaced to a Lincoln DC600 power supply. A Servo-Robot M-Spot 90 laser sensor with CAMI II controller is utilized for in-process sensing. The M-Spot 90 laser sensor was modified to output $100 \mathrm{~mW}$ visible laser in order to increase image-processing robustness during welding. An industrial Pentium ${ }^{\mathrm{TM}}$ based PC is utilized for the weld profile controller.

Three separate software programs were developed to run simultaneously on the three separate computers to comprise the weld profile control system. The robot program is written in the RAPID language and runs on the robot controller. The RAPID program controls the overall sequence of the welding operation along with manipulation of the robot arm and communicates with the ADAPT and WEGE programs. The modified fill algorithm, which runs in the feedforward loop, is written in the ADAPT language developed by Servo-Robot and runs on a dedicated digital signal processor (DSP) in the CAMI II system. The weld profile control program is written in $\mathrm{C}++$ and runs on the

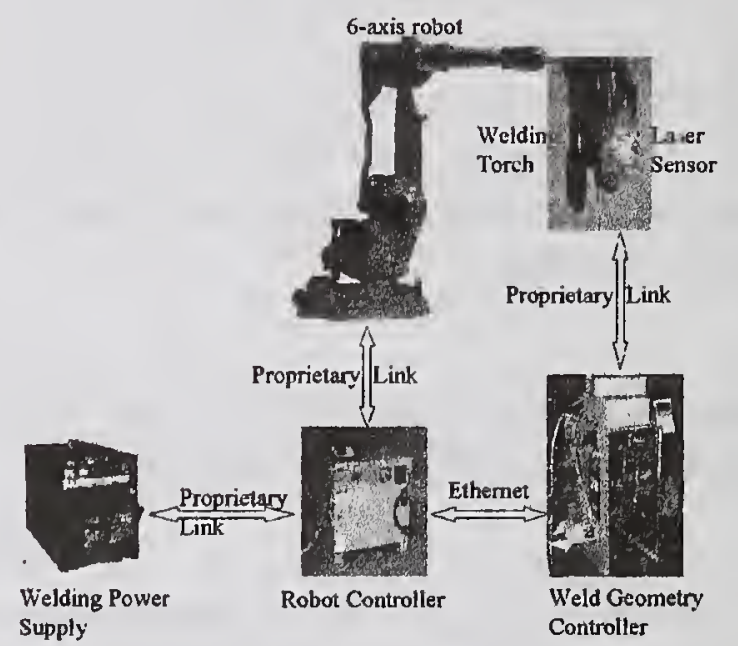

Figure 1 Components of weld profile control system industrial PC residing on an ISA bus within the CAMI II system.

The weld profile controller consists of separate but integrated feedforward and feedback control loops. The feedforward control loop performs the tasks of joint finding, joint tracking, CTWD regulation, and modified fill. The feedback control sub-system performs the task of weld symmetry control.

\section{Feedforward Control Loop}

A block diagram for the feedforward loop is shown in Figure 2. All four control tasks rely on sensory information from the laser-based, structured light machine vision sensor mounted parallel to the welding torch. The joint finding and joint tracking tasks utilize information about the joint centroid, calculated during a pre-weld scan of the weld joint to adjust the torch offset and contact-tip-to-work distance while welding. The modified fill task utilizes information about the joint such as tack size and gap area, along with the required fillet weld leg length to control deposition rate during welding by adjusting travel speed, wire feed speed, and arc voltage. All four tasks in the feedforward control loop utilize linear control with heuristics. 


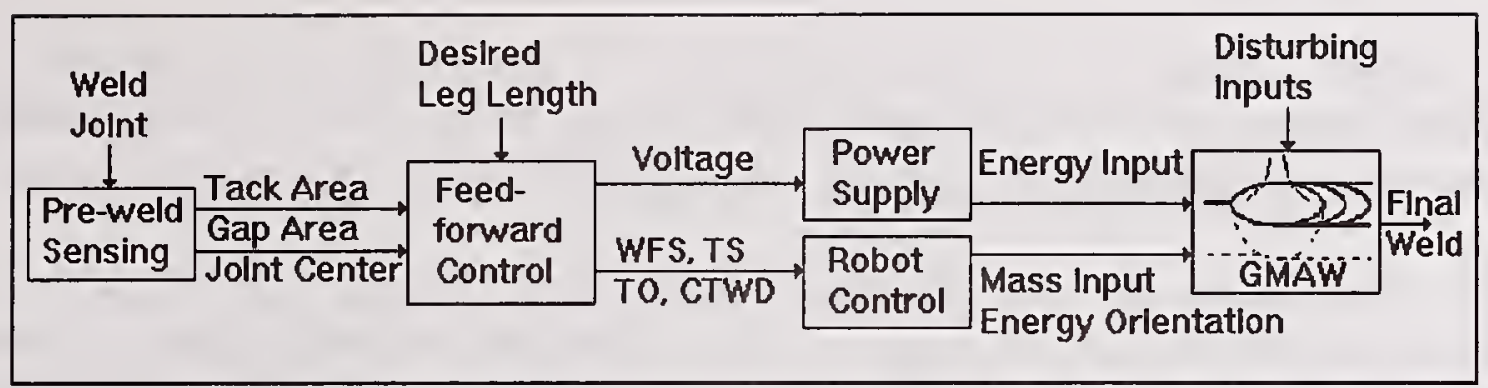

Figure 2 Feedforward control sub-system block diagram

One problem with automatic and robotic arc welding applications is maintaining the proper alignment of the welding arc (torch) with the weld joint. Dimensional tolerances of component parts, variations in edge preparation and fit-up, distortion during welding, and other dimensional variations can affect the exact position and uniformity of the weld joints from one assembly to the next. Joint finding coupled with joint tracking can overcome these limitations by adjusting the welding torch trajectory relative to the weldment as the welding torch proceeds along the joint. Joint finding and tracking technology is commercially available and will not be discussed in detail here ${ }^{3,4}$.

During the joint finding task, the robot scans near the perceived beginning of the weld joint in a pre-programmed pattern to locate the exact beginning of the weld joint. The entire preprogrammed robot path is then globally offset by the difference between the pre-programmed starting point and actual joint location.

Next the robot places the welding torch at the weld start location and conducts a pre-weld scan of the joint for the joint tracking and modified fill tasks. The robot moves in the pre-programmed direction while the laser sensor scans the weld joint, gathering imaging information about the joint location and volume. As the torch proceeds along the weld joint, the torch offset and contact-tip-to-work distance changes are continuously calculated and saved to a path file for later playback during welding. This new tool center point (TCP) is calculated every $2.5 \mathrm{~mm}(0.1 \mathrm{inch})$ along the joint. In addition to the joint tracking and CTWD calculations, information about the weld joint shape is processed and utilized for the adaptive fill task.

The image processing technique used for the joint finding and joint tracking tasks are diagrammed in Figure 3. The laser sensor scans the T-joint designated by the continuous plate (cp) and the discontinuous plate (dp). The image is processed to find two line segments, namely $1 \mathrm{~s} 1$ and $1 \mathrm{~s} 2$. At the intersection of these line segments is the joint root, which is designated as the tracking point. The tool center point or tcp is then calculated perpendicular to each line segment, and a fixed distance defined as the contact-tip-to-work distance from the tracking point.

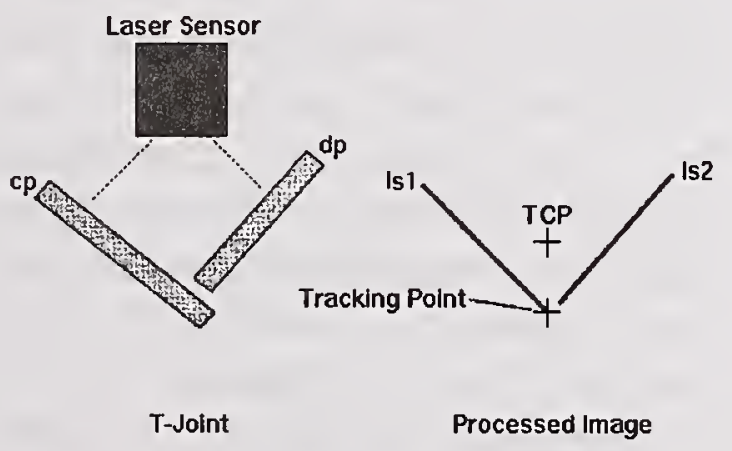

Adaptive fill control compensates for changes in the required volume of welds due to variations in part dimensions, edge preparation, fit-up, tacks, and distortion during welding. Utilizing machine vision, the adaptive fill controller measures the joint dimensions and volume, then 
determines changes needed in welding variables to compensate for changes in the joint geometry. Such changes in wire feed speed, arc voltage, and or travel speed adjust the weld deposition rate while maintaining arc stability and the desired weld metal transfer mode.

A customized version of adaptive fill control call "modified fill" was developed for this research. The modified fill algorithm operates similar to the standard adaptive fill algorithm in that travel speed, wire feed speed, and voltage are varied to compensate for changes in the required deposition area due gaps and tacks. The standard adaptive fill algorithm is designed to maintain a constant amount of weld reinforcement while maximizing productivity by utilizing the fastest travel speed feasible. The modified fill algorithm is designed to maintain the minimum required amount of weld reinforcement while producing an optimal weld profile, conducive to improved fatigue performance.

The image processing technique utilized in the modified fill algorithm is shown in Figure 4. As the laser sensor scans the T-joint formed by the continuous plate (cp) and the discontinuous plate (dp), it may encounter gaps or tacks. The imageprocessing algorithm detects the presence of gaps and tacks and measures their size. The modified fill algorithm then calculates the required deposition rate based on the desired fillet weld leg length, and the presence of a gap or tack.

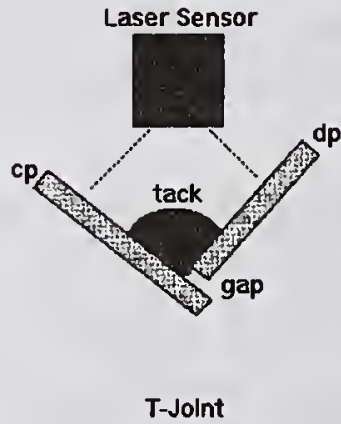

Figure 4 Processed image of T-joint showing modified fill attributes.

The required deposition area for a fillet weld with a specified leg length is calculated by:

$$
\text { deposition area }=0.5 \times \text { required leg lengt } h^{2}-\text { tack area }+ \text { gap area } .
$$

A $10 \mathrm{~mm}$ fillet weld in the absence of a gap or tack requires a $50 \mathrm{~mm}^{2}$ deposition area. When gaps ranging from $0 \mathrm{~mm}$ to $3 \mathrm{~mm}$ are encountered, the required deposition area increases from $50 \mathrm{~mm}^{2}$ to $84.5 \mathrm{~mm}^{2}$ in order to maintain a $10 \mathrm{~mm}$ leg length fillet weld (Figure 5). Likewise, when tacks ranging in size from no tack i.e. $0 \mathrm{~mm}$ to $8 \mathrm{~mm}$ leg lengths are encountered, the required deposition area decreases from $50 \mathrm{~mm}^{2}$ to $2 \mathrm{~mm}^{2}$. It should be noted that these calculations are ideal, made in two dimensions and assume a $100 \%$ deposition efficiency. Although the actual deposition rate is constant, the resulting weld bead dimensions and profile will vary along the length of the weld.

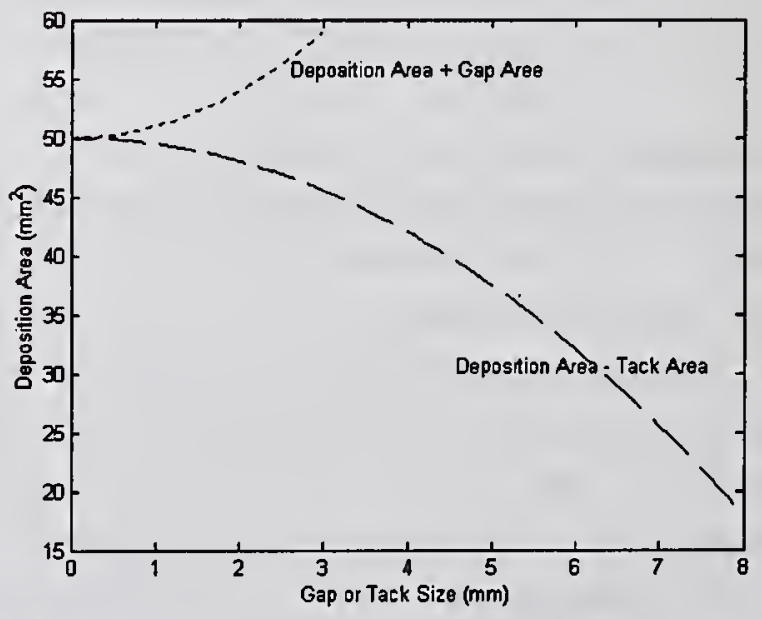

Figure 5 Change in required deposition area for a $10 \mathrm{~mm}$ fillet weld. 
The changes in wire feed speed and travel speed for a required deposition area are calculated by:

$$
\text { deposition area }=\frac{\text { wire feed speed } \times \text { wire area } \times \text { deposition efficiency }}{\text { travel speed }} .
$$

For this research, a $95 \%$ deposition efficiency was assumed while using a standard $1.32 \mathrm{~mm}(0.052$ inch) diameter ER70-S3 wire was used with $90 \%$ Argon $-10 \% \mathrm{CO}_{2}$ shielding gas. The optimal ranges for wire feed speed and travel speed to produce welds with the desired profiles were determined experimentally in prior research using a Design-Of-Experiments (DOE) approach. The range for wire feed speed was limited to 89 to 114 $\mathrm{mm} / \mathrm{sec}(350$ to $450 \mathrm{ipm})$ and travel speed to 3.0 to $5.08 \mathrm{~mm} / \mathrm{sec}$ ( 7 to $12 \mathrm{ipm})$. Arc voltage was varied linearly with wire feed speed within the range of 35 to 40 volts. It should be noted that this range for wire feed speed is considerably narrower than that typically utilized in the
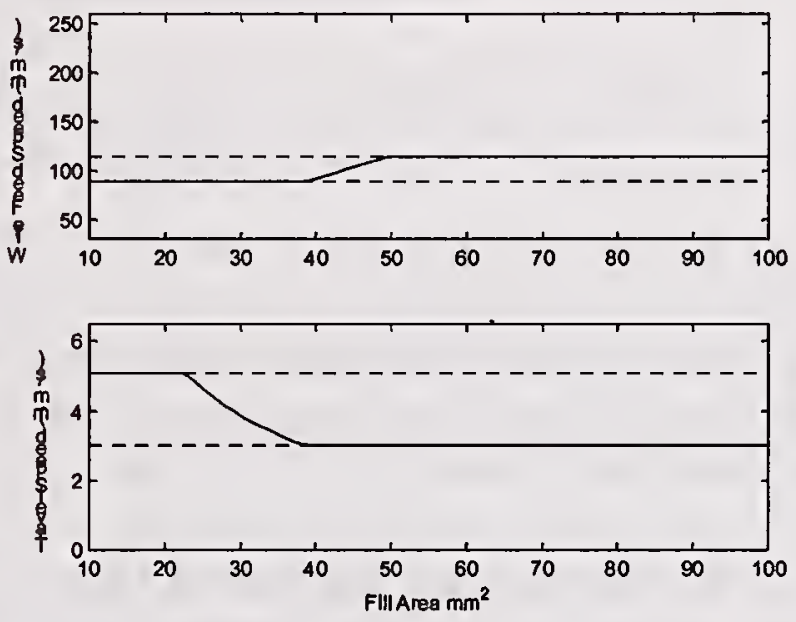

Figure 5 Characterization of the modified fill algorithm.

standard adaptive fill algorithm. Likewise, travel speed was slower and narrower, and arc voltage was narrower and higher. The characteristic behavior of the modified fill algorithm is shown in Figure 5. Initially, in the low deposition area, the algorithm minimizes wire feed speed and adjusts travel speed. Once the minimum travel speed is encountered, the algorithm begins to increase wire feed speed. Based on the requirements for a $10 \mathrm{~mm}$ leg length fillet weld discussed in the previous paragraph, the adaptive fill algorithm will slow travel speed in the presence of tacks upto about $3.4 \mathrm{~mm}$ in leg length where the limits were reached and minimal deposition occurs. For gaps, the algorithm provides maximum deposition with wire feed speed at its maximum and travel speed at its minimum.

\section{Feedback Control Loop}

The feedback control loop, shown in Figure 6, performs the task of weld symmetry control. The weld profile is measured in real-time during welding by the laser vision sensor. The inputs to the controller are the error in weld symmetry and its rate-of-change. The weld symmetry is quantified by measurements such as the difference in fillet weld lengths known as differential leg length (DLL). Adjustments to compensate for weld asymmetry are made by the controller to the torch offset (TO) and/or work angle (WA). The weld symmetry control task is performed by a fuzzy control algorithm. 


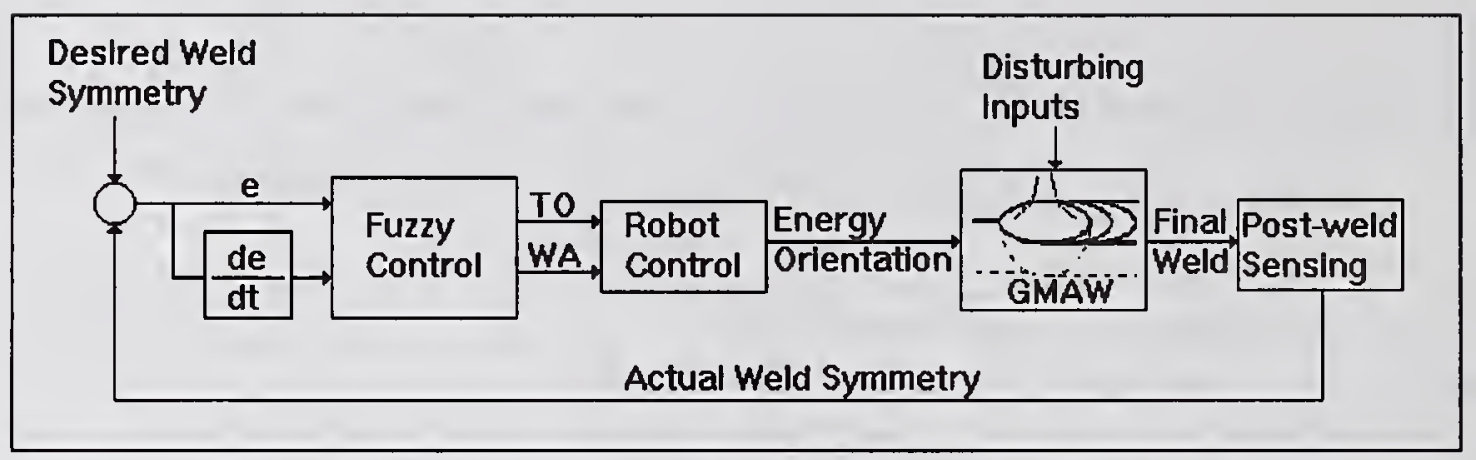

Figure 6: Feedback control sub-system block diagram.

During welding, the controller determines the degree of weld symmetry from a scan of the weld profile from the laser-sensor. The fuzzy controller then determines what the torch offset and/or work angle should be. Every $2.5 \mathrm{~mm}$ of weld, the robot controller polls the weld profile controller for corrective measures to the torch offset and work angle. The average overall control rate is 1.4 hertz.

The development of the rule base for this research relied on the evaluation of the data from the modeling effort described in Barborak, et. al. ${ }^{5}$. From this work, it was determined that torch offset and work angle were to be the control variables, whose physical limits were determined to be $+/-2.5 \mathrm{~mm}$ and $+/-5$ degrees respectively. The state variables were determined to be differential leg length (DLL), and rate of change in differential leg length (DDLL). These state and control variables were tested in various combinations of single-input/single-output (SISO) and multi-input/single-output (MISO) controllers.

An example of a SISO controller utilizes DLL as the input, and TO as the control variable. By studying the dynamic response surface produced under varying conditions, a simplified linear relationship of DLL with respect to the two control variables TO and WA can be surmised as shown in Figure 3. In order to construct a rule-base for control, this relationship is simply inverted.

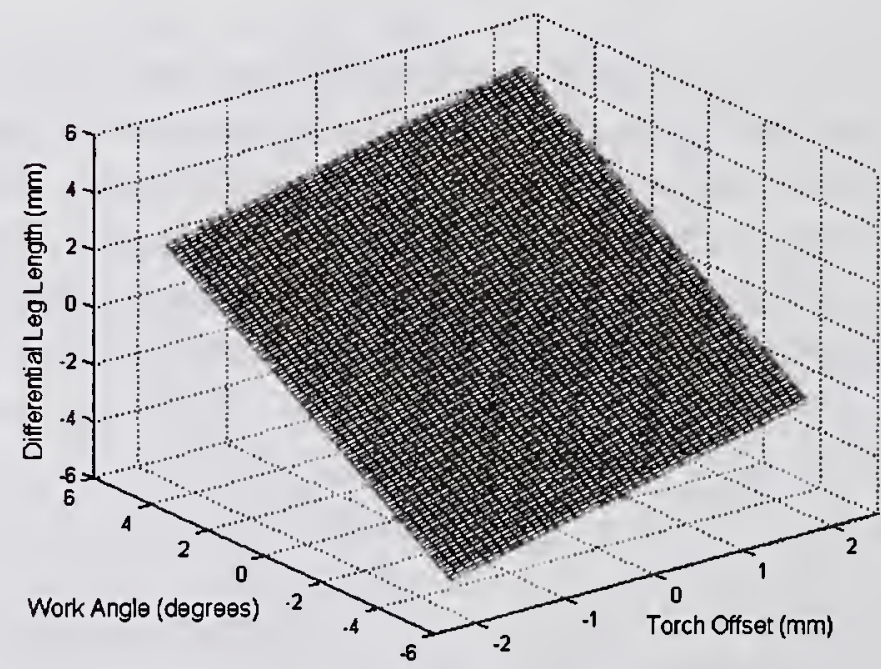

Figure 3 Generic linear response surface of the effect of work angle (WA) and torch offset (TO) on the resulting weld symmetry or differential leg length (DLL). 
The set of linguistic rules for this simple linear controller would be as follows:

1. If weld is Skewed Left Large then move torch Right Large.

2. If weld is Skewed Left Small then move torch Right Small.

3. If weld is Symmetric then move torch None.

4. If weld is Skewed Right Small then move torch Left Small.

5. If weld is Skewed Right Large then move torch Left Large.

Various combinations of input variables, output variables, number of membership functions, and gains were tested experimentally. See Passino and Yurkovich ${ }^{6}$ for a thorough treatment of fuzzy control.

\section{SUMMARY}

In this research, we developed a hybrid robotic weld profile control system. This control system employs an articulated arm welding robot interfaced with a laser-based, structured light machine vision sensor. The laser sensor is utilized in the feedforward controller by pre-scanning the joint prior to welding, and is then utilized in real-time in the feedback controller. The feedforward control sub-system integrates the tasks of joint finding, joint tracking, and modified fill. The controlled variables in the feedforward loop are travel speed, wire feed speed, arc voltage, torch offset, and contact-tip-work-distance. The feedback control sub-system performs the task of weld symmetry control and controls the variables torch offset and/or work angle. Linear control with heuristics is utilized in the feedforward sub-system while fuzzy control is utilized in the feedback sub-system.

\section{REFERENCES}

${ }^{1}$ A. Matting and M. Neitzel, "Evaluation of weld defects in fatigue testing," Welding Research Abroad, XII(7), Aug-Sept, 1966.

${ }^{2}$ A. Matting and M. Neitzel, "The influence of weld defects on the fatigue behavior of welded construction," British Welding Journal, 14(1), 1967.

${ }^{3}$ R.W. Richardson, "Robotic weld joint tracking systems - theory and implementation methods," Welding Journal, 65(11)43-51, 1986.

${ }^{4}$ R.B. Madigan, et. al. "Monitoring and Control of Welding and Joining Processes," Welding Handbook, $9^{\text {th }}$ ed., vol. 1, chapt. 10, pp:421-448, American Welding Soceity, 2001.

${ }^{5}$ D. Barborak, R. Richardson, D. Farson, and H. Ludewig, "Empirical models for GMAW fillet weld profiles", Proc. $9^{\text {th }}$ Int. Conf. On Computerization of Welding Information, NIST special publication, vol. 949, pp.76-87, 1999.

${ }^{6}$ Passino, K. and Yurkovich, S. "Fuzzy Control", Addison Wesley Longman Inc. ISBN \#0-20118074, 1997. 



\title{
WELD PENETRATION CONTROL DURING GTA WELDING USING WELD POOL OSCILLATION SENSING
}

\author{
M.J.M. Hermans*, B.Y. Yudodibroto ${ }^{*}$ and G. den Ouden ${ }^{*}$
}

\begin{abstract}
In this paper attention is given to on-line control of weld penetration during GTA welding by means of weld pool oscillation frequency measurements.

Weld pool oscillations can be triggered by applying short current pulses. Due to changes in arc length as a result of the oscillation of the weld pool, the frequency of the oscillation can be measured from the arc voltage signal. Different modes of oscillation can be distinghuised. For each mode the oscillation frequency depends on the weld pool geometry, the density of the liquid metal and the surface tension. It appears that a dramatic change in oscillation frequency occurs when a transition takes place from partial to full penetration or vice versa. This phenomenon is used as a sensing tool for penetration control.

By applying a feedback loop in the measuring and control system, a situation can be obtained where full penetration can be maintained by welding current adjustment.

Experiments were carried out both in bead-on-plate welding as in orbital tube welding, which show that good results can be obtained. It appears that shielding gas composition plays an important role as it influences the electric field strength in the arc and thus the detectability of the changes in arc voltage/length.
\end{abstract}

\section{KEYWORDS}

GTA welding, sensing and control, weld penetration control, weld pool oscillation

\section{INTRODUCTION}

Over the last decade a large amount of research has been carried out in the field of sensing and control during welding. Development in sensor systems for automation of welding processes is essential to increase productivity and to detect defects in an early stage of production. A large variety of tactile and non-tactile sensing systems have been developed for seam positioning, seam tracking, process control, penetration control and detection of weld pool dimensions [refs.1-10]. Especially through-the-arc-sensing is an elegant and relatively simple method, as it does not involve additional equipment.

This paper deals with penetration sensing and control during GTAW based on the occurrence of oscillations in the weld pool. The oscillation frequency of the weld pool is calculated from variations in the voltage. As the mode and the frequency of the oscillation are related with the

* Delft University of Technology, Joining Technology, Delft, The Netherlands 
state of penetration, this information can be used for penetration control. The benefits of the developed feedback system incorporate adjustment of the current in order to prevent overpenetration due to accumulation of heat or underpenetration, for example as a result of castto-cast variations, and makes additional NDT superfluous.

In order to obtain oscillations in the weld pool, a short current pulse superimposed on the welding current is applied, which increases the arc pressure temporarily. Due to the variation of the arc length and thus the arc voltage, the frequency of the oscillation can be calculated from the collected voltage data. It must be noted that as the sensor is based on measuring arc voltage variations, the detectability in the case of GTAW with helium gas shielding is better than with argon gas shielding, as the electric field strength in the arc column is higher (Ar: $\mathrm{E}=\sim 1 \mathrm{~V} / \mathrm{mm}$, He: $\mathrm{E}=\sim 2 \mathrm{~V} / \mathrm{mm}$ ).

A weld pool can oscillate in different modes depending on the external circumstances. The dominant modes in the case of a partially penetrated weld pool are schematically depicted in Figure 1. Mode 1 represents an up-and-down movement of the weld pool, whereas mode 2 represents a front-to-back movement. As these modes are surface oscillations, the oscillation frequencies are relatively high. They can be expressed by the following equations [2]:

$$
\begin{array}{ll}
\text { Mode 1: } & f_{1}=5.84\left(\frac{\gamma}{\rho_{l}}\right)^{1 / 2} D^{-3 / 2} \\
\text { Mode 2: } & f_{2}=3.37\left(\frac{\gamma}{\rho_{l}}\right)^{1 / 2} D^{-3 / 2}
\end{array}
$$

In these equations $\gamma$ represents the surface tension, $\rho$ the density of the liquid metal in the weld pool and D the (equivalent) diameter of the weld pool. It can be seen that the oscillation frequency decreases with increasing weld pool width and that mode 2 oscillation exhibits a lower oscillation frequency. A transition from mode 1 to mode 2 oscillation occurs when increasing trigger duration, trigger current and travel speed. Especially travel speed has a strong influence, as with increasing travel speed the weld pool becomes elongated and the position of the impulse of the arc pressure shifts towards the front part of the weld pool.

In the case of full penetration the weld pool oscillates as a membrane, see Figure 2. This bulk type of oscillation has a relatively low frequency, which can be expressed by:

$$
\text { Mode 3: } \quad f_{3}=1.08\left(\frac{\gamma}{H \rho_{s}}\right)^{1 / 2} D^{-1}
$$

with $\mathrm{H}$ the thickness of the workpiece. Higher order modes of oscillation in the full penetration situation are to be expected in case of a more eccentric position of the arc with respect to the centre of the weld pool [Ref. 11]. However, the amplitude of these higher order oscillation modes is expected to be relatively small. 


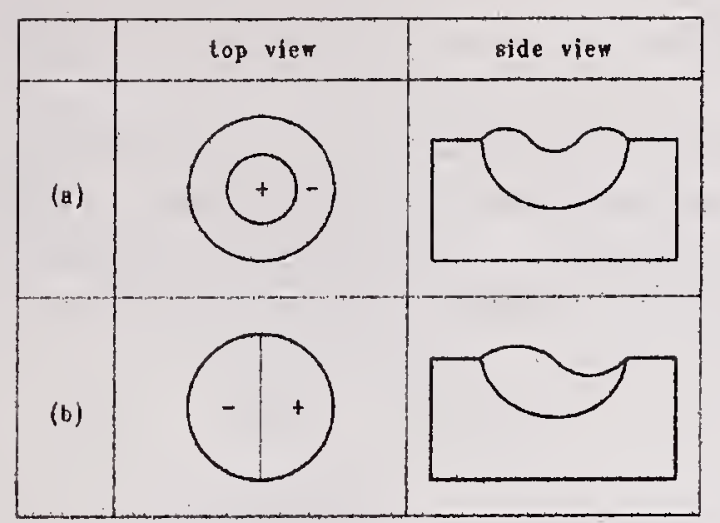

Figure 1: Top and side view of the oscillation modes in partially penetrated welds: a) mode 1, b) mode 2 .

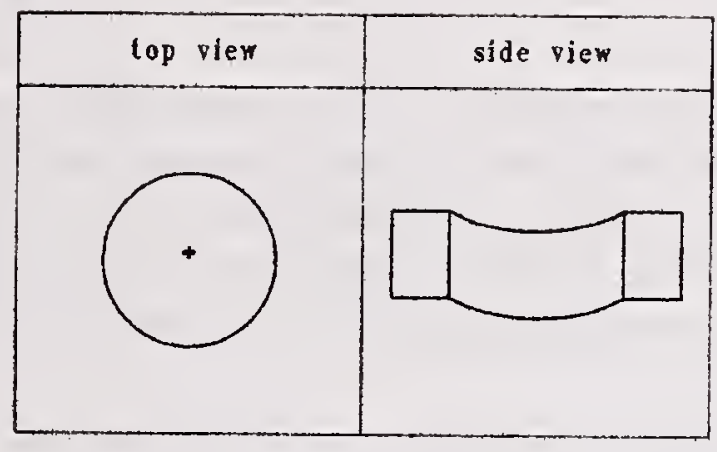

Figure 2: Top and side view of full penetration oscillation mode, mode 3 .

The principle of the weld pool oscillation based penetration sensing is that the oscillation frequency of the weld pool changes abruptly during the transition from a partially to a fully penetrated weld pool. By applying a simple feedback control system, the welding current can be adjusted in order to maintain correct penetration over the entire weld length. Overpenetration and underpenetration can both be detected and automatically repaired by adjusting the welding current.

To apply the penetration sensing method based on weld pool oscillations, two different approaches can be followed.

The first approach (single frequency approach) can be used in the case of constant current welding [Ref. 8]. The basic principle of this method is to predefine and maintain an optimal oscillation frequency (control frequency) for a correct fully penetrated weld pool. During welding the oscillation frequency is measured and welding parameters are adjusted in such a way that the requirements of the full penetration control frequency are met. The advantage of this method is the better detectability of the mode 3 oscillation compared to the partially penetrated oscillation modes (mode 1 and 2) as the amplitude and thus the variations in arc voltage are relatively high. This approach is therefore suitable for small weld pool top width, i.e. relatively high welding speed conditions, and for welding under argon gas shielding. Selecting the appropriate control frequency is the key to success of this approach.

The second approach (double frequency approach) can be applied in the case of pulsed GTAW [Ref. 7]. Basically, the procedure is similar to the single frequency approach. The oscillation in the weld pool is triggered by a short current pulse and the frequency is measured during both the pulse and the base period. Correct penetration is achieved when during the pulse period an oscillation frequency is measured corresponding to the oscillation frequency of a fully penetrated weld pool, while during the base period the oscillation frequency corresponds with the frequency of a partially penetrated weld pool. In this approach mode 1 oscillation should be monitored, as the difference between the magnitude of the mode 2 oscillation frequency and the mode 3 oscillation frequency is small. Therefore the travel speed has to be relatively low.

The focus of this paper will be on the single frequency approach.

Momentarily, research is carried out concerning the influence of metal transport on weld pool oscillations during GTAW with filler wire. 


\section{EXPERIMENTAL}

A large number of experiments are carried out on mild steel and stainless steel in the form of plate and tube of different dimensions. The physical properties of the metals are listed in Table 1. For welding a transistorized power source was used in constant current mode. Trigger pulses were superimposed on the welding current. As shielding gas both $\mathrm{Ar}$ and $\mathrm{Ar}-5 \% \mathrm{H}_{2}$ were used. The welding parameters are listed in Table 2.

Table 1: Physical properties of the steels used.

\begin{tabular}{|l|c|c|}
\hline Workpiece metal & Surface tension $\gamma_{0}(\mathrm{~N} / \mathrm{m})$ & Density $\rho\left(\mathrm{kg} / \mathrm{m}^{3}\right)$ \\
\hline Mild steel, Fe 360 & 1.0 & $7.0 \cdot 10^{3}$ \\
Stainless steel, AISI 316L & 1.2 & $7.5 \cdot 10^{3}$ \\
\hline
\end{tabular}

Table 2: Welding conditions.

\begin{tabular}{|l|l|}
\hline Electrode & $\mathrm{W}-2 \% \mathrm{ThO}_{2}, \varnothing 2.4 \mathrm{~mm}$, tip angle $60^{\circ}$ \\
\hline Arc length & $1.0-2.0 \mathrm{~mm}$ \\
\hline Travel speed & $7.5-24 \mathrm{~cm} / \mathrm{min}$ \\
\hline Shielding gas & $\mathrm{Ar}, \mathrm{Ar}-5 \% \mathrm{H}_{2} 10 \mathrm{l} / \mathrm{min}$ \\
\hline Base current & $30-150 \mathrm{~A}$ \\
\hline Trigger pulse frequency & $2 \mathrm{~Hz}$ \\
\hline Trigger current & $100-300 \mathrm{~A}$ \\
\hline Trigger duration & $2-3 \mathrm{~ms}$ \\
\hline
\end{tabular}

Labview (National Instruments) was used to control the power source and shielding gas supply, for applying the trigger pulses, for data acquisition and for feedback control. Figure 3 shows a data flow chart. The input parameters are: initial base current and trigger current, trigger duration, trigger frequency and control frequency. During welding, voltage data was collected with an acquisition rate of $20.48 \mathrm{kHz}$. During every cycle 4896 data points are captured, see Figure 4. From this set of data the first 400 data points (including data before the trigger pulse and the data of the trigger pulse) and the next 400 data points (just after the trigger pulse) are omitted for data analysis. Thus, a sub-set of 4096 voltage data points remain for determining the oscillation behaviour. The DC component is removed from the signal. This data was then filtered with a band pass filter and on-line analysed by means of Fast Fourier Transformation. The calculated oscillation frequency was compared with the selected control frequency. The outcome of this comparison was then used for current feedback.

The response rate in current adjustment depends on the difference in the measured oscillation frequency and the required control frequency. If necessary, current adjustments were carried out after each cycle. As an additional safety in the system the amplitude of the peak frequency is also taken into account. In the case that the amplitude is too low, for instance when generation of a proper oscillation fails, the signal is disregarded by the feedback system.

The raw and analysed data is stored on disk. Therefore, at any position in the weld, the data of the oscillation behaviour is traceable and provides information about penetration quality. 


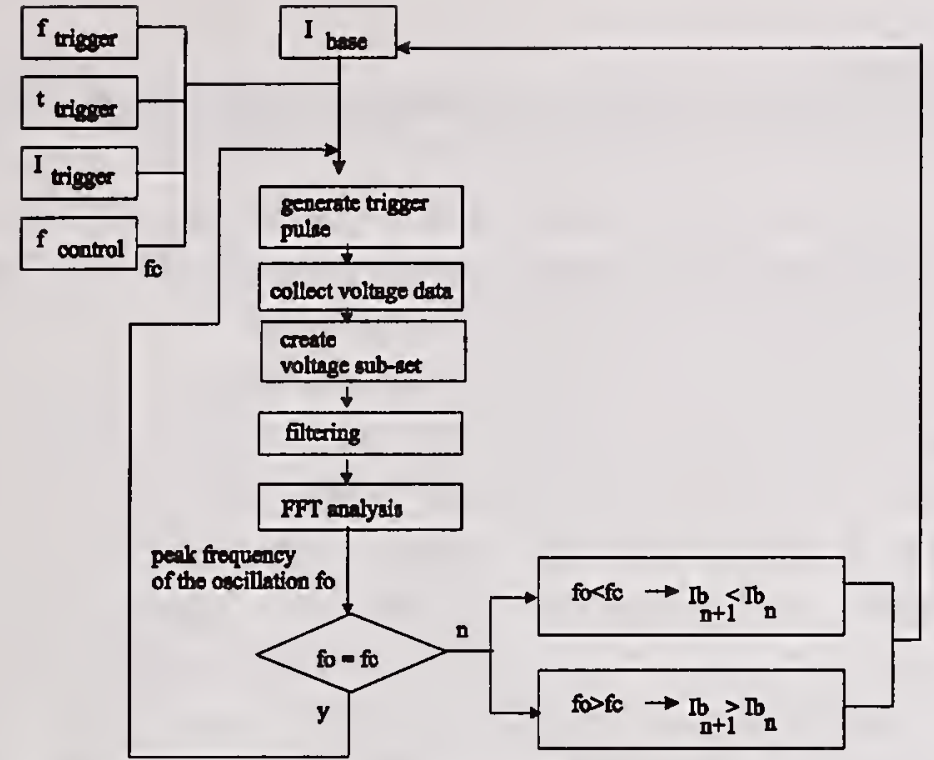

Figure 3: Flow chart of data analysis

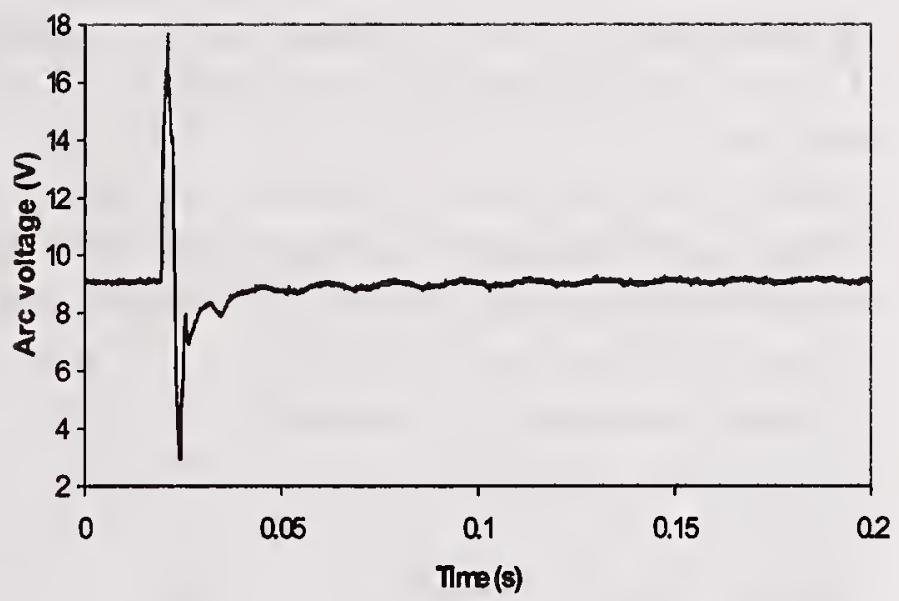

Figure 4: Example of collected voltage data

\section{RESULTS AND DISCUSSION}

\section{Data acquisition and analysis for partial and full penetration}

Oscillation frequency measurements were carried out under various experimental conditions. Figure 5 shows the signals obtained in the case of a partially penetrated weld pool during beadon-plate welding of mild steel. Figure 5a depicts the arc voltage as function of time after triggering when the weld pool is partially penetrated. A good oscillation signal can be directly identified by the occurrence of damping. This signal is processed by FFT, which is displayed in Figure $5 \mathrm{~b}$. The oscillation frequency of a partially penetrated weld pool is situated at the higher regions of the frequency domain and can be associated with surface oscillations.

The situation in which full penetration occurs is presented in Figure 6. In this case, the relatively low frequency, characteristic for bulk oscillation is observed.

The measured oscillation frequency, in both situations, is in good agreement with the calculated oscillation frequency (equation 1 and 3 ).

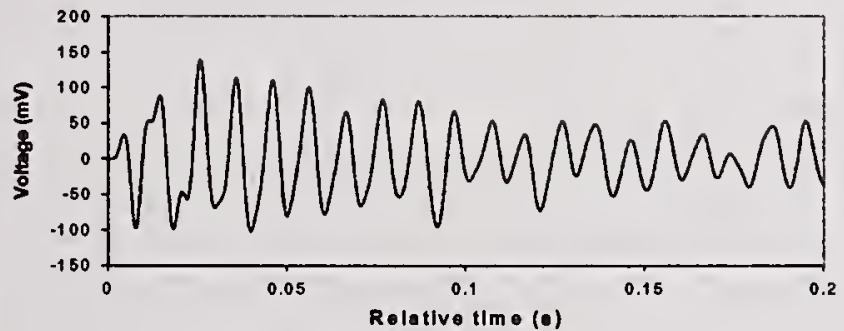

a)

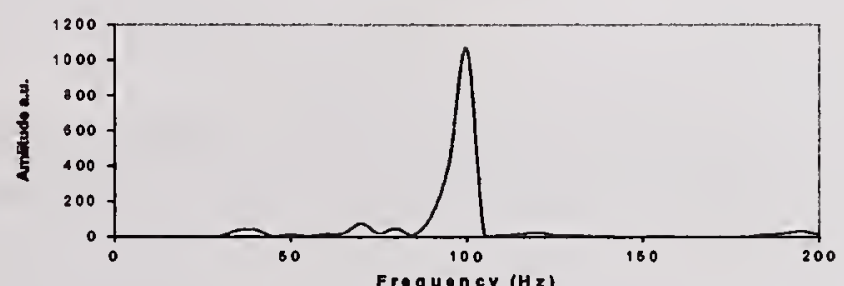

b)

Figure 5: Partial penetration, a) voltage oscillation signal, b) frequency domain.

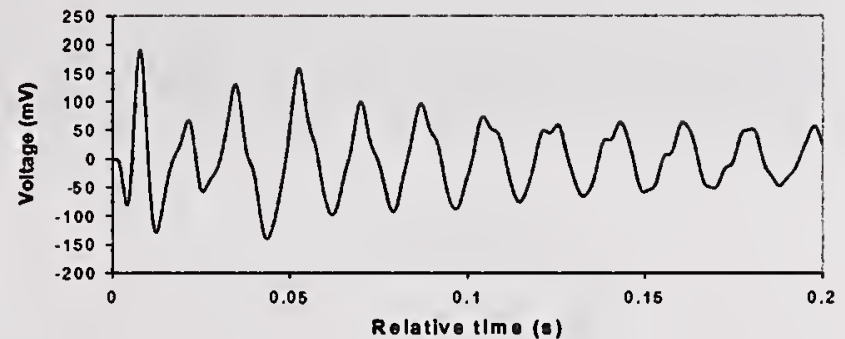

a)

b)

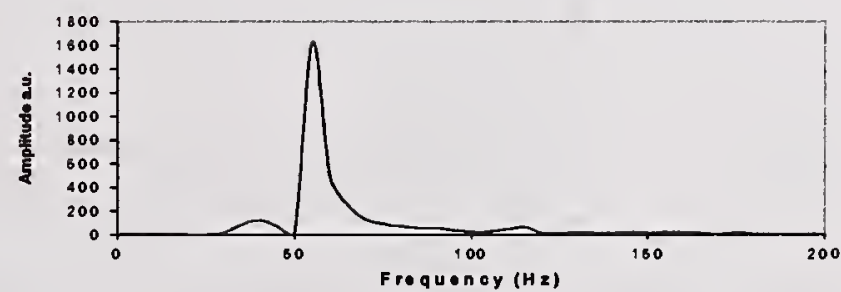

Figure 6: Full penetration, a) voltage oscillation signal, b) frequency domain. 


\section{Sensing and control during bead-on-plate welding}

For the single frequency approach a control frequency in the full penetration range has to be chosen.

As an example, Figure 7 shows the results of a bead-on-plate weld on a $3 \mathrm{~mm}$ thick mild steel workpiece welded at a travel speed of $2 \mathrm{~mm} / \mathrm{s}$. The initial base current was set at $115 \mathrm{~A}$. The control frequency is set at $50 \mathrm{~Hz}$. This value is calculated by means of equation 3 .

During the first part of the weld the feedback system was not activated. As can be seen the current is constant. At the start of welding a partial penetration situation exists and the oscillation process has to be stabilised. This period (until $t=6 \mathrm{~s}$ ) is associated with the occurrence of a relatively high frequency. As the weld pool size increases during welding, full penetration is obtained and the oscillation frequency starts to drop (from $t=6 \mathrm{~s}$ ). Finally, severe sagging of the weld pool occurs. At $t=22 \mathrm{~s}$ the feedback system is switched on. As can be seen in the figure, the base current is reduced in order to increase the oscillation frequency of the weld pool by reducing the weld pool bottom width. The measured oscillation frequency is increasing until the control frequency is reached. The width of the penetration remains constant when the feedback system is active.

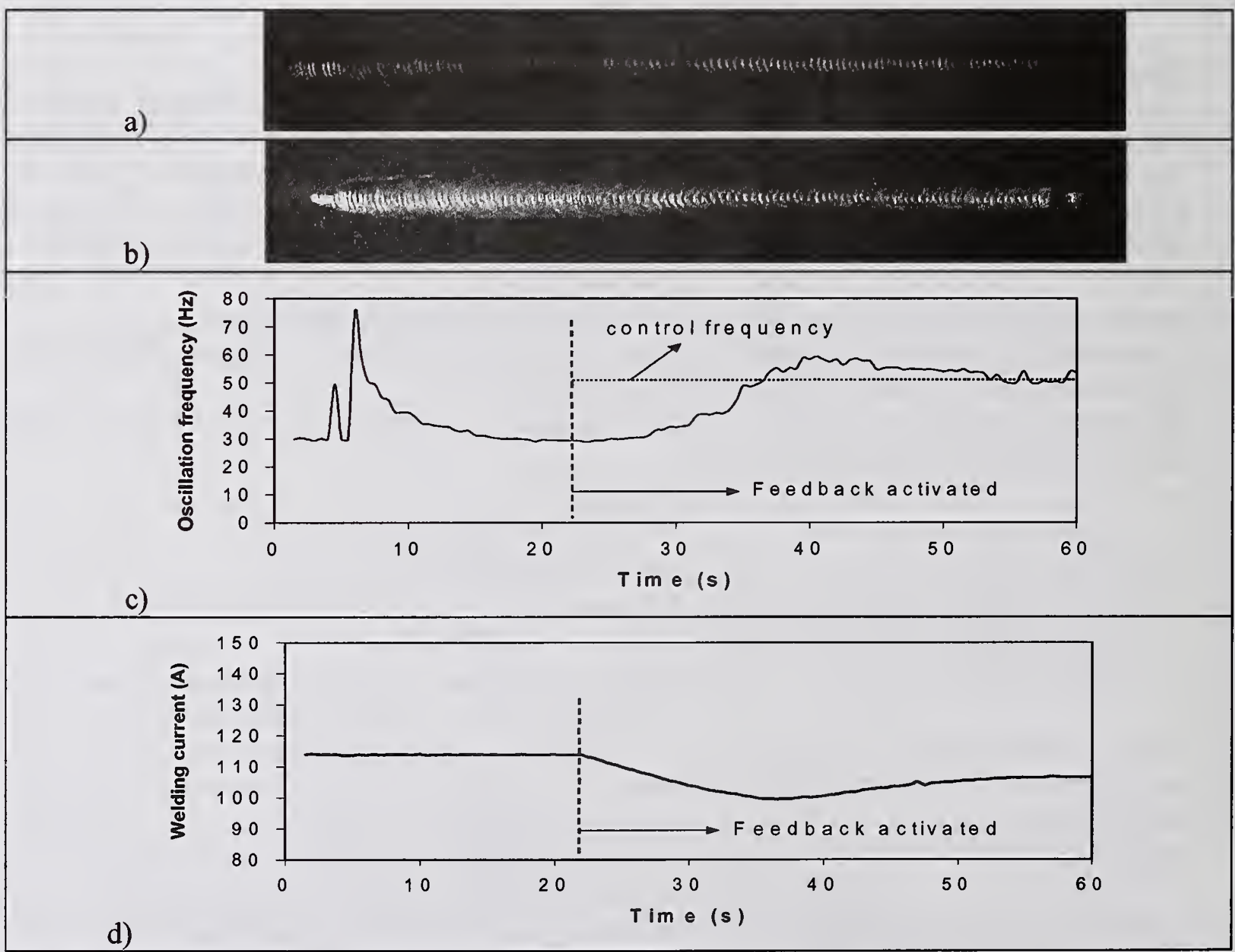

Figure 7: Bead-on plate weld on $3 \mathrm{~mm}$ thick mild steel; a) top view of the weld, b) bottom view of the weld, c) oscillation frequency as function of time, d) welding current as function of time. 


\section{Sensing and control during orbital tube welding}

The oscillation frequency and the current response were also measured during orbital tube welding on $2.0 \mathrm{~mm}$ thick stainless steel AISI 316L. The initial base current was $50 \mathrm{~A}$ and a control frequency of $40 \mathrm{~Hz}$ was selected. The feedback system was activated before welding started. An example of the results obtained is presented in Figure 8. Figure 8a shows the measured oscillation frequency as function of time. After full penetration is achieved, the oscillation frequency is maintained at the control frequency by small current adjustments (Figure $8 \mathrm{~b})$. The reduction in current in the final part of the weld is directly related with the accumulation of heat in the tube. Less current is required to maintain full penetration.

Inspection of the welds shows that correct penetration is achieved along the entire weld and that the overall quality is excellent, see Figure 8c.

It should be expected that the welding position, in the case of orbital welding, has an influence on the weld pool behaviour. However, it appears that the influence of gravity on the oscillation behaviour is limited and therefore can be neglected [2].

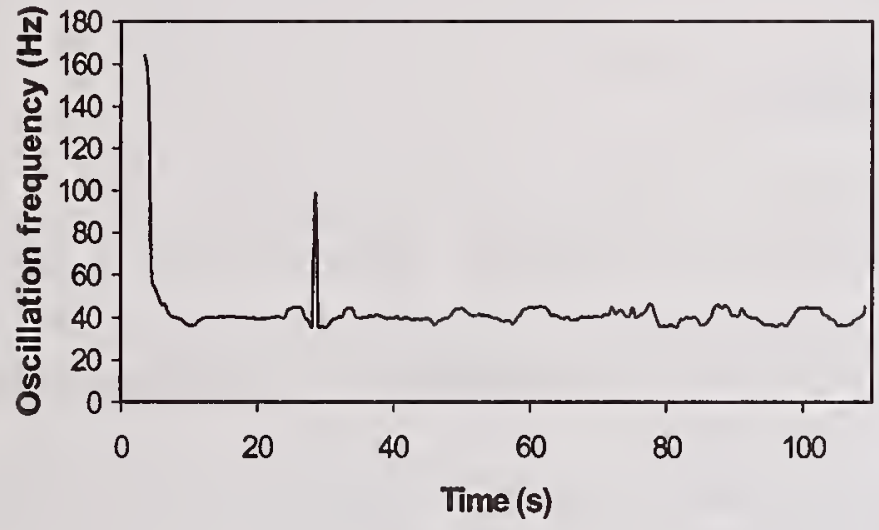

a)

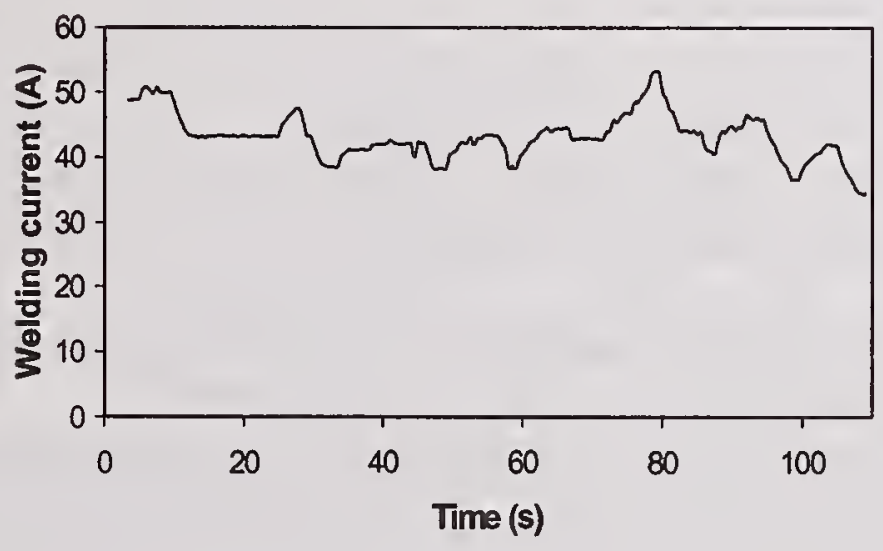

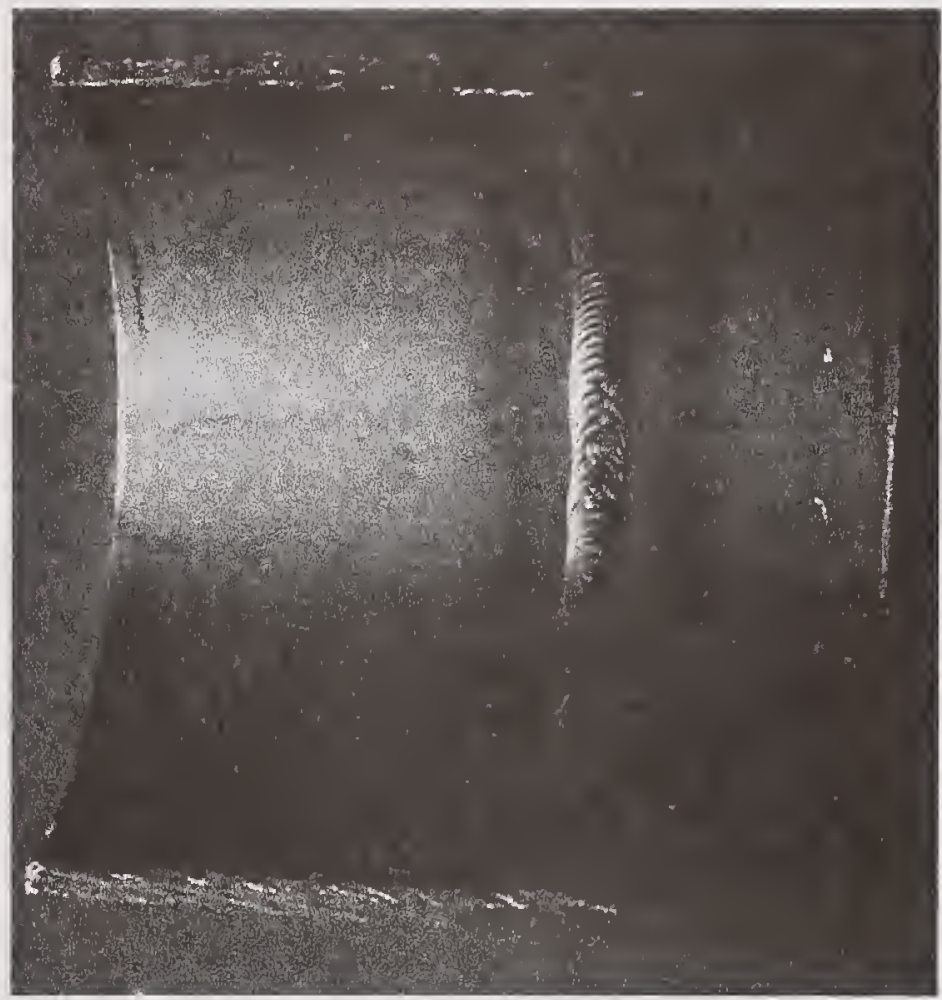

c)

b)

Figure 8) Orbital weld on $2 \mathrm{~mm}$ stainless steel; a) oscillation frequency versus time, b) welding current versus time, c) penetration appearance. 


\section{CONCLUSIONS}

On the basis of the results of the research carried out in the field of penetration sensing by means of weld pool oscillation monitoring the following conclusions can be drawn:

1. Partially and fully penetrated weld pools oscillate in different oscillation modes.

2. The oscillation frequency depends on the mode of oscillation, weld pool size and physical properties of the liquid metal.

3. The oscillation frequency drops when full penetration is achieved. This change in oscillation frequency can be used for in-process control of weld pool penetration.

4. The use of sensing in combination with feedback results in excellent weld quality.

\section{ACKNOWLEDGEMENTS}

The authors wish to express their gratitude to Y.H. Xiao, A.J.R. Aendenroomer and B. Hu for their contributions and to W.A.J. Brabander, F.J.A.M. Bosman for their technical assistance.

\section{REFERENCES}

1. Madigan R.B. and R.J. Renwick; 'Computer based control of full penetration GTA welds using pool oscillation sensing', Proc. $1^{\text {st }}$ Int. Conf. On Computer Technology in Welding, London, UK, 1986, 165-174.

2. Xiao Y.H. and G. den Ouden; 'A study of GTA weld pool oscillation', Welding Journal, Vol. 69, No.8, 1990, 289s-293s.

3. Sorensen C.D. and T.W. Eagar; Modeling of oscillation in partial penetration weld pools', Journal of Dynamic Systems, Measurement and Control, 1990, 112, 469-474.

4. Xiao Y.H. and G. den Ouden; Weld pool oscillation during GTA welding of mild steel', Welding Journal, Vol 72, No.8, 1993, 428s-434s.

5. Bicknell A., J.S. Smith and J. Lucas; 'Arc voltage sensor for monitoring of penetration in TIG welds', IEE Proc. Sci.Meas. Technol. Vol 141, No. 6, 1994, 513-520.

6. Anderson K., G.E. Coock, R.J. Barnett and A.M. Strauss; 'Synchronous weld pool monitoring and control', IEEE transactions on industry applications Vol. 33, No. 2, March/April 1997, 464-471.

7. Aendenroomer A.J.R. and G. den Ouden; 'Weld pool oscillation as a tool for penetration sensing during pulsed GTA welding', Welding Journal, Vol 77, 5, 1998, 181s-187s.

8. Hu B. and G. den Ouden; Weld penetration sensing and control during GTA welding using weld pool oscillation, in Proceeding Int. Conf. on trends in welding research, Pine Mountain, Georgia, USA, Juni 1998, 1125-1130.

9. Rehfeldt D. and T. Polte; 'Arc sensor as a quality assurance element for uniform full penetration welding', Schweissen und Schneiden, Vol. 52, No. 10, 2000, E228-E231.

10. H. Wohlfahrt, K. Thomas and S. Wiesner; 'Welding voltage measurements during TIG and MIG welding with the objective of controlling the weld penetration', Schweissen und Schneiden, Vol. 53, No. 2, 2001, E29-E31.

11. Maruo $\mathrm{H}$. and Y. Hirata; 'Natural frequency and oscillation modes of weld pools', Welding International, Vol. 7, No. 8, 1993, 614-619. 


\title{
REAL-TIME MOLTEN POOL AREA EXTRACTION FOR CONTROL OF GAS TUNGSTEN ARC WELDING
}

\author{
Y.Z. Wang, Z. Jandric and R. Kovacevic ${ }^{*}$
}

\begin{abstract}
This paper presents a real-time molten pool area extraction method for the control of gas tungsten arc welding (GTAW) with a wire filler in the hybrid rapid prototyping and tooling (RP\&T) technique based on welding and CNC milling. The molten pool shape and size were strongly influenced by the two-dimensional geometry of the built part. A coaxial machine vision unit is used to acquire images of molten pool and its surrounding area through the torch. After image processing, the boundary of the molten pool is extracted. Because the molten pool is partially hidden by the tungsten electrode, its boundary is incomplete. The molten pool area is selected as the feedback to the closed-loop control of the GTAW process. In order to calculate the area of the molten pool with an incomplete boundary, a neural network is used. By changing welding parameters, the neural network is trained for different sizes of molten pools. The testing of the neural network is performed by another group of molten pool images.
\end{abstract}

\section{INTRODUCTION}

Nowadays, industry is under constant and growing pressure for shortening the time-to-market lead time. Rapid prototyping (RP) techniques have been developed for fulfilling this demand. For many years, rapid prototyping techniques have been used almost exclusively on materials such polymers, waxes, or paper (Ref. 1). The rapid prototyped parts had limited dimensions, and they had a tendency to distort due to shrinkage.

Three-dimensional welding has the ability to rapidly produce strong, fully dense metallic parts in the form of layers (Refs. 2,3). To increase the surface quality and accuracy of the metallic parts made by the 3D welding, a promising hybrid rapid prototyping and tooling (RP\&T) technique based on gas tungsten arc welding (GTAW) and CNC milling has been under development at the Research Center for Advanced Manufacturing (RCAM) at Southern Methodist University, Dallas, TX (Ref. 4). This hybrid process allows the fabrication of metallic parts with high dimensional accuracy and with complex external and internal geometrical features.

In the hybrid RP\&T technique, the GTAW process with a wire filler provides depositing materials and energy to fuse the added material. For the needs of RP\&T, the welding process is required to provide lower heat input and lower bead penetration that is exactly opposite to the conventional welding process. In order to generate uniform and fairly flat beads and layers, a metal deposition rate and molten pool size should be invariant of the alternations of the welding

Research Center for Advanced Manufacturing, Southern Methodist University 1500 International Pkwy, Suite 100, Richardson TX 75081

* corresponding author.

Tel: 1-214-768-4865, Fax: 1-214-768-0812,Email: kovacevi@seas.smu.edu 
conditions. The geometry of the molten pool can provide abundant, accurate and instantaneous information about the welding process. Hence, closed-loop control of the welding process, with the information of the molten pool size as a feedback, is a necessity.

Although several indirect approaches, such as monitoring the pool oscillation, the ultrasonic technique, infrared sensing, and radiography, have been proposed to sense the geometry of the molten pool, machine vision, being non-intrusive, seems to be the most promising technique for obtaining the clear geometry of a molten pool.

Machine vision systems have been extensively used for the molten pool sensing and seam tracking for more than two decades, mostly with the idea to simulate the eye sensing and process actuation of experienced human welders. Previous research works for sensing the molten pool in GTAW with machine vision was conducted without a wire filler.

In the GTAW process with a wire filler, it is necessary to ensure that the wire is always fed in front of the moving arc. In order to avoid blocking the optical path between the molten pool and the CCD camera by the wire filler, a coaxial machine vision unit, developed by Richardson et al. (Ref. 5), is used in this paper. The lens of a CCD camera is coaxially mounted with the electrode. The electrode tip is used to block the bright core of the arc light from the overpowering exposure on the CCD target. After the image of the molten pool is captured, the Laplacian operator is used to detect the edges. Then, the image was segmented into a binary one with an adaptive threshold. The boundary of the molten pool is extracted from the binary image.

The molten pool size and shape in the hybrid RP\&T are strongly influenced by the twodimensional geometrical features of the part to be built. Fig. 1 demonstrates the molten pools at the flat surface (point 6), the edge (point 5), and the corners with different angles (points 1 to 4), while keeping the welding parameters constant. This paper is concentrated on the acquisition and processing of the shape of the molten pool at the flat surface (point 6).

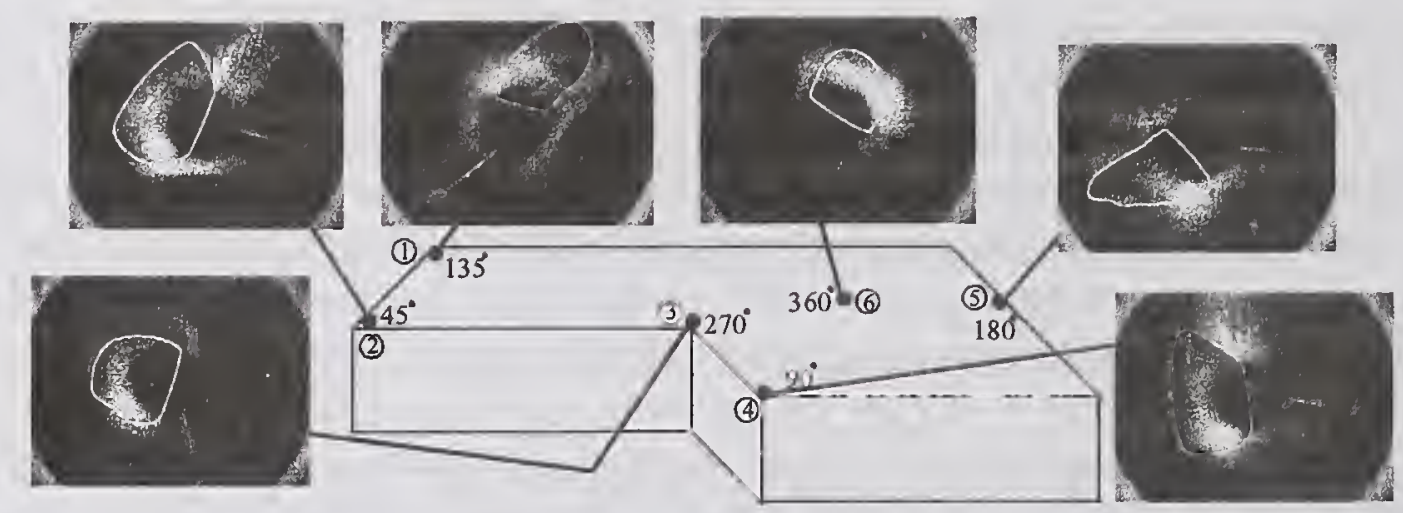

Figure 1. The shapes of the molten pool as a function of $2 \mathrm{D}$ geometrical features of substrate

\section{EXPERIMENTAL SET-UP AND EXPERIMENTAL CONDITIONS}

Fig. 2 illustrates the schematic presentation of the hybrid RP\&T machine, consisting of a central controller, a CNC milling machine, a GTAW unit, and a coaxial machine vision unit. The central controller acquires the measured data and controls the welding current, wire feeding speed, and 
wire feeder's position, while the workpiece motion is controlled by the CNC machine microprocessor. The filler metal is fed directly into the molten pool. The step motor 1 is used to ensure that the wire is always in front of the moving arc. The step motor 2 is used to control the filler metal feeding speed.

The coaxial machine vision unit used for the molten pool image acquisition, the image processing, and the molten area extraction, is composed of a computer equipped with the frame grabber, a CCD camera, and the image processing software. The image resolution is $640 \times 480$ pixels with 256 gray-levels.

The experiments are carried out with the following parameters:

- The workpiece traverse speed is 23 $\mathrm{cm} / \mathrm{min}$;

- The wire feeding speeds are 20 and 40 $\mathrm{cm} / \mathrm{min}$;

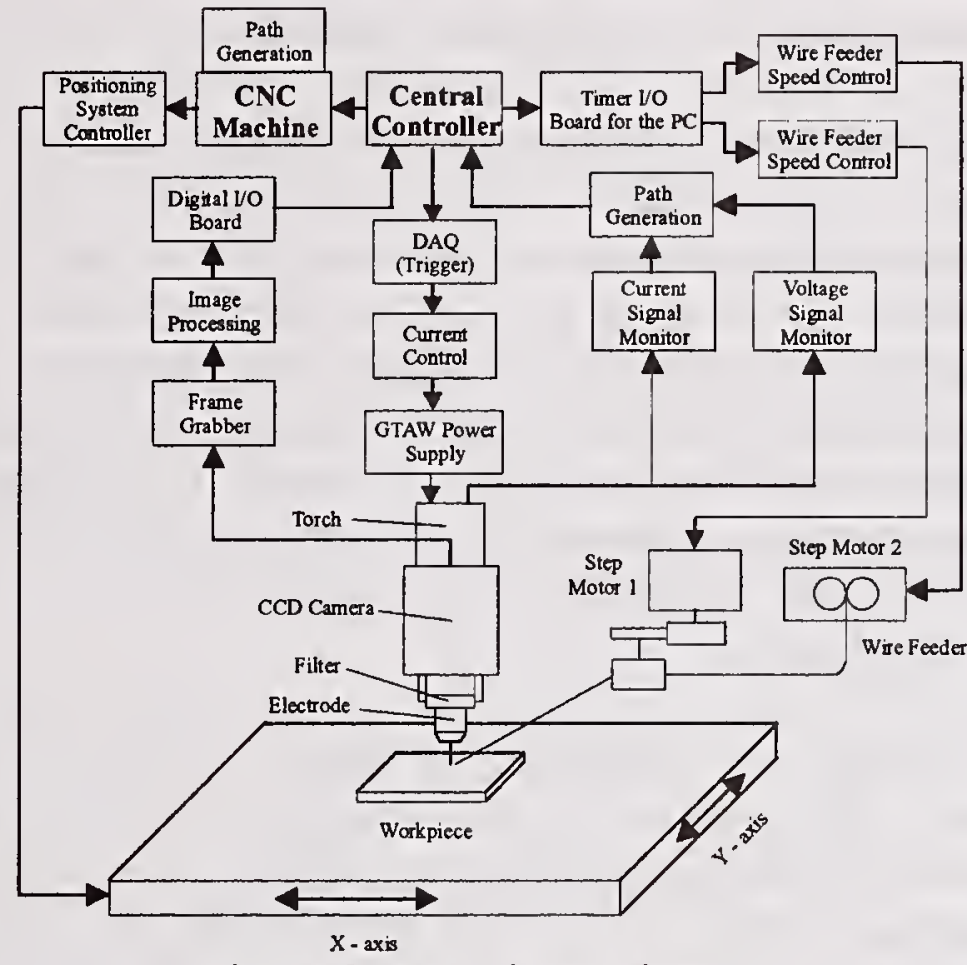

Figure 2. Experimental set-up

- The welding currents are changed from $100 \mathrm{~A}$ to $133 \mathrm{~A}$ with a 3-A interval.

- The welding voltages are changed from $9 \mathrm{~V}$ to $11 \mathrm{~V}$.

- The filler metal is mild steel with a diameter of $0.89 \mathrm{~mm}$.

\section{DESCRIBING MOLTEN POOL GEOMETRY}

In conventional GTAW without a wire filler, the shape of the front part of the molten pool always keeps semicircle with the half-maximum-width as the radius despite different welding conditions, but the sharpness of the rear of the molten pool is changed with the penetration. Also, the shape of the molten pool can be thought as symmetric along the welding direction (Ref. 6). The following geometrical parameters were used by different researchers to characterize the geometry of the molten pool: the maximum width, the maximum length, the half-length, the area, and the rear angle.

In the case of RP\&T based on the deposition by welding, the shape of the molten pool is no longer symmetric along the welding direction, as shown in Fig. 3. Thus, the width and length are no longer reliable parameters to represent the molten pool. In such conditions, the molten pool area is the only reliable parameter for the control of GTAW.

For a molten pool with a closed boundary, the area is equal to the number of the pixels inside the
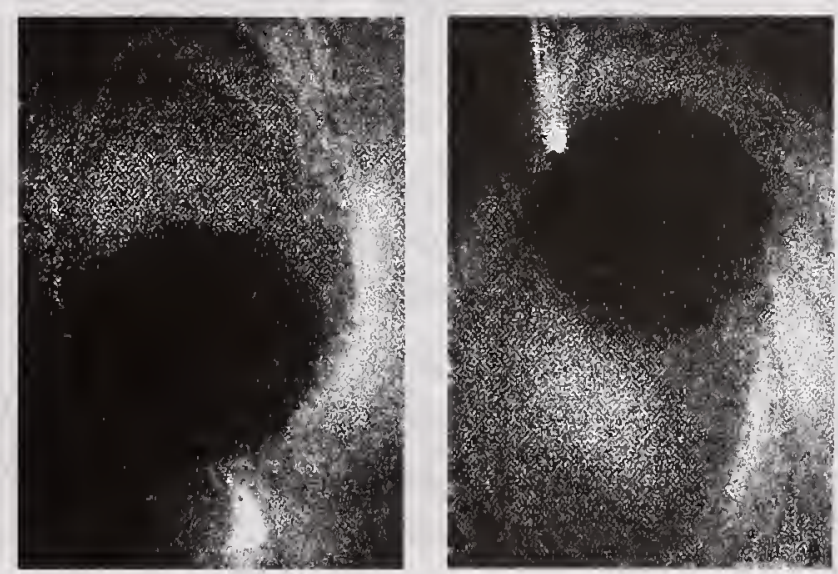

Figure 3. Asymmetric molten pools 
boundary. In order to calculate the area of a molten pool with an asymmetric shape and incomplete boundary, the molten pool must be characterized by the adequate parameters.

The selection of the characteristic parameters is crucial. Three criteria must be satisfied. First, all parameters must have a common reference point. Second, the area of the molten pool must be calculated using the selected characteristic

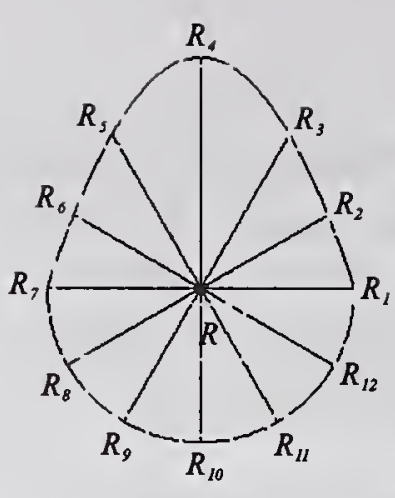

(a) Complete

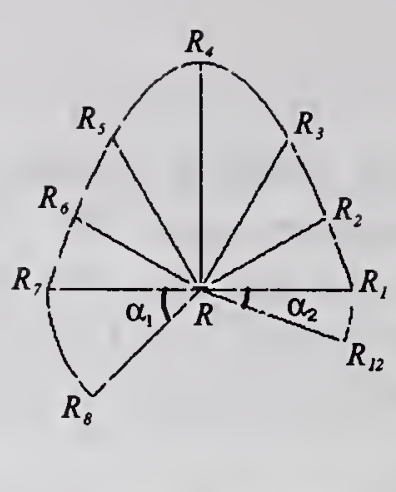

(b) Incomplete

Figure 5. The illustration of the selected characteristic parameters parameters. Third, although more parameters could describe the molten pool more accurately, the increase in the number of selected characteristic parameters may complicate the calculation and increase the calculation time. Thus, the number of parameters must be optimized.

Intuitively, the center of the molten pool is a suitable reference point. But because of the difficulty of finding the molten pool center, a relative fixed point is another choice. The position of the electrode shadow, the dark spot in Fig. 4, is unchanged in all images. The top point $L$ of the electrode shadow is always inside of the molten pool. Based on this position, the reference point $R$ is determined as follows:

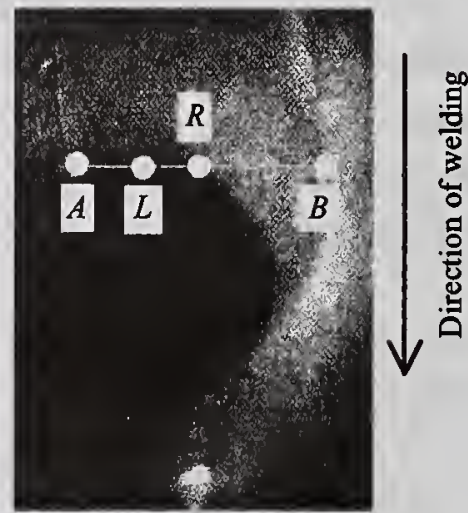

Figure 4. Definition of reference point

- Find the line segment $\overline{A B}$ that passes through point $L$ and is perpendicular to the direction of welding as shown in Fig. 4. $\overline{A B}$ intersects with the boundary of the molten pool at points $A$ and $B$, respectively;

- Find the middle point $R$ of $\overline{A B} . R$ is defined as the reference point for all selected characteristic parameters.

For a closed-contour of the molten pool, in which each radius vector from the center of the boundary intersects the boundary at only one point, a one-dimensional series of real numbers can be acquired by measuring the length of successive radius vectors that are angularly equispaced. This series is denoted as $\{R(\alpha)\}$, where $R(\alpha)$ is the radius vector at the angle $\alpha$.

In our case, the boundary of the molten pool is incomplete. A series of radius vectors is selected as the parameter to calculate the area of the partially hidden molten pool. Fig. 5(a) shows the simulation of a completed molten pool and a series of radius vectors composed of $R_{I} \sim R_{12}$. The angle between two adjacent radius vectors is $30^{\circ}$. From Fig. 4 , it can be seen that the front part of the molten pool is hidden by the electrode shadow. It was experimentally determined that only part of the radius vectors, $R_{1} \sim R_{7}$, can be extracted from every image. The rest, $R_{8} \sim R_{12}$, are not always available. Two radius vectors, denoted as $R_{8}$ and $R_{12}$ are used to connect the reference point $R$ and the two end points. As shown in Fig. 5(b), the angles $\alpha_{1}$ and $\alpha_{2}$ may not be $30^{\circ}$. Thus, $R_{1}$ to $R_{8}$ and $R_{12}$ plus $\alpha_{1}$ and $\alpha_{2}$ are the parameters used to calculate the area. 


\section{MOLTEN POOL BOUNDARY EXTRACTION}

After the image of the molten pool is taken, it is smoothed using a median filter. A Laplacian operator is used to detect the edges. Then, the image is enhanced and segmented into a binary one with adaptive thresholding. All possible edge points are detected. The molten pool boundary is then extracted by making use of the continuity of the molten pool boundary with the following steps as shown in Fig. 6:

- Draw a line that passes through the reference point $R$, defined in previous section, and is perpendicular to the direction of welding (Y direction) as shown in Fig. 6(a);

- Find all possible edge points that the line intersects. For example, points 1 6 in Fig. 6(a);

- Starting from these points, trace the connected edge points to a negative Y direction to form edges, while counting the lengths of all edges;

- Reserve the longest edge as the upper part of the molten pool boundary as shown in Fig. 6(b), and ignore the others;

- Starting from points 3 and 5, along the positive $Y$ direction, trace the possible edge points connected with the upper part of the boundary to find its lower part. The result of this operation is show in Fig. 6(c).

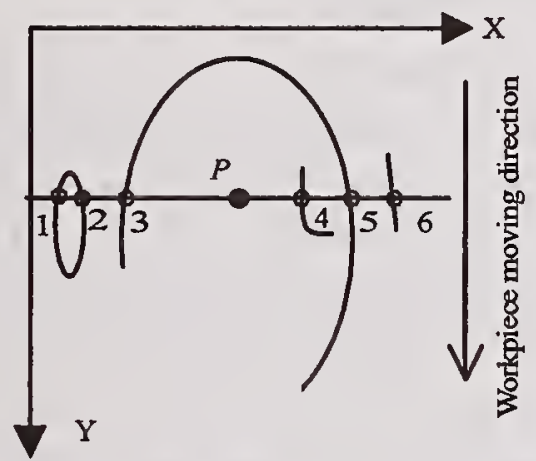

(a) starting points

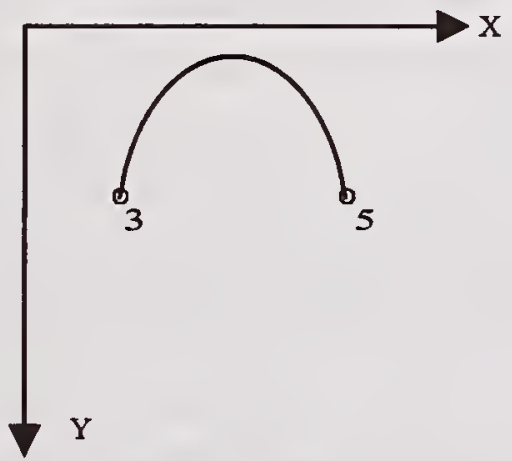

(b) the upper part

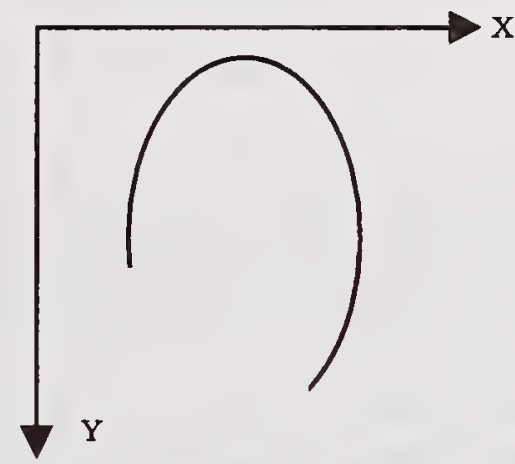

(c) the boundary

Figure 6. Molten pool boundary search

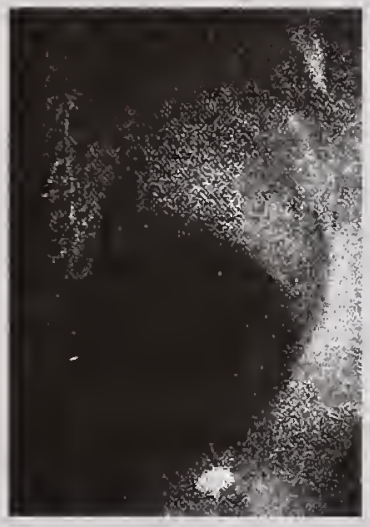

(a) original image

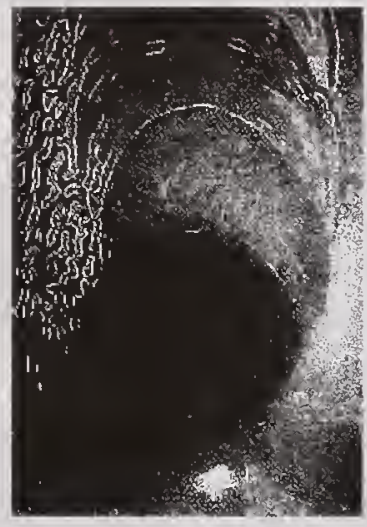

(b) possible edges

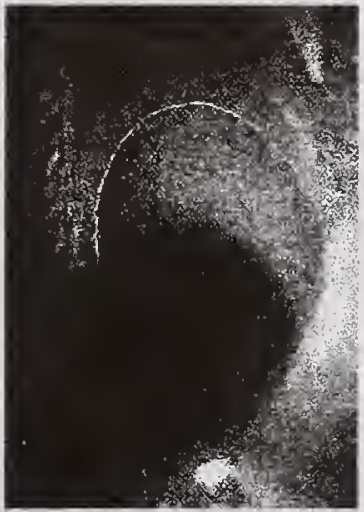

(d) extracted boundary

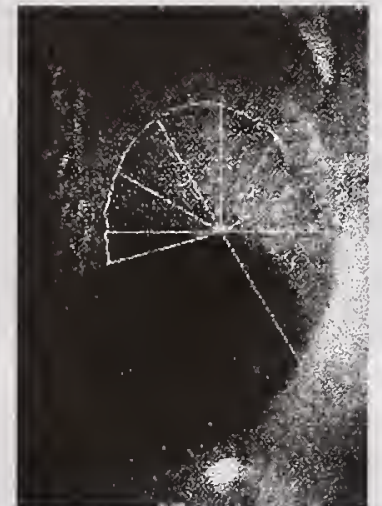

(e) radius vectors

Figure 7. Molten pool boundary extraction

Based on extracted boundary, nine radius vectors and two angles are determined to calculate the area of the molten pool. Fig. 7(a) shows the original image acquired under the following welding conditions: the workpiece traverse speed is $23 \mathrm{~cm} / \mathrm{min}$; the wire feeding speed is $40 \mathrm{~cm} / \mathrm{min}$; the welding current is $115 \mathrm{~A}$; the welding voltage is $10.5 \mathrm{~V}$; and the filler metal is mild steel with a 
diameter of $0.89 \mathrm{~mm}$. The images after each processing step are shown in Fig. 7(b)-(d). The values of the geometrical parameters of the molten pool are tabulated in Table 1. The image processing time is within $300 \mathrm{~ms}$.

Table 1. The nine radius vectors (pixel) and two angles (degree)

\begin{tabular}{|c|c|c|c|c|c|c|c|c|c|c|}
\hline$R_{0}$ & $\alpha_{1}$ & $R_{1}$ & $R_{2}$ & $R_{3}$ & $R_{4}$ & $R_{5}$ & $R_{6}$ & $R_{7}$ & $R_{8}$ & $\alpha_{2}$ \\
\hline 102.7 & 58.9 & 74.0 & 67.0 & 71.6 & 83.0 & 85.5 & 78.5 & 75.0 & 77.9 & 15.6 \\
\hline
\end{tabular}

\section{DEVELOPMENT OF NEURAL NETWORK MODEL FOR MOLTEN POOL AREA CALCULATION}

The area of the molten pool can be correlated using the nine radius vectors and the two angles. However, the correlation is non-linear. To approximate the complicated non-linearity, a twolayer multi-layer perceptron (MLP) neural network is used to build the correlation. Log-sigmoid function is applied as transfer function in both layers. The lengths of the nine radius vectors plus two angles are used as the input vector. The output of the network is the molten pool area.

Back propagation (BP) is a well-established method to train the MLP neural network. In order to ensure the convergence, a momentum method is applied in the BP learning algorithm. The training method is then called back propagation with momentum (BPM). Properly trained BP networks tend to give reasonable answers when presented with inputs that they have never seen before. Typically, a new input leads to an output similar to the correct output for the input vector used in training that is similar to the new input being presented. This generalization property makes it possible to train a network on a representative set of input/target pairs and get good results without training the network on all possible input/output pairs.
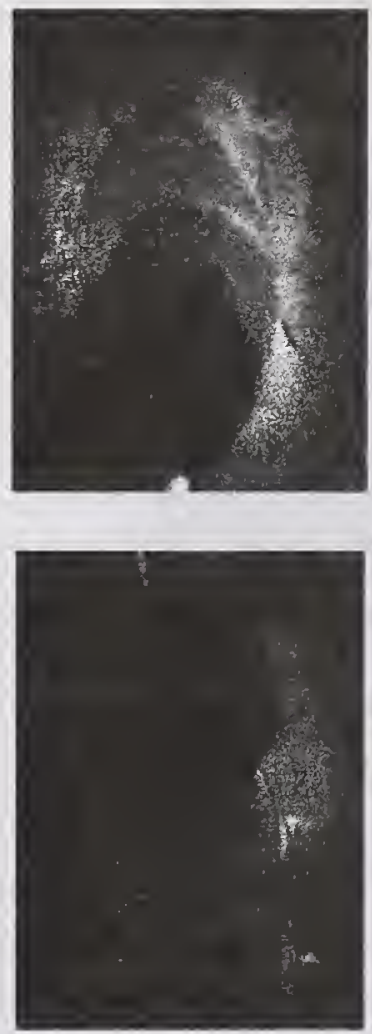

$\mathrm{I}=100 \mathrm{~A}$

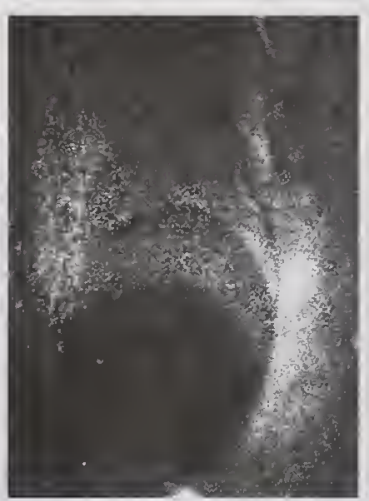

Wire feeding speed $=20 \mathrm{~cm} / \mathrm{mm}$
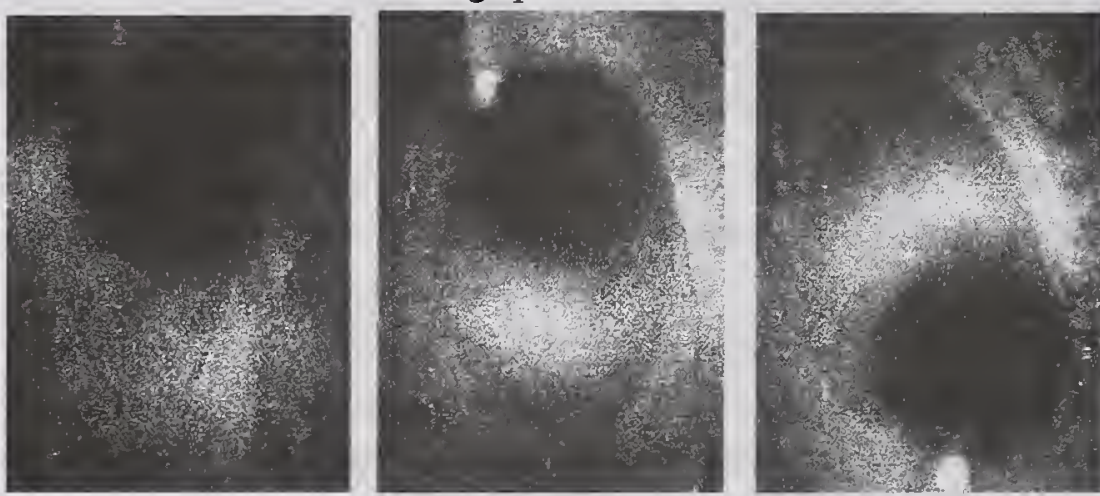

Wire feeding speed $=40 \mathrm{~cm} / \mathrm{mm}$

$\mathrm{I}=109 \mathrm{~A}$

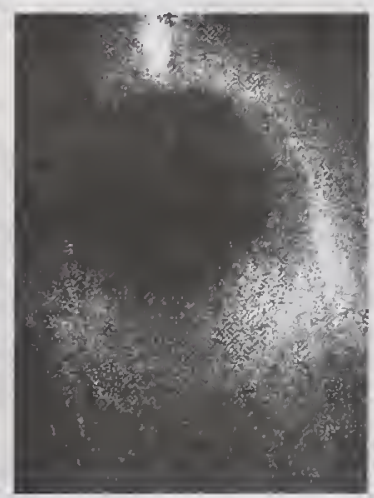

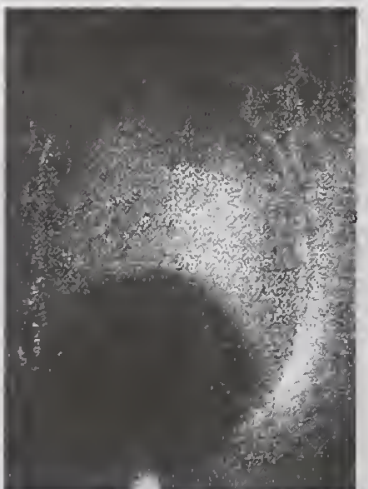
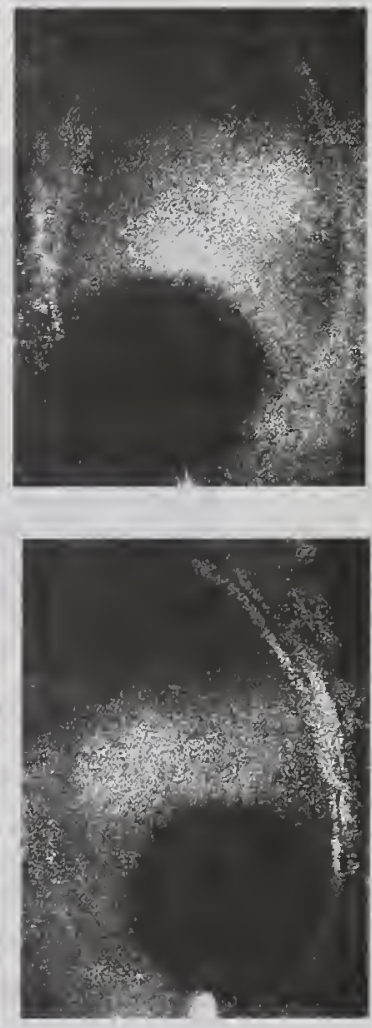

$\mathrm{I}=133 \mathrm{~A}$ 
Figure 8. Molten images under different wire feeding speeds and welding currents

A MATLAB program is developed to train the neural network by BPM using the molten pool images with different sizes as samples. While the workpiece travels to different directions, the molten pool images are acquired under the following experimental conditions: the filler metal is mild steel with a diameter of $0.89 \mathrm{~mm}$; the workpiece traverse speed is $23 \mathrm{~cm} / \mathrm{min}$; the wire feeding speeds are 20 and $40 \mathrm{~cm} / \mathrm{mm}$; and the welding currents are changed from $100 \mathrm{~A}$ to $133 \mathrm{~A}$ with a 3-A interval. Some of the acquired images are given in Fig. 8. It is clear that the molten pool size increases while the welding current increases.

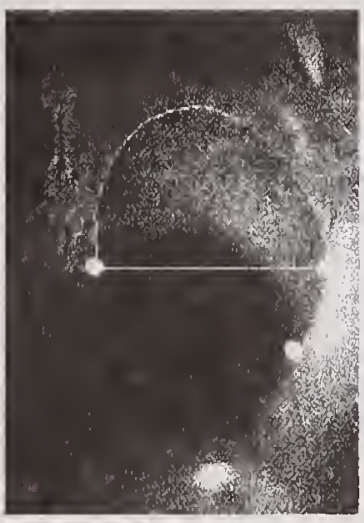

(a) Extracted boundary

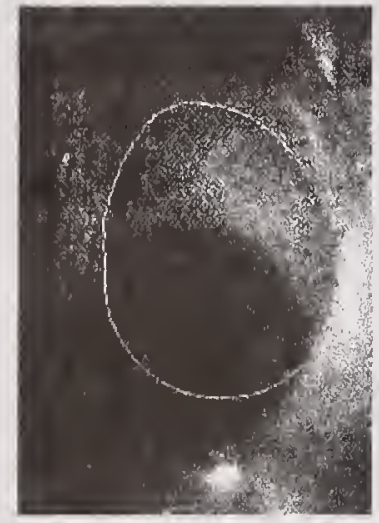

(b) Approximated boundary

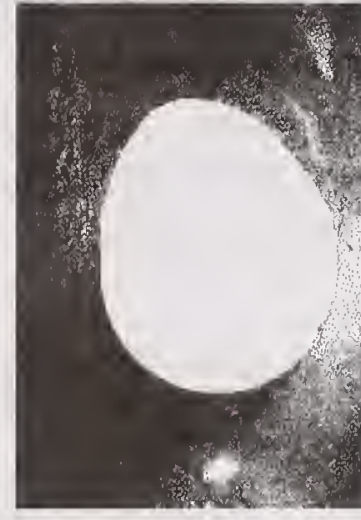

(c) The area

Figure 9. Molten pool boundary approximation

In order to measure the molten pool area, a part of a circle is used to approximate the incomplete molten pool boundary. Fig. 9(a) shows the incomplete boundary extracted from Fig. 7(a). A circle is determined by three points $a, b$, and $c$ on the boundary as shown in Fig. 9(a). Points $a$ and $b$ are the two ends of the extracted boundary. The segment $\overline{a c}$ is perpendicular to the welding direction. The approximated boundary is shown in Fig. 9(b). The molten pool area, the white area as shown in Fig. 9 (c), is then determined by counting the number of pixels.

The values of the geometrical parameters in Tab. 1 and the area, 20,346 pixels, is an input/output pair for the neural network. One hundred input/output pairs are calculated from the acquired molten pool images. Eighty input/output pairs, the smallest and the largest ones are 12,166 and 44,738 pixels, respectively, are used to train the neural network model. The other 20-input/output pairs test the trained neural network. Fig. 10 provides a comparison of the measured molten pool areas (presented by ' $o$ ') and the neural network calculated results (presented by ' $x$ '). There is a good agreement between the measured molten pool area and the calculated ones.

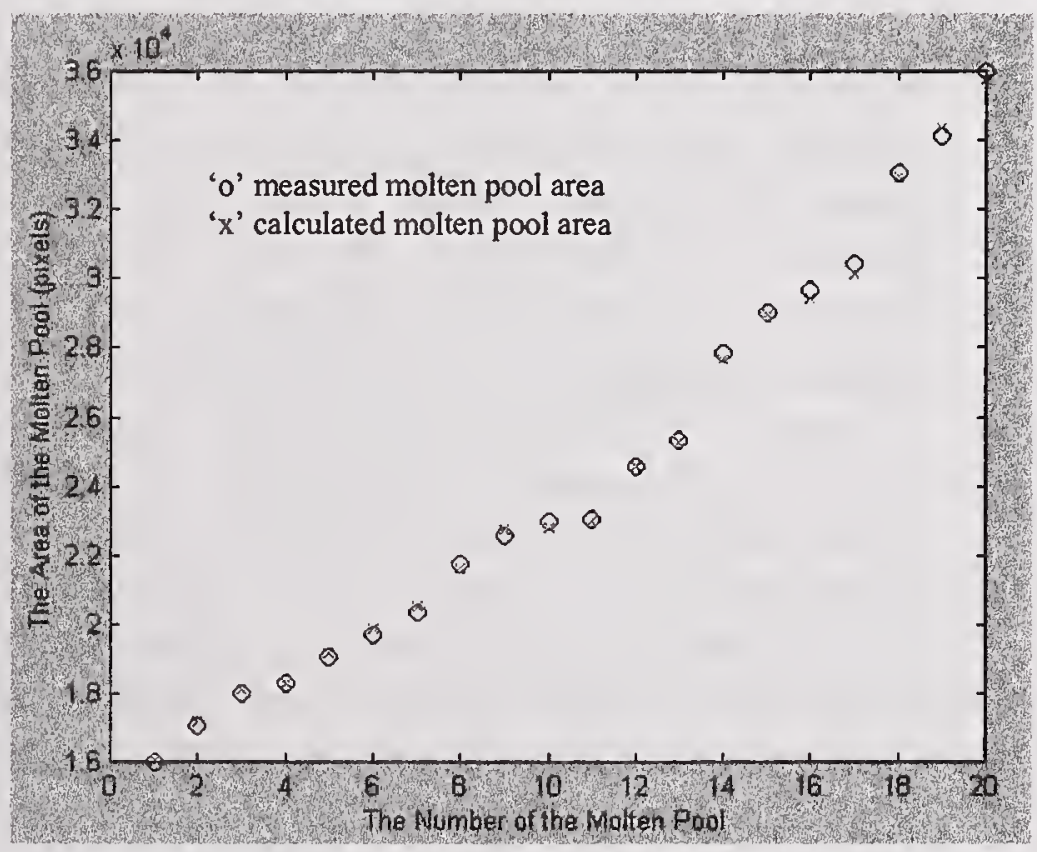

Figure 10. Comparison of measured molten pool areas with the calculated ones 


\section{CONCLUSION}

Machine vision is an efficient sensing method for monitoring the molten pool in the GTAW process. In order to control the welding process of GTAW with a wire filler for the need of the hybrid RP\&T technique based on welding and CNC milling, the area of the molten pool was used as feedback to control the GTAW process. The image of the molten pool and its surrounding area was acquired by a CCD camera coaxiallv integrated with the tungsten electrode. The boundary of the molten pool is extracted al $\iota_{1}$ the image processing. The boundary is incomplete because the molten pool is partially hidden by the electrode. A two-layer neural network is established using MLP to calculate the area of the molten pool with its incomplete boundary. The neural network was trained by back propagation learning with a momentum term using molten pool images acquired for different welding conditions. The testing results by another group of the molten pool images show that the neural network provides a good result.

\section{ACKNOWLEDGEMENTS}

This work was financially supported by THECB, Grant No. 003613-0022001999, NSF Grant DM1-9809198, and by the US Department of Education, Grant No. P200A-80806-98. The authors would like to express the gratitude to Weldware Inc. Columbus, $\mathrm{OH}$ for providing free of charge the GTAW torch with coaxial machine vision system for the need of this project. Assistance of Mr. I. S. Kmecko during the experiment is also gratefully acknowledged.

\section{REFERENCES}

1. Kruth, J. P.; Leu, M. C.; and Nakagawa, T. 1999. Progress in adaptive manufacturing and rapid prototyping. CIRP Annuals 47 (2): 525-540.

2. Beardsley, H. E.; and Kovacevic, R. 1999. New rapid prototyping technique based on 3-D welding. Technical Paper - Society of Manufacturing Engineers. PE, No. PE99-126, SME, Dearborn, MI, USA: PE99-126-1 - PE99-126-6.

3. Spencer, J. D.; Dickens, P. M.; and Wykes, C. M. 1998. Rapid prototyping of metal parts by three-dimensional welding. Proceedings of the Institution of Mechanical Engineers, Part B: Journal of Engineering Manufacture 212 (B3): 175-182.

4. Kovacevic, R. 2001. Rapid manufacturing of functional parts based on deposition by welding and 3D laser cladding. Proceedings of the Mold Making 2001 Conference, presented by Mold Making Technology Magazine: 263-276.

5. Richardson, R. W.; Gutow, D. A.; Anderson, R. A.; and Farson, D. F. 1984. Coaxial arc weld pool viewing for process monitoring and control. Welding Journal 63 (3): 43-50.

6. Kovacevic, R.; and Zhang, Y. M. 1995. Machine vision recognition of weld pool in gas tungsten arc welding. Proceedings of the Institution of Mechanical Engineers, Part B: Journal of Engineering Manufacture 209 (B2): 141-152. 
Session A3: Sensing and Control II: VPPAW 



\title{
MONITORING STABILITY OF KEYHOLE FORMATION IN VPPAW
}

\author{
G. Tao, H. Wang and R. Kovacevic*
}

\begin{abstract}
There are a number of factors that influence the quality of the weld formation in variable polarity plasma arc welding (VPPAW). Stability control of weld formation in VPPAW is required. One of the control strategies is to apply feedback control. The key for the successful application of feedback control is to acquire a characteristic signal that reflect the status of the controlled object. Airborne sound generated in the keyhole mode of VPPA welding process is used to characterize the presence of the keyhole and its size. Short-time spectrum analysis is used to study the frequency characteristics of the sound signal during the welding process. Short-time spectra are integrated along the frequency domain to obtain the power at different frequency intervals. The power is then used as the input to an artificial neural network (ANN) model to classify the keyhole diameter.
\end{abstract}

\section{KEYWORDS}

Sound signal, Time-frequency analysis, ANN, VPPAW

\section{INTRODUCTION}

Variable polarity plasma arc welding (VPPAW) is a promising welding technique for joining aluminum alloys. It has been used successfully in production, such as in the fabrication of the space shuttle external tanks (Ref. 1-3). VPPAW can generate high welding quality and high productivity at relatively low cost. However, the keyhole molten pool is very dynamic, and it can collapse and generate burn-through holes during welding, especially when welding plates with thicknesses above $4.0 \mathrm{~mm}$. Thus, the selection of the welding parameters and the implementation of controller to ensure the stability of the weld formation in real-time remain a challenge. Recently, it was found that the presence or absence of a keyhole could be determined by measuring the ratio of hydrogen to argon in the plasma arc column with an optical spectrometer (Ref.4). However, the size of the keyhole cannot be determined and the welding process cannot be distinguished from the cutting process according to that signal. At present, two problems are associated with sensing of the keyhole weld pool in VPPAW: 1) Inaccessibility from the frontside of the weld pool because of the limited torch standoff-distance and the interference produced by the strong arc light. 2) Detection of the keyhole from the back-side of the workpiece is related to the complexity of the weld structure, for instance, in the welding of pressure vessels.

Research Center for Advanced Manufacturing, Southern Methodist University 1500 International Pkwy, Suite 100, Richardson, Texas 75081

* corresponding author

Tel: 1-214-768-4865, Fax:1-214-768-0812,Email: kovacevi@seas.smu.edu 
Previous work shows that keyhole size captured by a machine vision system can be used as a feature signal to the feedback control of the weld formation in VPPAW (Ref.5). However, it is difficult to precisely monitor the keyhole size by machine vision when the keyhole is very small or very large. A new approach to characterize the presence and the size of the keyhole is needed. One of the potential solutions is to relate the signature of the airborne sound signal during welding to the geometrical characters of the molten pool. The acoustic signal has been used successfully in the monitoring of different welding processes. For example, the time-frequency characteristics of airborne signals were investigated in laser welding (Ref.6). It was found that the acoustic spectrum of good-quality, full-penetration welds could be differentiated from the spectra of poor-quality welds, defined as either partially penetrated welds or welds having a gap between the sheets being joined. Welding sound signature in GTAW was also studied to reveal the conditions that generate weld defects (Ref.7). It was found that the sound signature produced by GMAW contained information about the behavior of the arc column, the oscillation of metal in the molten pool, and the metal transfer mode. The analysis of the acoustic signal from arc welding, oxy-flame cutting, and water jet cutting were carried out also (Ref.8). In addition, acoustic emission monitoring of laser beam welding was investigated to detect laser misfiring, loss of power, and improper focus (Ref.9).

In this paper, the sound generated in the VPPAW is studied. The investigation is focused on the following: 1) Frequency and time-frequency analyses of the sound signal generated in VPPAW to study the welding status. 2) Establishment of the model to describe the relationship between the sound signal and the keyhole diameter of the weld pool.

\section{EXPERIMENTAL SET-UP AND PROCEDURES}

\section{Experimental Set-up}

The experimental set-up, as shown in Figure 1, consists of a VPPAW machine, a CNC positioning system, an ultra high-shutter-speed camera assisted with a pulsating nitrogen laser (Laser Strobe system) and a microphone with a data acquisition system. The Laser Strobe system is used to monitor the keyhole size from the

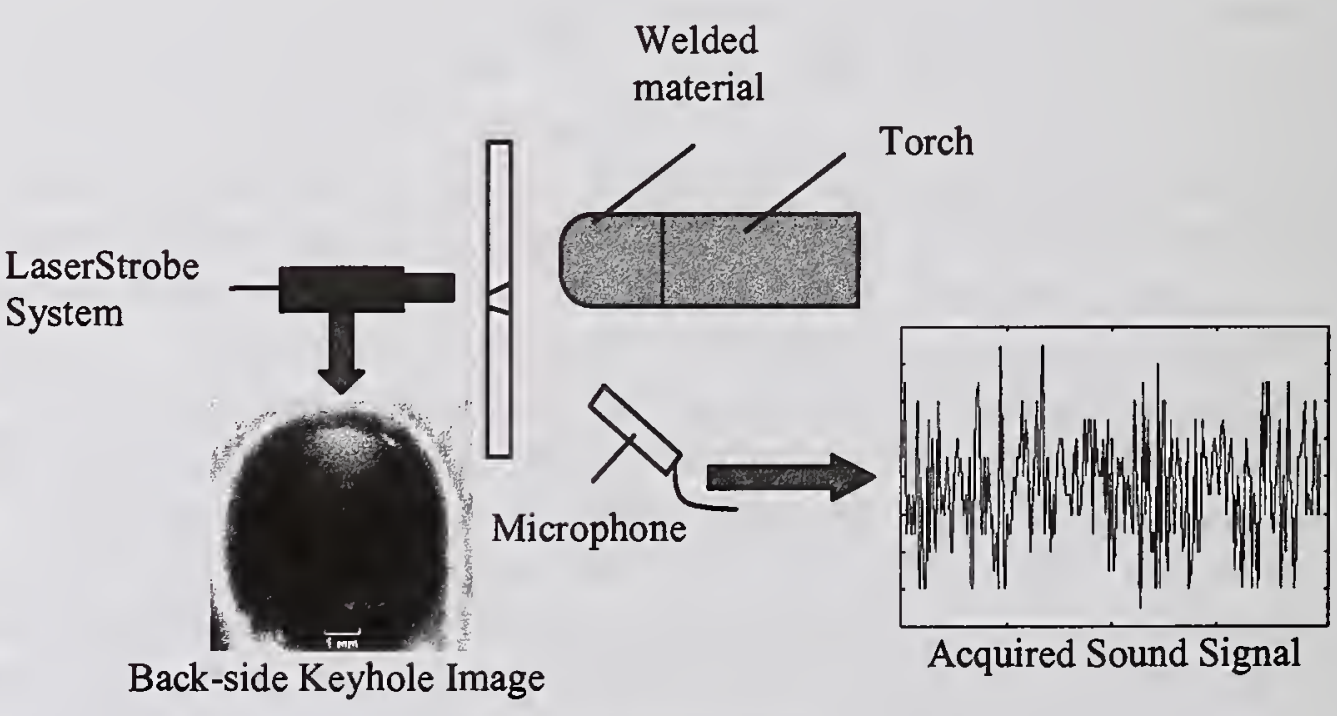

Figure 1. Experimental set-up backside of the workpiece as a reference. The image acquisition and sound signal acquisition is synchronized. The frequency of image acquisition is $2 \mathrm{~Hz}$ while the frequency of the sound signal acquisition is $50 \mathrm{kHz}$. 


\section{Experimental Procedures}

The experiments are performed in several steps:

1) The keyhole is generated by a stationary torch in order to study the sound signal at different welding modes.

2) A copper plate with pre-drilled holes is used to study the relationship between the size of the hole and the generated sound signal by the plasma.

3) A set of experiments is performed in order to study the relationship between the keyhole diameter and the level of sound signal simulating the real welding conditions.

\section{SPECTRUM ANALYSIS AND TIME-FREQUENCY ANALYSIS}

Spectrum analysis is used to study the process characteristics. In this paper, an averaged periodical diagram is used to obtain the spectral analysis to speed up the calculation and to increase the resolution. The data is separated into several segments with overlapping. For each data segment, $\mathrm{p}$, the periodicity can be calculated by equation (1) :

$$
I_{M, p}\left(\omega_{k}\right)=2 / M\left|\sum_{i=1}^{M} x_{p, i} w_{M}(i) e^{-j r k / M}\right|^{2}, \mathrm{k}=1,2, \ldots \mathrm{M} / 2 \text { or }(\mathrm{M}-1) / 2
$$

The Hann window is used for truncation to decrease the power leakage and to get a smoother result. Finally, the average periodicity is obtained by averaging the periodicity of each segment, $p$, for $p=1: L$

$$
\overline{I_{M}}\left(\omega_{k}\right)=1 / L \sum_{p=1}^{L} I_{M, p}\left(\omega_{k}\right)
$$

Thus, the expectation of the standard deviation of the spectral analysis can be decreased to $1 / \mathrm{L}$.

Due to the variation of the VPPAW welding process, the acoustic signal is dynamic and time dependent, so the time-frequency distribution is an effective tool for studying this process. The time-frequency distribution can be viewed as a transform which represents the energy or density of a signal simultaneously in both time and frequency domains. It reveals the time-dependent features that the ordinary spectrum analyses fail to show. In this paper, a short-time spectrum analysis is used to study the time-frequency character of the VPPAW. Equation (3) shows the expression of the short-time spectrum analysis:

$$
\operatorname{SSPEC}_{x}(t, f)=\left|\operatorname{STFT}_{x}(t, f)\right|^{2}=\left|\int_{T} x\left(t^{\prime}\right) w\left(t^{\prime}-t\right) e^{-j 2 \pi f t^{\prime}} d t^{\prime}\right|^{2}
$$

Where $x(t)$ is the acoustic signal windowed by a suitable function $w(t)$ (in this paper, it is the Hann window), and $T$ is the interval of integration. Equation (3) can be expressed in a discrete version of the short-time spectrum analysis:

$\operatorname{SSPEC}_{x}(i, k)=\left|\operatorname{STFT}_{x}(i, k)\right|^{2}=\left|2 / M \sum_{j=1}^{M} x(j+i) w(j) e^{-j 2 \pi k j / M}\right|^{2}$

The short-time spectrum at time $i$ (the $i$ the second of the sound signal) is obtained by averaging the periodical diagram. The results of the short time spectrum are integrated in different frequency intervals from $f_{k 1}$ to $f_{k 2}$ in order to present the frequency properties around the central frequency, $f_{k}$, in the same time (equation (5)): 


$$
\operatorname{INSSPE}_{x}\left(i, f_{k}\right)=\sum_{k=f_{k 1}}^{f_{k 2}} \operatorname{SSPEC}_{x}(i, k)
$$

In this paper, the real-time spectrum analysis is performed for the duration of one second by averaged periodicity. The next second, the previous data should be discarded in order to generate data for a new short-time spectrum analysis.

\section{SOUND GENERATION FOR DIFFERENT VPPAW MODES}

During welding, the pulsation of the arc plasma, and the oscillation of the weld pool are the major sources of the sound signal. The formation of the weld pool in VPPA welding passes through three stages: a no-keyhole weld pool or a fusion weld pool, a transition weld pool, and a keyhole weld pool. In the case of the no-keyhole weld pool, there is a layer of unmelted metal below the weld pool that allows for strong stirring of the weld pool. So, the weld pool generates slightly stronger oscillations at low frequencies. In the case of the transition mode, the keyhole is not formed, but the weld pool is generated along the entire thickness of the welded material. In this weld mode, the natural oscillation frequency of the weld pool is low. So, resonance could occur and the sound signal at the low frequency should be the strongest among the all three welding modes. In the case of the keyhole mode, there is a keyhole at the center of the weld pool and the stirring effect of the jet is decreased. So, the intensity of the sound signal at the lower frequencies is less pronounced than in the case of the transition mode. It is expected that the analysis of the intensity of the sound signal will be useful in distinguishing the modes of the weld pool in VPPAW.

\section{EXPERIMENTAL RESULTS}

\section{Generation of the Keyhole by Stationary Torch}

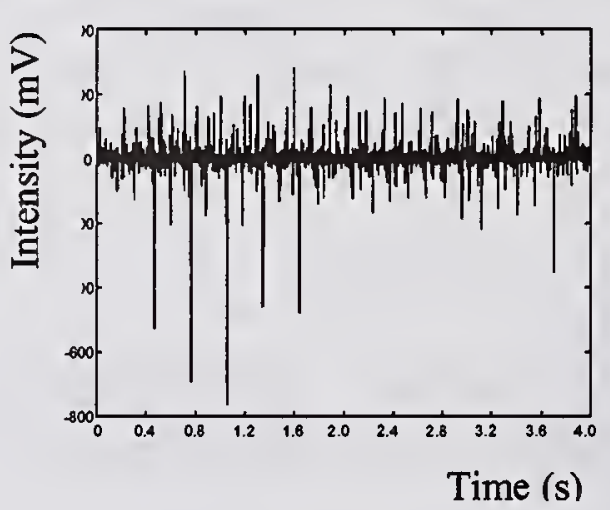

a)

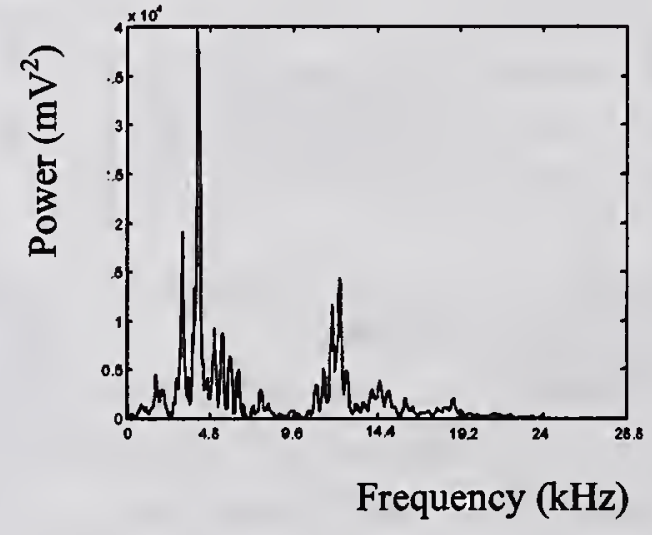

b)

Figure 2. Sound signal for stationary torch welding
a) Time domain
b) Frequency domain

In the case of keeping a fixed position of the VPPAW torch with respect to the aluminum plate with a thickness of $3.18 \mathrm{~mm}$, all three modes of the molten pool are formed by controlling the intensity of the welding current. The signature of the sound in the time domain (Fig.2 a) does not 
show too much variation and only a number of randomly occurring singularities are present. Figure $2 \mathrm{~b}$ shows the power spectrum of the sound signal. There are several dominating spectra
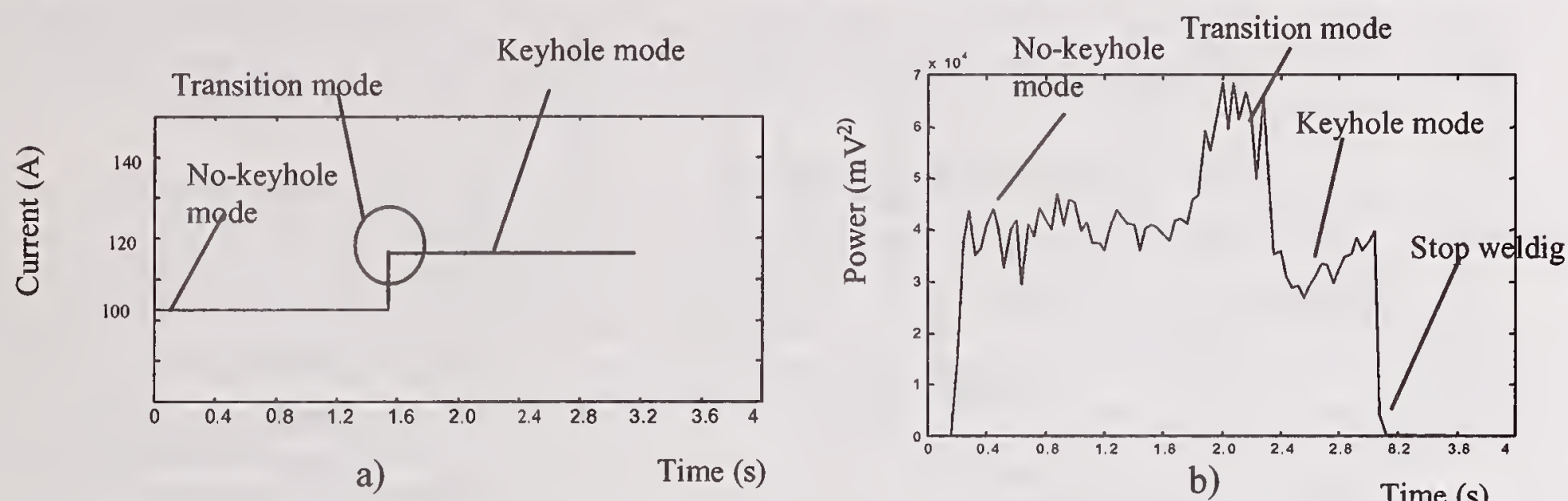

Figure 3. Diagram of the welding current (a) and short-time spectrum analysis results of keyhole formation process at the frequency of $2.2 \mathrm{kHz}(b)$

peak values at the frequencies such as: $2.2 \mathrm{kHz}, 3.9 \mathrm{kHz}, 8 \mathrm{kHz}, 12 \mathrm{kHz}$.

The sound signal is studied by integrating a short-time spectrum analysis around these peak values. The time-frequency analysis around the frequency of $2.2 \mathrm{kHz}$ shows that there is a strong relationship between the power spectrum of the sound signal and the phases of the molten pool formation (Figure. 3).

In this experiment, based on the changing of the welding current, the transition mode and keyhole mode will happen at 1.6 and 2.5 seconds respectively. Figure 3 shows the changing of the welding current and the short-time spectrum analysis results during the stationary torch welding at the frequency of $2.2 \mathrm{kHz}$. It is shown that the power spectrum at the frequency of $2.2 \mathrm{kHz}$ will increase during the transition mode and then will drop back down when the keyhole is generated. It is clear that the spectral analysis of the sound signal provides a useful tool in distinguishing the welding pool modes in VPPAW.

\section{Simulation of the Keyhole Size Effect on the Sound}

In order to study the relationship between the intensity of the air-borne-generated sound by plasma passing through pre-drilled holes and the diameters of these holes, a number of experiments are performed. For these experiments, a special device is designed and constructed.

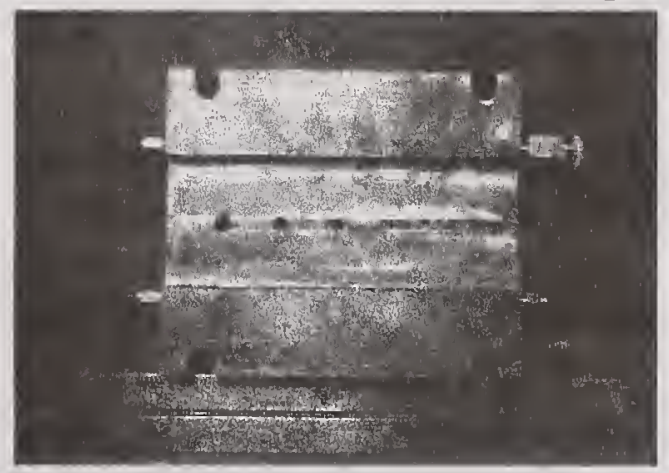

a)

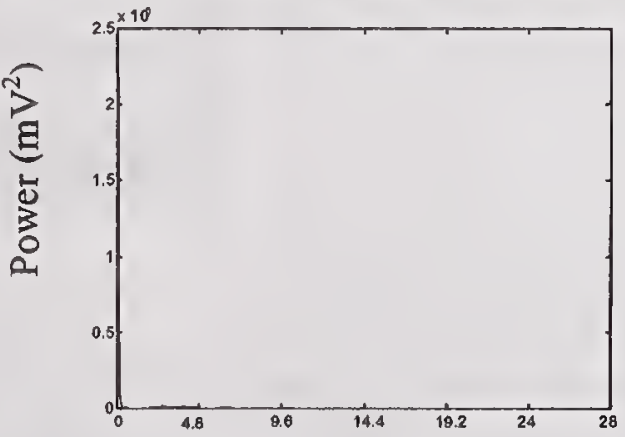

Frequency $(\mathrm{kHz})$

b)

Figure 4. Copper plate with cooling channels (a) and power spectrum of sound signal for hole diameter of $3.18 \mathrm{~mm}$ 


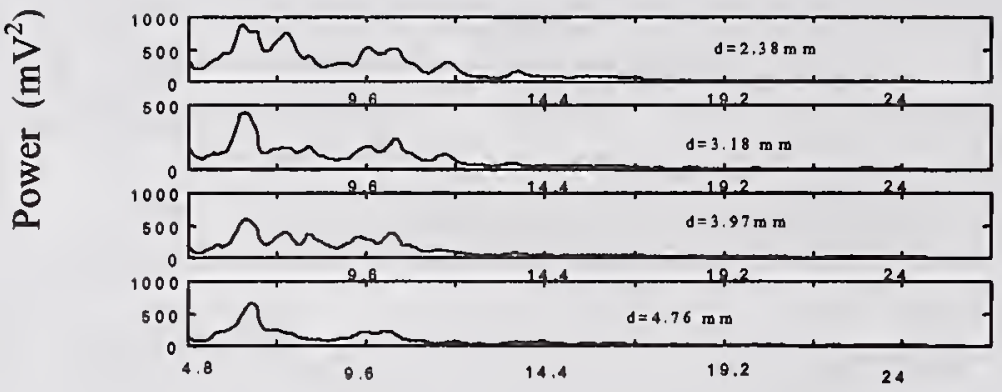

Frequency $(\mathrm{kHz})$

Figure 5. Power spectra of sound for different hole diameters

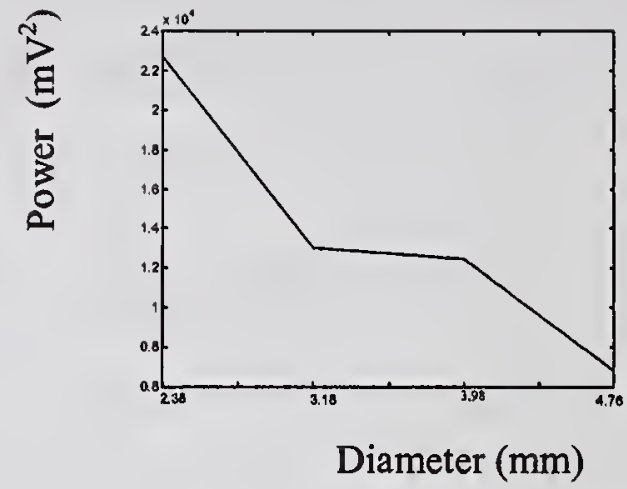

Figure 6. Relationship between power spectrum and hole diameter at the frequency of 7.2

The device is in the form of a copper plate equipped with cooling channels and with a set of through-drilled holes of different diameters. Cooling of the copper plate prevents the degradation of the holes by the plasma flow. There are four holes tested with different diameters: $2.38 \mathrm{~mm}$, $3.18 \mathrm{~mm}, 3.98 \mathrm{~mm}$, and $4.78 \mathrm{~mm}$. The cooling copper plate and the sound signal power spectra are shown in Figure $4 \mathrm{a}$ and $4 \mathrm{~b}$. The spectrum of sound obtained for this experiment is much different from the spectrum obtained in the stationary keyholing. The energy of the power spectrum is mainly focused on the very low frequency interval. Figure 5 shows the spectral results from all four hole diameters. The area under the curve decreases with an increase in the diameter. The power spectrum of the sound at frequency of $7.2 \mathrm{kHz}$ (as representative of low frequencies spectra) as a function of the hole diameter is shown in Figure 6. The results show that there is a relationship between the hole diameter and the intensity level of the sound generated by flowing plasma through the holes.

\section{Classification of Keyhole Diameter by ANN Model in Case of Continuous Keyhole Welding}

In order to simulate the change of the size of the keyhole, a bead-on plate with changing heat transfer conditions is used. The two $25 \mathrm{~mm}$ slits are placed parallel to the welding line. The constant welding parameters: welding current, flow rate of inert gas, welding speed, wire feeding speed, etc.) are optimized in order to guarantee a keyhole mode. The keyhole diameter enlarges when the torch passes through the region with the slits. This is caused by worsening of the heat transfer from the welding zone. The diameter of the keyhole at the back-side of the plate as well as the air-borne sound generated by the plasma at the top face of the plate are simultaneously
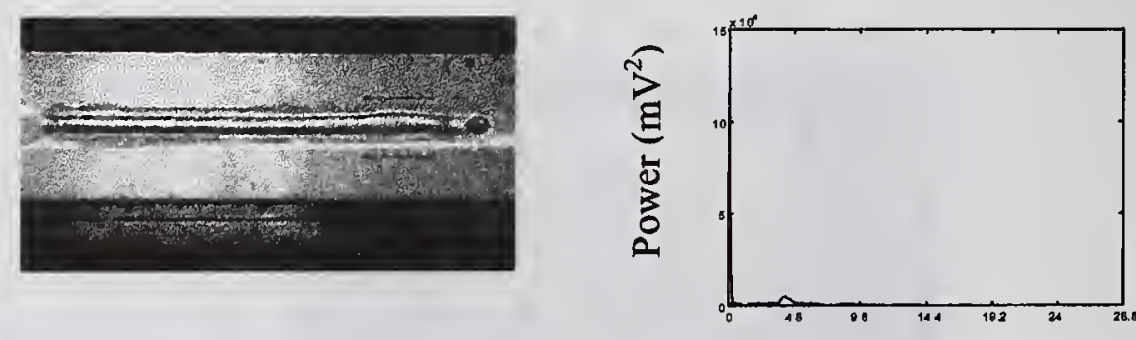

Frequency $(\mathrm{kHz})$

Figure 7. Welded sample with corresponding power spectrum of sound signal for continuos keyhole welding

recorded during the welding. A high-shutter-speed camera assisted with a pulsing nitrogen laser is used to acquire the images of the back-side of the keyhole with frequency of two images per second. The welded sample with the power spectrum of sound is shown in Fig 7. 
The classification of GMAW transfer modes using the spectrum analysis results of the sound signal was previously reported (Refs 10,11 ). In this paper, the analysis of the sound signal is used to classify the keyhole diameter into classes: small, medium, and large. The three layer neural network is designed to perform this classification.

The input layer and hidden layer each have 20 nodes, and the output layer has three nodes. For each second, the sound signal generated by the plasma is processed by a short-time spectral analysis, and the power spectra uniformly distributed at 20 central frequencies along the whole spectra are used as the network input. The output has three binary nodes that indicate three classes of the keyhole diameter (small, medium and large). After the diameter class is determined, the output node corresponding to this class is set to one and the other two nodes are set to zeros.

A back-propagation (BP) algorithm is used to minimize the loss function. In order to speed up the training process, the convergence fast algorithm proposed by Chan and Fallside (Ref.12) is used to train the network based on dynamically changing the learning rate. Figure $8 \mathrm{a}$ shows a real time measured diameter and the corresponding diameter classification. The diameter of the keyhole acquired by the high shutter speed camera from the back-side of the welding plate is classified as small, medium, and large by thresholds of 5.4 $\mathrm{mm}$ and $6.6 \mathrm{~mm}$. (up to $5.4 \mathrm{~mm}$--small, from 5.4

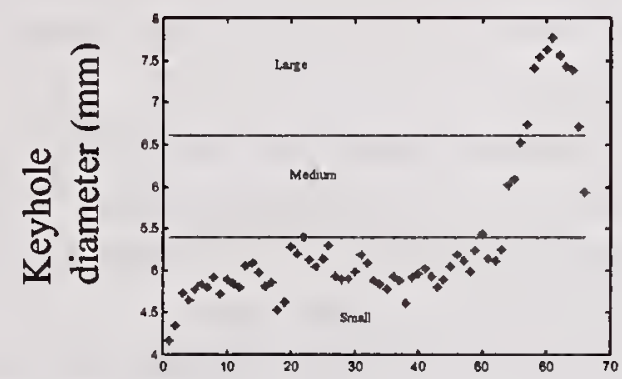

a)

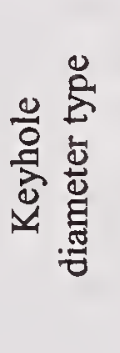

Time (s)

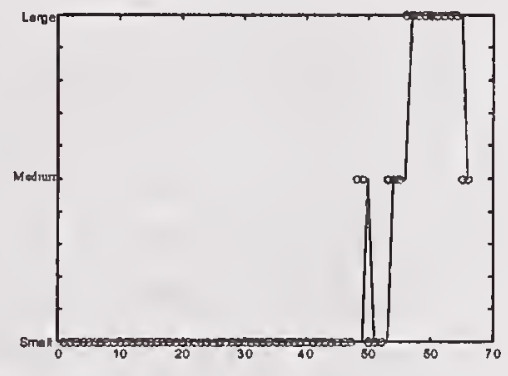

b) Time (s)

Figure 8. Classification of keyhole diameter $\begin{array}{ll}\text { a) by measuring } & \text { b) } B y A N N\end{array}$ $\mathrm{mm}$ to $6.6 \mathrm{~mm}$ including the boundary--medium, and over $6.6 \mathrm{~mm}$ large). Figure $8 \mathrm{~b}$ shows the test results of the neural network. The "o" presents the keyhole diameter class predicted by the neural network. From the test results, the classification error is less than $10 \%$. So, based on the sound signal analysis, the ANN output could be used to predict the keyhole diameter in the continuos keyhole VPPA welding and is potentially useful as a feedback signal to control the keyhole diameter.

\section{CONCLUSION}

A sound sensing technique is used to classify the keyhole size in VPPAW into small, medium, and large. The experimental results show that the sound signal can be used to determine the instant when the keyhole is established as well as to clearly distinguish among the modes of the molten pool: no-keyhole mode, transition mode, and keyhole mode. 


\section{ACHNOWLEDGEMENT}

This work was financially supported by NSF's Grant No. DMI-9900011 and by the U.S. Department of Education, Grant No. P200A80806-98, and by the Brown Foundation.

\section{REFERENCES}

1. Nunes A. C. 1984. Variable polarity plasma arc welding on space shuttle external tank, Welding Journal 63(4): 27 -s to 35 -s.

2. Martinez Luis F. 1994. Effect of weld gases on melt zone size in VPPA welding of Al 2219. Welding Journal 10: 50-s to 55-s.

3. Howard Woodward. A. 1996. U.S. Contractor for the International space station, Welding Journal 3: 35-s to 40-s.

4. Martinez L. F. 1992. Front side keyhole detection in aluminum alloys, Welding Journal 5: 49$\mathrm{s}$ to 52 -s.

5. Zheng, B., Wang, H., Wang, Q., and Kovacevic, R. Control for weld penetration in VPPAW of aluminum alloys using the front weld pool image signal, Welding Journal/Research Supplement, Dec.2000, p363s-370s.

6. D. Farson, K. Hillsley, J. Sames and R. Young. 1996. Frequency-time characteristics of airborne signals from laser welds, Journal of Laser Application, 7., p33-p42.

7. D. Saini and S. Floyd. 1998. An investigation of gas metal arc welding sound signature for on-line control, Welding Journal/Research Supplement, Apr., p172s-179s.

8. T. Morita, Y. Ogawa and T. Sumitomo. 1995. Analysis of acoustic signal on welding and cutting, 1995 OMAE-Volume III, Materials Engineering ASME, p547-p552.

9. M. C. Jon. 1985. Non-contact acoustic emission monitoring of laser beam welding, Welding Journal, Sep, p43s-p48s.

10. Metteson,A., Morris, R., \& Raines, D.,An 1992. Optimal artificial neural network for GMAW arc acoustic classification Proc. 3rd International Conference Trends in Welding Research, Gatlinburg, TN.

11. L. Quintino. R.Ribeiro. J.Raria. 1999. Classification of GMAW transfer modes using neural networks" Int. J for the Joining of Materials, 11(1), 6-8.

12. Chan, L.W. and Fallside, F.1987 An adaptive training algorithm for backpropagation networks, Computer Speech and Language, 2, 205-218. 


\title{
SYSTEMS TECHNOLOGY FOR VARIABLE POLARITY \\ PLASMA ARC WELDING OF ALUMINUM ALLOYS
}

\author{
B. Zheng*
}

\begin{abstract}
This paper summarized further researches in variable polarity plasma arc welding of aluminum alloys on the issues of arc behavior, monitoring of a keyhole, and real-time closed-loop control for weld formation. Development of a unique welding power source system, configuration of a machine vision system to monitor a keyhole weld pool, and implementation of an intelligent closed-loop control for weld formation have been made. The results achieved by both experiment and theoretical analysis show an efficient way to systematically improve the weld quality.
\end{abstract}

\section{INTRODUCTION}

Aluminum is one of the important structural materials in industry. However, the tenacious oxide film existing on its surface strongly impedes the flow of molten metal in the weld pool during welding and easily leads to the formation of poor welds. Also, the hydrogen that is easily absorbed in the weld pool becomes trapped to form porosity as it solidifies (Ref. 1-2).

Variable Polarity Plasma Arc Welding (VPPAW) of aluminum alloys has been developed and applied to the fabrication of key products such as space shuttle external fuel tanks and nuclear magnetic resonance devices. This welding technology features higher quality and productivity at relatively lower cost: it removes the oxide film on the workpiece surface and generates a unique keyhole-mode weld pool that forces the porosity to be flushed out of the weld pool (Ref. 3-12). However, there are still some crucial problems to be solved, such as severe deterioration of the tungsten electrode, short service life of the nozzle, arc instability, unstable penetration state, and inconsistency of weld formation (Ref. 4-5 and 12-20).

This paper presented further research of VPPAW technology on arc behavior, monitoring of a keyhole, and real-time closed-loop control for weld formation.

\section{EXPERIMENTAL PROCEDURE}

The experimental setup consists of a welding system and a monitoring and control system for weld quality. The welding system are the following: an AC plasma arc power source, a DC pilot arc power source, a welding current redistribution device, a programmable sequence controller, a plasma gas controller, an all-position supporter for the workpiece, a computer-controlled wire feeder, and a water-cooled plasma torch rated at 300 amperes. The hardware of the monitoring and control system includes a CCD camera with a band-pass filter, a monitor, a frame grabber, a VCR, a data acquisition system, and a PC-Pentium computer. The camera is attached to and positioned in front of the plasma arc torch with its axis at an angle $(\phi)$ of $43^{\circ}$ from the workpiece surface plane. The software functions as a system platform that integrates the welding system

\footnotetext{
"Edison Welding Institute, 1250 Arthur E. Adams, Columbus, OH 43221
} 
and the monitoring and control system for weld quality. Main modules that were designed are: welding operation routine, arc current redistribution control, welding parameters and weld pool image acquisition, image processing routine, keyhole monitoring, and control decision making. Butt-jointed welds of 5.0-10.0 mm-thick 2024 aluminum plates are made under the shielding of pure argon with wire feed. Welding parameters have the following ranges: a DCEN to DCEP time duration of $13-22 \mathrm{~ms}$ to $3.0-7.0 \mathrm{~ms}$, DCEN current 80-210 A, DCEP current 80-240 A, welding voltage $25-33 \mathrm{~V}$, pilot arc current $15-30 \mathrm{~A}$, plasma gas flow rate $4.5-5.5 \mathrm{~V} / \mathrm{min}$., shielding gas flow rate $6.0-8.0 \mathrm{~V} / \mathrm{min}$, welding speed $80-100 \mathrm{~mm} / \mathrm{min}$, torch stand-off distance 5.0-6.0 mm, angle between workpiece surface plane and the horizontal plane $85^{\circ}-90^{\circ}$, orifice diameter $3.2-4.0 \mathrm{~mm}$, orifice length $3.5-4.0 \mathrm{~mm}$, tungsten electrode diameter of $4.0 \mathrm{~mm}$, and a tungsten electrode setback of $3.2-4.0 \mathrm{~mm}$.

\section{RESULTS AND DISCUSSIONS}

\section{Arc behavior}

Arc behavior explored in this paper includes the stability of a combined arc (consisting of an AC plasma arc and a DC pilot arc) and the characteristic of heat and force generated from a plasma arc. The combined $\mathrm{AC}$ arc welding can guarantee the initiation of a plasma arc in the beginning of each period of direct current electrode positive (DCEP). However, in the rest part of each DCEP period, partial current from an AC power source may flow through a DC pilot arc power source and partial current from the pilot arc power source may also flow through the $\mathrm{AC}$ power source. The existence of this type of current flow will result in double arc that makes the weld formation worse and sometimes damage the rectifier inside the pilot arc power source. Although a resistor with the variable resistance can be added to the output circuit of the pilot arc power source to limit this type of current flow, the potential of the nozzle that contacts the arc column will be changed under this condition. Since the directions of both electric field and current for the pilot arc are completely adverse to those for the plasma arc in the DCEP period, the change of the nozzle potential may lead to an extremely unstable arc discharge (arc interference) because of the neutralization of electric charges (Ref. 13). To guarantee the weld quality, a trade-off between initiation of each DCEP plasma arc and avoidance to the arc interference must be electrically made. A unique solution to this is shown in Fig. 1, which makes use of the pilot arc and the arc between the nozzle and the workpiece at the transition from the DCEN period to the DCEP period during the welding process. The DC power source 1 is also used as a continuous welding power source, but its output current is changed into an $\mathrm{AC}$ current with the help of transistors GTR $_{1}$ through GTR $_{5}$, which forms an H-bridge. The

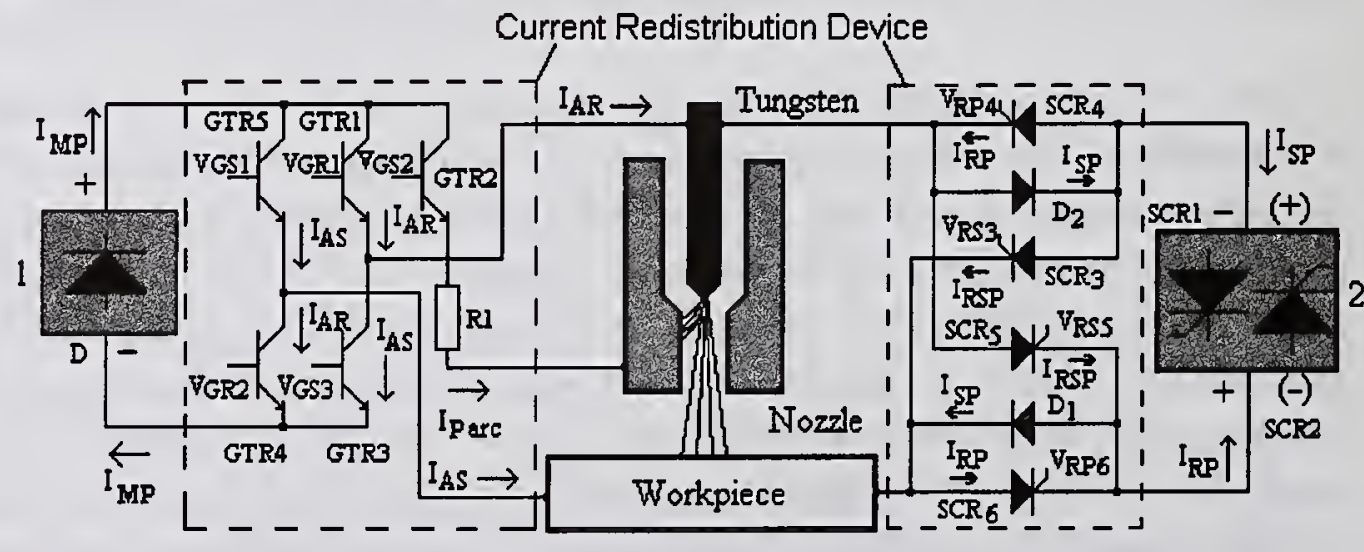

Fig. 1 A Push-pull-arc Solution to Arc Interference in AC PAW 


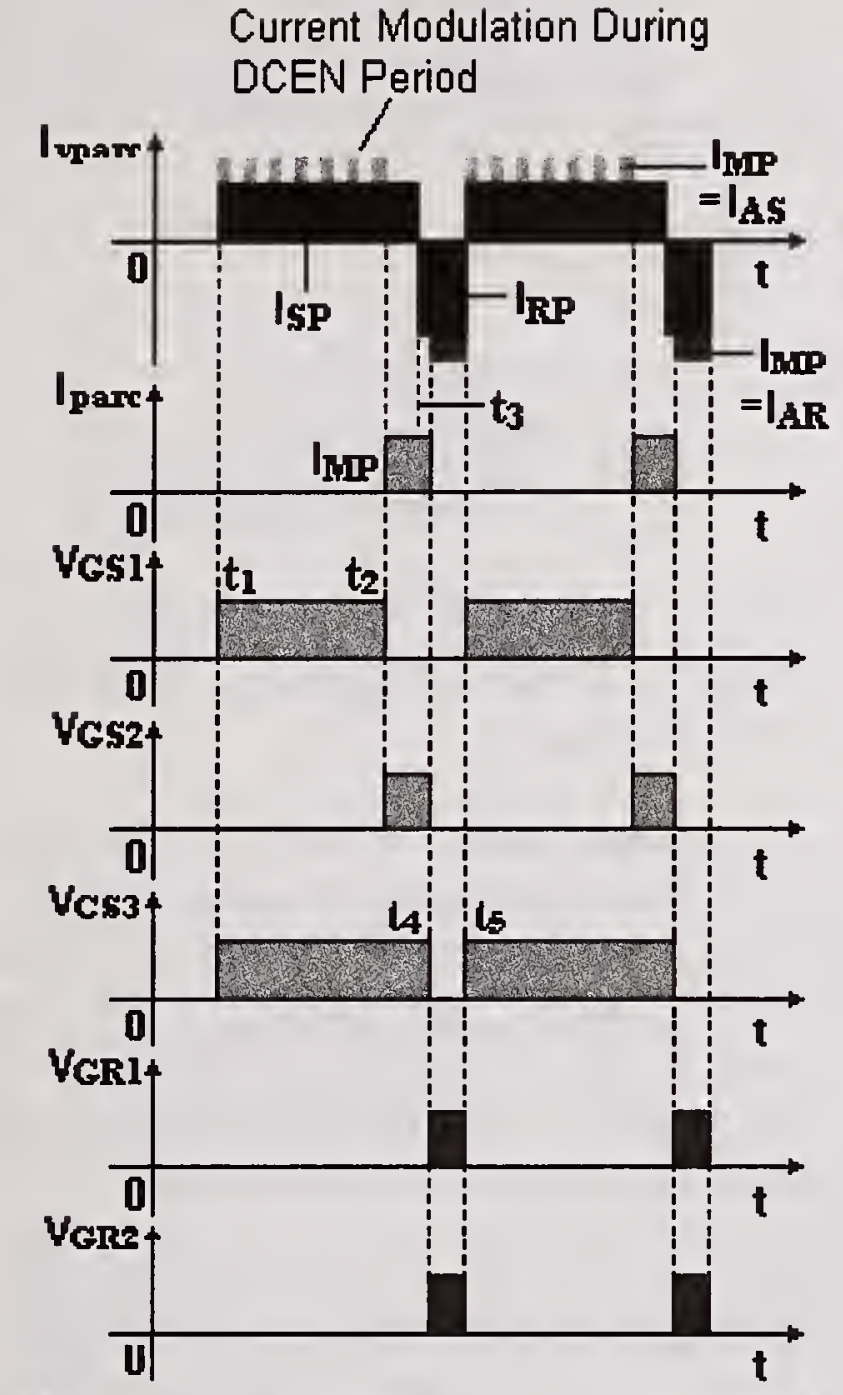

Fig. 2 Waveforms of Welding Currents and Voltages Related to Push-pull-arc Solution

comes from the plasma arc column to heat and melt the workpiece. 2) More heat is generated in the DCEN period than in the DCEP period. In applications, a maximum-width cathodic cleaning area is accepted as a rule if no special consideration should be given to the deterioration of the tungsten electrode. To evaluate and better take advantage of an AC plasma arc as well as improve the weld quality, the following investigation is made. Welding currents with different $\mathrm{AC}$ frequencies shown in Fig. $3(50 \mathrm{~Hz}, 25 \mathrm{~Hz}, 16.7$ $\mathrm{Hz}, 12.5 \mathrm{~Hz}$, and $4.54 \mathrm{~Hz}$ ) are used by enabling $\mathrm{SCR}_{3} \sim \mathrm{SCR}_{6}, \mathrm{D}_{1}$, and $\mathrm{D}_{2}$ in Fig. 1. The corresponding ratios of current $I_{P 1}$ from power source 1 is used as a pulse current for initiating the plasma arc at the beginning of each DCEP period. This means that the arc between the tungsten electrode and the nozzle is a pilot arc for a very short period: from $t_{2}$ to $t_{4}$, as shown in Fig. 2. So, the pilot arc is a discontinuous arc. After the plasma arc is initiated, during the following time (from $t_{5}$ in the DCEP period to $t_{2}$ in the next DCEN period), the current from the DC welding power source 1 is also used as a part of the plasma arc current. The current will flow through the conducting plasma arc column between the tungsten electrode and the workpiece. Consequently, the pilot arc and the plasma arc consist of a push-pull arc: the DCEP plasma arc is initiated with the help of the discontinuous pilot arc that is initiated with the help of the DCEN plasma arc.

A weld bead with a high depth-to-width ratio is preferable for AC plasma arc welding (PAW) of aluminum alloys in the keyhole mode, which is directly related to the heat and force generated from an arc. For AC PAW of aluminum alloys, two statements have been made. 1) The temperature of the plasma arc column is higher than that for other types of arc columns. Therefore, compared with the cathodic and anodic regions, much more heat

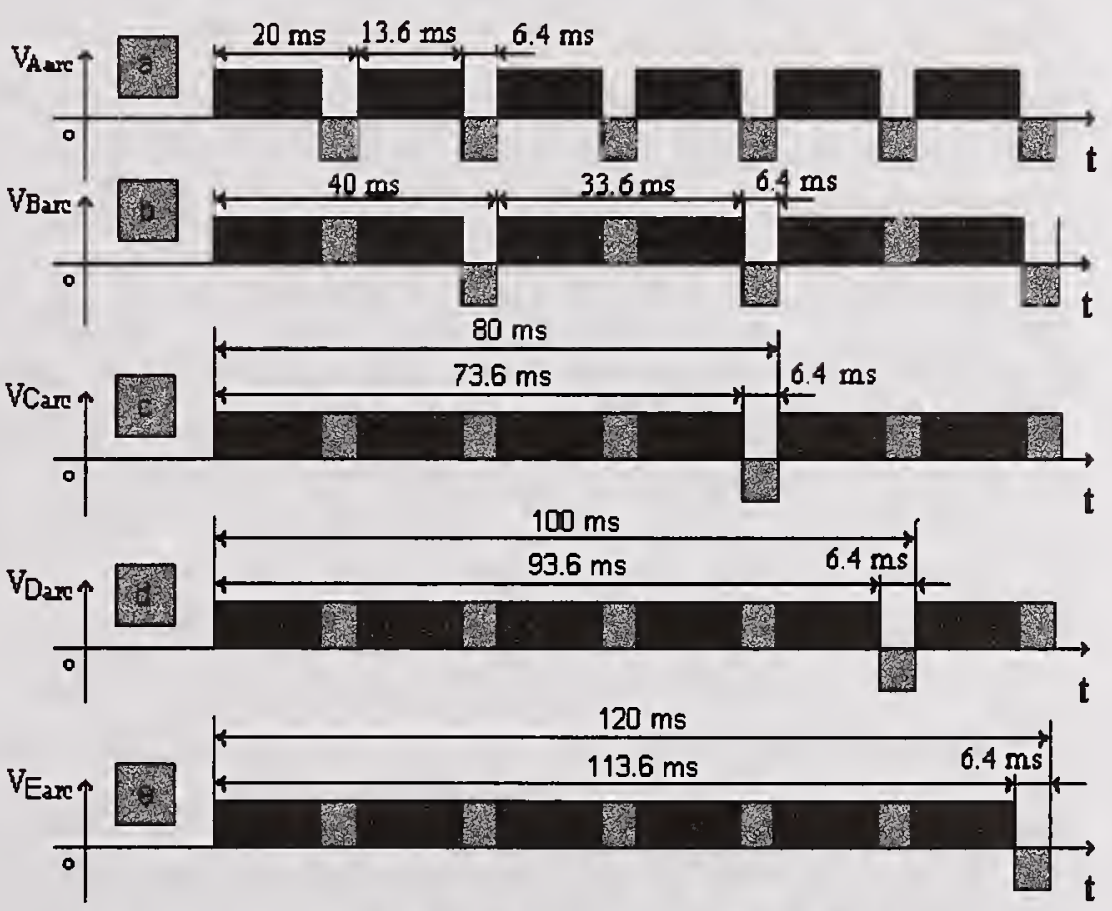

Fig. 3 AC Voltage Waveforms with Different Frequencies
a. $50 \mathrm{~Hz}$
b. $25 \mathrm{~Hz}$
c. $16.70 \mathrm{~Hz}$
d. $12.5 \mathrm{~Hz}$
e. $4,54 \mathrm{~Hz}$ 
DCEP duration to DCEN duration are $47.06 \%$, $19.05 \%, 11.94 \%$ and $8.70 \%$, respectively. The voltage for each of the weld beads was initially an $\mathrm{AC}$ frequency of $50 \mathrm{~Hz}$, and then it was switched to an $\mathrm{AC}$ frequency corresponding to Fig. 3. The resultant widths of both the weld beads and the cathodic cleaning area versus the $\mathrm{AC}$ frequency are shown in Fig. 4. It can be seen that the width and penetration of a weld bead decrease as the voltage frequency decreases. When the frequency of the voltage is changed to $12.5 \mathrm{~Hz}$, the workpiece is not penetrated by the arc. It should be mentioned that the width of the cathodic cleaning area, in this case, is enough to form a weld bead with a smooth surface. In other words, the weld bead with the narrower width and partial penetration resulted from less energy input, not from an insufficient cathodic cleaning area. Another interesting phenomenon observed is that the cathodic region spots on the workpiece surface move freely and quickly in a half circular edge ahead of the keyhole weld pool. Thus, this area may get more heat because the DCEP current goes through it into the workpiece and is larger than the area heated by a DCEN arc due to the scattering DCEP arc. The above results reveal that the cathodic region also plays the main role in the heating and melting of the workpiece. Calculated results for the corresponding experiment show that although the heat supplied to the plasma arc by the power sources in the DCEN period is more than that in the DCEP period, the heat resulting from the cathodic region potential $(11 \sim 13 \mathrm{~V})$ is more than that from the anodic potential $(1 \sim 2 \mathrm{~V})$. During the welding, the role of the DCEP arc, scattered on the semi-circular edge in front of the keyhole weld pool, is not only to bombard the oxide film on the workpiece but also to provide the coming DCEN arc with heat for melting and penetrating the workpiece. The DCEN arc concentrates on the central spot of the semi-circular edge to pierce through the workpiece. Therefore, if a maximum-DCEN-duration approach is used, enough heat must be generated in the cathodic cleaning region during the DCEP period in order to achieve a high depth-to-width ratio weld bead. For a keyhole AC PAW of aluminum alloys, good weld formation can not be guaranteed if the cathodic cleaning area is too wide.

The capability of deep penetration from a plasma arc is attributed to the higher arc pressure resulting from the nozzle orifice, cooling arc column, and radial electromagnetic force inside an arc. However, the mechanism of this arc pressure generation has not been completely disclosed (Ref. 17-19). The velocity of a plasma arc jet is a direct measurement of its pressure: the higher its velocity, the larger its pressure. The exit velocity $\mathrm{V}_{2}$ of a plasma arc jet coming out from an

$$
V_{2}=\frac{Q p}{A_{2}} \times \frac{T_{2}}{T_{1}} \times \frac{M_{2}}{M_{1}} \times \frac{P_{2}}{P_{1}} \times(1+\alpha)
$$

where $Q_{p}$ is the plasma gas flow rate, $A_{2}$ is the cross-sectional area of the orifice at the exit, $T_{1}$, $M_{1}, P_{1}$ and $T_{2}, M_{2}, P_{2}$ stand for the temperature of plasma arc jet, molecular weight of the plasma gas, and atmosphere pressure, respectively at the entrance and exit of the orifice, and $\alpha$ is the 
ionization intensity of the plasma arc jet. According to this formula and the experimental data, different calculated velocities of the plasma jet under different conditions are listed in Table 1.

Table 1 Calculated Velocity of a Plasma Arc Jet under Various Arc Conditions

\begin{tabular}{|c|c|c|}
\hline Different Conditions & $\begin{array}{l}\text { Velocity of Plasma Arc Jet } \\
\qquad(\mathrm{m} / \mathrm{s})\end{array}$ & Common Conditions \\
\hline An arc is not initiated & 5.31 & \multirow{4}{*}{$\begin{array}{l}\mathrm{T}_{1}=298^{\circ} \mathrm{K}, \\
\mathrm{T}_{2}=13500^{\circ} \mathrm{K}, \\
\mathrm{P}_{1}=1.0133 \times 10^{5} \mathrm{~N} / \mathrm{m}^{2}, \\
\mathrm{P}_{2}=1.0133 \times 10^{5} \mathrm{~N} / \mathrm{m}^{2}, \\
\mathrm{M}_{1}=39.95 \mathrm{~g} / \mathrm{mol}, \\
\mathrm{M}_{2}=39.95 \mathrm{~g} / \mathrm{mol}, \\
\mathrm{Q}_{\mathrm{gas}}=4.0 \times 10^{-3} \mathrm{~m}^{3} / \mathrm{min}, \\
\text { Orifice diameter and length } \\
\text { are respectively } 4.0 \mathrm{~mm} \text { and } \\
4.0 \mathrm{~mm},\end{array}$} \\
\hline $\begin{array}{l}\text { An arc is initiated, but no } \\
\text { consideration of plasma gas } \\
\text { ionization is made }(\alpha=0) \text {. }\end{array}$ & 240.55 & \\
\hline $\begin{array}{l}\text { An arc is initiated and the } \\
\text { consideration of plasma gas } \\
\text { ionization is made }(\alpha=0.46)\end{array}$ & 351.30 & \\
\hline $\begin{array}{l}\text { An arc is initiated and the } \\
\text { consideration of both plasma gas } \\
\text { and arc static electromagnetic } \\
\text { force is considered. }\end{array}$ & 386.43 & \\
\hline
\end{tabular}

The results show that the velocity of the plasma arc jet depends heavily on its temperature that relates to the level of the welding current. This temperature-related component of the velocity is the major contribution to the arc pressure. The arc pressure is generated by the dynamic pressure resulting from the arc temperature, the static pressure from the electromagnetic force inside an arc, and the restricted orifice dimensions that provides a mechanical compression on an arc column. To simplify the control for weld formation, efficiently dynamic regulation of the arc pressure is required. However, the necessity of constant heat input to a weldment with certain thickness limits the regulation of arc pressure to adjusting the welding current level upon which the dynamic pressure of a plasma arc jet depends. Also, no quick adjustment to the arc pressure during a welding process can be achieved by regulating the plasma gas flow rate that influences the temperature of an arc column. In addition, it is impractical to change the orifice dimensions during a welding process. Consequently, the only way to regulate arc pressure is changing the static arc pressure resulting from the electromagnetic force of an arc. Accordingly, the current redistribution device, shown in Fig. 1, is designed to modulate welding current (see Fig. 2) during the DCEN period so that static arc pressure can be regulated. The results achieved showed that the crown and root bead widths significantly decrease as the modulation frequency for DCEN current increases up to $13 \mathrm{KHz}$, which was very favorable to maintaining a weld pool with small dimensions when the total heat input to the workpiece was kept nearly constant.

A VPPAW process can be divided into three segments: (1) start-up segment, during which the keyhole is generated; (2) main body segment, during which the keyhole is dynamically maintained; and (3) the terminal segment, during which the keyhole collapses and the crater is filled. Very often, a smooth transition can not be made from the start-up segment to the main body segment and the weld fails because the keyhole weld pool is not properly generated. The keyhole weld pool during the start-up segment can be categorized as one of three modes: heat conduction mode, strong force mode, or heat and force mode. The reason for generating a heat 
conduction mode is that the weld pool melts through mainly by the heat accumulation in the workpiece. A higher plasma gas flow rate accompanied by a lower initial welding current and shorter welding current rise time will result in a higher and more concentrated arc force, which will generate a strong force mode weld pool. The heat and force mode can be achieved using the appropriate heat accumulation and proper temperature gradient between the weld pool and heat affected zone. The efflux through the keyhole can not expand freely because of the limited sizes of the weld pool and the keyhole. Using this mode (achieved by increasing both the plasma gas flow rate and the welding current from their initial values to the values for main body segment) the transition from the start-up segment to the main body segment is smooth and the as-welded result is good.

\section{Visual sensing a kevhole weld pool}

The keyhole is actually a cavity through the weld pool, with a profile resembling a trumpet. The cross section of this cavity varies both in diameter and shape (irregular ellipse). The maximum cross sectional diameter occurs at the top surface of the workpiece (top section diameter). The cross section with the smallest diameter is located about $1.5-2.5 \mathrm{~mm}$ up from the bottom surface of the 6.0 $\mathrm{mm}$ thick workpiece (throat section diameter). The diameter of the cross section on the bottom surface of the workpiece is larger than the throat diameter, but less than the top section diameter. Therefore, the keyhole weld pool is a deformed weld pool, and is much different from the weld pool in GTAW and GMAW. A typical image of a keyhole weld pool with wire feed is shown in Fig. 5, the black trace line on which is

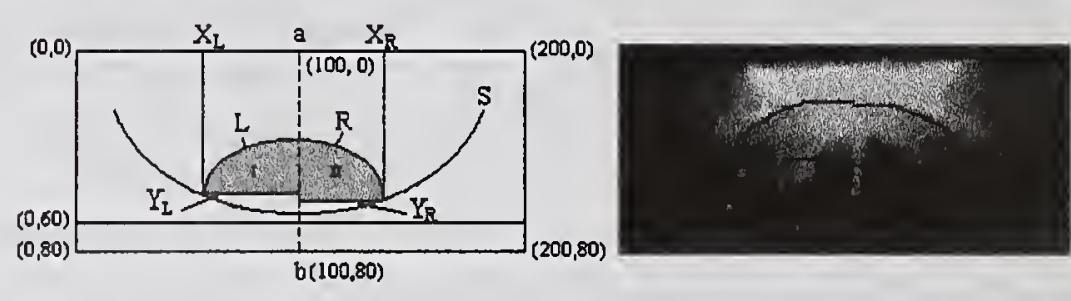

a

b

Fig. 5 Schematic for Image Processing

a. Image division

b. A keyhole traced by the algorithm for image processing

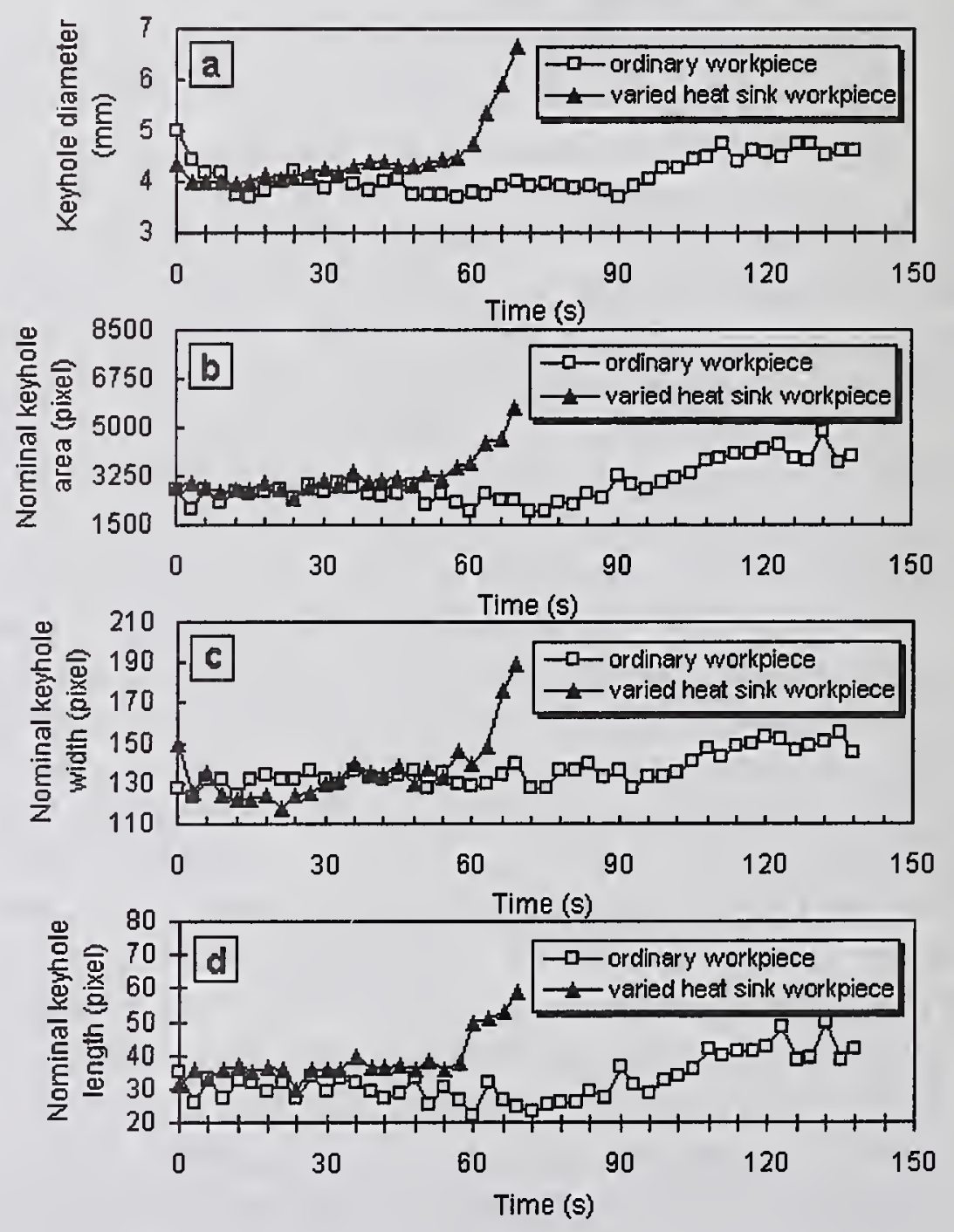

Fig. 6 Variation of Keyhole Parameters
a. Bottom diameter of a keyhole
c. Nominal keyhole width
b. Nominal keyhole area
d. Nominal keyhole length 
achieved after image processing operation and stands for the boundary of an enclosed keyhole. The thermal state of a weld is the most crucial and complex factor influencing weld formation because of its variability, immeasurability, and uncontrollability. In a keyhole welding process, the cutting usually occurs easier than in the melting-in mode welding process. Once a cutting process occurs, the weld bead is not acceptable and must be repaired. Thus, avoiding a cutting process that results mainly from the variation in thermal conditions is the first priority and a prerequisite for achieving consistent uniformity of a weld bead (Ref. 13, 19-23). Fig. 6 shows the variation of the bottom diameter of a keyhole with the time when the thermal conditions of a workpiece are changed. The origin of the time axis represents the instant when workpiece movement begins after the keyhole size reaches a certain value. The off-line measured width between the bottom oxide films along a weld bead, which is the trace of the arc efflux, is used for representing the bottom diameter of a keyhole. It can be seen that the variation in bottom diameter of a keyhole agrees well with the monitored keyhole size acquired from an sensed image.

\section{Weld formation control}

An artificial BP neural network of three layers with 9 parameters as inputs and the bottom diameter of a keyhole as an output are used to identify the penetration state. The number of elements in the hidden layer is experimentally determined as six based on the training results for the model.

The keyhole size may be regulated by adjusting the wire feed speed and the heat input may be controlled by changing the welding current. The wire feed speed is selected as the first controlling variable for the control model to avoid the occurrence of a cutting

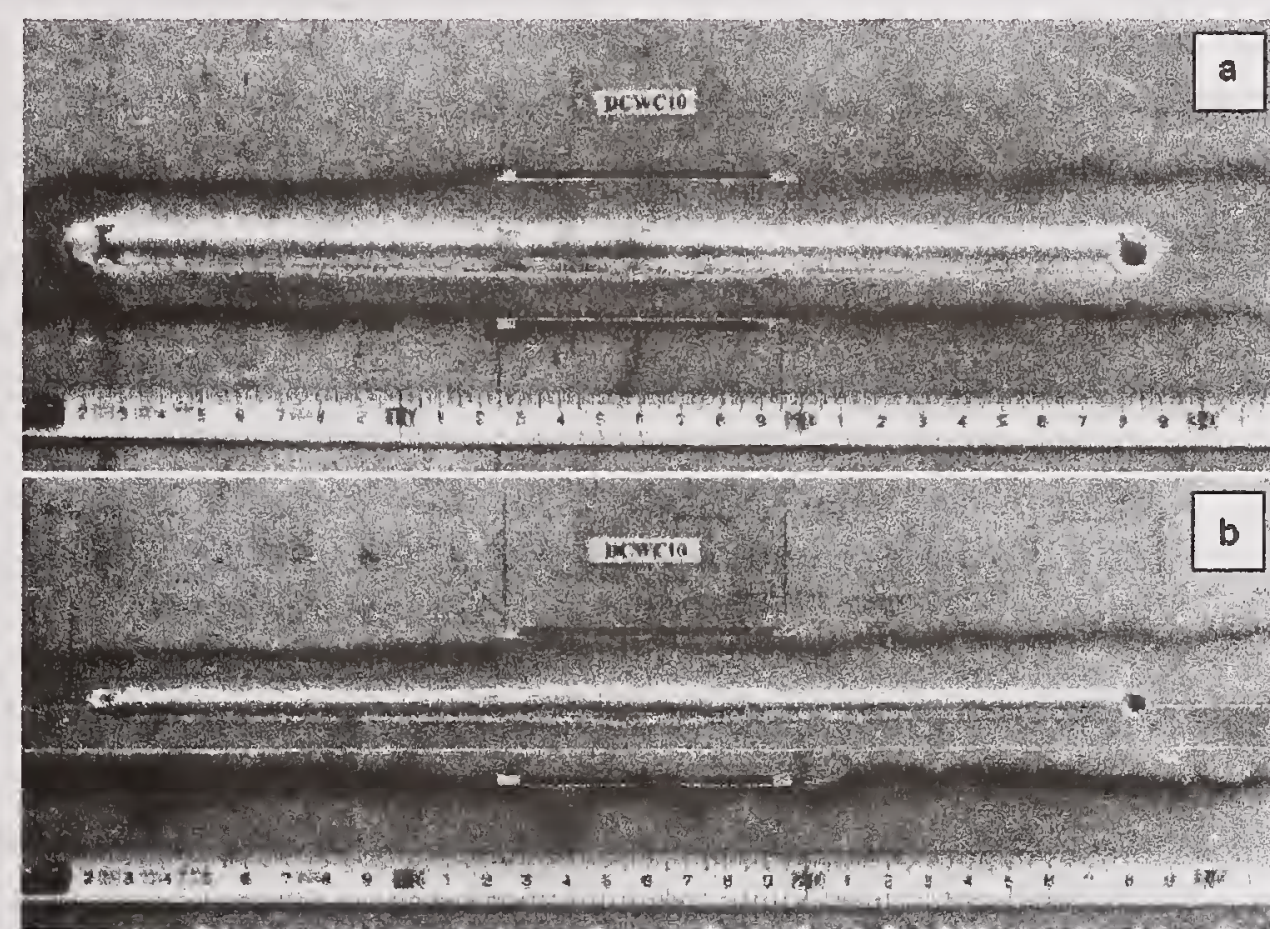

Fig. $7 \mathrm{~A}$ Weld Bead in a Butt Joint with the Closed-loop Control of Consistent Weld Formation Using both Wire Feed Speed and Welding Current as Controlling Variables

a. Top face b. Bottom face

process. To achieve the consistent uniformity of a weld bead during a welding process, the welding current should be controlled for regulating the heat input to the workpiece. Corresponding photographs of the weld bead, shown in Fig. 7, show that the consistent uniformity of a weld bead in VPPAW of aluminum alloys by the keyhole mode can be made in real time by closed-loop feedback control. 


\section{CONCLUSIONS}

Problems related to quality welds in variable polarity plasma arc welding of aluminum alloys are filmy surface oxide, deterioration of tungsten electrode, unstable penetration state, arc instability, short service life of a nozzle, inconsistent weld formation. To solve these problems, arc behavior, online front face sensing and monitoring of a keyhole, and real-time closed loop control for consistent uniformity of weld formation have to be addressed. Arc performance can be improved greatly by applying an innovative push-pull variable polarity arc. The establishment of a quality keyhole weld pool during the start-up segment is one of the key techniques. This can be implemented by ramping up both welding current and plasma gas flow rate. The dynamic keyhole dimensions measured accurately reflect the state of weld pool. The real-time intelligent control using both wire feed speed and welding current as controlling variables can guarantee the consistent uniformity of weld formation even when thermal conditions of a butt-jointed weldment change.

\section{ACKNOWLEDGEMENT}

The author expresses his great acknowledgement to the supports of National Key Laboratory of Advanced Welding Production Technology, P. R. China and Edison Welding Institute, Columbus, Ohio.

\section{REFERENCES}

1. Craig E.. 1988. The Plasma arc process-A review. Welding Journal 2: 19 to 25.

2. Tuttle W.. 1991. Understanding aluminum welding. Welding Journal, 70: 43 to 46.

3. Vancleave B. P.. 1981. Keyhole plasma arc welding of aluminum. American Welding Society. 2501 N. W., Seventh St. Miami, Fla, 33125, [Proc. Conf. 1980].

4. Tomsic M. and Barhost S.. 1984. Keyhole plasma arc welding of aluminum with variable polarity power. Welding Journal 63(2): 25 to 27.

5. Nunes A. C. and Bayless E. O.. 1984. Variable polarity plasma arc welding on space shuttle external tank. Welding Journal 63(4): 27 to 35.

6. Howard Woodward. 1996. A U.S. contractor for the international space station. Welding Journal 3:35 to 40 .

7. Irving B.. 1992. Joining experts meet to exchange ideas about welding in space. Welding Journal 1:67 to 69 .

8. Barhorst S.. 1985. The cathodic etching technique for automated aluminum tube welding. Welding Journal 5: 28 to 31.

9. Kyselica S.. 1987. High-frequency reverse arc switch for plasma arc welding of aluminum. Welding Journal 1: 31 to 35.

10. Eagar T. W.. 1989. Recent trends in welding science and technology.. ASM International. S. A. David, Ed. P. 341, Materials Park, Ohio

11. Pang Q. and et al.. 1994. Workpiece cleaning during variable polarity plasma arc welding of aluminum. Transactions of ASME, Journal of Engineering for Industry 116(11): 463 to 466. 
12. Torres M. R.. 1992. Gas contamination effects in variable polarity plasma arc welded aluminum. Welding Journal 4:123 to 130.

13. Zheng B.. 1995. The stability of weld formation in keyhole plasma arc welding of aluminum alloys. Ph. D. dissertation, Harbin Institute of Technology, P. R. China

14. Martinez L. F.. 1994. Effect of weld gases on melt zone size in VPPA welding of Al 2219. Welding Journal 10:50 to 55.

15. Jiang H. Z.. 1988. Arc welding and slag welding: Beijing, P. R. China: Mechanical Industry Publications.

16. Lancaster J. F.. 1986. The physics of welding: 2nd ed. Edition: New York: Pergamon Press.

17. Ando K.. 1985. Arc phenomena: Beijing, P. R. China: Mechanical Industry Publication (Translated version from Japanese).

18. Martinez L. F.. 1992. Front side keyhole detection in aluminum alloys. Welding Journal 5: 49 to 52.

19. Metcalfe J. C. and Quigley M. B. C.. 1975. Keyhole stability in plasma arc welding. Welding Journal 11: 401 to 404.

20. Steffens H. D.. 1972. Automatic control for plasma arc welding with constant keyhole. Welding Journal 6:40 to 45 .

21. Agapakis E. and Bolstad J.. 1991. Vision sensing and processing system for monitoring and control of welding and other high luminosity processes. International Robots \& Vision Automation Conference: 23-29.

22. Nakata S; Huang J.; and Tsuruha Y.. 1988. Visual sensing system for in-process control of arc welding process. Welding International 12: 1086 to 1090.

23. Hoffman T.. 1991. Real-time imaging for process control. Advanced Materials \& Processes 9: 37 to 43 . 

Session A4: Imaging 



\title{
CHARACTERIZING THE MICROSTRUCTURE OF A GTA WELD IN-PROCESS USING HIGH-SPEED, HIGH-MAGNIFICATION, DIGITAL IMAGING
}

\author{
A.C. Hall ${ }^{*}$, G. A. Knorovsky ${ }^{*}$, C. V. Robino*, J. Brooks ${ }^{+}$, \\ D. O. Maccallum ${ }^{*}$, M. Reece ${ }^{*}$ G. Poulter ${ }^{*}$
}

\begin{abstract}
A high quality zoom lens and a high-speed CCD camera have been used to image gas tungsten arc (GTA) welds in stainless steel. Both the trailing (solidifying) edge and the leading (melting) edge of the weld pool have been observed. A number of solidification phenomena have been captured including: dendrite growth, melting, and weld ripple formation. Significant information about the evolution and structure of the solid-liquid interface can be extracted from these videos using computerized image analysis techniques. This information can be directly related to the microstructure of the finished weld. Video clips will be presented, techniques for extracting microstructural information from those clips will be discussed, and the extracted information will be related to the microstructure of the finished weld.
\end{abstract}

\section{KEYWORDS \\ WELD VISUALIZATION, MACHINE VISION, WELD RIPPLE, SOLIDIFICATION, MELTING}

\section{INTRODUCTION}

Over the last decade, significant advances have been made in digital photography and related computer technology. Combination of these technologies has given rise to a field known as machine vision. It is now relatively straightforward to capture and process images using a computer. Robust software exists that allows the user to extract quantitative measurements from digital images. This technology has found widespread application in the world's manufacturing industries [1-3]. Parts are routinely inspected using machine vision technology to see that they have been assembled properly or manufactured to desired tolerances. Surprisingly, this technology has not been widely adopted in the scientific community. It affords the researcher with a powerful new tool set that allows large amounts of information about dynamic physical processes to be accessed and extracted in an automated fashion. Solidification of metals is a field that is ripe for the exploitation of such technology.

Conventional techniques for inspecting the solid-liquid interface in a metal typically involve rapidly quenching a sample and then polishing, etching, and examining the quenched sample [46]. Experimental techniques based on such schemes are very useful but have a significant

\footnotetext{
"Sandia National Laboratories; Albuquerque, NM

${ }^{+}$Sandia National Laboratories; Livermore, CA
} 
limitation. They only provide the researcher with a "snapshot" of the solid-liquid interface. No direct information about the motion or the evolution of the solid-liquid interface is available from a quenching experiment. The only experimental schemes that do provide information about the motion and the evolution of the solid-liquid interface are based on visualization of transparent materials (like succinonitrile) that solidify in a manner analogous to metals $[7,8]$. These experiments have provided a wealth of information about dendrite growth and the evolution of the solid-liquid interface. However, these experiments have a significant limitation. Transparent materials often do not exhibit the complex phenomena that occur during the solidification of multi-component engineering alloys. Solidification mode transformations, which are commonly observed in stainless steels [9], are an example of complex phenomena that are not exhibited by transparent materials. Complex phenomena like this demand new experimental techniques that can provide information about the dynamic behavior of solid-liquid interfaces in engineering materials.

In this paper, we describe a technique for using machine vision technology to characterize the behavior of the solid-liquid interface in a gas tungsten arc (GTA) weld. Dendrite growth, melting, and weld ripple formation have all been observed at high spatial and temporal resolution. A large amount of quantitative data can be extracted from these experiments in a relatively straightforward manner. An example showing how data is extracted and related to the microstructure of the finished weld is discussed in detail.

\section{EXPERIMENTAL PROCEDURE}

The solid-liquid interface in a GTA weld was filmed using a Kodak EktaPro camera equipped with a Navitar ${ }^{\circledR}$ Zoom 6000 lens. The Kodak EktaPro camera is capable of capturing digital images at up to 12,000 frames / second. In practice, we have found that filming at 2000 frames / second provides temporal resolution that is more than adequate for a GTA weld. The Navitar ${ }^{\circledR}$ Zoom 6000 lens is designed specifically for machine vision. It is a parafocal zoom lens system capable of imaging objects within fields of view ranging from $146.6 \mathrm{~mm}$ to $0.03 \mathrm{~mm}$ on each side.

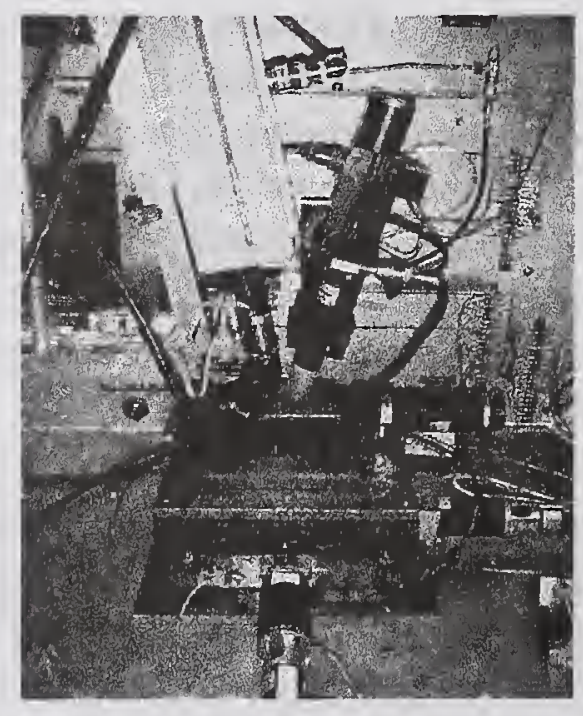

Figure 1: Camera, lens, X-Y- $\theta$ stage, and welding torch 
The camera and welder are fixed with respect to each other and the sample is translated below them. See Figure 2. This arrangement keeps the solid-liquid interface stationary with respect to the camera.

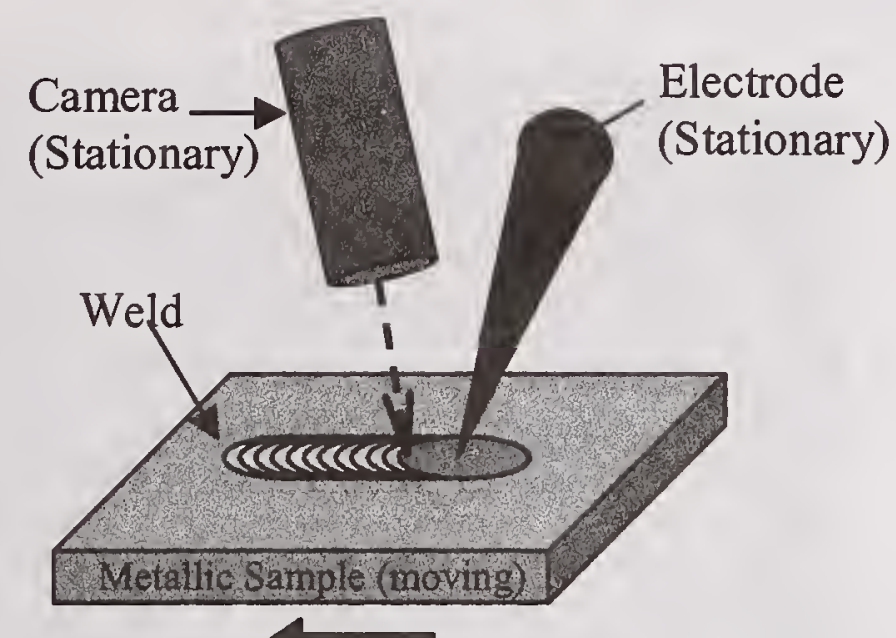

Figure 2: Schematic showing the arrangement of the camera, sample, and welding torch

The entire apparatus (camera, motion control stage, and welding torch) is contained inside a glove box so that an inert atmosphere can be maintained around the weld. This prevents oxide from forming on the surface of the weld pool and obscuring the phenomena of interest. Welds were made at a variety of amperage, arc length, and speed combinations. Solidification and melting phenomena were filmed by pointing the camera at the trailing or leading edge of the weld pool.

\section{RESULTS AND DISCUSSION}

Figure 3 is a still image taken from a video of a 150 amp GTA weld in 304 stainless steel traveling at $4.2 \mathrm{~mm} / \mathrm{sec}$. Dendrites can be seen at the solid-liquid interface.

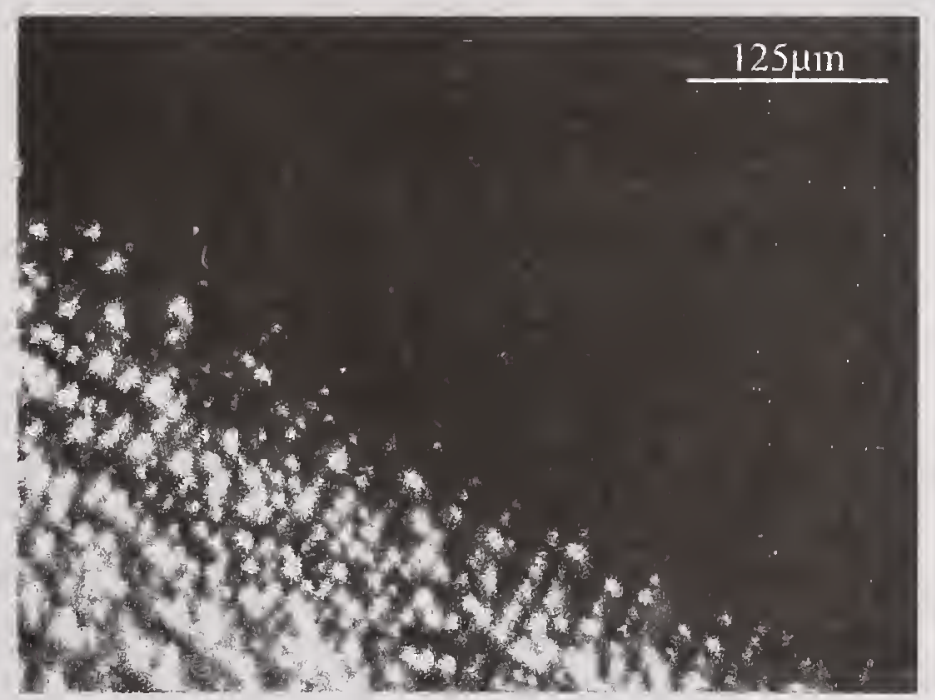

Figure 3: Dendrites at the solid-liquid interface in a GTA weld in 304 stainless steel 
Figure 4 is a still image showing melting in Inconel 718. Liquation of the interdendritic laves and/or niobium carbide eutectic-like constituents can be seen at the solid-liquid interface. Figure 5 is a still image showing weld ripples in 304 stainless steel. Multiple weld ripples can be seen behind the solid-liquid interface.

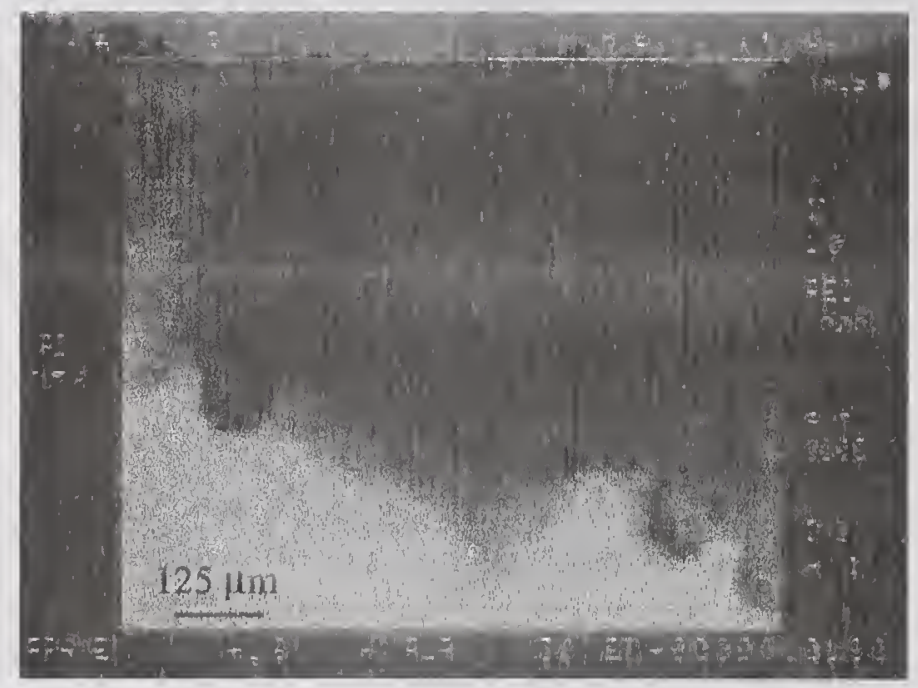

Figure 4: Melting at the solid-liquid interface in Inconel 718, liquation can be seen at the interface

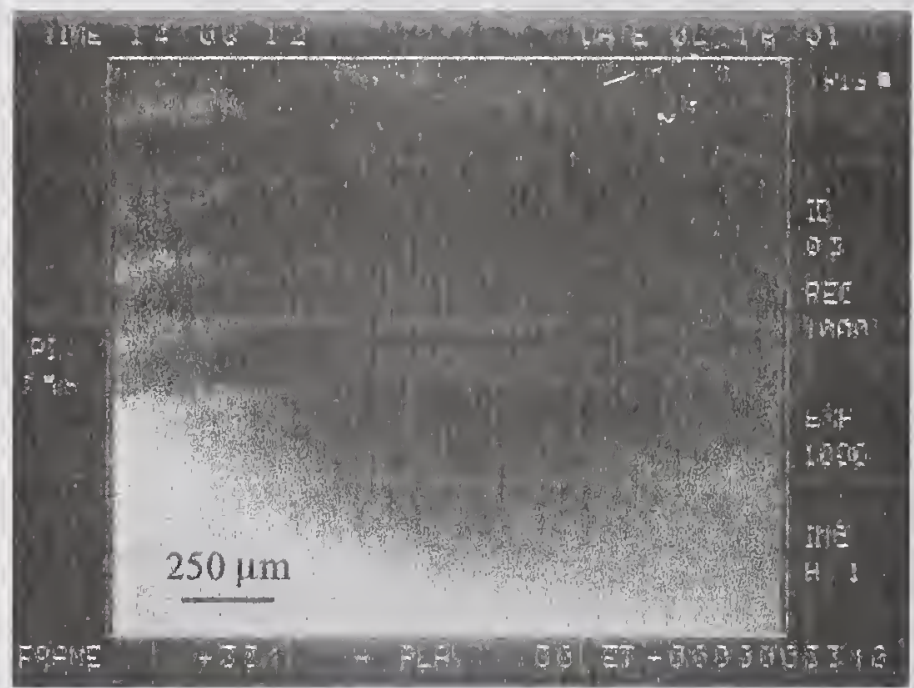

Figure 5: Weld ripples at the trailing edge of a GTA weld in 304 stainless steel

\section{Data Extraction}

A large amount of information about the solid-liquid interface and its motion with time is present in these video recordings. Quantitative information can be extracted from these video clips in a relatively straightforward manner using computerized image analysis schemes. We have accomplished this using National Instruments ${ }^{\mathrm{TM}} \mathrm{Lab}$ View and IMAQ Vision software.

Our image analysis scheme proceeds as follows. First, a digital movie is recorded as a series of image files. Each movie typically contains between one and two thousand image files each of 
which are $500 \mathrm{~KB}$ in size. Image analysis software is used to open each image file in sequence and measure a feature of interest (a dendrite arm spacing, the location of an interface, etc...). All measurements are made in a pixel coordinate system that uses the top left corner of the image as an origin. After each measurement is made, it is associated with the frame number of the image and written to an output file. Frame number and pixel coordinates must then be converted into meaningful units like time and position. Frame number is easily translated into time by multiplication with the framing rate. A dimensional measurement like primary arm spacing requires a simple unit conversion to an appropriate length measurement. Filming an accurate scale provides this, as well as a velocity calibration. A measurement like interface velocity requires a frame of reference correction in addition to a unit conversion. A frame of reference correction is necessary because all measurements are made in the camera frame of reference. If information like interface velocity is to be translated into the sample frame of reference the stage velocity must be measured accurately and accounted for.

\section{Example: Weld Ripple}

In the video that Figure 5 was extracted from the motion of the solid-liquid interface that is associated with the formation of a weld ripple was seen. That motion was quantified by measuring the position of the solid-liquid interface in each frame of the video. The interface position is determined from a marked intensity change in the digital image. Figure 6 is a graph of this information. The upper curve shows interface position in pixels coordinates with respect to time.

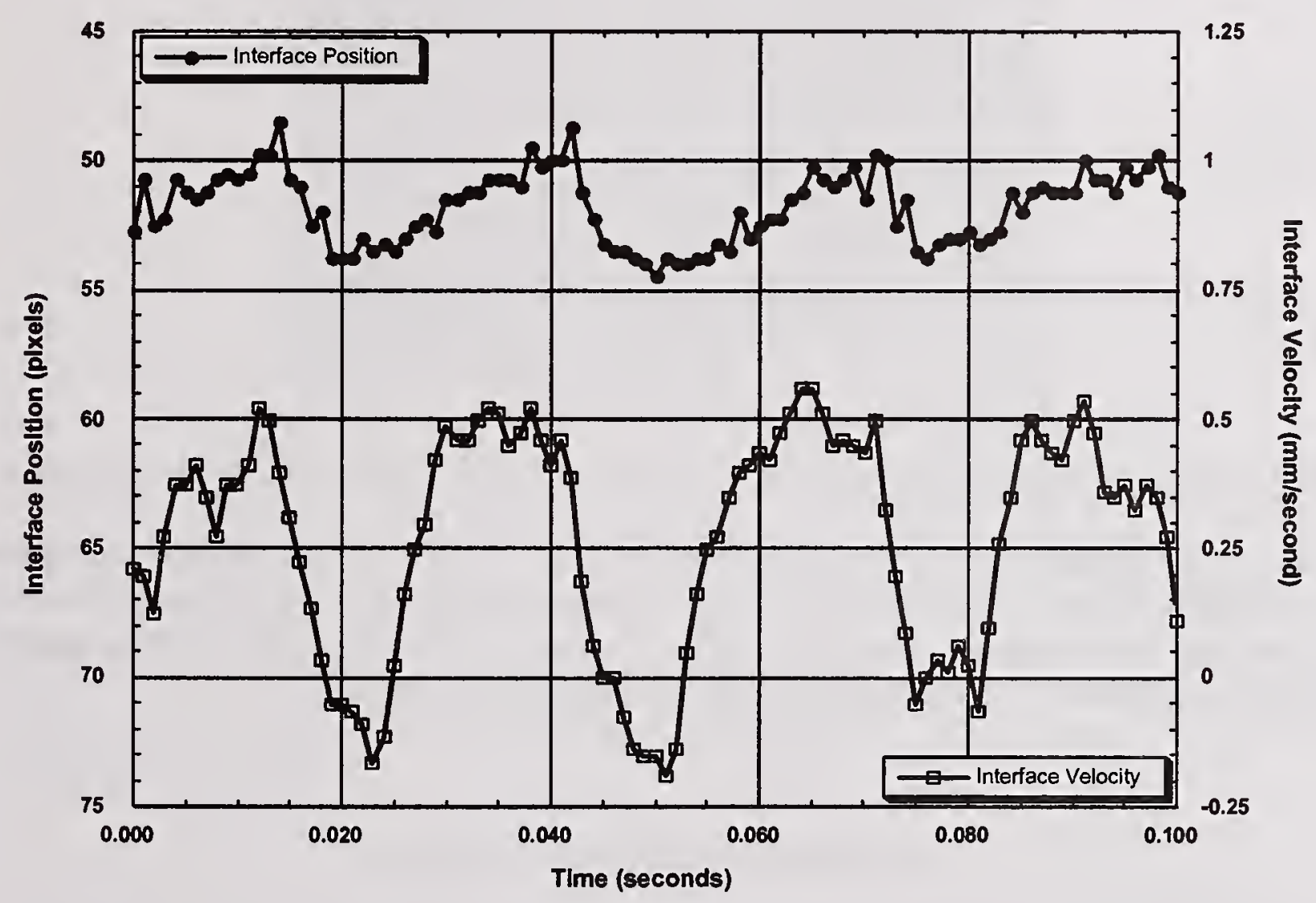

Figure 6: Data from machine vision analysis of a rippling weld.

The raw data associated interface position with frame number. Converting frame number to time was simple. This video was filmed at 1000 frames / second with an exposure time of 1/1000 
second. Dividing the frame number by 1000 converted the $\mathrm{X}$-axis to time in seconds. Converting the $\mathrm{Y}$-axis was also straightforward. At this magnification 360 pixels is equivalent to $2 \mathrm{~mm}$, so dividing the $\mathrm{Y}$-axis coordinates by 180 gave the interface position in $\mathrm{mm}$. This position was measured relative to the camera. To measure interface velocity in the frame of reference of the sample we had to account for the stage velocity. This was done by numerically differentiating the position versus time data and adding a vector representing the stage velocity to the differentiated curve. The addition of this vector placed the data in the frame of reference of the sample. The converted data is also shown in Figure 6. The interface behavior associated with the formation of a weld ripple can now be clearly seen. When a ripple is formed the solidliquid interface appears to go thorough a cycle of advancing and remelting.

Evidence of this change in interface velocity can be seen in the microstructure of the finished weld. Figure 7 is a micrograph showing the transverse cross section of a rippled weld in 304 stainless steel. Light colored bands in the shape of the solid-liquid interface can be seen in the image. Similar features can be seen in longitudinal cross sections of rippled welds. These features are associated with the ripple formation seen in the video. As shown in Figure 7, the ripple bands extend all the way to the bottom of the weld pool.

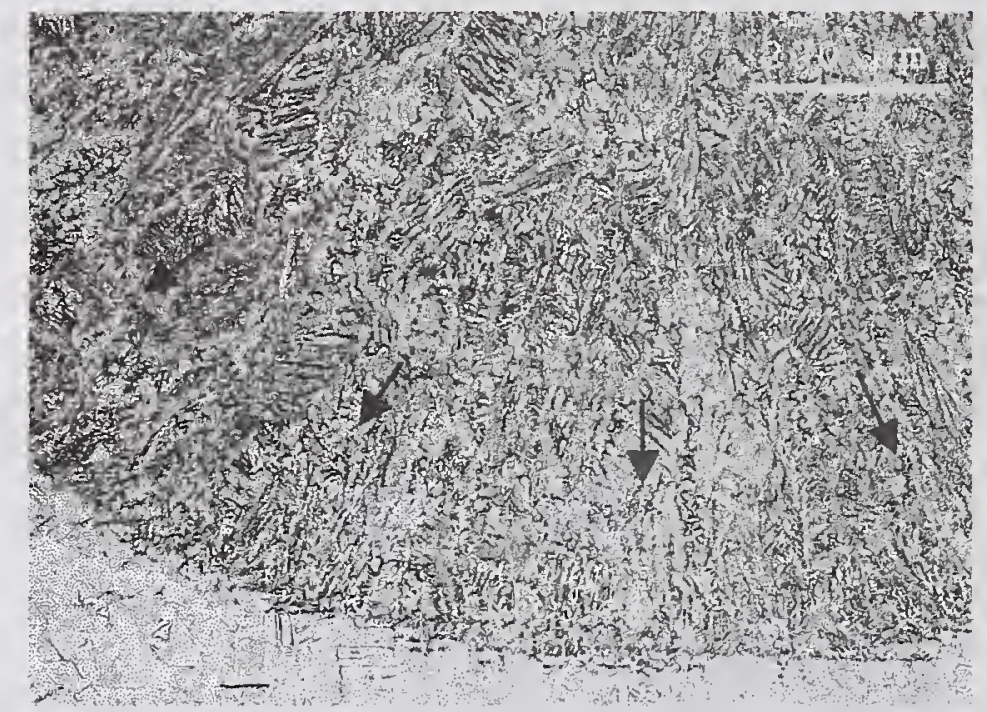

Figure 7: Cross section of a GTA weld showing evidence of rippling in the microstructure.

Like the metallographic cross section shown in Figure 7, the video clips mentioned above are two-dimensional representations of three-dimensional phenomena. Microstructural evidence of cyclic interface motion deep within the weld pool suggests that data gathered at the surface of the pool can be correlated with phenomena that occur deeper in the pool.

\section{SUMMARY AND CONCLUSIONS}

The example given above illustrates how machine vision technology can be used to measure the solid-liquid interface velocity associated with weld ripple formation. Computerized image analysis techniques were used to process a large numbers of images. This allowed solid-liquid interface velocity to be measured with high temporal resolution. A cyclic solid-liquid interface 
velocity was observed and was associated with microstructural features present throughout the depth of the weld. This suggested that data collected from this type of experiment can provide information about phenomena deep within a weld pool. By applying the same technique to the other videos mentioned in this paper, primary and secondary dendrite arm spacing, and local melting velocity can also be measured as a function of time and welding conditions. Clearly machine vision has tremendous potential as a data collection technique for scientific experiments. This potential is not limited to solidification science; in fact machine vision could be used to study almost any dynamic phenomena. In the case of solidification science it has allowed us to view and quantitatively measure the dynamic behavior of the solid-liquid interface in an engineering material under actual welding conditions.

\section{ACKNOWLDEGMENT}

Sandia is a multiprogram laboratory operated by Sandia Corporation, a Lockheed Martin Company, for the United States Department of Energy under Contract DE-AC04-94AL85000

\section{REFERENCES}

1. Zuech, N., When you're hot, you're hot: Machine vision and the semiconductor roller coaster. Advanced Imaging, 2000. 15: p. 3.

2. Babb, M., Machine Vision Moves into The Mainstream of Manufacturing. Control Engineering, 1995(July 1).

3. Hogarth, S., Machines with vision. Manufacturing Engineering, 1999. 122: p. 102-104.

4. Brooks, J.A., N.Y.C. Yang, and J.S. Krafcik, On the origin of ferrite morphologies of primary ferrite solidified stainless steel welds. Int. Trends Weld. Sci. Technol., Proc. Int. Conf. Trends Weld. Res., 3rd, 1993: p. 173-80.

5. DuPont, J.N., et al., Solidification of Nb-bearing superalloys: Part II. Pseudoternary solidification surfaces. Metallurgical and Materials Transactions a-Physical Metallurgy and Materials Science, 1998. 29(11): p. 2797-2806.

6. Huang, X.B., et al., Effect of solidification rate on the microstructure of a Ni-base superalloy. Materials Transactions Jim ;, 1997. 38(11): p. 1016-1021.

7. Glicksman, M.E., et al., Dendritic Growth of Succinonitrile in Terrestrial and Microgravity Conditions As a Test of Theory. Isij International, 1995. 35(6): p. 604-610.

8. Glicksman, M.E., et al., The isothermal dendritic growth experiment. Solidification and Gravity, 1996. 215: p. 179-189.

9. Elmer, J.W., S.M. Allen, and T.W. Eagar, Microstructural Development during Solidification of Stainless Steel Alloys. Metallurgical Transactions A, 1989. 20A (October): p. 2117-2131. 



\section{Vision-based Weld Defect Detection and Depiction}

\section{Overview}

- Principles of Laser-Based Machine Vision Technology

- Software Aspects and Involvement

- Inspection Examples

- Pipeline Corrosion

- Fillet Weld Surface Quality 


\section{Why improve on Visual Testing?}

- Advantages of VT

- Efficient, little operator training, relatively inexpensive

- Disadvantages of VT

- Limited to detection of surface discontinuities

- Subjectivity on assessment of weld

- Existing codes and specs may lack sufficiently clear and objective criteria for all weld surface conditions encountered

\section{Laser-Based Machine Vision Technology}

- Principles of Triangulation

- Assembling 3D measurements

- Types of Laser Sensors

- Spot

- Laser Line/ Spray of Light 


\section{Principle of Triangulation}

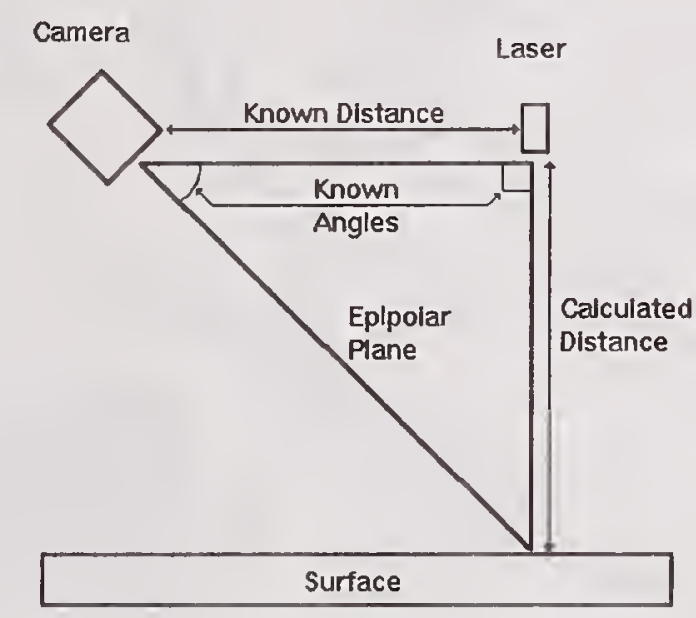

\section{Laser Spot (1D) \& Laser Line (2D)}
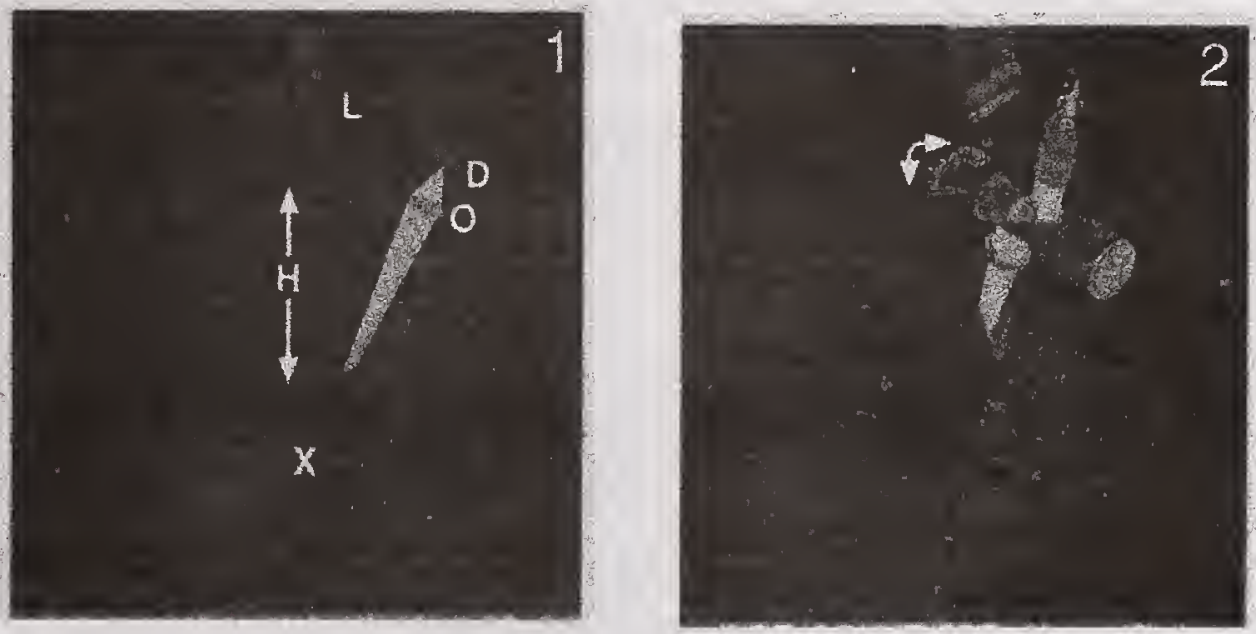


\section{Laser-Line System}

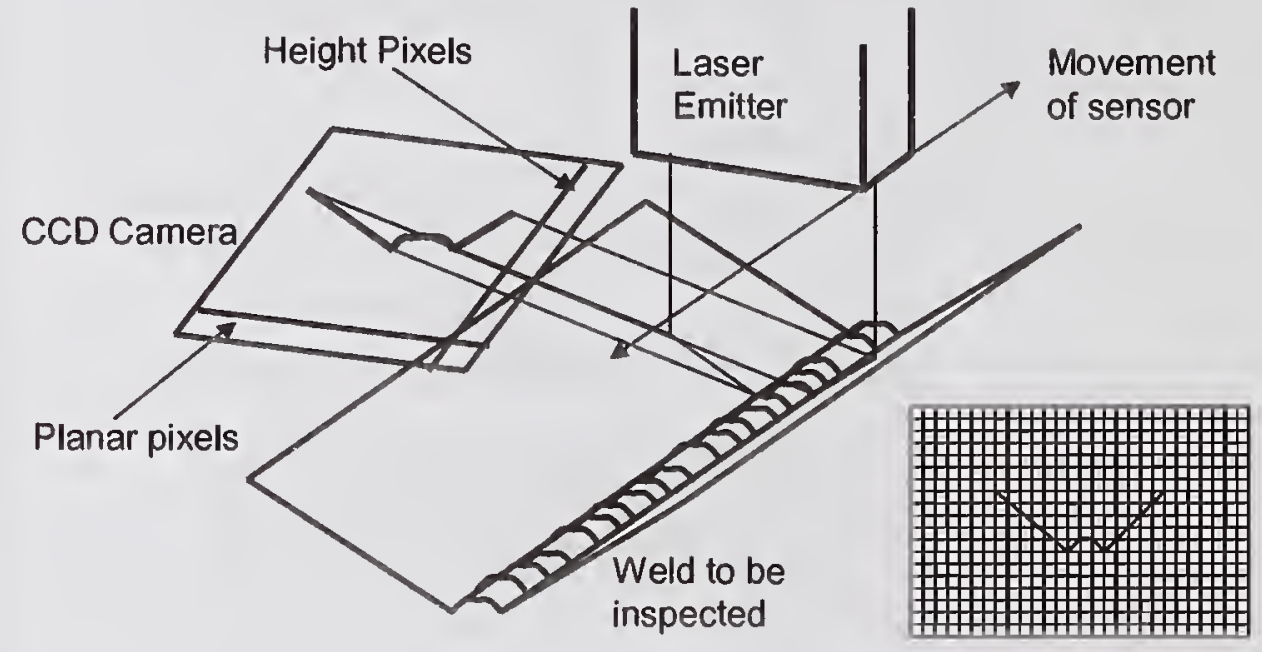

EUI

\section{Principles of Operation}

- CCD Camera

- Laser light is captured by the "detector" which is a grid of charge-coupled devices (CCD)

- Two Types of Data Obtained From CCD Detector:

- Range Data-Quantitative Data used for measurements of surface profile

- Intensity Data-Light Reflectance Level Data used for reconstructing image 


\section{CCD Image of Fillet Weld}

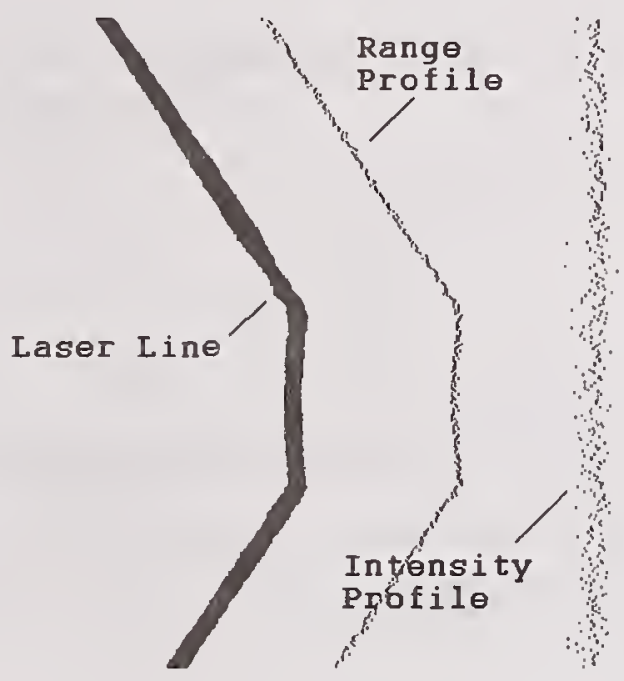

\section{Data Processing}

- Software is the Key

- The laser is simply the tool to gather data

- Software is the means for transforming the data into actual measurements

- Examples of Processing the Range Data

- Segmentation

- Curve Fitting 


\section{Software Examples}

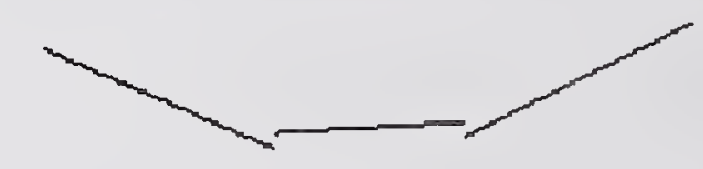

- Segmentation of Fillet Weld Range Data

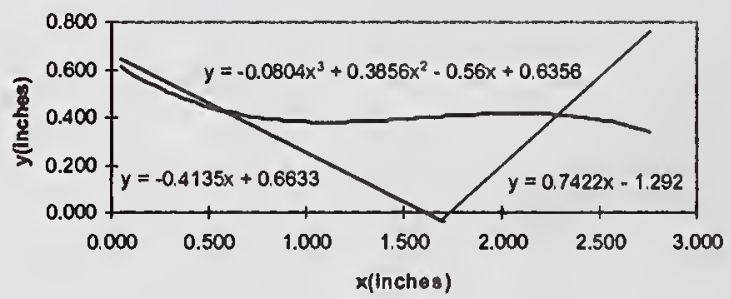

- Line Segmentation \& Curve Fitting

\section{Inspection Example: Pipeline Crawler with Spot Sensor}

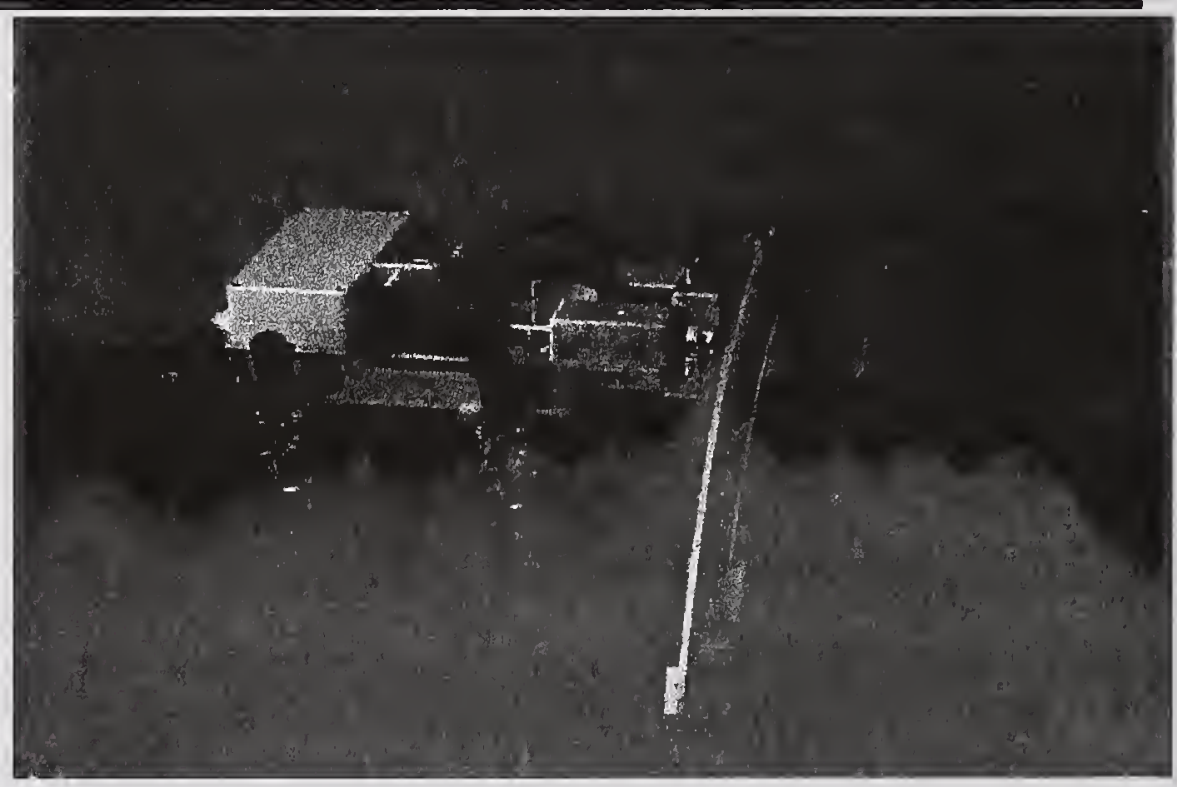




\section{Principles of Operation of Crawler}

- Small, portable robotic vehicle with magnetic wheels

- Motorized circumferential slide

- On-board computer

- Field-ruggedized for harsh environments

- 100-ft umbilical cable attached to workstation

- Laser sensor moves along circumferential slide taking measurements

\section{Software Display for Pipeline Corrosion}

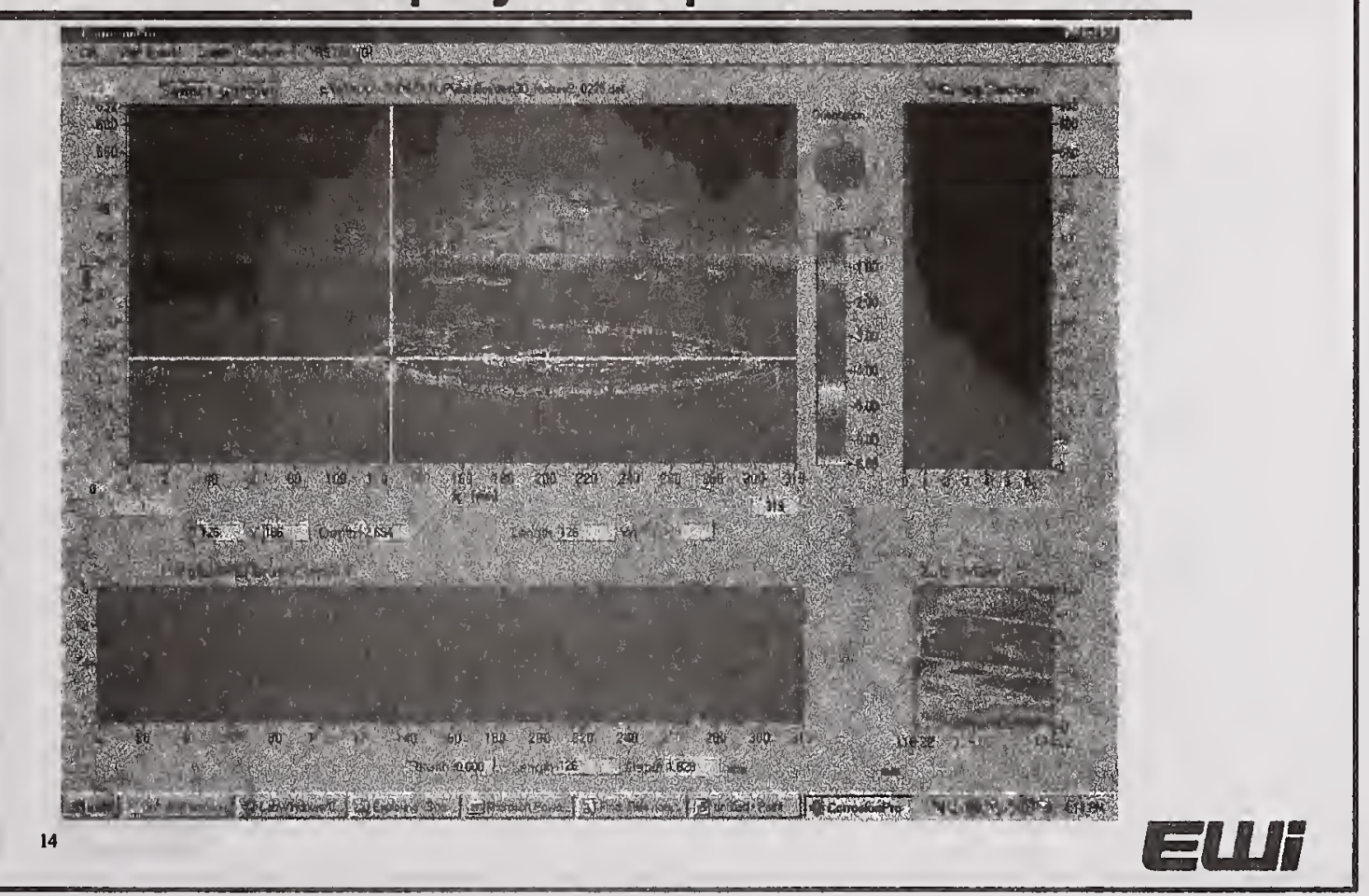




\section{Benefits of Pipeline Inspection System}

- Data collection Efficiency/Reduced Inspection and Data Assessment Time

- Operator Independence

- Increased Data Quality and Integrity

- Baseline for Comparison with Other Technologies (calibration for other tools)

\section{Fillet Weld Inspection}

- Industrial Need for Automated Inspection

- Advantages of a Laser-based System

- Previous Developments

- Current Developments 


\section{Previous Developments}

- Portable System for Inspection of Fillet Welds for Shipbuilding Industry

- Hand-held system

- Measured 2 and 3-dimensional defects

- Development of System for Inspection of Girth Weld on Pipe

- Measured Toe Angles and Radii

- Measured Misalignment

\section{Previous Developments}

- Semi-automatic System for Inspection of Fillet Welds for Automotive Industry

- Tube to plate fillet weld

- Measures 2 and 3-dimensional defects

- Indicates defect type, size and location

- Improved upon previous technologies 


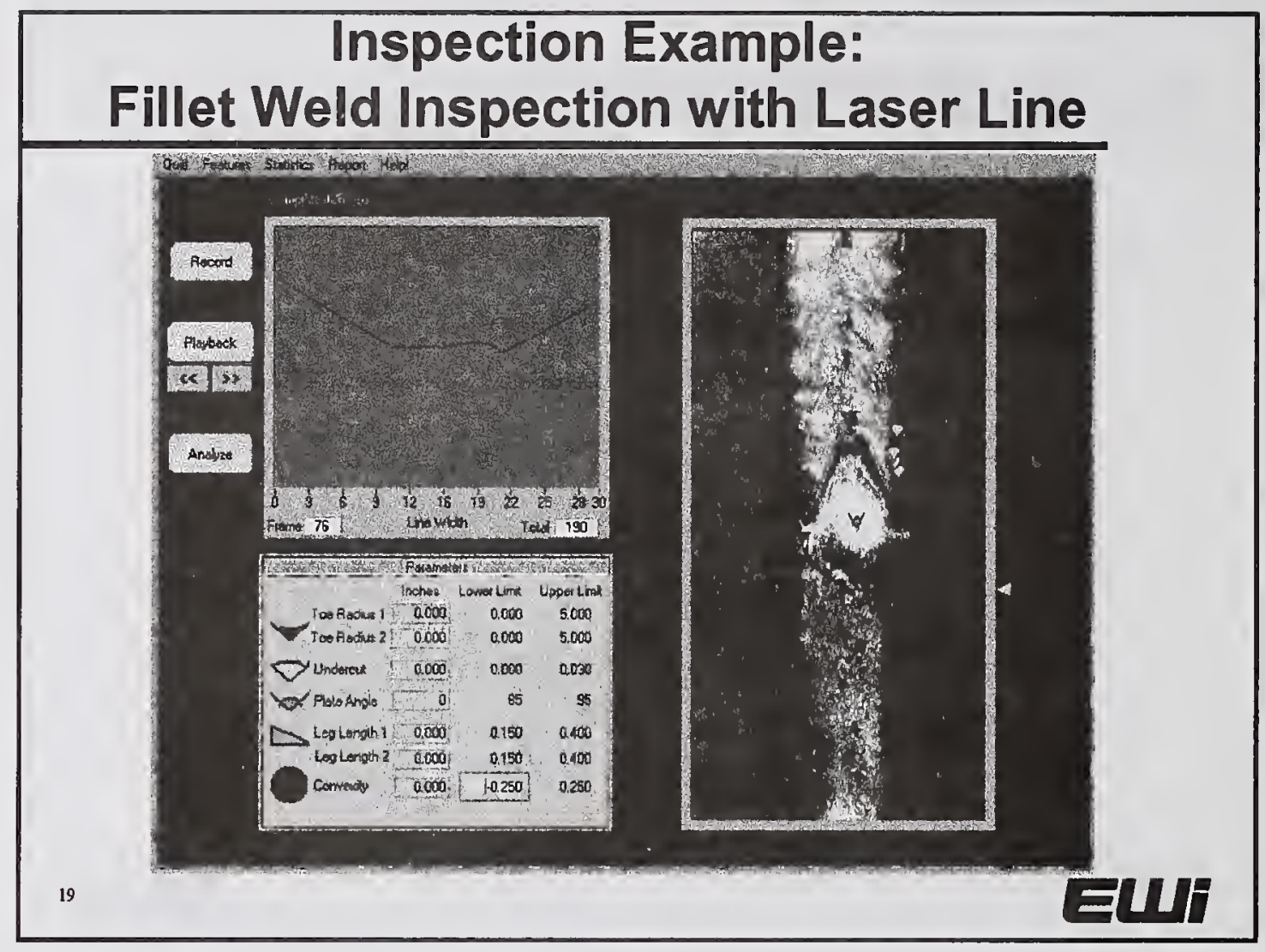

\section{Two-Dimensional Measurements}

- Leg Lengths

- Weld Face Widths

- Weld Toe Angles

- Theoretical Throat

- Convexity/Concavity

- Plate Angle 


\section{Three-Dimensional Measurements}

- Surface Porosity

- Surface Cracks

- Undercut

- Spatter

\section{Current Developments}

- Adaptive Welding

- Bug-based system with adaptive control of welding parameters using laser-based sensing for:

» Pre-weld joint tracking

»Weld joint template selection

»Post-weld inspection 



\section{COMPUTER MICRO-IMAGE ANALYSIS IN THE \\ HEAT-AFFECTED ZONE OF HQ130 HIGH STRENGTH STEEL}

Li Yajiang* and Wang Juan*

\section{ABSTRACT}

The microstructure and grain degree in the heat-affected zone (HAZ) of HQ130 high strength steel, which was welded by gas shielded metal arc welding, are researched by means of microscope and computer technique. A series of sample are cut from the heat affected zone of HQ130 steel to make metallographic analysis. The relative content of different microstructure in the HAZ is researched by means of XQF-2000 micro-image analyzer. Fine structures in the HAZ are analyzed by means of Transmission Electron Microscope (TEM) and electron diffraction. Experimental results indicate that with the increase of weld heat input $(E)$, cooling time $\left(t_{8 / 5}\right)$ is longer and impact toughness deteriorates. The main microstructure in the HAZ changes from lath martensite $(M L)$ and low bainite $(M L+B L)$ to lath martensite, low bainite and upper bainite $(\mathrm{ML}+\mathrm{BL}+\mathrm{Bu})$, and the grain becomes coarser, especially, the austenitic grain diameter increases to $158 \mu \mathrm{m}$ when weld heat input $(E)$ is $22.3 \mathrm{~kJ} / \mathrm{cm}$. By controlling weld heat input under $16 \mathrm{~kJ} / \mathrm{cm}$ to form $\mathrm{ML}+\mathrm{BL}$ in the HAZ, avoiding producing $\mathrm{Bu}$ and preventing grain coarse, the microstructure and performance in the HAZ of HQ130 steel can be ensured.

\section{KEY WORDS}

High strength steel, Heat-affected zone, Microstructure and Micro-image

\section{INTRODUCTION}

HQ130 steel is newly developed low alloy high strength steel used in welded structures, the tensile strength of which is $1300 \mathrm{MPa}$. The various parts in the heat affected zone (HAZ) of HQ130 steel experience different weld thermal cycle. The HAZ is considered as a weak part in the welded joint of the high strength steel (Ref.1,2). It is very important to ensure the microstructure and mechanical properties of welding zone, especially for the low alloy high strength steel. Previously researchers made some qualitative analysis in the heat-affected zone of high strength steel by means of traditional metallography (Ref.3-5).

*School of Materials Science and Engineering, Shandong University, Jinan 250061, P.R.China

E-mail: yajli@jn-public.sd.cninfo.net 
Operators observe the microstructure under the metallograph with slow velocity and high requirement for the operator's skill and the result with inaccuracy. The use of advanced computer technology to analyze the microstructure in the weld zone, will be significantly increase the accuracy of determining the microstructure in the HAZ.

In this paper, the toughness in the HAZ of HQ130 has been studied through the weld heat simulated test. We have measured the relative content of different microstructure and grain degree in the HAZ by means of XQF-2000 micro-image analyzer, which will decrease the artificial influence to analyze the microstructure. We also make further analysis of fine microstructure in the HAZ by means of Transmission Electron Microscope (TEM) and Scanning Electron Microscope (SEM), which provide experimental and theoretical basis for the determining welding technology parameters.

\section{EXPERIMENTAL METHODS}

Chemical compositions and mechanical properties of HQ130 steel after the heat treatment are listed in Table 1. The thickness of the base metal is $12 \mathrm{~mm}$ in the test. Using gas shielded metal arc welding (GSMAW) with single $\mathrm{V}$ groove at the place of butt joint and with different weld heat input $(E)$, such as smaller $(9.6 \mathrm{~kJ} / \mathrm{cm})$, middle $(16.0 \mathrm{~kJ} / \mathrm{cm})$ and $\operatorname{larger}(22.3 \mathrm{~kJ} / \mathrm{cm})$.

Table 1 Chemical compositions and mechanical properties of HQ130 high strength steel

\begin{tabular}{|c|c|c|c|c|c|c|c|c|}
\hline \multicolumn{1}{|c|}{ Chemical compositions $/ \%$} \\
\hline $\mathrm{C}$ & $\mathrm{Si}$ & $\mathrm{Mn}$ & $\mathrm{Cr}$ & $\mathrm{Mo}$ & $\mathrm{Ni}$ & $\mathrm{B}$ & $\mathrm{S}$ & $\mathrm{P}$ \\
\hline 0.18 & 0.29 & 1.21 & 0.61 & 0.28 & 0.03 & 0.0012 & 0.006 & 0.025 \\
\hline \multicolumn{8}{|c|}{ Mechanical properties } \\
\hline$\sigma_{\mathrm{b}} / \mathrm{MPa}$ & $\sigma_{\mathrm{s}} \mathrm{MPa}$ & $\delta / \%$ & $\Psi / \%$ & $\mathrm{HRC}$ & Akv/J \\
\hline 1370 & 1313 & 10 & 43 & 40.5 & $64\left(20^{\circ} \mathrm{C}\right)$ \\
\hline
\end{tabular}

The weld thermo-simulation test of HQ130 steel was carried out by means of a Gleeble-1500 test machine. The test parameters were: peak temperature $(T \mathrm{p}) 1350^{\circ} \mathrm{C}$ and cooling time $\left(t_{8 / 5}\right) 5 \mathrm{~s}$, $10 \mathrm{~s}, 20 \mathrm{~s}, 40 \mathrm{~s}$. A series of sample are cut from the heat-affected zone of HQ130 to make metallographic analysis. The relative content of microstructure in the HAZ is measured by means of XQF-2000 micro-image analyzer. The relative content of different microstructure in the HAZ is calculated using the computer according to the percent that the microstructure represents by each color covering the field of vision.

The grain degree was measured by the cutting-line method. The formula of grain degree index is:

$$
\mathrm{G}=-3.2877+6.6439 \lg (\mathrm{m} \times \mathrm{N} / \mathrm{L})
$$

Where: $\mathrm{L}$ is the gauged lattice length (mm); $\mathrm{M}$ is the magnifying multiple; $\mathrm{N}$ is the cutting dot number on the gauged lattice. 
Thin slice from the HAZ by line-cutting machine was made into film sample. The fine microstructure was analyzed using TEM and electron diffraction in the chosen zone.

\section{EXPERIMENTAL RESULTS AND ANALYSIS}

\section{Impact toughness in the HAZ}

Impact work and hardness in the simulated $\mathrm{HAZ}$ have close relationship between peak temperature $(T \mathrm{p})$ and cooling time $\left(t_{8 / 5}\right)$. When $\mathrm{Tp}=1350^{\circ} \mathrm{C}$, the result of thermo-simulation test for impact work and hardness in the HAZ of HQ130 steel is listed in Table 2.

Table 2 Impact toughness and hardness in the simulated HAZ of HQ130 steel $\left(\mathrm{Tp}=1350^{\circ} \mathrm{C}\right)$

\begin{tabular}{|c|c|c|c|c|}
\hline$t_{8 / 5} / \mathrm{s}$ & 5 & 10 & 20 & 40 \\
\hline$E / \mathrm{kJ} \cdot \mathrm{cm}^{-1}$ & 9.2 & 13.1 & 18.6 & 26.4 \\
\hline $\mathrm{Akv} / \mathrm{J}$ & $65.7,56.1,65.4$ & $55.9,51.7,53.4$ & $60.3,55.0,46.8$ & $23.7,22.6,33.2$ \\
& $(62.4)$ & $(53.7)$ & $(54.0)$ & $(26.6)$ \\
\hline $\mathrm{HV}$ & $(372)$ & $(365)$ & $(318)$ & $(255)$ \\
\hline
\end{tabular}

Note: 1) Relationship between $t_{8 / 5}$ and $E$ was calculated by using the D.Uwer formula (Ref.3);

2) Number in the bracket is the test average value.

When $T \mathrm{p}=1350^{\circ} \mathrm{C}$, which is corresponded to the coarse grain heat-affected zone(CGHAZ) and $t_{8 / 5}=40 \mathrm{~s}$ (with considerable weld heat input), impact work decreases obviously. The test results indicate that $t_{8 / 5}$ should be controlled smaller than $20 \mathrm{~s}$, because the larger the weld heat input is, the longer $t_{8 / 5}$ and the easier the deterioration of impact toughness in the $\mathrm{HAZ}$ is.

In practice, most welded joints are formed by the multi-layer welding technology. The heat-affected zone will undergo multiple weld thermal cycles. Impact work in the HAZ of multi-layer welding is obviously increased, which is favorable to welding of HQ130 high strength steel. The test result of impact work in the practical welded joint zone of HQ130 steel is shown in Table 3.

Table 3 Impact work in the practical HAZ of HQ130 high strength steel

\begin{tabular}{|c|c|c|c|}
\hline Positions & Coarse grain zone & The fusion zone & Base metal \\
\hline \multirow{2}{*}{ Akv /J } & $80.5,91.8,84.2$ & $97.1,99.6,111.8$ & $60.5,63.2,68.8$ \\
& $(85.4)$ & $(102.8)$ & $(64.2)$ \\
\hline
\end{tabular}

Note: Impact work in the fusion zone virtually included a part of weld metal and HAZ.

\section{Microstructure in the HAZ of HO130}

The austenite grain in the CGHAZ near the fusion zone for HQ130 steel grows up obviously and grows into even-distributed austenite, which will change into coarse low carbon lath martensite (ML) when cooling rapidly after the welding (see Fig.1a). The coherent lattice interface lies between the ML and the former austenite, which is non-diffusion phase transform. Fig.1b shows the feature of the lath martensite (ML) in the CGHAZ and the fusion zone of HQ130 observed under SEM. The main feature is that the low-carbon martensite lath is distributed in bigger angle 
and Matensite laths are nearly parallel to form a martensite zone. If the former austenite grain is finer the width of the martensite lath would be smaller and toughness would be better.

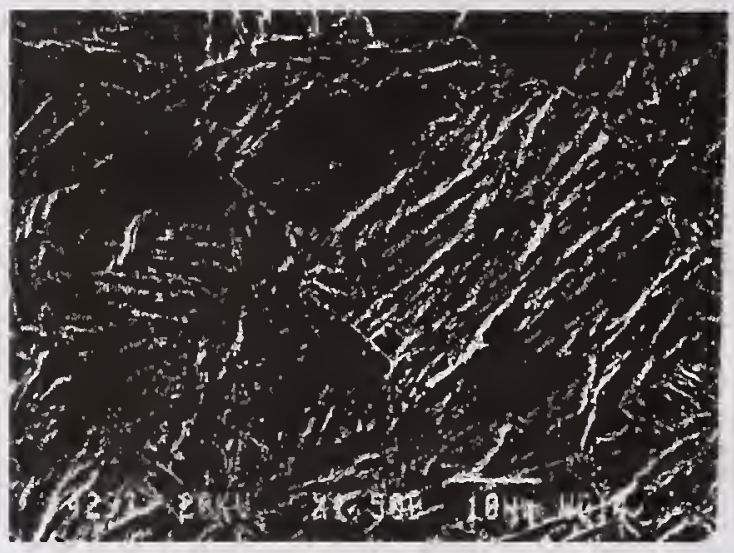

a) CGHAZ $1500 \times$

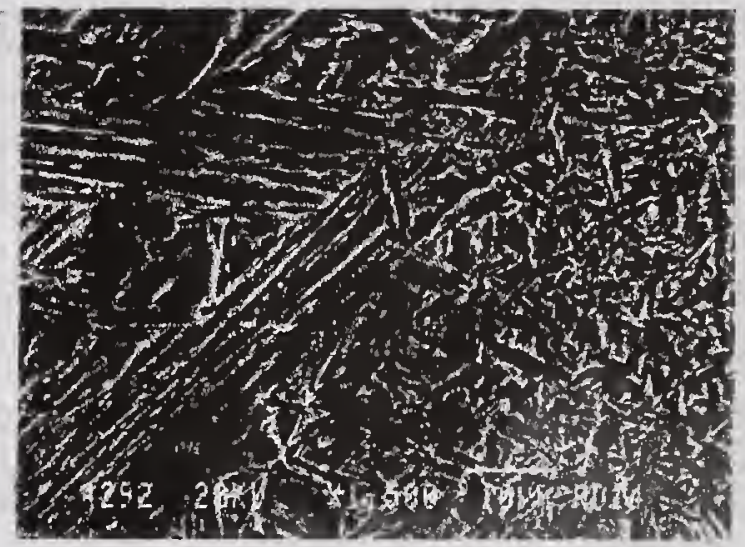

b) CGHAZ and the fusion zone $1500 \times$

Figure 1 Feature of microstructure in the HAZ of HQ130 high strength steel

Metallographic analysis shows that there is low banite $(\mathrm{BL})$ in the coarse grain heat affected zone (CGHAZ) of HQ130 steel besides a large number of ML. Some of BL are parallel to ML, others transverse martensite lath and cut apart the austenite grain. When austenite changes into ML during the cooling, more than ten closed lathes have the same orientation and make up a bond of lathes. When BL+ML mixture microstructure is produced in the CGHAZ, the first separated BL would carve the former austenite effectively, which makes more opportunities for ML core and limits the growth of ML. In contrast to a unitary ML, the mixed microstructure of BL+ML has more larger angle boundary. Crack developing is hindered on the border of ML bunch or ML-BL border and changes direction, the length of crack developing in unit becomes shorter, so the impact toughness increases obviously.

\section{The relative content of microstructure and grain degree}

The relative content of various microstructures is not easy to be determined accurately. The contents of the $\mathrm{BL}$ and $\mathrm{Bu}$ are very important to toughness in the HAZ. The type of microstructure and their relative contents in the HAZ with different weld heat input were analyzed by means of XQF-2000 micro-image analysator. The result of quantitative analysis is listed in Table 4. Effect of weld heat input on microstructure is showed in Fig.2. The larger weld heat input is, the more easily the $\mathrm{Bu}$ would be produced in the HAZ. When the weld heat input $(E)$ is controlled under $16.0 \mathrm{~kJ} / \mathrm{cm}$, the mixed microstructure of ML+BL may be acquired in the CGHAZ where there is no Bu. A certain angle exists among BL lath and separates martensite lath, which leads to the increase of sub-boundary and the decrease of crack sensitivity and make the toughness in the HAZ increase obviously.

When the weld heat input is $22.3 \mathrm{~kJ} / \mathrm{cm}$, the main microstructure in the $\mathrm{HAZ}$ is $\mathrm{ML}+\mathrm{BL}+\mathrm{Bu}$, among which there is 9.8 percent $\mathrm{Bu}$. The orientation of crystallization among $\mathrm{Bu}$ lath is very small, cleavage crack can run through $\mathrm{Bu}$ lath continuously, corresponding to lower cleavage 
fracture stress, which makes the toughness decrease in the HAZ. The weld heat input in welding of HQ130 steel should be limited $(E \leqslant 16 \mathrm{~kJ} / \mathrm{cm})$ in order not to produce $\mathrm{Bu}$ in the $\mathrm{HAZ}$. Table 4 Relative content of microstructure in the HAZ of HQ130 steel

\begin{tabular}{|c|c|c|c|c|}
\hline $\begin{array}{c}\text { Weld heat input } \\
/ \mathrm{kJ} \cdot \mathrm{cm}^{-1}\end{array}$ & $\mathrm{ML} / \%$ & $\mathrm{BL} / \%$ & $\mathrm{Bu} / \%$ & $\begin{array}{c}\text { Main } \\
\text { microstructure }\end{array}$ \\
\hline 9.6 & $\begin{array}{c}92.9,93.0,93.6 \\
(93.2)\end{array}$ & $\begin{array}{c}4.6,5.0,5.1 \\
(4.9)\end{array}$ & 0 & $\mathrm{ML}$ \\
\hline 16.0 & $\begin{array}{c}85.1,83.8,83.6 \\
(84.2)\end{array}$ & $\begin{array}{c}10.2,11.4,12.7 \\
(11.4)\end{array}$ & 0 & $\mathrm{ML}+\mathrm{BL}$ \\
\hline 22.3 & $\begin{array}{c}60.2,68.8,68.5 \\
(65.8)\end{array}$ & $\begin{array}{c}22.2,18.3,19.2 \\
(19.9)\end{array}$ & $\begin{array}{c}9.6,10.1,9.9 \\
(9.8)\end{array}$ & $\mathrm{ML}+\mathrm{BL}+\mathrm{Bu}$ \\
\hline
\end{tabular}

Note: Number in the bracket is the average value of three version field

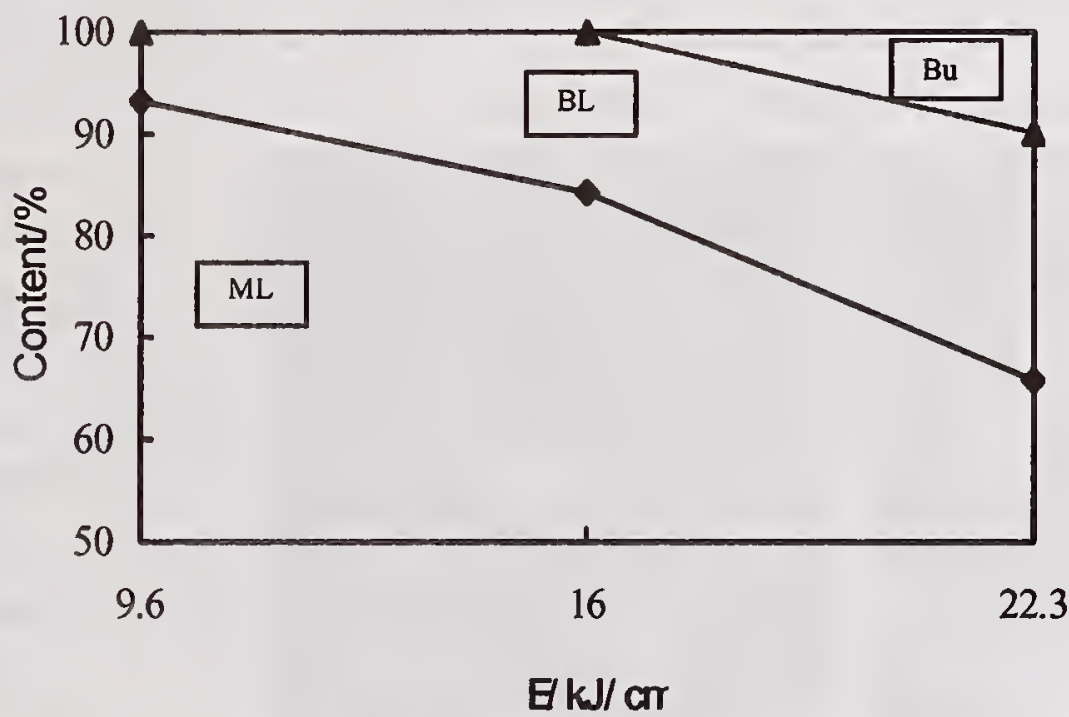

Figure 2 Effect of weld heat input $(E)$ on the relative content of microstructure in the HAZ

Affected by the peak temperature, the austenite grains become coarse in the CGHAZ of HQ130 steel. The width of the CGHAZ and the diameter of austenite grain are different using different weld heat input. Under the condition of different weld heat input, the width of the CGHAZ and austenite grain degree is evaluated using XQF-2000 micro-image analyzer, and the grain diameters are calculated by means of the computer. The analysis results are shown in Table 5.

Table 5 the width, the grain degree and diameter in the CGHAZ of HQ130 steel

\begin{tabular}{|c|c|c|c|}
\hline $\begin{array}{c}\text { Weld heat input } \\
/ \mathrm{kJ} \cdot \mathrm{cm}^{-1}\end{array}$ & $\begin{array}{c}\text { Width of CGHAZ } \\
/ \mathrm{mm}\end{array}$ & $\begin{array}{c}\text { Grain degree } \\
\text { /grade }\end{array}$ & $\begin{array}{c}\text { Maximum grain } \\
\text { diameter } / \mu \mathrm{m}\end{array}$ \\
\hline 9.6 & $\begin{array}{c}1.03,1.10,0.98 \\
(1.00)\end{array}$ & $\begin{array}{c}3.0,3.5,4.2 \\
(3.6)\end{array}$ & 105 \\
\hline 16.0 & $\begin{array}{c}1.28,1.16,1.29 \\
(1.24)\end{array}$ & $\begin{array}{c}2.9,3.2,3.7 \\
(3.3)\end{array}$ & 115 \\
\hline 22.3 & $\begin{array}{c}1.58,1.56,1.52 \\
(1.55)\end{array}$ & $\begin{array}{c}2.4,2.0,2.5 \\
(2.3)\end{array}$ & 158 \\
\hline
\end{tabular}

Note: Number in the bracket is the average value of three version field 
From the measured data we can see that the larger the weld heat input is, the broader CGHAZ is and the larger the size of austenite grains are. Especially when $E=22.3 \mathrm{~kJ} / \mathrm{cm}$, the maximal diameter of austenite grain in the CGHAZ reaches $158 \mu \mathrm{m}$. So in order to prevent the grain from being coarse in the CGHAZ, the weld heat input must be limited, which ensure the impact toughness and avoid producing brittleness and crack.

\section{Fine microstructures in the CGHAZ}

Fine microstructures in the HAZ of HQ130 steel have great effect on the impact toughness. Thin slice was cut out from the HAZ by the liner-cutting machine, then abraded into thin slice sample and finally made into film sample slighted by electrolysis double-spurt method. Using the TEM and electron diffraction to conduct analysis for the film sample. The feature of $M L$ and BL under TEM in the HAZ of HQ130 steel taken from [110] direction, the electron diffraction pattern and schematic index diagram are showed in Fig.3a,b,c.

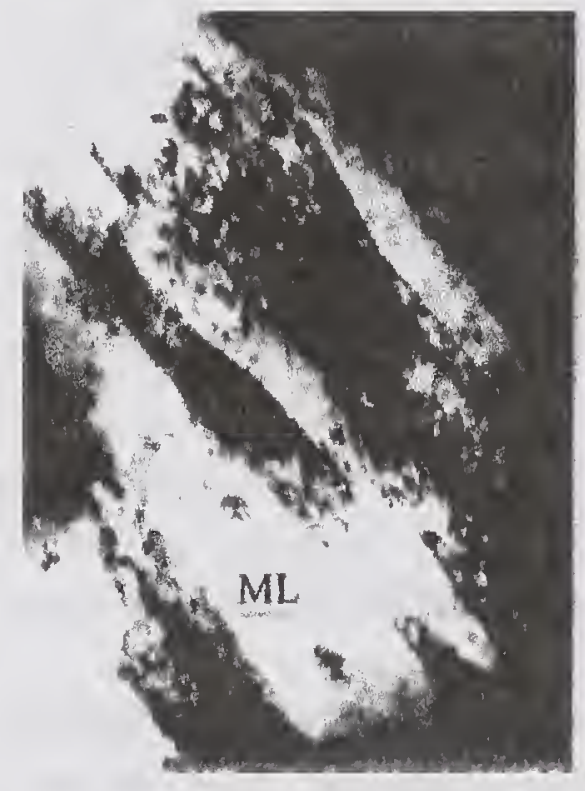

a) TEM morphology $40000 \times$

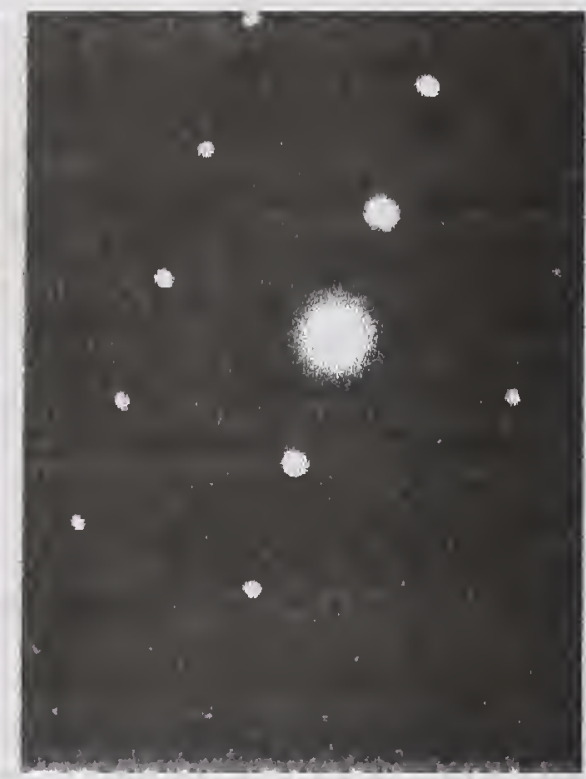

b) Electron diffraction pattern

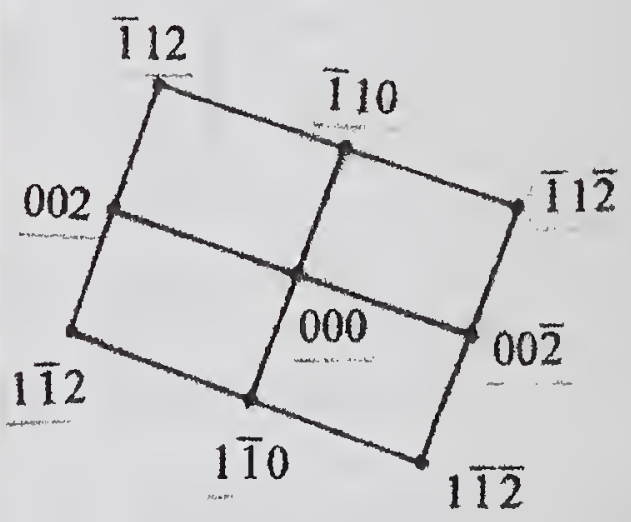

$\mathrm{B}=[110]$

c) Schematic index diagram

Figure 3 Feature of the fine structure in the CGHAZ of HQ130 steel (TEM)

The sub-structure in the HAZ of HQ130 steel observed under TEM is martensite lath with dislocation, whose dislocation density about $(0.3 \sim 0.9) \times 10^{12} / \mathrm{cm}^{2}$. Some carbide distributes inside the ML lath and on the boundary. There is a part of lattice distortion region near dislocation where the diffraction strength is different from other parts. So we can observe the lining shadow of the dislocation. $\gamma \rightarrow M L$ forms dislocation sub-structure in microcosmic and exists crystallographic orientation relationship of $(111)_{\curlyvee} / /(011)_{a}$ and $[101]_{\curlyvee} / /[111]_{a}$ between origin and new phase.

When the weld heat input is $9.6 \mathrm{~kJ} / \mathrm{cm}$, self-tempering phenomenon appears in the $\mathrm{HAZ}$ even cooling rapidly. Under TEM, carbide dispersed inside the grain can be clearly observed. A little of twin martensite can be also observed besides dislocation ML because of cooling rapidly, which is testified by electronic diffraction analysis. The twin martensite in the ML lath of low 
carbon quenched and tempered steel is distinct from that of high carbon alloy steel. Only in the local parts of covering ML appears the twin, others are high density dislocation line, while the twin in high carbon martensite is distributed with concentrated arrangement (Ref.4)

The toughness of low carbon lath mantensite depends on the sub-structure of high-density dislocation inside ML lath. The TEM analysis shows that there are a lot of dislocations in the sub-structure of lath martensite in the HAZ of HQ130 steel. This perhaps because of "self-tempering" phenomenon that supersaturated carbon in the new-produced martensite in the HAZ can still spread, assemble and separate out in form of the carbide particle. Self-tempered lath martensite has certain toughness itself. If the self-tempered ML is produced in the HAZ of high strength steel, the performance to resist cleavage fracture can be increased and the impact toughness in the HAZ can be ensured.

\section{CONCLUSION}

When weld heat input $(E)$ changes from $9.6 \mathrm{~kJ} / \mathrm{cm}$ to $22.3 \mathrm{~kJ} / \mathrm{cm}$, the microstructure in the HAZ of HQ130 steel changes from ML to $M L+B L$ and then to $B L+M L+B u$, the width of $H A Z$ increases, and size of austentite grain becomes larger. Even when $E=22.3 \mathrm{~kJ} / \mathrm{cm}$, the content of upper bainite $(\mathrm{Bu})$ arrived to $9.8 \%$ and the maximum diameter of relevant grain in the CGHAZ of HQ 130 steel is $158 \mu \mathrm{m}$. In order to prevent the toughness deterioration in the HAZ because of the formation of $\mathrm{Bu}$, the weld heat input should be limited under $16 \mathrm{~kJ} / \mathrm{cm}$. The TEM observation and analysis of electron diffraction indicate that there are high density dislocation about (0.3 0.9) $\times 10^{12} / \mathrm{cm}^{2}$ in sub-structure inside low carbon lath martensite in the HAZ of HQ130 steel. The closed lathes inside ML lath bundle have small orientation difference and have no obvious twin structure.

\section{ACKNOWLEGEMENT}

The work was supported by the foundation (05170104) of Key Laboratory of Liquid Structure and Heredity of Materials, Ministry of Education, People's Republic of China.

\section{REFERENCES}

1. Tian D.W, Qian B.N, Si Z.Y and Yang H.X. 1995. Effect of Boron on microstructure and toughness of simulated CGHAZ of HSLA steel. China Welding. 4(1): 37-s to 43-s.

2. Yang Y.X. 1992. Behavior of transformation induced plasticity in simulated CGHAZ for HT-80 steel. Proceedings of the International Conference. Beijing, (2): 71-s to 74-s

3. Zou Z.D, Li Y.J and Yin S.K. 1998. Toughness and TEM analysis for the CGHAZ of HQ130 steel. Transactions of the China Welding Institution. 19(3): 141-s to 146-s.

4. ${ }^{-}$Tan Y.X. 1985. TEM analysis for low carbon martensite steel. Acta Metallurgic Sinica. 21(3): 181 -s to 186 -s.

5. ' Li Yajiang, Zou Zengda et al. Effect of weld thermo-cycle on microstructure and performance in the heat-affected zone of HQ130 steel, Acta Metallurgic Sinica. 32(5): 532-537 

Session A5: Fabrication Quality and Standards 



\title{
VIRTUAL AND AUGMENTED REALITY FOR QUALITY IMPROVEMENT OF MANUAL WELDS
}

\author{
P. Tschirner", S. Nordbruch" ${ }^{*, *}$, A. Gräser
}

\begin{abstract}
The large problem to create manual welds of constant high quality results from missing optical information during the actual welding process. Due to the extreme brightness conditions e.g. in arc welding and the use of protective glasses even experienced welders can hardly recognize details of the welding pool, the welding seam and the environment. This paper describes a new research project for the development of a system for the support of the welder.
\end{abstract}

\section{KEYWORDS}

Virtual and augmented reality, manual arc welding, quality control

\section{INTRODUCTION}

Welding is one of the most important industrial manufacturing methods. The creation of manual welds is often used in production of unique pieces where an automation is impossible or uneconomic, e.g. in shipbuilding with its complicated hull geometry. A common welding method is the inert-gas arc welding e.g. manual gas tungsten-arc welding (GTAW) or manual gas metal-arc welding (GMAW). In all these processes the electrical arc transfers energy to the welding seam. This arc has an extraordinary high brightness and ultraviolet radiation and for observation a welding helmet with suitable protective glasses is necessary. These glasses absorb the radiation and darken the entire scene, so even experienced welders can hardly recognize details of the welding pool, the welding seam and the environment. Figure 1 shows a view of the welder recorded with a CCD camera through standard protective glasses.

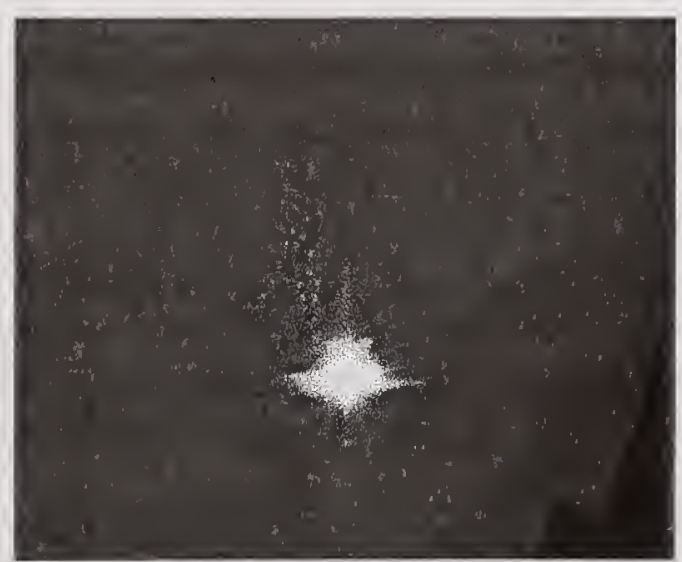

Figure 1: Simulated view of the welder recorded with a CCD camera and standard protective glasses.

\footnotetext{
- University Bremen, Institute of Automation (IAT), Kufsteiner Str. NW 1, 28359 Bremen, Germany

* FWBI Friedrich-Wilhelm-Bessel-Institut Forschungsgesellschaft m.b.H, Postfach 106364, 28063 Bremen, Germany
} 
A further disadvantage from protective glasses is the restricted field of view of the welder. Furthermore the welder has no additional information, e.g. the actual parameters of the welding power supply. This is particularly of importance if the welding had to be done in far distance from the welding power supply.

During the welding process the requirements at the concentration and reliability of the welder are very high because of the insufficient optical information. Due to these missing optical information the creation of manual welds with constant high quality becomes very difficult.

To increase the manufacturing quality and economic efficiency a support system for the welder is required. This can be achieved by improving the visual information for the welder as well as by supplying additional information with methods of virtual and augmented reality. The described system is based on the approach for the optimization of PGMAW using visual online observation of the droplet transfer in combination with recording of electrical welding parameters described by Nordbruch, Tschirner and Gräser e.g. in [1], [2].

\section{VIRTUAL AND AUGMENTED REALITY}

Virtual reality is a new technology, which uses a computer model to generate and present an artificial environment to a person, evoking the impression of actually moving in this environment.

Augmented reality is a new form of human-machine interface, which inserts information via head-mounted displays in the users field of view. The insertion is context dependent, i.e. compatible to and derived from the observed object, e.g. the real field of view of a mechanic is extended by the insertion of instruction sheets. Apart from this the use of wearable computers opens new augmented reality application fields, in which high mobility as well as actual process values, measured or simulated data are required.

\section{DESCRIPTION OF THE SYSTEM}

The system combines a conventional welding helmet with modern technology. The system consists of

- a welding helmet combined with

- two High-Dynamic-Range-CMOS-cameras (HDRC) for observation of the welding scene

- a head-mounted display for visualization of the welding scene

- a wearable computer for image recording and image processing

- a computer for measuring electrical welding parameters and automatic calculation of characteristic welding process parameters

The basic set-up of the system is shown in figure 2 . 


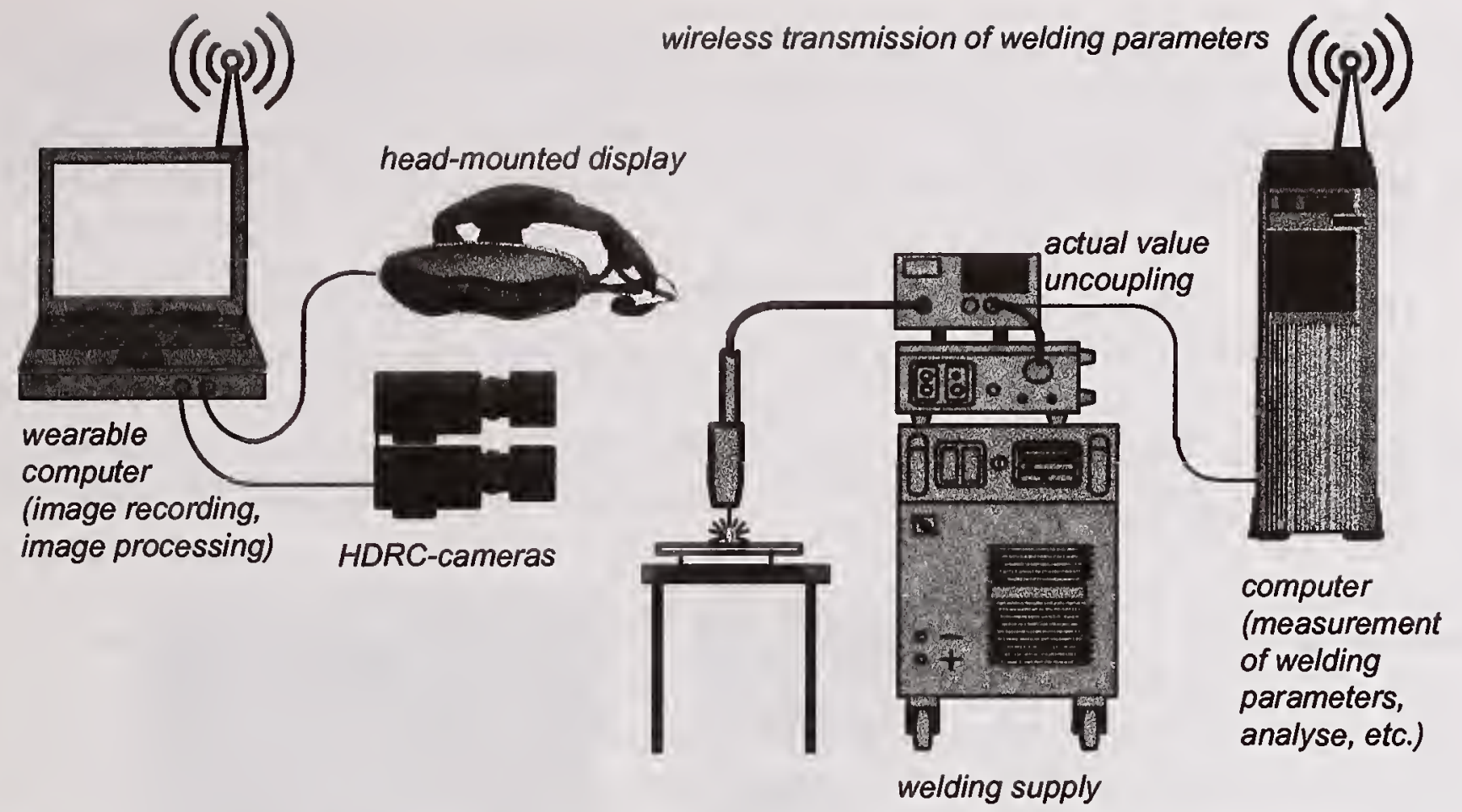

Figure 2: Principle sketch of the system, depicted without the welding protective mask

Figure 3: System

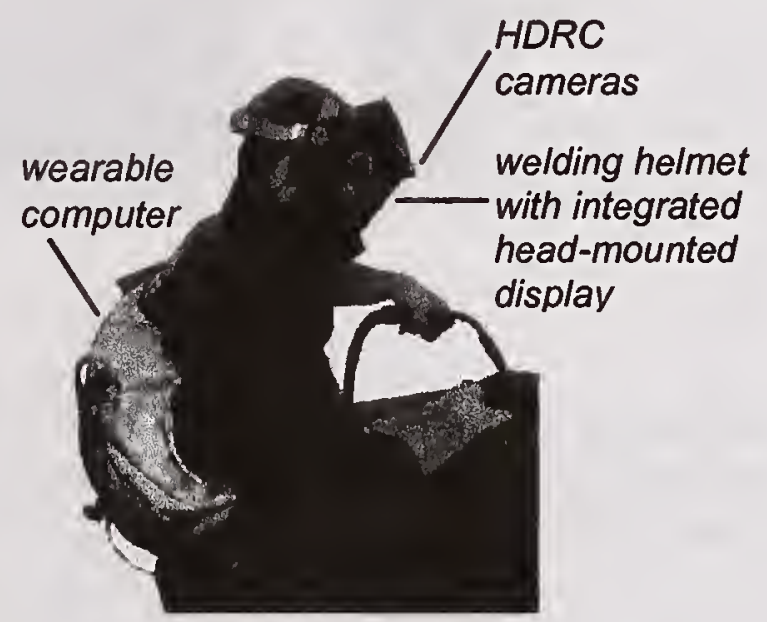

A welding helmet is combined with two HDRC cameras for the observation of the welding scene. A portable computer enhances the image quality and displays the images on the headmounted display, which is also integrated in the welding protective mask.

By methods of virtual and augmented reality, additional information can be inserted depending on the application. This information can be derived from the welding power supply (e.g. electrical welding parameters, wire feed), directly extracted from the images (dimensions of the arc) or transferred by another computer (constructional details, like work piece geometry).

\section{High-Dynamic-Range-CMOS Camera (HDRC)}

The principle of HDRC-cameras can be described as follows. The intensity levels in an image are essentially dependent on the irradiance and reflection properties of an object. The information of an image only depends on the contrasts resulting from varying reflections, and fewer on the absolute intensity values caused by irradiancy because the reflection property of a surface is independent of its irradiancy. 
A CCD-camera maps the absolute intensity values caused by the irradiance of an object. These light intensities can indicate a relation of up to $10^{6}: 1$, i.e. in high-energy processes as welding. Due to the technology the dynamic range of a $C C D$ camera is approximately $4000: 1$, which is not sufficient in order to represent the high light intensities of the welding process.

The image sensor of the HDRC camera consists of CMOS transistors. Before further processing, the input signal is compressed logarithmically in every cell of the image sensor. Due to this compression the HDRC camera maps the contrast caused by different reflections between two side-by-side object points and the information content of the image is not reduced. Thus the dynamic range of a HDRC camera is circa $10^{6}: 1$. This corresponds approximately to the light intensities of the welding process.

a.

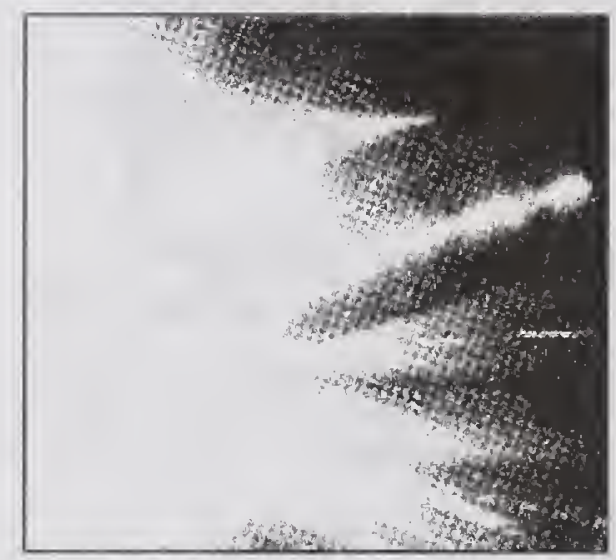

b.

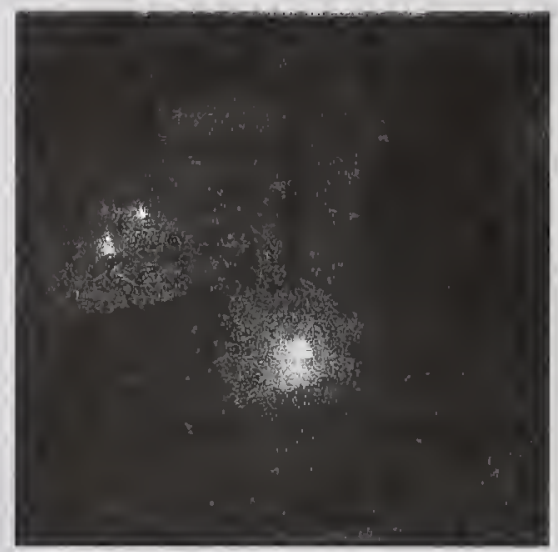

Figure 4: Welding scene recorded with a. CCD camera b. HDRC camera

\section{Signal Processing}

The signal-processing unit allows the measurement of specific parameters of the welding supply (e.g. welding current, welding voltage and wire feed) and the automatic calculation of typical characteristic process parameters from of the measured current and voltage signals [1], [2].

\section{Image processing}

The image processing unit allows the recording and further processing of the images. Additionally relevant welding parameters, e.g. geometry of the arc, can be automatically extracted from the image data.

Due to the extreme brightness conditions of the welding process and the characteristics of the camera the images are of low contrast. In order to give the welder a detailed view both on his environment and the welding process (e.g. melting of the electrode and the welding pool) a further processing of the images is necessary. The contrast has to be adapted to the different image areas, so that the resulting image contains all image areas with sufficient contrast. Standard image processing techniques cannot cope with the extreme brightness conditions and the characteristics of the camera. Objects in far distance the arc may differ only by few gray levels from the background. In these image areas a contrast enhancement is necessary. Objects near the are clearly visible against the background. In these areas a contrast enhancement can lead to a degradation of the image quality (e.g. by exceeding the range of the gray level images). Therefore the image processing procedure needs to be robust against varying image contents (e.g. brightness). An adaptive procedure has been developed [4], that 
"recognizes" the high-contrast areas which have to be maintained and the low-contrast areas which have to be improved. For contrast enhancement the procedure Contrast Enhancement Using the Laplacian-of-a-Gaussian Filter from Neycenssac [3] was adapted to the problem. In this procedure the enhanced image $g(x, y)$ is obtained by the convolution of the original image $f(x, y)$ with a LoG filter $\operatorname{LoG}(x, y)$, multiplication of the resulting image $g^{\prime}(x, y)$ by a contrast factor $\beta$ and subtraction from the original image $f(x, y)$ :

$$
g(x, y)=f(x, y)-\beta \cdot f(x, y) * \operatorname{LoG}(x, y)
$$

For the implementation of an adaptive contrast enhancement the procedure was modified by adapting the contrast factor $\beta$ to the image content. To generate a priority function for $\beta$ a gradient operator is applied to the original image in order to determine the contrast of the image areas. The resulting gradient image $f_{S o b}(x, y)$ is low-pass filtered to create a continuous transition between the areas. Within high-contrast areas, the low-pass-filtered gradient image $f_{\text {Sob,TP }}(x, y)$ contains high values, and $\beta$ must be small in order to maintain the contrast. Within low-contrast areas, the low-pass-filtered gradient image $f_{S o b, T P}(x, y)$ contains low values, and $\beta$ must be high in order to enhance the contrast. The following relationship for $\beta$ was experimentally determined:

$$
\beta=200-40 \cdot \ln \left[f_{\text {Sob,TP }}(x, y)\right]
$$

Figure 5 shows the concept of the procedure. An example for a result of image processing with uniform contrast in all image areas is shown in figure 6.

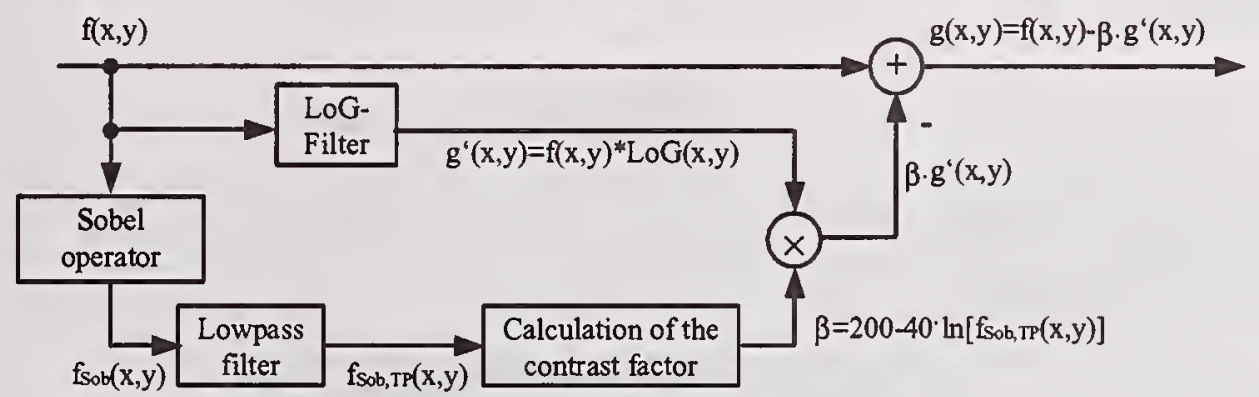

Figure 5: Flow of the adaptive contrast enhancement procedure

a.

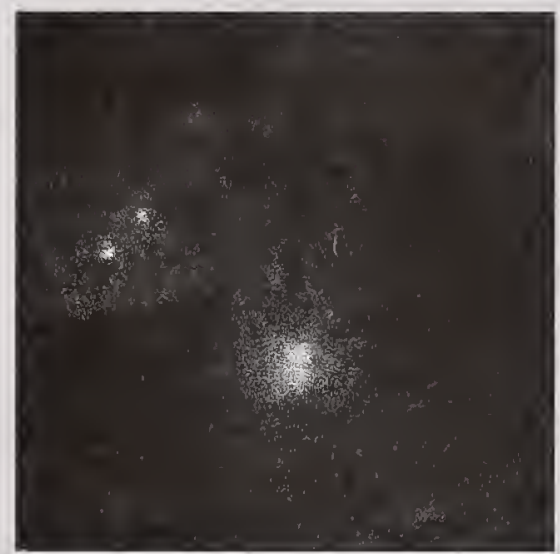

b.

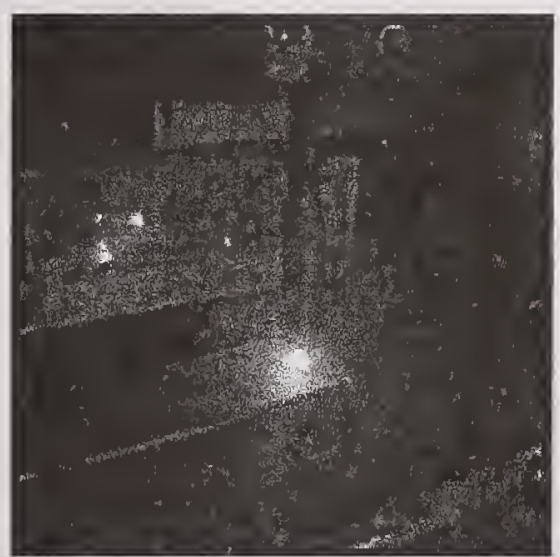

Figure 6: a. Original image; $b$. Result of adaptive contrast enhancement 


\section{POSSIBLE APPLICATIONS}

In the following the possible areas of application of the system are presented. They represent the current status of the requirement specification for this research project and were determined in cooperation with experienced welders.

\section{Welding preparation}

The first application possibility is the use during welding preparation. Constructional details can be displayed, e.g. the material type, desired values of the welding power supply or the desired welding seam.

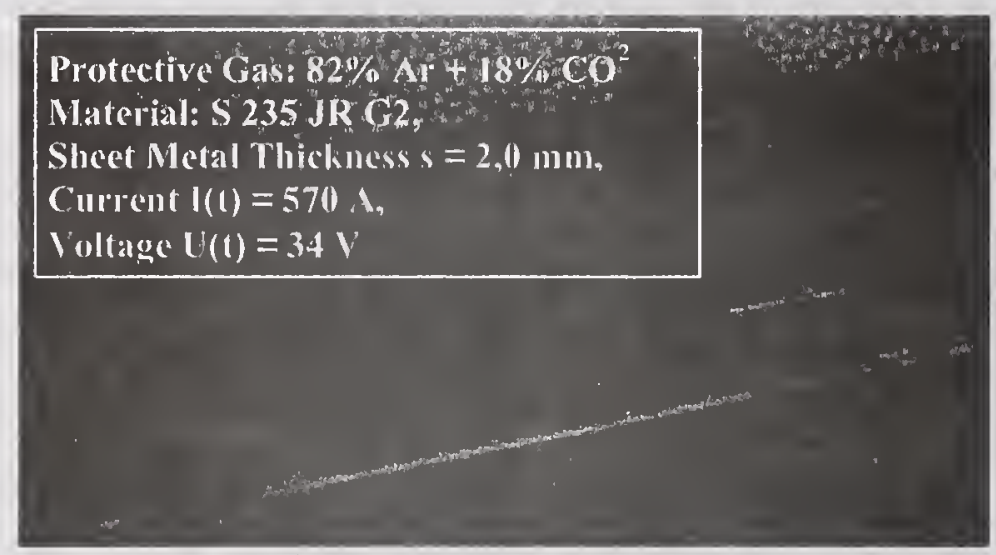

Figure 7: Possible insertions: welding seam and welding parameters

\section{Welding Process}

During the welding process an online quality control is possible utilizing the parameters extracted from the image data, e.g. the measurement of the welding seam geometry. This also allows an online adaptation of the welding parameters when welding long seams with changes in the work piece geometry.

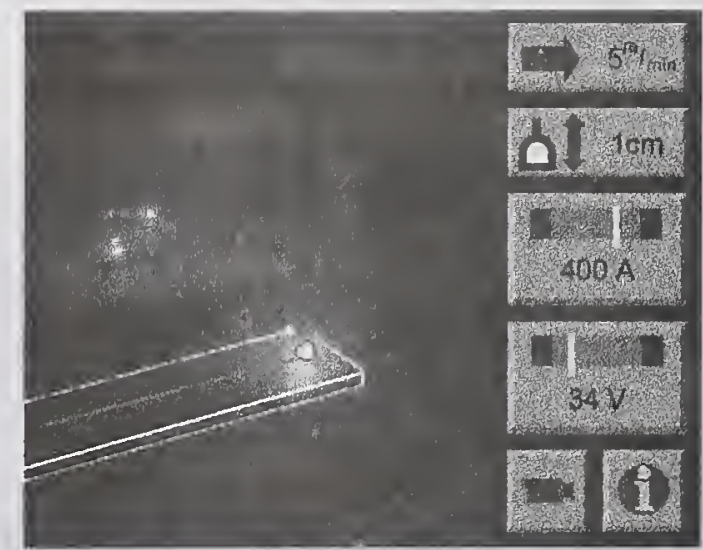

Figure 8: Possible insertions: work piece geometry, welding seam, splashes, wire feed, height of the arc, welding current and welding voltage

\section{Quality control}

The system can also be used for quality control. At present this is done via radiometric examination after the welding process, which is very complex and expensive. Additionally this method does not allow any conclusions on occurrences during the welding process. 
With the new system during safety-relevant welds the images can be stored together with relevant welding parameters, which can be easily assigned to each individual picture [1], [2]. The example shows splashes caused by a to high welding current.

a.

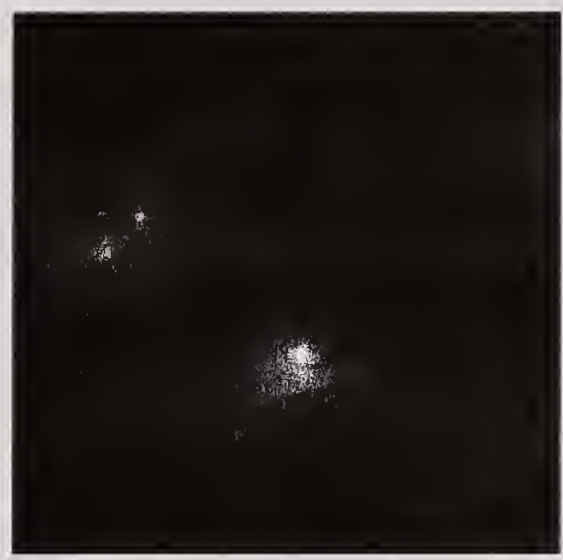

b.

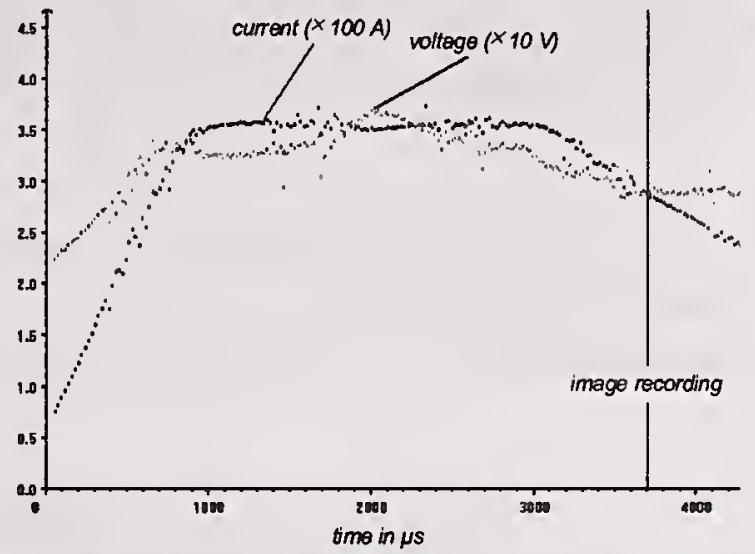

Figure 9: a. Image of the welding process; b. Synchronized and simultaneously measured electrical welding parameters current and voltage including the time of the image recording

\section{Education}

A further possible application is the use in education of welders. By using of a second headmounted display, teacher and trainee can observe the same welding process. The influence of changing welding parameters on the weld quality can be directly examined.

\section{SUMMARY}

The system described in this paper combines a conventional welding helmet with a headmounted display and two HDRC cameras. The welding scene is observed with the cameras. A portable computer provides further enhancement of the image quality and displays the images in real time in the head-mounted display. The welder has a detailed view on the welding pool, welding seam and his environment. By methods of virtual and augmented reality additional information (e.g. welding parameters or constructional details) can be inserted depending upon the application. The system can be used for getting information about the welding task before the beginning of the welding process, for the observation of the welding process including online quality control, for quality control after the welding process and for education.

\section{CONCLUSION}

By the visual observation of the welding process in combination with the representation of relevant welding parameters the welder receives a completely new status of information and is able to do his work much more precisely. Thus the simplification of manual welding and an improvement of the welding result are achieved, leading to a better and more constant quality and therefore to a reduction of the inspection expenditure in quality control 


\section{ACKNOWLEDGEMENT}

The presented research project is supported by the German Federal Ministry for Education and Research $(\mathrm{bmb}+\mathrm{f})$ under 01IRA07A. This research project is executed in cooperation with

- research institutes: Institute of Automation, University of Bremen, Germany; Institute of Industrial Engineering and Ergonomics, RWTH Aachen, Germany

- a shipbuilder: Lürssen Yachts - Super Yachts, Sail and Motor Boats, Bremen, Germany

- a manufacturer of welding supplies and devices: EWM HIGHTEC Welding GmbH, Mündersbach, Germany

- a manufacturer of autodarkening welding masks: Optrel AG, Wattwil, Switzerland

\section{REFERENCES}

1. Nordbruch, S.; Tschirner, P.; Gräser, A. 2000. Visual Online Monitoring of PGMAW without a Lighting Unit. International Sheet Metal Welding Conference IX, 18.-20. October 2000, Detroit, Michigan, USA. Paper Nr. 4-4

2. Nordbruch, S.; Tschirner, P.; Gräser, A. 2000. Visual Online Optimization of Pulse Gas Metal Arc Welding with a HDRC-Camera without a Lighting Unit. UKACC International Conference Control 2000. 4.-7. September 2000, University of Cambridge, UK. Paper Nr. 190

3. Neycenssac, F.; 1993. Contrast Enhancement Using the Laplacian-of-a-GaussianFilter. Computer Vision, Graphics, and Image Processing, No. 55, pp. 447-463

4. Tschirner, P.. 1999. Visuelle Analyse von HDRC-Kamerabildern des Werkstoffübergangs des MSG-Schweißprozesses. Diploma Thesis, Institute of Automation, University of Bremen, Germany 


\title{
A9 Mission, Current Activities and
}

\section{What Should We Do Next?}

\author{
Bill Rippey, A9 Chairman
}

- A9 Charter

- AWS Vision

- A9 Status

- Some examples from other industries

- Shipbuilding

- Electronics Manufacturing

- Machining

- Discrete parts inspection

- Discussion - where does A9 go from here?

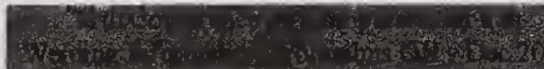


- A9 currently has a small number of projects in the committee.

- Other manufacturing industries have extensive efforts underway in digital information specifications - should we follow their examples?

- A9 wants your input on needs, and your participation if something matters to you!

- Issues involving information manipulated by computers

- Original projects related to database software used for WPSs, PQRs, WQRs.

- Scope could include CAD product and weldment design, CAM process planning and programming, automated weld cell control, inspection data, equipment control integration. 
"Major Challenges"

- "Welding will be better integrated into the production cycle"

- Vision (for 2010)

- "drivers" will include "... use of information technology .... in which technologies for design, fabrication, and inspection are seamlessly integrated with welding technology where they are needed."

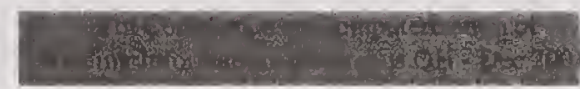

- "Strategic Goals

- Cost/productivity/markets/applications reduce the average cost of welding by one-third, by providing better process selection guidance, increasing the use of automation and robots, and lowering reject and repair rates; increase the use of welding by $25 \%$." 
"Process technology

- Enhance the use of welding in manufacturing and construction operations by integrating welding with other manufacturing and construction disciplines, at the engineering level and also at the operational level."

- "Developments in Information Technology

- Welding processes based on rigorous engineering analysis, numerical modeling, and computer-based automated manufacturing will be widely used, and will depend heavily on information technology." 


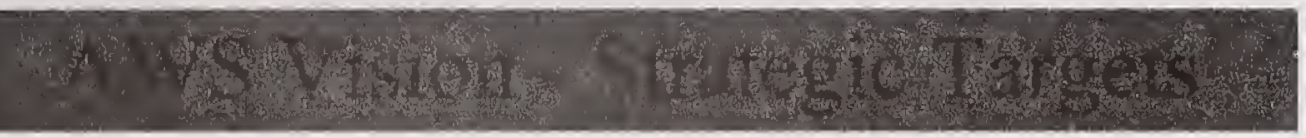

- "Integrate welding within entire factory..... upstream and downstream"

- "Move to an 'open architecture' and automation in welding technology. Make $80-90 \%$ of equipment compatible (plug and play) by developing an industry standard"

- "Among the techniques foreseen are integration of design, sensing, and process control, and development of modular processing equipment to ensure compatibility of various systems."

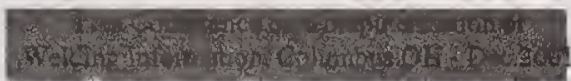

- A9A Subcommittee hosts this conference

- A9.1 - Data Dictionary

- A9.3 - Activity Model

- A9.4 - Network Communications for IntraCell Components

- Past submissions of CAD<->OLP, OLP<->Robot, Interface to Weld Procedure Database. 


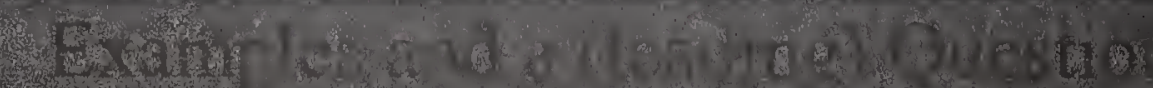

- Other industries have begun efforts in integration of computer information

- Is the welding industry part of some of these industries (e.g. shipbuilding)?

- Question: is the welding industry enough like these that we should make similar efforts?

- STEP AP 215 - Ship Arrangement

- STEP AP 216 - Ship Moulded Forms

- STEP AP 218 - Structural Systems Data for ship predesign, design, production, and inspection/survey

- STEP AP 226 - Ship Mechanical Systems

- STEP AP 212 - Electrical Design and Installation

- STEP AP 227 - Piping

- Currently no STEP AP for welding. 
- IPC - Association Connecting Electronics Industries

- NEMI - National Electronics Manufacturing Initiative

- SEMI - Semiconductor Equipment and Materials International

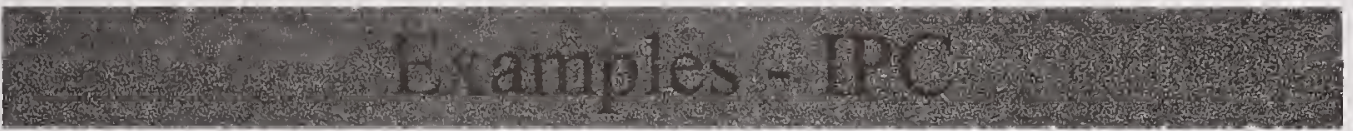

- CAMX standards Computer Aided Manufacturing using the eXtensible Mark-up Language (XML).

- provides for factory floor communication.

- standardizes the language used by assembly placement and test equipment,

- reduce the amount of time and cost that it takes to integrate a new piece of electronics assembly equipment into a shop floor environment and start collecting data and controlling that equipment.

- improved interoperability, ie. "plug and play" capability between equipment also allows manufacturers to use the best piece of equipment for the job, regardless of vendor.

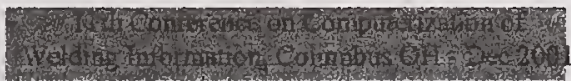


- IPC-2510 Product Data (GenCAM)

- IPC-2520 Product Data Quality

- The IPC-2524 - facilitates grading the quality of product data packages delivered to a printed wiring board fabricator. It defines a checklist of information required in a complete data package.

- IPC-2530 Process Data (Standard Recipe File Format)

- The standard recipe file format (SRFF) defined in the IPC-2531 was created to standardize the instruction file format for controlling assembly equipment.

- IPC-2540 Shop Floor Communication

- vendor-independent plug and play environment for electronics assembly, inspection, and test equipment.

- quickly integrate new pieces of electronics assembly equipment into a shop floor line management system and how to manage the vast amounts of data available in today's electronics manufacturing environment.

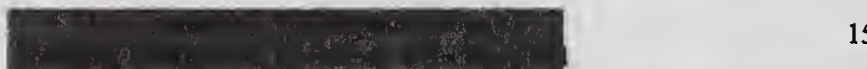

- IPC-2550 Execution System Communication communication protocols and information requirements for Manufacturing Execution Systems (MES) within a factory.

- IPC-2560 Enterprise Communication - enterprise resource planning system standards.

- IPC-2570 Supply Chain Communication - supply chain messages that are passed between trading partners, including requests for quotes, engineering change orders, and product genealogy reports. 
- VFIIP builds on the work of NEMI's Plug \& Play Factory Project, which was completed in December 1999.

- While Plug \& Play focused on interoperability on the factory floor, Virtual Factory

- extends interoperability from within an enterprise to information systems distributed across business partners.

- is addressing the bidirectional exchange of information between OEMs and EMS providers for board and final assembly, centering on the exchange of technical engineering and manufacturing data.

- SEMI E4-0699 - SEMI Equipment Communications Standard 1 Message Transfer (SECS-I)

- SEMI E5-0701 - SEMI Equipment Communications Standard 2 Message Content (SECS-II)

- SEMI E54-0997 - Sensor/Actuator Network Standard 


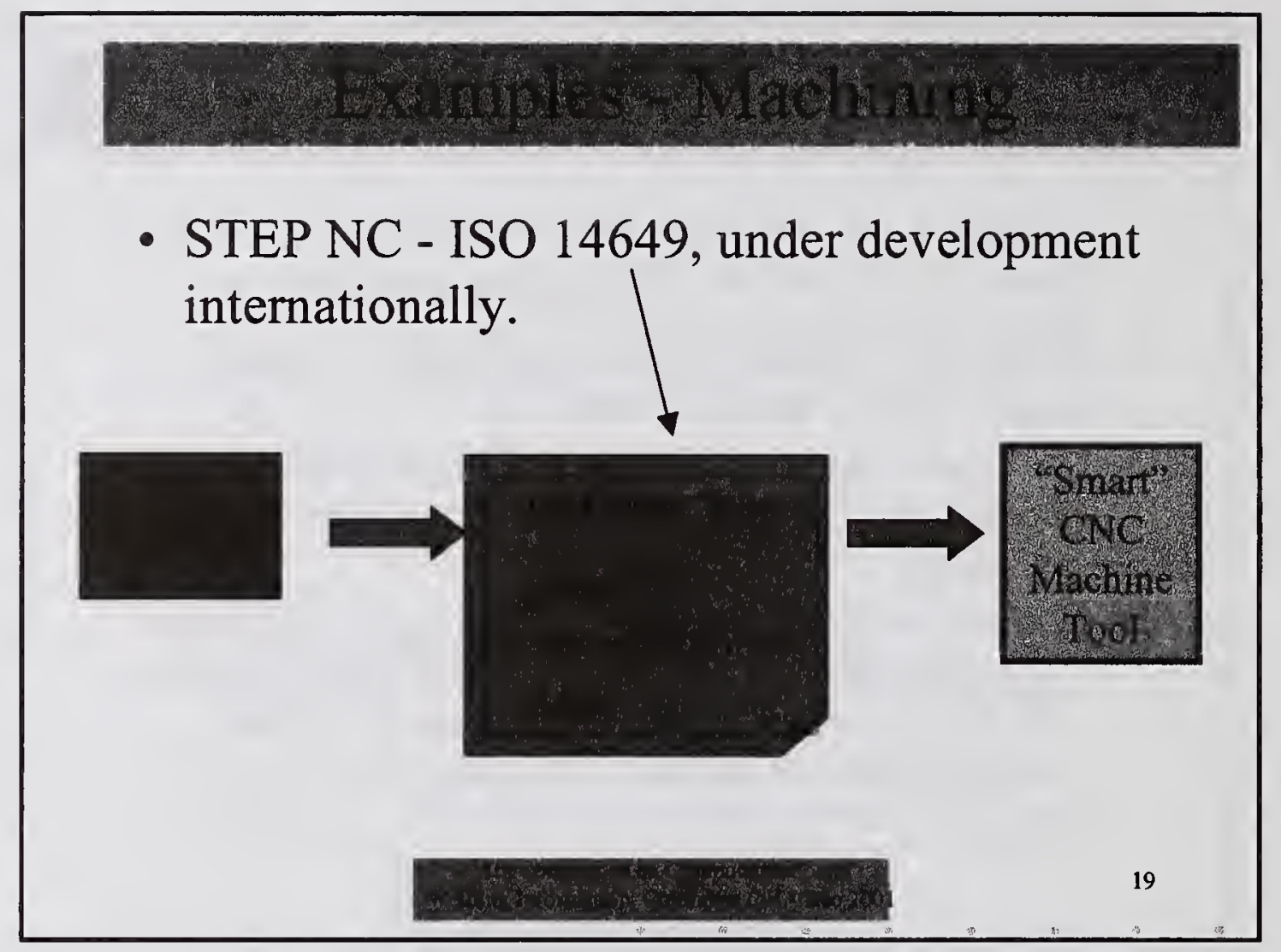

- Metrology Interoperability Consortium scope includes computerized data describing part design, inspection plans and programs, and data gathering and analysis.

- DMIS - machine-independent language for programming inspection (e.g. by CMM) 

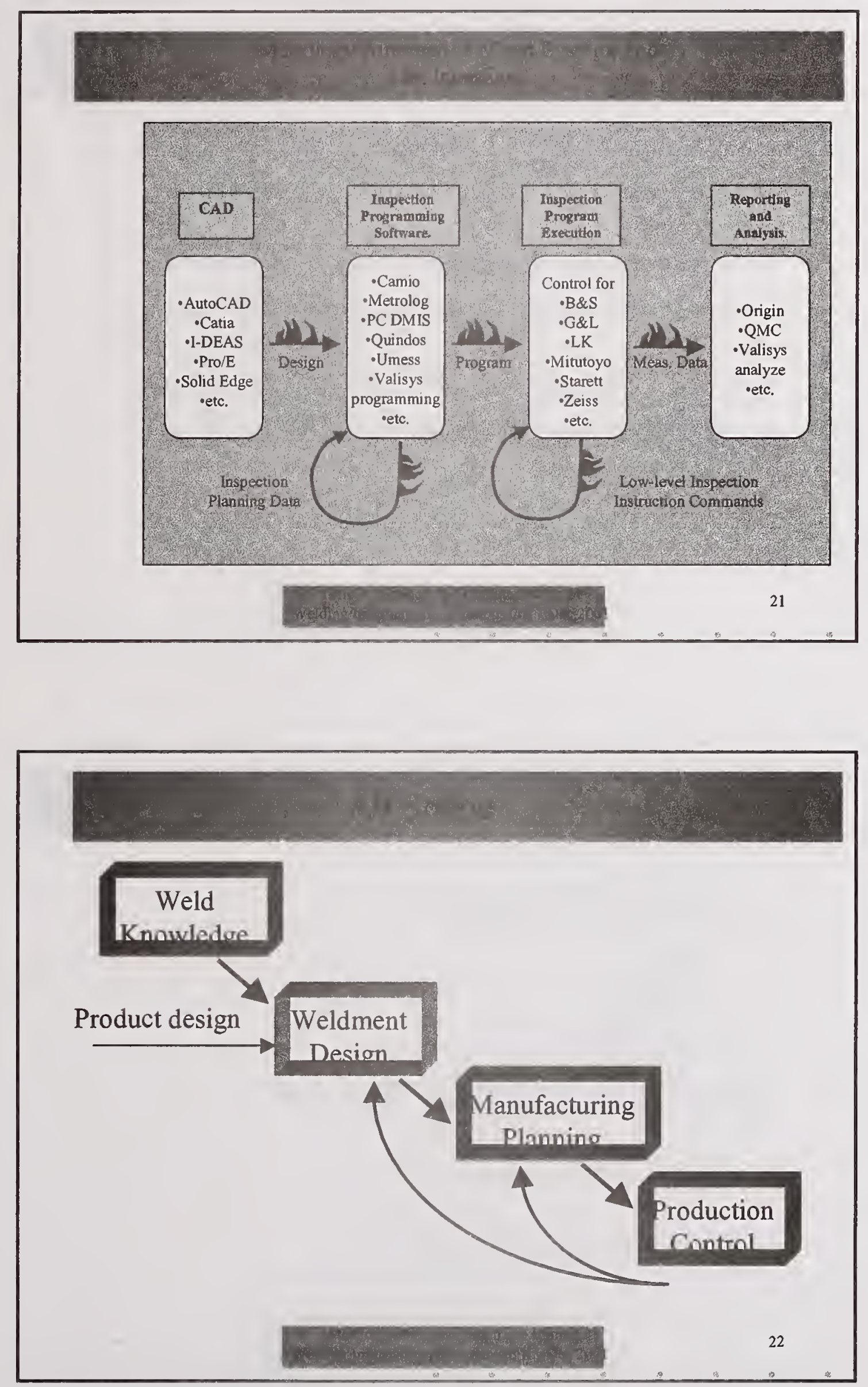
- Other industries have begun efforts in integration of computer information

- Is the welding industry part of some of these industries (e.g. shipbuilding)?

- Is the welding industry enough like these that we should make similar efforts? Is there a need?

- What are the needs of

- users

- vendors

- integrators?

- Should A9 Do More? Do Less?

- Who Needs What?

- Who will contribute? 
- Chairman, Bill Rippey

- NIST, 301-975-3417

- william.rippey@nist.gov

- AWS Secretary, Len Connor

- 800-443-9353 x302

- 1connor@aws.org

- Next A9 meeting, January/February 2002, Florida 


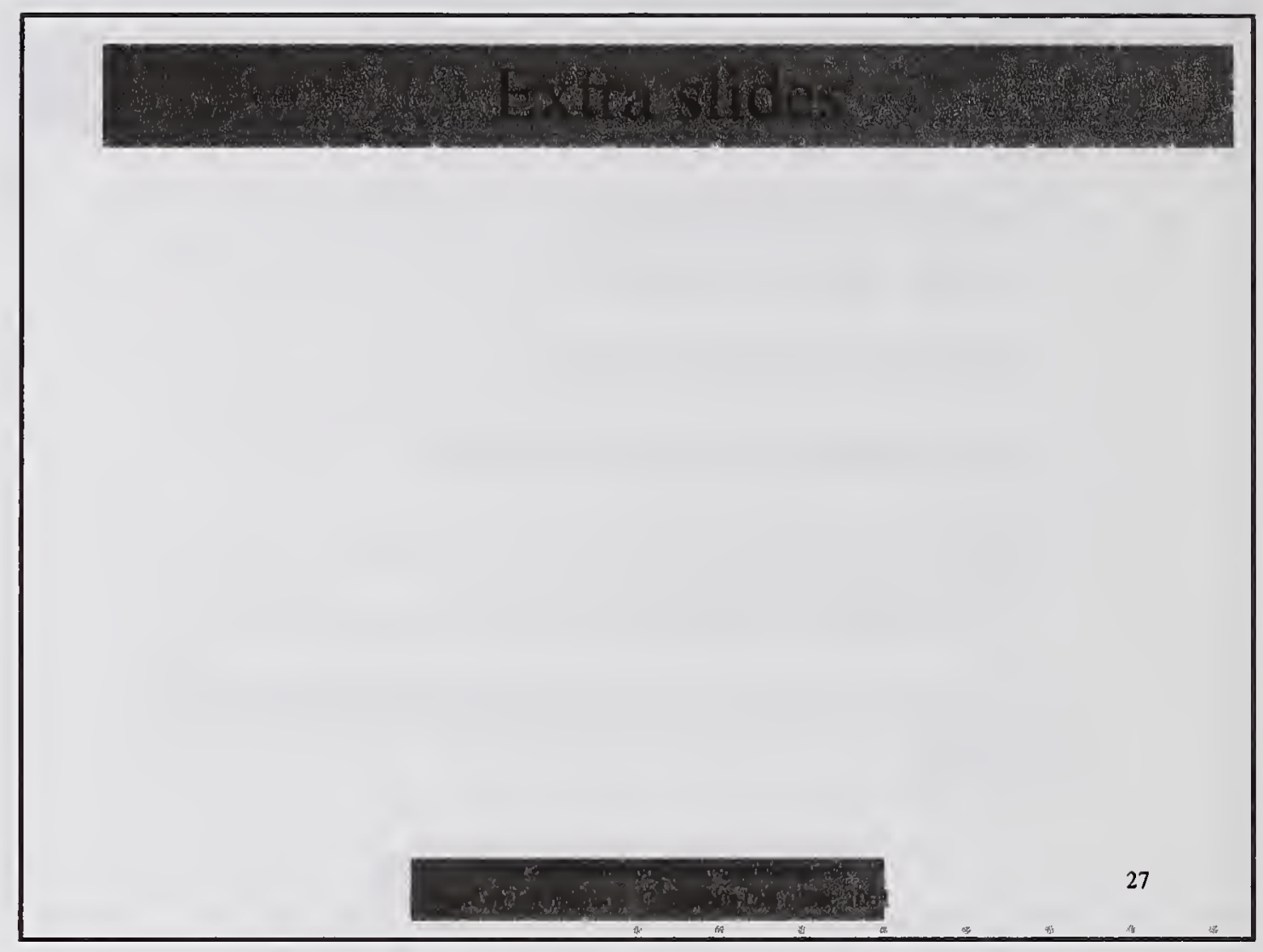

- Capture/record/exchange inspection results, manual and automated.

- Shop floor communications for better \& easier integration?

- Weldment design data?

- Enterprise level data flow?

- ??? 


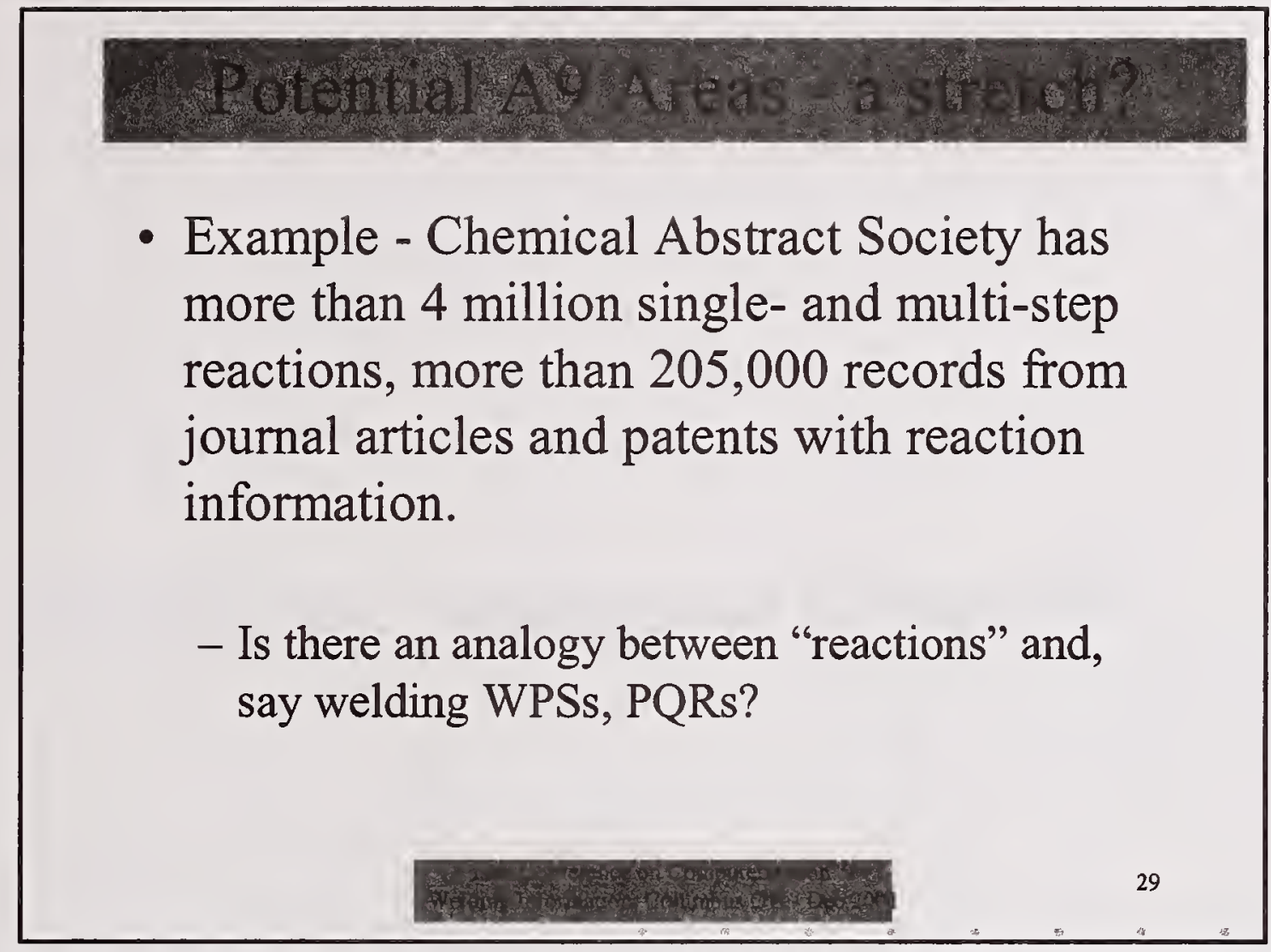



Session A6: Sensing and Control IV 



\title{
ON-LINE WELD QUALITY DIAGNOSTICS IN FRICTION STIR WELDING
}

\author{
D. Jandric, J.H Ouyang, M. Valant and R. Kovacevic ${ }^{\text {* }}$
}

\begin{abstract}
The temperature distribution on the plate surface in front of the shoulder and around the pin very close to the workpiece surface is analyzed in this paper. Consideration is given to the influence of the welding parameters, the traverse speed and the rotational speed on the temperature distribution and their effect on weld quality. Infrared sensing is applied in order to collect relevant data for this analysis.
\end{abstract}

\section{KEYWORDS}

Infrared Sensing, Surface Temperature Distribution, Welding Parameters and Weld Quality

\section{INTRODUCTION}

Friction Stir Welding (FSW), invented and patented by The Welding Institute, Cambridge, England, in 1991, has recently captured the attention of the welding community for fabricating high-quality joints of aluminum alloys. This is an energy efficient, environment friendly and versatile process. Friction Stir Welding is a very attractive technology because of its simplicity and applicability to a wide range of alloys. It offers a variety of new design and production welding opportunities and should be of particular interest to the shipbuilding, aerospace, and automotive industries.

The basic principle of FSW is relatively simple. A rotating pin is inserted in the material to be joined and traversed through the joint line while the shoulder is in contact with the top surface of

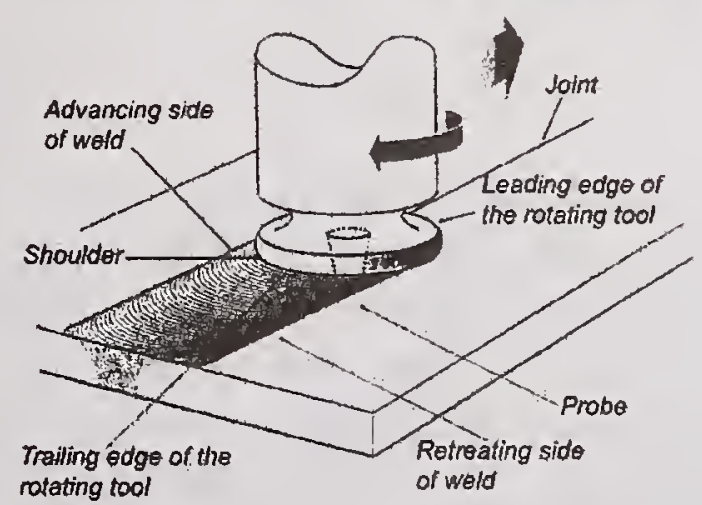

Figure 1. Friction Stir Welding Principle the workpiece. Rotary motion generates heat, which creates a softened and plasticized region around the pin and contacting surface. A weld joint is produced by extrusion of the plasticized material from the leading side to the trailing side of the tool, Fig.1. (Ref. 1). Welds with higher quality and finer microstructure than the parent material can be achieved by FSW.

Recently, several studies of the relationship between the welding parameters and the temperature distribution in the friction stir welded joints have been reported. The

Research Center for Advanced Manufacturing

Southern Methodist university, 1500 International Pkwy. Suite \# 100, Richardson, TX 75081

"Corresponding author - tel.: 214-768-4865. Email: kovacevi@engr.smu.edu 
temperature around the pin was measured by thermocouples embedded into the workpiece in order to get the temperature history during the welding process. Simultaneous sensing is performed by an infrared camera and by force/torque sensors.

Sensing the surface temperature in front of the tool using an infrared camera will provide conditions for controlling the welding parameters during the friction stir welding process. Very little has been done in FSW for process monitoring with an infrared camera, but based on research results from other welding techniques, infrared sensing can be successfully applied to FSW. This process monitoring technique is used in the analysis of the surface temperature distribution during the laser beam welding process, (Ref. 2). In Gas Tungsten Arc Welding, infrared process monitoring represents the basis for weld quality control during the welding. Variations in the welding process parameters such as bead width, penetration depth and torch position produce an infrared radiation response, (Ref. 3). In general, the infrared thermal imaging system is a valuable tool that may be used to pinpoint problem areas and to avoid costly delays.

Monitoring the pin tool depth during the FSW process is one of the most difficult tasks, but it is possible using the force/torque sensors, (Ref. 4). The relationship between the axial load and the pin tool depth during the welding was observed to be constant for all welds made with the same tool pin geometry and the pin tool depth. By controlling the axial load, the pin tool depth is also controlled. NASA has introduced (Ref. 5) a new tool known as Retractable Pin-Tool. This tool will assist in developing the closed-looped force control system capable for performing defect free welding.

The microstructure of the joint made by the FSW technique has been the subject of several investigations (Ref. 6). The extrusion and forging operation, coupled with the tool rotation, are collectively responsible for the characteristic microstructure of friction stir welded joints. Microstructural analyses gave information about the weld quality and also provided proof that FSW is a solid state process.

The present paper focuses on the effects of the welding parameters, rotational speed and traverse speed on weld quality. It is shown that infrared sensing is feasible technique for nondestructive monitoring of the FSW process.

\section{OUTLINE OF STUDY AND METHODS}

\section{Experimental set-up}

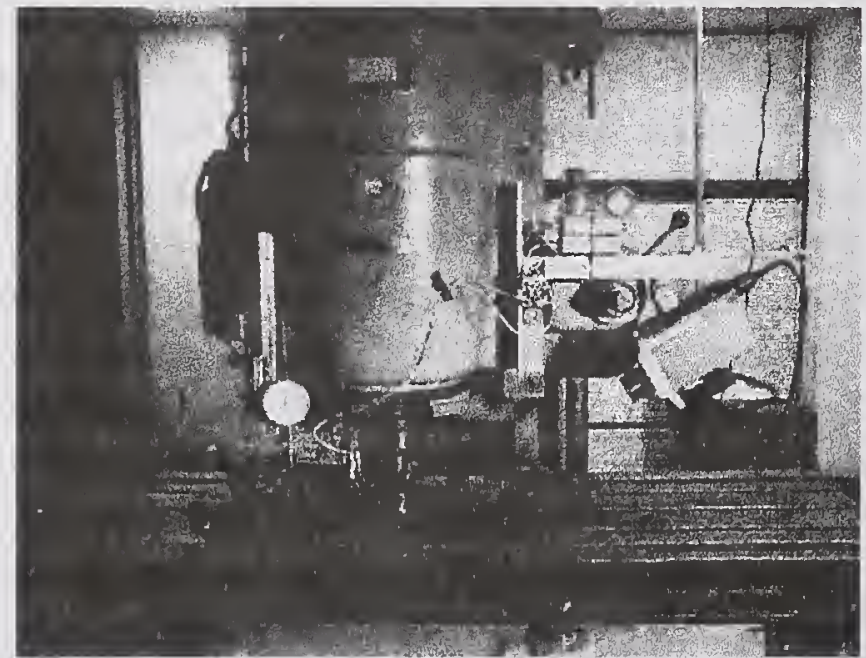

Figure 2. Experimental Set-Up
The experimental set-up consists of a powerful vertical-milling machine equipped with in-house-designed and built tooling and fixturing. The tool consists of a shoulder and a pin with the ratio of their diameters of $3: 1$. The workpiece is clamped onto a backing bar and secured against vertical, longitudinal and lateral movements. In order to measure peak temperatures during the welding, thermocouples placed at different distances from the pin center are used. An infrared camera mounted on an extension arm 
records the infrared radiation in front of the shoulder on the plate surface, Fig.2. Additional software is developed for storing and processing recorded data.

\section{Experimental procedure}

A number of FSW experiments are carried out to obtain the optimum parameters by adjusting the rotational and traverse speeds. Experiments are performed on 6061-T6 aluminum plates, $280 \times 200 \times 12.7 \mathrm{~mm}$ in size. Temperatures during the Friction Stir Welding process were measured by $\mathrm{K}$ type $\mathrm{NiA} / \mathrm{NiCr}$ thermocouples placed in a series of small holes equidistant from the specimen's surface. It was found that the existence of these holes does not significantly affect the temperature field during the welding process, (Ref. 7). Peak temperatures were measured for five different rotational tool speeds $(151 \mathrm{rpm}, 344 \mathrm{rpm}, 416 \mathrm{rpm}, 637 \mathrm{rpm}$ and 914 $\mathrm{rpm}$ ) and five different welding speeds (linear workpiece speeds, $95 \mathrm{~mm} / \mathrm{min}, 140 \mathrm{~mm} / \mathrm{min}, 190$ $\mathrm{mm} / \mathrm{min}, 229 \mathrm{~mm} / \mathrm{min}$ and $330 \mathrm{~mm} / \mathrm{min}$ ). The temperatures were recorded digitally using the embedded thermocouples and the data acquisition system.

In the effort to understand the process parameter/joint quality relationship, surface temperatures near the edge of shoulder, at the centerline of the nugget zone, are recorded by an infrared camera. The recorded infrared data is processed to obtain the temperature distribution on the top side of the workpiece in front of the tool shoulder. A frame grabber captures images, and image processing is performed using LabView and Matlab programming. The stored images are digitized into $640 \times 480$ matrices which are composed of gray level values from the infrared image.

The cross-sections of the weld beads are etched by a modified Keller's etchant. Microstructural analyses are performed using a high-resolution microscope.

\section{Results and discussion}

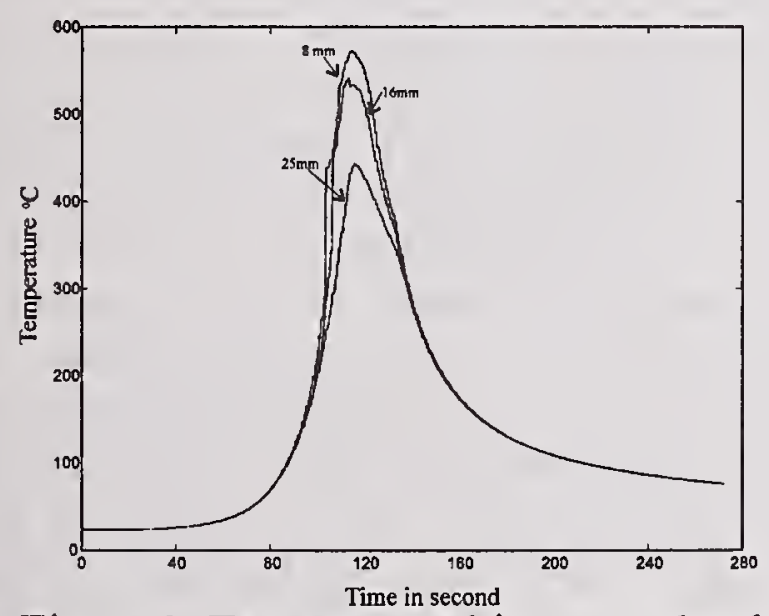

Figure 3. Temperature history under the shoulder for the weld made at $637 \mathrm{rpm}$, $95 \mathrm{~mm} / \mathrm{min}$

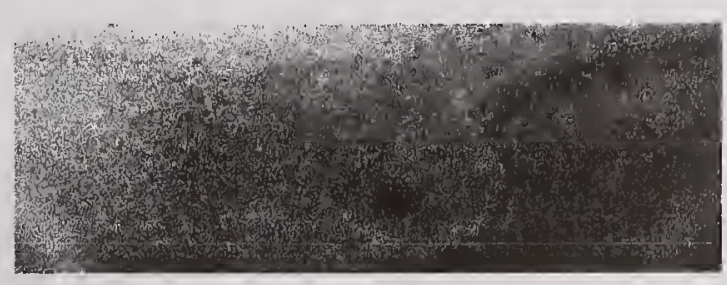

Figure 4. Typical microstructure of the weld joint
As a solid state process, FSW operates below the melting temperature, which is $585^{\circ} \mathrm{C}$ for $\mathrm{Al} 6061$ T6, (Ref. 8). Typical welding temperature histories for different distances from the pin center $(8 \mathrm{~mm}$, $16 \mathrm{~mm}, 25 \mathrm{~mm}$ ) and at the same depth from the top surface $(0.5 \mathrm{~mm})$ measured by thermocouples are shown in Fig. 3. It is shown that the welding temperature at $0.5 \mathrm{~mm}$ from the top surface is about $570{ }^{\circ} \mathrm{C}$, which is still below the melting temperature. Microstructural analysis shows that there is no melting in the body of the weld during the FSW process, Fig. 4. (Ref. 9). The weld is characterized by a fine-grained, hot-worked condition with no entrapped oxides or porosity.

The temperature distribution in front of the shoulder on the plate surface is obtained by recording with the infrared camera and correlating gray levels from isothermal images with the temperatures measured by the thermocouples. Matching the time recorded by the infrared camera 
with the time when thermocouples registered the temperature peak, the thermocouples' positions are found on the isothermal images. A typical result is shown in Fig.5. The measured temperatures are not the real surface temperatures but they can be treated as such because the thermocouples are placed very close to the plate surface.

The temperature of $410{ }^{\circ} \mathrm{C}$, which is about $75 \%$ of the peak temperature, was measured $25 \mathrm{~mm}$ from the center of the pin. This corresponds to a gray level of 220 . The surface temperature in front of the shoulder, being $70-80 \%$ of the peak temperature, as measured by thermocouples very close to the nugget, could be a good indicator of the process, measured by an infrared camera placed in front of the tool. Optimization of the welding parameters relative to the gray level intensity in the thermal images gives a basis for on-line control of the FSW process.

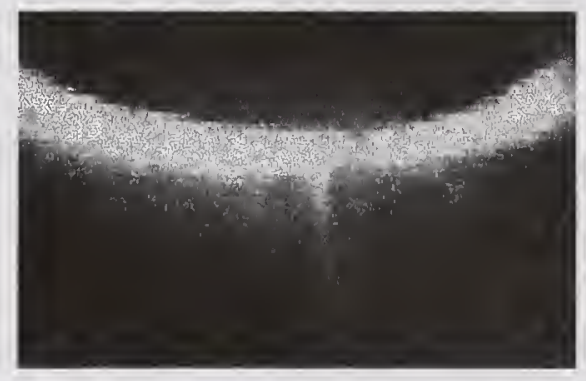

Unprocessed image

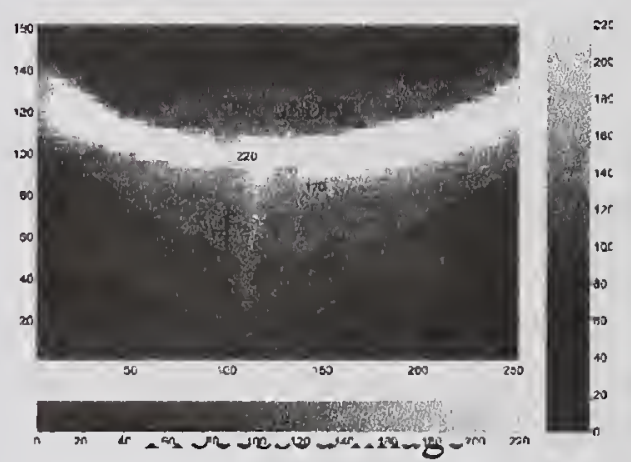

Figure 5. Temperature distribution in front of the tool shoulder

\section{Influence of welding speed on temperature distribution}

Welding speed, one of the most important parameters in the FSW process, affects temperature distribution on the plate surface in front of the tool shoulder. The temperature as a function of the distance from the shoulder is shown in Fig. 6. This temperature is obtained by processing the original images recorded by an infrared camera. It follows that the temperature in front of the shoulder will decrease if the welding speed increases for a constant value of heat input. In a real welding situation this eventually leads to pin fracture and the formation of weld defects as the

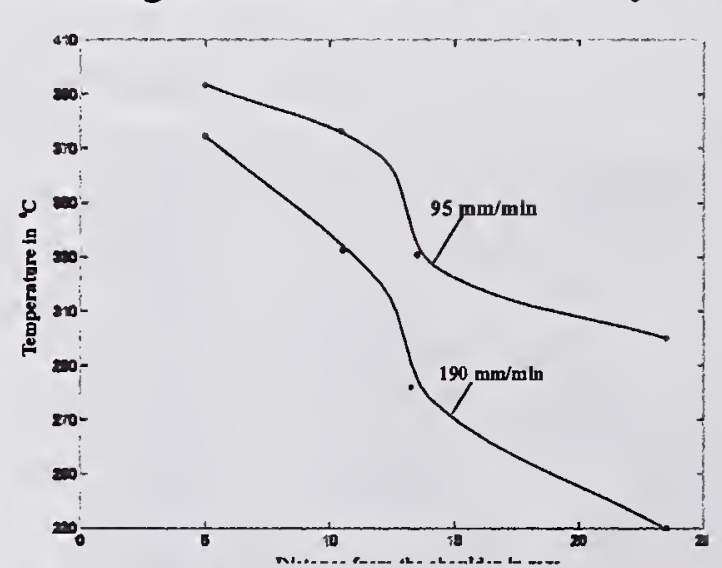

Figure 6. Temperature in function of distance from the shoulder material becomes less formable, (Ref. 10). On the other hand, the temperature on the shoulder edge is measured by thermocouples as well. It can be shown that for welding speed of $95 \mathrm{~mm} / \mathrm{min}$ temperature around the shoulder is $405^{\circ} \mathrm{C}$ and for welding speed of $190 \mathrm{~mm} / \mathrm{min}$ that temperature is $382{ }^{\circ} \mathrm{C}$. When comparing the temperature results from thermocouples with results obtained from thermal images on the shoulder edge and by extrapolating the curve, see Fig. 6 , small difference in temperature on the shoulder edge can be expected because of the $0.5 \mathrm{~mm}$ depth from the top surface where the thermocouples are placed.

\section{Influence of rotational speed on the temperature distribution}




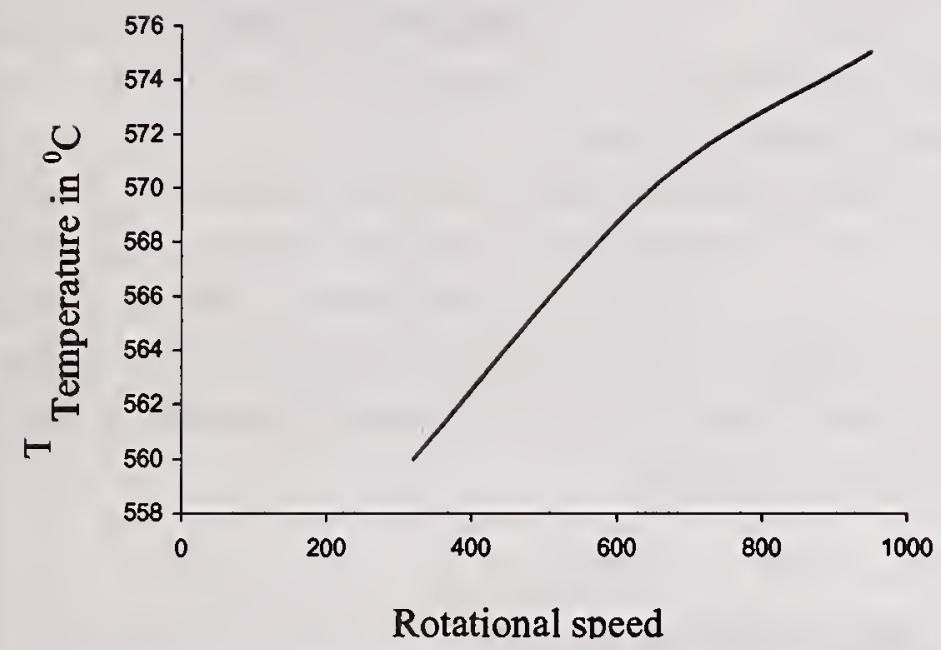

Figure 7. Temperature in function of rotational tool speed under the shoulder
In the welding process, heat may come from friction between the workpiece and the tool as well as from the plastic deformation of the workpiece. The rotational speed of the pin tool may change each of these. The influence of the pin tool rotational speed on the temperature field under the shoulder is shown on the Fig.7. It is clear that the rotational speed has a modest effect on the peak temperature measured by the thermocouples, (Ref. 7). The temperature is increased only $15{ }^{\circ} \mathrm{C}$ when the rotational speed increase from $314 \mathrm{rpm}$ to $914 \mathrm{rpm}$. With the higher rotational speed the temperature should increase, but increased

temperature reduces both metal flow stress and input torque, which limits the power generation increase. Because the temperature change is very small with a relatively large change in rotational speeds, in this paper the rotational speeds' influence on surface temperature in front of the shoulder is not analyzed.

\section{Determination of cooling rate}

As mentioned before, by processing the original images, the temperature distributions in front of the shoulder can be obtained in the form of contour plots. Using a LabView program it can be shown that, close to the shoulder, temperature distribution is nearly linear with the distance from the shoulder, so in this paper that assumption will be used, Fig. 8. The cooling rate can be calculated using contour plots and known welding parameters. The distance between two isotherms (s), shown in Fig.9, and welding speeds (v) are given, so the traveling time between two isotherms can be calculated as $\mathrm{t}=\mathrm{s} / \mathrm{v}$.

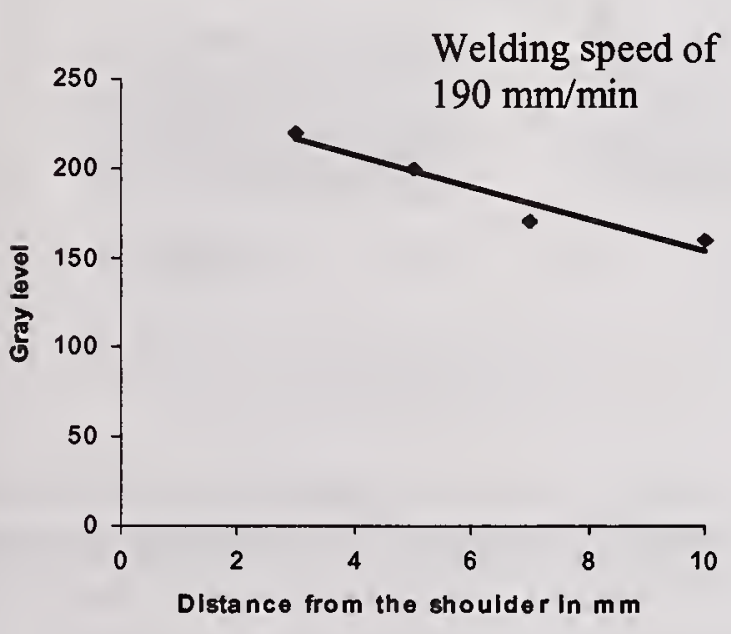

Figure 8. Temperature distribution with respect to the measuring point from the tool shoulder

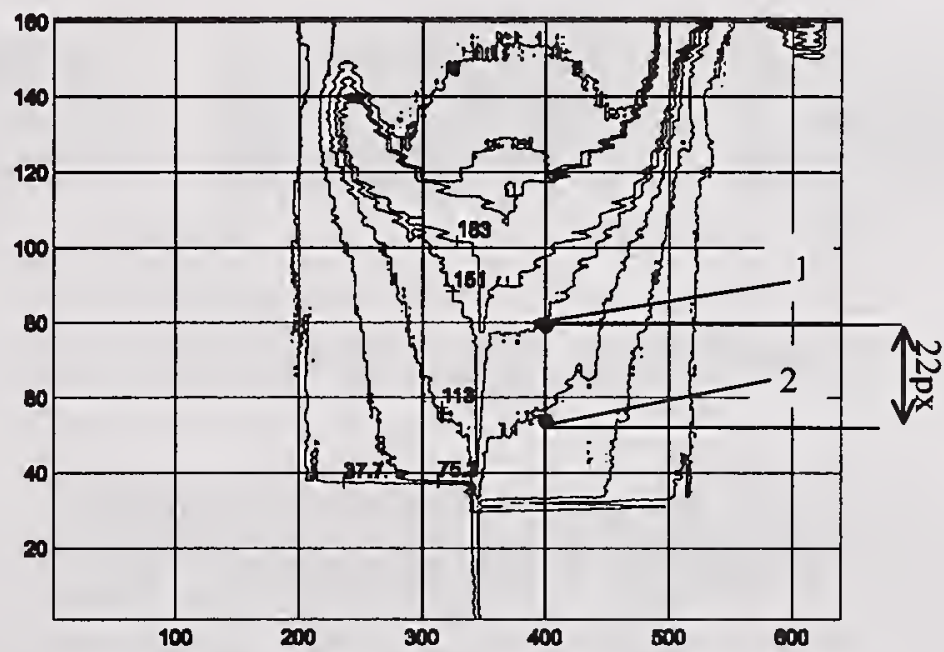

Figure 9. Contour plot for $v=190 \mathrm{~mm} / \mathrm{min}$ 
By correlating the length in pixels with the length in centimeters using the edge of the end of the plate as a reference, the measurement relationship is found to be $10 \mathrm{~mm}=19$ pixels (px). If $\mathrm{v}=$ $190 \mathrm{~mm} / \mathrm{min}$ and $\mathrm{s}=22 \mathrm{px}=11.5 \mathrm{~mm}, \mathrm{t}=3.65 \mathrm{sec}$. The temperature difference can be expressed in pixels, Fig .9, so that the distance between isotherms 1 and 2 is 38 pixels for the traveling time of $3.65 \mathrm{sec}$. that the tool takes to pass between isotherms.

Using the assumption that, near the shoulder, temperature linearly decreases as the distance from the shoulder increases, the cooling rate is obtained. For the welding speed of $190 \mathrm{~mm} / \mathrm{min}$ the cooling rate is $20^{\circ} \mathrm{C} / \mathrm{sec}$, but only in the narrow region around the shoulder. Although the temperature in front of the shoulder decreases when the welding speeds increases, it can be shown, using the same procedure that the cooling rate rises when the welding speed increases due to the lower average temperature of the surrounding material.

\section{Influence of the weld pitch on the temperature field and weld quality}

In real welding situations the surface temperature at the centerline of the nugget in the welding direction, is the most critical (Ref. 10). The temperature in front of the shoulder is shown in Fig. 10 as a function of the weld pitch (the spindle rotational speed divided by the welding speed). In general, as the weld pitch increases, the maximum temperature in the weld increases as well. The temperature may saturate at some value of the weld pitch. It is important to realize that as the weld pitch is increased by reducing the welding speed, the time that the material spends at or near the maximum temperature will increase: the slower the weld, the longer the time the weld spends at or above some particular temperature (Ref. 11). As the weld pitch increases from 0.6 to $3.0 \mathrm{rev} / \mathrm{mm}$, the surface temperature increases rapidly. This heat generation reduces the quantity of defects and improves the joint quality of the weld. As the weld pitch continues to increase to $9-11 \mathrm{rev} / \mathrm{mm}$, the surface temperature increase slowly and tends to saturate at some value of the weld pitch. A surface temperature in excess of 120 grayness level is required for the pin to accomplish the required forging, see Fig. 11.

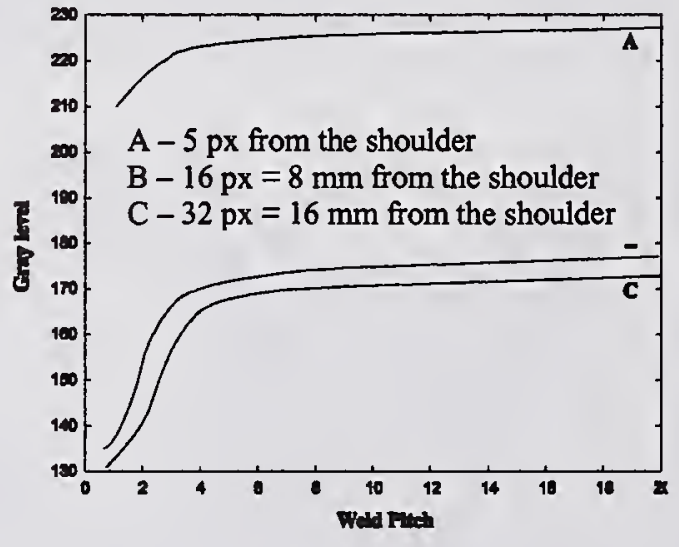

Figure 10. Maximum temperature in front of the shoulder as function of weld pitch

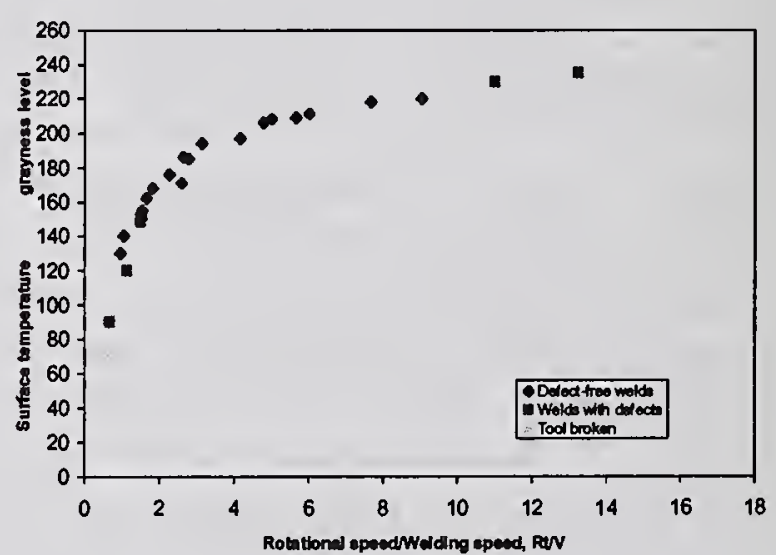

Figure 11. Surface temperature with the weld quality defined, as a function of the weld pitch 
Excessive weld pitch results in overheating with localized melting of the surface material. It can be calculated from Fig.11 that welds of good quality are obtained in the optimized range from 2.0 to $9.0 \mathrm{rev} / \mathrm{mm}$, (Ref. 12).

The operating parameters of friction stir welding for 6061-T6 Al plate is shown in Fig. 12. The quality of the joints is judged from the weld appearance and presence of any internal defects. Low rotational speeds do not produce stable welds because of the insufficient heat generation. On the other hand, high rotational speed produces excessive heat and causes unsatisfactory appearance and internal defects.

Typical weld appearance under different welding conditions is shown in Fig.13. Good weld joints are obtained for rotational speeds ranging from 344 to $637 \mathrm{rpm}$ and welding speeds from $95 \mathrm{~mm} / \mathrm{min}$ to $330 \mathrm{~mm} / \mathrm{min}$. For the welding parameters $151 \mathrm{rpm}$ and $229 \mathrm{~mm} / \mathrm{min}$ heat input is too low to produce sufficient heat to induce the plastic flow of material. This can cause additional force to be applied to the traversing pin, leading to pin failure, Fig13 (b). Figures 13 (c) and 13 (d) show the weld appearance with severe surface defects caused by excessive heat input under the conditions of $914 \mathrm{rpm}$ and $140 \mathrm{~mm} / \mathrm{min}$, and $914 \mathrm{rpm}$ and $330 \mathrm{~mm} / \mathrm{min}$.

The cross-sectional morphologies of the friction

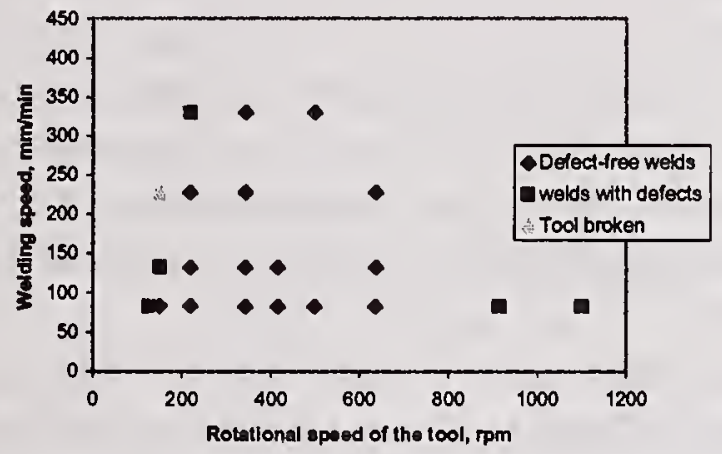

Figure 12. Operating parameters for FSW stir welds for different welding parameters, shown in Fig.14. An onion-ring-type inner structure consisting of concentric ovals proves that the nugget is well-developed, Fig.14 (a). A small void is found on the cross section of the welds created under the welding conditions of $914 \mathrm{rpm}$ and $95 \mathrm{~mm} / \mathrm{min}$ Fig. 14 (c, d). Tensile test results show that the good weld quality is obtained for the rotational speed from 344-637 $\mathrm{rpm}$ and welding speeds $95-330 \mathrm{~mm} / \mathrm{min}$, (Ref. 13).

The shapes and sizes of all of the different zones (HAZ, TMAZ plus weld nugget) are very hard to predict because they depend on the tool design and the welding parameters, and particularly on the welding speed (Ref. 12).

a)

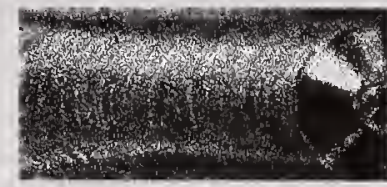

b)

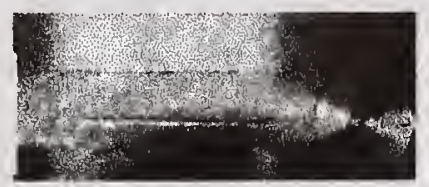

c)

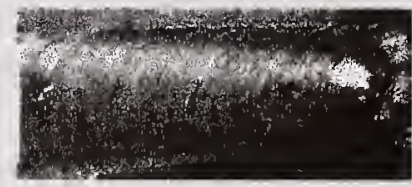

d)

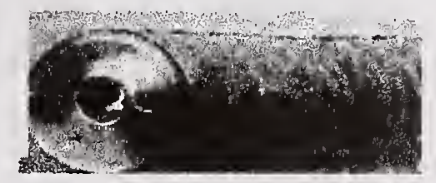

a) $637 \mathrm{rpm}$ and $190 \mathrm{~mm} / \mathrm{min}$; b) $151 \mathrm{rpm}$ and $220 \mathrm{~mm} / \mathrm{min}$; c) $914 \mathrm{rpm}$ and $140 \mathrm{~mm} / \mathrm{min}$ d) $914 \mathrm{rpm}$ and $330 \mathrm{~mm} / \mathrm{min}$

Figure 13. Typical weld appearances of the friction stir welds under different welding conditions

It is found that the welding speed has a larger effect on the weld zone size than the rotational speed. The width of the zones (HAZ, TMAZ, and nugget) is the largest, about $60 \mathrm{~mm}$ under the conditions of $914 \mathrm{rpm}$ and $95 \mathrm{~mm} / \mathrm{min}$, while the smallest is about $45 \mathrm{~mm}$ under the conditions of $344 \mathrm{rpm}$ and $330 \mathrm{~mm} / \mathrm{min}$. 


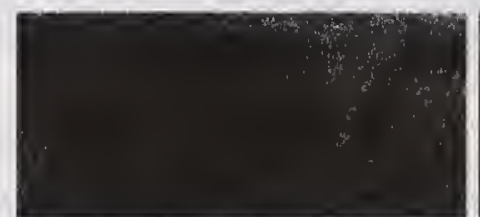

a)

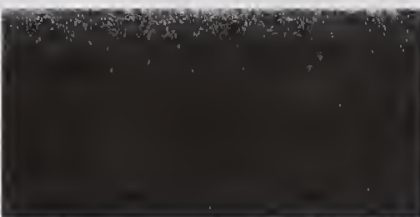

b)

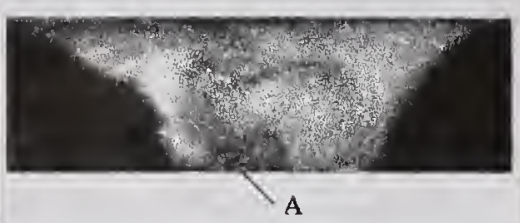

c)

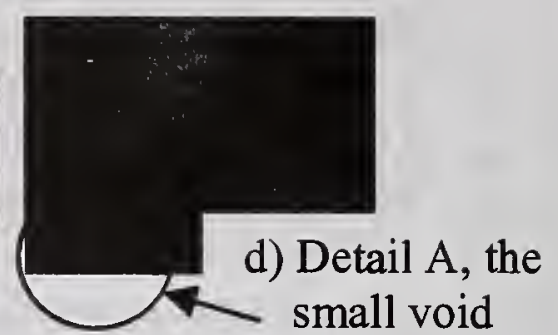

d) Detail A, the

a) $416 \mathrm{rpm}$ and $133 \mathrm{~mm} / \mathrm{min}$; b) $637 \mathrm{rpm}$ and $95 \mathrm{~mm} / \mathrm{min}$;) $914 \mathrm{rpm}$ and $95 \mathrm{~mm} / \mathrm{min}$

\section{CONCLUSION}

Using an infrared camera, the temperature distribution in front of the shoulder can be obtained for different welding parameters. Rotational and linear speeds can be adjusted during the welding in order to keep the temperature in front of the shoulder constant. The optimal range of parameters that produce satisfactory weld quality includes rotational speeds from $344 \mathrm{rpm}$ to 673 $\mathrm{rpm}$ and welding speeds from $95 \mathrm{~mm} / \mathrm{min}$ to $330 \mathrm{~mm} / \mathrm{min}$.

The influence of the rotational speed and the linear speed on the temperature changes is presented. The temperature change is very small for variations in rotational speed from 95 to $330 \mathrm{~mm} / \mathrm{min}$. The welding speed affects the surface temperature in front of the shoulder, and the temperature $0.5 \mathrm{~mm}$ from the surface under the shoulder, more than does the rotational speed. The influence of the linear speed on the cooling rate and zones' (HAZ, TMAZ plus weld nugget) sizes is also observed.

It has to be pointed out that this work is still in its embryonic stage. Some other sensing techniques will also be investigated. These are very attractive topics for future research in Friction Stir Welding and also very helpful for industrial applications.

\section{ACKNOWLEDGEMENTS}

The authors would like to express sincere thanks to Dr. M. Song for providing help in performing the calculations. This work was financially supported by the U.S Department of Education, Grant No. P200A80806-98. and the American Welding Society through the Graduate Fellowship Grant.

\section{REFERENCES}

1. E. D. Nicholas and W. M. Thomas, A review of friction processes for aerospace applications, Int. J. of Materials and Product Technology, Vol.13, Nos 1/2, 1998.

2. G. Bruggemann and Th. Benziger, The combination of thermography and image treatment for monitoring of quality assurance during laser welding, Independent nondestructive evaluations 33 , no. $7 ; 453$

3. Lukens, W. E. and Morris, R. A., Infrared temperature sensing of cooling rates for arc welding control, Welding Journal, Vol. 61, No.1, pp.27-33 (1982) 
4. Z.Loftus, R. Venable and Glynn P. Adams, Development and Implementation of a LoadControlled Friction Stir Welder, The First International Symposium on Friction stir welding, CA, USA, 14-16, June 1999.

5. R. Jeffrey Ding, Force Characterization on the welding pin of a friction stir welding retractable pin-tool using Aluminum-Lithium 2195, The Second International Symposium on Friction stir welding, Gothenburg, Sweden 26-28 June 2000.

6. Mahoney, M. W. and C. G. Rhodes, J. G. Flintoff, R. A. Spurling, and W. H. Bingel, Properties of Friction Stir Welded 7075 T651 Aluminum, Metallurgical and Materials Transaction A, 29A: 1955-1964, 1998.

7. W. Tang, X. Guo, J. c. McClure and L. E. Murr, Heat Input and Temperature Distribution in Friction Stir Welding, Journal of Materials Processing and Manufacturing Science, v7, $\mathrm{n} 2,1998$

8. J.R. Davis, ASM Specialty Handbook-Aluminum and Aluminum Alloys, ASM International

9. J.H. Ouyand and R. Kovacevic., Material Flow and Microstructure in the Friction Stir Butt Welds of the Same and Dissimilar Aluminum Alloys, Accepted to the Journal of Material Engineering and Performance, July 2001

10. O. Frigaard, O Grong and O.T. Midling, Modeling of the Heat Flow Phenomena in Friction Stir Welding of Aluminum Alloys, Seventh International Conference Joints in Aluminum - INALCO '98, Cambridge, UK, 15-17, April 1998.

11. A. P. Reynolds, W. D. Lockwood and T.U. Seidel, Processing-Property Correlation in Friction Stir Welds, Materials Science Forum, Vols. 331-337 (2000), pp. 1719-1724.

12. J. H. Ouyang, R. Kovacevic. M. Vlant and D. Jandric, Experimental Studies on Friction Stir Welding of Aluminum Alloys, submitted to the Journal of Materials Science

13. P. Threadgill, Friction Stir Welds in Aluminum Alloys - Preliminary microstructural assessment, TWI Bulletin, March/April 1997 



\title{
A REAL-TIME MONITORING AND CONTROL SYSTEM \\ FOR RESISTANCE SPOT WELDING
}

\author{
K. Matsuyama*, R. Obert\#, J-H. Chun*
}

\begin{abstract}
A new monitoring and control algorithm has been developed based on an integral form of an energy balance model to realize a low-cost real time monitoring and control system for resistance spot welding. The system captures welding voltage, welding current, and total plate thickness to calculate the mean temperature of a weld during welding. It predicts both weld diameter for the non-destructive evaluation of weld quality and splash occurrence for improvement of the working environment. After training with two stack welds of equal plates, the system can handle two stack welds of unequal thickness, multi stack welds, and other thickness welds without modification of the program parameters.
\end{abstract}

\section{KEYWORDS}

Resistance spot welding, Prediction of weld diameter, Prediction of splash occurrence, Integral form, Energy balance model, Quality monitoring, Improvement of working environment

\section{INTRODUCTION}

Industry belief has it that the occurrence of splash, or expulsion, yields good information on weld melting. Its evaluation has been used as quality assurance in resistance spot welding. Splash, however, causes some deterioration of the working environment and the quality of welds. Making a weld for comparison purposes is also wasteful of expensive energy. Furthermore, maintenance costs are higher than necessary because the metal powders caused by the splash degrade the moving parts of production robotics.

A new procedure addresses the problems inherent in splash during resistance spot welding. It solves deterioration issues based on the idea that splash or expulsion occurs when a weld part is overheated, even shortly, during welding.

The authors analyze the facts of splash with numerical simulations and experiments (Ref. 1). The article demonstrates that splash occurs when the corona bond zone at the faying interface suddenly melts. The article also suggests that continuous monitoring or prediction of weld part temperature, i.e., of the dynamic behavior of the temperature rising pattern, is important in predicting splash caused by overheating.

Temperature patterns and history can be continuously predicted if a monitoring procedure is associated with a prediction system based on a numerical simulation program as an identification routine of the weld part temperature (Ref. 2). The system described in the referenced paper

\footnotetext{
* Mechanical Engineering, Massachusetts Institute of Technology, 77 Massachusetts Ave, Cambridge, MA 02139 \#MS Student, Massachusetts Institute of Technology, 77 Massachusetts Ave, Cambridge, MA 02139
} 
addresses welding phenomena, current distributions, temperature distributions, and variations over time for each tested weld. In addition to the physical property data of the workpiece, the system only requires the monitoring of welding voltage and current.

The procedure, however, has a very high processing cost when using a standard CPU. The length of the calculation time is 100 to 1000 times that of the actual weld time. The system is a powerful tool for predicting the weld diameter and temperature history of welds if a high speed CPU, i.e., a high speed Digital signal processing, DSP, device with a large amount of memory, is installed on a welding controller. Therefore, the system is comparatively quite expensive and so used only in special cases (Ref. 3).

Calculation times are dictated by the procedure time required to solve the heat conduction differential equation. A finite differential method requiring many mesh points to get accurate temperature patterns is commonly used. The same is true when the finite element method or finite boundary method is employed.

A new integral form of the heat equation is induced for achieving a real time treatment. The new concept calculates the energy balance in a weld part to simplify the relationships among heat input to the weld, heat loss from the weld part, and the temperature rise in the weld.

This paper describes fundamentals of nugget formation process to understand the basic concept, an energy balance model, new governing equations derived from the new concept, configuration of monitoring and control system, and experimental results of the adaptation. The effort has developed a new low cost monitoring/control system to simultaneously end splash occurrences and estimate weld diameters.

\section{NUGGET FORMATION PROCESS IN RESISTANCE SPOT WELDING}

Figure 1 illustrates a typical nugget formation process for resistance spot welding (Ref. 4). Similar nugget growth patterns occur regardless of the type of material used as the workpiece (Ref. 2). Figure 1(a-1) to (a-3) illustrates cross sections of welds at different weld times. $d_{\mathrm{e}}$ is the contact diameter at the electrode-workpiece interface and $d_{\mathrm{c}}$ the diameter at the faying interface. Nugget diameter and nugget penetration are $d_{\mathrm{n}}$ and $p_{\mathrm{n}}$, respectively. Figure 1(b) shows the nugget growth pattern with contact diameter data at the electrode-workpiece interfaces and at the faying interface. The observation timings with arrows can also be found in Figure1(b). Broken lines in Figure 1(b) show the molten diameter and molten thickness of the nugget. After the solid and broken lines separate, solidification has begun, although the current conduction continues due to increasing contact diameters during welding.

The contact diameters are inconstant during welding. This variation in diameter has a large influence on the nugget formation process (Ref. 5). Measurement of the contact diameters shows that the contact diameter at the beginning stage of the weld cycle before forming a nugget is smaller than at the end. Furthermore, the contact diameter of tips used for many welds is usually larger than those used with the new electrodes. This change causes the weldable current range to increase. A similar effect causes a shift in the weld lobe for zinc-coated sheets. The initial contact diameters are also larger than those for uncoated sheets in spot welding zinc-coated steels. 


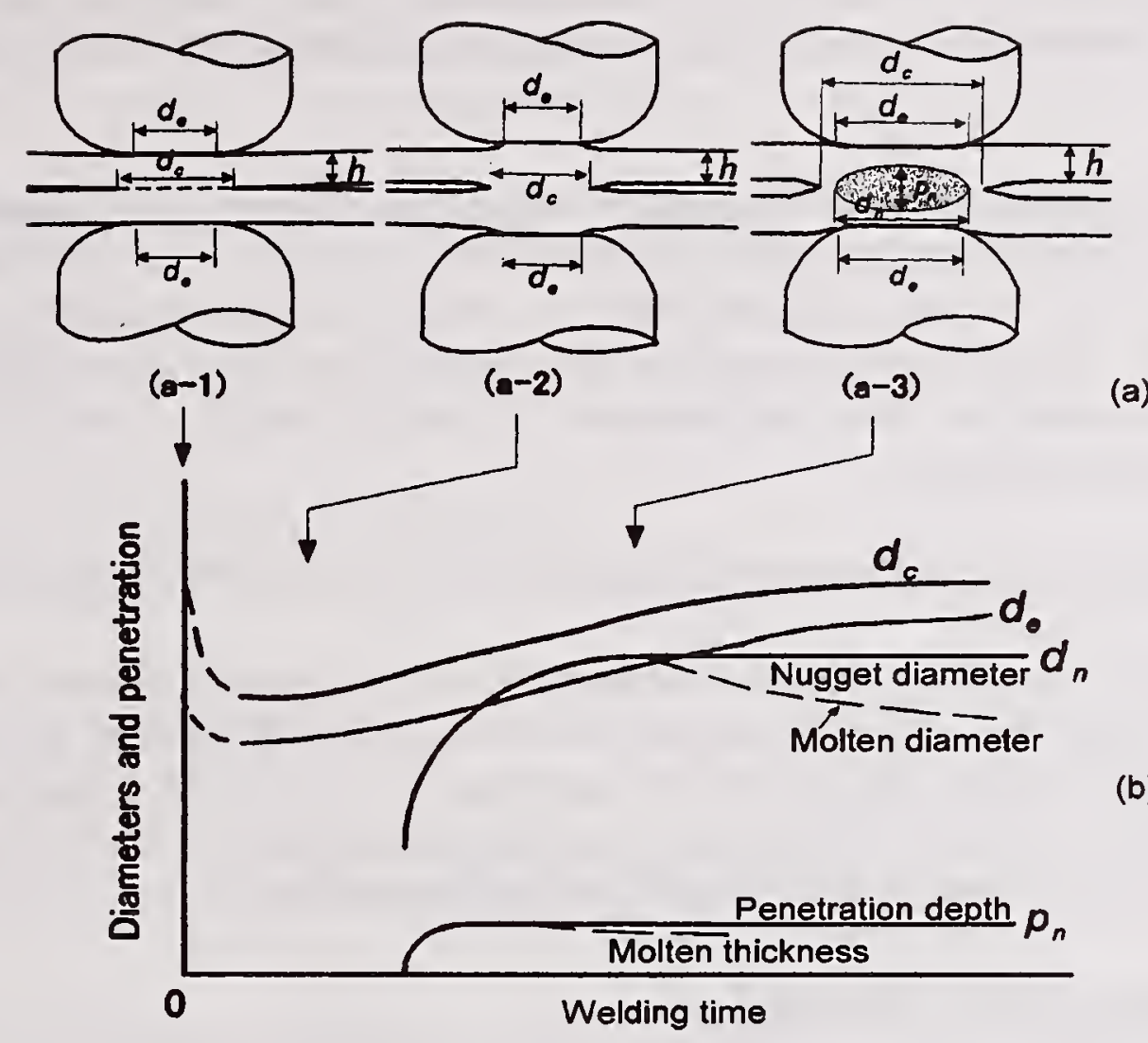

Figure 1: Schematic Illustration of Nugget Formation Process

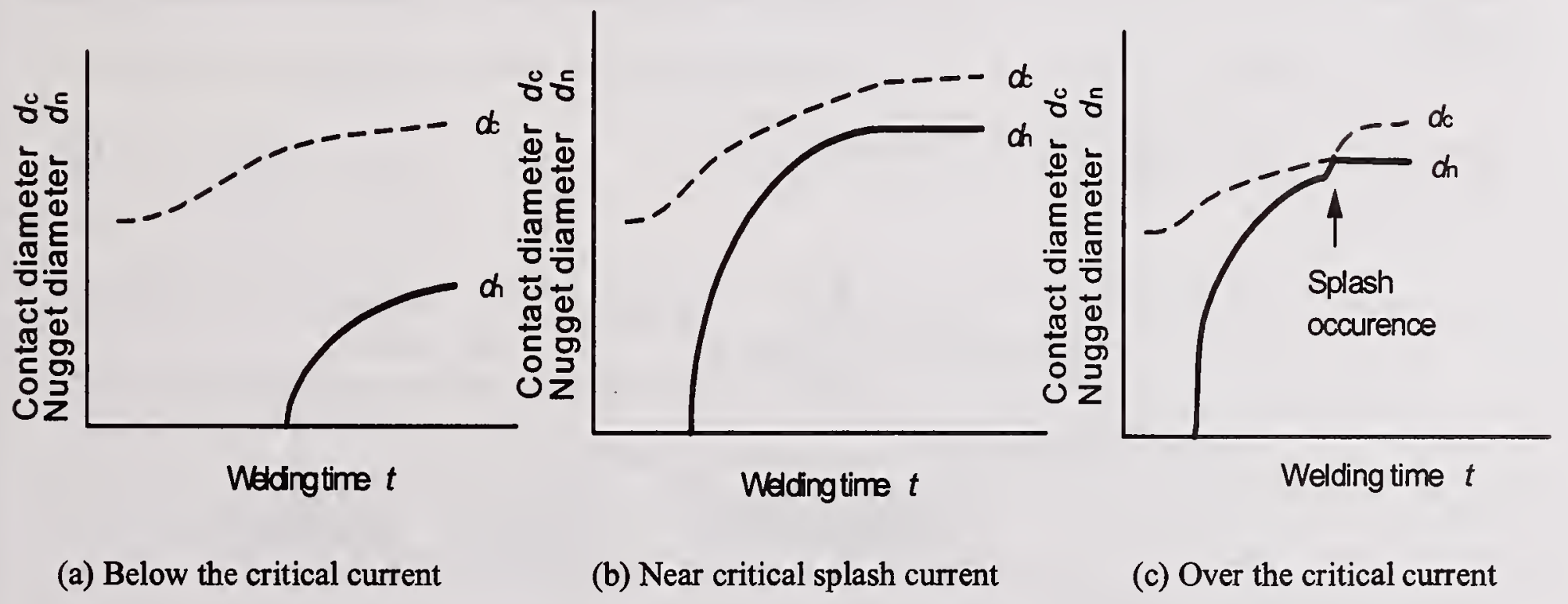

Figure 2: Relationship between Nugget and Contact Diameters at Various Setting Current Levels

Changes in the contact diameter have such a large influence because the heat generation density in resistance spot welding is a function of the contact diameter raised to the fourth power. For this reason, the variable contact diameter concept needs to be considered.

Figure 2 illustrates the growth patterns of nugget and contact diameters at various welding current levels. Figure 2(a) shows a pattern in lower current condition than the critical splash current 
condition. Figure 2(b), just under the critical splash current condition, and Figure 2(c) in a splashing current condition over the critical current. All figures show at the same electrode force condition.

The difference between the contact diameter and nugget diameter is greatest in a low current condition. This suggests that the mean temperature of a weld, defined as the weld zone bounded by the contact diameter and both interfaces between workpieces and electrode tips, is lowest among these three cases. The mean temperature in the splashing condition is the highest. This suggests that information about the mean temperature of a weld is useful to predict the welding state, including splash occurrence.

\section{MATHEMATICAL CONSIDERATIONS BASED ON AN ENERGY BALANCE MODEL}

The mean temperature of a weld can be estimated with both a finite difference method and two sets of monitoring data of the welding current and resistance between tips (Ref. 2). The latter procedure, however, requires a huge investment in numerical calculation. An expensive digital signal processor (DSP) is required to process the data. The problem can be resolved by employing a new algorithm based on an integral form of heat conduction equation.

\section{Basic Equation written in Integral Form}

The mean weld temperature is calculated in a target volume defined as a square zone enclosed by the four broken lines, shown in Figure 3, where $d_{c}$ is the contact diameter at electrode-workpiece interfaces. The following heat $Q$ is contained in the target volume at a time $t$ after starting current flow:

$$
Q=\int_{0}\left(v \cdot i \cdot f(d / h)+2 K_{e} \frac{\partial T}{\partial z} \pi d^{2} / 4+\pi d h_{t} K \frac{\partial T}{\partial r}\right) d \tau
$$

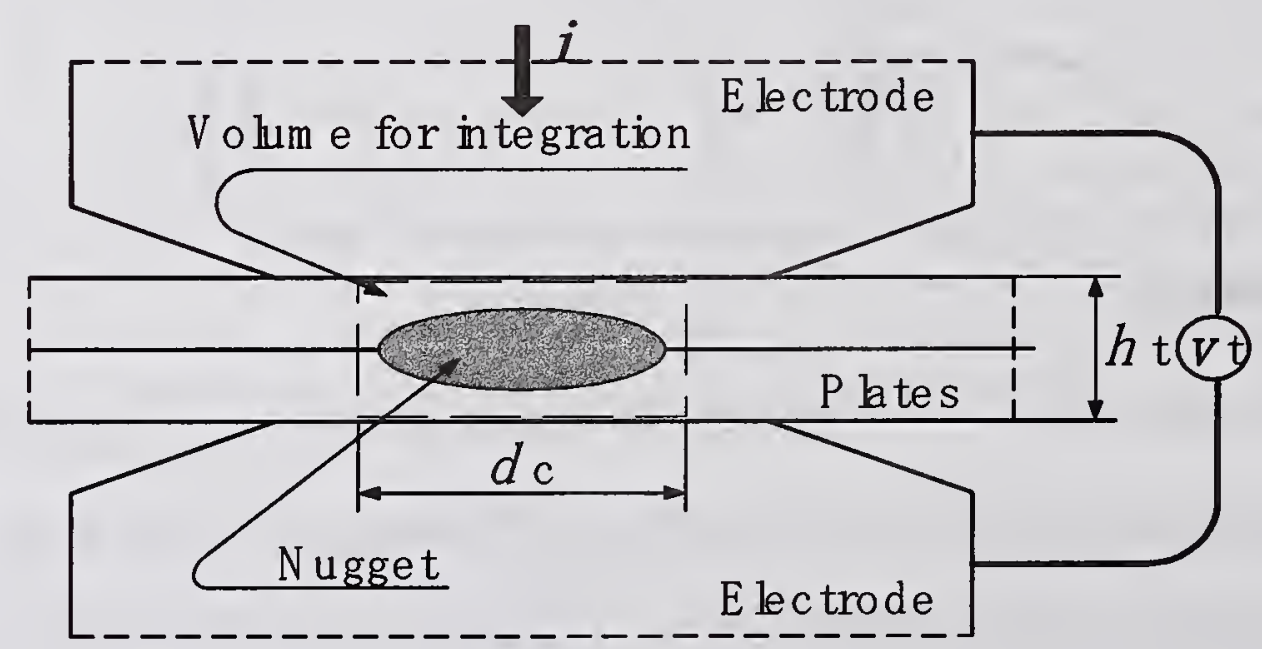

Figure 3: Schematic Illustration of Energy Balance Model

where $Q$ is the heat amount contained in the target volume, $t$ is the time, $v$ is the voltage between plate surfaces (measured voltage between tips [drop in tips], induced voltage by inductance), $i$ is the welding current, $K_{\mathrm{e}}$ is the heat conductivity in electrode tip, $K$ is the heat conductivity in the 
workpieces, $d$ is the contact diameter at plate-electrode interface at time $t, T$ is the temperature, $h_{\mathrm{t}}$ is the total plate thickness, $f(d / h)$ is the correction factor of current density by fringing effect, $h$ is the plate thickness of one plate, $r$ is the radius direction, and $z$ is the plate thickness direction.

A mean temperature of weld $\bar{T}$ in the weld part is defined by the following equation:

$\bar{T}(t)=\frac{Q}{h_{t} C \sigma \pi d_{c}^{2} / 4}$

where $C$ is the specific heat and $\sigma$ is the density:

$\bar{T}(t) \square \frac{1}{h} \int_{h_{h}-h}^{h_{h}} T(0, z, t) d z$

Thus, the following equation is yielded:

$\bar{T}(t)=\frac{4}{h_{t} C \sigma \pi d_{c}^{2}} \oint_{0}^{c}\left\{v \cdot i \cdot f(d / h)+\frac{\pi d^{2} K_{e}}{2} \frac{\partial T}{\partial z}+\pi d h_{t} K \frac{\partial T}{\partial r}\right\} d \tau$

This is the basic equation to predict the mean temperature in the workpiece as a function of welding time.

\section{Estimation of Temperature History during Welding}

The resistance between both electrode tip surfaces can be expressed with the following equation:

$\frac{\nu}{i}=R=\frac{4 \rho h_{t}}{\pi d^{2}} f(d / h)$

where $\rho$ is the resistivity.

The above equation combined with Equation 3 yields:

$\bar{T}(t)=\frac{4}{C \sigma d_{c}^{2}} \int_{b}\left\{\frac{v^{2} d^{2}}{4 \rho h_{t}^{2}}+\frac{K_{e} d^{2}}{2 h_{t}} \frac{\partial T}{\partial z}+K d \frac{\partial T}{\partial r}\right\} d \tau$

where $d_{\mathrm{c}}$ is the diameter at the time $t$, and $d$ is the diameter as a function of $\tau$.

Equation 5 can be used for the real time prediction of mean temperature during welding using only welding voltage as the monitoring data. The welding current, however, should be measured simultaneously to make the appropriate corrections for the effects of electrode tip resistance and mutual inductance between the secondary welding current circuit and the measuring circuit.

In actual monitoring, the voltage used in Equation 5 is calculated with: 
$\nu=v_{t}-\Sigma R_{e} \cdot i-M \frac{d i}{d t}$

where $v_{\mathrm{t}}$ is the voltage between electrode tips captured/measured, $R_{\mathrm{e}}$ is the resistance drop in electrode tips, $M$ is the mutual inductance between the secondary welding current circuit and the lead cable for measuring welding voltage $v_{\mathrm{t}}$.

Equation 5, however, requires the setting of every heat loss term for applying other stack conditions. That problem can be resolved if the equation yielded is based on a heat loss criterion at the electrode-workpiece interface, although the detail is not described here. The relation can be expressed as Equation 7 . The heat loss terms are defined only from measuring results for the stack condition of total plate thickness $h_{0}$.

$\bar{T}(t)=\frac{4}{C \sigma d_{c}^{2}} \int_{\delta}\left\{\frac{v^{2} d^{2}}{4 \rho h_{t} h_{0}}+\frac{K_{e} d^{2}}{2 h_{0}} \frac{\partial T}{\partial z}+K d \frac{\partial T}{\partial r}\right\} d \tau$

Equation 7 is the same as Equation 5 when the total plate thickness $h_{\mathrm{t}}=h_{0}$.

\section{Scheme for the Calculation}

Equation 8 is deduced as a discrete form of Equation 7 because the heat loss terms " $A$ " is constant according to the above result:

$\bar{T}_{w}(t+\Delta t)=\left\{\frac{d^{2}(t)}{d^{2}(t+\Delta t)}-2 A\right\} \bar{T}_{w}(t)+\frac{v^{2}(t+\Delta t)}{C \sigma \rho h_{t} h_{0}(1+A)} \Delta t$

where:

$$
\begin{aligned}
& A \equiv \frac{2}{C \sigma}\left(\frac{\alpha_{1}}{4 h_{0}}+\frac{\alpha_{2}}{d}\right) \Delta t \\
& \alpha_{1} \bar{T}=-\left.K_{e} \frac{\partial T}{\partial z}\right|_{z=h_{1} / 2} \\
& \alpha_{2} \bar{T}=-\left.K \frac{\partial T}{\partial r}\right|_{r=d / 2}
\end{aligned}
$$

where $h_{0}$ is the total plate thickness when the heat-loss term " $A$ " is trained with two-sheet stack of same thickness $h$. ( $\left.h_{0}=2 h\right), \alpha_{1}, \alpha_{2}$ is defined in Equations 10-a and 10-b for two stack type of plate thickness $h$, and $d^{2}(t) / d^{2}(t+\Delta t) \square 1.0$ because $\Delta t$ is very small.

Equation 8 can be solved by an ordinary progressive procedure, assuming that values $\alpha_{1}$ and $\alpha_{2}$ are constant during each weld sequence. This procedure matches the synchronized treatment with the 
capturing procedure of monitoring data. Equation 8 shows that the system requires only welding voltage. The welding current, however, should be also simultaneously measured to correct the magnetic flux effect on the measuring cables of welding voltage. Measurement of electrode tip movement is also effective to capture the actual total plate thickness in Equation 8. Measurement of the electrode force is useful to adjust the deflection value of the welding gun when the dynamic electrode force changes during welding.

\section{Feature of the Calculated Results by the New Procedure}

Figure 4 schematically illustrates a typical result calculated with Equation 8. After setting some physical properties of materials used and training heat loss term " $A$ ", welding voltage $v$ as a function of welding time and total plate thickness $h_{\mathrm{t}}$ are read/captured as the input data for calculation of the mean temperature.

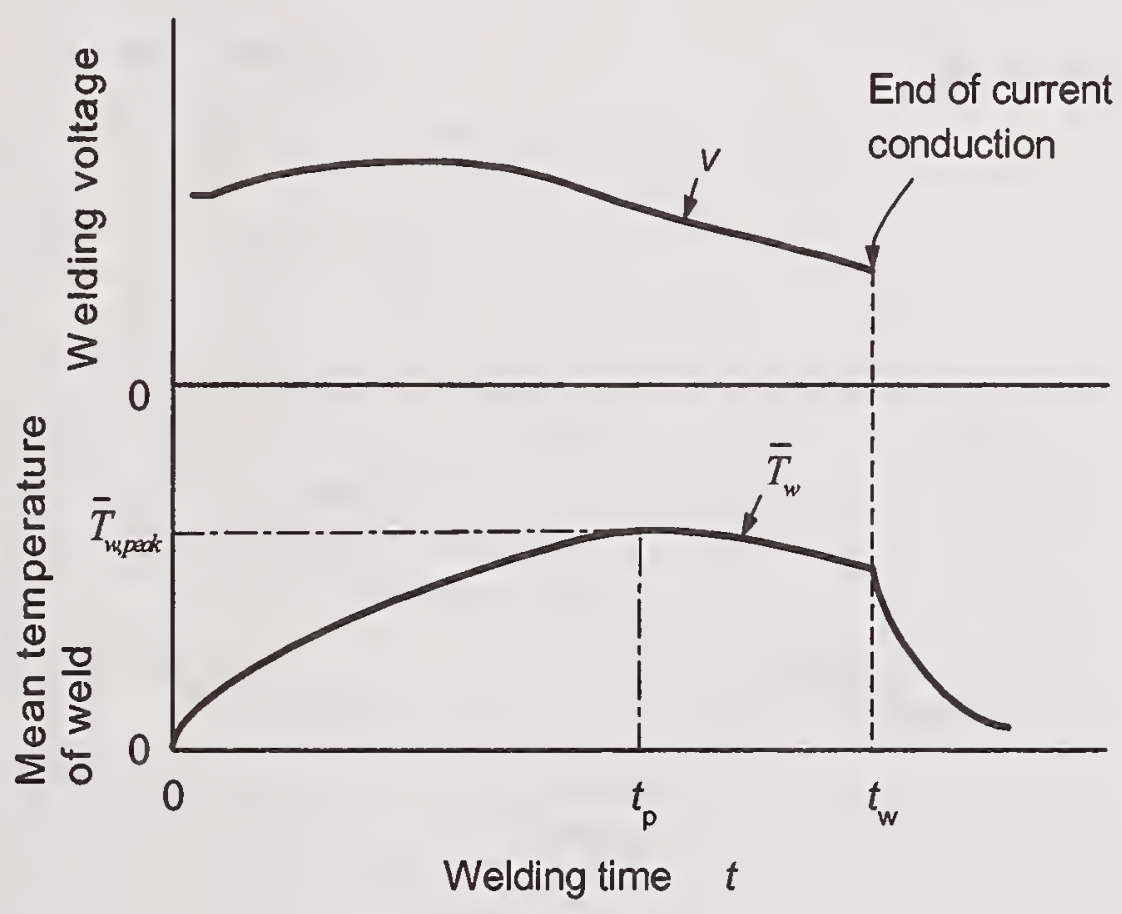

Figure 4: Schematic Illustration of Calculated Mean Temperature of Weld

The calculated mean temperature of weld increases with welding time to reach a peak value at welding time $t_{\mathrm{p}}$ if the weld time $t_{\mathrm{w}}$ is enough long. The time $t_{\mathrm{p}}$ corresponds to the starting time of solidification, as indicated with the separation of solid and broken lines in Figure 1. This means that the weld diameter for each weld can be estimated from this peak value of the calculated mean temperature. When the weld time $t_{\mathrm{w}}$ is shorter than the peak time $t_{\mathrm{p}}$, the time $t_{\mathrm{p}}$ equals to the weld time $t_{\mathrm{w}}$.

Figure 5 shows the principle of predicting weld diameters and splash occurrence with the mean temperature of weld. If the heat loss term " $A$ " could be set in an adequate and suitable value, all monitored data as shown in Figures 5(a) and 5(b) can be arranged with only one curve as shown in Figure 5(c). 
$\mathrm{U}$ and $\mathrm{L}$ in Figure 5(c) indicate threshold values of the critical splashing condition and the forming of the minimum weld diameter required by the customer or specifications.

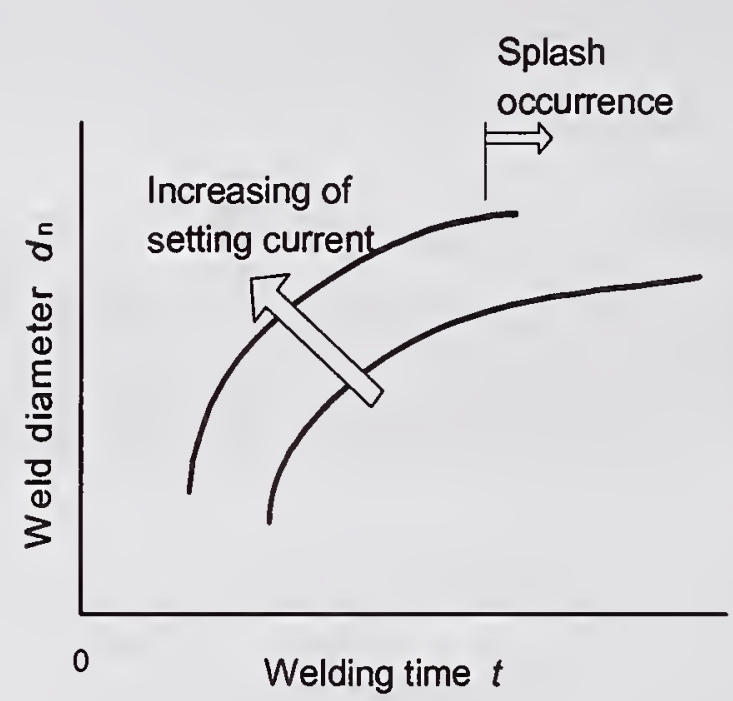

(a)

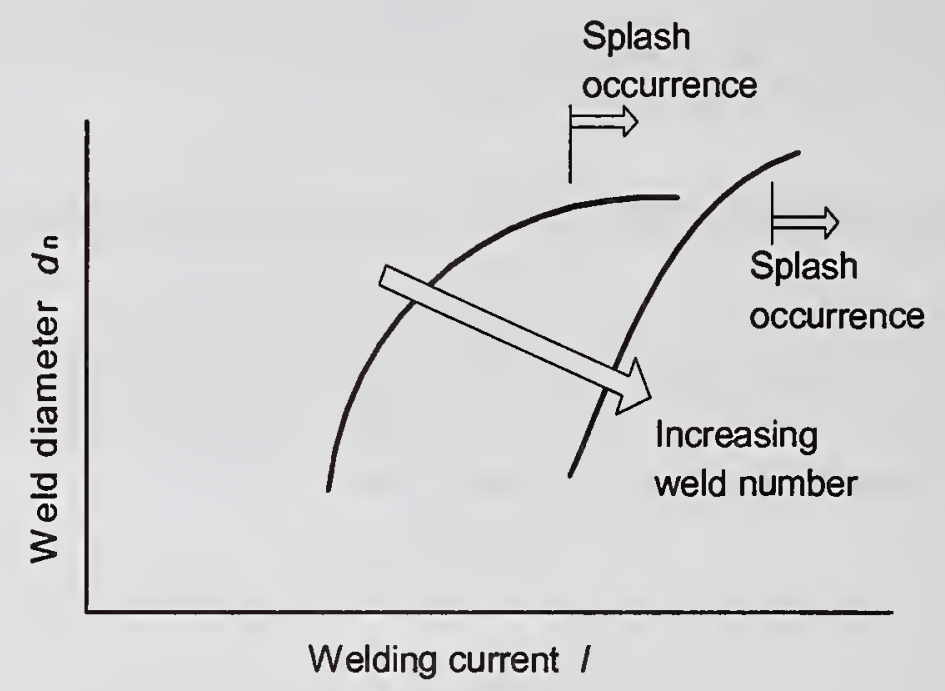

(b)
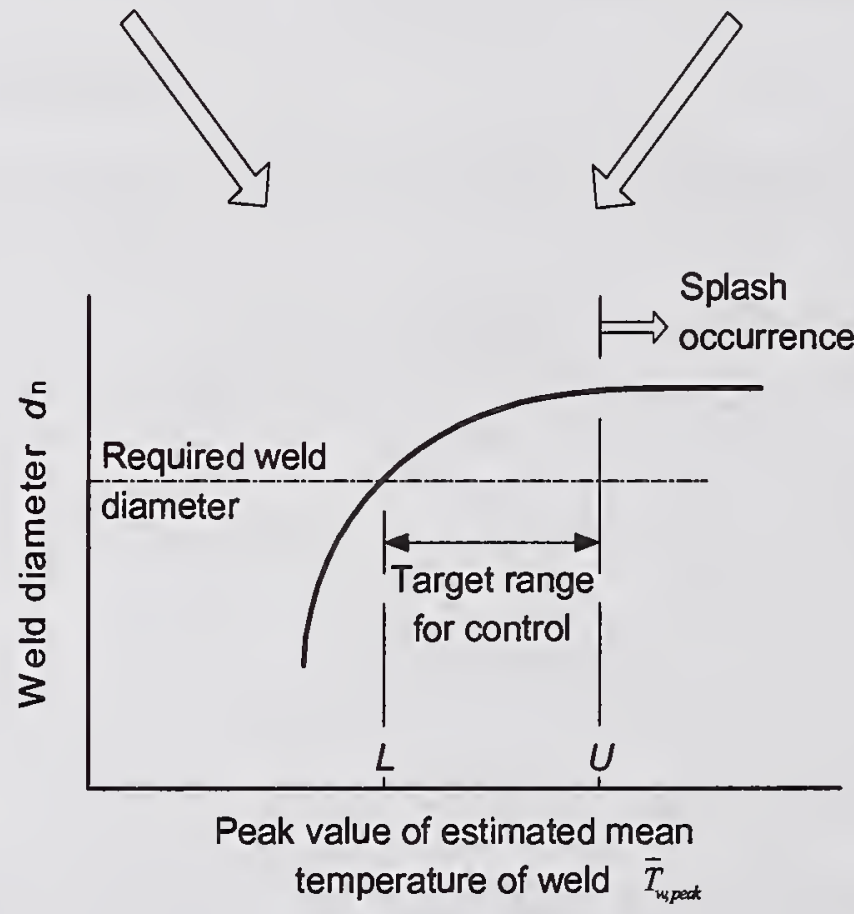

(c)

Figure 5: Prediction of a Welding State from Peak Values of the Predicted Mean Temperature 


\section{SYSTEM CONFIGURATION FOR MONITORING AND CONTROL}

Figure 6 shows an example of the system configuration. The system minimally consists of a welding controller including power control unit and transformer, an electrode force control unit, a welding gun, a data capturing and treatment computer, an $\mathrm{AD}$ converter board, and some sensing devices including a current sensor. The $\mathrm{AD}$ converter board connects to four sensing cables; welding current, welding voltage, electrode force, and electrode tip movement, although Equation 8 directly requires only one monitoring data of welding voltage.

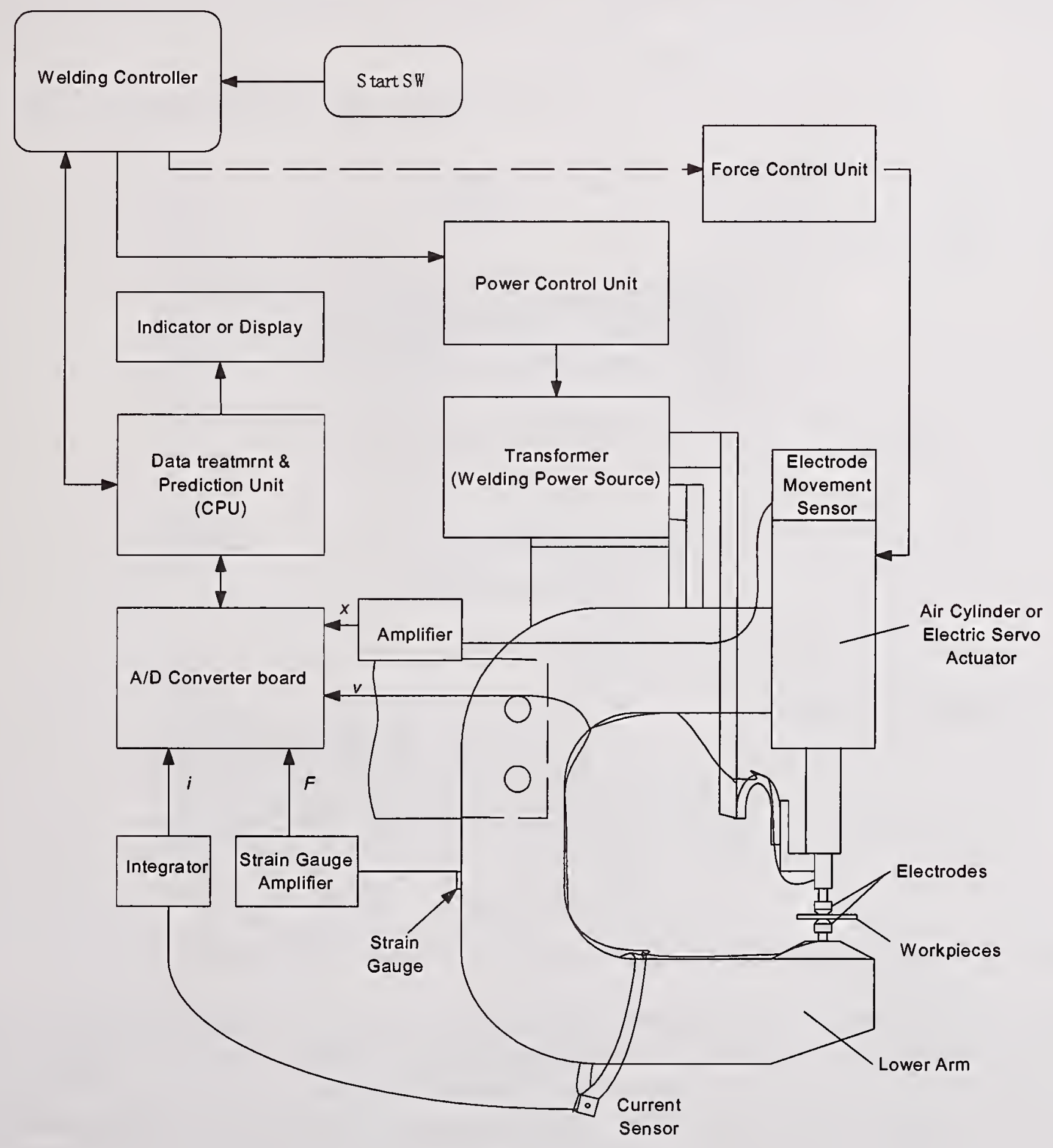

Figure 6: Typical configuration of the New Monitoring/Control System 
The welding current corrects the influence of electrode tip drop and induced voltage on the measured welding voltage by the secondary welding current. The information on electrode force and electrode tip movement is employed to estimate the dynamic behavior of total plate thickness. Alternatively, the later two monitoring parameters can be replaced with the nominal value of total plate thickness.

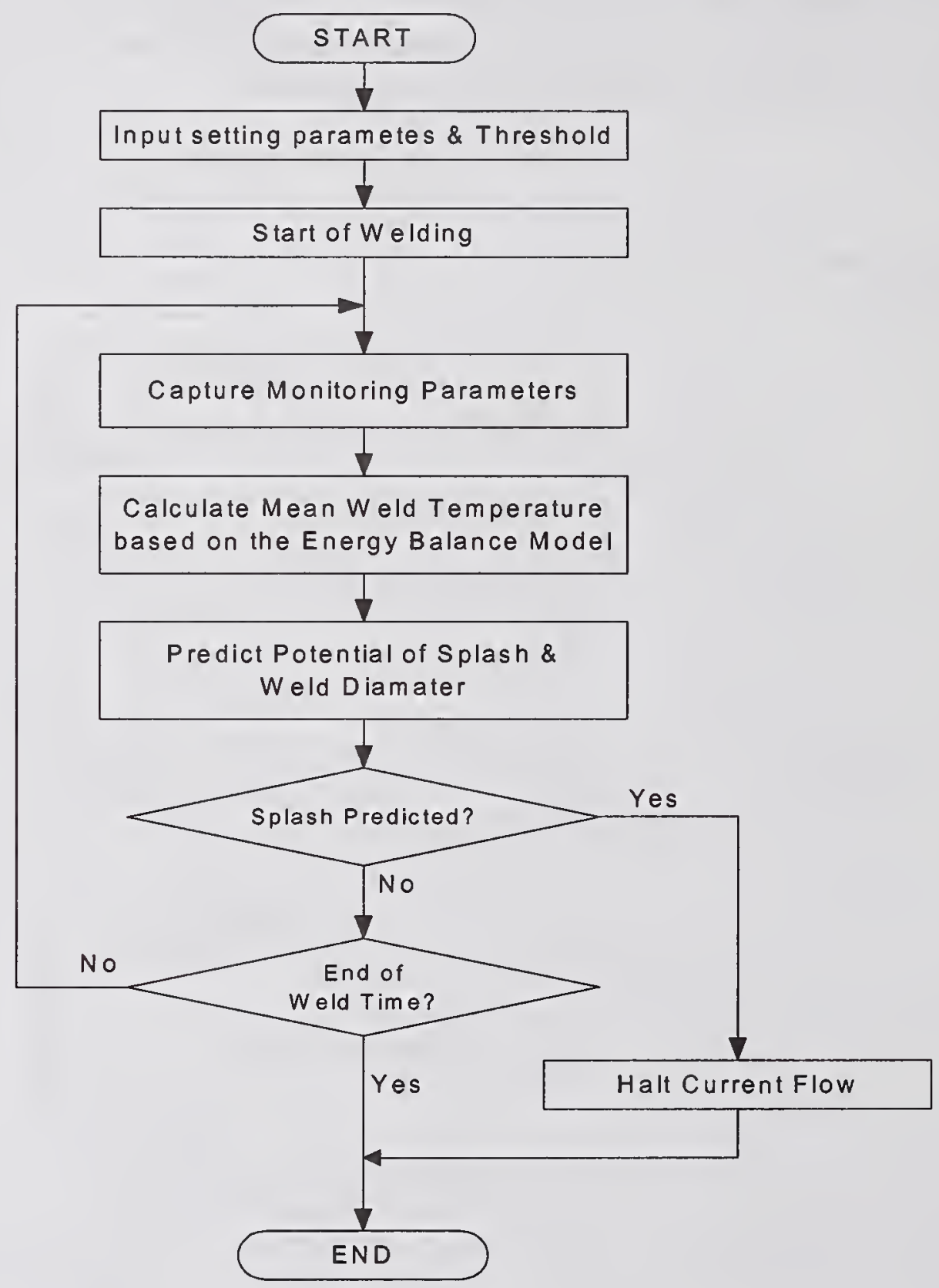

Figure 7: A Flowchart to Monitor and Control to Eliminate Splash Occurrence

Figure 7 is a flowchart to predict the weld diameter and eliminate the splash occurrence in real time during welding. The weld diameter and the possibility of the splash occurrence are predicted in the stage of "Predict potential of splash \& weld diameter" as future values of one or a half cycle later after the capturing time to control the next ignition angel for current flow. In the prediction of the next half or one cycle of welding current conduction, the calculation is achieved under an assumption that the same welding voltage waveform is repeated.

The program is in Visual Basic, run on a Windows-based computer with a $66 \mathrm{MHz}$ bus and a 
$300 \mathrm{MHz}$ CPU. Data sampling is repeated in $2.4 \mathrm{kS} / \mathrm{s}$. Total CPU time including data capturing, data treatment, and machine control is less than $60 \%$ of the total welding time. This means the procedure can be realized as a low cost real-time system.

\section{ANALYSIS OF THE NEW PROCEDURE: A REAL TIME PREDICTION SYSTEM OF SPLASH OCCURRENCE AND WELD DIAMETERS}

The new monitoring procedure was experimentally examined to understand how to predict the splash occurrence and nugget formation process. The experiments were carried out with an AC spot welding machine mounted pneumatic gun. Two types of zinc-coated steels were used as the test pieces.

\section{Prediction of Splash Occurrence during Welding}

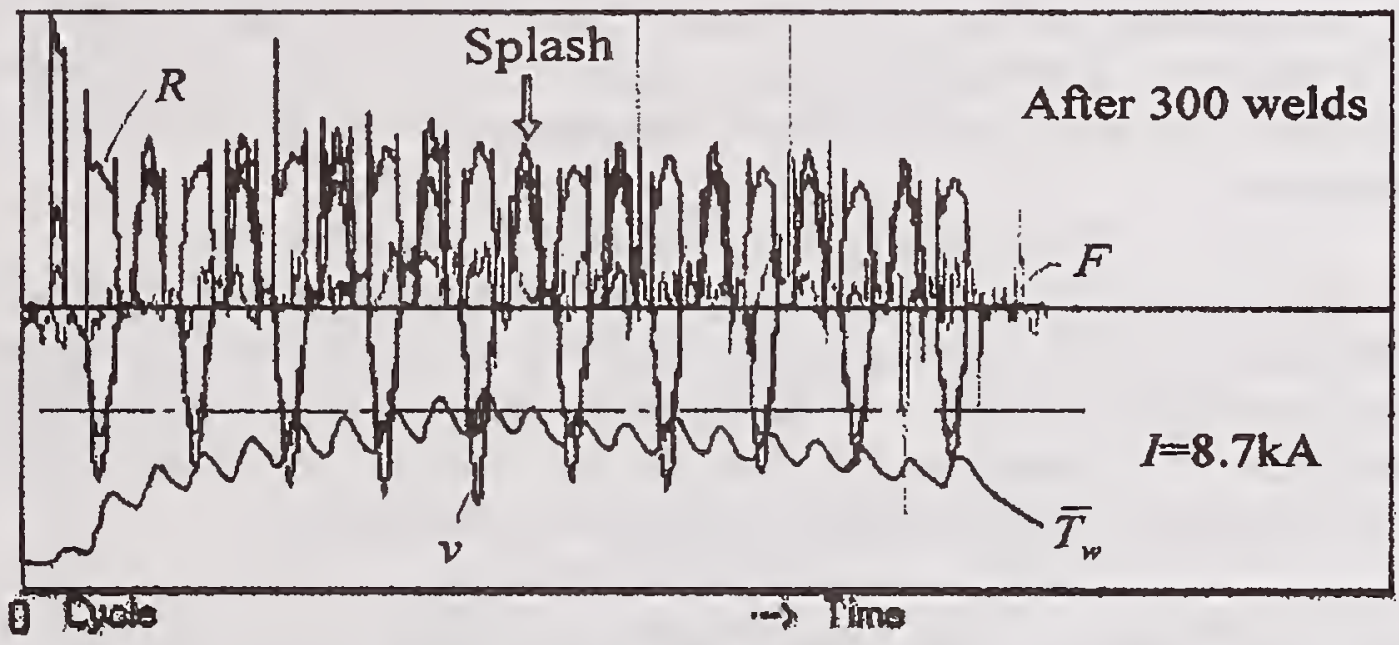

(a) Splashing during welding

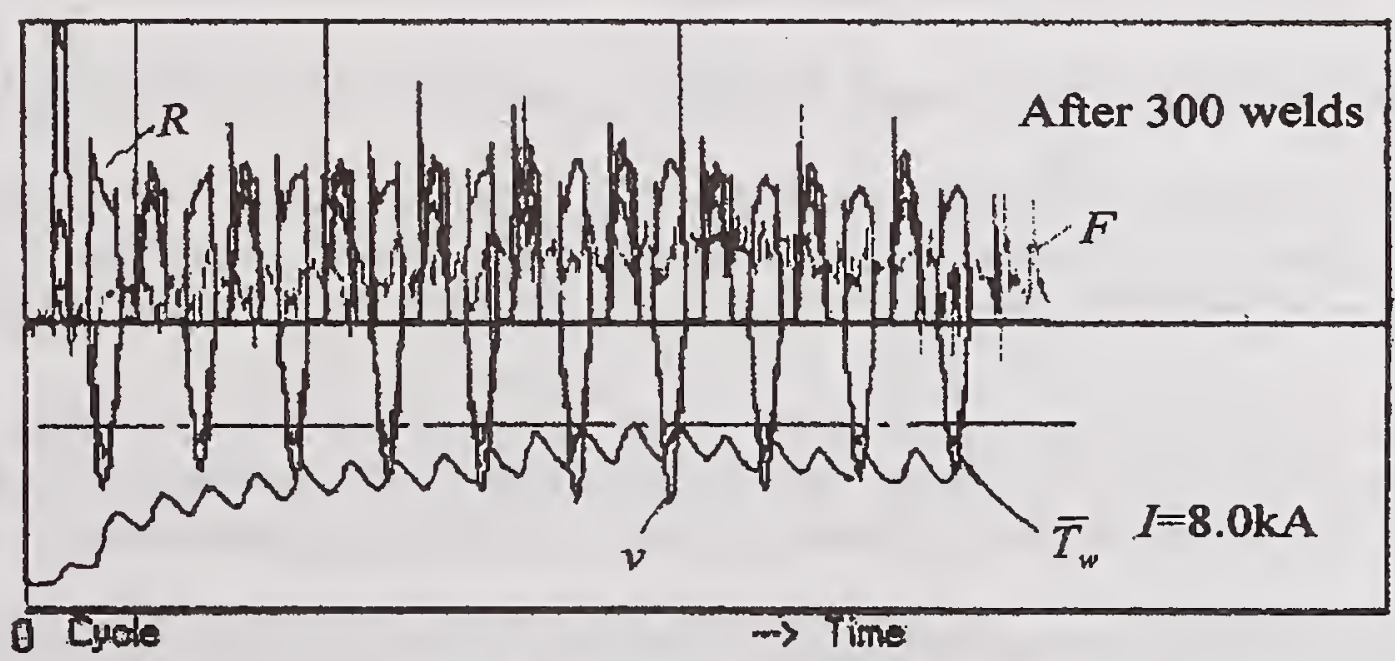

(b) No splashing and making sound weld

Figure 8: Prediction of the Mean Temperature History during Welding with the New Procedure $(P=200 \mathrm{daN}, t=10$ cycles of $60 \mathrm{~Hz}, h=1 \mathrm{~mm} \times 2$ : Organic zinc coated sheets) 
Workpieces for the first trial were steel with a one-sided organic-zinc coating. Plate thickness was $1 \mathrm{~mm}$. The test pieces were made from two stacked sheets. Electrode tips were the $16 \mathrm{~mm}$ dome type.

The experiments were done with coupons of $300 \mathrm{~mm} \times 300 \mathrm{~mm}$ at $200 \mathrm{daN}, 10$ cycles, and $8.0 \mathrm{kA}$. Weld diameters were measured with small coupons of $30 \mathrm{~mm} \times 100 \mathrm{~mm}$ to observe shifting of the weld lobe during electrode tip life test. The weld lobe test was administered every 1000 welds. In the weld lobe test, the welding current was varied between a no nugget condition to a current value higher than the critical splashing condition.

Examples of the predicted result with the new monitoring procedure based on Equation 8 are shown in Figure 8. The parameter " $A$ " was determined with a few training data captured for a two-sheet stack type. A measured welding current $i$, a modified weld voltage $v$ after reduction of induced voltage and tip resistance drop, a resistance $r$ between both plate surface calculated with the current and modified voltage, a dynamic electrode force $F$, and a predicted mean weld temperature $T$ are shown. An arrow indicates the time of splash. A dotted line illustrates the critical temperature level. The sum of plate thickness stacked is input as the total plate thickness in the present calculation

Figure 8(a) shows an example of splash. A splash occurs when the temperature is over the threshold line indicated with a one-dotted line. There is no splash shown in Figure 8(b); the peak value of mean temperature is lower than the threshold line. The same relationships for other weld number specimens. This demonstrates that the new concept described in Figures 4 and 5 effectively correlates the splash occurrence with the mean temperature.

Furthermore, the difference of peak times indicates variations in the incubation time needed for nugget growth. If the peak period does not appear by the end of the current conduction, the weld time can be increased to produce a bigger nugget.

\section{Arrangement of Weld Diameters and Splashing Conditions with the Weld Temperature}

Measured weld diameters are plotted in Figure 9(b). They are shown as a function of the peak value of the predicted temperature in the weld part. Solid marks in the figure indicate a splash condition. Figure 9(a) shows the same data arranged as a function of the RMS welding current.

There is good agreement between the peak value of temperature during welding and measured weld diameter in Figure 9(b), although the relationship between the welding current and the weld diameter is not close. Figure 9(b) presents all monitored data arranged on a single curve.

Splash/expulsion (expressed as solid marks) occurs only at temperatures higher than about $1700^{\circ} \mathrm{C}$ for organic zinc coated steels. This suggests that the new concept and procedure is applicable not only to predict splash, but also to predict weld quality, and that to build up a new adaptive control system would be an easy task. 


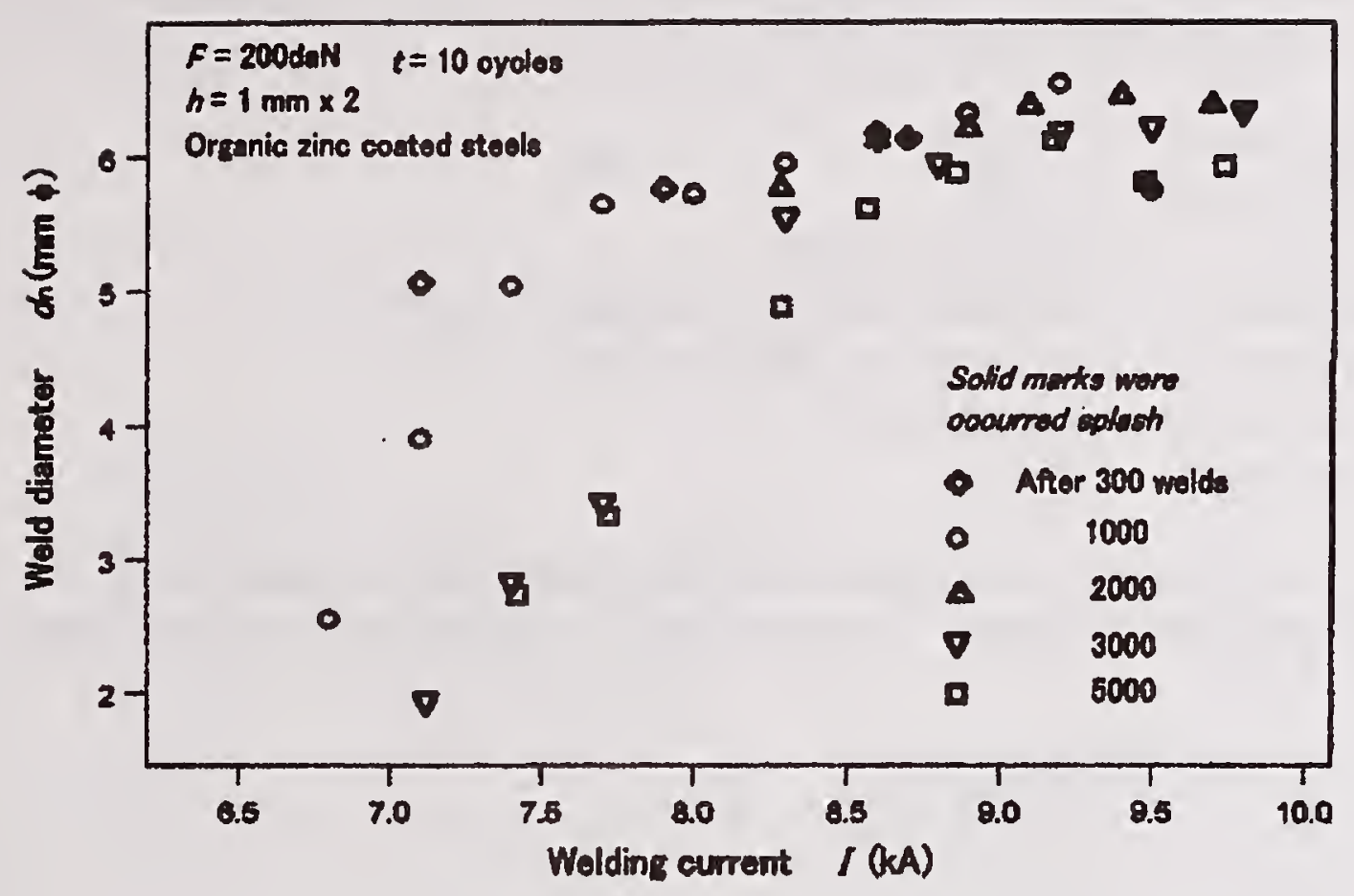

(a) Weld diameter vs. RMS value of welding current

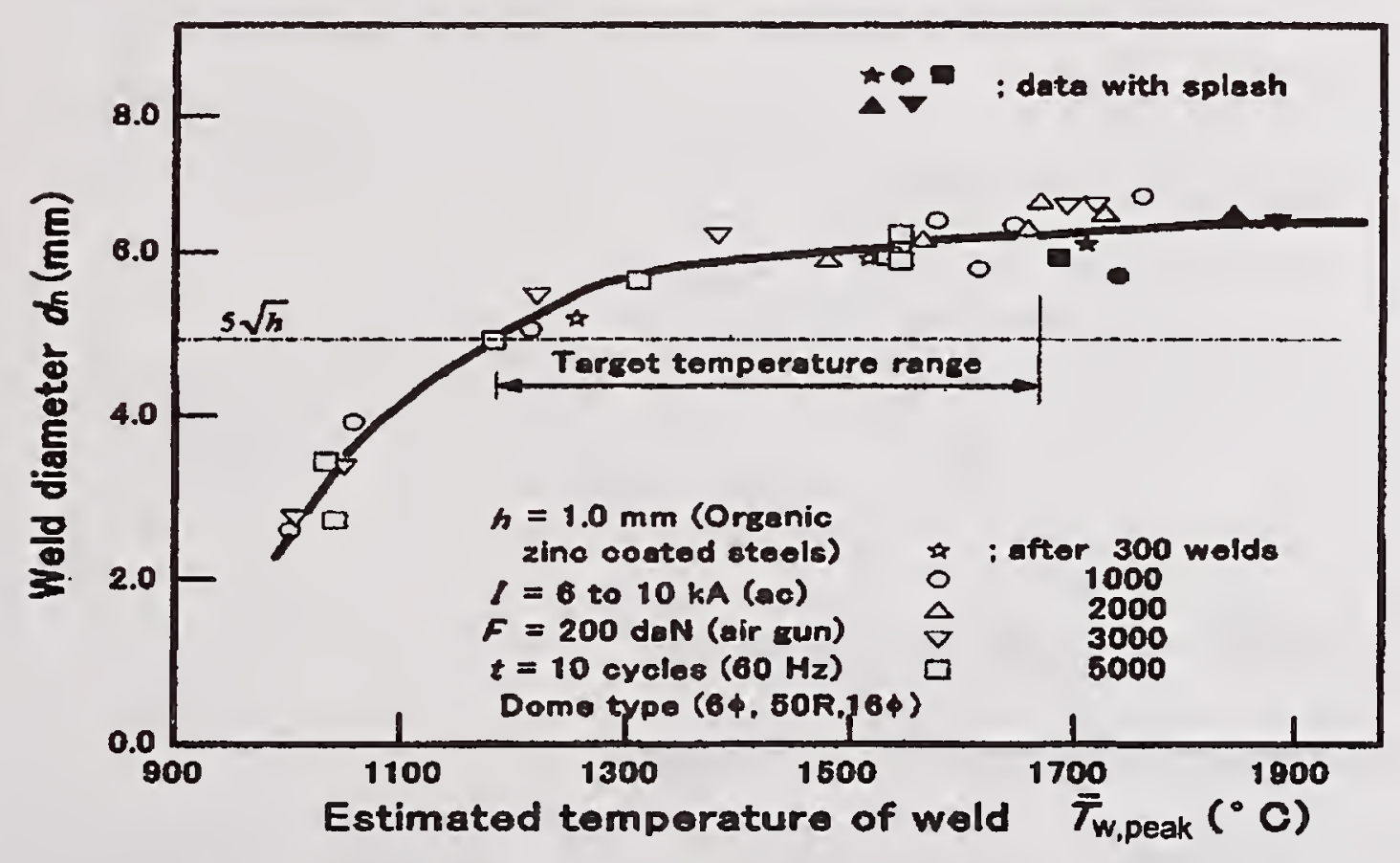

(b) Arrangement of the weld diameter data with the peak temperature predicted by the new procedure during welding

Figure 9: Adaptability of the New Prediction Procedure of Splash Occurrence and Weld Diameters

\section{Adaptability for Other Stack Conditions}

Galvannealed steels coated on both sides were also examined as the second trial for various combination stacks. The tests were completed during an electrode tip wear test. The electrode tip 
wear test was carried out in the following condition with $1.0 \mathrm{~mm}$ thick coupons of $300 \mathrm{~mm} \times 300$ $\mathrm{mm}$. The wear test condition is shown below:

$\begin{array}{ll}\text { Welding current } & 8 \mathrm{kA}(\mathrm{AC}) \\ \text { Electrode force: } & 200 \text { daN } \\ \text { Weld time: } & 10 \text { cycles of } 60 \mathrm{~Hz} \\ \text { Test materials: } & \text { Galvannealed steels sheets }\left(60 / 60\left[\mathrm{~g} / \mathrm{m}^{2}\right]\right) \\ \text { Electrode tips: } & \text { Dome type tips }(8 \mathrm{R}, 6 \phi-50 \mathrm{R}) \\ \text { Welding rate : } & 1 \text { weld } / 3 \mathrm{~s} \\ \text { Cooling water: } & 3.5 \mathrm{l} / \mathrm{min}\end{array}$

Six combinations of plates were examined after about 100 welds and after every 1000 welds with the test conditions shown in Table.1. Figure 10 shows configurations of the test pieces.

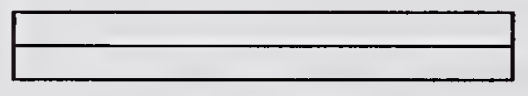

(a)

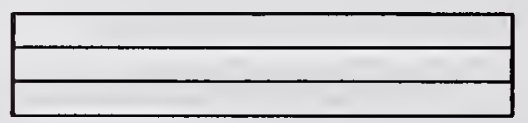

(b)

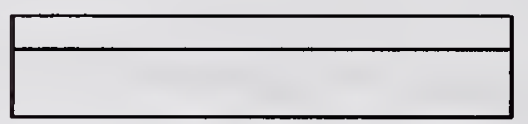

(c)

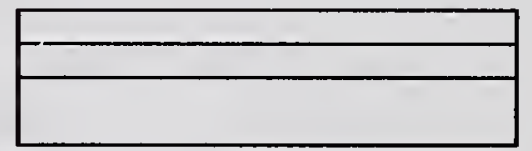

(d)

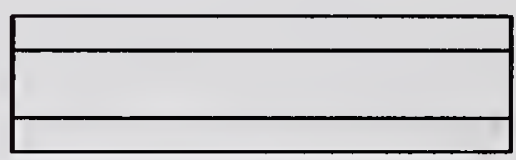

(e)

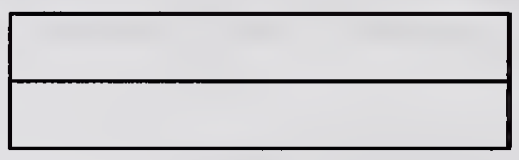

(f)

Figure 10: Configuration of Stacks used for the Tests

Table 1: Size of the Test Pieces and Testing Conditions

\begin{tabular}{|l|c|c|c|c|}
\hline Plate $(\mathrm{mm})$ & $I(\mathrm{kA})$ & $F(\mathrm{daN})$ & $t_{\mathrm{w}}$ (cycles) & Pre-weld \\
\hline $0.8+0.8$ & Varied & 200 & Varied & No \& exist. \\
\hline $0.8+0.8+0.8$ & Varied & 200 & 10 & No \\
\hline $0.8+1.8$ & Varied & 200 & 10 & No \\
\hline $0.8+0.8+1.8$ & Varied & 200 & 10 & No \\
\hline $0.8+1.8+0.8$ & Varied & 200 & 10 & No \\
\hline $1.8+1.8$ & Varied & 400 & 20 & No \\
\hline
\end{tabular}

Weld diameters were measured at each interface between the top plate and the next after a peel or torsion test. The size of small coupons are $30 \mathrm{~mm} \mathrm{x} 100 \mathrm{~mm}$ for $0.8 \mathrm{~mm}$, and $50 \mathrm{~mm} \times 150 \mathrm{~mm}$ for $1.8 \mathrm{~mm} \times 2$. Carbon prints of electrode tips are shown in Table 2 . 
Table 2: Carbon Print of the Electrode Tips during the Test

\begin{tabular}{|l|c|c|c|c|}
\hline Observed & After 100 welds & After 1000 welds & After 2000 welds & After 3000 welds \\
\hline $\begin{array}{l}\text { Upper } \\
\text { electrode tip }\end{array}$ & 0 & 0 & & \\
\hline $\begin{array}{l}\text { Iower } \\
\text { electrode tip }\end{array}$ & 0 & 0 & & 5 \\
\hline
\end{tabular}

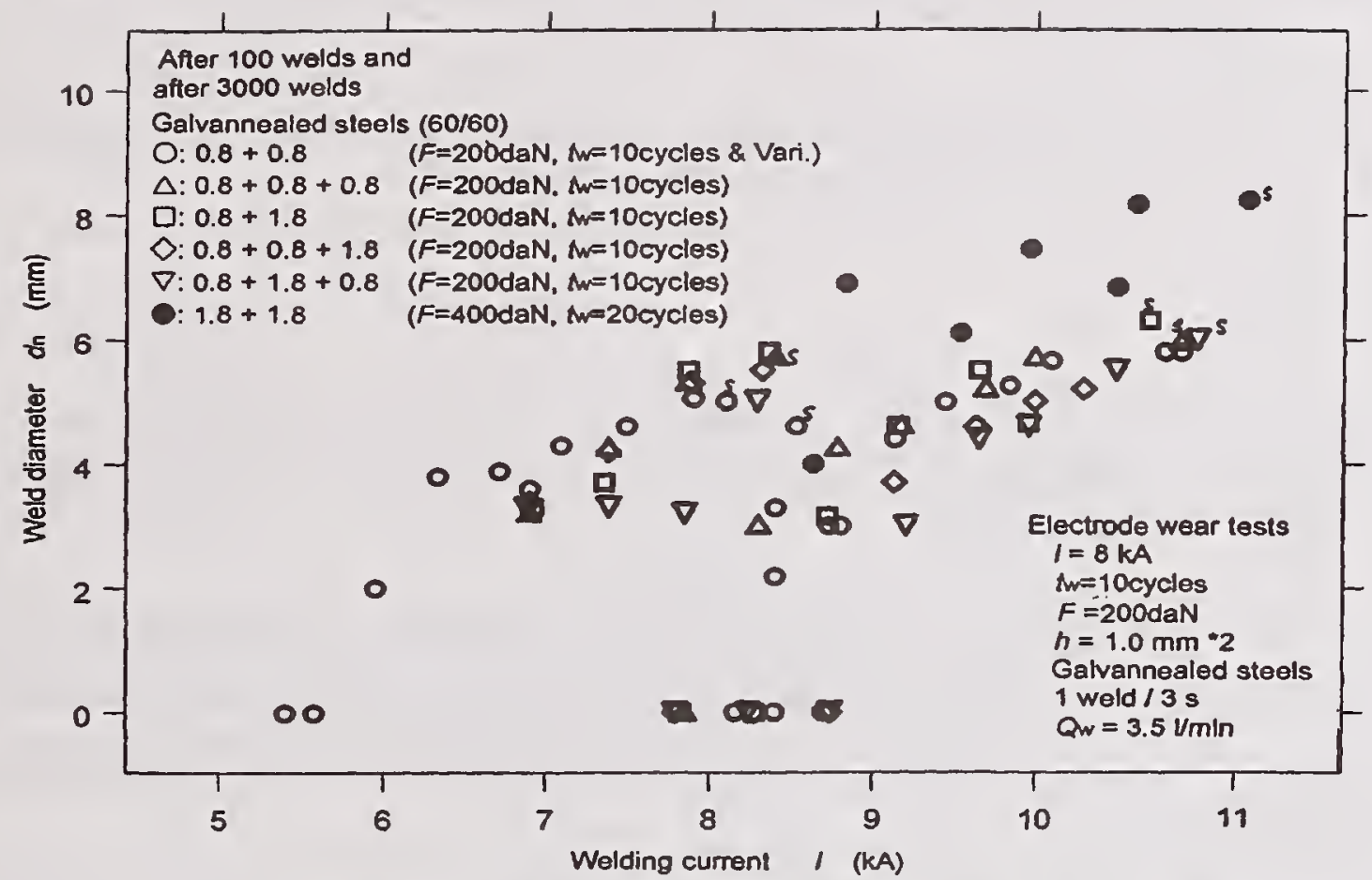

(a) Weld diameter vs. RMS value of welding current

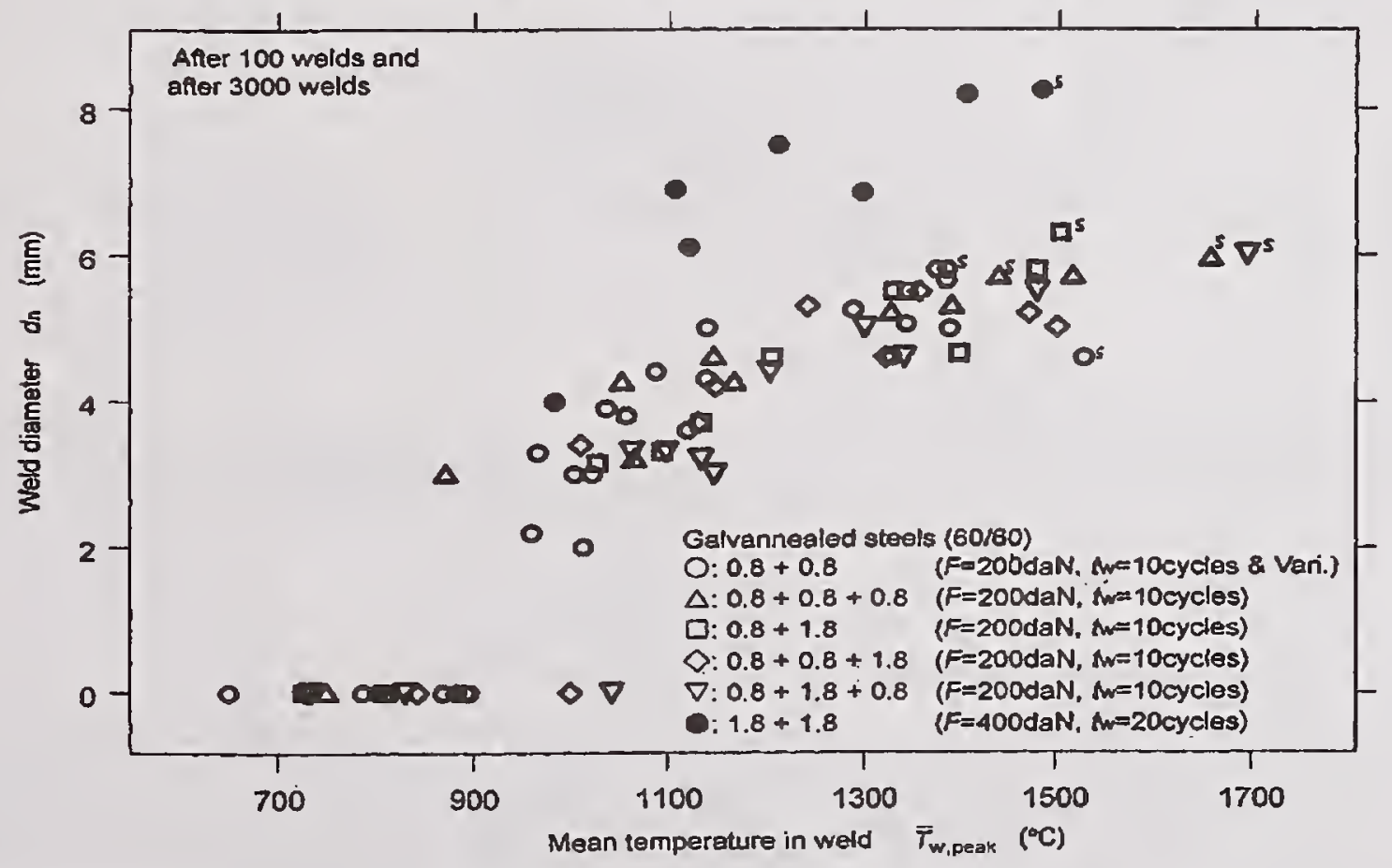

(b) Arrangement of the data with weld temperature

Figure 11: Test results for Galvannealed Steels of Various Stack Conditions

Figure 11(a) shows the test results as a function of welding current. The weld diameters were 
measured only for welds after 100 welds and after 3000 welds although the wear tests were done after 4000 welds. Marks with a character " $\mathrm{s"}$ " in the figure indicate that a splash occurred during welding. Scatters are observed in the figure when the measured weld diameters are arranged with the welding current value.

Figure 11(b) shows the relationship between the measured weld diameters and the peak values of estimated mean weld temperature calculated by Equation 8 . The data were separated into two groups: One for the thinnest stack thickness, $0.8 \mathrm{~mm}$ with a $200 \mathrm{daN}$ electrode force; the other for the two stack type of $1.8 \mathrm{~mm}$ with a $400 \mathrm{daN}$ electrode force.

The contact diameter $d_{c}$ varies depending on the square root of electrode force $F$ applied on the weld part because of the contact diameter described by (Ref. 6):

$$
F=C_{s} \sigma_{Y} S \cdot g\left(d_{c} / h\right)
$$

where $F$ is the electrode force applied, $C_{\mathrm{s}}$ is a constant $(\approx 2.6-3.0), \sigma_{\mathrm{Y}}$ is the mean yield stress of weld part, $S$ is the contact area $\left(\pi d_{\mathrm{c}}{ }^{2} / 4\right)$, and $\mathrm{g}\left(d_{\mathrm{c}} / h\right)$ is the correction function. The value is supposed to be equal to 1.0 in this case.

This equation suggests that the measured weld diameter should be normalized with the ratio $\sqrt{F / F_{0}}$ to arrange all data on one curve. Figure 12 plots the normalized data according to the above consideration. The maximum scatter of weld diameter is less than $\sqrt{ } h$. Galvannealed steels also had good agreement.

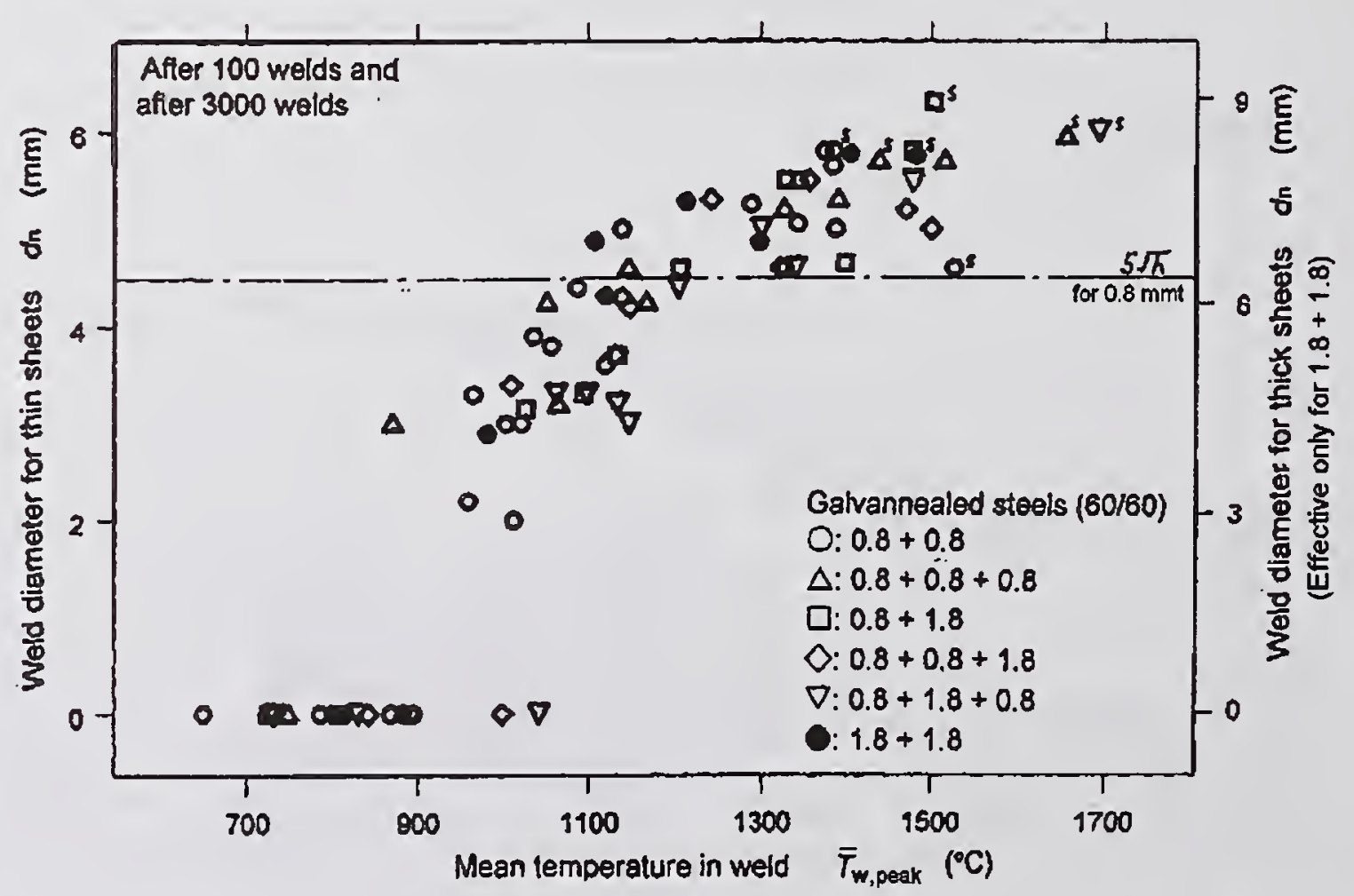

Figure 12: Arrangement of Normalized Weld Diameters with the Predicted Mean Temperature

All weld diameters are greater than the required weld diameter; $5 \sqrt{h}$ when the weld temperature is 
higher than $1200^{\circ} \mathrm{C}$. The minimum threshold value remains constant in spite of the coated materials, as shown in Figure 11(b), while splash occurs when the estimated weld temperature exceeds $1400^{\circ} \mathrm{C}$. The upper threshold value is lower than that shown in Figure 9 (b) for organic zinc coated steels. This suggests that the surface condition can influence the upper threshold temperature of the critical splash condition.

Alternatively, the normalized weld diameter can be estimated by the dynamic resistance curve calculated with the welding voltage and welding current because the contact diameter $d_{\mathrm{c}}$ is related to the dynamic resistance based on Equation 4. This alternative is especially effective on the low current and long weld time conditions like the class $\mathrm{C}$ conditions described on the RWMA (Resistance Welding Manufacturing Association) table.

Table 3: Threshold Value for the Critical Splash Condition vs. the Stack Configuration

\begin{tabular}{|l|c|c|}
\hline $\begin{array}{c}\text { Stack configuration } \\
(\mathrm{mm})\end{array}$ & $\begin{array}{c}\text { Corrected } \bar{T}_{w, \text { splash }} \text { based on the } \\
\text { stack configuration* }\left({ }^{\circ} \mathrm{C}\right)\end{array}$ & $\begin{array}{c}\bar{T}_{w, \text { splash }} \text { shown in Fig. 12 } \\
\left({ }^{\circ} \mathrm{C}\right)\end{array}$ \\
\hline $0.8+0.8$ & 1400 & 1400 \\
\hline $0.8+0.8+0.8$ & 1488 & 1450 \\
\hline $0.8+1.8$ & 1515 & 1500 \\
\hline $0.8+1.8+0.8$ & 1652 & 1700 \\
\hline
\end{tabular}

* Threshold values were calculated based on the value for the $0.8+0.8$ case and information on the stack configuration of each test piece.

The variation of the critical splash current values depend on the stack condition of workpieces can be explained by the temperature distribution along the plate thickness direction during welding. If the analytical solution can be applied for estimation of the temperature distribution, the peak temperature at the faying surface of each stack condition can be estimated with the experimental result on a two stack condition, and faying interface position of the stack. Estimated results of critical splash current for each stack are shown in the middle column of Table 3. The values were calculated based on the measured value under two sheet stack of $0.8 \mathrm{~mm}+0.8 \mathrm{~mm}$. Temperatures in the left column in Table 3 show the measured results in each stack conditions. Each corresponding values closely coincide with each other.

This suggests that if the information on the stack configuration of workpieces, the accuracy of threshold value for each critical splash condition expressed as the temperature can be improved.

\section{CONCLUSION}

A new monitoring and control system was developed based on the energy balance model in a weld part during resistance spot welding. Mathematical analysis deduced a new governing equation and procedure. The new concept, described in integral form, was proved with prior data and experimental results. This concept realizes a new adaptive control system that not only predicts splash occurrences in real time but also estimates weld diameter. Important conclusions of the present research are listed below: 
1) A new governing equation of integral form was developed based on an energy balance model in a weld part during welding.

2) A new discrete equation can be applied to real time prediction of weld diameter and splash with at least two monitoring data, i.e., welding voltage, welding current, and one pre-measured value of total plate thickness.

3) If the measured weld size is normalized by the square value of the electrode force, there is good agreement between the normalized weld diameter and the peak value of mean weld temperature.

4) The maximum weld diameter is closely related to the applied electrode force for welding.

5) Only the upper threshold for defining critical splash current is influenced by the surface condition of workpieces. The lower threshold for minimum weld diameter is influenced by physical properties of the bulk materials.

6) Threshold values for splash occurrence in various stack configuration condition can be estimated with information on stack configuration and position of faying interface in the stack based on a measuring result of the mean weld temperature for two stack condition of equal sheets.

\section{ACKNOWLEDGEMENT}

The authors are very grateful for the support provided by Kawasaki Robotics (USA) Inc., Osaka Denki Co., Ltd, Dengensha Mfg. Co., Ltd., and Robotron Inc.

\section{REFERENCES}

1. Nishiguchi, K, Matsuyama, K., et al., 1987, Influence of current wave form on nugget formation phenomena when spot welding thin steel sheet, Welding in the World, 25 (11/12), pp 222-244.

2. Matsuyama, K. et al., 1990, Computer-Aided Monitoring System of Nugget Formation Process, The fifth Symp. of Japan Welding Society (JWS) in Makuhari, pp 577-582.

3. Fujii, K., Matsuyama, K., et al., 1995, Resistance Spot Welding Monitors, Automotive Manufacturing International '95, pp 168-173.

4. Matsuyama, K., Obert, R., Chun, J-H., 2001, Inverse method for measuring weld temperatures during resistance spot welding, $\underline{\mathrm{SAE}}, \mathrm{SP}-1621$, pp.131-137

5. Nishiguchi, K., Matsuyama, K., et al., 1978, A study on nugget formation process in resistance spot welding of multi stack plates, Com. on Resistance welding in Japan Welding Society, Doc. RW-148-78 (in Japanese).

6. Matsuyama, K., Chun, J-H., 2000, Splashing mechanism in resistance spot welding, Proc. of International Sheet Metal Welding Conference in Steringheight IX, Paper. 5-4 
Session B1：Modeling I: Distortion and Stress 



\title{
AN INTEGRATED FEA BASED PROCEDURE FOR WELD FIXTURE DESIGN
}

\author{
Z. Yang*, X. Chen*', Y. Dong* \\ E. Martin", D. Michael
}

\begin{abstract}
As mechanical forces and welding induced thermal stress are applied during welding, the strength of the weld fixture is one of the crucial factors determining a successful or unsatisfactory welding practice. In the present study, an integrated FEA based procedure for welding fixture design was established. In the integrated FEA procedure, the welding induced stress and distortion were simulated by sequentially coupling thermal and mechanical modeling using a comprehensive welding simulation package. The welding simulation results showed that thermal induced reaction forces were significant at the constraint locations, which must be considered for weld fixture design. The magnitudes of reaction forces varied depending on constraint locations during welding process. The obtained maximum reaction forces were then input into weld fixture structural analysis. The predicted stress and distortion from the fixture structure analysis provided a guideline for the weld fixture design.
\end{abstract}

\section{KEYWORDS}

Welding Simulation, FEA, Stress and Distortion, Fixture Design

\section{INTRODUCTION}

Residual stresses and distortions are two of the major concerns in welded structures. The welding induced residual stress can exceed the yield strength of the material and is detrimental to the integrity and fatigue life of the welded parts. Welding can also cause the welded products to distort significantly from their original shape, which may require costly post-weld treatment such as machining or straightening. In recent years, tremendous efforts have been made to reduce weld distortion. Various methods have been proposed to accomplish this, including precambering, pre-bending, preheating, and thermal tension.

Thermal-mechanical FEA of the welding process is an emerging and rapidly maturing technique. Computer aided design of the welding process is becoming an efficient and effective approach to achieve high quality weld products in industry (Ref. 1-5). Welding simulation helps to optimize welding procedure (welding parameters, sequence, and weld joint geometry) and apply appropriate mechanical or thermal methods to reduce welding induced residual stress and distortion. In recent years, welding computer models have demonstrated the capability to reduce fabrication costs, improve weld quality and increase service durability by optimizing the weld process.

\footnotetext{
- Technica/Service Division, Caterpillar Inc., 1311 East Cedar Hills Dr., Mossville, IL 61656-1875

* Mining and Construction Equipment Division, Caterpillar Inc., 27 Pershing Rd., Decatur, IL 62525-1817
} 
The weld fixture is normally used to facilitate assembly of parts and to hold the parts in a fixed relationship for welding fabrication. In welding industry, it is common to employ techniques prior to the welding processes that induce distortions in the material that are essentially opposite to the distortions induced by the welding process. Pre-cambering is such a method that places the material into a weld fixture, which holds the material in the desired distorted shape until welding is completed. The intent of the induced distortions is to mitigate the distortion induced by welding to maintain the desired shape after welding.

Weld fixtures have typically been designed based on empirical data and past experience. Many trial-and-error tests are needed for designing a new weld fixture, which leads to not only costly design but also delays in production. Furthermore, a weld fixture for pre-cambering must be designed to withstand reaction forces from the mechanical distortions induced on the material, and from thermal distortions produced by the weld process. However, the thermal induced reaction forces were usually ignored or not fully understood in previous fixture design due to the lack of knowledge in this area. As a result, the designed weld fixture might deform or even fracture during welding, which significantly affected the quality and reliability of welded product.

In this paper, an integrated FEA based procedure for weld fixture design is proposed. In this procedure, the thermal and mechanical reaction forces were considered in combination to design weld fixtures for welding processes, especially for pre-cambering technique. Welding induced distortion and thermal reaction forces were calculated by using a comprehensive welding simulation tool (Ref. 1-5), which has been proven to be very efficient and effective in prediction of weld distortion and residual stress in large fabricated structures in industry.

\section{OUTLINE OF STUDY AND METHODS}

\section{Modeling Algorithm}

The algorithm of the integrated FEA based fixture design procedure is shown in Fig. 1. The procedure can be categorized into three steps: (1) model preparation, (2) welding simulation, and (3) fixture strength analysis. Steps (1) and (3) are common in FEA for structure analysis. Step 2, welding simulation, is critical in the present study. It calculates the thermal distortion in the

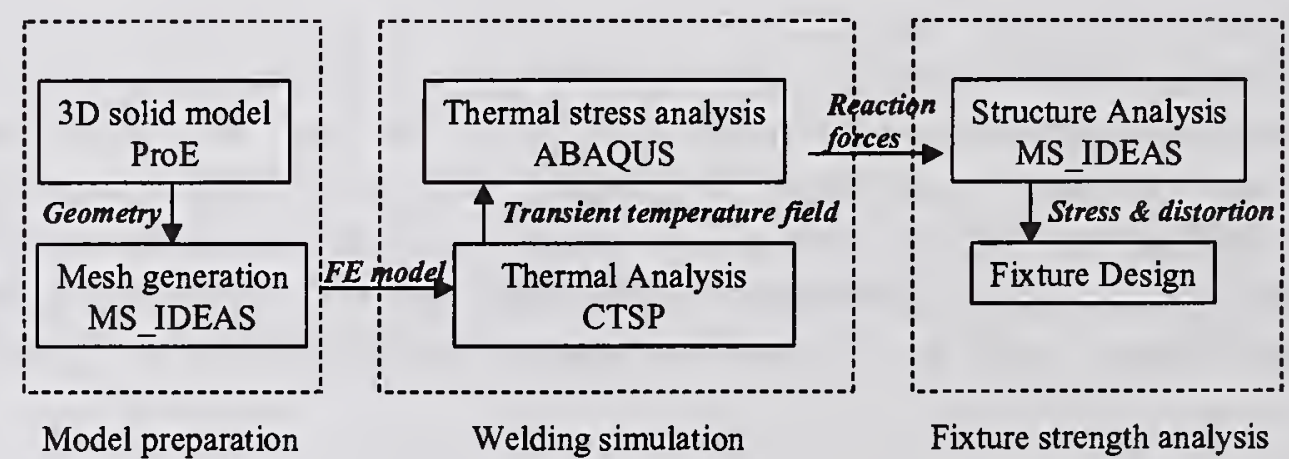

Fig. 1: Flow chart of FEA based weld fixture design. 
weld products and reaction forces to the fixture during welding process. The reaction forces were then input into weld fixture model for stress analysis and distortion prediction in order to examine the stiffness of the designed weld fixture.

\subsection{Welding Thermal Analysis}

The welding simulation was done by sequentially coupling thermal and stress analysis. Two approaches, numerical or analytical solution, can be taken for thermal analysis. Numerical solution is the commonly used approach in FEA thermal-mechanical analysis. However, it requires extensive manpower and computer resources to analyze complex weld structures. In the present study, an analytical based Comprehensive Thermal Solution Procedure (CTSP) was used, which is able to simulate temperature-time history for various types of weld joints (V-groove, Tfillet and lap joint) in complex welded structures (Ref. 1,2). The details of CTSP are given in literature and only its essential features are given here (Ref. 1):

$$
\begin{aligned}
& T(\zeta, y, z, t)=T_{s s}(\zeta, y, z) \cdot F(\zeta, y, z, t) \\
& T_{s s}(\zeta, y, z, t)=\frac{Q}{4 \lambda \pi R} \exp \left(-\frac{v \zeta+\nu R}{2 \alpha}\right) \\
& F(\zeta, y, z, t)=\frac{1}{2}\left[\exp \left(\frac{v R}{\alpha}\right)\left(1+\operatorname{erf}\left(\frac{v t+R}{2 \sqrt{\alpha t}}\right)\right)+\left(1+\operatorname{erf}\left(\frac{v t-R}{2 \sqrt{\alpha t}}\right)\right)\right]
\end{aligned}
$$

where $T(\zeta, y, z, t)$ is the transient temperature field, $T_{s s}(\zeta, y, z, t)$ is the quasi-static state temperature field, $F(\zeta, y, z, t)$ is the transient transform function, $Q$ is welding heat input, $\lambda$ is conductivity, $R$ is the radius from point heat source, $v$ is welding speed, $\alpha$ is thermal diffusivity, erf is error function, and $\zeta=x-v t$, with the heat source traveling in the $x$ direction. It should be noted that equations (1), (2), and (3) are valid for an infinitely thick plate. For plates with finite thickness, it is assumed that the heat loss from the surfaces is negligible in comparison with that due to conduction in an infinite body and a number of reflected heat sources are used to balance heat loss at surfaces.

\subsection{Welding Stress Analysis}

The weld residual stress analysis was done using commercial FEA software - ABAQUS. A special user material subroutine (UMAT) was developed to account for most of the essential features in welding process, which include the effects of stress/strain history annihilation due to material melting/remelting, weld metal deposition, and material phase changes. The details for the weld UMAT and its numerical implementation are given in literature (Ref. 3, 4). The material properties critical to weld residual stress analysis (yield strength, thermal expansion coefficient, true stress-strain curves) were determined experimentally.

\subsection{Fixture FEA Structural Analysis}

The objective of fixture FEA structural analysis was to evaluate the stress and distortion of the fixture due to the reaction forces when parts are pre-cambered and welded in the fixture. The analysis used the loosely coupled method. The reaction forces at various clamping locations were obtained from the welding simulation where the clamping locations were assumed to be 
fixed. The forces were then applied to the fixture model and the stress and distortion were assessed using linear FEA structural analysis.

\section{Case Study}

In this case study, a pre-cambering robotic welding process was used to control welding distortion to eliminate post-weld treatment such as straightening and machining. Welding simulation and weld fixture stiffness analysis were the major tasks to define a pre-cambering robotic welding process that would hold the flatness tolerance after welding.

\subsection{Welding experiments}

A large fabricated structure, as shown in Fig. 2, was robot welded using GMAW process. The weld material is equivalent to ASTM A572 steel. There are total 26 welds in consideration in the product, as indicated by the red arrows in Fig. 2. The total welding length is about 20 meters. The welding sequence was optimized for minimum distortion based on welding simulation results. Single-pass $10 \mathrm{~mm}$ and $6 \mathrm{~mm}$ fillet welds were used on top and bottom sides, respectively. The welding parameter for $10 \mathrm{~mm}$ welds were: $33.7 \mathrm{~V}, 400 \mathrm{~A}$, and $4.8 \mathrm{~mm} / \mathrm{s}$. The welding parameters for $6 \mathrm{~mm}$ welds were $23.5 \mathrm{~V}, 265 \mathrm{~A}$, and $7.0 \mathrm{~mm} / \mathrm{s}$. Prior to welding, the structure was tack welded and pre-cambered in the fixture. The magnitudes and direction at various pre-cambering locations varied depending on the predetermined results from the welding simulation.

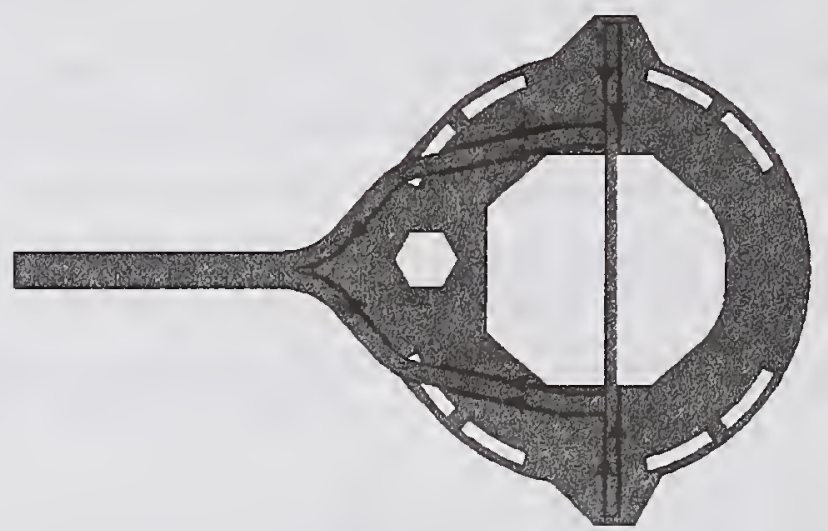

(a)

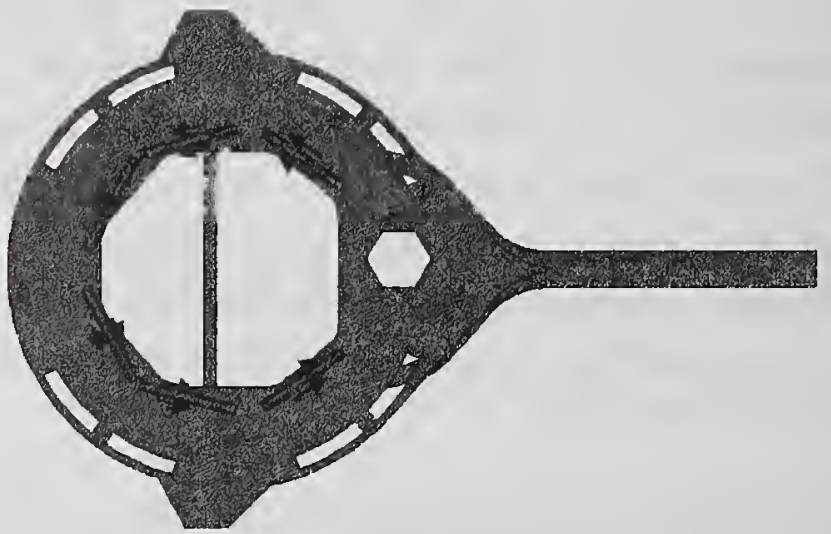

(b)

Fig. 2: Welds in the studied structure: (a) top side and (b) bottom side.

\subsection{FEA models}

The FEA models for the weld product and weld fixture are shown in Fig. 3(a) and (b), respectively. The FEA model for the weld product was built with hexahedral solid elements. There were 19,820 elements and 25813 nodes in the weld product model. The size of elements within and near the welds was very fine so that welding induced thermal and mechanical features can be captured. In contrast, the size of the elements far away from weld line was very coarse for efficient computation. The weld fixture FEA model consisted of three types of elements - solid, shell, and beam elements. There were 74,374 elements and 80,260 nodes in the weld fixture model. 


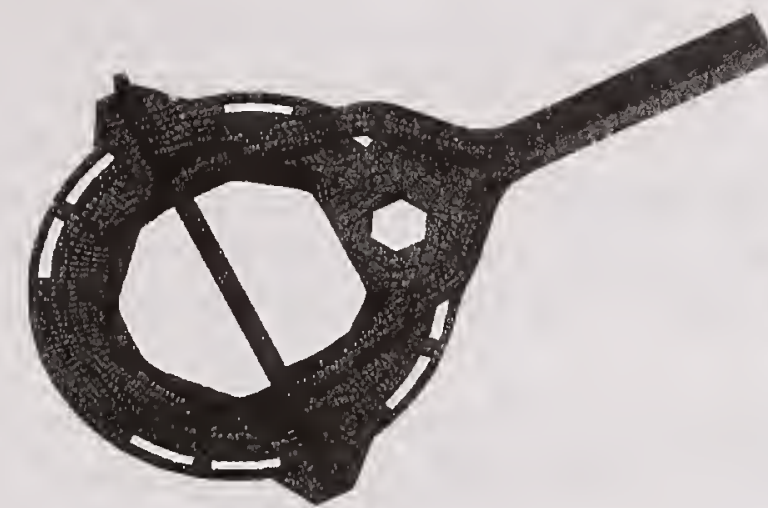

(a)

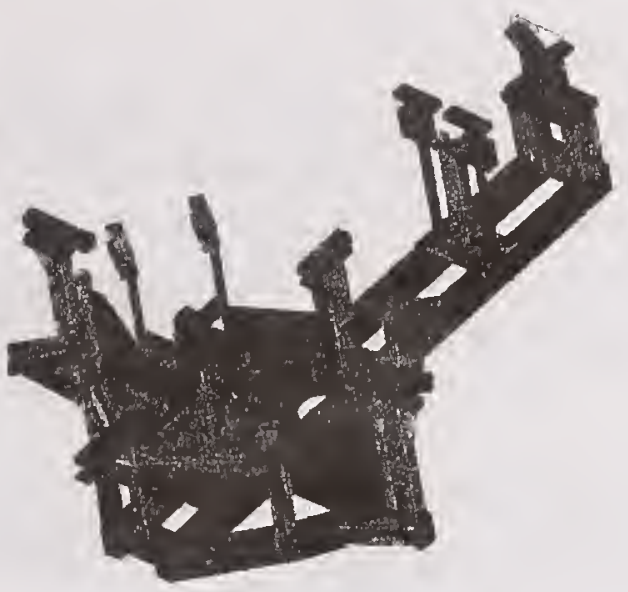

(b)

Fig. 3: FEA models for (a) weld product and (b) weld fixture.

\subsection{Results and discussions}

\subsubsection{Temperature and stress fields}

Examples of the calculated transient temperature and stress fields during welding are shown in Fig. 4(a) and (b). As shown in Fig. 4(a), the transient temperature field due to the moving welding heat source is well demonstrated. The welding induced residual stress is in the magnitude of the yield strength of the material (around $450 \mathrm{MPa}$ ). This predicted residual stress magnitude is reasonable and comparable with those reported in literature.

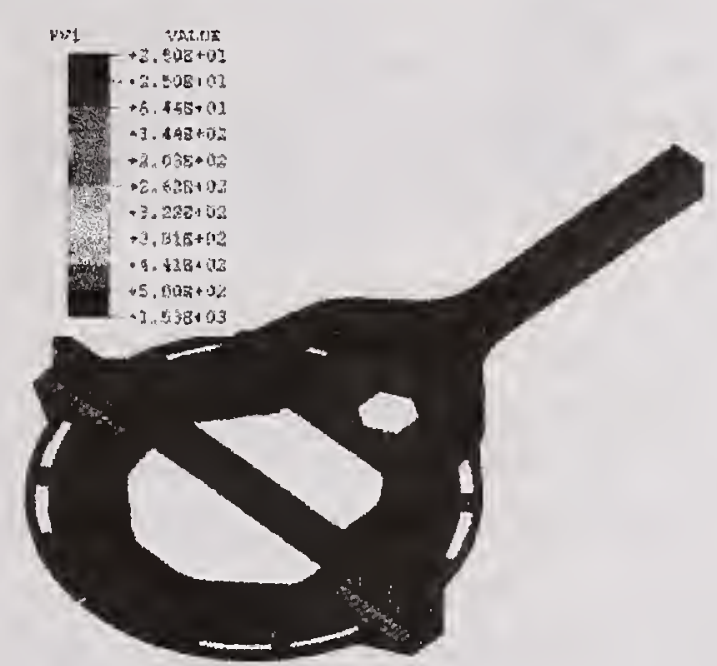

(a)

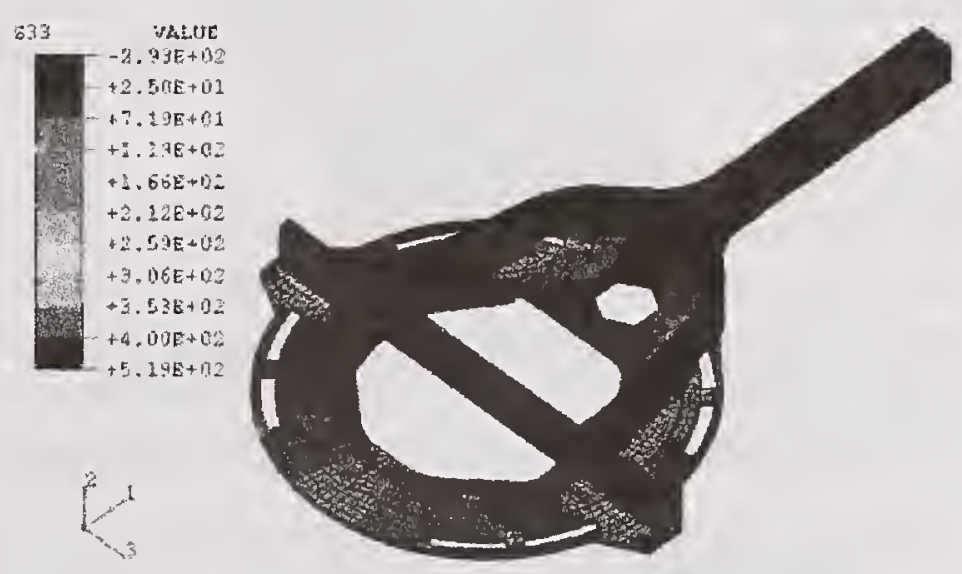

(b)

Fig. 4: (a) calculated temperature field and (b) calculated stress field.

\subsubsection{Reaction forces on weld fixture}

In the welding simulation, the clamping locations were assumed to be fixed in the welding model. The reaction forces on weld fixture at various pre-cambering locations during and after welding are shown in Fig. 5. As stated in previous sections, the reaction forces during welding in this case study were the combination of the mechanical force from pre-cambering and the thermal induced force from welding. The mechanical forces due to pre-cambering are shown at time zero in Fig. 5, where the welding process was not yet started. The values of the mechanical forces at various locations depend on the magnitudes and directions of pre-cambering at the corresponding positions. 


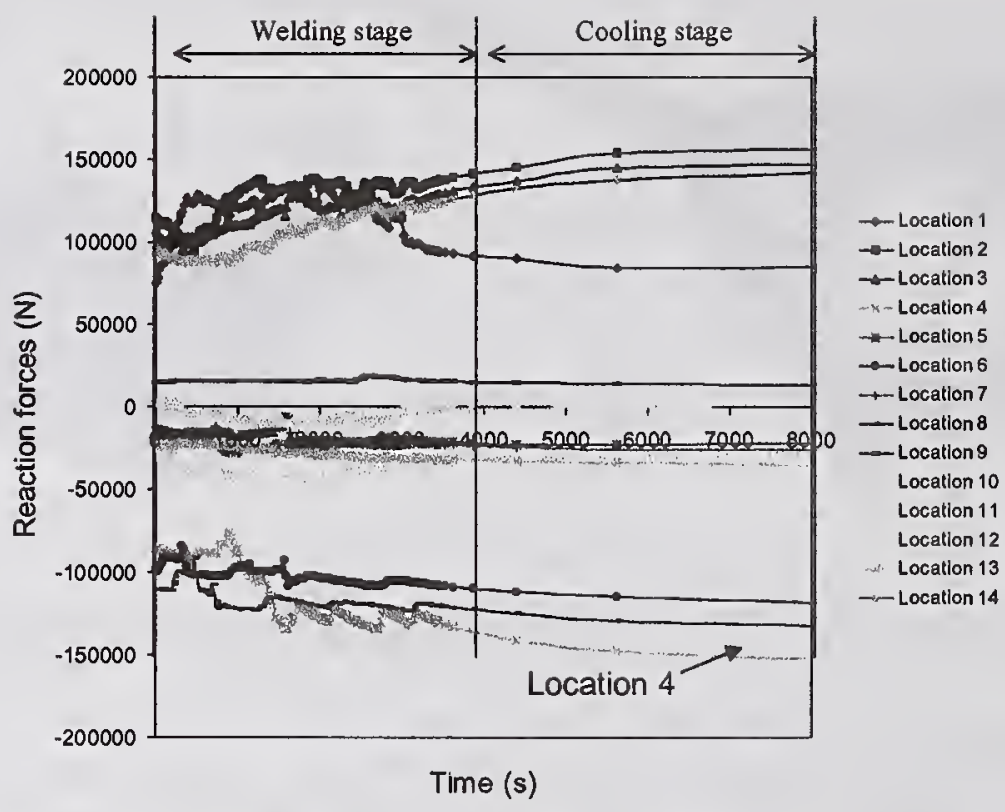

Fig. 5: The reaction forces at various clamping locations during welding process

From Fig. 5, it can be observed that the reaction forces varied significantly during the welding and subsequent cooling stage. Such variations resulted from welding induced thermal stress. It can be seen that the reaction forces at some locations were almost doubled due to welding induced thermal reaction forces. For example, the reaction at location 4 before welding (time $=0$ ) was around $-85 \mathrm{kN}$ and its value reached $-153 \mathrm{kN}$ after welding. Therefore, the fixture would have been significantly under-designed without considering the thermal induced reaction force.

\subsubsection{Fixture Stiffness Analysis}

The stiffness of weld fixture was examined through linear static analysis, in which the reaction forces at the final cooling stage from the welding simulation were applied to the fixture FEA model. This is because the maximum reaction forces at most of the pre-cambering locations were reached at the final cooling stage, as shown in Fig. 5. The results from the structural analysis show that the fixture would be significantly deformed, as shown in Fig. 6(a), and the maximum

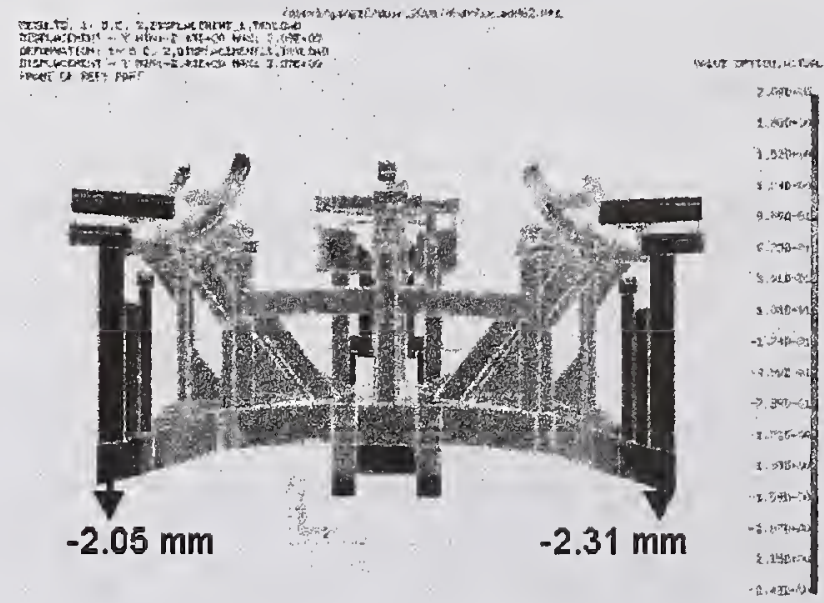

(a)

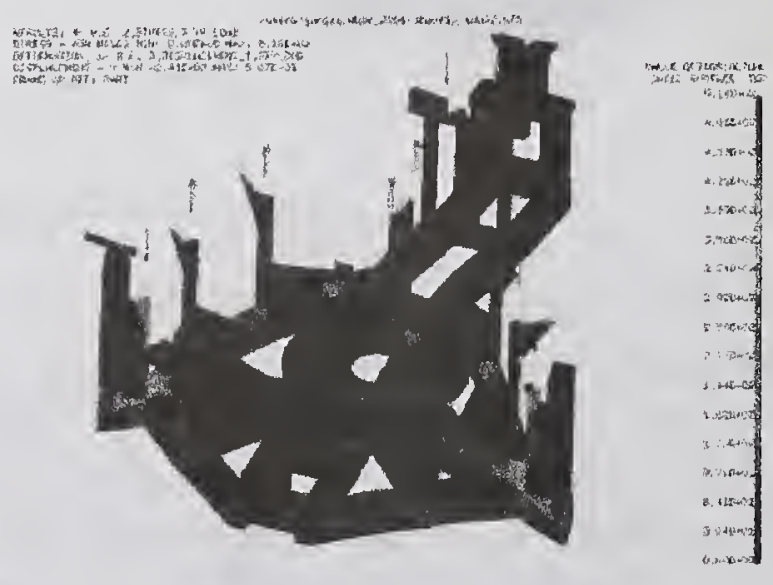

(b)

Fig. 6: (a) Deformation of weld fixture and (b) Stress distribution in weld fixture. 
stress in the "hot" spot in the model is $518 \mathrm{MPa}$, as shown in Fig. $6(\mathrm{~b})$, which is much higher than the yield strength of the material. This implies that the original fixture was under-designed. During welding experiments, it was also observed that some clamps for pre-cambering were broken during welding due to significant thermal induced reaction forces. The failure of the clamps in the fixture and its global deformation would deviate the defined pre-cambering scheme and thus affected the flatness of the bottom plate after welding.

Based on the results of the fixture structure analysis, the weld fixture was modified to increase the stiffness in order to account for welding induced additional reaction forces. The deformation and stress distribution in the modified weld fixture is shown in Fig. 7 (a) and (b), respectively. Compared to that in original fixture, the deformation in the modified fixture was significantly reduced. Also, the stress in the modified fixture model was reduced to $298 \mathrm{MPa}$. Therefore the modified weld fixture is strong enough to withstand the combination reaction forces and maintain the pre-cambering scheme.
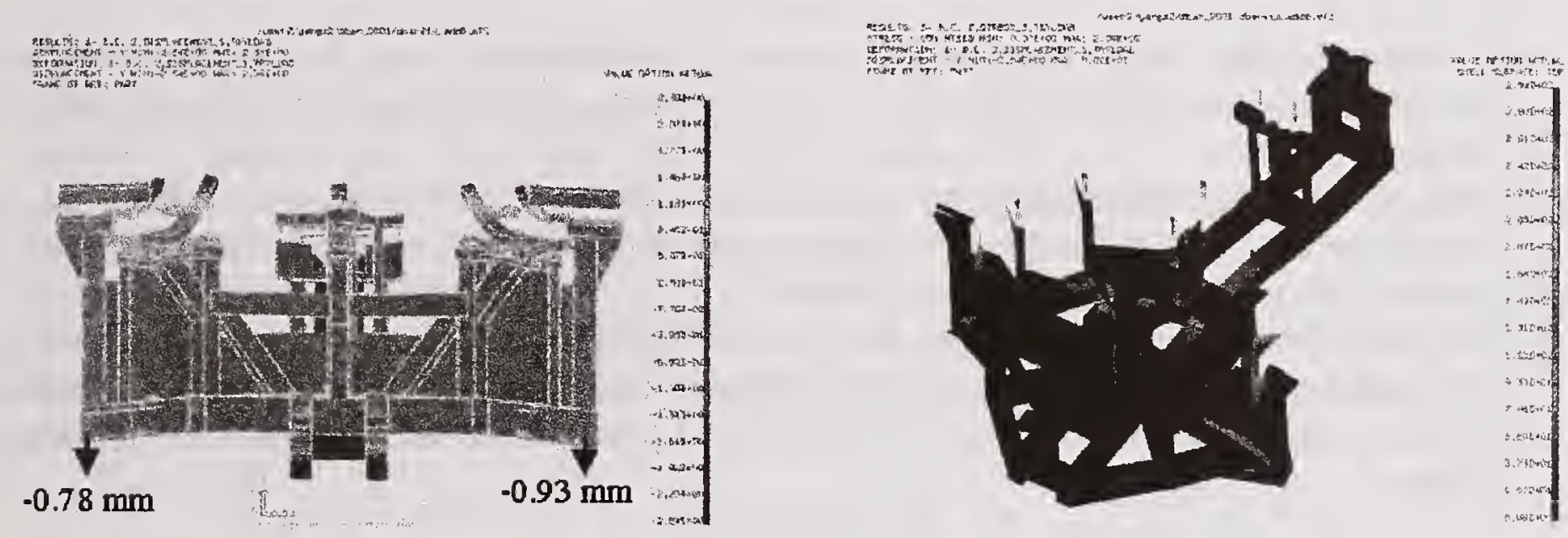

Fig. 7: (a) Deformation of weld fixture and (b) Stress distribution in the modified weld fixture.

\subsubsection{Effect of weld fixture stiffness on product flatness}

The distortions at critical points on the bottom plate of the welded parts were measured after the parts were cooled to room temperature and released from the fixture. The flatness check was

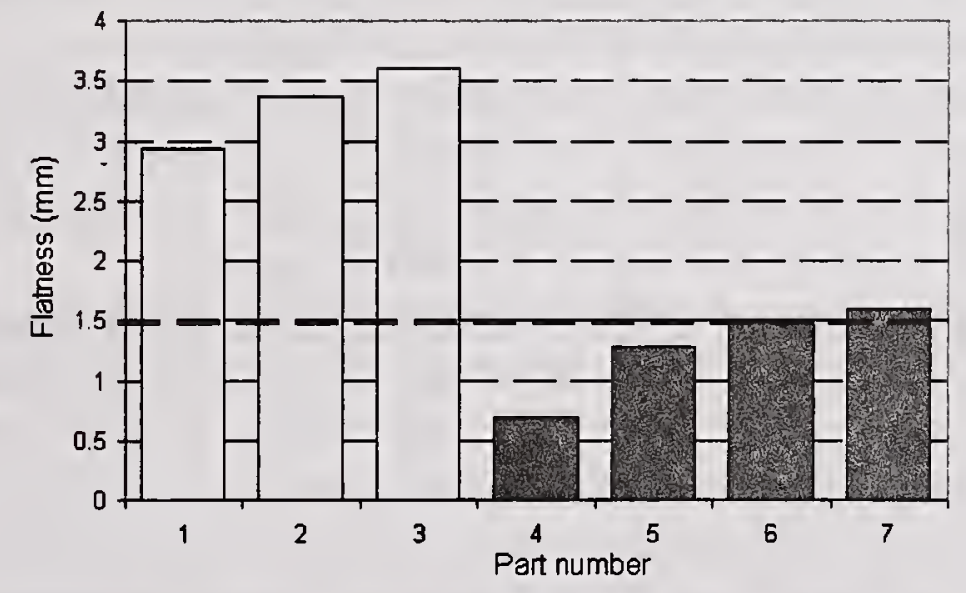

Fig. 8: Distortions in weld products using original and modified weld fixtures

(Parts 1-3 used original fixture and parts 4-7 used modified fixture) 
made for seven weld parts, of which three were welded using the original weld fixture and four were welded using the modified weld fixture. The degree of flatness in these weld parts are compared in Fig. 8. It can be observed that the distortions in the three parts using the original fixture were much larger than the flatness tolerance $(1.5 \mathrm{~mm})$. As mentioned previously, this is primarily due to the failure of the parts in the fixture (clamps) and the global deformation of the fixture during welding. In contrast, the distortions were significantly reduced after using the modified weld fixture that was stiff enough to support the pre-cambering scheme. Almost all four parts meet the flatness requirement.

\section{SUMMARY AND CONCLUSIONS}

An integrated FEA based procedure for weld fixture design was proposed in the present study. The procedure was applied to examine the strength of an existing weld fixture by taking into account both the mechanical forces from pre-cambering and the welding induced thermal reaction forces. The following conclusions can be made based on the present study.

(1) Welding induced thermal reaction forces are significant and must be considered in weld fixture design, especially when the fabricated structure is large and the number/length of welds increases.

(2) Weld fixtures may deform or even break during welding if the reaction forces from thermal distortions are not considered. The deformation of the fixture itself will directly affect the pre-cambering process for distortion control.

(3) Welding simulation is critical in the integrated FEA based procedure for weld fixture design. The welding simulation tool used in this study has demonstrated the capability to effectively predict residual stress, distortion, and reaction forces, which are critical for weld fixture design.

\section{REFERENCES}

1. Cao, Z., Brust, F. W., Nanjundan, A., Dong, Y., and Jutla, T.: Advances in Computational Engineering and Sciences; Eds. S. N. Atluri and F. W. Brust, p. 630, Tech Science Press, 2000.

2. Cao, Z., Brust, F. W., Nanjundan, A., Dong, Y., and Jutla, T.: A Comprehensive Thermal Solution Procedures for Multiple Pass and Curved welds, 2000 ASME Pressure Vessels and Piping Conference, Seattle, Washington, July 23-27,2000.

3. User Manual for UMAT-CAT - A Welding Specific User Material Routine Interfaced with ABAQUS, Version 3.1, Caterpillar Inc., Peoria, IL, September 1999.

4. Brust, F. W., Dong, P., and Zhang, J.: Advances in Computational Engineering Science, Eds. S. N. Atluri, and G. Yagawa, p.51, Tech Science Press, 1997.

5. Brust, F. W., Yang, Y., Dong, Y., and T. Jutla: Advances in Computational Engineering and Sciences; Eds. S. N. Atluri and F. W. Brust, p. 714, Tech Science Press, 2000. 


\title{
WELDING INDUCED DISTORTIONS MODELING OF LARGE PLATE STRUCTURES
}

\author{
R. D. Everhart ${ }^{*}$
}

\begin{abstract}
A method for predicting the welding induced distortions of large plate structures is described. Predictive, three-dimensional modeling of large structures presents some special numerical challenges. As models become large (as required by accuracy considerations) demands on computer resources grow quickly. Run times can grow to days and weeks. Computer memory can be overrun quickly, and the simulation becomes impractical. To overcome some of these challenges, a method that is accurate and numerically efficient is detailed. The methodology is incorporated in a new computer program called EFFECT (Efficient, Fast First-principle's Engine for Combined Thermal-mechanical). EFFECT incorporates a very efficient thermal solver. Temperature distributions are generated "on the fly" to drive the mechanical solution. Finite element shell elements are used to model deformation of the plate structures. The BelytschkoTsay large displacement, geometrically non-linear shell element is employed. A selection of sophisticated plasticity models that accurately deal with material melting and solidification are available. Explicit time integration is used for the mechanical solution. The shell element that is used is computationally efficient, and the explicit time integration greatly reduces computer memory requirements. A novel time scaling technique is used to deal with the thermal and mechanical time scale differences. This method is especially useful for predicting the buckling and out-of-plane warping of large plate structures. Model results are compared with distortion test data from large steel test structures. The model is able to accurately predict buckling and warping behavior.
\end{abstract}

\section{KEYWORDS}

Welding, modeling, finite-element analysis, distortion, explicit time integration.

\section{INTRODUCTION}

A method for predicting the welding induced distortions of large plate structures is introduced in this paper. Some background on the problem will be given, the computational methods will be described, then two examples involving warping and buckling will be presented.

\section{BACKGROUND}

As pointed out by Brown and Song [1], accurate distortion modeling of large, complex structures requires three-dimensional models. Three-dimensional finite element models of these structures include many thousands of degrees of freedom. This presents a considerable computational resource challenge even for modern computers. It is not practical for an analyst to wait several days, or even weeks, for the results of an analysis. A reasonable goal for analysis turn-around time is for an analysis to run over-night. An analyst can submit an analysis before leaving work

\footnotetext{
Edison Welding Institute, 1250 Arthur E. Adams Drive, Columbus, OH 43221
} 
for the evening and have the results waiting the next morning. Run times of this order are the goal of the present development effort.

In order to meet the computational performance goals of this development effort, a paradigm shift was made in the solution strategy for these types of structures. Implicit time integration was discarded in favor of explicit time integration. Explicit time integration is used in such wellknown finite-element analysis codes such as LS-DYNA [2,3].

The use of explicit finite-element analysis techniques produces two benefits that are critical to the practical analysis of large structures. First, the in-core (RAM) memory requirement is vastly reduced. Explicit methods do not require the formation and storage of a stiffness matrix. Second, the explicit solution method is very efficient for large, highly non-linear problems. The implicit solution method convergence difficulties for highly non-linear material behavior are avoided.

This added performance comes at a cost however. Time scales must be carefully managed. The major inhibitor to the use of explicit time integration methods for these class of problems is that they are conditionally stable and are subject to the Courant condition [3]. The end result is that time steps are on the order of a fraction of a microsecond. It is easy to see that for welding simulations (typically several hundreds of seconds) many millions of time steps would be required for a simulation. This is clearly unacceptable. Therefore, time-scaling methods are employed to manage these time scale disparities. This technique and others are introduced in the computational methods section.

\section{COMPUTATIONAL METHODS}

The computational methods used for modeling welding induced distortions for large plate structures have been implemented in an analysis code called EFFECT (Efficient, Fast Firstprincipals Engine for Combined Thermal-mechanical). EFFECT can be thought of as a numerical test-bed for the consideration of explicit distortion analysis methods. In the following sections, some of the more critical numerical methods employed in EFFECT will be introduced.

There are two types of finite elements used. The plates that make up the structures are modeled with shell elements. The shell elements follow the formulation by Belytschko and Tsay [4]. A five-point numerical integration scheme is used in order to give good non-linear response performance. The weld beads are modeled explicitly with hexahedron continuum elements. They follow the formulation of Flanagan and Belytschko [5]. Both element types have a long history of successful application in such codes as LS DYNA $[2,3]$.

The thermal solution is performed concurrently with the mechanical solution in the same explicit time integration loop. They are fully coupled. In order to deal with the disparity between time scales for the mechanical and thermal solutions (the stable time step for the thermal solution is much larger), the thermal solution uses sub-cycling. That is, several mechanical solution steps are performed for each thermal solution step. Using this strategy, the thermal solution time ends up being a small percentage of the total solution time. 
The thermal solution algorithms used are similar to those developed by Liu and Belytschko [6]. The method is extended to hexahedron continuum elements and shell elements. The shell elements use a split integration technique. A two-dimensional transient heat conduction solution is performed in the plane of the element. A one-dimensional transient heat conduction solution is performed through the thickness of the element. This allows for full through-thickness effects to be included. This is important in weld zones.

The heat energy from welding is input to the system in two ways. First, the weld bead goes in "hot" (as it does in real life). The hexahedron elements that make up the weld bead are activated at a pre-determined temperature. Usually, this is the melting temperature of the material. The elements are activated according to the motion of the welding torch (speed and path). Second, welding energy is input to the base material to form the remainder of the heat affected zone. The Goldak (et. al.) double ellipsoidal heat source [7] is used to map the welding energy into the base metal.

Sophisticated material models are available for both hexahedron continuum elements and shell elements. Virtually all of the mechanical and thermal properties of the material may be input as a function of temperature. Phase changes are easily considered, so that melting and solidification are treated seamlessly in the analysis. To increase the flexibility of the models, information is input in a piece-wise linear fashion (tabular).

Proper time scale treatment is critical for application of explicit methods to welding simulations. The time scale differentials between the thermal and mechanical solutions are overcome using sub-cycling. There are several mechanical time steps for every thermal time step. Mass scaling is used to manage the mechanical solution time scale. This technique is common in metal stamping simulations for instance [8]. This technique takes advantage of the fact that the welding operation is a quasi-static process. Material velocities are vastly smaller than the sound speed of the material (on which the stable time step size is based). By artificially manipulating the masses, and thus the stable time step size, the time scale may be manipulated. This may only be reliably taken to the limit that material velocities remain at least a factor of 10 less than the artificial sound speed of the material. A check of this condition is made throughout the solution to insure compliance. This technique allows the character of the solution to be preserved while promoting computational economy in the solution.

These techniques form the core of the method. The method has been successfully applied to large plate structures, two of which are detailed in the following section.

\section{RESULTS AND DISCUSSION}

The explicit finite-element analysis methodology in EFFECT has been used to simulate the welding induced distortions in large plate structures. Two examples are presented in this paper. The first example involves out of plane warping, and the second example involves large scale buckling.

The out of plane warping of a steel plate structure with a welded tee joint (base plate 20 " $\times 20$ " $\mathrm{x} 0.5$ ") was analyzed with a full three-dimensional EFFECT analysis. A schematic of the 
process is shown in Figure 1, and a photograph of the experimental setup is shown in Figure 2. A tee joint is welded on both sides simultaneously using a GMAW process. Temperature measurements were taken with thermocouples, and displacement measurements were taken with LVDTs. A comparison between measured temperature time histories and calculated temperature time histories are shown in Figure 3. The calculation is shown to accurately predict temperature profiles for this experiment. A comparison between measured displacement time histories and calculated displacement time histories are shown in Figure 4 . The calculation accurately predicts the out of plane warping of the base plate. This calculation was run on a Pentium III Intel processor operating at a clock speed of $800 \mathrm{Mhz}$ in an overnight run, satisfying the computational performance goal.

The second example considers the bucking of a very large steel plate structure ( $8 \mathrm{ft} . x 20 \mathrm{ft} . x 3 / 16$ ") induced by welding three tee stiffeners using a GMAW process. Photographs of the experimental setup are shown in Figures 5 and 6 . The stiffeners are welded on both sides, and they are welded in succession. The welded structure suffers extensive buckling along the free long edges as evidenced in Figure 7. A full three-dimensional EFFECT analysis was performed on this configuration. A rendering of the result is shown in Figure 8. Severe buckling is clearly evident in the calculation results. The method was successful in predicting the onset and evolution of buckling. This calculation was also run on a Pentium III Intel processor operating at a clock speed of $800 \mathrm{Mhz}$ in a run time of approximately 24 hours. This is larger than the performance goal. In is anticipated that future performance gains (hardware and software) will result in meeting computational performance goals for this problem.

\section{SUMMARY AND CONCLUSIONS}

An explicit finite-element based method has been developed to simulate the welding induced distortions in large plate structures. A computer code called EFFECT has been written that incorporates those methodologies. The goal of the effort was to produce a computational scheme that is computationally efficient enough to approach this class of problems on existing computer platforms. A goal of overnight execution times was established. Two example problems are presented which demonstrate the successful application of the methodology.

\section{ACKNOWLEDGEMENTS}

The author of this paper would like to acknowledge the support of the United States Navy and the Naval Joining Center.

\section{REFERENCES}

1. Brown, S., Song, H., Finite Element Simulation of Welding of Large Structures, Journal of Engineering for Industry, 1992, Vol. 114, p. 441-451.

2. LS-DYNA Keyword User's Manual, Version 960, Livermore Software Technology Corporation, Livermore, California, March, 2001. 
3. Hallquist, J. O., LS-DYNA Theoretical Manual, Livermore Software Technology Corporation, Livermore, California, May, 1998.

4. Belytschko, T., Lin, J. I., Tsay, C. S., Explicit Algorithms for the Nonlinear Dynamics of Shells, Computer Methods in Applied Mechanics and Engineering, Num. 42, 1984, p. 225-251.

5. Flanagan, D. P., Belytschko, A Uniform Strain Hexahedron and Quadrilateral With Orthogonal Hourglass Control, International Journal for Numerical Methods in Engineering, Vol. 17, 1981, p. 679-706.

6. Liu, W. K., Belytschko, T., Efficient Linear and Nonlinear Heat Conduction With a Quarilateral Element, International Journal for Numerical Methods in Engineering, Vol. 20, 1984, p. 931-948.

7. Goldak, J., Chakravarti, A., Bibby, M., A New Finite Element Model for Welding Heat Sources, Metallurgical Transactions B, Vol. 15B, June, 1984, p. 299-305.

8. Jung, D. W., Study of Dynamic Explicit Analysis in Sheet Metal Forming Processes Using Faster Punch Velocity and Mass Scaling Scheme, Journal of Materials Engineering and Performance, Vol. 7, No. 4, August, 1998, p. 479-490.

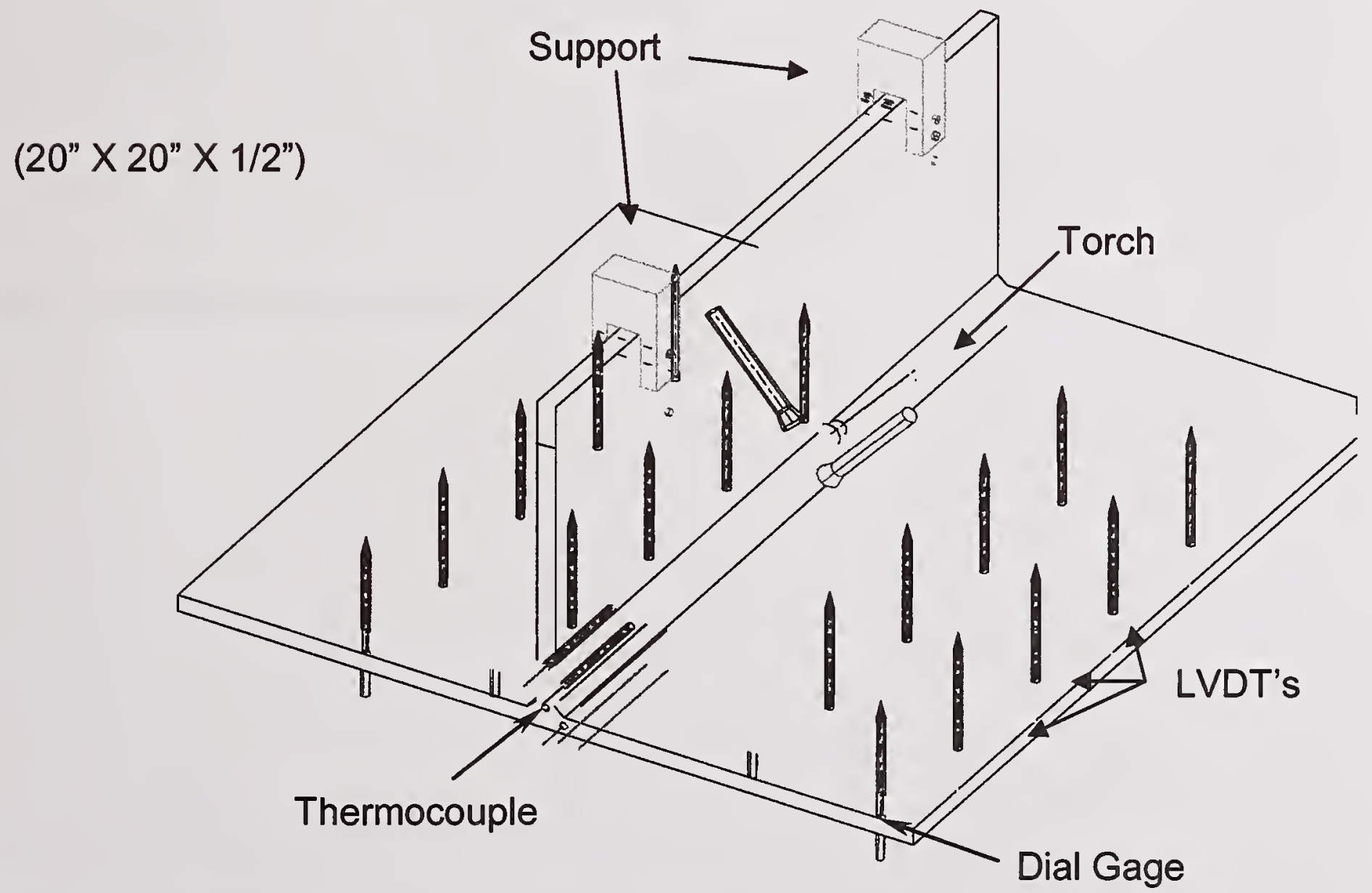

Figure 1 Schematic of Welded Tee Joint Experiment 


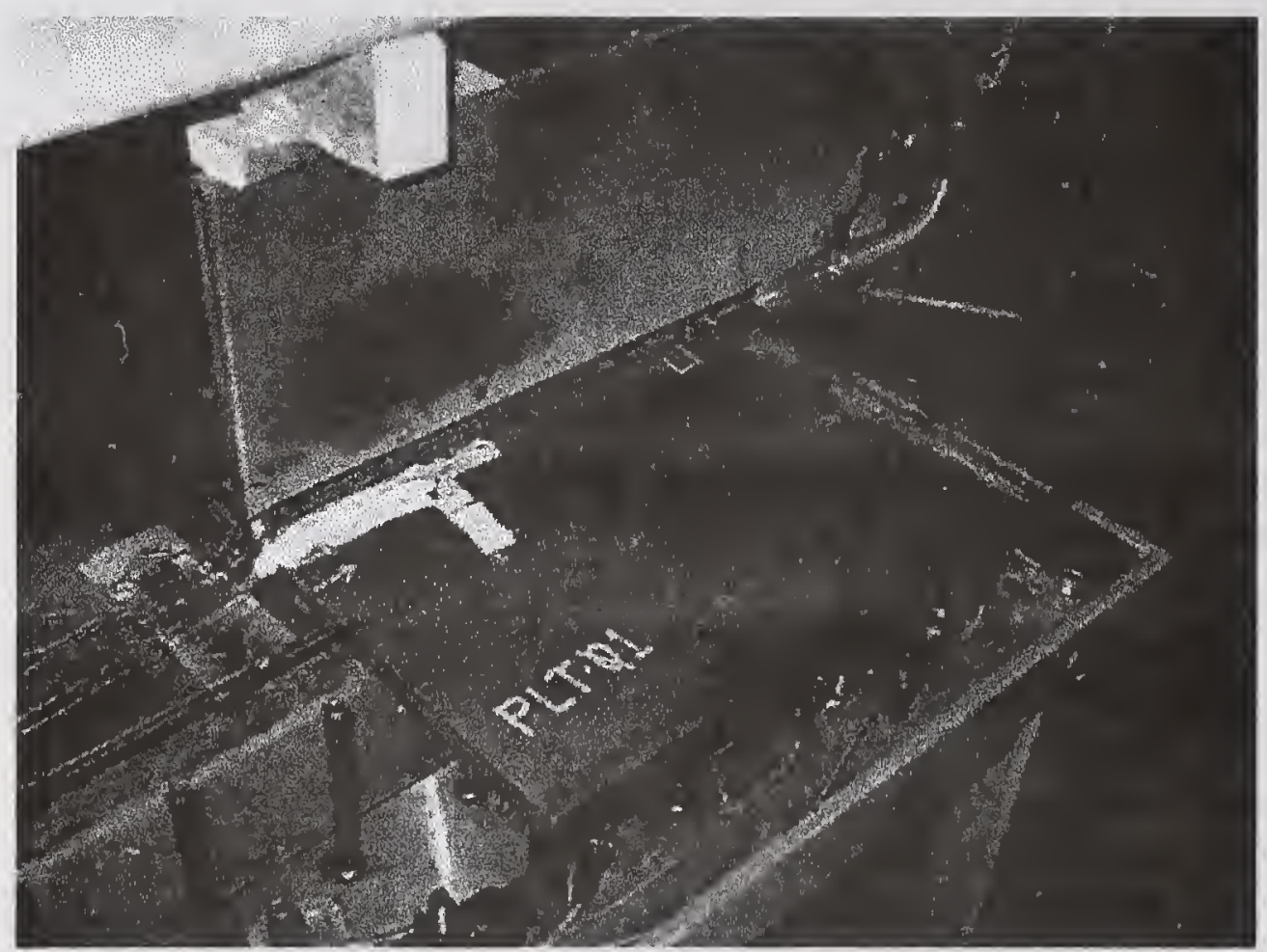

Figure 2 Image of Welded Tee Joint Experiment 


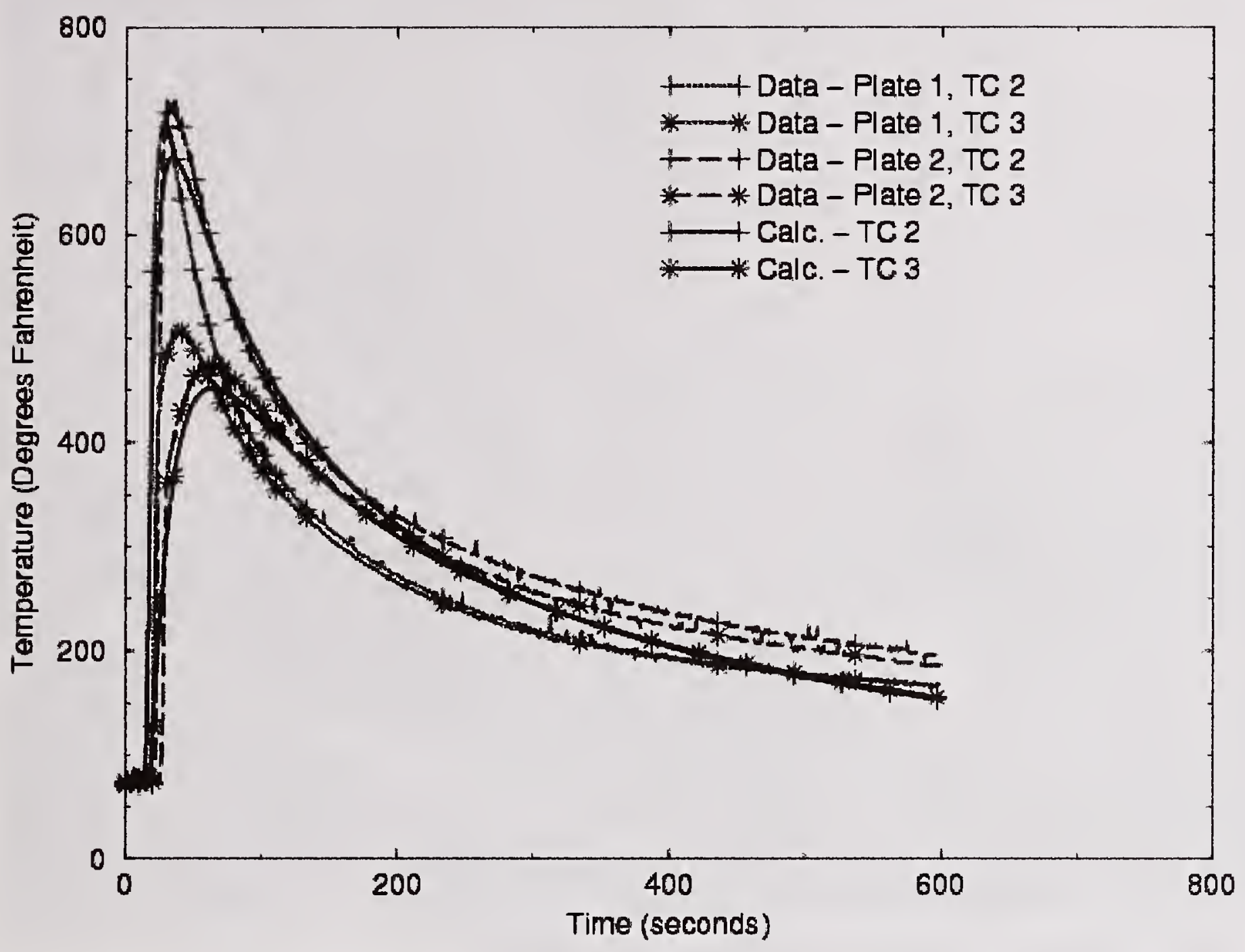

Figure 3 Temperature Time Histories 


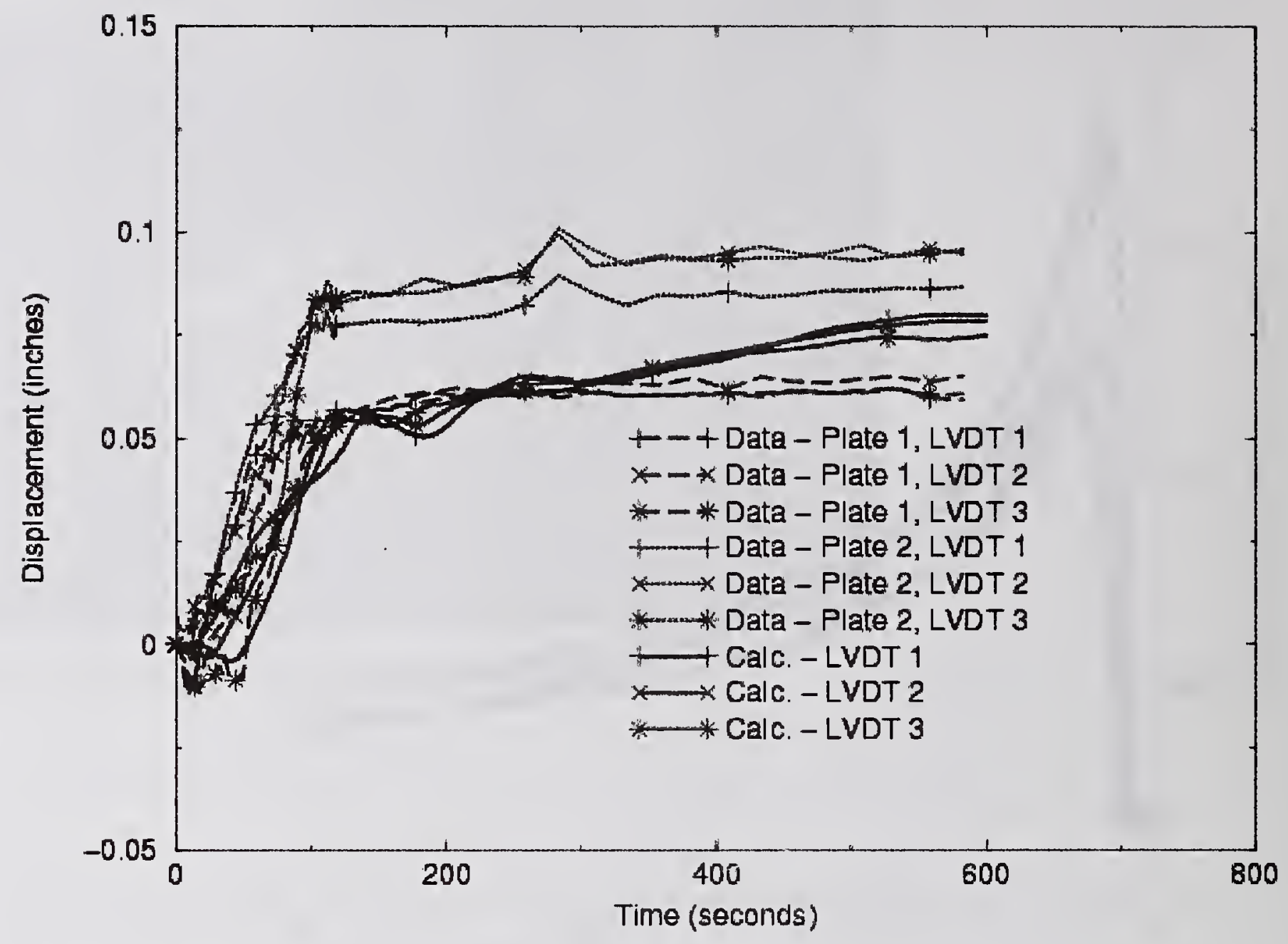

Figure 4 Displacement Time Histories (Up is Positive) 


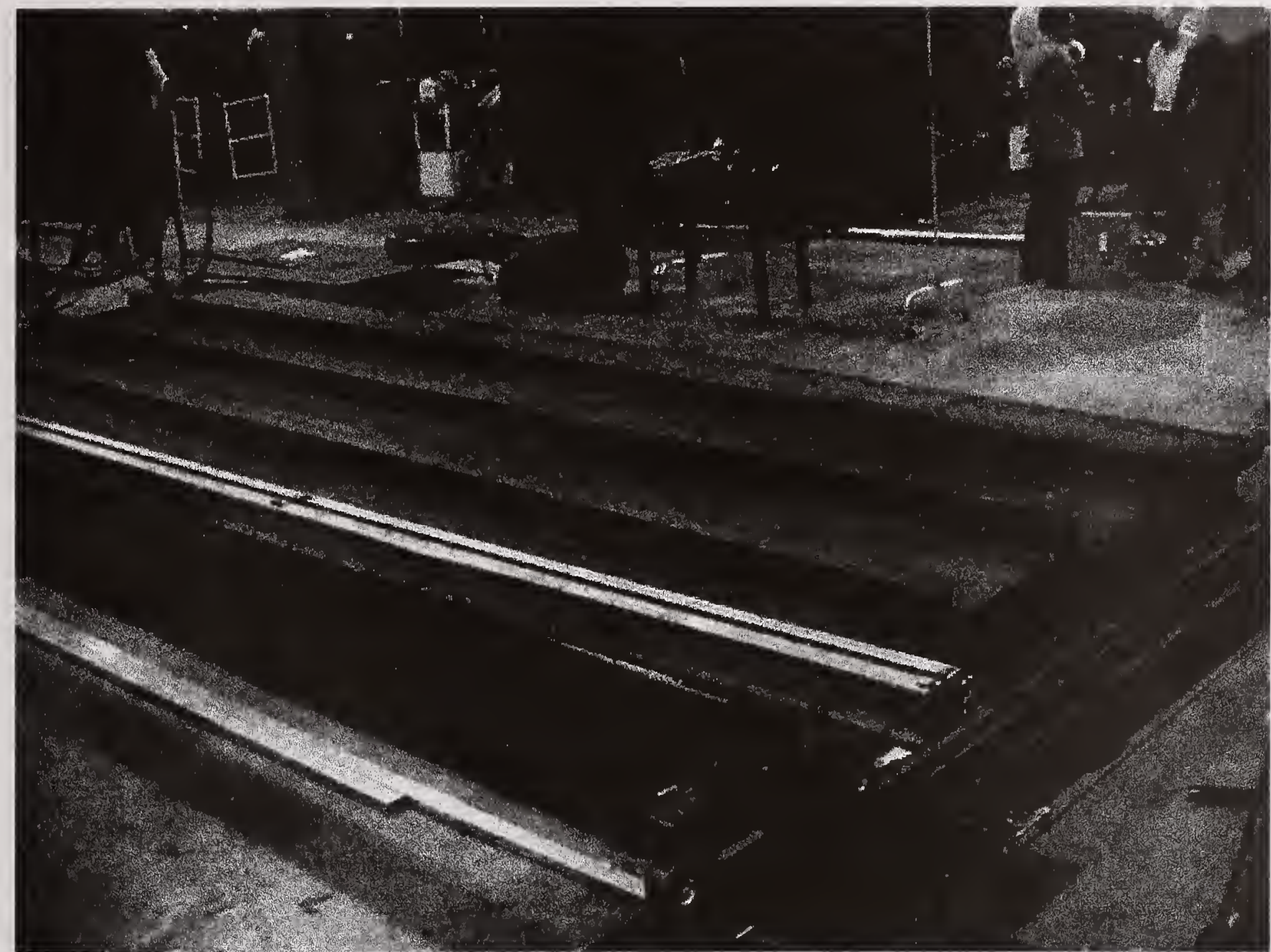

Figure 5 Photograph of $8^{\prime} \times 20^{\prime} \times 3 / 16^{\prime \prime}$ Plate Experiment 


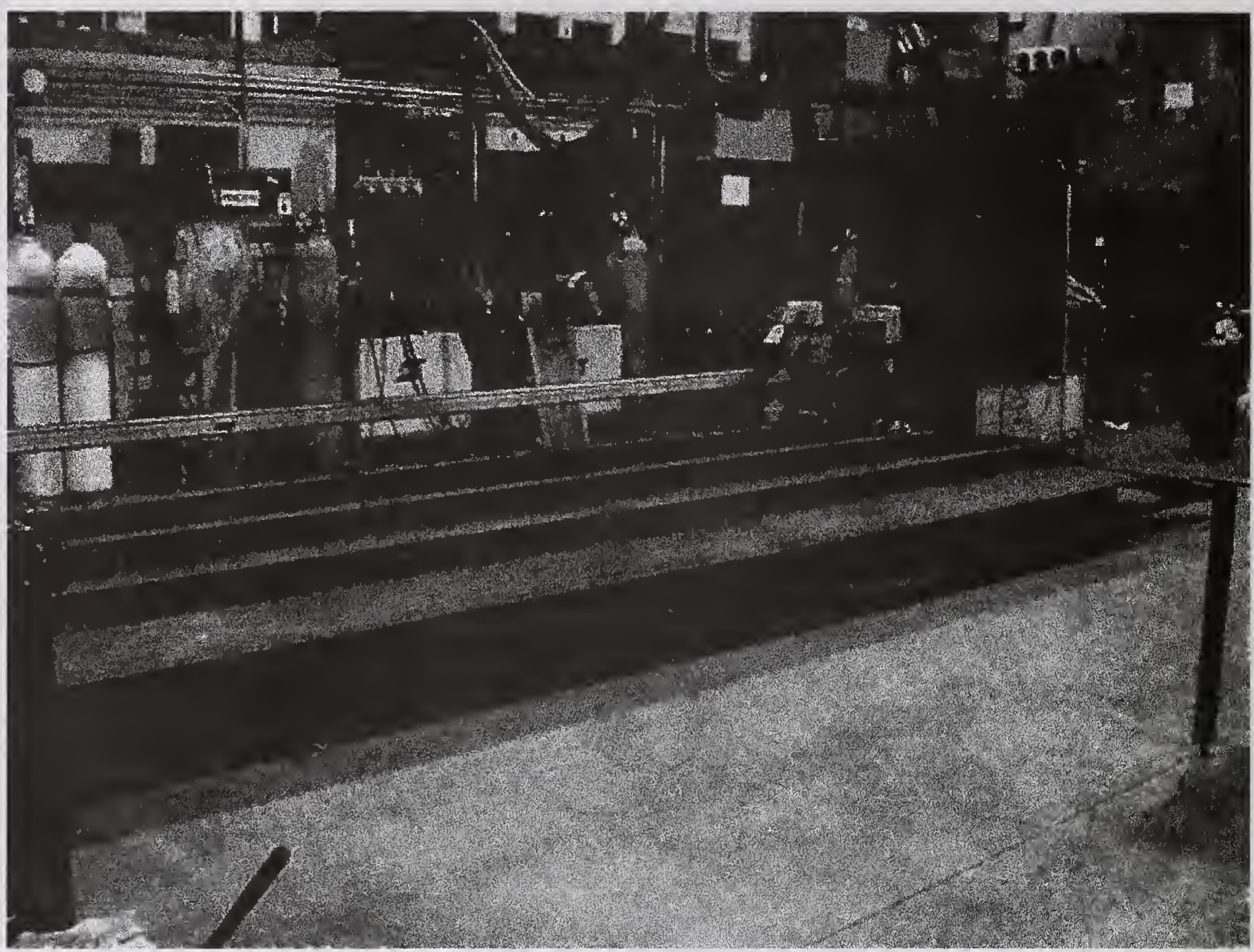

Figure 6 Photograph of $8^{\prime}$ x 20' x 3/16" Plate Experiment

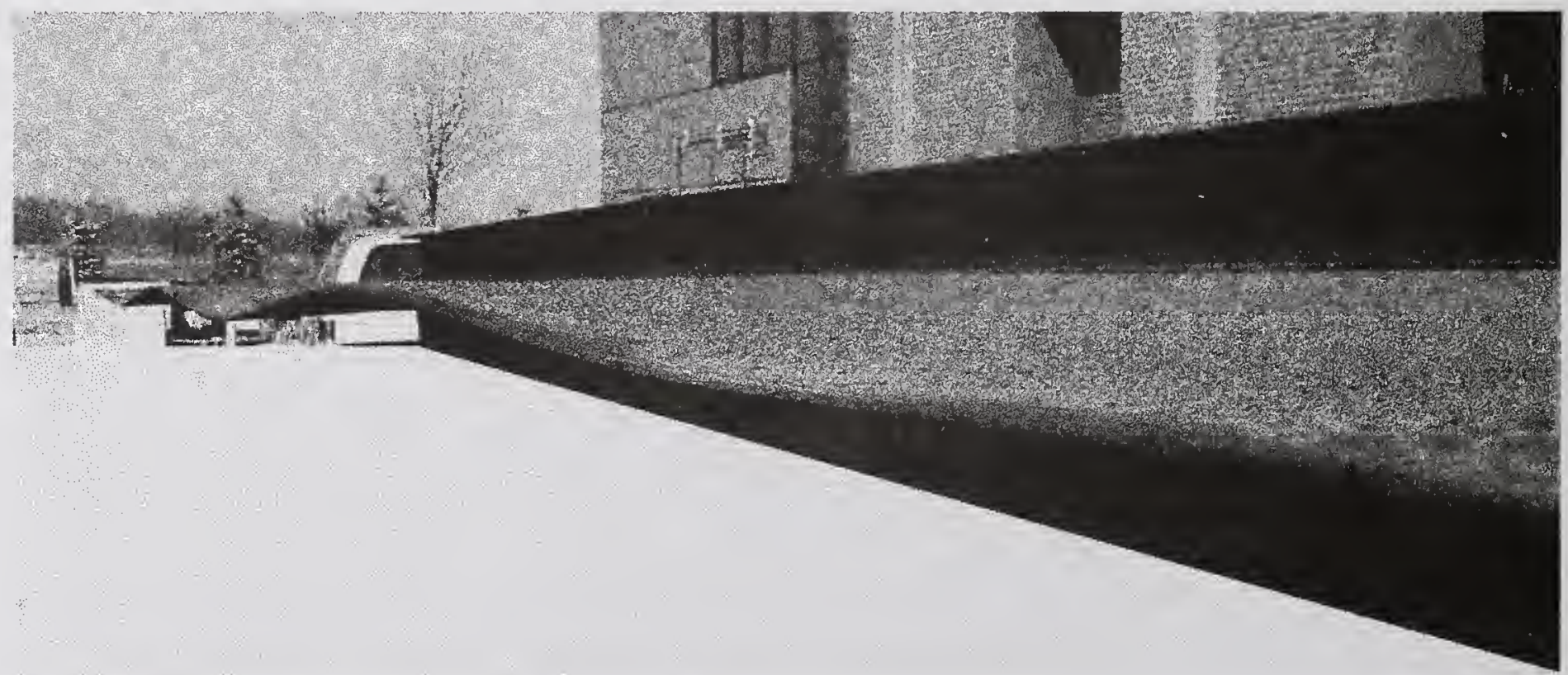

Figure 7 Photograph of $8^{\prime}$ x 20' x 3/16" Plate - Severe Buckling 


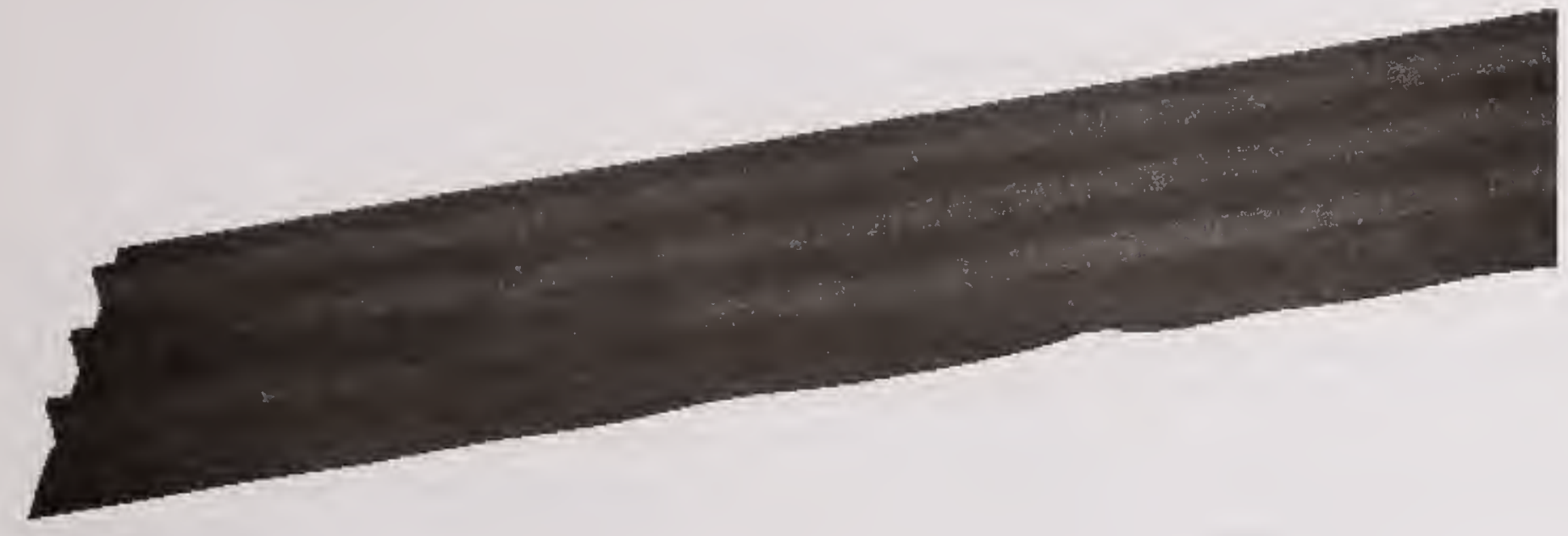

Figure 8 Rendering of Calculation Results. Buckling is Clearly Evident 



\title{
COMPUTATIONAL MODELING OF RESIDUAL STRESS IN WELDS
}

\author{
S. Yushanov ${ }^{*}$ and K. C. Koppenhoefer ${ }^{*}$
}

\begin{abstract}
Accurate prediction of stresses, strains, and residual stresses that are generated as a result of welding is important for a number of practical issues. Hydrogen-induced cracking, stresscorrosion cracking, distortion, as well as fatigue strength of welded structures can all be affected significantly by the residual stresses that are generated around welds. Due to the complexity involved in the experimental measurement of residual stresses, numerical simulation procedures are increasingly used for estimating the residual stresses that arise from welding. Commercially available simulation software readily allows one to account for the non-linearities due to variation of material properties and heat transfer coefficients with temperature. However, the material constitutive models available in most commercial packages do not account for some of the unique features associated with the welding process, e.g., material melting/remelting as different weld passes are deposited, solid phase transformation effects, etc.
\end{abstract}

These features of the welding process influence the prediction of residual stress and distortion. However, the open literature does not include a systematic study of these factors. The work reported here uses a multiple-pass butt weld in a cylindrical pipe to study in detail the effects of advanced material modeling methods on residual stress. Deposition of material during the welding is carried out in multiple weld passes. At the melting temperature, all accumulated elastic and plastic strains are set to zero. The interaction of this strain zeroing with the isotropic and kinematic hardening is examined in terms of the effect on the distribution of residual stress and welding-induced distortion.

\section{KEYWORDS}

Residual stress, kinematic hardening, isotropic hardening, strain accumulation, welding, finiteelement analysis.

\section{INTRODUCTION}

Accurate modeling of the material response to welding represents a significant challenge in welding simulations ([1] and [2]). The large temperature change associated with welding generates a complex material response. These temperature changes produce difficulties in both the thermal and mechanical analysis in a welding simulation. However, the material response in a mechanical analysis represents a greater challenge than the thermal response to the numerical analyst. The mechanisms of plastic deformation and evolution of the yield surface during welding continues to be a point of discussion among organizations conducting welding simulations.

To determine the influence of different techniques for modeling plastic deformation during welding, the International Institute of Welding (IIW) conducted a round robin of residual stress

"Edison Welding Institute, 1250 Arthur E. Adams Drive, Columbus, OH 43221 
prediction. Eleven well-recognized organizations conducted analyses of a butt girth weld in a pipe. The IIW provided each participant with the finite-element mesh, the net heat input of welding, and the temperature-dependant material properties. The residual stress predictions from these 11 organizations contained significant scatter [3]. A detailed review of the analysis techniques used by the participants suggests the plasticity routines generated the majority of the scatter. The work reported here examines the effect of two critical components of welding simulation, namely the effects of the hardening model (isotropic versus kinematic) and zeroing the plastic strain during material melting.

\section{COMPUTATIONAL MODELS}

The analyses conducted here utilize an axisymmetric idealization of a pipe girth butt weld. The finite-element mesh used in the IIW round robin forms the basis for all analyses conducted in this work. The mesh in the weld region contains significant refinement with a coarsening as the mesh moves away from the weld (see Figure 1).

The analyses consist of a sequentially coupled technique where the thermal analysis is conducted first. All analysis used the commercial code ABAQUS. A double-ellipsoid function with a Gaussian distribution of heat flux [4] models the arc heating effects during welding. User routine DFLUX is utilized to implement a moving body heat input due to welding. Surface convection is applied at the inner and outer surfaces of the pipe. Radiation heat transfer effects are not accounted for in this analysis. The initial, and interpass, temperature of the model was room temperature.

Each weld pass is modeled discretely using an element-rebirth technique. In this technique, the element sets representing each weld pass are generated along with the finite-element analysis meshes for the base material, see Figure 1. During analysis, elements associated with higher numbered passes are first removed and then reactivated at the beginning of the appropriate weld pass.

When a group of weld pass elements is activated, specified initial temperatures are imposed for all nodes associated with the weld pass elements. In reality, rebirth of the weld pass elements occurs at the melting temperature. However, numerical studies indicate [5] that predicted residual stress distributions do not seem sensitive to prescribed initial conditions for the weld metal. Thus, rebirth of the higher pass elements occurs at room temperature in the present analysis.

The material is assumed to follow the von Mises yield criterion and associated flow rules. Linear isotropic and kinematic hardening models were analyzed. Strain rate effects and phase transformation effects are not considered in the present analysis. Temperature-dependent thermal-physical properties used in thermal analysis and material properties for stress analysis are taken from Reference [3]. These analyses utilize a strain resetting technique, included into ABAQUS via a UMAT that eliminates all accumulated plastic strains for material once it exceeds the solidus temperature. 


\section{RESULTS AND DISCUSSION}

The first set of results presented in this section show the evolution of different variables at Point $A_{1}$ located within the weld filler metal that are deposited during Pass 1 (see Figure 1). This point experiences melting during Pass 1 and re-melting during Pass 2, see Figure 2. As indicated previously, the solidus temperature for this material equals $1455^{\circ} \mathrm{F}$. Thus, Point $\mathrm{A}_{1}$ remains in the molten state over $0.5 \mathrm{sec} \leq t \leq 4 \mathrm{sec}$ for Pass 1 , as indicated by the region between the dashed lines in Figure 2(a). Point $A_{1}$ becomes molten again for approximately $5 \mathrm{sec}$ $(5,012 \mathrm{sec} \leq t \leq 5,017 \mathrm{sec})$ during Pass 2, as shown by the dashed lines in Figure 2(b).

Figure 3 presents the transient equivalent plastic strain at Point $A_{1}$. Each figure compares an analysis where the strains accumulated through the entire welding cycle (dashed line) with an analysis for which the strains are reset (solid line) at all points where the temperature exceeded the solidus temperature of the material. The resetting algorithm effectively reduces the equivalent plastic strain for Point $A_{1}$ to zero while the material melts and re-melts. Thus, the analyses that do not include resetting accumulate significantly more plastic strain than the analyses with resetting. The plastic strains presented in Figure 3 appear independent of the hardening model (isotropic or kinematic).

Figure 4 shows the variation of the plastic strain in the hoop direction, $\varepsilon_{\theta \theta}^{p l}$, as a function of time at Point $A_{1}$. The resetting algorithm influences this plastic strain component in a similar manner as the equivalent plastic strain. However, the examination of $\varepsilon_{\theta \theta}^{p l}$ permits a physical interpretation of the results to determine the applicability of the resetting algorithm. Introducing the resetting algorithm changes the direction of the hoop plastic strain from compression to tension. The tensile plastic strain represents a more physically reasonable result. The weld metal must contract on cooling after each pass. Thus, the weld metal should experience a tensile strain. As with the equivalent plastic strain, the strains shown in Figure 4 appear independent of the hardening model.

The hoop stress computed from these strains using an isotropic hardening model appear in Figure 5 (all stresses are normalized by the room-temperature yield strength, $\sigma_{0}$ ). As Pass 1 heats the material, a large compressive stress initially develops $\left(\sigma / \sigma_{0}=-1.1\right)$. Additional heating during Pass 1 decreases the stress to near zero due to the decrease in yield strength and Young's modulus of the material. After the material solidifies, the hoop stress becomes tensile and attains a value of $\sigma / \sigma_{0}=1.5$ at the end of Pass 1. The evolution of stress at Point $A_{1}$ for Pass 2 appears in Figure 5(b). During Pass 2, Point $A_{1}$ experiences re-heating, re-melting, and cooling. Reheating drives the hoop stress from a tensile value of $\sigma / \sigma_{0}=1.5$ to a maximum compressive value of $\sigma / \sigma_{0}=-0.7$ before the material loses strength and stiffness. In the re-melting region, the hoop stress at Point $\mathrm{A}_{1}$ approaches zero. Cooling of the material generates a large tensile stress $\left(\sigma / \sigma_{0}=1.2\right)$.

A direct comparison of the analyses with and without the resetting algorithm indicates strain resetting does not influence the residual stress at any point during the analyses, for the case of 
isotropic hardening shown in Figure 5. Resetting the strains does not influence the residual stress due to the lack of influence of these strains on the yield surface, as shown in Figure 6. By definition, the yield surface may not shift for isotropic hardening. Thus, the yield surface for isotropic hardening remains unchanged during the resetting of the strains. This unchanged yield surface produces a constant residual stress since the stress state must remain on the yield surface during plastic flow.

Figure 7 shows the variation of hoop stress with time for the kinematic hardening case. Figure 7 (b) shows the dramatic influence of strain resetting on residual stress prediction. For kinematic hardening, the yield surface radius does not change with plastic flow. Instead, the yield surface translates. Figure 8 shows the evolution of the back stress for Passes 1 and 2. For the case of strain resetting, the yield surface origin returns to the coordinate origin due to an elimination of the back stress. This modification of the yield surface origin produces the difference in hoop stress observed in Figure 7.

Figure 9 summarizes the effect of strain resetting on the hoop stress for both isotropic and kinematic hardening models. Figure 9(a) shows there is no influence of strain resetting on the hoop residual stress over the entire welding cycle for isotropic hardening. The stresses at Point $A_{1}$ attain a value of approximately 1.5 times the yield stress early in the welding and maintain that value (except in the transients during welding). Figure 9(b) shows the strong influence of strain resetting over time. For Pass 1 , the residual stress attains a value of approximately 2.0 times the yield stress. However, the addition of new material reduces the residual stress to yield level at the completion of the weld.

Figure 10 shows the final hoop and axial residual stresses along the weld centerline for all cases. The kinematic hardening results show the influence of the strain resetting technique used here. The isotropic case and kinematic case with resetting show the closest agreement for the hoop stress. All three cases show reasonable agreement for the axial stress case.

In additional to residual stress, welding simulations may provide predictions of distortion. Figure 11 shows the axial and radial displacement along the internal diameter of the pipe for all cases considered. For both plots, the origin of the $x$ axis occurs at the symmetry plane of the weld. Strain resetting does not influence the residual stress for analyses using isotropic hardening. However, the axial and radial displacements show an effect of strain resetting. The effect of resetting strains appears much greater for kinematic hardening. Without strain resetting, isotropic and kinematic hardening provide dramatically different displacements. However, resetting the strains drives the two analyses towards each other.

\section{SUMMARY AND CONCLUSIONS}

This work examines the effect of strain resetting during melting in welding simulations. The resetting procedure does not influence the residual stress prediction for isotropic hardening. The resetting procedure does not alter the yield surface radius. Thus, the residual stress predicted from the simulation does not change. For kinematic hardening, the modification of the back stress produces a strong effect on the residual stress. Strain resetting does alter the predicted 
welding distortion. By resetting the strain, the difference in distortion predictions for isotropic and kinematic hardening decreases significantly.

\section{ACKNOWLEDGEMENTS}

The authors of this paper would like to acknowledge the help and support of the members of the IIW Commission X-XV Joint Working Group on Residual Stress and Distortion Prediction and the organizations who participated in Phase 1 of the Round Robin; TWI, EWI, British Energy, British Steel, Paton Institute, Technical University - Braunschweig, Technical University - Graz, SAQ Kontroll AB, Institut of Applied Mechanics - BRNO, Institut de Soudure, and the Ohio State University. In additional, the authors would like to acknowledge Professor R. H. Dodds for his many useful discussions.

\section{REFERENCES}

1. Goldak, J. Modeling thermal stresses and distortions in welds. in Proceedings of the 2nd International Conference on Trends in Welding Research. 1989.

2. Lindgren, L.-E. The Use of Simulations and the Need of Experiments in Material Processing. in The sixth Cairo International MDP Conference. 1996.

3. Koppenhoefer, K.C., Gordon, J.R., IIW Round Robin on Residual Stress and Distortion: Phase 1 Results. 2000, IIW.

4. Goldak, J.A., Chakravarti, A., and Bibby, M.J, A New Finite Element Model for Welding Heat Sources. Trans. AIME, 1984. 15B: p. 299-305.

5. Hong, J.K., Tsai, C.-L., and Dong, P.,, Assessment of Numerical Procedures for Residual Stress Analysis of Multipass Welds. Welding Journal, 1988: p. 372s-382s. 

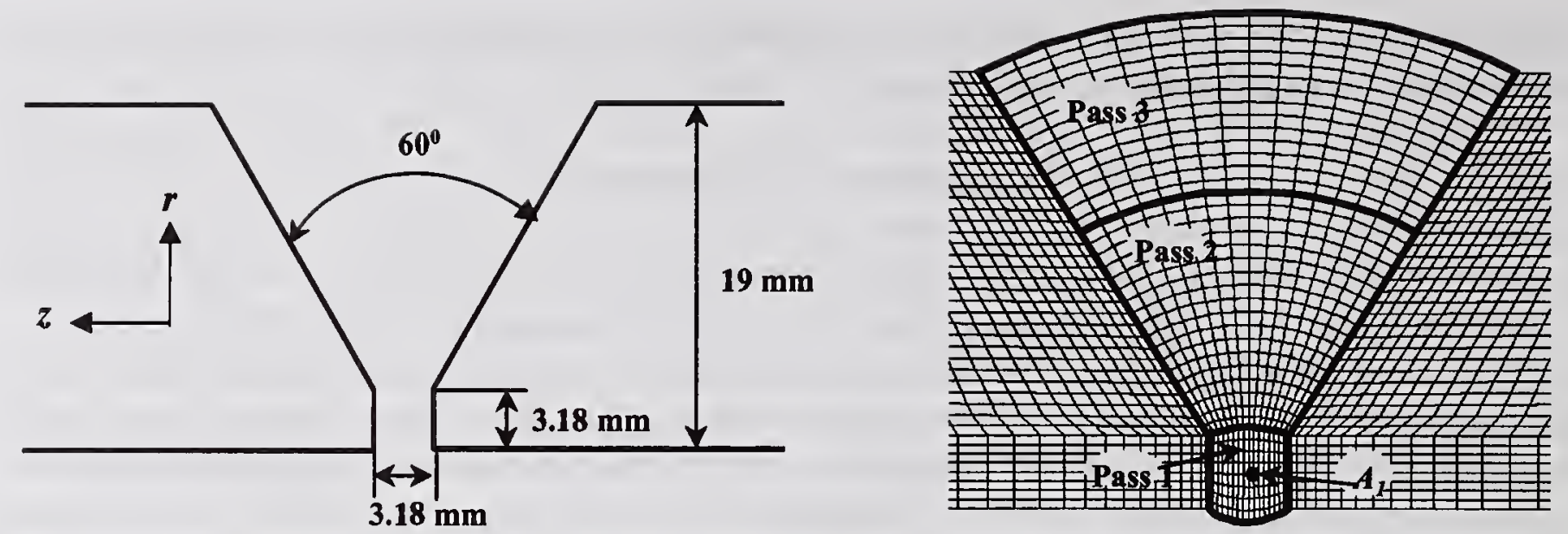

Figure 1. Weld joint geometry and finite-element model with Point $\boldsymbol{A}_{1}$ specified.
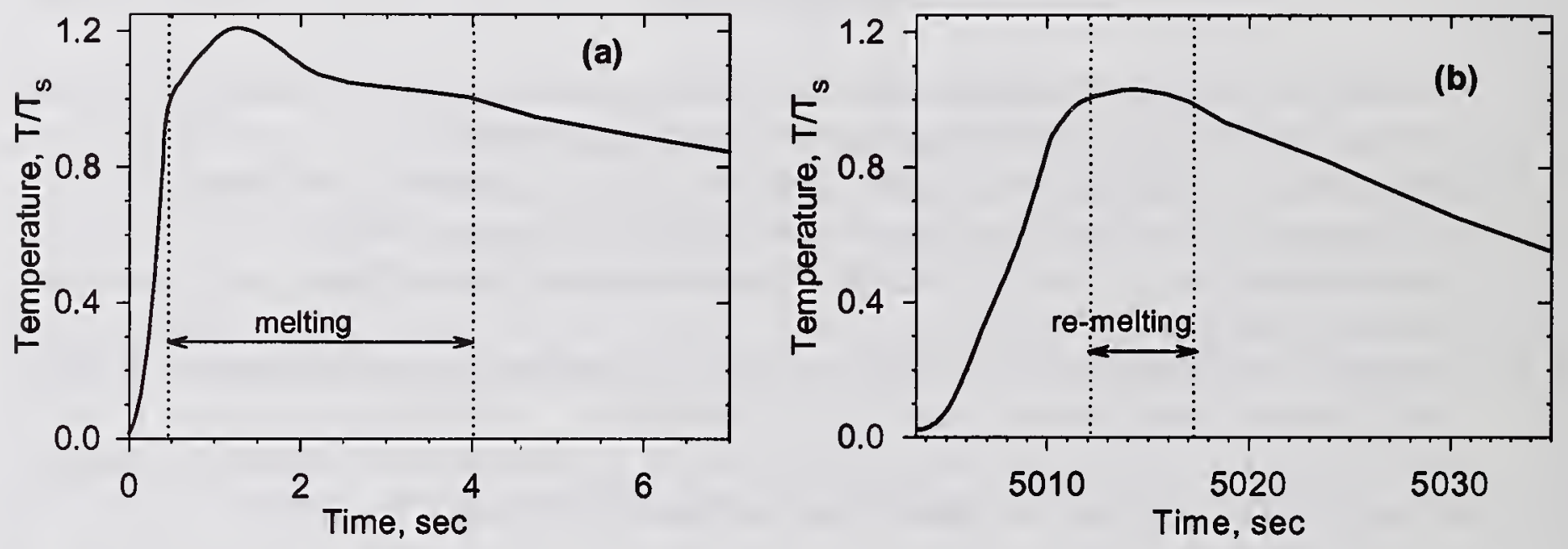

Figure 2. Variation of temperature with time for Point $\boldsymbol{A}_{1}$ during (a) Pass 1, and (b) Pass 2.
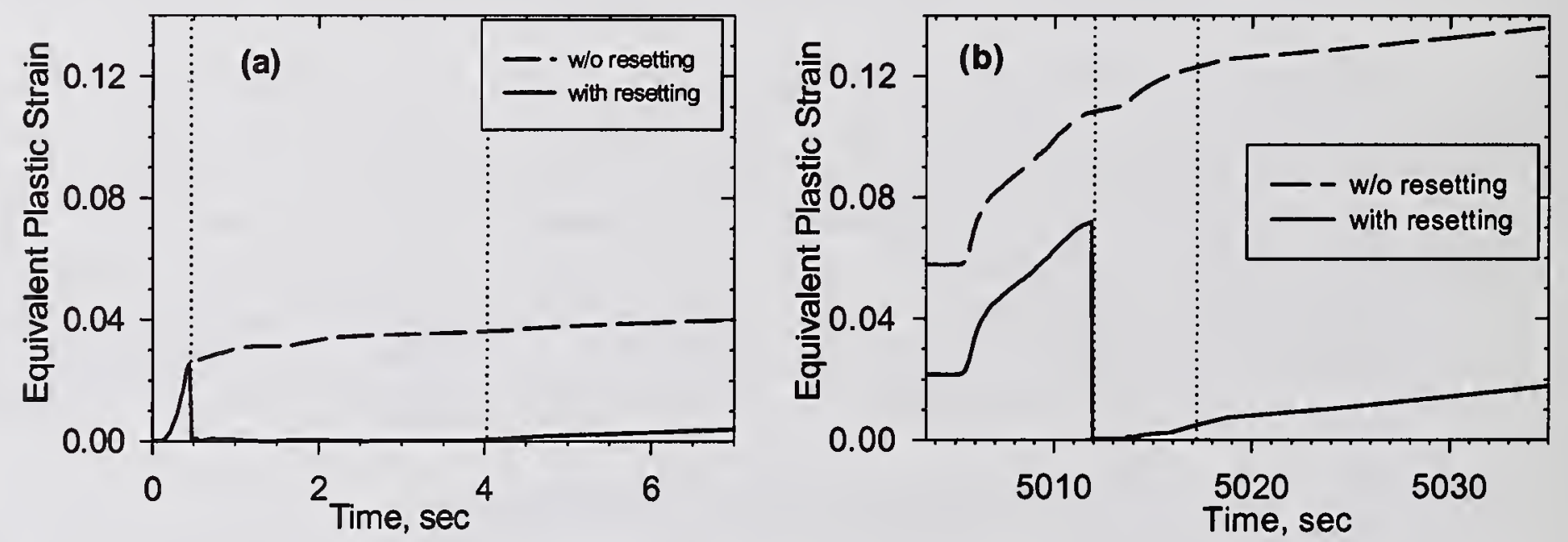

Figure 3. Variation of equivalent plastic strain, $\bar{\varepsilon}^{p l}$, with time for Point $A_{1}$ during (a) Pass 1, and (b) Pass 2. (All analyses used an isotropic hardening model.) 

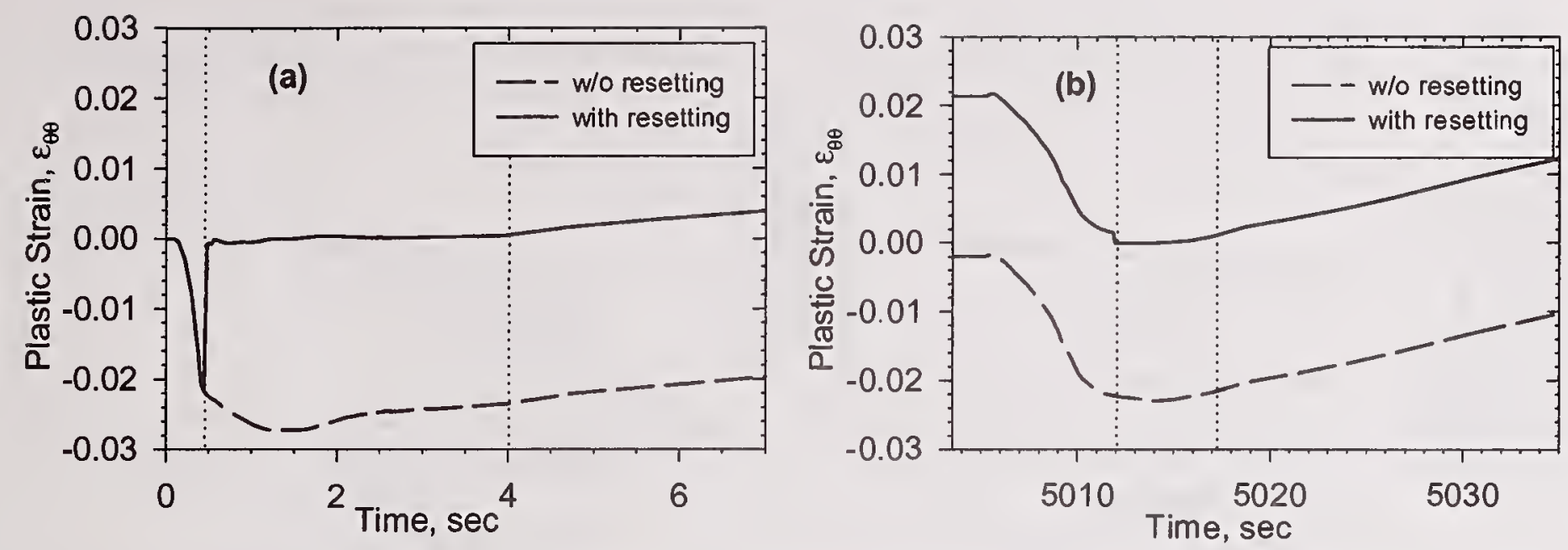

Figure 4. Variation of plastic strain in hoop direction, $\varepsilon_{\theta \theta}^{p l}$, with time for Point $A_{1}$ during (a) Pass 1 , and (b) Pass 2.
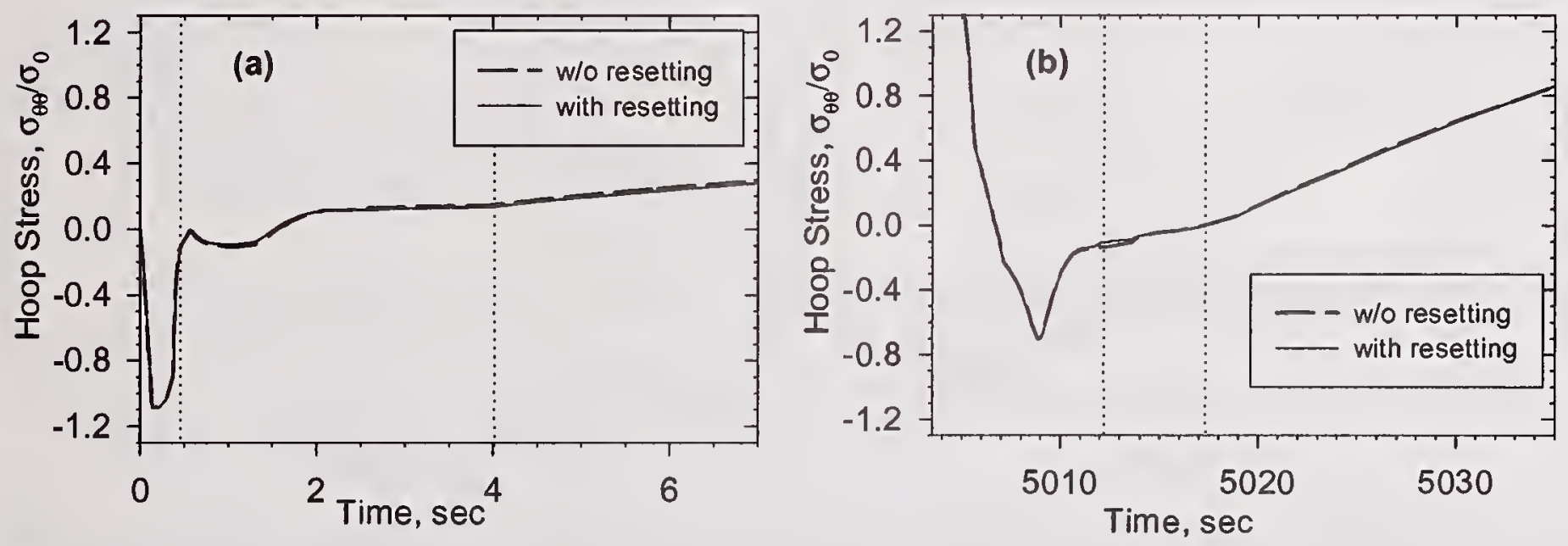

Figure 5. Variation of hoop stress, $\sigma_{\theta \theta}$, with time for Point $A_{1}$ during (a) Pass 1, and (b) Pass 2. (Isotropic hardening.)
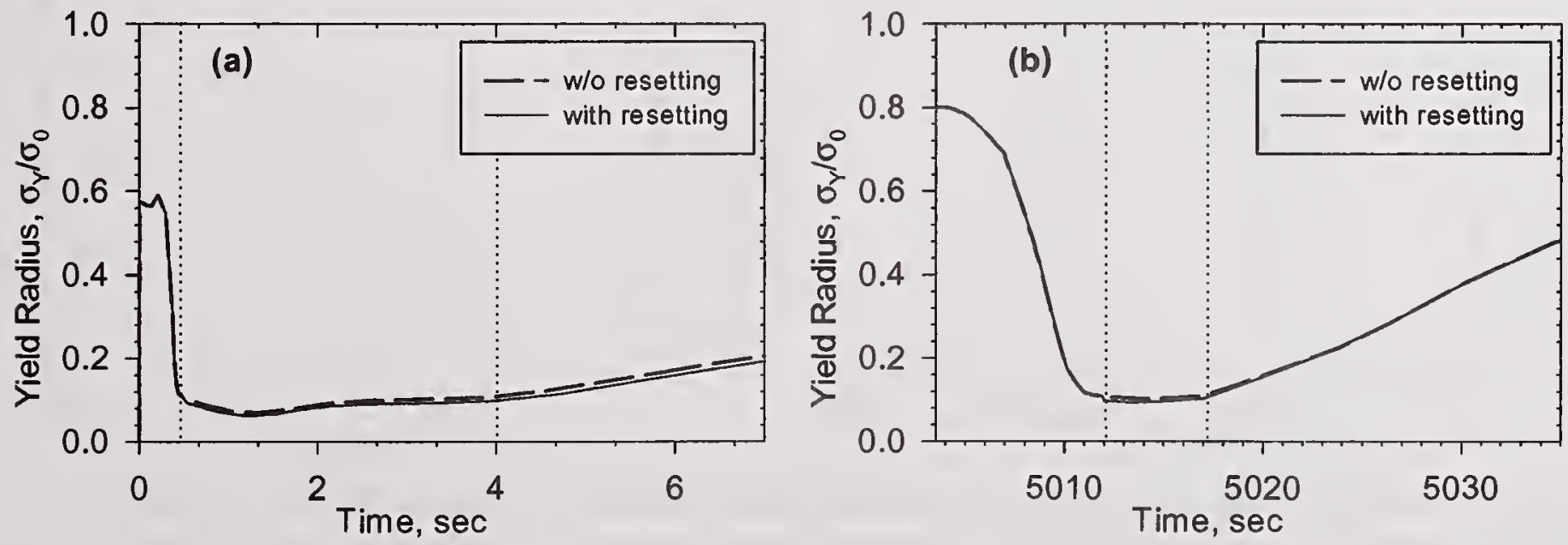

Figure 6. Variation of the radius of the yield surface with time for Point $A_{1}$ during (a) Pass 1, and (b) Pass 2. (Isotropic hardening.) 

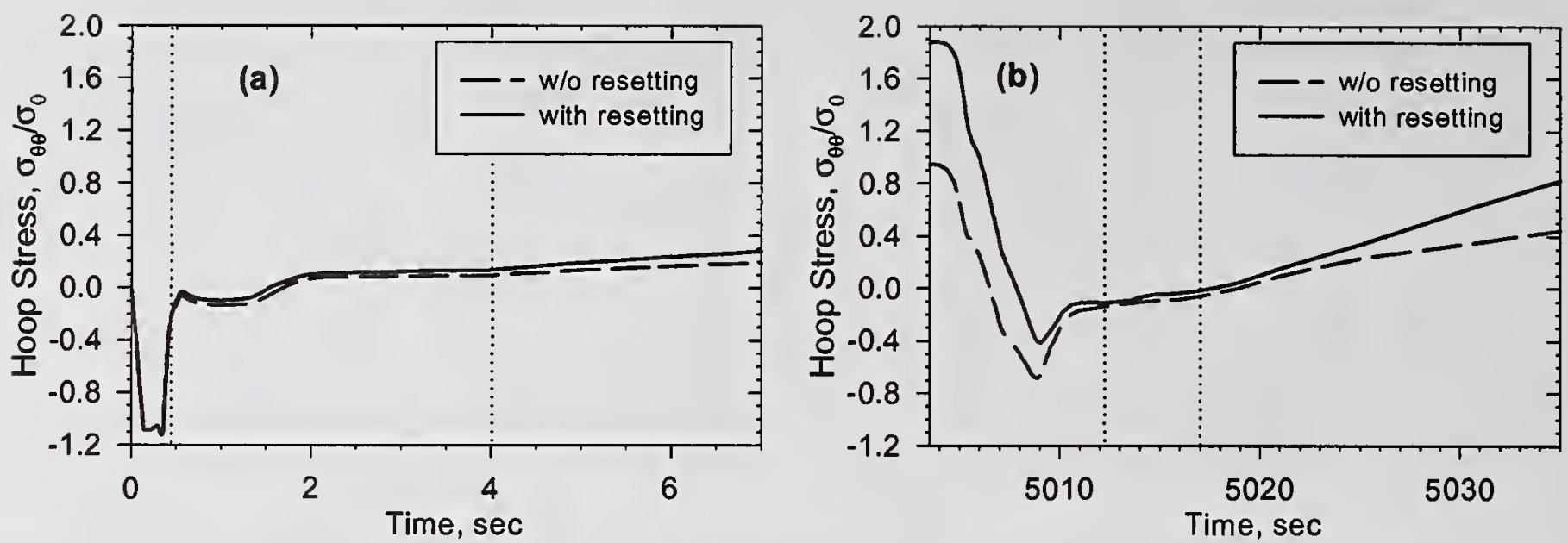

Figure 7. Variation of hoop stress, $\sigma_{\theta \theta}$, with time for Point $A_{1}$ during (a) Pass 1 , and (b) Pass 2. (Kinematic hardening.)
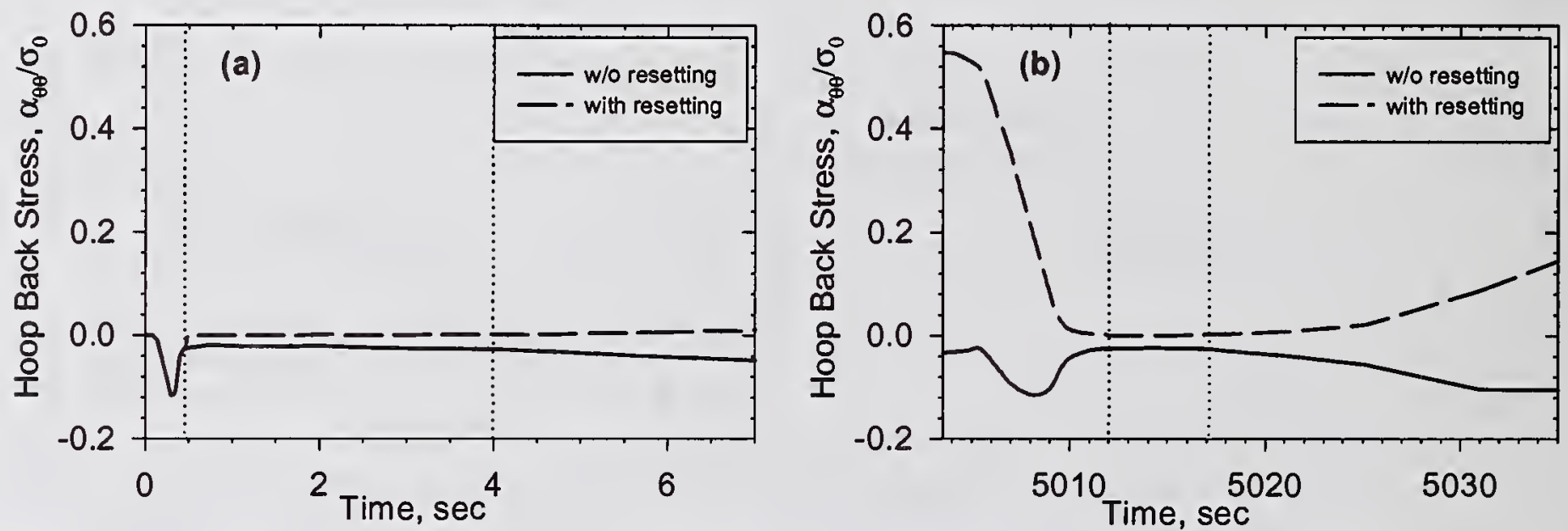

Figure 8. Variation of the back stress with time for Point $A_{1}$ during (a) Pass 1 , and (b) Pass 2. (Kinematic hardening.)
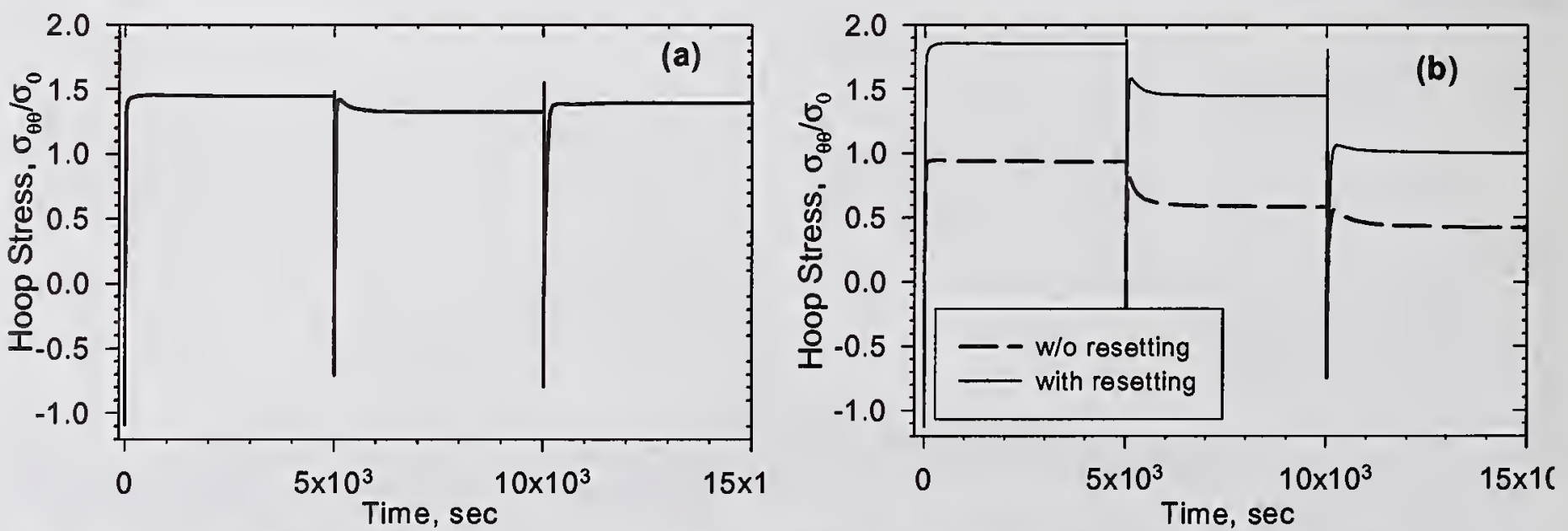

Figure 9. Variation of hoop stress, $\sigma_{\theta \theta}$, with time for Point $A_{1}$ during three passes for (a) isotropic hardening resetting, (b) kinematic hardening without resetting strains, (c) kinematic hardening with resetting strains. 

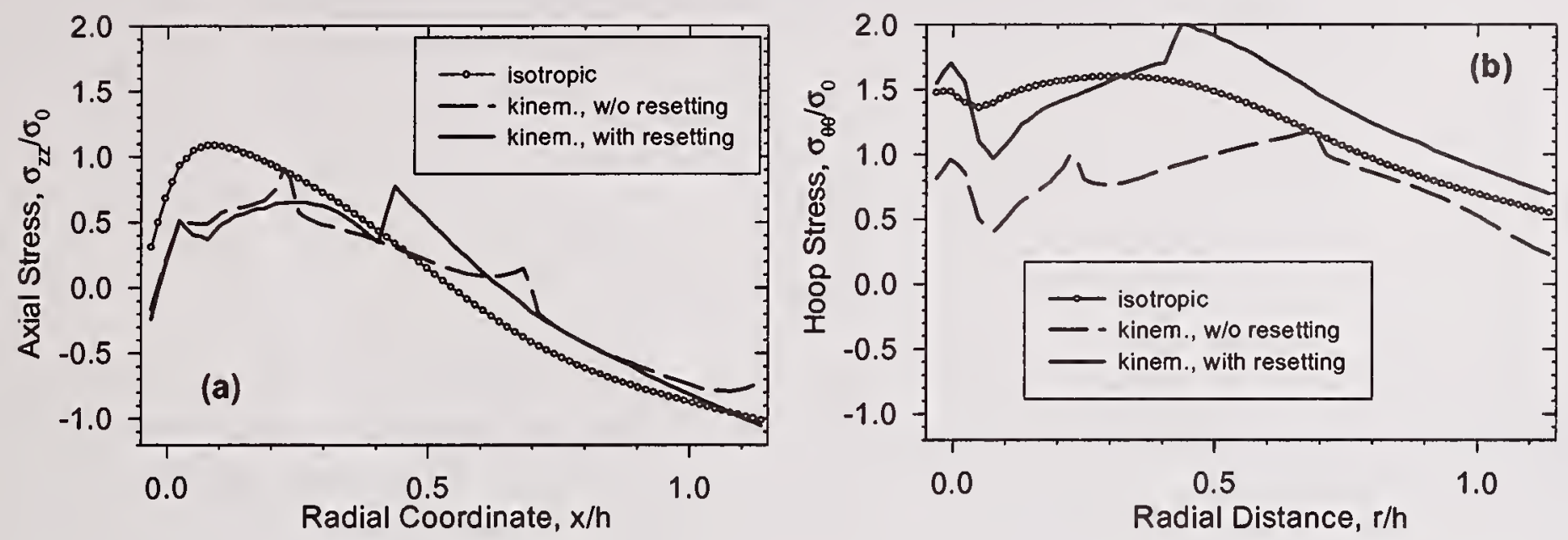

Figure 10. Variation of stress at centerline of weld for isotropic and kinematic hardening with and without strain resetting: (a) axial stress, (b) hoop stress.
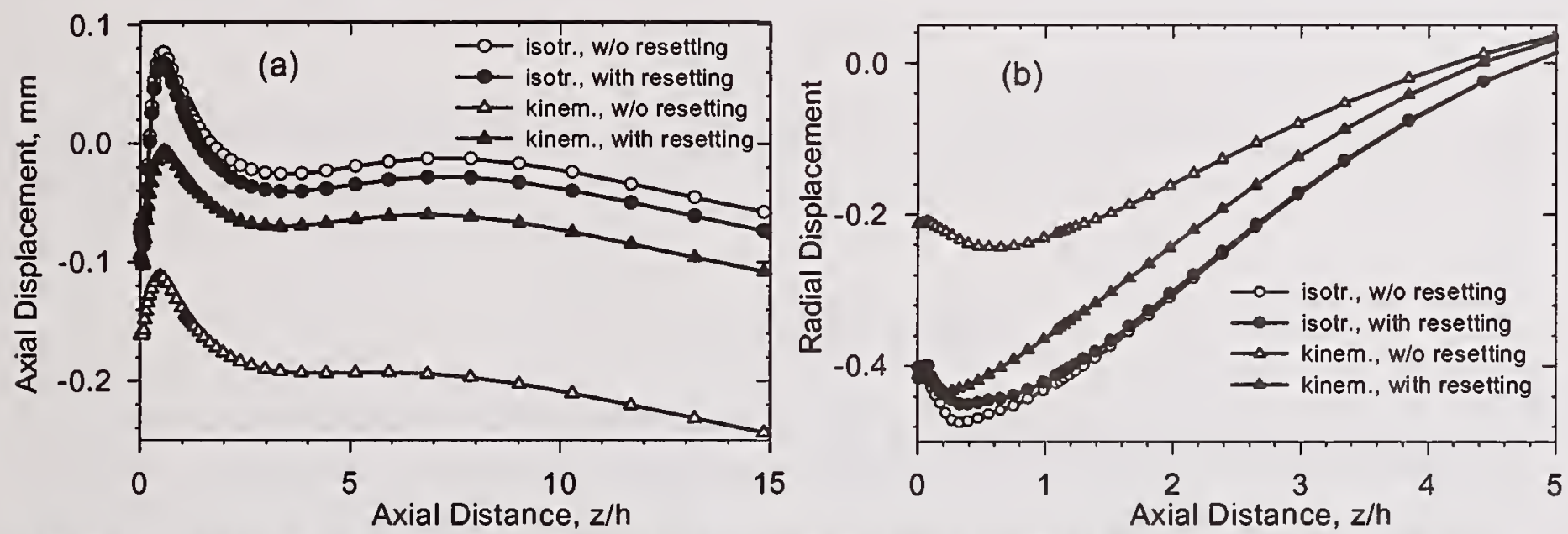

Figure 11. Variation of (a) axial and (b) radial displacement along internal diameter of pipe. (Origin of abscissa equals the centerline of the weld.) 



\title{
A STUDY ON THE PREDICTION OF WELDING DISTORTIONS OF HULL IN SHIPBUILDING (I)
}

\author{
S.B. Shin*, J.G. Youn*
}

\begin{abstract}
In order to evaluate principal factors controlling welding distortions in particular angular distortion, both dimensional analysis and Finite Element Analysis (FEA) have been performed. The principal factors were found to be the heat intensity, rigidity and restraint, which were verified by experimental results. Angular distortion both at the fillet and at the butt weldment of hull structure can be predicted by an equation expressed with function of heat intensity, bending rigidity and internal restraint.
\end{abstract}

\section{KEYWORDS}

Angular Distortion, Heat Intensity, Bending Rigidity, Restraint, Dimensional Analysis and Finite Element Analysis (FEA)

\section{INTRODUCTION}

Welding process has been widely used in fabricating steel structures such as ships and pressure vessels. However, some inherent problems of the welding still need to be solved (Ref.1-3). A major problem is welding distortion. This is attributed to incompatible strains over the welded joint, which is developed by uneven temperature distribution during/after welding. Welding distortions adversely affect the service behavior of welded structure including static and dynamic stability and buckling characteristics. In order to reduce and prevent distortions, lots of researches have been performed but welding distortion for actual structures has not yet been controlled. This may be associated to the fact that the previous studies have been limited generally in a laboratory scale with simple welding variables. Welding distortions at actual structures like ships depend not only on welding process and conditions, but also on internal and external restraint acting on the weldment. It is, therefore, necessary to establish proper predictive and control methods of welding distortion for actual structures on the basis of these factors, combined with material itself.

This study has been tried to identify the principal factors controlling welding distortion of the hull structure, in particular angular distortion and transverse shrinkage both at the fillet and butt weldment by the dimensional analysis and Finite Element Analysis (FEA). Comprehensive experiments were also carried to verify the results of the dimensional analysis and FEA. Based on these results, a predictive equation of welding distortion applicable to actual hull structures has been proposed. In this study, results and discussion on the angular distortion are described

* Material Research Dept., Hyundai Heavy Industries Co. Ltd., Jeon Ha Dong, Dong Gu, Ulsan, South Korea 


\section{ANALYSIS AND EXPERIMENT PROEDURE}

Dimensional analysis was performed to identify the principal factors controlling welding distortion as given in below. If effects of material properties including yield strength and thermal expansion coefficient were not taken into consideration because of their temperature dependence, residual angular distortion of fillet and butt weldment could be expressed as shown in Eqn (1).

$$
\delta_{b}=f\left(Q, D_{b}, W\right)=f\left([Q]^{a},\left[D_{b}\right]^{b},[W]^{c}\right)
$$

Where, $Q$ is heat intensity and $D_{b}$ and $W$ are bending rigidity and width of weldment respectively. Here, equation (1) is rearranged as following equation by substituting the dimensional formula.

$$
[\mathrm{L}]=\left([\mathrm{FL}]^{\mathrm{a}}[\mathrm{FL}]^{\mathrm{b}}[\mathrm{L}]^{\mathrm{c}}\right)
$$

The equation corresponding to the dimensional homogeneity is defines as follow:

$$
\begin{aligned}
& F: a+b=0 \\
& L: a+b+c=1
\end{aligned}
$$

Solving Eqn (3), the following exponents are obtained

$$
a=1 b=-1 c=1
$$

Substituting these values into Eqn (1), the dimensionless form of angular distortion is defined as following equation

$$
\phi=\frac{\delta_{\mathrm{b}}}{\mathrm{W}}=f\left(\frac{\mathrm{Q}}{\mathrm{D}_{\mathrm{b}}}\right)
$$

As shown in Eqn (5), the angular distortion could be defined as a function of heat intensity (Q) and bending $\left(D_{b}\right)$. It is, therefore, deduced that the principal factors controlling welding distortion are the heat intensity and rigidity.

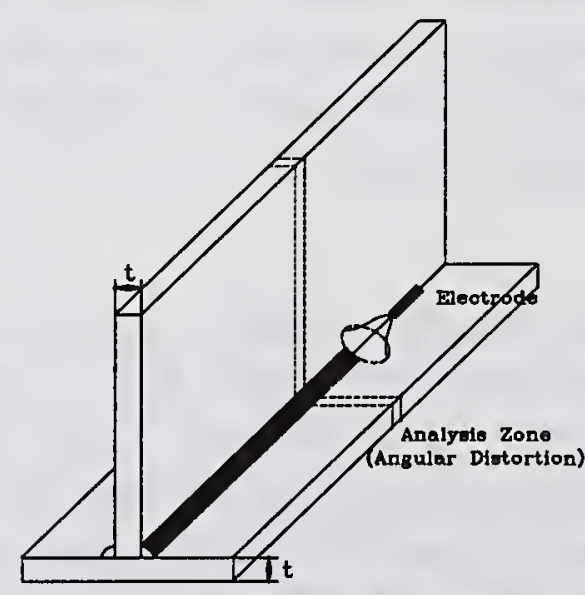

(a) Fillet Weldment

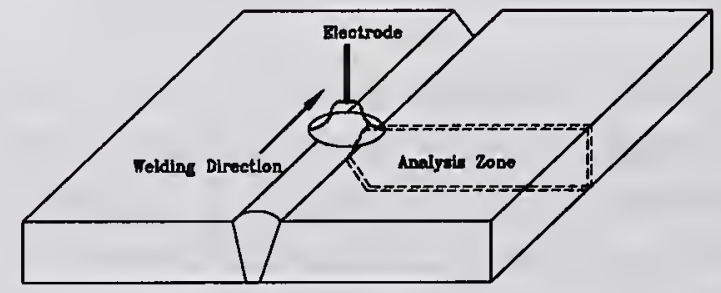

(b) Butt Weldment

Fig. 1 Schematic diagram of fillet and butt weldment 
From the results of the dimensional analysis, the variables for FEA and experiment were selected to establish a predictive equation for welding distortion for actual weldment as shown in Table 1. Fig. 1 shows the schematic configuration of the models for FEA and experiment. Welding parameters used in this study were typical FCAW (Flux Cored Arc Welding) and SAW (Submerged Arc Welding) conditions for actual fillet and butt weldment. In order to evaluate the effect of internal restraint on the angular distortion, width of weldment is varied from $700 \mathrm{~mm}$ to $4000 \mathrm{~mm}$. External restraint condition at the weldment was simulated by attaching temporary fixture to the lateral side of the weldment as shown in Fig. 2. The amount of external restraint is defined as a function of bending or in-plane rigidity and restraint length (Ls). (Ref. 4, 5)

Table 1 Variables used For FEA and experiment

\begin{tabular}{|c|c|c|c|c|c|c|c|}
\hline \multirow{2}{*}{ Base Metal } & \multirow{2}{*}{$\begin{array}{c}\text { Heat Intensity } \\
{[\mathrm{cal} / \mathrm{mm}]}\end{array}$} & \multicolumn{3}{|c|}{ Flange [mm] } & \multicolumn{3}{c|}{ Web [mm] } \\
\cline { 3 - 8 } & Thickness & Width & Length & Thickness & Height & Length \\
\hline A grade & $224-522$ & $12-25$ & $\begin{array}{c}500 \\
-4000\end{array}$ & $\begin{array}{c}\text { Infinite(FEA) } \\
1000(\mathrm{EXP})\end{array}$ & $12-25$ & 250 & $\begin{array}{r}\text { Infinite(FEA) } \\
1000(\mathrm{EXP})\end{array}$ \\
\hline
\end{tabular}

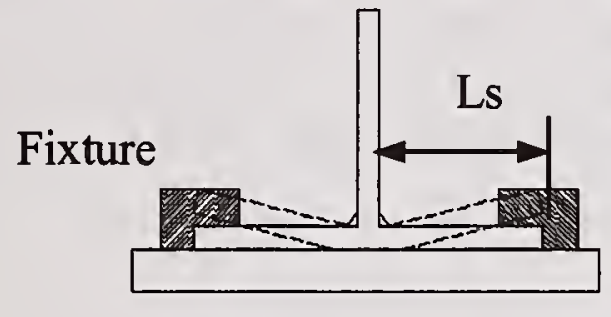

a) Fillet Weldment
Tack weld

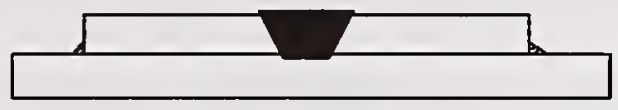

(b) Butt Weldment

Fig. 2 Schematic diagram of external restraint of fillet and butt weldment

\section{Finite Element Analysis}

The transient temperature distribution was calculated based on the assumed quasi-stationary condition. (Ref. 6) The volume heat source of welding arc was specified by a Gaussian distribution. Heat loss at all surfaces of the solution domain was governed by natural convection. Thermal properties of the material used depend on the temperature and an effective conductivity may be assumed at the temperature above the melting point. To consider the effect of latent heat at the phase change, the specific heat in the solidification range was modified to be very high. (Ref. 7)

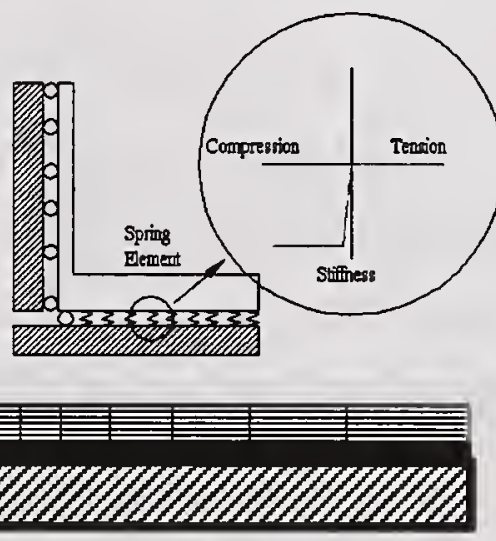

Fig. 3 Mesh design of fillet and butt weldment used for FEA 
Mesh design used for thermo-mechanical stress analysis consisted of 8-nodes plane element with general plane strain condition and spring element as shown in Fig. 3. Spring elements attached to bottom surface of fillet and butt weldment are to prevent reverse angular distortion during the welding and cooling stage. (Ref. 8) While thermo-mechanical strains along the longitudinal direction of welding line were assumed to be uniform, free expansion and bending in the transverse direction of welding line were allowed. When the temperature of elements reached the liquidus temperature, the plastic strains accumulated by that time were assumed to be relieved. In this region above the melting temperature, thermal strain increment was set to zero. Mechanical properties of weldment were postulated to behave as an isotropic, elasto-plastic and strain-hardening continuum. Yielding of material was assumed to be governed by von-Mises criteria.

\section{RESULTS AND DISCUSSION}

\section{Verification of Principal Factors}

Fig. 4 shows the variation of angular distortion at the fillet and butt weldment as a function of the heat intensity $(\mathrm{Q})$ and bending rigidity $\left(\mathrm{D}_{\mathrm{b}}\right)$. Angular distortion at the fillet and butt weldment is strongly dependent on the function of heat intensity and bending rigidity. Good agreements between calculated results and measured results for angular distortion are found. This verifies that heat intensity $(\mathrm{Q})$ and bending rigidity are the principal factors controlling the angular distortion, which was already proposed from the dimensional analysis described in the previous section.

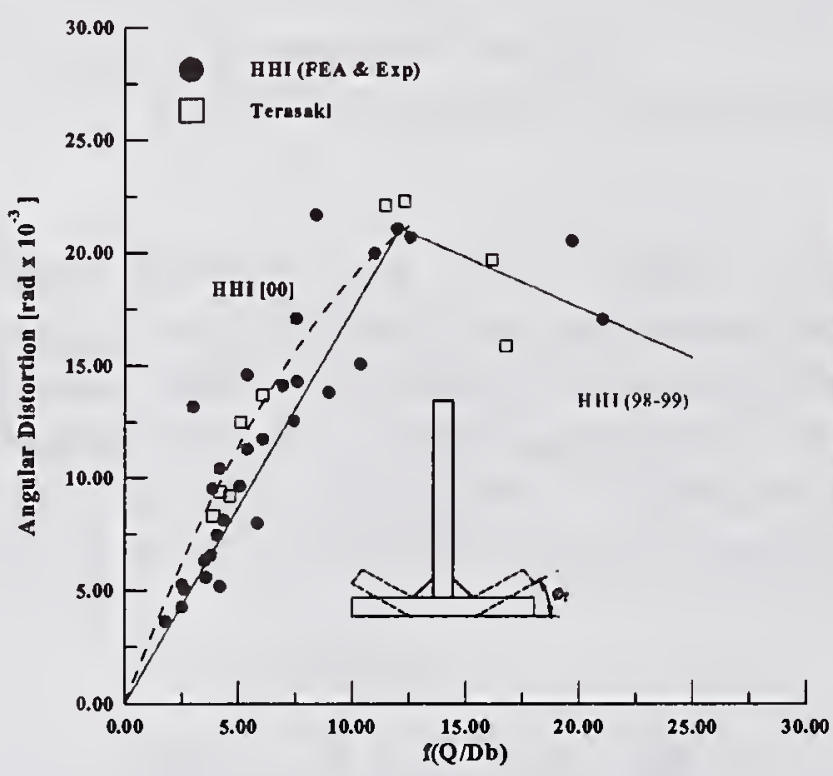

(a) Fillet weldment

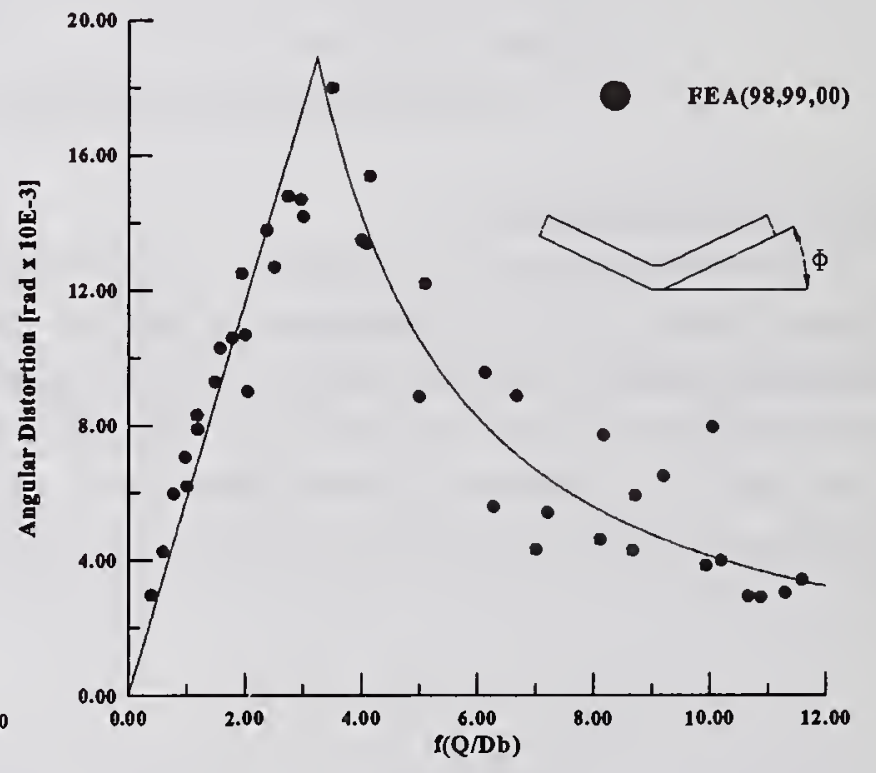

(b) Butt weldment

Fig. 4 Variation of the angular distortion at the fillet and butt weldment with the ratio of $Q$ and $\mathrm{Db}$

\section{Internal Restraint}

Welding distortion of the actual welded structure is different from that of small size welding specimen in terms of amount and deformed shape. Welding distortion of actual weldment could be over-estimated by a predictive method established by the small size weldment. Fig. 5 
(a) and (b) show the variation of the dimensionless parameters of angular distortion at the fillet weldment $\left(\zeta_{f}\right)$ and the butt weldment $\left(\zeta_{b}\right)$ with reference to weight per unit weld length ( $\left.\mathrm{W}_{\mathrm{UL}}\right)$. Here, dimensionless parameter was defined as the ratio of angular distortion according to existence of self-weight effect. At the fillet weldment, $\zeta_{f}$ decreases with an increase in $\mathrm{W}_{\mathrm{UL}}$ but the reduction rate of $\zeta_{f}$ is constant irregardless of the ratio of $Q$ and $D_{b}$. However, the reduction rate of $\zeta_{b}$ for the butt weldment strongly depends on the ratio of $Q$ and $D_{i}$ as shown in Fig. 5 (b). As the ratio of $Q$ and $D_{b}$ increases, the reduction rate of $\zeta_{b}$ increases.

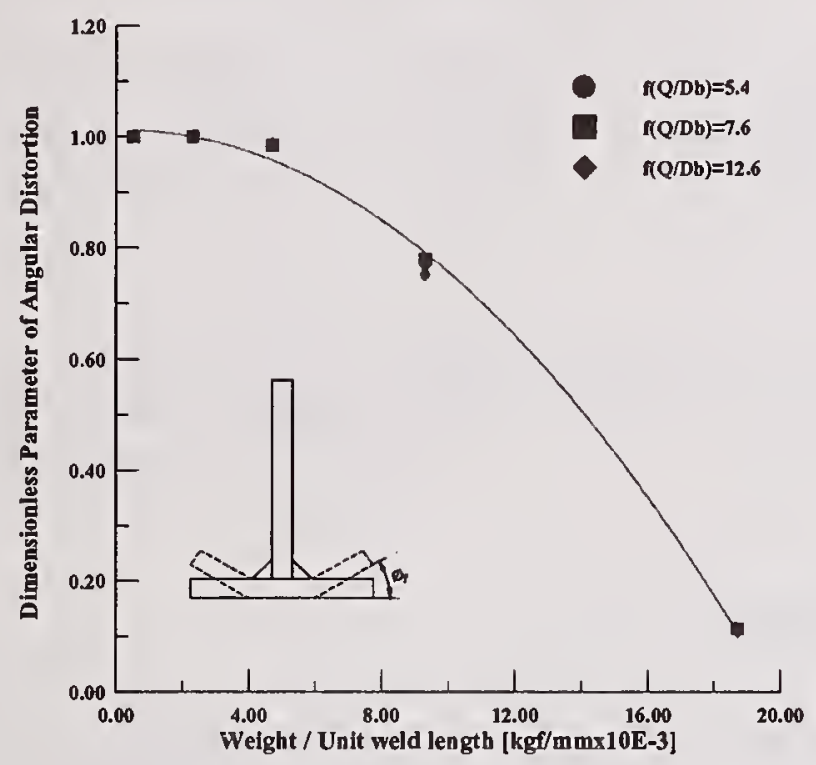

(a) Fillet weldment

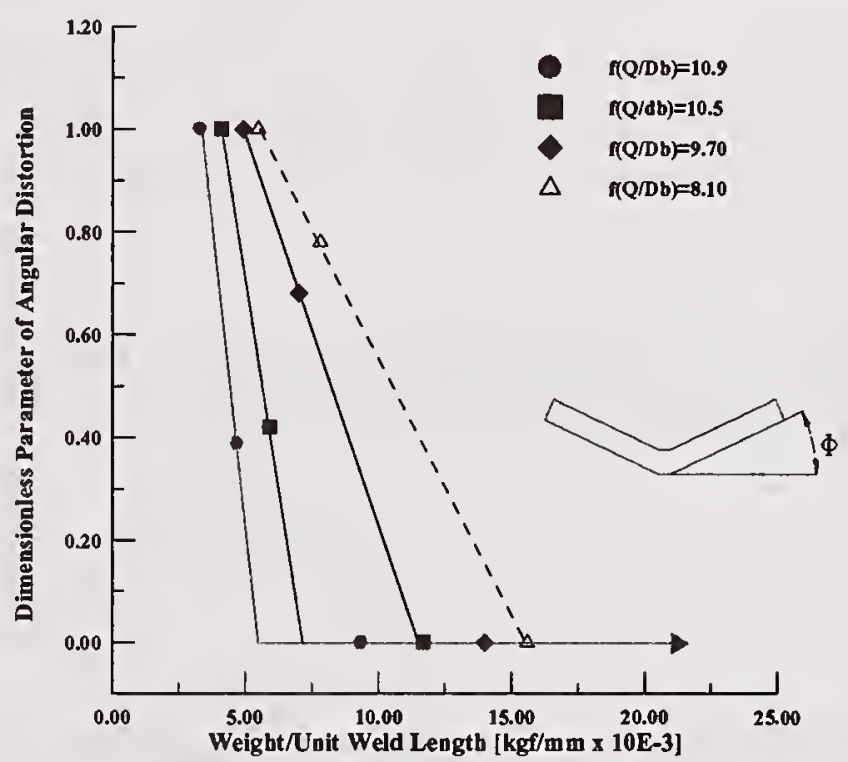

(b) Butt weldment

Fig. 5 Variation of dimensionless parameter of angular distortion at the fillet and butt weldment with $\mathrm{W}_{\mathrm{UL}}$

\section{$\underline{\text { External Restraint }}$}

External constraint using a temporary fixture or jig was widely used for controlling welding distortion. It is, therefore, important to understand how external constraint affects the welding distortion at the weldment. Fig. 6 (a) and (b) show the variation of angular distortion at the fillet and butt weldment with bending restraint intensity $\left(K_{\theta}\right)$. In this figure, the fuction of heat intensity $(\mathrm{Q})$ and bending rigidity $\left(\mathrm{D}_{\mathrm{b}}\right)$ are 7.54 for the fillet weldment and 10.67 for the butt weldment, respectively. Angular distortion of the fillet and butt weldment decreases with an increase in bending restraint intensity. This may be resulted from the increase in the reaction stress of the weldment owing to the increase of bending restraint intensity.

Variation of the angular distortion at the fillet and butt weldment subjected to a given external restraint ( $K_{\theta}=8640 \mathrm{kgf} / \mathrm{rad}$ ) is shown in Fig. 7 (a) and (b) as a function of the ratio of Q and $\mathrm{D}_{\mathrm{b}}$ and compared with that obtained under free condition. Angular distortion of the butt weldment decreased by an external restraint regardless of the ratio of $Q$ and $D_{b}$. If bending restraint intensity $K_{\theta}$ is sufficiently large, the angular distortion of the butt weldment could be perfectly controled. For the fillet weldment, effect of external restraint on the angular distortion strongly depends on the ratio of $Q$ and $D_{b}$. Suppression of the angular distortion at the fillet weldment by an external restraint could be neglected when the ratio of $Q$ and $D_{b}$ is smaller than 4 . This is attributed to the fact that the amount of elastic spring back decreases when the ratio of $Q$ 
and $\mathrm{D}_{\mathrm{b}}$ is smaller than the speicfied critical value depending on $\boldsymbol{K}_{\boldsymbol{\theta}}$.

Based on the above results, it is possible to propose a predictive equation of angular distortion for actual steel structures as follow.

$$
\phi_{\mathrm{b}}=f\left(\frac{\mathrm{Q}}{\mathrm{D}_{\mathrm{b}}}, \mathrm{W}_{\mathrm{UL}}, \frac{1}{\mathrm{~K}_{\theta}}\right)
$$

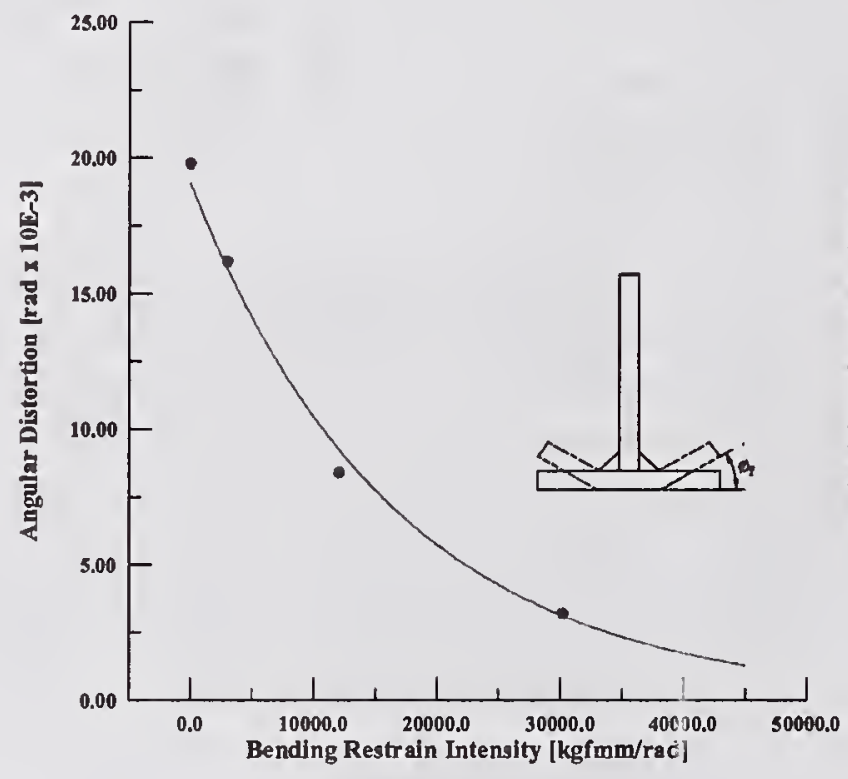

(a) Fillet weldment

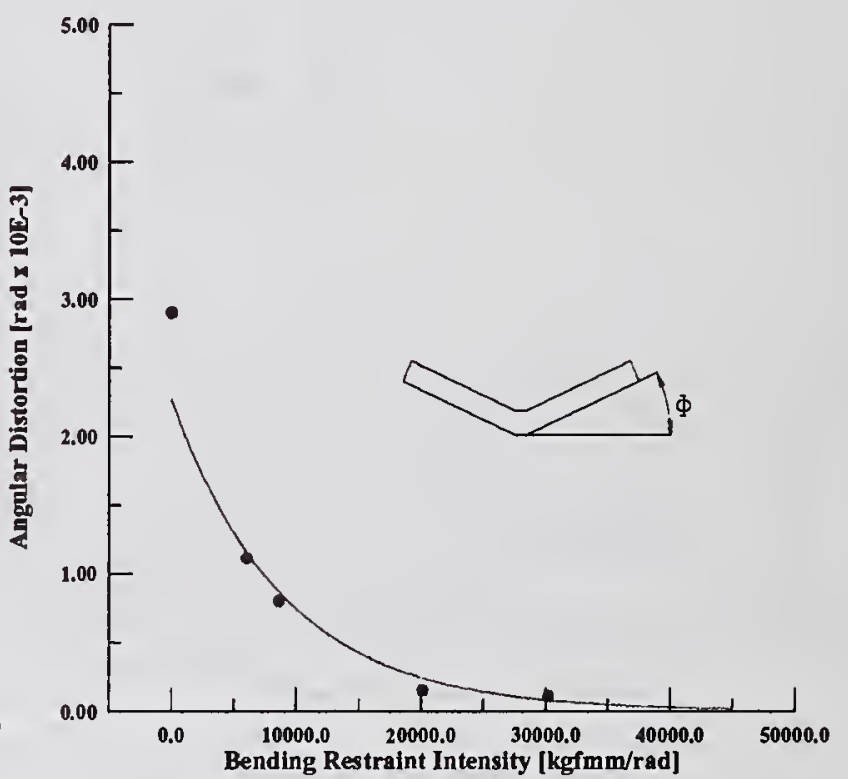

(b) Butt weldment

Fig. 6 Variation of the angular distortion at the fillet and butt weldment with $\boldsymbol{K}_{\boldsymbol{\theta}}$

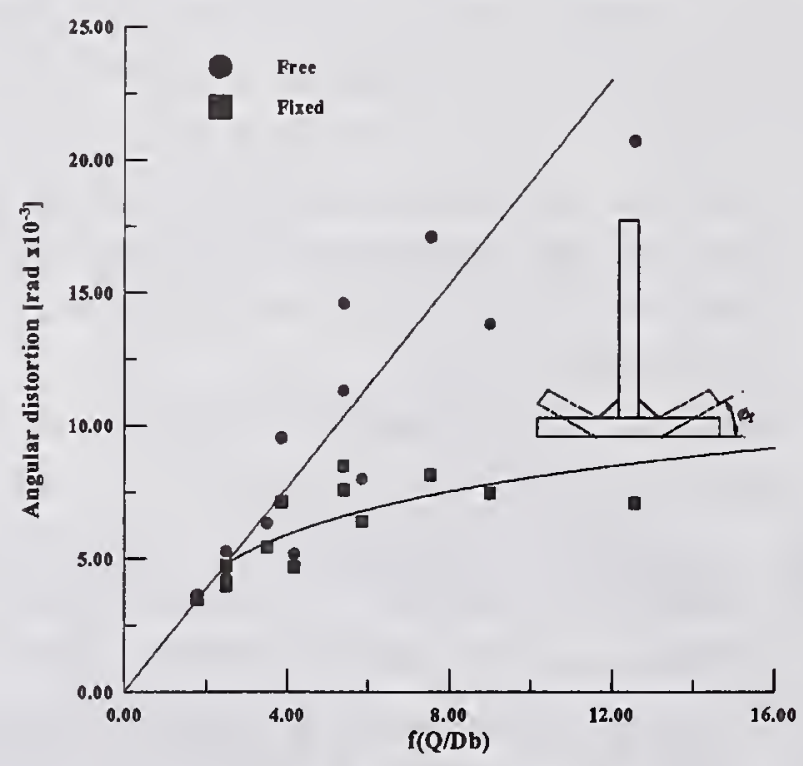

(a) Fillet weldment

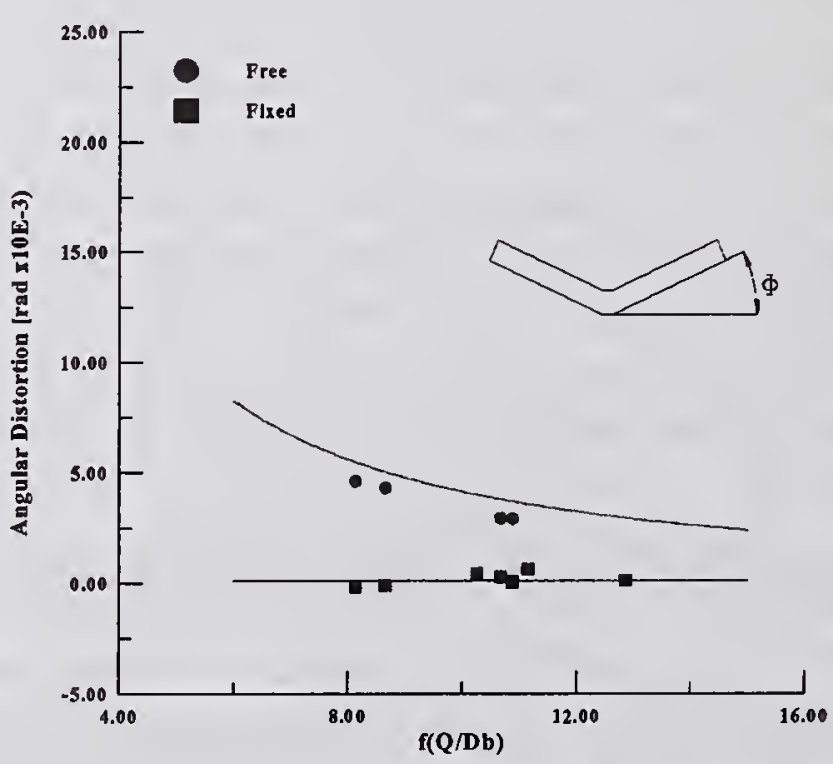

(b) Butt weldment

Fig. 7 Variation of the angular distortion at the fillet and butt weldment with $Q / D_{b}$ 


\section{CONCLUSION}

Dimensional analysis, FEA and experimental studies have been performed with reference to various variables such as welding condition, rigidity and restraint to identify the principal factors controlling welding distortion and to establish a predictive equation of welding distortion for hulls. The main results obtained are summarized as follow.

1. Angular distortion at the fillet and butt weldment was defined as a function of heat intensity, bending rigidity, and internal and external restraint condition.

2. Angular distortion decreased by effects of internal restraint. However, reduction rate of angular distortion at the fillet weldment depends on restraint intensity alone, while that of angular distortion is determined by internal restraint and ratio of heat intensity and bending rigidity.

3. The control effect of external restraint (by jig) on the angular distortion increased as the function of ratio of heat intensity and bending rigidity and bending restraint intensity increased.

\section{REFERENCES}

1. N. R. N. Rao and L. Tall, 1961, "Residual stress in welded plates", Welding Journal, Vol.40, p. $468 \mathrm{~s}-480 \mathrm{~s}$.

2. Rodgers and Fetcher, 1938, "Determination of internal stress from the temperature history to a butt-welded plate", Welding Journal, Vol. 17, p.4-7s

3. Griffith and H. R. George, 1941, "Residual stress in butt-welded steel plates", Welding Journal, Vol.20, 1941, p.410s-414s

4. Satoh K., Matsui S., 1967, "Reaction stress and weld cracking under hindered condition", Technological Reports of the Osaka Univ., 1967

5. Satoh K., 1972, "An analytical approach to the problem of restraint intensity in slit weld", IIW, Document X-661-72,

6. Yukio Ueda and Taketo Yamakawa, 1971, "Analysis of thermal elastic-plastic stress and strain during welding by finite element analysis", Trans. of JWS, Vol. 2, No. 2, p.90s-98s

7. E. Friedman, 1975, "Thermo-mechanical analysis of the welding process using the finite element method", Trans. of ASME, p.209s-213s

8. Satoh K., Terasaki T., 1976, "Effect of welding conditions on welding deformations in welded structural materials", Trans. of JWS, Vol. 45, No. 4, p.54s-60s 



\title{
NUMERICAL SIMULATION OF SHEAR STRESS DISTRIBUTION IN THE FILLET OF AN Al-Al $\mathrm{O}_{3}$ SOLDERING ASSEMBLY
}

\author{
X. Ma ${ }^{*}$ Y.Y. Qian ${ }^{\#}$
}

\begin{abstract}
By means of finite element numerical simulation, the shear stress distribution in the $\mathrm{Al}-\mathrm{Al}_{2} \mathrm{O}_{3}$ soldering assembly and the effect of the coefficient of thermal expansion of interlayer alloy and the fillet geometry had been analyzed. The calculating results showed that, maximum level of shear stress occurred at the soldering fillet and the interface between $\mathrm{Al}_{2} \mathrm{O}_{3}$ ceramic and coated $\mathrm{Cu}$. Meanwhile, in order to get better shear stress distribution, the coefficient of thermal expansion of interlayer alloy should match with the Al base metal and the optimized geometry of soldering fillet is the concave shape with extruding length a little more than the gap height.
\end{abstract}

\section{KEYWORDS}

Finite element numerical simulation, $\mathrm{Al}_{-}-\mathrm{Al}_{2} \mathrm{O}_{3}$ soldering, shear stress, coefficient of thermal expansion, fillet geometry

\section{INTRODUCTION}

Metal-ceramic soldering assembly has been widely used in industrial applications [Ref. 1-2]. As an assembly with different materials joining, cooling stage after soldering will lead to thermal stress in the assembly due to the mismatch of coefficients of thermal expansion (CTE) of different base materials. Such thermal stress will further cause crack at the joining interface in some cases and failure of the assembly [Ref. 3-4]. With the consideration of the special geometry of soldering seam, the mechanical response which is most affected is the shear stress distribution in the soldering fillet [Ref. 5]. Undoubtedly, the CTE of base materials and the geometry of soldering fillet will play important roles on the shear stress distribution. Similar problem has been investigated in reliability evaluation of surface mount solder joints used in microelectronic packaging [Ref. 6-7]. But few works had been done for metal-ceramic soldering assembly. In

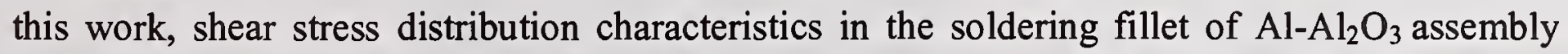
caused by cooling stage has been studied by finite element numerical simulation, the effects of the CTE of base materials and the geometry of the soldering fillet had been illustrated.

\footnotetext{
"National Key Laboratory of Reliability Physics of Electronic Product, CEPREI, Guangzhou 510610, P.R.China

" National Key Laboratory of Welding, Harbin Institute of Technology, Harbin 150001, P.R.China
} 


\section{FINITE ELEMENT MODEL}

Figure 1 is the schematic of $\mathrm{Al}-\mathrm{Al}_{2} \mathrm{O}_{3}$ soldering assembly. $\mathrm{Cu}$ with $0.2 \mathrm{~mm}$ thickness was coated on the surface of $\mathrm{Al}_{2} \mathrm{O}_{3}$ ceramic in order to improve the solderability. The interlayer filler is Sn-based, low melting point $\left(180-190^{\circ} \mathrm{C}\right)$ alloy with $0.1 \mathrm{~mm}$ thickness. The corresponding two-dimensional finite element model with 8 nodes isoparametric elements is shown in Figure 2. Since it is axial symmetric, only half part of the assembly was modelled in this simulation.

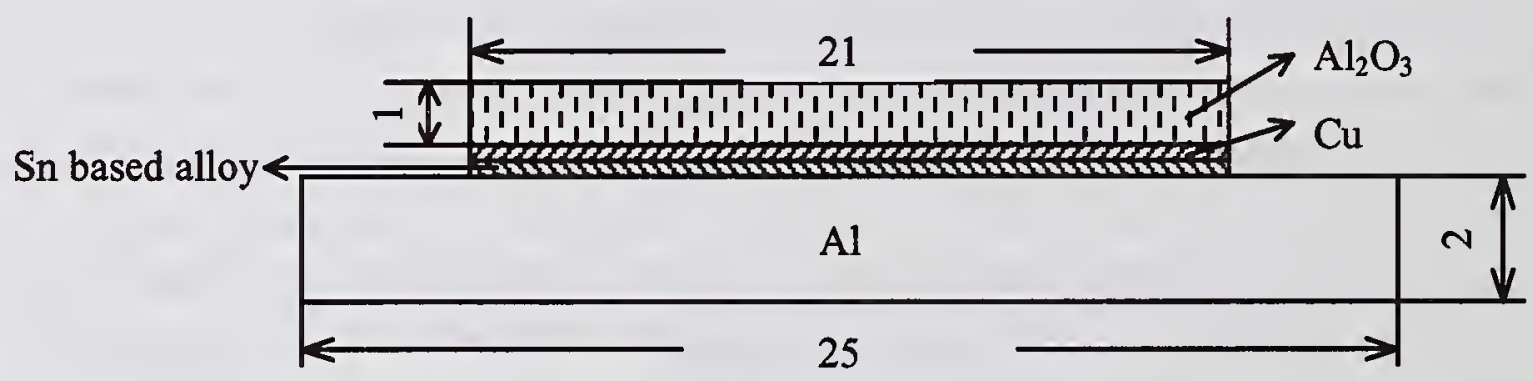

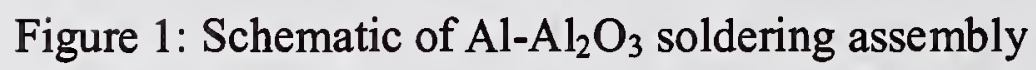

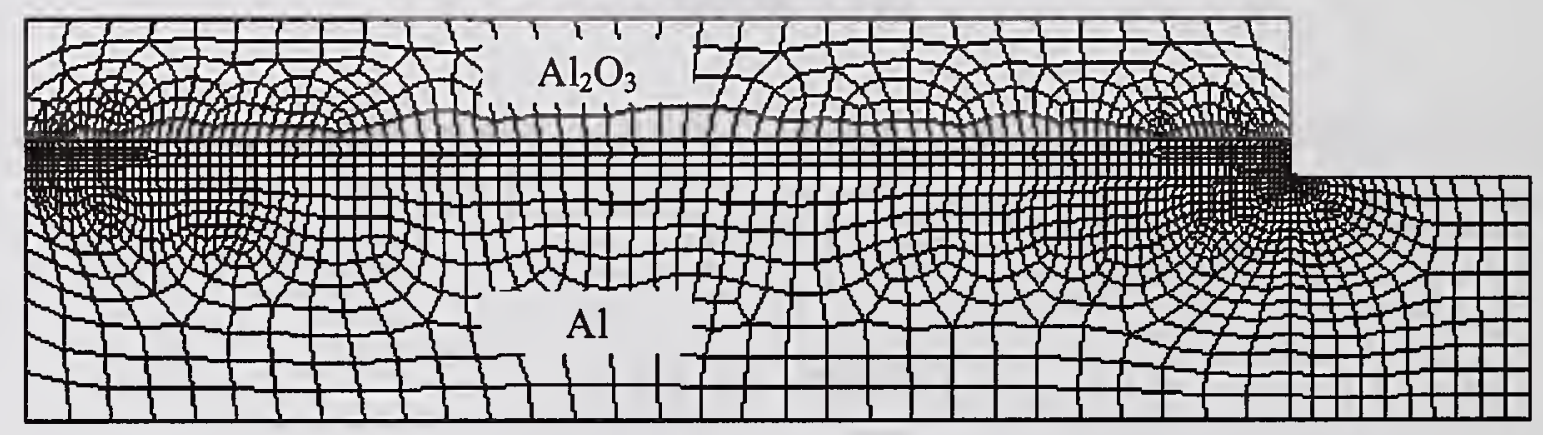

Figure 2: Two-dimensional finite element model of $\mathrm{Al}-\mathrm{Al}_{2} \mathrm{O}_{3}$ soldering assembly

Commercial finite element analysis software ANSYS5.6 was used in this work. All the materials were assumed as elastic and the materials properties used in this calculation is listed in Table 1. Cooling stage is the only outer thermal loading, cooling temperature range is from $180^{\circ} \mathrm{C}$ to 20 ${ }^{\circ} \mathrm{C}$ since the melting point of interlayer alloy is rather low.

Table 1 Material parameters used in FEM calculation

\begin{tabular}{cccc}
\hline Material & $\mathrm{E}(\mathrm{MPa})$ & $v$ & $\alpha\left(10^{-6} \mathrm{~K}^{-1}\right)$ \\
\hline $\mathrm{Al}$ & 70000 & 0.33 & 25.1 \\
Sn-based alloy & 43251 & 0.36 & 25.4 \\
$\mathrm{Cu}$ & 117000 & 0.34 & 16.7 \\
$\mathrm{Al}_{2} \mathrm{O}_{3}$ & 300000 & 0.23 & 6.7 \\
\hline
\end{tabular}




\section{EFFECT OF SOLDERING FILLET SHAPE}

From the point of view of geometry, the shape of soldering fillet can be divided into three types: concave, $45^{\circ}$ line style and convex. Their corresponding finite element models are shown in figure 3.

The shear stress distribution in the above three types soldering fillets are shown in figures 4, 5 and 6, respectively. By comparing the shear stress level, it can be seen that convex fillet is the worse shape because the maximum shear stress in the fillet is 50MPa more than concave shape and $45^{\circ}$ line style. As to the latter two fillet shapes, the shear stress level is similar, but the site of maximum shear stress is a little different. The maximum shear stress occurs at the fillet centre for concave shape while at the upper side of fillet for $45^{\circ}$ line style. Furthermore, it can be seen from the shear stress contours that stress concentration is more serious in $45^{\circ}$ line style than concave shape. Therefore, the mechanical response in the concave fillet shape can be considered as the best.

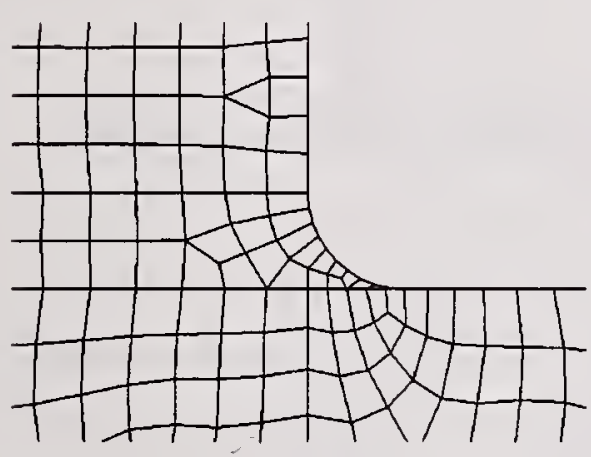

(a) Concave fillet

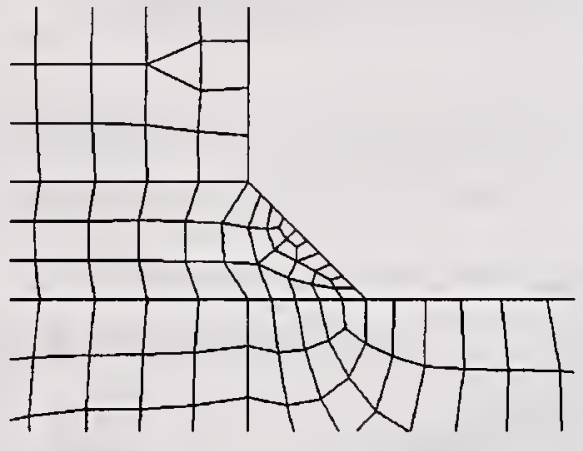

(b) $45^{\circ}$ line style fillet

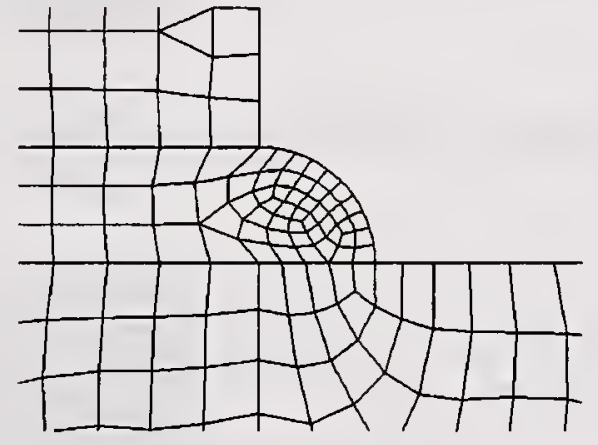

(c) Convex fillet

Figure 3: Finite element models of different soldering fillets geometry

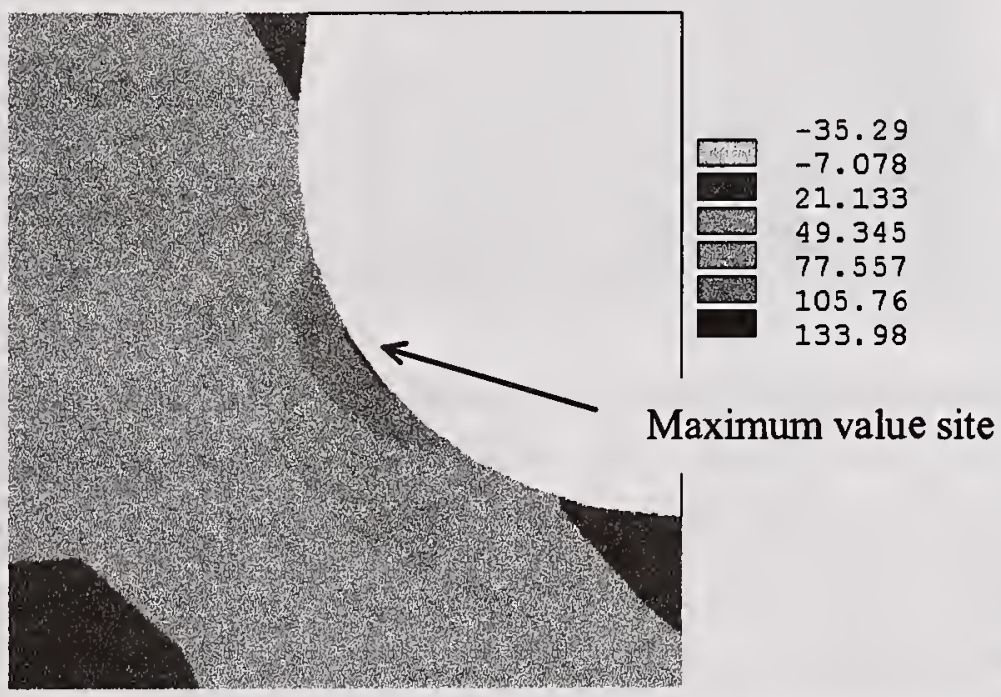

Figure 4: Shear stress distribution corresponding to concave soldering fillet (Unit: MPa) 


\section{EFFECT OF CTE OF INTERLAYER ALLOY}

The thermal stress caused by cooling stage is due to the mismatch of the four materials in the soldering assembly. There is the global mismatch between the $\mathrm{Al}_{2} \mathrm{O}_{3}$ ceramic and $\mathrm{Al}$ base metal, and also the local mismatch between the adjacent layers. Usually, the properties of the materials

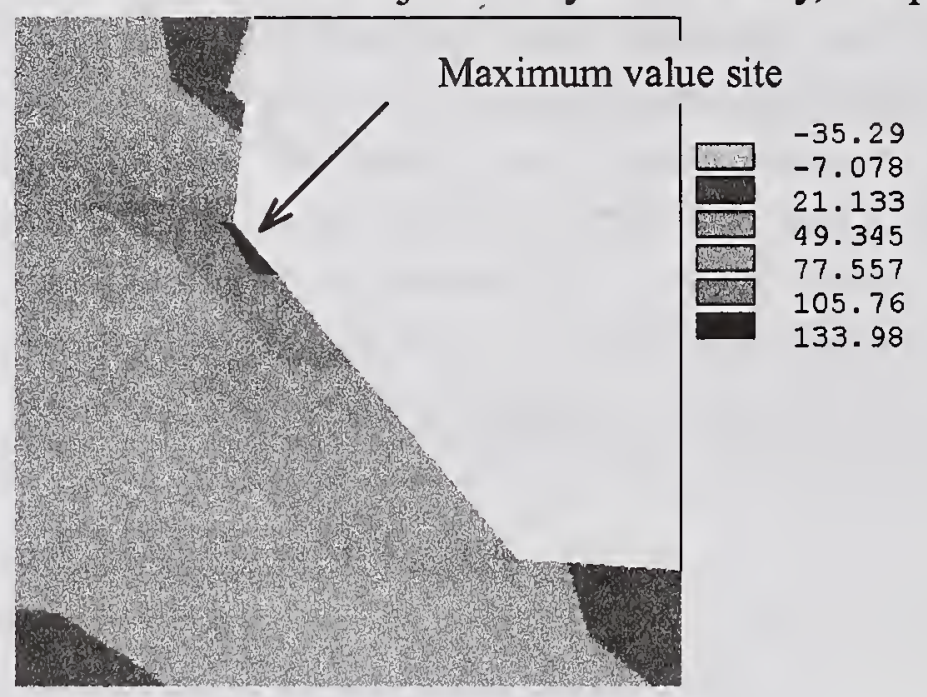

Figure 5: Shear stress distribution corresponding to $45^{\circ}$ line style soldering fillet (Unit: MPa)

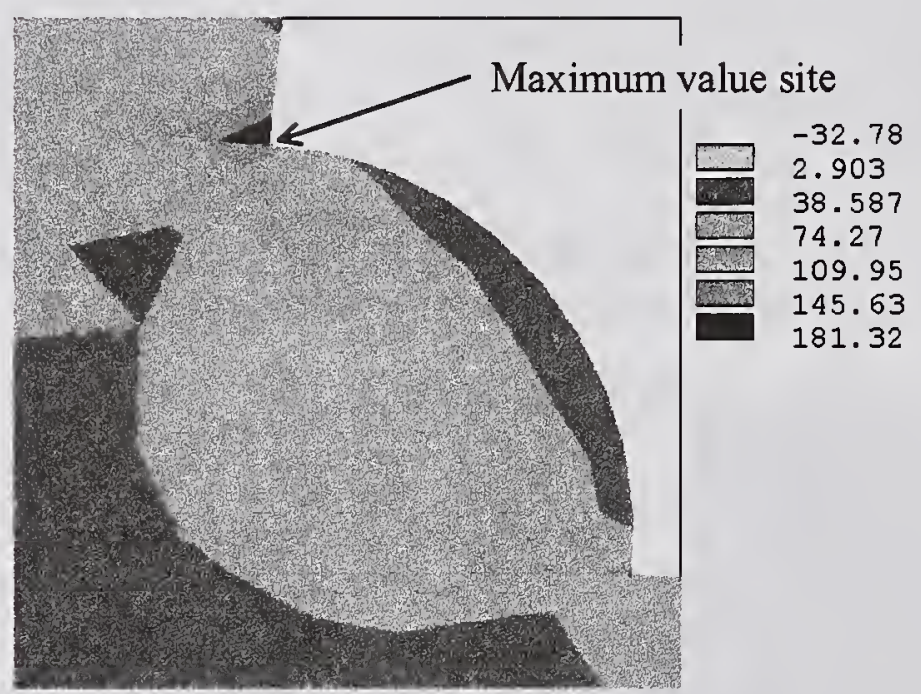

Figure 6: Shear stress distribution corresponding to convex soldering fillet (Unit: MPa)

to be joined are fixed, and we only can change the property of interlayer material by alloy design. In this work, the effect of the CTE of interlayer alloy on the maximum shear stress in the soldering fillet has been studied.

The soldering fillet shape used in this calculation is concave type as shown in figure 3(a). The calculating results are shown in figure 7. The maximum value of CTE of interlayer alloy is assumed to be more than $\mathrm{Al}$ and the minimum value less than $\mathrm{Al}_{2} \mathrm{O}_{3}$. The results showed that there is minimum shear stress level in the soldering fillet when the CTE of interlayer alloy 
matched with Al. This is to say, for the multilayer assembly with different materials, the CTE of the interlayer alloy should match with the Al base metal which has the maximum CTE value. At this time, the shear stress in the soldering fillet caused by cooling stage could be minimized. Such results also indicate that the interlayer alloy (see Table 1) that we used in soldering experiments is a good choice.

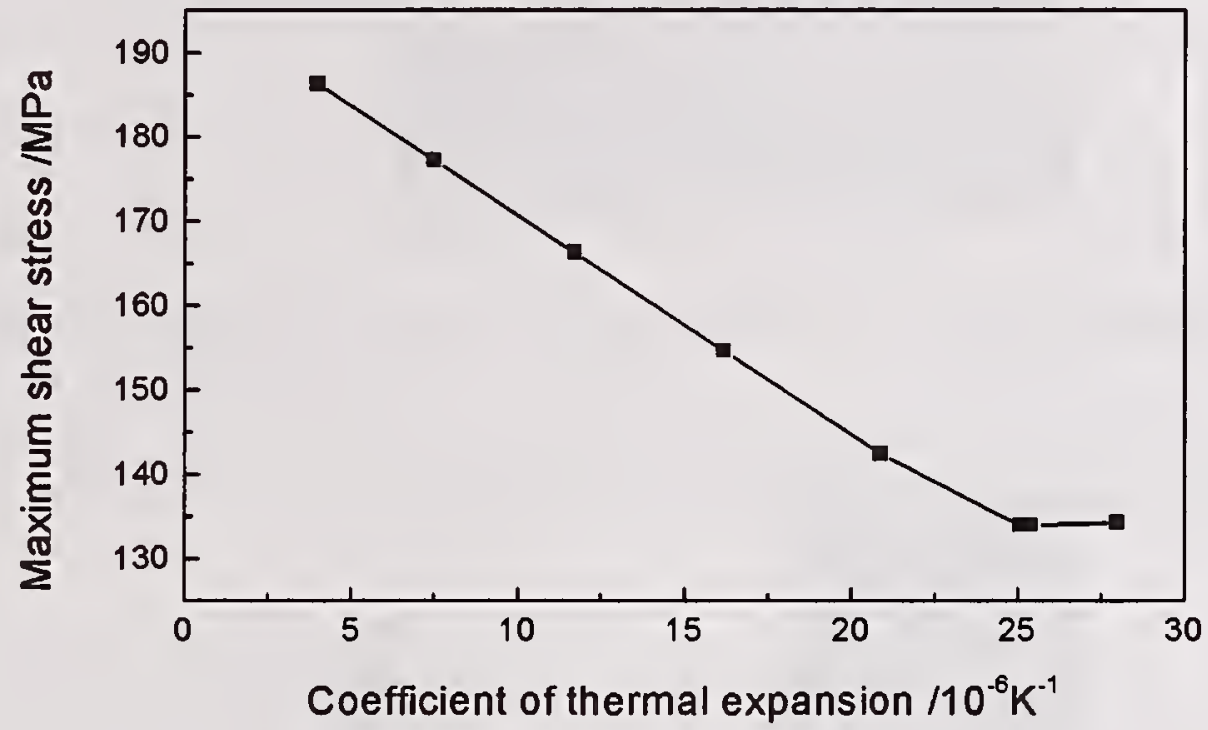

Figure 7: Effect of CTE of interlayer alloy on the maximum shear stress in the soldering assembly

\section{EFFECT OF GEOMETRIC PARAMETER OF CONCAVE FILLET}

As to the concave fillet shape, the most important geometric parameter is the ratio of the fillet extruding length $\mathrm{L}$ to the gap height $\mathrm{H}$. Figure 4 (corresponding to figure 3(a)) shows the shear stress distribution for the case of $\mathrm{L} / \mathrm{H}=1$. Let the gap height $\mathrm{H}$ invariable, figures 8 and 9 show the shear stress distribution in the soldering fillet for the case $\mathrm{L} / \mathrm{H}=0.5$ and 1.5 , respectively. Using $\mathrm{L} / \mathrm{H}=1$ as reference geometry, it can be seen that the maximum shear stress level and its location change little when the fillet extruding length decreases. On the other hand, the maximum shear stress level is reduced by $10 \mathrm{MPa}$ when the fillet extruding length increases, meanwhile, its location changes from fillet center to the upper side. 


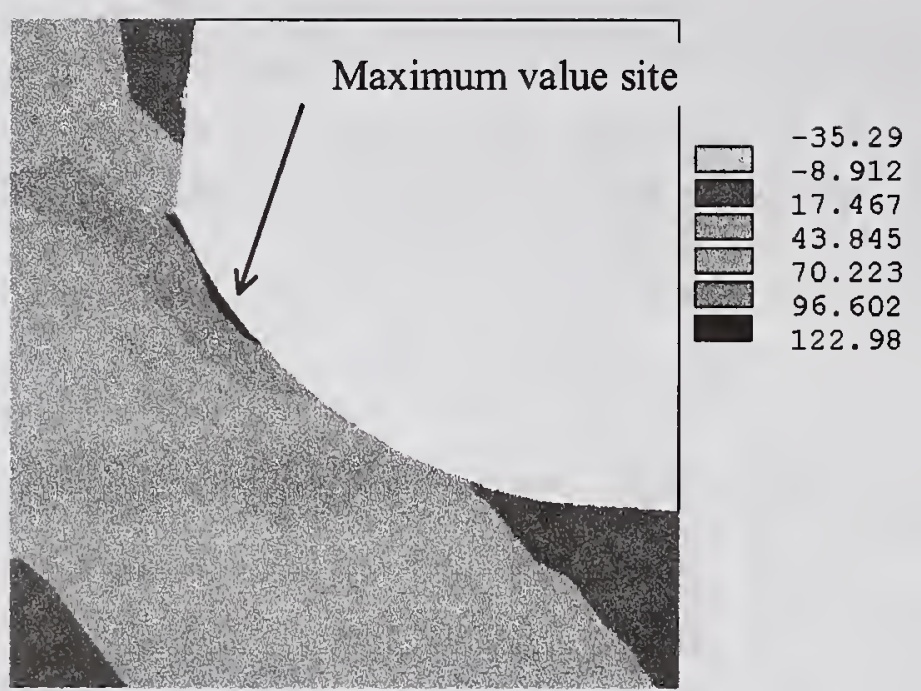

Figure 8: Shear stress distribution corresponding to concave soldering fillet with $\mathrm{L} / \mathrm{H}=0.5$ (Unit: $\mathrm{MPa})$

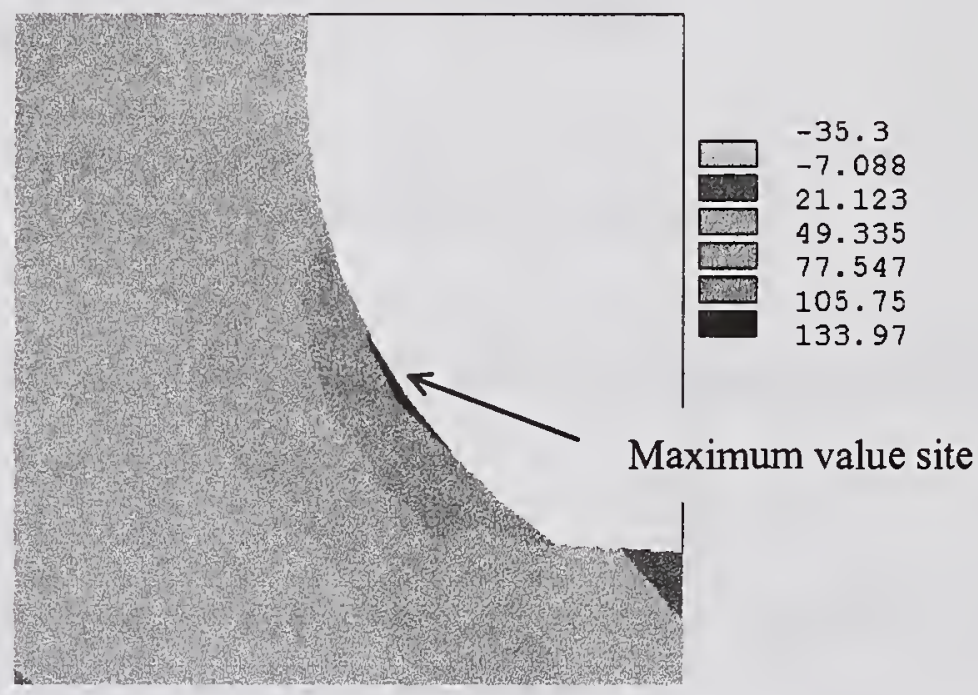

Figure 9: Shear stress distribution corresponding to concave soldering fillet with $\mathrm{L} / \mathrm{H}=1.5$ (Unit: $\mathrm{MPa})$

\section{CONCLUSIONS}

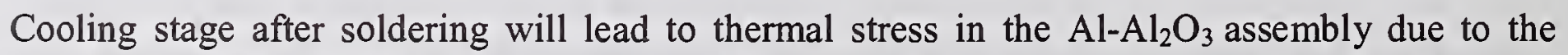
mismatch of CTE of different materials. The CTE of interlayer alloy and the fillet geometry play important roles on the shear stress distribution. In order to minimize the shear stress level in the soldering fillet, the CTE of interlayer alloy should match with the Al base metal, meanwhile, the best fillet shape is the concave type with extruding length a little more than the gap height 


\section{REFERENCES}

1. James, I. 1989. Challenge of bonding metals to ceramics. Machine Design 61(24): 95-100.

2. Ohmori, A.; Zhou, Z.; Suzumura, A.; and Arata, Y. 1993. Bonding of ceramics to metals with activated coating layers made by plasma spraying. Materials and Manufacturing Processes 8(2): 183 to 201.

3. Qian, Z.; and Akisanya, A.R. 1998. An experimental investigation of failure initiation in bonded joints. Acta Mater. 46: 4895 to 4904.

4. Reimanis, I.E.; Dalgleish, B.J.; and Evans, A.G. 1991. The fracture resistance of a model metal/ceramic interface. Acta Metall. Mater. 39: 3133 to 3141.

5. Yang, Y.Y.; Munz, D.; and Schaller, W. 1997. Effect of the stress jump at the interface of a joint on the failure behavior. International Journal of Fracture 87: L113 to L118.

6. Heinrich, S.M.; and Liedtke, P.E. 1993. Effect of chip and geometry on solder joint formation in SMT. ASME Journal of Electronic Packaging 115: 443 to 449.

7. Charles, H.K.; and Clatterbaugh, G.V. 1990. Solder joint reliability design implications from finite element modeling and experimental testing. ASME Journal of Electronic Packaging 112: 135 to 146. 

Session B2: Modeling II: Predicting Microstructure and Performance 



\title{
ADVANCES IN ALUMINUM WELD SIMULATIONS APPLYING WELDSIM
}

\author{
H. G. Fjær ${ }^{(*)}$, O. R. Myhr ${ }^{(* *)}$, S. Klokkehaug ${ }^{(* *)}$, E. J. Holm ${ }^{(*)}$
}

\begin{abstract}
This paper describes recent developments and applications of the advanced simulation model WELDSIM. This model is applicable for welding of age hardening aluminum alloys, and computes the evolution of temperatures, microstructure, residual stresses and distortions. The model is today extensively applied in seeking adequate welding procedures in the fabrication of welded automotive parts in aluminum, and it has become an attractive alternative to the traditional procedure of trial and error based optimization of the welding parameters. In the present work, results from weld simulations have been compared to corresponding measurements during welding, and a very good agreement has been obtained. The model has also been applied investigating the possibilities of minimizing the distortions in the welding of automotive parts. The simulations have shown how the fixture design, the weld sequence as well as the welding parameters, significantly affects the resulting weld distortions.
\end{abstract}

\section{INTRODUCTION}

Extruded aluminum profiles are to an increasing extent applied in automotive components like space frames, engine cradles and windshield frames where weight saving is essential. Welding is a key operation in the manufacturing of such parts, and robotic GMA welding is by far the most commonly applied process for high volume production. A major problem associated with welding is the thermally induced deformations caused by the intense non-homogeneous heating and cooling of the material. These deformations are unavoidable in welding, but can usually be minimized to an adequate level by proper selection of the welding parameters and the fixture design. In order to obtain weld deformations within the geometrical tolerance limits, two principally different approaches can be applied, as schematically outlined in Figure 1. The loop on the left hand side of the diagram (i.e. the "physical welding" loop) illustrates the traditional trial and error based procedure applying welding experiments and a robotic welding unit. The welding is followed by measurements of the resulting distortions and the corresponding deviations from the nominal geometry. If the distortions are outside the tolerance limits, some adjustments are done for the welding conditions, the fixture- or the geometric design as indicated in the rectangular window of the figure before another component is welded. The welding is followed by measurements of the resulting geometry, and this loop is repeated until a certain combination yields weld distortions that are acceptable.

The loop on the right hand side (i.e. the "virtual welding" loop) utilizes a computer instead of a welding cell, where the simulation results provide direct information on the positions the distortions are outside the tolerance limits. It is easy to change the input data systematically in order to analyze their individual effect on the resulting local and global distortions. A post-

(*) Institute for Energy Technology, Box 40, N-2027 Kjeller, Norway

(**) Hydro Automotive Structures Raufoss, Box 15, 2831 Raufoss, Norway 
processor tool can be applied to view animations of the time evolution of the thermally induced deformations. Such illustrations of the process give valuable insight and increased understanding of the process. This provides a basis for generating rules for a better design of both the fixture and the welding sequence.

A complete replacement of real welding experiments by simulations in the fabrication of welded components is not realistic in the foreseen future, but there is no doubt that the trend is irreversible moving towards a more extensive utilization of computer simulations. The impetus for this development is the substantial potential for saving both costs and time in the preproduction stage. This has been the motivation for developing the weld simulation model WELDSIM (Ref. 1-4), which provides a mathematical description of the relation between the main welding parameters and the subsequent distortions and weld properties. The model has been developed and refined to a stage where it can be applied for realistic simulations of welding of complex aluminum structures and capture important effects of the geometric design and the weld parameters, as well as material related variables like alloy composition and base metal temper condition. Moreover, the last years significant developments on computer hardware has made it possible to carry out simulations on an ordinary PC within an acceptable amount of time. The WELDSIM model is today extensively applied in the pre-production phase in seeking adequate welding procedures in fabrication of welded automotive parts in aluminum. The present paper illustrates recent developments of the model. The accuracy of the simulations is illustrated by comparisons between simulation results and measurements, and an example from a case study on welding of an automotive component is presented at the end of the paper.

\section{Physical wolding Virtual wolding}

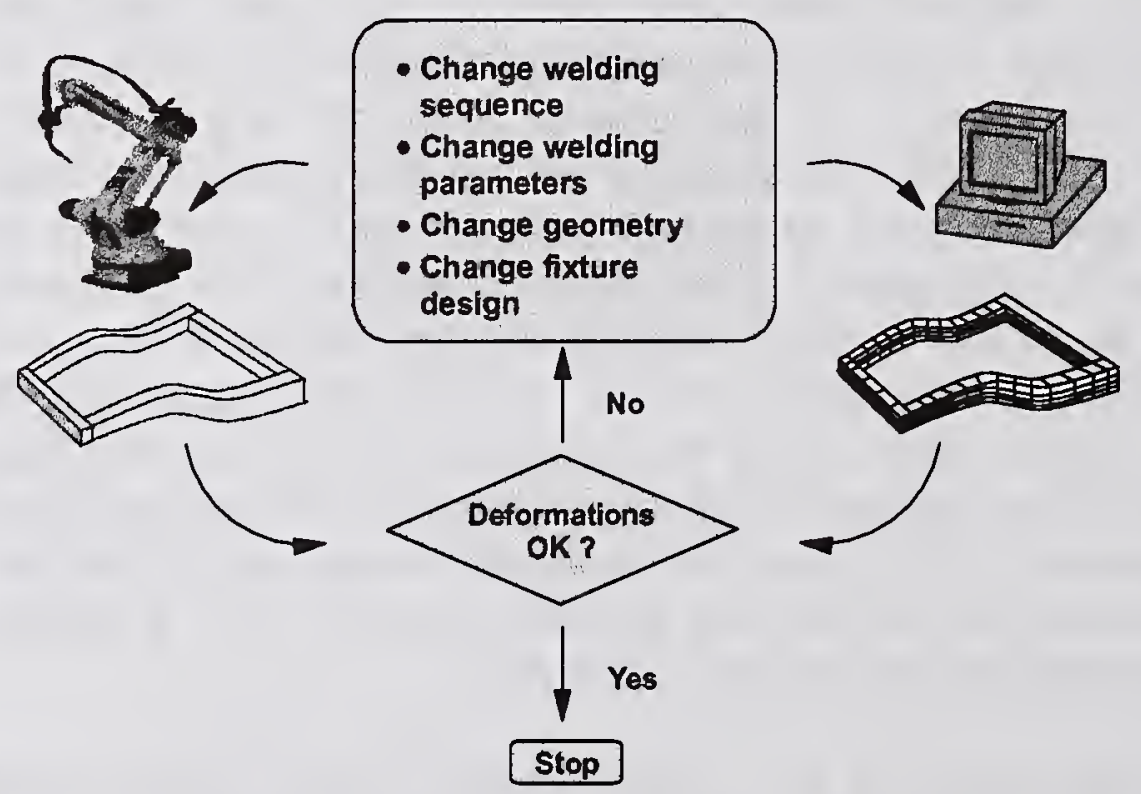

Figure 1: Two different approaches for satisfying geometric tolerances in fabrication of welded structures. 


\section{MODEL OUTLINE}

WELDSIM consists of a thermal, a microstructure and a mechanical sub-model that are connected as illustrated in Figure 2, and implemented in a finite element method (FEM) software framework. The thermal field influences both the microstructure evolution as well as the evolution of the thermally induced stresses and strains. At the same time there is a link between the microstructure and the mechanical sub-model to account for the important effect of softening of the heat affected zone due to reversion of hardening particles. This is taken into account by a separate (internal state) variable $f / f_{0} . f / f_{0}$ is computed by the microstructure model and transmitted to the mechanical model as a field variable. There is no "back-coupling" from the mechanical model to the microstructure model.

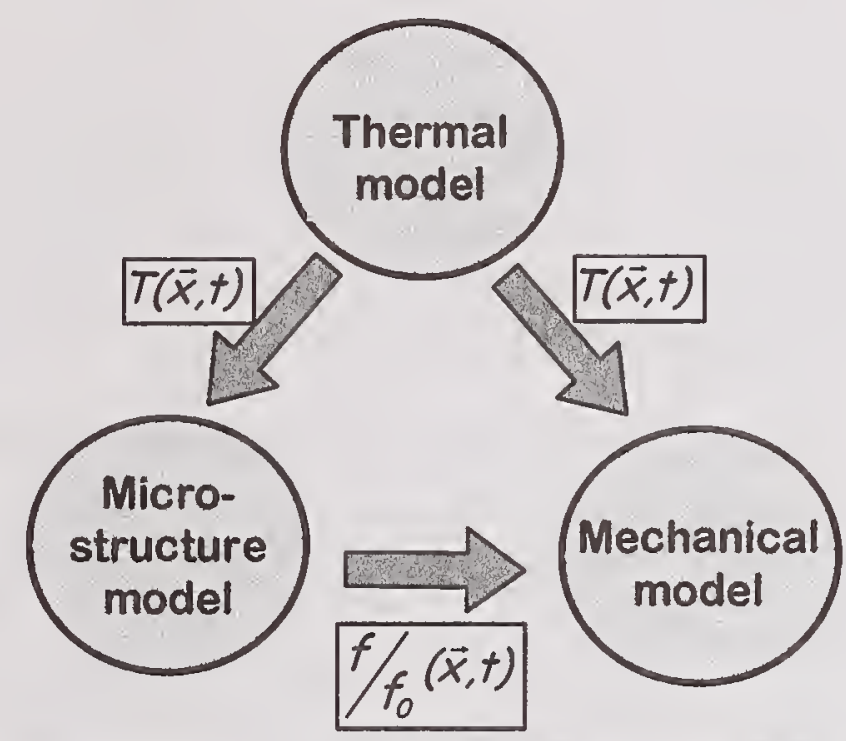

Figure 2: The connection- and transition of data between the different sub-models of WELDSIM.

\section{The Thermal Model}

The thermal model is a fully 3D FEM solver with an implicit time integration scheme, where the specific enthalpy is the basic unknown. In WELDSIM, the heat source is modeled as a traveling double ellipsoid heat distribution function. In order to obtain realistic temperatures, the heat distribution function has been calibrated against measurements of the weld pool geometry. Moreover, the model has a correction algorithm for compensating numerically induced deviations in the total heat input. If present, the addition of filler wire material is modeled by a continuous activation of the finite elements in the reinforcement regions when they are approached by the heat source. The dimensions of these regions must, however, be predefined.

\section{The Microstructure Model}

Heat-treatable aluminum alloys obtain their main strengthening contribution from the precipitation of a fine dispersion of small particles from a supersaturated solid solution. These small particles are not resistant to the high temperatures imposed by the welding, and will partly or fully dissolve at short distances from the weld. This process is usually referred to as reversion, and is the main reason for the HAZ softening taking place during welding of heat-treatable aluminum alloys. In order to quantify this effect, the model considers the evolution of the volume fraction $f$ of the small hardening particles. The state of the precipitate structure can then be described by the single normalized state variable $f / f_{0}$, where $f_{0}$ refers to the initial volume fraction of the particles before the welding starts. As shown in Ref. 1, f/f $f_{0}$ can be calculated as: 


$$
\frac{f}{f_{0}}=1-\left[\int \frac{d t}{t_{1}^{*}}\right]^{n_{1}}
$$

Here, $n$ is a time exponent $(<0.5)$, and $t_{1}^{*}$ the time taken for complete particle dissolution at a given temperature. The corresponding local hardness $H V$ is assumed to vary linearly with $f / f_{0}$, i.e.:

$$
\frac{f}{f_{0}}=\frac{H V-H V_{\min }}{H V_{\max }-H V_{\min }}
$$

Here $H V_{\min }$ corresponds to the hardness in the fully reverted state whereas $H V_{\max }$ represents the original base material strength for the actual temper condition (e.g. artificially aged (T6) or naturally aged (T4) material). For alloys within the $6 \mathrm{xxx}$ series (i.e. Al-Mg-Si alloys) in the T6 temper conditions, the hardening phase consists of needle-shaped $\beta$ ''-particles, while in the T4 temper condition, the precipitates are the fully coherent and significantly smaller GP-zones. The width of the HAZ depends both on the temperature distribution and on the thermal stability of the hardening phase. Since the GP-zones revert at lower temperatures than the $\beta$ " particles, a wider HAZ is obtained for an alloy in the T4 than in the T6 temper (Ref. 4).

A recently developed precipitation model for aluminum alloys within the 6xxx series (Ref. 5) predicts both the density and the size distribution of the hardening precipitates, and correlates this to the room temperature yield stress through established dislocation theory. This offers the possibilities of predicting the microstructure throughout the different operation steps and thermal treatments, including both the pre- and post-weld heat treatment. This more advanced microstructure model will be implemented in the next version of WELDSIM.

The Mechanical Model

In WELDSIM, the metal is described as an isotropic and elastic-viscoplastic material according to the following formula:

$$
\sigma=F(T) G\left(f / f_{0}, T\right)\left[H\left(\phi, f / f_{0}\right)\right]^{n(T)}\left(\dot{\varepsilon}_{p}\right)^{m(T)} .
$$

Here the flow stress is expressed as a product of a solely temperature dependent function $F$, a function $G$ incorporating the effect of hardening particles, a function $H$ representing the work hardening and finally the viscoplastic strain rate $\dot{\varepsilon}_{p}$. Eq. (3) requires elevated temperature data, which are only to a limited extent given in the literature. Therefore, material characterization of some relevant alloys and temper conditions has been carried out on a Gleeble ${ }^{1}$ thermal simulator. Samples were tested at different temperatures and strain rates. The effect of particles on the flow stress was investigated by performing tests both on samples with- and without an initial precipitate structure at a given temperature. In order to remove the particles before the testing, a solution heat treatment was prescribed as part of the pre-defined thermal program. The flow stress was generally seen to be strongly dependent on the temperature and the volume fraction of precipitates, and less dependent on the work hardening and strain rate. For alloy or temper conditions not covered by the Gleeble testing, the $F$ and $G$ functions in Eq. (3) are scaled by

${ }^{1}$ Gleeble is a registered trademark of Dynamic Systems Inc. 
assuming a linear relationship between the stress and the hardness, and by inserting reasonable values for $H V_{\min }$ and $H V_{\max }$ in Eq. (2).

The mechanical boundary conditions representing the fixture or clamping is an essential part of the model. These can be imposed as a distributed force or spring stiffness. The boundary conditions are allowed to vary with time, and the spring stiffness can be dependent on the computed displacement. These boundary conditions can also be applied at the interface between parts of the solution domain, making modeling of gaps or mechanical contact possible. In the simulations, the interface boundary conditions are different in front of and behind the moving heat source in order to account for the cohesion, i.e. the ability of the interface to withstand tensile forces, depending on whether a prescribed threshold temperature is reached.

\section{Applying the Model}

CAD drawings are used as a basis for generating appropriate geometries for the FEM meshing. Commercial pre-processors are applied for generating the mesh and a necessary set of labels for the different volume or surface domains. These labels are then combined with data sets representing the thermal, microstructure and mechanical properties of the actual alloy(s), as well as the thermal and mechanical boundary conditions reflecting the fixture design. Data describing the welding process and the welding sequence must be specified, as well as parameters governing the time stepping and the numerical schemes.

Modeling the welding process involves solving time-dependent coupled non-linear problems with a moving, localized heat source. Such computations require extensive CPU-times. Generally, much larger time steps (typically a few tenths of a second) are applied for solving the mechanical problem compared to the time steps of the order of milliseconds for the thermal/microstructure problem. Output from WELDSIM includes the temperature evolution, the microstructure and resulting material strength, as well as the residual stresses and the distortions of the assembly.

\section{COMPARISON BETWEEN MODEL SIMULATIONS AND MEASUREMENTS}

A series of welding experiments were carried out at Hydro Automotive Structures Raufoss. Robotic GMA welding was performed on extruded aluminum tubes, of the alloys 6063 and 6082. The assembly, corresponding to a corner of a frame, is shown in Figure 3(a). Two welds were deposited in order to join the two tubes, as illustrated in Figure 3(b). The starting point of the welds was at the exterior side of the corner. The cross section of the extruded tubes was 40 by 60 $\mathrm{mm}$, while three different values of the wall thickness were used, i.e. 3,4 and $5 \mathrm{~mm}$. The welding speed was $16.1 \mathrm{~mm} / \mathrm{s}$ at the straight portions of the welds and $21 \mathrm{~mm} / \mathrm{s}$ at the curved corners. The voltage was about $21.5 \mathrm{~V}$ and the mean amperage was estimated to be $147 \mathrm{~A}$.

The distortions were registered by 4 extensometers. An example of displacements recorded from one welding experiments, applying $3 \mathrm{~mm}$ thick 6082-T6 extrusions, is shown in Figure 4(a). Compression of the extensometers corresponds to positive displacement values. Corresponding computed displacements are shown in the right part of the figure. A very good agreement is seen. However, all measurements suffered from noise during welding, due to the $\mathrm{AC}$ power supply for 
the welding arc. When the clamps are released (after 1200s in the experiments, after 500s in simulations) the displacements develop similarly, but the elastic spring boundary condition remaining at clamp a (conf. Figure 3(a)) cause the computed displacements at extensometer positions 1 and 2 to become zero.

(a)

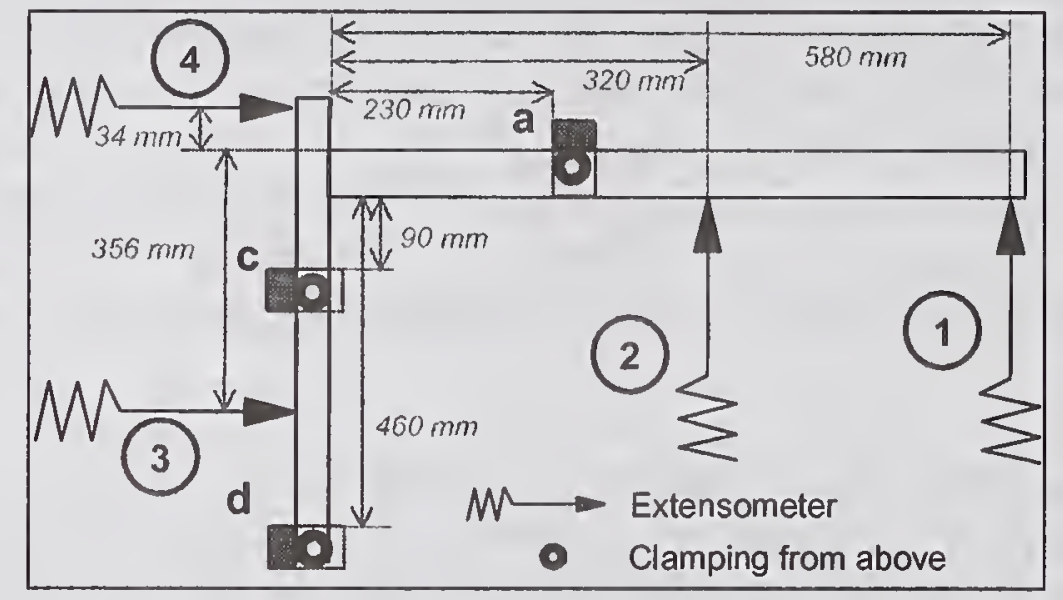

(b)

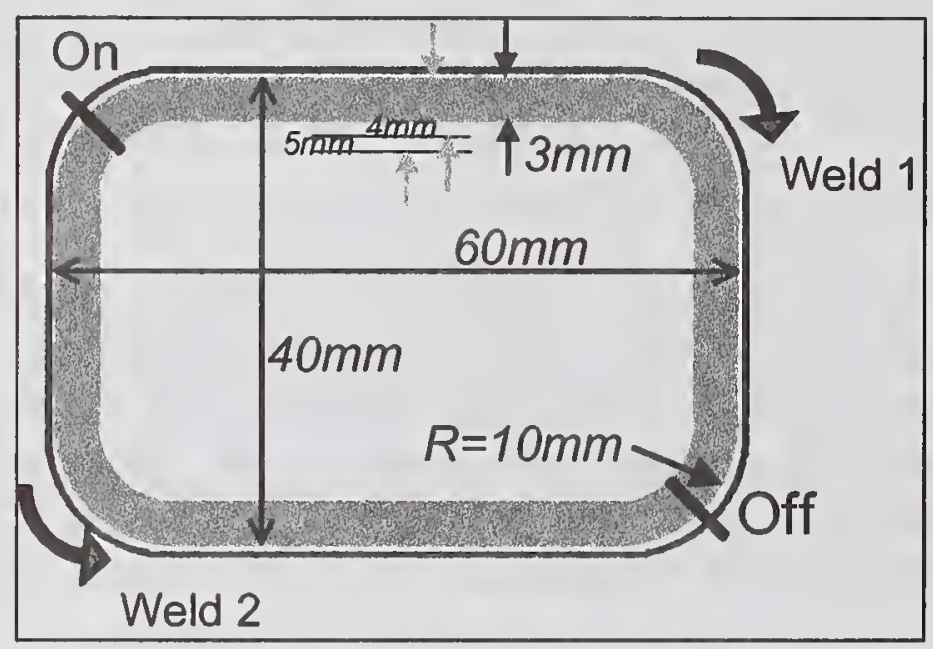

Figure 3: (a) Top view of the experimental set-up showing locations of clamps and extensometer positions. (b) The cross-section of the tubes and the trajectory of the weld path.

The measured displacements are affected both by rigid body translation and rotation. In fact, only one distortional mode can be extracted from the measurements. This corresponds (closely) to the tilt angle $\theta$ illustrated in Figure 5(a). Also of interest is the bending of the extrusion tube A, represented by the angle $\varphi$. Figure 5(b) shows final computed distortions, magnified by a factor of 20. The tilt angle is clearly larger than would be expected from an orthogonal attachment of tube B on a bent tube A. Hence plastic deformation must have been induced at the weld joint interface due to the welding.

A comparison between the calculated and measured permanent tilt angels is shown in Figure 6 . A very good agreement is found for the $3 \mathrm{~mm}$ thick tubes of the $6082 \mathrm{~T} 6$ alloy. In the case referred to as "strongly clamped", an extra clamp acting horizontally was applied on tube B. This clamping is clearly seen to reduce the tilt angle. The deformations resulting from varying the tube thickness for the 6063 alloy, as shown in Figure 6(b), also reveals a reasonable agreement between calculated and measured values. These results clearly show that the distortions are reduced as the profile thickness increase due to a corresponding increase in the profile stiffness. 
(a)

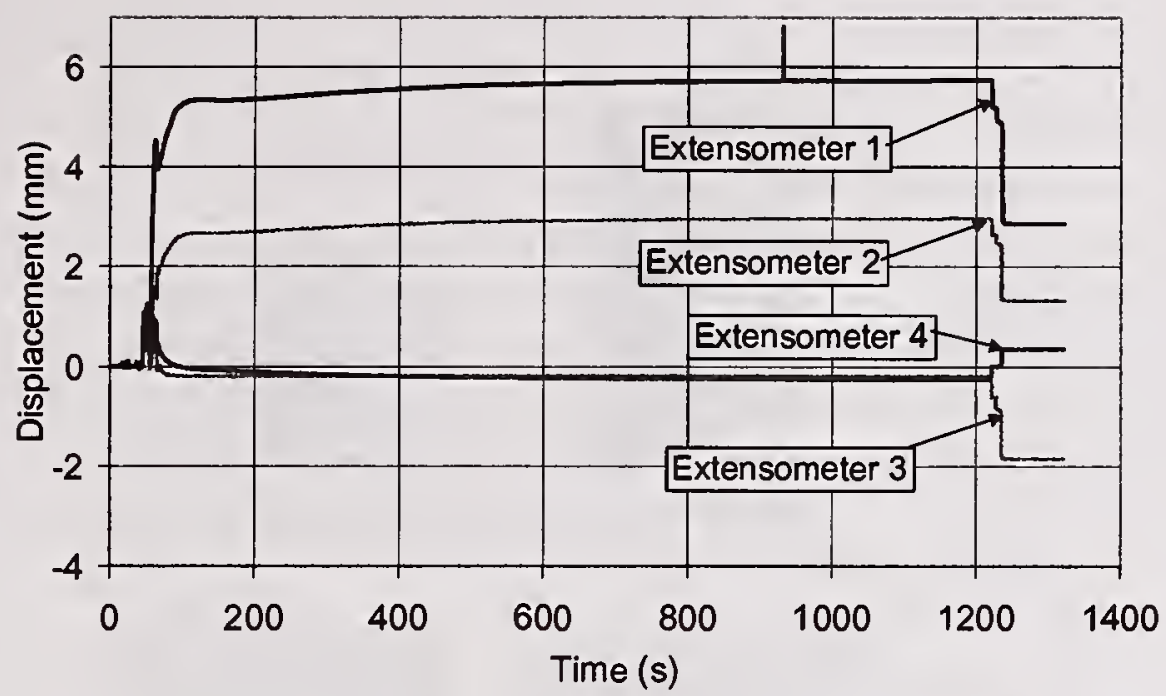

(b)

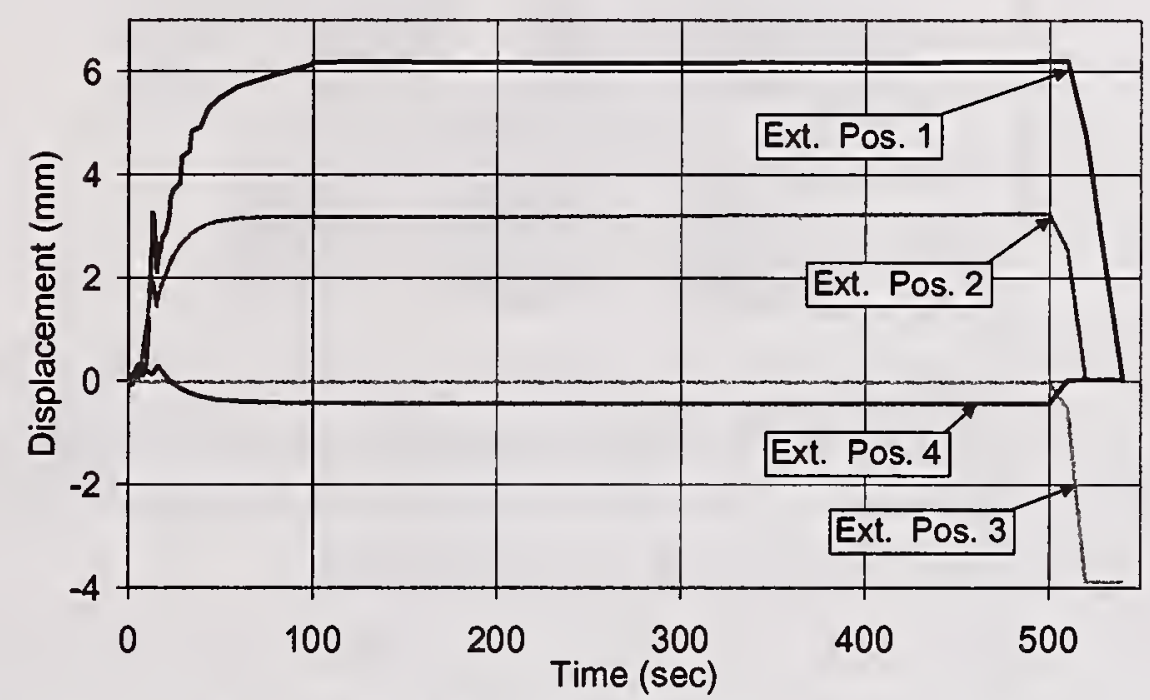

Figure 4: (a) Measured and (b) computed displacements during welding and subsequent cooling of $3 \mathrm{~mm}$ thick extruded tubes of the alloy 6082-T6.

(a)

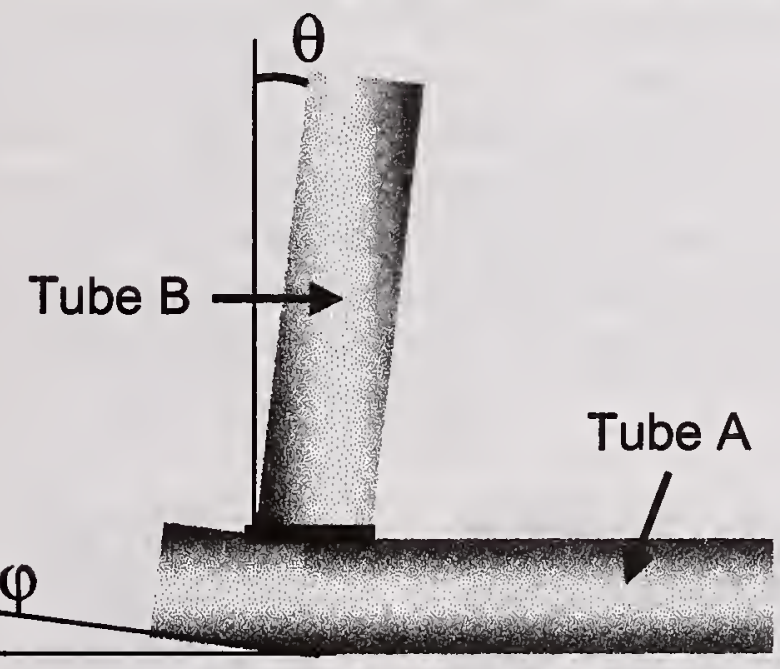

(b)

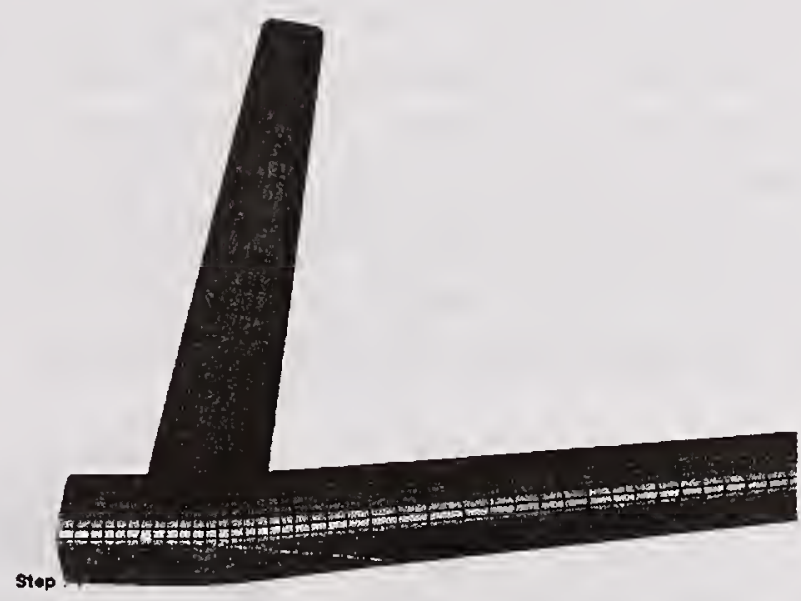

Figure 5: (a) Illustration of the tilt angle $\theta$ and the bending angle $\varphi$. (b) Computed final distortions magnified by a factor of 20 . 
(a)

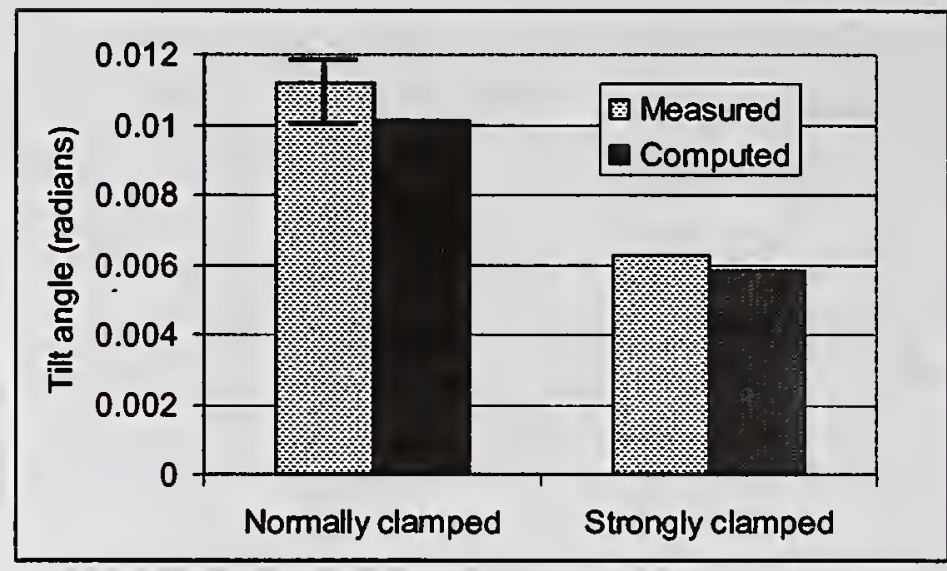

(b)

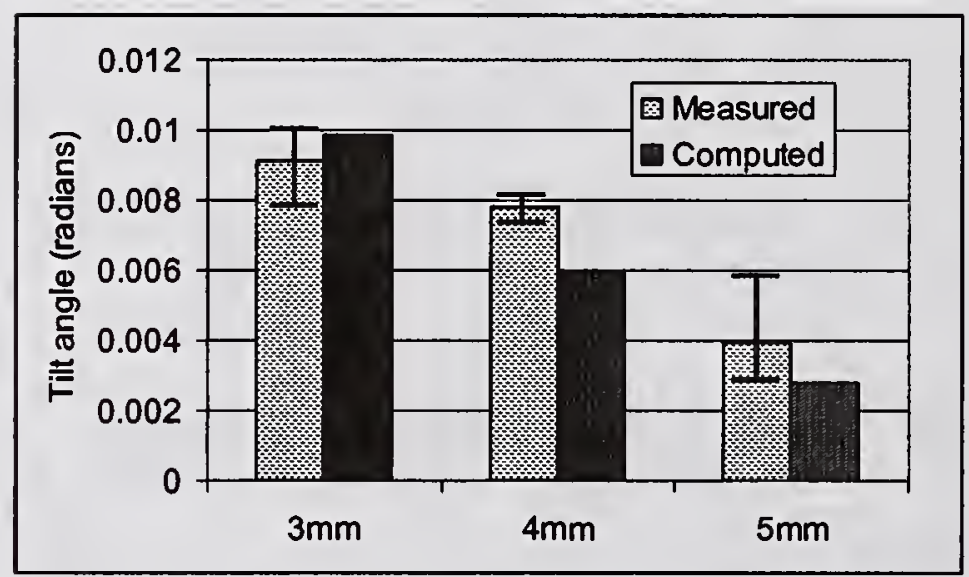

Figure 6: Comparison of computed and measured tilt angles. (a) Effect of different clamping for $3 \mathrm{~mm} 6082 \mathrm{~T} 6$ alloy tubes. (b) Effect of varying wall thickness of $6063 \mathrm{~T} 6$ alloy tubes. The error bars indicate the range of measured values.

\section{SIMULATING THE WELDING OF AUTOMOTIVE PARTS}

A detail from an actual windshield frame, made from extruded profiles of the aluminum alloy AA6082 in T6 temper conditions, has been subject for a series of simulations. Only a minor part of the frame, containing an upper corner was included in the solution domain, as illustrated schematically in Figure 7. The actual CAD geometry of this corner contained numerous finer details, making it unsuitable as basis for meshing with solid brick elements. Instead, a finite element mesh was constructed on a simplified geometry. This geometry deviated somewhat from the actual shape, and no reinforcement regions were included. It was nevertheless considered to be an adequate basis for the simulations.

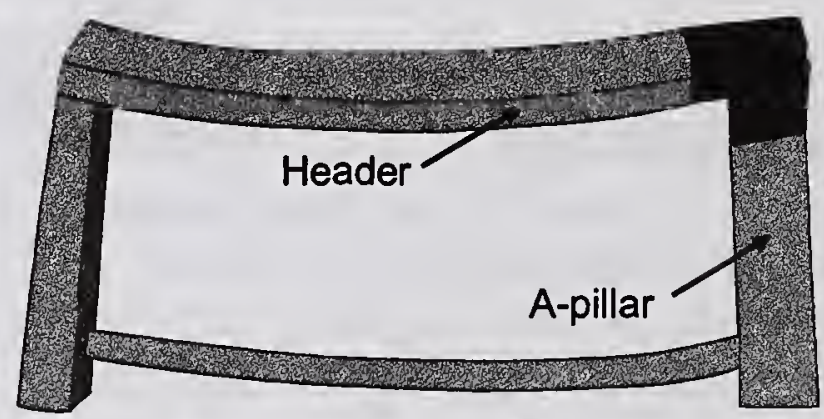

Figure 7: Schematically drawn figure illustrating the windshield frame including the solution domain for the simulations located at the upper right corner. 
In these simulations, a constant welding speed of $3.8 \mathrm{~mm} / \mathrm{sec}$ and a net heat input of $1250 \mathrm{~W}$ were generally assumed. The welding sequence 1 depicted in Figure 8 was applied in most of the simulations. The welding was completed after about 85 seconds. The clamping was released 60 seconds later, and the simulations were stopped after 400 seconds. The two end boundaries of the solution domain, shown in Figure 8, correspond approximately to the nearest clamping positions. The effect of the clamping was simulated by use of linear spring boundary conditions at these boundaries. Generally, a rather rigid clamping was imposed. A displacement of $0.1 \mathrm{~mm}$ resulted in stresses of $10 \mathrm{MPa}$ and $1 \mathrm{MPa}$ in the normal and transversal directions, respectively. An initial gap between the parts of $0.5 \mathrm{~mm}$ was assumed. This was achieved by imposing mechanical boundary conditions entailing an initial offset displacement.
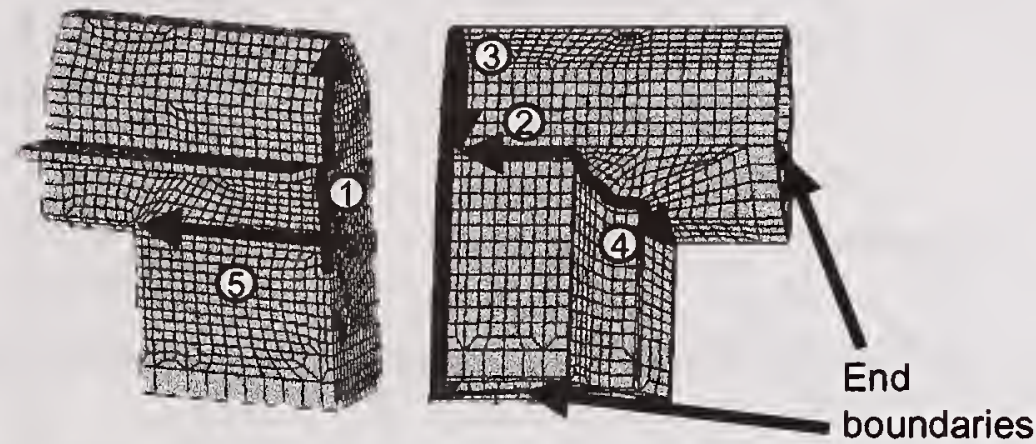

Figure 8: Welding sequence 1 superimposed on the solution domain containing the finite element mesh. At the end boundaries, indicated by arrows, the boundary conditions represent the clamping.

In the following the results from the simulations listed in Table 1 will be discussed. Two alternative welding sequences that have been tested in the simulations are illustrated in Figure 9.

Table 1: The simulation cases for the windshield frame corner. For the cases B-F the difference from case $\mathrm{A}$ is described.

\begin{tabular}{|l|l|}
\hline Case & Description \\
\hline A & The reference case with welding sequence 1 \\
\hline B & Welding heat source $2 \mathrm{~mm}$ offset into the header \\
\hline C & Heat input increased by $25 \%$ \\
\hline D & Boundary condition stiffness reduced by a factor of 100 at A-pillar cross section \\
\hline E & Welding sequence 2 \\
\hline F & Welding sequence 3 \\
\hline
\end{tabular}

(a)

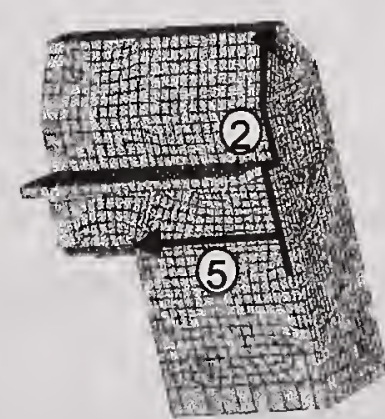

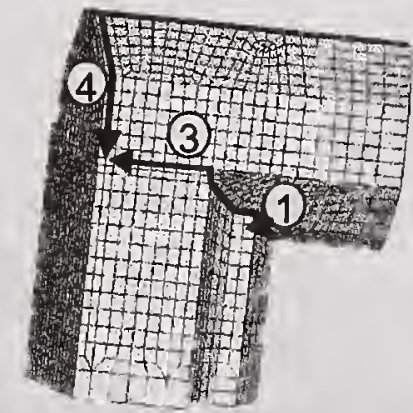

(b)

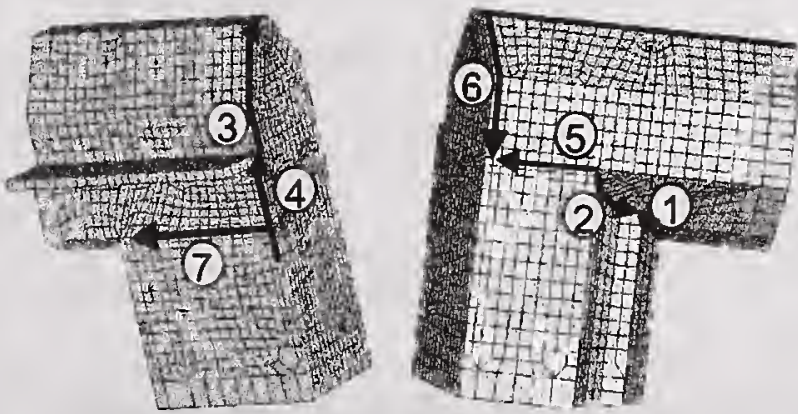

Figure 9: (a) Welding sequence 2 applied in case E. (b) Welding sequence 3 applied in case F. 
In order to simplify the interpretation of the computed displacements, the displacements have to be transformed into a few quantities representing the particular distortions of interest, i.e. the inplane tilt angle $\theta$ and the out-of-plane torsional distortion angle $\phi$ as illustrated in Figure 10.
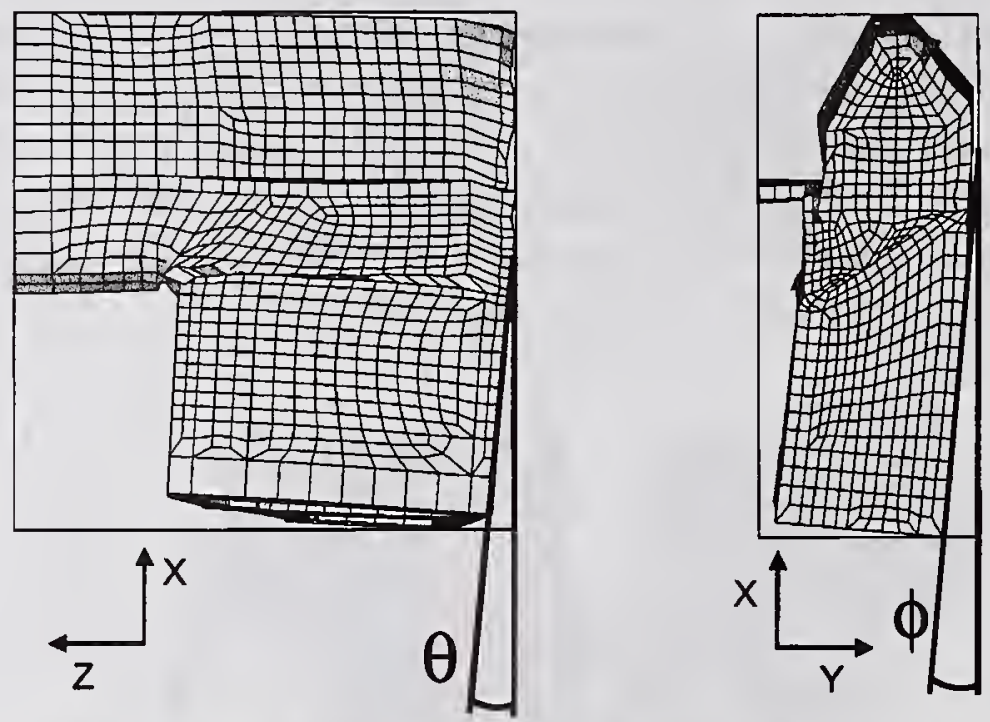

Figure 10: Definition of the tilt angle $\theta$ and the torsional angle $\phi$ quantifying the distortions.

The thermally induced strains in the weld joint zone also entail longitudinal contractions of the extruded profiles. However, due to the size of the windshield frame, the longitudinal contractions are assumed to have less significance than the angular distortions. The illustrated distortions appear as the result of imposing zero displacement at the end boundary of the header and releasing the corresponding end boundary of the A-pillar. On the other hand, if the A-pillar is kept fixed, the angle $\phi$ represents a rotation of the header. As the header actually has a pronounced curvature, this rotation would entail a considerable displacement at a distance from this windshield frame corner. The computed angular distortions are shown in Figure 10. As a windshield frame has a typical dimension of $1 \mathrm{~m}$, some results indicate displacements of several $\mathrm{mm}$. (Angle values in radians multiplied by 1000 gives distortions expressed in $\mathrm{mm} / \mathrm{m}$.)

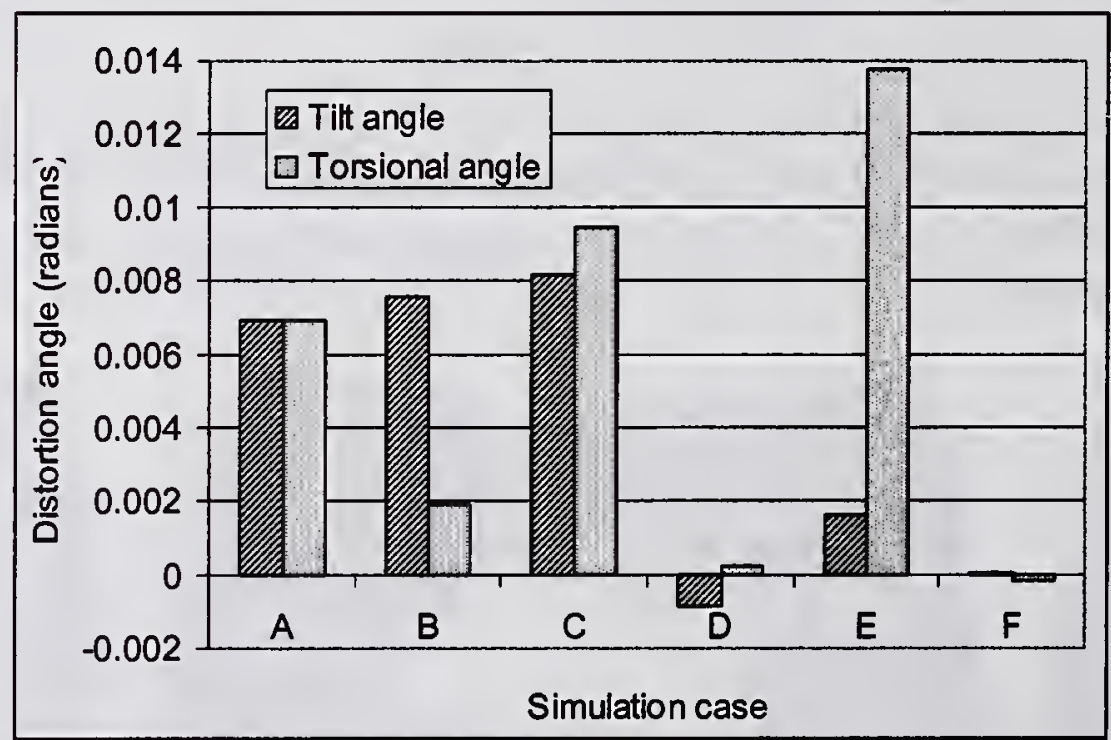

Figure 11: Computed values of the distortion angles at the end of the simulations. 
In case $B$, where the heat input was given an offset of $2 \mathrm{~mm}$ into the header, the tilt angle is seen to be about $10 \%$ larger than in case $\mathrm{A}$. This is associated with the larger computed plastic strains at the lower side of the header, compressive in the profile's longitudinal direction. The displacement of the heat source also resulted in a lack of fusion at parts of the weld seam. This is probably the major cause for the low values of the predicted torsional distortion. The results from case $\mathrm{C}$ show that the distortions are sensitive to the total amount of welding heat input. The relative increase in the torsional distortion is even larger than the relative increase of heat.

Notable, the more significant compliance of the clamps in case D nearly eliminated the predicted distortions. This strong effect of the clamping forces therefore represents an interesting possibility of manipulating the weld distortions, and the underlying mechanism behind this effect will be explained in the following. In Figure 12 the computed angular distortions as function of time is shown for cases $\mathrm{A}, \mathrm{D}$ and $\mathrm{F}$.
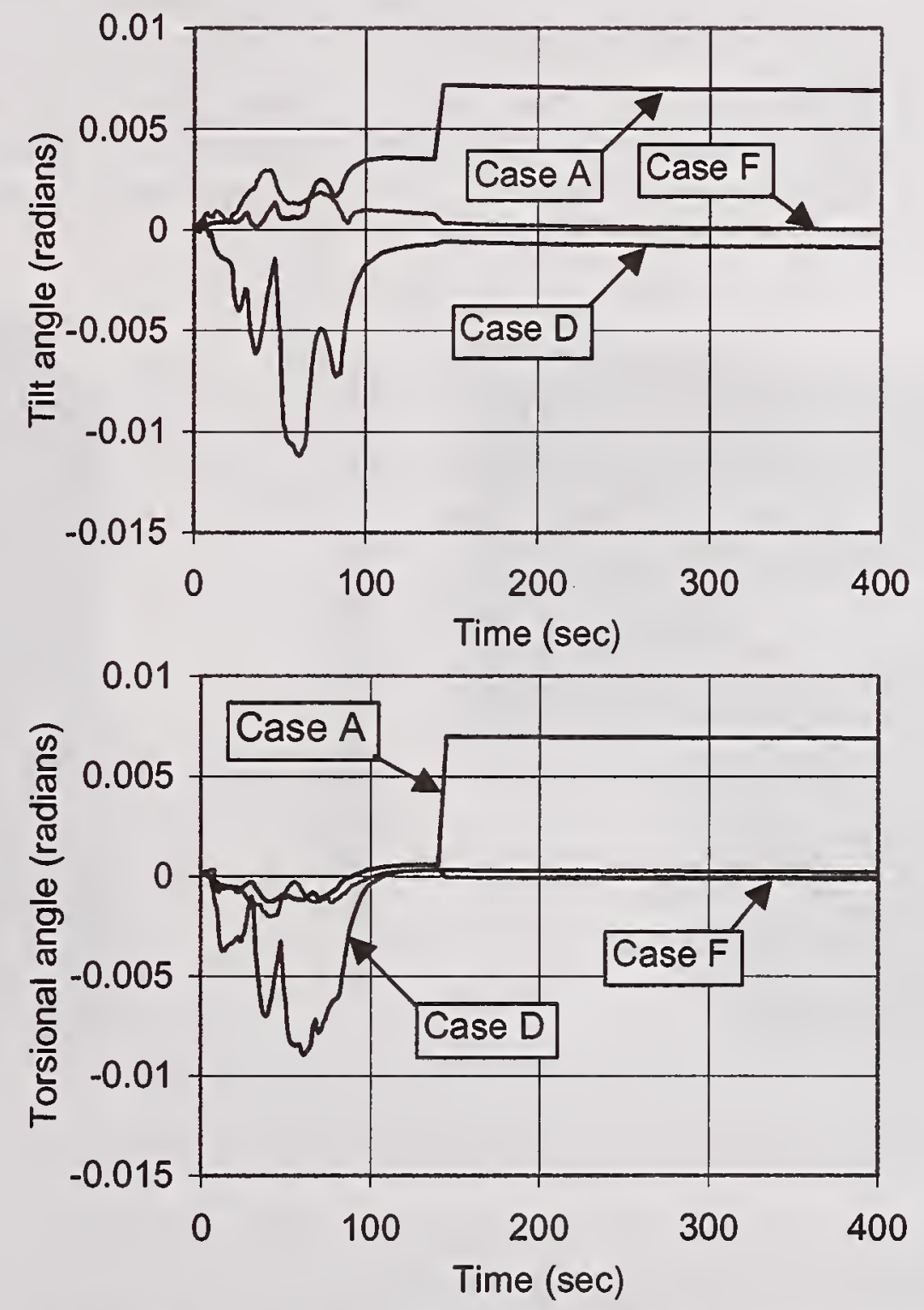

Figure 12: Computed angular distortions as function of time for case A, D and F.

The lower stiffness of the clamps assumed in case D, compared to case A and case F, allows for much larger distortions during welding. In particular, after about 51 seconds, a rapid evolution of the tilt angle distortion takes place. This can be associated with the heating/expansion resulting 
from the onset of weld segment 4, whereas the region close to weld segment 3 undergoes cooling, as illustrated in Figure 13(a). This compensates for the subsequent distortion caused by the weld segment 4 , involving a transversal weld across the header. Considering the torsional distortion, it seems important to allow for a large (negative) angle at the time the welding of the last segment starts. This is the situation depicted in Figure 13(b). The cooling after welding of this segment will induce considerable thermal contractions at the regions at the right part of this figure, corresponding to the computed development between 80 and 100 seconds of the torsional distortion angle seen in Figure 12.

The results from cases $E$ and $F$ illustrate clearly that the welding sequence may have a pronounced influence on the distortions. Use of welding sequence 2 (case E) resulted in a significantly reduced tilt angle compared to case $\mathrm{A}$. The bending of the header induced by the first weld segment is seen to be "absorbed by" the gap between the parts. However, the torsional angle is predicted to be very large in case $E$, and therefore an improved welding sequence was looked for by performing a series of simulations. After a few trials, the welding sequence 3 applied in case $\mathrm{F}$ was found to be optimal with respect to both kinds of angular distortions. The splitting of the first weld into two segments (compared to sequence 2) was essential in order to obtain a uniform gap distance prior to local fusion along these weld segments. This, in turn, prevented torsional distortion.

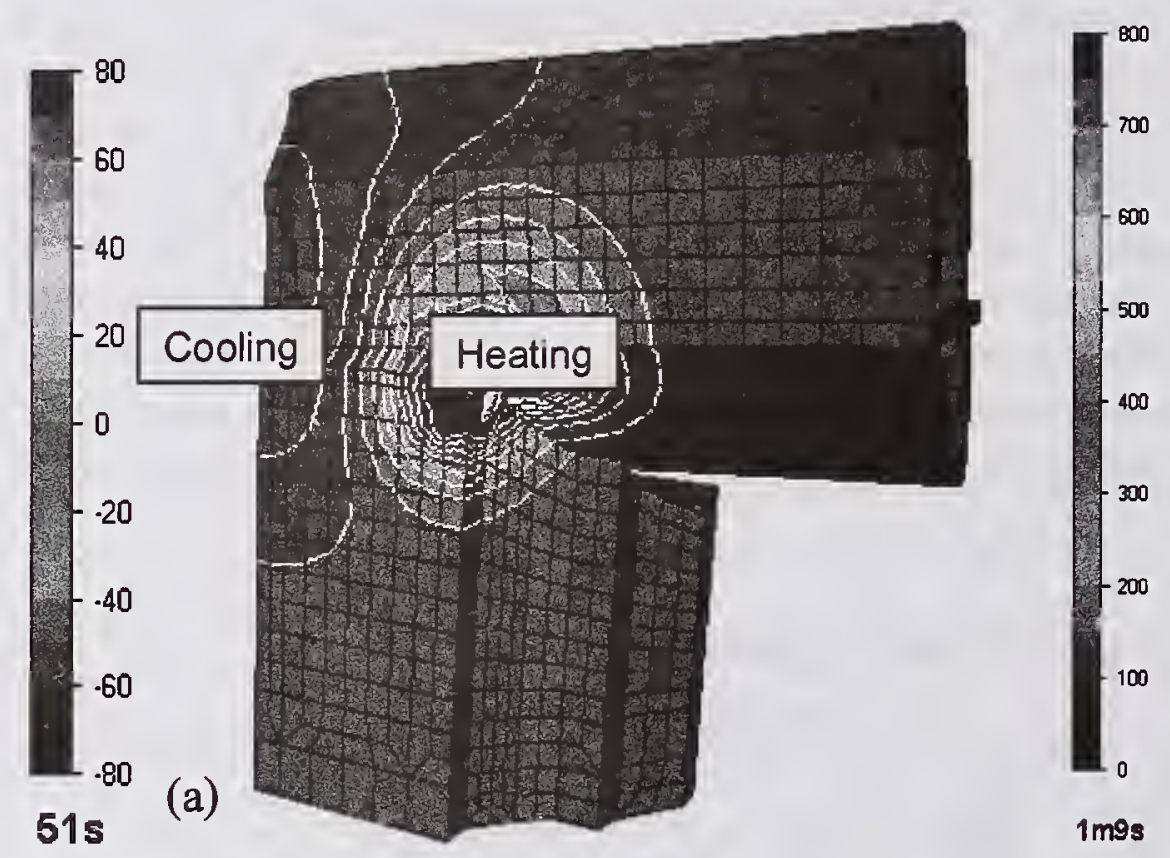

(b)

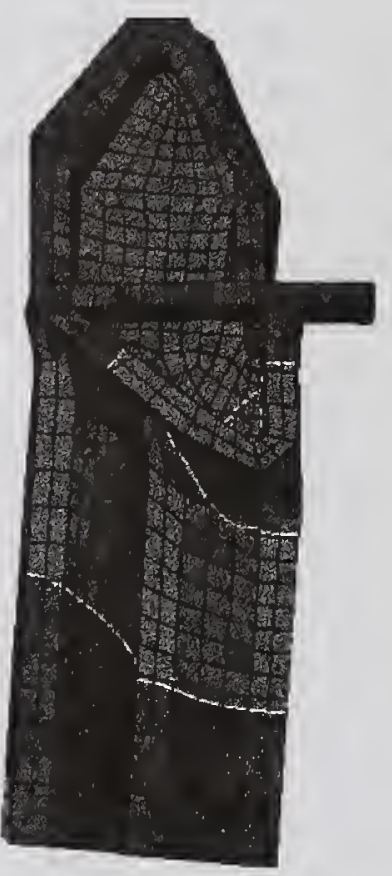

Figure 13: (a) Fringe plot of heating/cooling rate after 51 seconds. (b) Temperatures after 69 seconds (right) from case D. Distortions are magnified by a factor of 10 .

In general, it is possible to compensate for the resulting weld distortions by mounting the parts in the fixture in an offset position, unless the welded parts form a closed loop. The exact magnitude of the offset positions can be determined from practical welding experiments, or preferably, by weld simulations provided that the latter are sufficient accurate. Canceling the distortions, by applying weak clamping or by applying an optimal welding sequence, can be an attractive alternative. Generally, it is considered as advantageous to apply a welding sequence, which implies that the distortions at the interface (including generation or closure of gaps) can compensate for the welding induced deformations of each of the welded parts. 


\section{SUMMARY}

In this article a process model (WELDSIM) for welding of age-hardening aluminum alloys has been outlined. The model consists of a thermal, a microstructure and a mechanical sub-model implemented in a FEM framework.

Examples have been shown on verification of the simulation model against distortion measurements on real welding experiments. Very good agreement was obtained between computer simulations and welding experiments.

Results from a modeling case study on welding of a windshield frame have been presented. A given welding sequence resulted in considerable angular distortions when rigid clamping was assumed. However, by selecting an alternative welding sequence and keep the rigid clamping, the computed distortions were almost eliminated. Alternatively, the distortions were also eliminated, by applying the original welding sequence combined with a more compliant clamping. This case study illustrates how a simulation model like WELDSIM can be a valuable tool in the pre-production phase in fabrication of welded automotive parts in aluminum.

\section{ACKNOWLEDGEMENTS}

The authors wish to acknowledge the financial support from Hydro Automotive Structures and The Research Council of Norway. Moreover, Mr. Peter B. Jacobsen and Mr Flemming Damborg at Hydro Automotive Structures Tønder, Denmark is acknowledged for their support regarding the automotive case study presented in the paper.

\section{REFERENCES}

1. Myhr, O.R.; Grong, Ø.; Klokkehaug, S.; Fjær, H.G.; Kluken, A.O. 1993. A Process Model for Welding of Al-Mg-Si Extrusions - Part I: Precipitate Stability, Science and Technology of Welding and Joining, Vol 2, (6), 245-253.

2. Myhr, O.R.; Klokkehaug, S.; Fjær, H.G.; Grong, Ø.; Kluken, A.O. 1998. Modelling of Microstructure Evolution, Residual Stresses and Distortions in 6082-T6 Aluminum Weldments, Welding Journal, Vol 77, (6), 286-292.

3. Myhr, O.R.; Klokkehaug, S.; Fjær, H.G.; Grong, Ø.; Kluken, A.O. 1999. Modelling of microstructure evolution and residual stresses in processing and welding of 6082 and 7108 aluminium alloys, Proceedings of the 5th International Conference on Trends in Welding Research, ASM International, 233-238.

4. $\quad$ Myhr, O.R.; Fjær, H.G.; Klokkehaug, S.; Holm, E.J.; Grong, Ø.; Kluken, A.O. 1999. WELDSIM - An advanced simulation model for aluminium welding. The Ninth International Conference on Computer Technology in Welding, Detroit 1999, 52-63.

5. Myhr, O.R.; Grong, Ø.; Andersen, S.J.; 2001. Modelling of the Age Hardening Behaviour of Al-Mg-Si Alloys, Acta Materialia, Vol 49, 65-75. 



\title{
A COMPUTATIONAL MODEL FOR HEAT AFFECTED ZONE OF REACTOR PRESSURE VESSEL STEEL
}

\author{
J.S. Kim*, S.G. Lee, J.S. Park and T.E. Jin
}

\begin{abstract}
ASTRACT
A computational model for the heat affected zone(HAZ) of reactor pressure vessel(RPV) steel is developed to predict microstructures and material properties. The microstructures of HAZ are predicted by a combination of the temperature analysis considering multi-pass welding and PWHT, and the thermodynamics-kinetics models for prior-austenite grain growth, austenite decomposition and carbide coarsening. Also, the hardness, yielding strength and tensile strength are estimated by the prediction results for microstructures and the empirical relations. Finally, these prediction results are compared with experimental ones and show the reasonable agreement.
\end{abstract}

\section{KEYWORDS}

Reactor Pressure Vessel Steel, Circumferential Narrow Gap Weld, Heat Affected Zone, Temperature Analysis, Microstructure, Mechanical Strength

\section{INTRODUCTION}

The metallurgical microstructures of HAZ are changed due to repeated thermal cycle during multi-pass welding and carbides coarsening during PWHT. Especially, in case of SA508 steel, which has been widely used for pressure vessel because of good mechanical properties, the mechanical properties of HAZ may be degraded due to the microstructure change. Therefore, the some studies(Ref. 1 4) has been performed to more detailedly estimate the microstructures and material properties for HAZ of SA508 steel. However, these studies used the experimental approaches, so it has the economic problems to apply the methodologies used in these studies to various welding processes and geometric shapes.

Accordingly, the purpose of this study is to develop a computational model predicting the microstructures and material properties for the HAZ of SA508 Gr.3 Cl.1, which has been often used for RPV. The microstructures of HAZ are predicted by a combination of the temperature analysis considering multi-pass welding and PWHT, and the thermodynamics-kinetics models for prior-austenite grain growth, austenite decomposition and carbide coarsening. Also, the aswelded hardness, yielding strength and tensile strength are estimated by the prediction results for microstructures and the empirical relations.

* Welding Integrity in Nuclear Structures Laboratory (www.wins.re.kr), Korea Power Engineering Company

Zip Code 449-713, 360-9, Mabuk-ri Kusong-eup Yongin-si, Kyunggi-do, Republic of Korea, kimjs@kopec.co.kr 


\section{COMPUTATIONAL MODEL}

Fig. 1 shows a computational model predicting the microstructures and mechanical properties for the HAZ of SA508 Gr.3 Cl.1. As shown in Fig. 1, firstly, thermal analysis is performed to determine the peak temperature and cooling rate in $\mathrm{HAZ}$ considering real welding processes such as multi-pass welding and PWHT. Secondly, the microstructures of HAZ are estimated considering various metallurgical factors such as prior-austenite grain growth and austenite decomposition during welding. In additions, the carbide coarsening during PWHT is also estimated. Finally, the changes of mechanical properties due to microstructure changes are predicted.

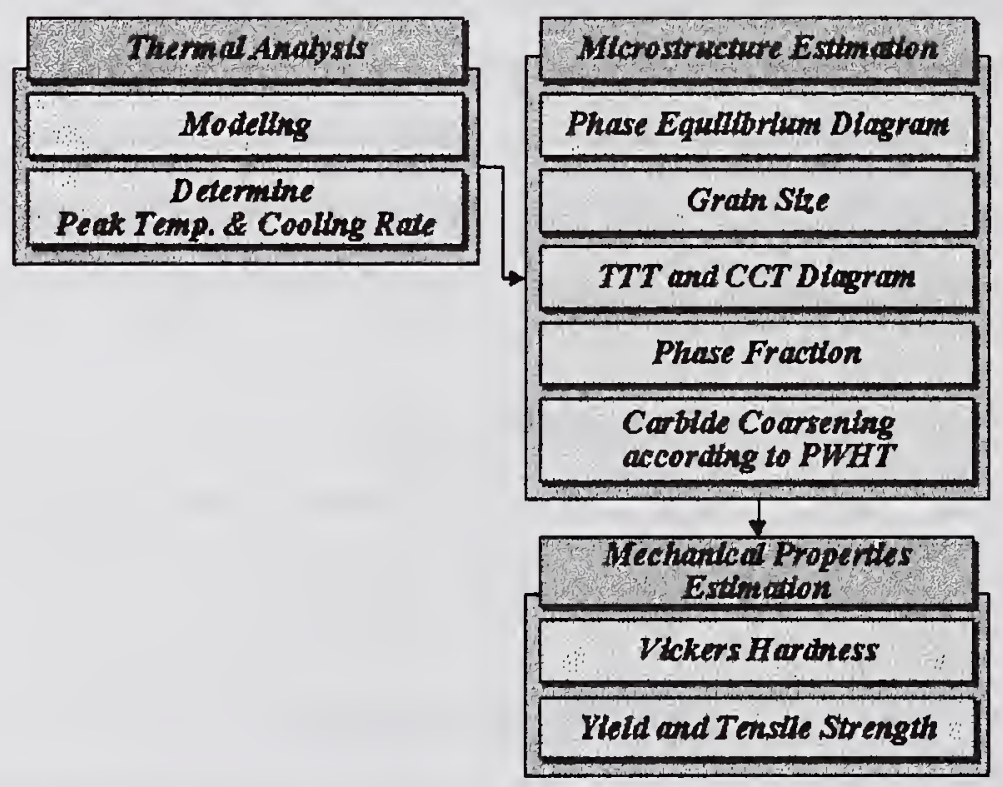

Fig. 1. Computational model to predict microstructures and material properties.

\section{TEMPERATURE ANALSYS}

\section{Analysis Model}

Fig. 2 shows the analysis model of RPV circumferential narrow gap weld. Base material is SA $508 \mathrm{Gr} .3 \mathrm{Cl} .1$ and filler material is L-TEC 44. Submerged arc welding(SAW) method is used in the narrow gap weld. Welding is performed with 81 passes and 39 passes for inner and outer part respectively. Table 1 summarizes the chemical compositions of SA 508 Gr.3 Cl.1 for RPV. And Table 2 summarizes the welding parameters of this model.

\section{Finite Element Model}

The finite element for an analysis model is shown in Fig. 3. The numbers of elements and nodes are 830 and 940 respectively. The element property is a 4-node axis-symmetric element (Ref. 5). By using the lumped model(Ref. 6), the finite element model is simplified as the one with 8 and 5 weld layers for inner and outer weld part respectively. Model change technique(Ref. 5) is adopted to simulate multi-pass welding process. 


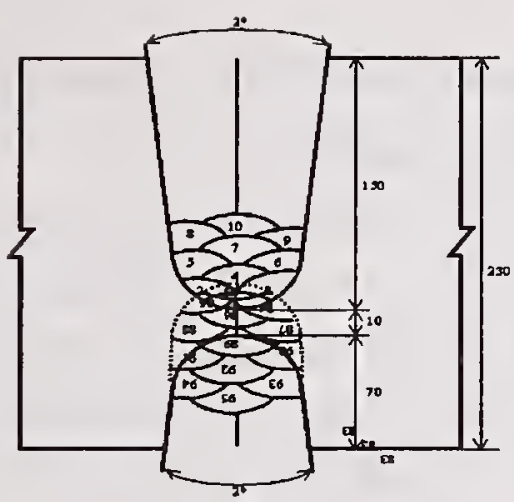

Fig. 2. Analysis model.

Table 1. Chemical composition of SA508 Gr.3 Cl.1

\begin{tabular}{|c|c|c|c|c|c|c|c|c|}
\hline Element & $\mathrm{C}$ & $\mathrm{Si}$ & $\mathrm{Mn}$ & $\mathrm{P}$ & $\mathrm{S}$ & $\mathrm{Ni}$ & $\mathrm{Cr}$ & $\mathrm{Mo}$ \\
\hline wt.\% & 0.19 & 0.08 & 1.35 & 0.006 & 0.002 & 0.82 & 0.17 & 0.51 \\
\hline
\end{tabular}

Table 2. Specifications of welding parameters

\begin{tabular}{|c|c|c|c|c|c|c|}
\hline Wire Size & Current & Voltage & Speed & Preheat temp. & Interpass temp. & PWHT \\
\hline $4 \mathrm{~mm}$ & $500 \sim 600 \mathrm{~A}$ & $28 \sim 32 \mathrm{~V}$ & $30 \sim 40 \mathrm{~cm} / \mathrm{min}$ & $121^{\circ} \mathrm{C}(\min )$. & $200^{\circ} \mathrm{C}(\max )$. & $615^{\circ} \mathrm{C} / 40 \mathrm{hr}$ \\
\hline
\end{tabular}

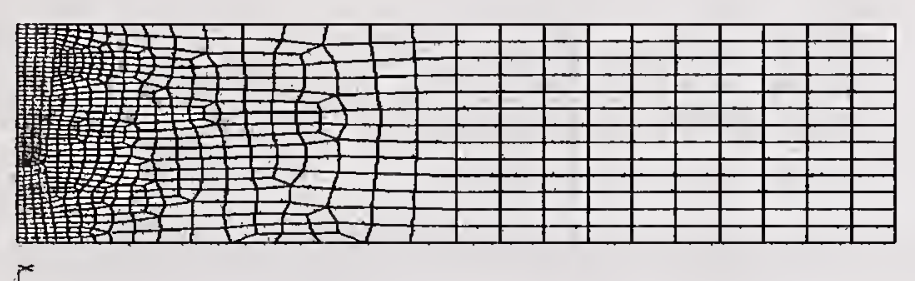

Fig. 3. Finite element model.

\section{Analysis Results}

As shown in Fig. 4, temperature cycle in $\mathrm{HAZ}$ is the saw-tooth pattern because of repeated heating and cooling during multi-pass welding.

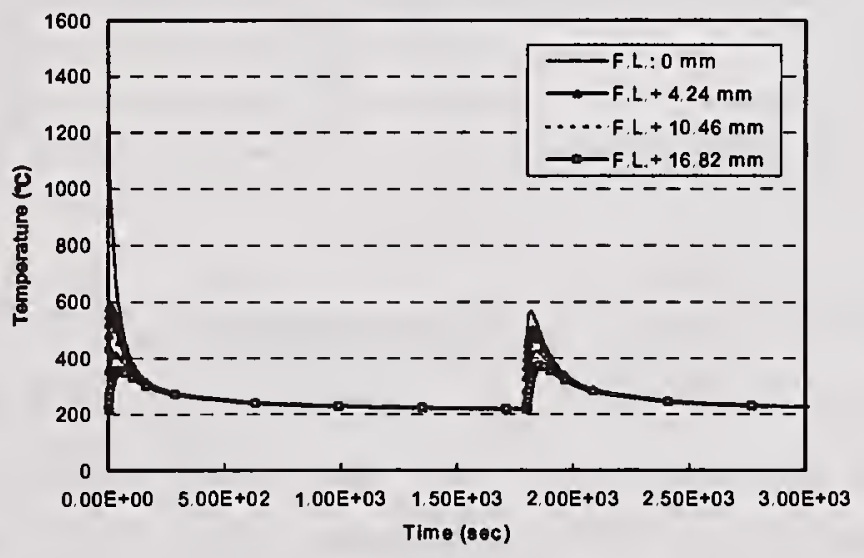

Fig. 4. Peak temperature change vs. time. 


\section{MICROSTRUCTURE PREDICTION}

\section{Determination of Equilibrium Phase Diagram}

Fig. 5 shows the equilibrium phase diagram of SA508 Gr.3 Cl.1 according to temperature calculated by Thermo-Calc(Ref. 7). As shown in Fig. 5, the thermodynamically stable carbides are $\mathrm{MC}, \mathrm{M}_{2} \mathrm{C}, \mathrm{M}_{7} \mathrm{C}_{3}, \xi$-carbide and cementite $\left(\mathrm{M}_{3} \mathrm{C}\right)$. But, it is difficult that the $\mathrm{MC}$ type carbide precipitates because of relatively low temperature. These results show the good agreement with TEM analysis results that the carbides of $\mathrm{M}_{2} \mathrm{C}$ and $\mathrm{M}_{3} \mathrm{C}$ type are observed. Table 3 presents the equilibrium phase transformation temperatures from ferrite to austenite, $A_{e 1}$ and $A_{e 3}$, determined from Fig. 5. From Table 3, these calculation results have a few differences in quantities from the previous results that are phase transformation temperatures during heating.

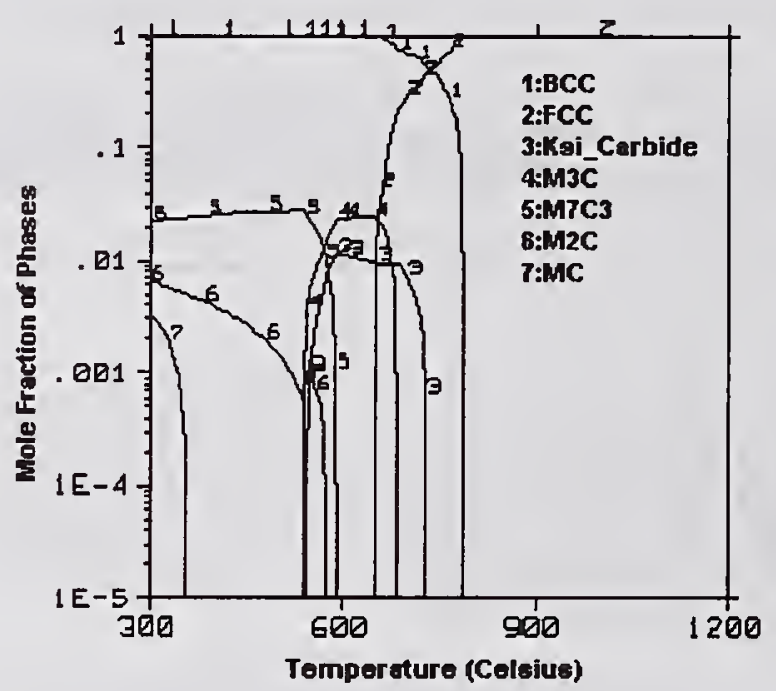

Fig. 5. Equilibrium mole fractions of phases vs. temperature for the SA508 Gr.3 Cl.1.

Table 3. Phase transformation temperatures from ferrite to austenite.

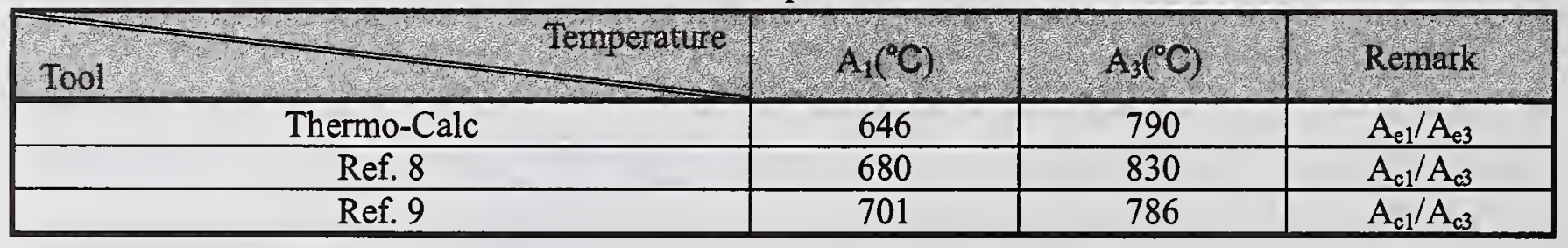

\section{Estimation of Grain Size}

Grain size is an important factor to predict the microstructure because grain boundaries provide nucleation sites and diffusion paths. Therefore, the grain sizes of HAZ have to be estimated according to distance from fusion line before determination of TTT (Time Temperature Transformation) and CCT (Continuous Cooling Transformation) diagrams. The grain sizes are predicted by using the following Svensson's equations (Ref. 10):

$$
d_{r}^{1 / n_{1}}=d_{r 0}^{1 / n_{1}}+K_{0} \alpha \tau e^{-Q / R T_{p}}
$$




$$
\begin{aligned}
& \alpha=2 \sqrt{\left(\pi R T_{p} / Q\right)} \\
& \tau=\left(q^{\prime} / v d\right) /\left[2 \pi \lambda \rho e\left(T_{p}-T_{0}\right)^{2}\right.
\end{aligned}
$$

where, $d_{r}$ is the time-dependent grain size, $d_{r 0}$ is the initial grain size, $n_{1}$ is an exponent, $Q$ is the activation energy, $T_{p}$ is the peak temperature, $\lambda$ is thermal conductivity, and $\rho$ is density.

As shown in Fig. 6, there is no grain growth for the case that peak temperature is $900^{\circ} \mathrm{C}$. Also, the final sizes of prior austenite grain are determined as $30 \sim 38 \mu \mathrm{m}$ by using the temperature analysis results.

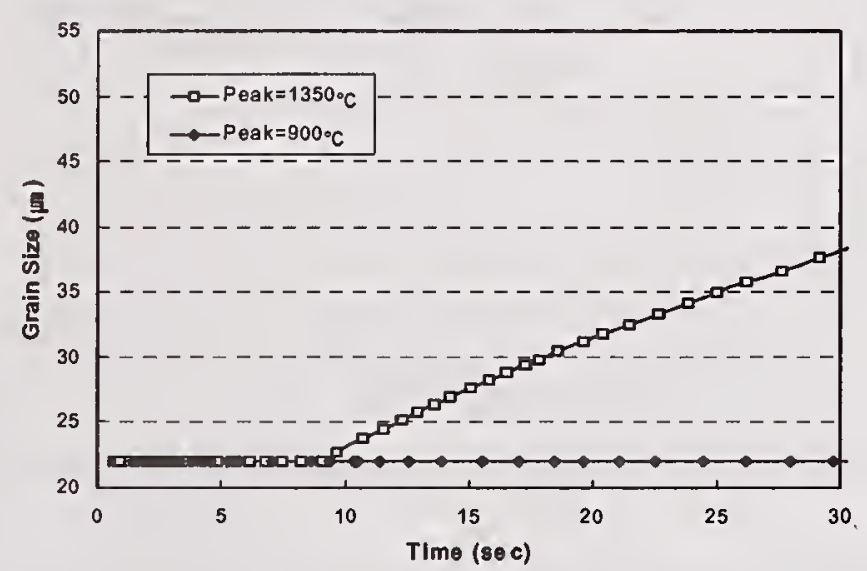

Fig. 6. Prior austenite grain growth according to peak temperature.

\section{Prediction of CCT Diagram}

To predict the CCT diagram of SA508 Gr.3 Cl.1, TTT diagram is determined by using the grain growth results and Li's reaction kinetics model(Ref. 11). And then the CCT diagram is determined by using the TTT diagram and Scheil-Avrami addivity rule(Ref. 12, 13). Fig. 7 shows the predicted CCC diagram of SA508 Gr.3 Cl.1.

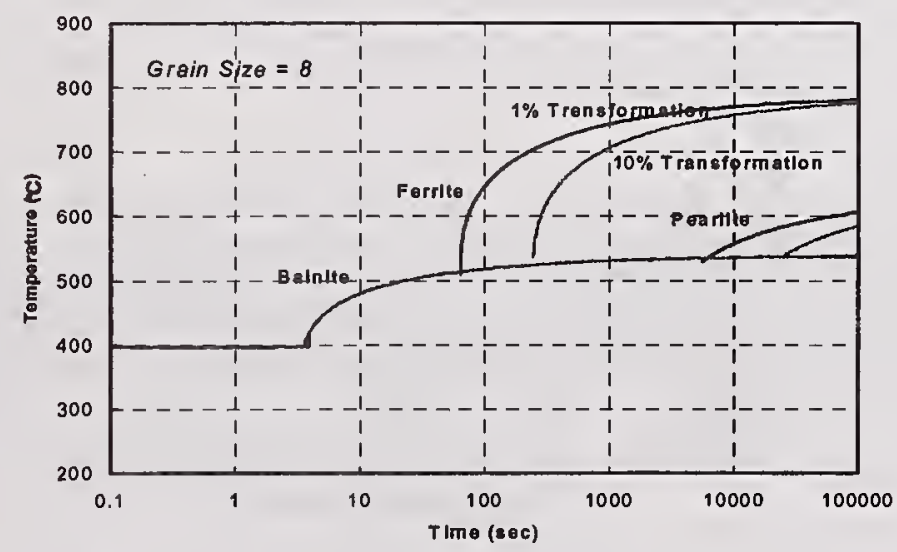

Fig. 7. Predicted CCT diagram of SA508 Gr.3 Cl.1. 


\section{Prediction of Precipitation Phases}

By superposing the cooling curves obtained from temperature analysis on the predicted CCT diagram, the precipitation phases are determined according to cooling rate or distance from fusion line. As shown in Fig. 8, we can expect that only bainite and martensite will be formed over HAZ and the volume fraction of martensite will decrease with the increase of distance from fusion line.

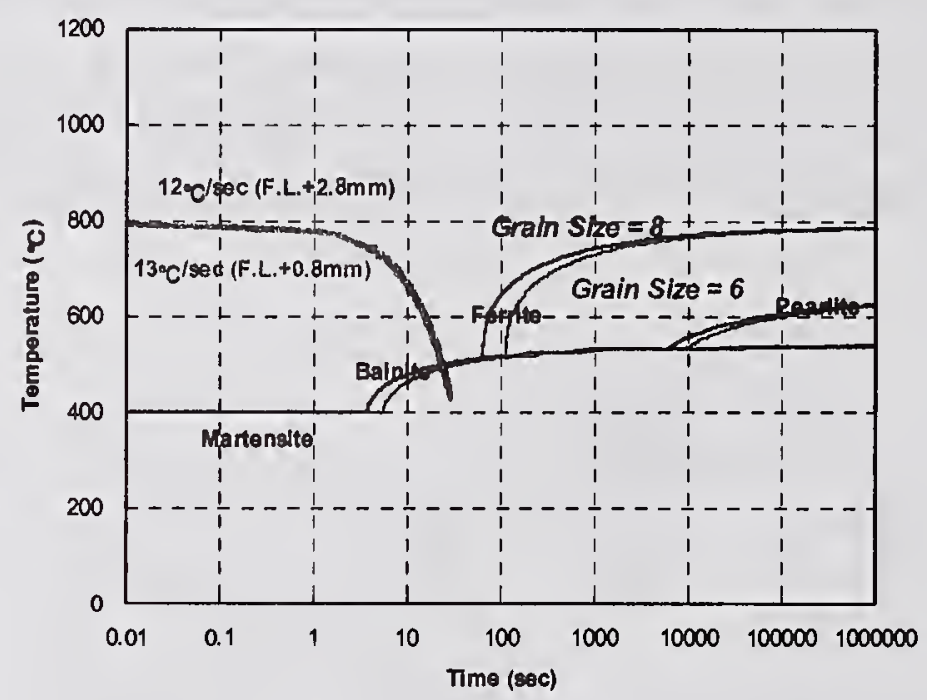

Fig. 8. Prediction of precipitation phases for SA508 Gr.3 Cl.1.

\section{Estimation of Carbide Coarsening due to PWHT}

Carbide coarsening during PWHT, which causes bainite microstructure to degrade hardness and ductility, is estimated by DICTRA(Ref. 14). Fig. 9 shows the estimation results of $\mathrm{Mo}_{2} \mathrm{C}$ coarsening during PWHT. As shown in Fig. 9, we can expect that the carbide size will increase by $30 \%$ as compared with initial size.

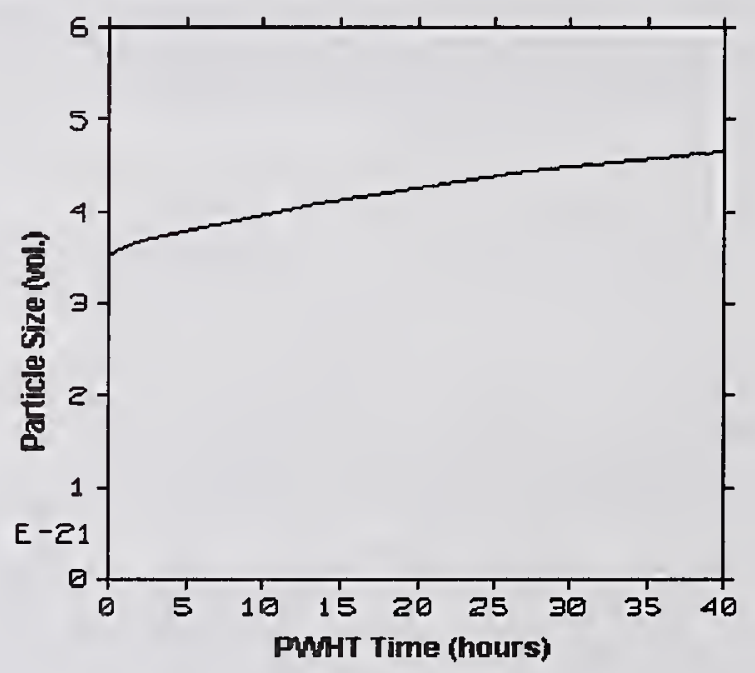

Fig. 9. Carbide coarsening due to PWHT. 


\section{MATERIAL PROPERTIES PREDICTION}

\section{Prediction of Hardness}

The hardness of HAZ before PHWT is predicted by using the volume fractions of precipitation phases and the empirically based formulas (Ref. 15). Fig. 10 shows that the hardness decreases with the increase of distance from fusion line. Also, comparing with the experimental results(Ref. 16), the difference from the ones increases with the increase of distance from fusion line.

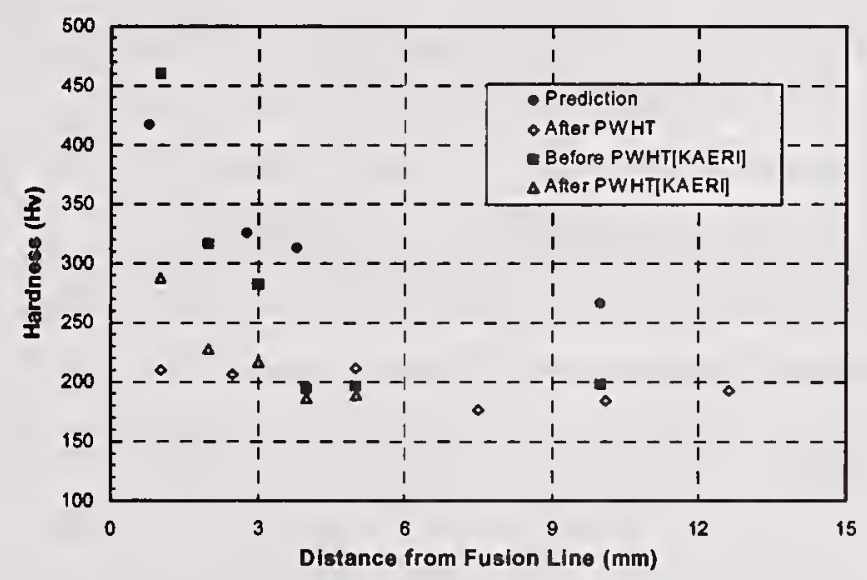

Fig. 10. Vickers hardness distribution according to distance from fusion line.

\section{Estimation of Mechanical Strength}

Yielding and tensile strength are estimated by the following relations (Ref. 17) between Vickers hardness and mechanical strengths:

$$
\begin{aligned}
& \sigma_{y}=3.1 H_{v}(0.1)^{n}-80 \\
& o_{u}=3.5 H_{v}(1-n)\{12.4 /(1-n)\}^{n}-92 \\
& n=0.065\left(\Delta t_{800 / 500}\right)^{0.17}
\end{aligned}
$$

From Table 4, we can expect that the predicted results will be greater than the experimental results.

Table 4. Comparison between the predicted and the experimental mechanical strengths.

\begin{tabular}{|c|c|c|c|c|c|}
\hline Location & $H_{v}$ & $\Delta t_{800} / 500$ & \multicolumn{2}{|c|}{ Prediction (MPa) } & Experiment (Ref. 18) \\
\cline { 4 - 6 } & & & $\sigma_{y}$ & $\sigma_{u}$ & $\sigma_{u}$ \\
\hline $\mathrm{FL}+0.8 \mathrm{~mm}$ & 417.6 & $23 \mathrm{sec}$ & 923.1 & 1648.2 & 1286 \\
\hline $\mathrm{FL}+2.8 \mathrm{~mm}$ & 325 & $25 \mathrm{sec}$ & 697.8 & 1265.8 & 1039 \\
\hline
\end{tabular}

\section{SUMMARY}

In this study, a computational model for the HAZ of RPV steel is developed to predict the microstructures and material properties for $\mathrm{HAZ}$ of SA508 $\mathrm{Gr} .3 \mathrm{Cl} .1$ by using the temperature analysis, the thermodynamics-kinetics models and the empirical relations. The 
prediction results by applying this model show the reasonable agreement with the experimental results.

\section{CONCLUSIONS}

Based on the prediction results obtained from this study, the following conclusions can be made:

- Temperature cycle in HAZ is the saw-tooth pattern because of repeated heating and cooling during multi-pass welding.

- There is no grain growth for the case that peak temperature is $900^{\circ} \mathrm{C}$ and the final sizes of prior austenite grain are also determined as 30 38 $\mu \mathrm{m}$.

- Only bainite and martensite are formed over HAZ and the volume fraction of martensite decreases with the increase of distance from fusion line.

- The carbide size increases by $30 \%$ during PWHT as compared with initial size.

- The as-welded hardness decreases with the increase of distance from fusion line.

- The predicted results for mechanical strengths are greater than the experimental results.

\section{REFERENCES}

1. Suzuki, K., et al., 2001, Nuclear Engineering and Design, 206, 261-278.

2. Kim, J.K. and Yoon, E.P., 1998, Journal of Nuclear Materials, 257, 303-308.

3. Alberry, P.J. and Lambert, J.A., 1982, The Welding Metallurgy of SA508 Cl.II Heat Affected Zones, Central Electricity Generating Board, TPRD/M/1223/R82.

4. Kussmaul, K., et al., 1982, Int. Conference on Welding Technology for Energy Applications, Gatlinburg, Tennessee, May 16-19, 17-69.

5. HKS Inc., 1998, ABAQUS User's Manual, Version 5.8.

6. Dong, P. and Brust, F.W., 2000, J. of Pressure Vessel Technology, 122, 329-338.

7. Thermo-Calc AB, 1999, Thermo-Calc User's Manual, Version M.

8. Atkins, M., 1980, Atlas of CCT Diagrams for Engineering Steels, ASM.

9. Hanjung Heavy Industry, 1996, Determination of Material Properties for SA508 Cl.III.

10. Svensson, L.E., 1993, Control of Microstructures \& Properties in Steel Arc Welds, CRC Press.

11. Li, M.V., et al., 1998, Metallurgical and Materials Transactions B, 29B, 661-672.

12. Scheil, E., 1935, Arch. Eisenhuttenwes, 8, 565.

13. Avrami, M., 1939, J. Chem. Phys., 7, 1103.

14. Thermo-Calc AB, 1998, DICTRA User's Manual, Version 2.0.

15. Maynier, P., et al., 1978, Hardenability Concepts with Applications to Steels, AIME, 163176.

16. Kang, S.Y., et al., 1999, J. of Korean Institute of Metals \& Materials, 37(4), 423-434.

17. Akselsen, O.M., et al., 1990, Material Science and Technology, 6, 383.

18. Kim, J.H. and Hong, J.H., 2001, The $2^{\text {nd }}$ Meeting for Welding Integrity Evaluation. 


\title{
NOVEL COMPUTER-AIDED METHOD OF WELDING MATERIALS DESIGN
}

\author{
V. Mazurovsky ${ }^{1}$, M.Zinigrad $^{2}$, A. Zinigrad ${ }^{1}$
}

\begin{abstract}
A novel welding materials design method is described. New CAMD (Computer-Aided Materials Design) software based on the mathematical simulation is proposed.

At present there is no integrated design procedure available to the welding materials developers. The authors have developed same, based on the following:

1) mathematical modeling of the technological process of welding (taking into account non-equilibrium crystallization and strengthening-phase-formation processes in the weld);

2) structural analysis of the problems relating to the design of new welding materials.

The mathematical models used as a basis for the method developed are described. These mathematical models enable the following to be performed:

a) predicting the composition, structure and properties of the weld based on the structural analysis and structuring the tasks defined according to the requirements of the customer;

b) calculating the composition of welding materials which will ensure obtaining of the weld metal with required properties.

Such integrated approach allows developing new welding materials according to the specific requirements of the customer.
\end{abstract}

\section{NOVEL METHODOLOGY OF THE MATERIALS DESIGN}

We understand design as a process of developing the package of technical documentation for the new product manufacturing - starting from the technical specification formulation and proceeding with its realization.

Design is complex system built as hierarchy and sequence of operations having respective links, priorities, tasks and intermediate solutions.

The optimal way to realize such a system (while automating the process) calls, for our opinion, for the following:

1. System approach (with the design process as the object of analysis)

2. Mathematical modeling (allowing automating the process of forming intermediate solutions and final solution)

\section{SYSTEM APPROACH}

System approach is only a means to structure a problem, to establish links and the order of priorities, to structure data, etc., using structural analysis. System approach as a multi-

${ }^{1}$ CWC Ltd., Ariel, Israel

${ }^{2}$ College of Judea and Samaria, Ariel, Israel 
purpose method uses abstract notions, which in our (as in any particular) case acquire a specific practical application:

- $\quad$ subject domain - specific fabrication process for a specific customer;

- subject domain element - an object or a process (belonging to the subject domain and having specific features, functions and links with other objects and processes) selected during the structural analysis. In our application, those will be the following:

- technological process of welding (hardfacing) itself;

- welding equipment;

- tools;

- environment, etc.

- design object - a subject domain element, whose design (development) is an objective of the task being structured. In our application, these are new welding materials.

A brief review of the major stages of structural analysis of the welding materials design problems is presented below:

1. Definition of the design stages (stage of the technical specification formulation; stage of the technical specification realization).

2. Structuring the stage of the technical specification formulation

2.1. Determining the structure of the design object

2.2. Determining the structure of the subject domain.

2.3. Determining of the input parameters for the design:

2.3.1. Characteristics of the design object elements;

2.3.2. Characteristics of the environment.

2.4. Determining the output parameters: characteristics of the design object.

2.5.Establishing functional relationship between the design object and the subject domain elements (direct and reverse connections).

2.6.Establishing connections between the subject domain and the design tool (expert system).

2.7.Determining the operational algorithm structure and the subject domain representation method for the design tool.

3. Structuring the stage of technical specification realization

3.1. Analysis of the technical specification. Formulating the task (specification) for CAMD;

3.2. Forming design operations and procedures as well as priorities and links;

The design tool (CAMD system) provides the designer with the solution of the task formulated (in our case: development of the welding material which provides for obtaining welds with required properties - as defined by the customer).

Flow-chart of the stage of technical specification realization and forming the final solution using CAMD system is shown on figure1. 

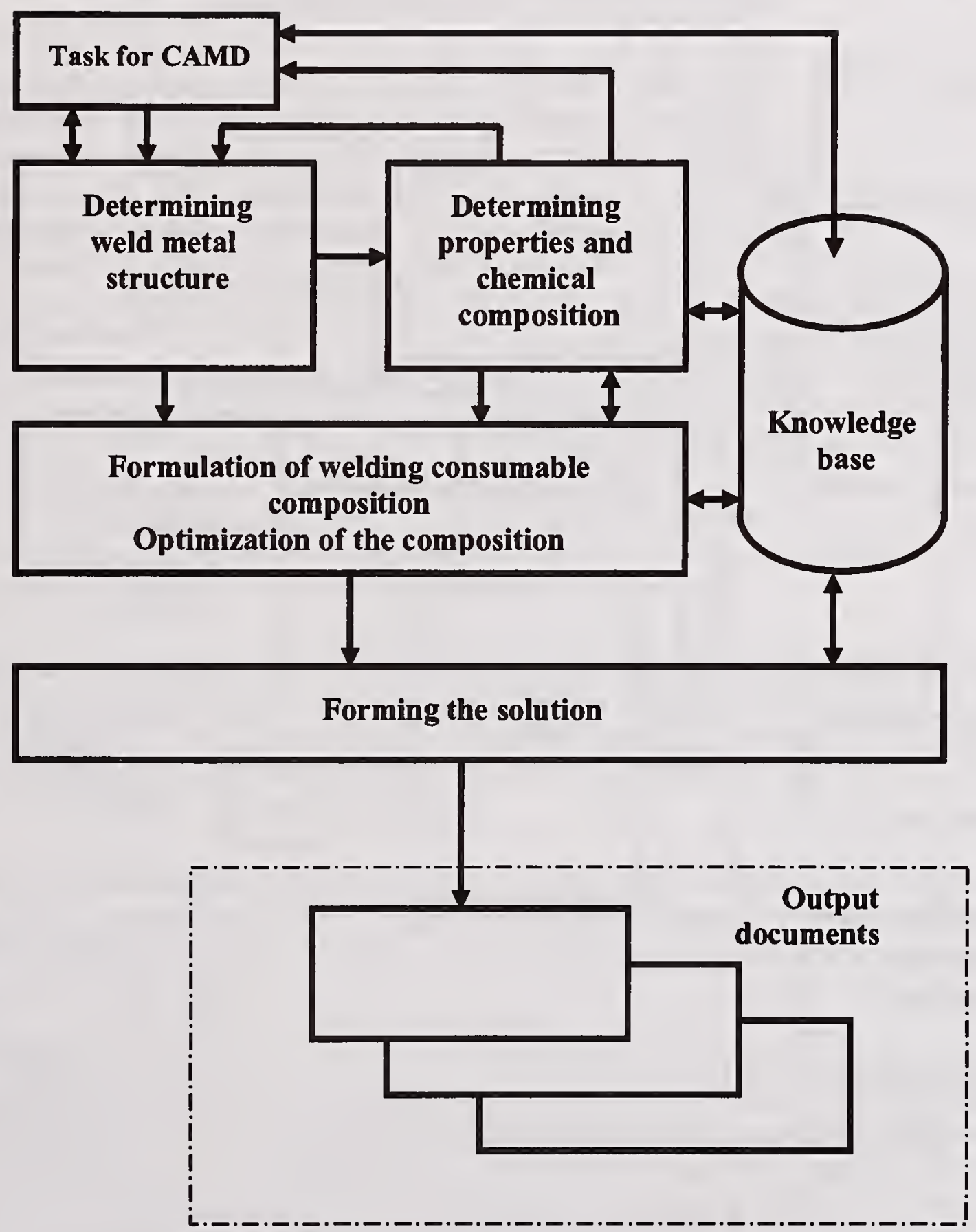

Figure 1: Flow-chart of the stage of technical specification realization 


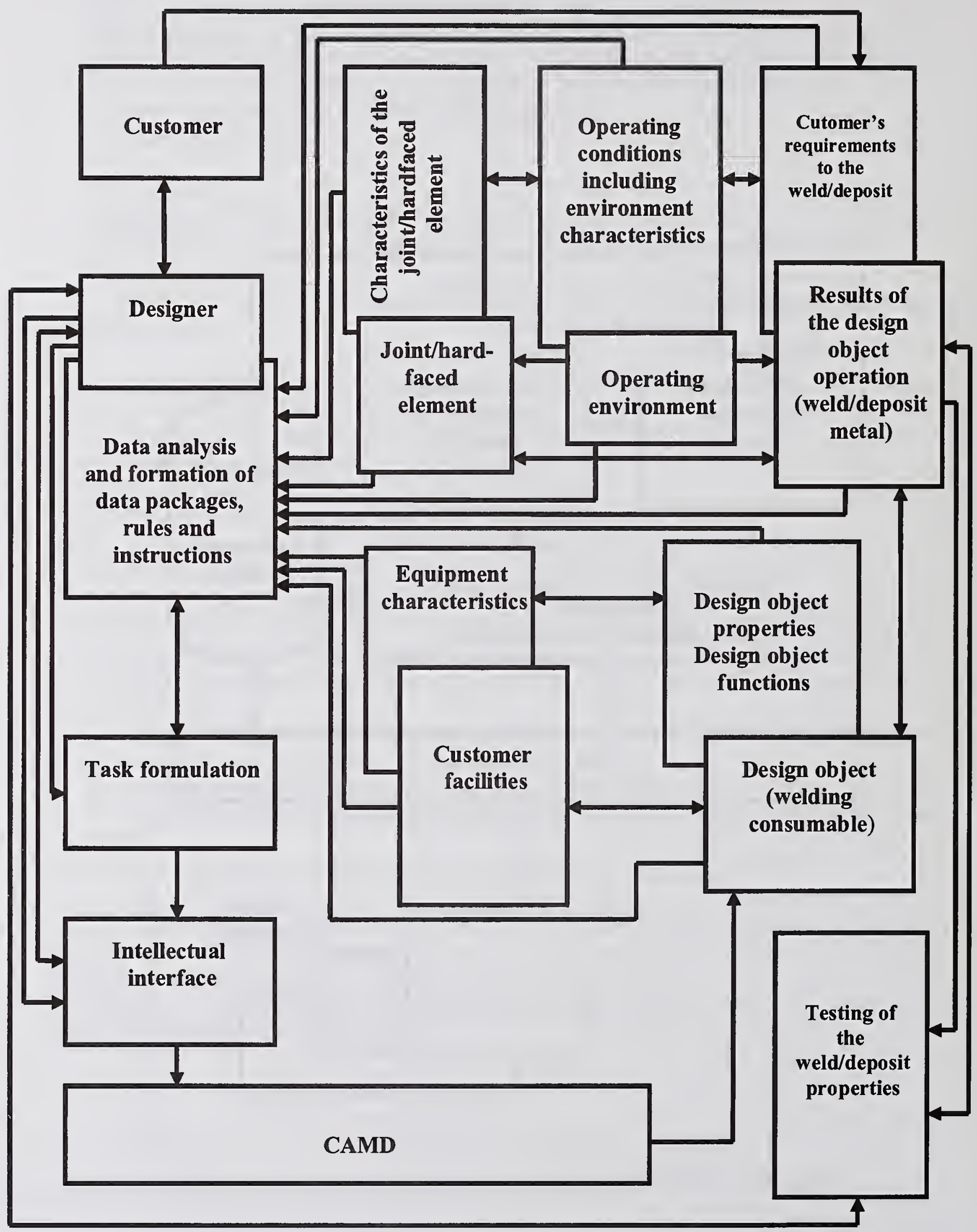

Figure 2: General flow-chart of the design process 


\section{MATHEMATICAL MODELING}

The basis for the technical specification realization according to the approach proposed is the CAMD system, based on the software package.

This software was developed on the base of the mathematical models of the technological process of welding and the process of non-equilibrium weld/deposit metal crystallization. The above models provide for the possibility of calculation of the composition of welding material, which will ensure obtaining of the weld metal with required composition, structure and properties.

\section{MODELING PHASE INTERACTION}

The method of the mathematical modeling of the phase interaction during welding and some examples of its implementations are presented, for instance, in [1].

The mathematical model of the processes involved in the physicochemical interaction of the phases is based on the method for the kinetic analysis of the interaction of multicomponent metallic and oxide melts previously developed with participation of one of the authors of the present work [2]. It is used to solve the most complex problems in modeling, viz., consideration of the rates of transfer of all the elements through the phase boundaries, as well as consideration of the mutual influence of all the chemical reactions taking place on these boundaries. On the basis of this method it is possible to take into account the complex interactions between all components, i.e. their interactions with each other [2]. At present in the physicochemical literature there are a lot of data on the thermodynamic and kinetic parameters of high temperature processes involving different metals, oxides and gases and physical properties of these phases which are necessary for computation. The missing data is obtained in the present paper. The authors of the work have the large experience in the field of experimental obtaining of the physicochemical constants [3 -7].

The method described provided good results in the modeling of the bucket refining of steel $[8,9]$. We developed a general scheme for the mathematical modeling of processes, whose investigation will be the subject of the present work, and we have compiled the database needed to create the model (which includes thermodynamic, kinetic, and diffusion constants, as well as a large body of technological data).

A fairly large amount of experimental data on the kinetics of chemical reactions on a metalslag interface has been obtained. Both general laws and individual steps of processes have been investigated while taking into account the influence of the temperature, composition, mixing rate, and other factors.

In most technological processes involved in the production of metals and alloys, the principal reactions determining the final composition of the products take place on the boundary of the metal with the slag. They are primarily redox reactions involving alloying elements and harmful impurities, as well as vaporization, gas-adsorption, and other processes. When the kinetics are analyzed, special attention is focused on revealing the nature of the individual rate-limiting steps of the overall heterogeneous reaction.

We note that the analysis of the kinetics and mechanism of individually occurring reactions does not present any special difficulties at the present time and that, as a rule, its results faithfully describe the real processes. A kinetic analysis of the interaction of multicomponent 
metallic and slag melts with consideration of the mutual influence of reactions taking place in parallel is considerably more complicated.

Let us briefly describe the method for the kinetic analysis of diffusion-controlled reactions that we previously developed [2]. The theoretical basis of the method consists of two assumptions:

1) under diffusion-controlled conditions the concentration ratio on the phase boundary for each reaction is close to the equilibrium value;

2) the rate of transfer of the reactants to the phase boundary or away from it is proportional to the difference between their concentrations in the bulk and on the boundary of the metallic and oxide melts.

The oxidation of elements in a metallic melt can be represented by the reaction:

$$
\frac{n}{m}\left[E_{i}\right]+(F e O) \rightarrow \frac{1}{m}\left(E_{i_{n}} O_{m}\right)+F e,
$$

In this equation $\mathrm{E}_{\mathrm{i}}$ are the elements dissolved in the liquid metal pool (Mn, $\mathrm{Si}, \mathrm{W}, \mathrm{Mo}, \mathrm{V}$, etc.), and $E_{i n} O_{m}$ are their oxides in the slag phase. The problem reduces to calculating the rates of reactions of type (1). We note that the problem of determining the rate of such a reaction for each element individually does not create special difficulties today. However, such an approach, i.e., analyzing each reaction individually, does not correspond to the situation in an industrial welding process. In the real case the mutual influence of the metal and slag components, as well as the mutual influence of all the heterogeneous reactions occurring in this complex system, are observed.

Our approach permits the determination of the rates $V_{E_{i}}$ of reactions of type (1) for all the metal components with taking into consideration their mutual influence:

$$
V_{E_{i}}=\frac{x^{m}-K_{i}^{m} \frac{\left(E_{i n} O_{m}\right)}{E_{i}}}{\frac{x^{m}}{V_{E_{i}}^{l}}+\frac{K_{i}^{m}}{\left[E_{i}\right]} \frac{\left(E_{i n} O_{m}\right)}{V_{E_{i n} O_{m}}^{l}}}
$$

Here $x$ is the ratio between the concentration of ferrous oxide $(\mathrm{FeO})$ in the slag and the concentration of iron in the metal [Fe] at the phase boundary; $V_{E_{i}}^{l}$ and $V_{E_{i n} O_{m}}^{l}$ are the limiting diffusion rates of the components; $\left[E_{i}\right]$ and $\left(E_{i n} O_{m}\right)$ are the initial concentrations of the elements and their oxides in the metal and the slag, respectively; $K_{i}$ is the equilibrium constant of the reaction involving $\left[E_{i}\right]$; and $\mathrm{n}$ and $\mathrm{m}$ are the stoichiometric coefficients. In other words, $V_{E_{l}}$ is the rate of passage of any element from the slag to the metal (or in the opposite direction), and the further problem reduces to calculating the concentration of that element as a function of time. After solving this problem, we become able to determine both the time-variant composition of the liquid metal pool and the final composition of the weld metal. Since the rates of passage of elements through the phase boundary $V_{E_{l}}$ depend significantly on the temperature, composition, hydrodynamic conditions, and some other factors, the correct determination of the technological parameters of a welding process would be of great value.

The concentration of element $i$ in the weld metal in the case, for example, of flux-core arc welding, can be written in the form [1] 


$$
\left[E_{i}\right]^{\tau}=\frac{V_{d} \cdot\left[E_{i}\right]_{d} \cdot \Delta \tau+V_{b m} \cdot\left[E_{i}\right]_{b m} \cdot \Delta \tau+V_{E_{i}} \cdot M_{E_{i}} \cdot A_{p} \cdot \Delta \tau \cdot 100}{m_{p}^{\tau}},
$$

where $A_{p}$ is the area of the interface between the metal and the slag, and $m_{p}$ is the mass of the weld pool at the time $\tau$.

The system of equations $(2,3)$ along with the equations deriving from the stoichiometry of the reaction (1), comprises a mathematical model, which describes the interaction between the phases in real welding (in this case FCAW) process. The model connects the composition of the weld metal with the composition of the welding consumables thus allowing calculating the composition of the welding consumable providing for the obtaining of the weld metal with required composition.

\section{MODELING NON-EQUILIBRIUM CRYSTALLIZATION PROCESS}

The model of non-equilibrium weld crystallization process proposed by the authors [10], constitutes mathematical description of the process of formation of the strengthening phases and calculation of chrome and nickel equivalents thus connecting the content of the strengthening phases and structure of the matrix with the composition of the metal (for weld metals over a broad range of levels of alloying).

The Schaeffler diagram has been modified taking into account the following factors:

- nonequilibrium crystallization;

- the formation of strengthening phases;

- mutual influence of the alloying elements.

The model includes the parametric dependencies connecting the weldability and mechanical properties of the weld with its structure and composition.

\section{DESIGN PROCEDURE}

Based on the structural analysis and mathematical modeling, the authors have developed a design method comprising a sequence of design stages (with specific instructions and rules for each stage): from data package formulation to the solution development phase.

Structure of the design process with direct and reverse connections between the stages, functional dependencies, and procedures of forming of input and output parameters are presented in the flow-chart of the design process (See figure 2).

As seen from the figure 2 , the proposed method comprises a close-circuit system with a comprehensive coverage of reverse connections, thus providing the following:

1. structuring the problem, defined according to the actual application (as defined by the customer);

2. forming data packages and technical specification 
3. forming instructions and rules based on the above, presentation thereof as a task definition for the CAMD system;

4. solving the task in a dialogue mode between the designer (expert) and the CAMD system;

5. formulating the solution as:

a) formula of the welding material needed to obtain the weld metal required;

b) predicting the composition, structure and properties of the weld;

c) welding/ hardfacing parameters.

\section{CONCLUSIONS}

The novel welding materials design method developed constitutes a comprehensive system allowing for the developing of custom welding consumables.

\section{REFERENCES}

1. Detroit, MI. 1999. Computer modeling of metallurgical technologies. Proc. Ninth International Conference "Computer Technology in Welding", M. Zinigrad, V. Mazurovsky: $164-171$, NIST Special Publication 949.

2. Boronenkov V.; Shanchurov S.; and Zinigrad M. 1979. Kinetics of the interaction of multicomponent metal with slay under diffusion conditions. Izvestiva Ac. Nauk USSR. Metal, 6: 21-s to 27-s.

3. Panphilova L.; Zinigrad M.; and Barmin L. 1978. Effect of surface concentration of oxygen in Me-S melts on the kinetics of its transfer through a sulfur-oxide melt interface. Journal Phys.Chem.: 5, 10, 2491-s to 2494-s.

4. Flyagin A.; Zinigrad M.; and Barmin L. 1979. Kinetics of ion exchange between an ironcarbon-aluminium melt and an oxide electrolyte. Electrochem.: , 5, 12, 1858-s to 1861-s.

5. Panphilova L.; Zinigrad M.; and Barmin L. 1981. Quick stage kinetics of oxygen ion discharge on the boundary of sulfide melts and liquid oxides. Electrochem.: 17, 9 , 1346 -s to 1349 -s.

6. Zinigrad M.; Phephelov A.; Barmin L.; and Shalimov M. 1986. Kinetics of the interaction of a boron containing metal melt with an oxide electrolyte. Electrochem.: $22,1,74-\mathrm{s}$ to $76-\mathrm{s}$.

7. Zinigrad M.; Okolzdajev A.; and Flyagin A. 1988. Limiting stage of anodic oxidation of tungsten at the boundary of metallic and oxide melts. Rasplavy: 2, 3, 46-s to 51-s.

8. Boronenkov V.; Zinigrad M.; and Shalimov M. 1983. Mathematical modeling of metal and slag- processes interaction in a ladle. IZV VUZ. Tcher. metallurg: 1, 36-4.

9. Sendai, Japan. 1992. Simulation of metal and slag interaction for optimization and development of technological processes, Proc. of the $4^{\text {th }}$ Molten Slags and Fluxes International Conference. M. Zinigrad: 125.

10. Ariel, Israel. 2000. Predicting weld structure using modified Schaeffler constitution diagram. Proc. of the International Conference Mathematical Modeling and Simulation of Metal Technologies. V. Mazurovsky, A. Zinigrad, and M. Zinigrad: 540-545. College of Judea \& Samaria Press. 


\title{
Finite Element Analysis of Creep Strength Mis-matched Dissimilar Welded Joints
}

\author{
Chen Hui", Chen Zigang** \\ * Department of Materials Engineering, Southwest Jiaotong University, Chengdu, China, \\ 610031
}

** Dept. of Materials Science and Engineering, Dalian Railway Institute, Dalian, China, 116028

The finite element analysis (FEA) of mismatched welded joints with a $30^{\circ}$ groove angle was performed to study the mechanical behavior of DMWJs (dissimilar metal welded joints). It is concluded that the distribution of stress triaxiality in the DMWJs is uneven, especially near the bond lines. The degree of creep strength mismatch has remarkable effect on the distribution. The higher the level of mismatch is, the more uneven the distribution is and the easier for premature failure to occur in the joint.

Key Words: dissimilar metal welded joints, finite element analysis, creep strength mismatch, stress and strain 


\section{Introduction}

The premature failure in of dissimilar metal welded joints (DMWJs) frequently occurs during elevated temperature services ${ }^{[1,2]}$. So far the predominant causes of the failure have not been clear, although some research workers have reported that the mismatch of creep strengths between the weld metal and base metals is the predominant cause of the failure ${ }^{[3]}$. The mechanical behavior of DMWJs is not clear, and the factors that accelerate the failure remain to be determined.

In the investigation, finite analysis was carried out to study the high temperature behavior of mismatched DMWJs with different creep strengths. The effect of creep strength mismatch on the distribution of triaxality in the joints is discussed particularly.

\section{Materials and Analysis Model}

\subsection{Materials and creep law}

$9 \mathrm{CrMoVNb}, 12 \mathrm{Cr} 2 \mathrm{MoWVTiB}$ and $12 \mathrm{Cr} 1 \mathrm{MoV}$ steels are selected respectively as high strength metal $(\mathrm{H})$, moderate strength metal $(\mathrm{M})$ and low strength metal $(\mathrm{L})$.

Bailey-Norton Creep law is used in the analysis, which is

$$
\bar{\varepsilon}=A \bar{\sigma}^{m} \cdot t^{n}
$$

where:

$A, m$ and $n$ are constants of the material varying with temperature;

$\bar{\varepsilon}$ is the effective strain; $\bar{\sigma}$ is the effective stress;

$\bar{\varepsilon}$ and $\bar{\sigma}$ are defined by

$$
\bar{\varepsilon}=\frac{\sqrt{2}}{3} \sqrt{\left(\varepsilon_{1}-\varepsilon_{2}\right)^{2}+\left(\varepsilon_{2}-\varepsilon_{3}\right)^{2}+\left(\varepsilon_{3}-\varepsilon_{1}\right)^{2}} \text { and } \bar{\sigma}=\frac{\sqrt{2}}{2} \sqrt{\left(\sigma-\sigma_{2}\right)^{2}+\left(\sigma_{2}-\sigma_{3}\right)^{2}+\left(\sigma_{3}-\sigma_{1}\right)^{2}} \text {, }
$$

where $\varepsilon_{1}, \varepsilon_{2}$ and $\varepsilon_{3}$ are the principle strains; $\sigma_{1}, \sigma_{2}$ and $\sigma_{3}$ is the principle stresses.

The mechanical properties and creep constants of test materials at elevated temperature $\left(560^{\circ} \mathrm{C}\right)$ are given in Table $1^{[5]}$.

\subsection{Parameters and model}

In this paper, stress triaxiality $\sigma_{\mathrm{t}}$ is the main factor to be studied, which is defined by the following formula:

$$
\sigma_{\mathrm{t}}=\sigma_{\mathrm{h}} / \bar{\sigma} \text {, }
$$

where: $\sigma_{\mathrm{h}}$ is the hydrostatic stress, defined as

$$
\sigma_{\mathrm{h}}=\left(\sigma_{1}+\sigma_{2}+\sigma_{3}\right) / 3
$$

and $\bar{\sigma}$ is the effective stress.

$\sigma_{\mathrm{h}}$ is a parameter relative to brittle fracture or rupture with low ductility. Under high hydrostatic stresses, materials will not be difficult to deform, which may lead to brittle fracture.

The effective stress is relative to plastic deformation. The higher the effective stress is, the more a material can deform, which may lead to plastic fracture.

$\sigma_{\mathrm{t}}$ increases with $\sigma_{\mathrm{h}}$ and decreases with $\bar{\sigma}$. The greater the $\sigma_{\mathrm{t}}$ is, the more likely the material fractures under low ductility. 
Table 1 The mechanical properties and creep constants of test materials at $560^{\circ} \mathrm{C}$.

\begin{tabular}{|c|c|c|c|c|c|c|c|}
\hline $\mathrm{N}$ & Material & $\begin{array}{c}\sigma_{\mathrm{S}} \\
(\mathrm{MPa}\end{array}$ & $\begin{array}{c}\sigma_{\mathrm{b}} \\
(\mathrm{MPa}\end{array}$ & $\begin{array}{c}\mathrm{E} \\
(\mathrm{GPa})\end{array}$ & $\mathrm{A}$ & $\mathrm{m}$ & $\mathrm{n}$ \\
\hline $\mathrm{H}$ & $9 \mathrm{CrlMoVNb}$ & 478 & 568 & 175.5 & $3.27 \times 10^{-9}$ & 2.17 & 0.58 \\
$\mathrm{M}$ & $12 \mathrm{Cr} 2 \mathrm{MoWV}$ & 318 & 435 & 175.3 & $3.81 \times 10^{-12}$ & 3.40 & 0.68 \\
$\mathrm{~L}$ & $\mathrm{TiB}$ & 183 & 302 & 175.1 & $2.79 \times 10^{-17}$ & 6.13 & 0.78 \\
& $12 \mathrm{CrlMoV}$ & & & & & & \\
\hline
\end{tabular}

The joints analyzed in the FEA are shown as in Fig 1.

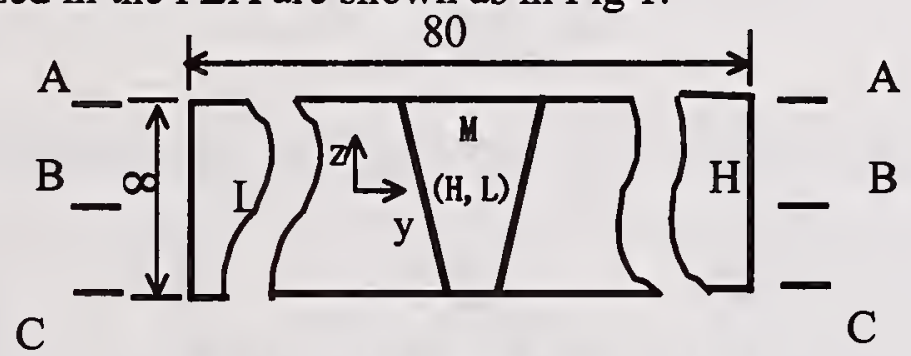

Fig.1 The joints used in FEA

The base metals in all the joints analyzed were respectively $12 \mathrm{Cr} 1 \mathrm{MoV}(\mathrm{L})$ and $9 \mathrm{CrMoVNb}$. $12 \mathrm{Cr} 1 \mathrm{MoV}(\mathrm{L}), 12 \mathrm{Cr} 2 \mathrm{MoWVTiB}(\mathrm{M})$ and $9 \mathrm{CrMoVNb}$ were selected as the weld metal respectively in the low, moderate and high strength matched joints. $\mathrm{L}, \mathrm{M}$ and $\mathrm{H}$ means low, moderate and high creep strength.

The joints analyzed in FEA operate at the fixed temperature of $560^{\circ} \mathrm{C}$ with initial tensile stress of $120 \mathrm{MPa}$ along the transverse direction of the joints (direction of $Y$ axis).

The FEA is performed under the plane stress state.

\section{Analysis Results and Discussion}

\subsection{The distribution of stress triaxiality in DMWJs}

The distribution of stresses in the joint of L-M-H after different creep times is shown as Fig. 2. In Fig. 2, it can be observed that the distribution of stresses $\left(\sigma_{y}, \sigma_{\mathrm{h}}, \sigma_{\mathrm{t}}\right)$ is even at the time without creep deformation ( $t=0 \mathrm{~h}$.). The distribution becomes uneven under creep condition and the degree of this uneven distribution increases with the extension of the creep time due to creep deformation. It should also be noted that there is a great fluctuation of the distribution nearby the interface of $12 \mathrm{Cr} 1 \mathrm{MoV}$ to $12 \mathrm{Cr} 2 \mathrm{MoWVTiB}$ or $12 \mathrm{Cr} 2 \mathrm{MoWVTiB}$ to $9 \mathrm{CrMoVNb}$.

2.2 The effect of creep strength on the distribution of stress triaxality

The distribution of $\sigma_{\mathrm{t}}$ in mismatched joints of steels with different creep strength was studied after the joints have undergone $1000 \mathrm{~h}$ creep deformation. The distribution of $\sigma_{\mathrm{t}}$ on the section $A-A, B-B$ and $C-C$ in L-L-H joint is shown respectively in Fig. 3(a), (b) and (c). The distribution of $\sigma_{\mathrm{t}}$ near the interface of $\mathrm{L}$ and $\mathrm{H}$ is uneven, and the highest value and the most uneven distribution of stress triaxiality is at the weld toe.

The distribution of $\sigma_{\mathrm{t}}$ on the section $A-A, B-B$ and $C-C$ of $\mathrm{L}-\mathrm{H}-\mathrm{H}$ joint is shown respectively as Figure 4(a),(b) and (c). It can be noted that the distribution of $\sigma_{\mathrm{t}}$ near the interface of $\mathrm{L}$ to $\mathrm{H}$ is uneven, and the highest value and the most uneven distribution of stress 
triaxiality is at the location corresponding to weld root and the location adjacent to the bond line on the $B-B$ section.

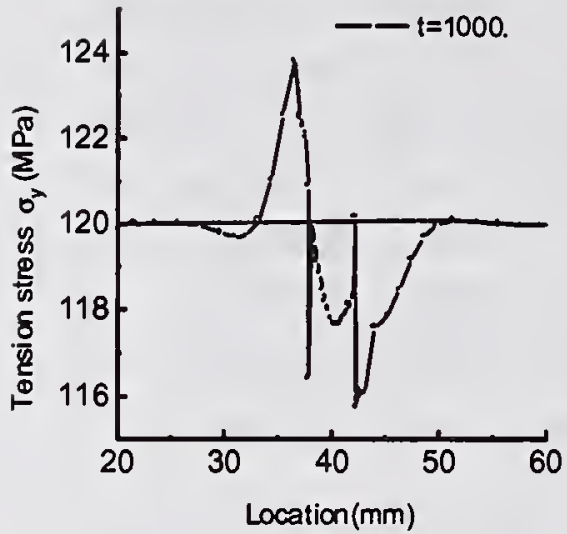

(a)

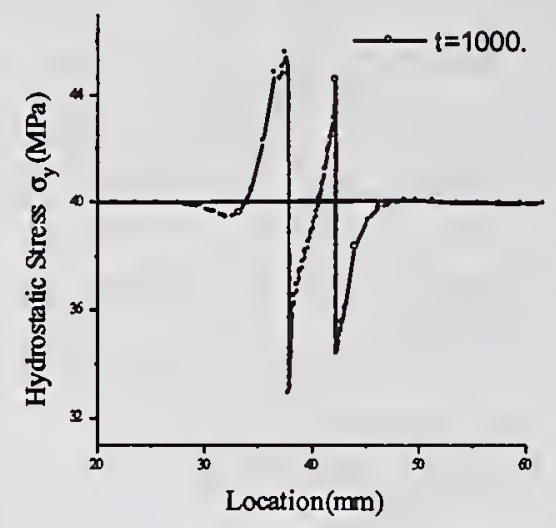

(b)

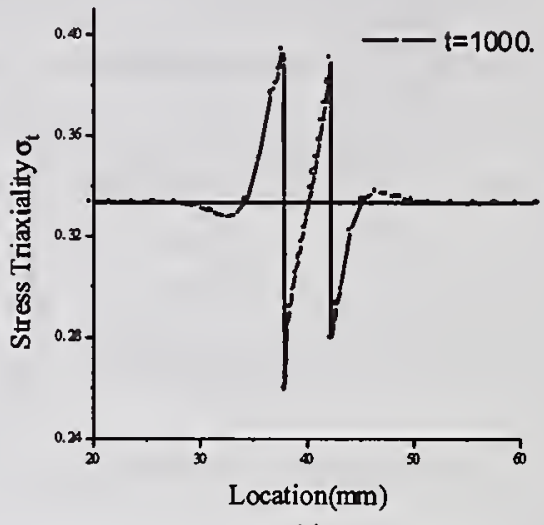

(c)

Figure 2 The distributions of stress on $B-B$ section in the joint of L-M-H

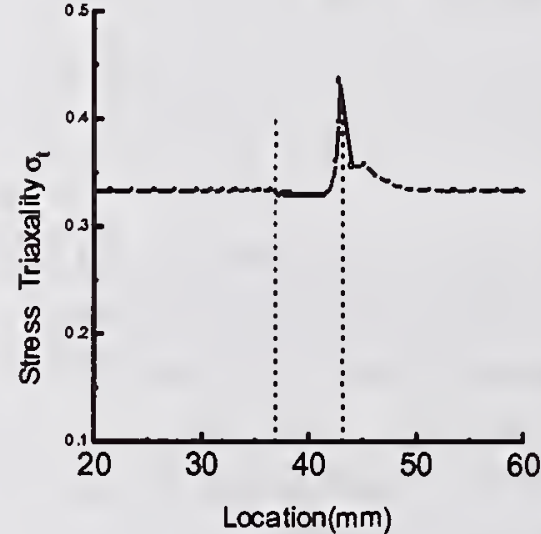

(a)

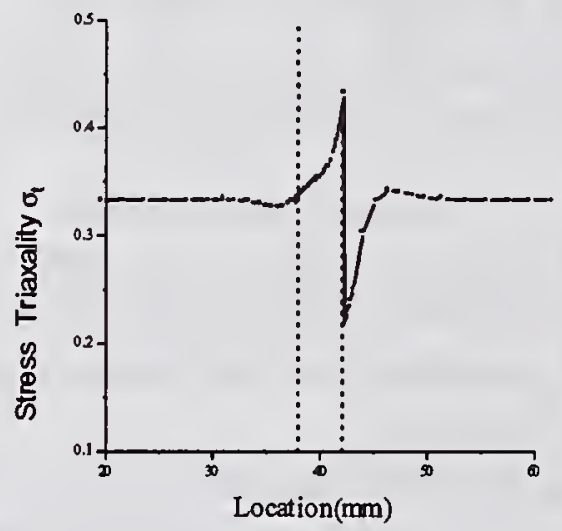

(b)

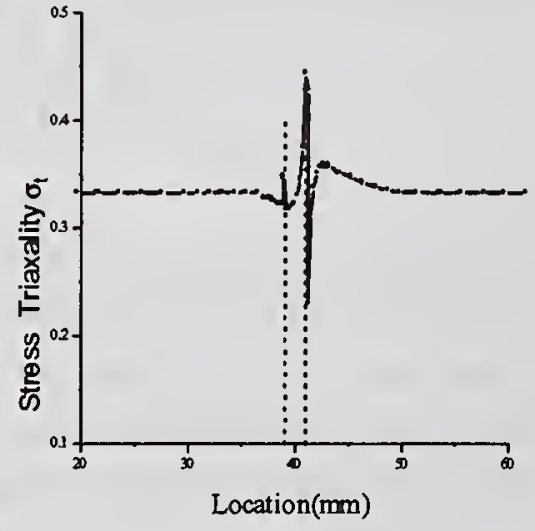

(c)

Figure 3 The distribution of $\sigma_{\mathrm{t}}$ on the section $A-A, B-B$ and $C-C$ in L-L-H joint

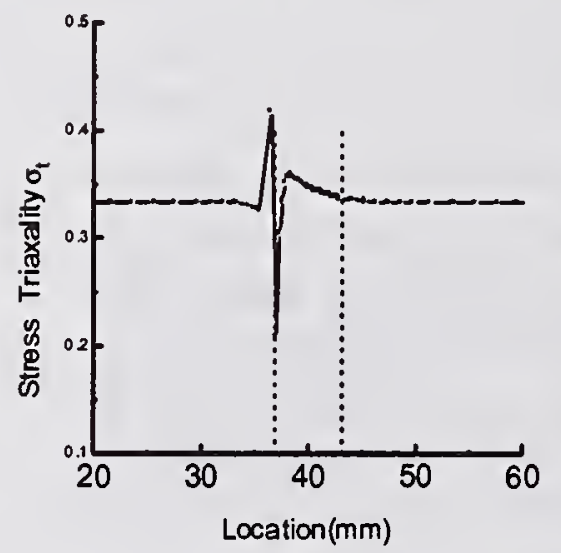

(a)

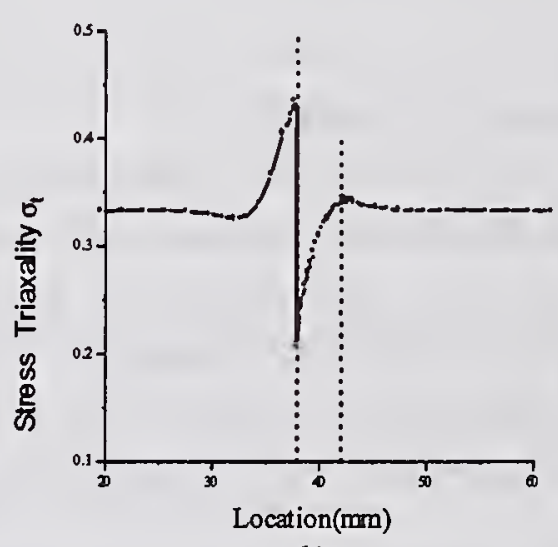

(b)

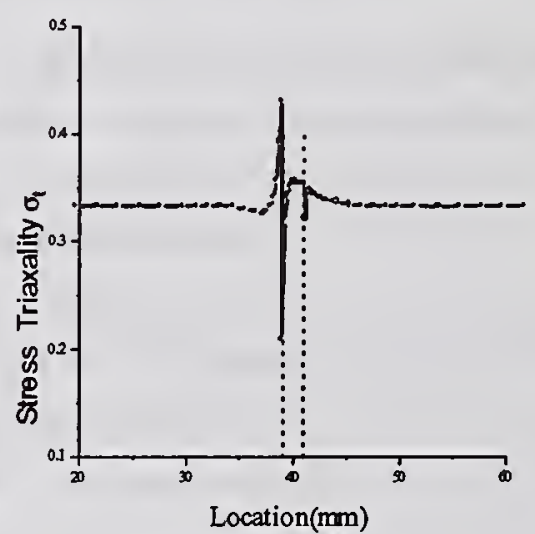

(c)

Figure 4 The distribution of $\sigma_{\mathrm{t}}$ on the section $A-A, B-B$ and $C-C$ in $\mathrm{L}-\mathrm{H}-\mathrm{H}$ joint

The distribution of $\sigma_{\mathrm{t}}$ on section $A-A, B-B$ and $C-C$ of L-M-H is shown respectively in Fig. $5(\mathrm{a}), \quad 5(\mathrm{~b})$ and $5(\mathrm{c})$. It can be seen that the distributions of $\sigma_{\mathrm{t}}$ near the interface of L-M and that of $\mathrm{M}-\mathrm{H}$ are uneven, but the values are much lower, and the unevenness is not so significant, as compared with that near the interfaces in L-L-H or L-H-H joint.

$\sigma_{\mathrm{t}}$ increases with $\sigma_{\mathrm{h}}$ and decreases with $\bar{\sigma}$. The greater the $\sigma_{\mathrm{t}}$ is, the more likely the material fractures under low ductility, which is corresponding to typical creep rupture. Stress triaxiality is the factor related to the creep cavity formation and linking ${ }^{[4]}$. Ref. [5] presented that stress triaxality is the dominant factor to accelerate the brittle creep rupture. So the degree 
of risk of the joint increases with the peak value and the level of uneven distribution of the stress triaxiality near the inhomogenous interface.

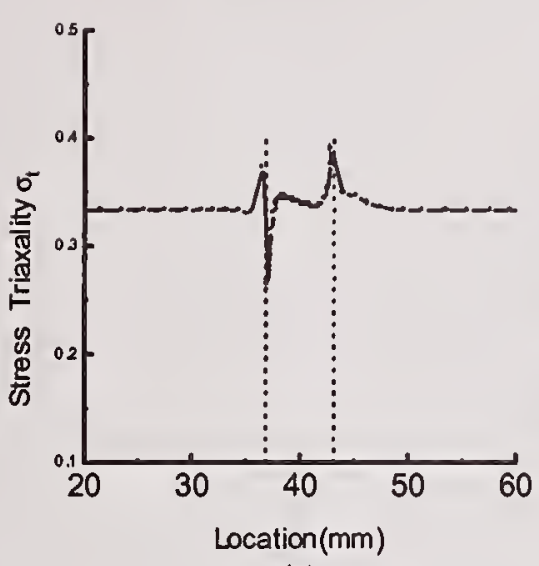

(a)

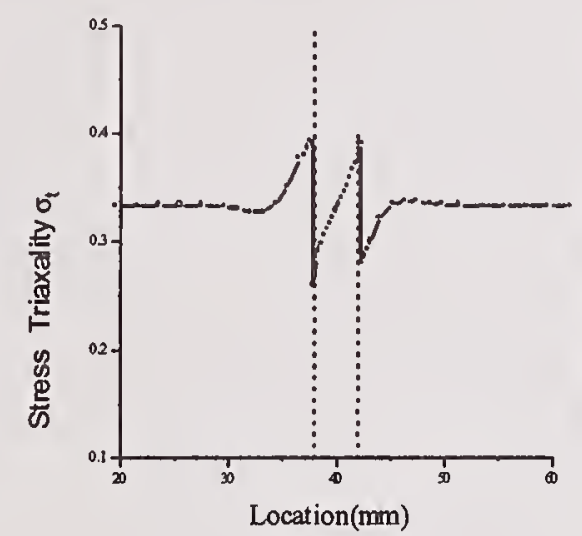

(b)

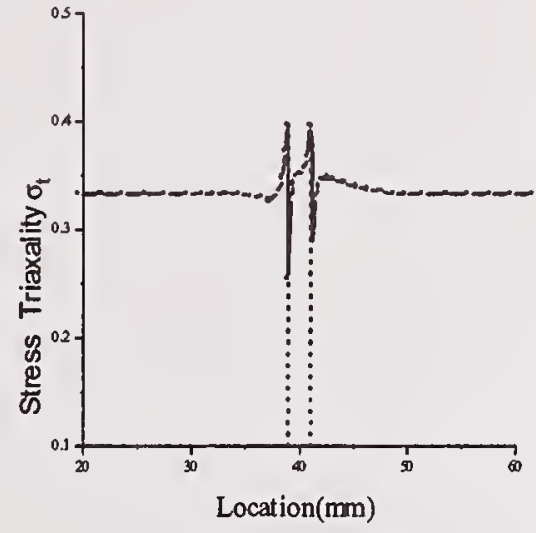

(c)

Figure 5The distribution of $\sigma_{t}$ the section $A-A, B-B$ and $C-C$ of L-M-H joint

As a summary, the potential risk location is closely related to the mismatch of creep strength. However, the location of the highest stress triaxality and unevenness of stress triaxality distribution are at the root for an L-H-H joint, but at the toe for an L-L-H joint, although both locations are close to the L-H interfaces, and are the potential risk locations where creep cracking easily occurs. The cause of the phenomenon needs further study.

\section{Conclusions}

(1) The distribution of stress triaxiality in DMWJs is uneven, especially near the inhomogenous interface. The degree of the uneven distribution increases with the extension of creep time.

(2) The degree of the uneven distribution increases with the increase in mismatch of creep strengths on the inhomogenous interface. The value and the uneven distribution of stress triaxiality near L-H interface is much higher than the ones near L-M and M-H interfaces.

(3) The value and the uneven distribution of stress triaxiality in moderate mismatched joint is low. The moderate mismatched joints are recommended for application in engineering.

\section{References}

1 Kefer D. W., Reuter W. G., Smartt H. B., et al, Needed: verified models to predict the fracture of weldments, Welding Journal, 1993, Vol.72, No.9, 73-78.

2 Holko K.H., Li C.C., Dissimilar weld failure analysis and development program, International Conference on Welding Technology for Energy Applications, Gatlinburg, Tennessee, May 16-19, 1982, (?)

3 Ye S.Y., Cong X.Z., Study on creep rupture of dissimilar welded joints, The 7th. Chinese Welding Annual Assembly, June,1993, Vol.2, 236-240 (in Chinese).

4 Nath B., Creep rupture and creep crack growth behaviour of transition joints, Int. Conf. on Welding Technology for Energy Application, Gatlingburg, May, 1982, 597-621.

5 Shi C.Y., Tian X.T., Chen Z.G., et al., On Mechanical parameter controlling creep brittle rupture along interface of dissimilar metal welded joints, Transactions of the China Welding Institute, Vol. 16(4), 1995,12 (in Chinese). 

Session B3: Modeling III 



\title{
LASER WELD KEYHOLE DYNAMICS: SIMULATION AND EXPERIMENT
}

\author{
M.H. Cho", D. Farson ${ }^{* *}$, J.Y. Lee ${ }^{* * *}$, C.D. Yoo ${ }^{* * * *}$,
}

\begin{abstract}
The dynamics of a keyhole formed by high energy density laser-material interaction is studied by $x$-ray videography experiments and by simulation. The molten material lining the sides of the keyhole periodically collapses due to the surface tension. The collapse of the keyhole due to the surface tension traps a void at the bottom of the keyhole. This phenomenon causes the instability of the keyhole during the laser beam welding. As the laser radiation continues to impinge on the material, the keyhole is subsequently forced open again and then re-collapses at a later time. This cyclical collapse behavior is verified qualitatively by high-speed $\mathrm{x}$-ray images of laser keyholes. The first keyhole is produced about $1.75 \mathrm{~ms}$ after the laser beam impact on the material and then it takes $0.88 \mathrm{~ms}$ to collapse again after the first keyhole collapse, which happens at $1.97 \mathrm{~ms}$ welding time. Also, the flow of the melt is calculated using the Volume of Fluid technique in order to compare the results for the simulation of keyhole to the experimental results. The evaporation recoil pressure, calculated from the thermodynamic relations, is the key driving input that causes motion of the melt layer. The propagation of the Gaussian laser beam within the keyhole is calculated using a ray-tracing model. According to the simulation, it takes approximately $0.2 \mathrm{~ms}$ between the formation of protrusions and the keyhole collapse. This is the same as the experimental results. The simulation predicts that the laser-induced keyholes are unstable. The simulation also predicts the formation of "protrusions" on the keyhole wall due to non-linear interactions between the melt dynamics and laser power density on the keyhole wall.
\end{abstract}

\section{INTRODUCTION}

The laser welding process is one of the most attractive methods for joining two materials up to $32 \mathrm{~mm}$ thickness with a single pass. The laser welding process can minimize the distortion of the materials due to the minimum heat input for fusing the metal and also increase the productivity due to the high travel speed. ${ }^{1}$ The laser is easily automated by computer control, so the precision of the weld joint is much better than the one used by the conventional electrical arc process.

The keyhole formed by a high energy density laser-material interaction is one of important aspects in the laser welding process. The keyhole dynamics have been studied for a long time, but it has been very difficult to observe and predict it. According to previous publications, the keyhole is not constant but dynamically fluctuating during the laser welding process, so this is one of the factors to have the welding defects. When the laser beam impinges on the workpiece with the power densities on the order of $10^{6} \mathrm{~W} / \mathrm{cm}^{2}$, the keyhole is created due to the highdensity energy and is dynamically fluctuating. During the fluctuation of the keyhole, the keyhole

\footnotetext{
* Graduate Student in Welding Engineering department at the Ohio State University

** Professor in Welding Engineering department at the Ohio State University

*** Graduate Student in Mechanical Engineering department at KAIST in Korea

${ }^{* * * *}$ Professor in Mechanical Engineering department at KAIST in Korea
} 
is collapsing and reopening periodically due to the continuous beam and the high surface tension of the material. The flow of melting material has important effects on the keyhole dynamics. The direct observation of the keyhole is the one of the best ways to trace the flow of the melt. The keyhole was observed by x-ray videography experiments. ${ }^{2,3}$ The charge coupled device (CCD) camera with a recording rate of 4500 frames per second was used to capture the keyhole formation because the keyhole is fluctuating rapidly. In this work the simulation of the keyhole calculated by Volume of Fluid technique is used to predict the formation of the keyhole and the results are compared to the pictures taken by $\mathrm{x}$-ray videography in order to verify the keyhole dynamics.

\section{OUTLINE OF STUDY AND METHODS}

The dynamics of a keyhole formed by high energy density laser-material interaction was investigated by $\mathrm{x}$-ray videography experiments and by numerical simulation. In the $\mathrm{x}$-ray videography experiment, the images of keyhole formation during the laser welding implementation were captured by a high speed CCD camera. In the simulation, the effect of multiple reflections in the keyhole was modeled using the ray tracing method, and the free surface profile, flow velocity and temperature distribution of the keyhole were calculated numerically using a volume-of-fluid (VOF) technique.

\section{Experimental procedure}

The experimental setup for direct observation of the keyhole formation is shown in Figure 1. The dynamic behavior of the keyhole formed by the $500 \mathrm{~W} \mathrm{CO}$ laser interaction on the edge of the plain carbon steel plate is observed by a micro-focus x-ray imaging system. (Figure 1) A Feinfocus ${ }^{\circledR} 225 \mathrm{kV} \mathrm{X}$-ray unit with $5 \mu \mathrm{m}(0.0002$ in.) focal size was used as an X-ray source. The positioning system was used for the manipulation of the sample. This system has three linear axes with two-micron resolution and one rotating axis with $0.01^{\circ}$ resolution. The magnification of the image is defined as $\mathrm{d}_{i} / \mathrm{d}_{\mathrm{o}}$, so the magnification of the image is larger as the sample is close to the x-ray source. In this experiment the distance $\left(d_{0}\right)$ from the $x$-ray source to the sample was $1.1875 \mathrm{in}$, and the distance $\left(\mathrm{d}_{\mathrm{i}}\right)$ from the $\mathrm{x}$-ray source to the intensifier was 28.1875 in. Therefore, the magnification of the image came out to be 23.7 times $(28.1875 \mathrm{in} / 1.1875 \mathrm{in})$. At this high magnification, a small x-ray source size is needed to minimize image unsharpness.

The CCD camera, EKTAPRO HS Motion Analyzer, was used for taking pictures of the image, which is the optical image converted from $x$-ray image by the intensifier. This CCD camera is located at the end of the intensifier and captured the keyhole image inside intensifier. This CCD camera was able to record the pictures up to $40500 \mathrm{frames} / \mathrm{sec}$, and the capacity of the memory can store 1024 full frames. In this experiment, 4500 frames/sec recording rate was used which was able to capture the images every $0.22 \mathrm{~ms}$. This fast recording rate was able to clearly image the collapse of the keyhole and permit observation of the keyhole dynamics.

Parameters for $\mathrm{CO}_{2}$ laser were $500 \mathrm{~W}$ power, $20 \mathrm{kHz}$ frequency pulse, $50 \mu$ s period, and $26 \mu \mathrm{s}$ pulse width. The $20 \mathrm{kHz}$ pulsed frequency was chosen to be well above the natural frequencies of the molten keyhole. The laser focus spot size was $0.13 \mathrm{~mm}$ diameter. The thickness of the 
plain carbon steel was $1 \mathrm{~mm}$. Sample thickness is critical because the attenuation of x-ray through the thick plate is large so the keyhole cannot be observed well. Samples thinner than $1 \mathrm{~mm}$ are very difficult to manipulate and weld on the edge of the plate.

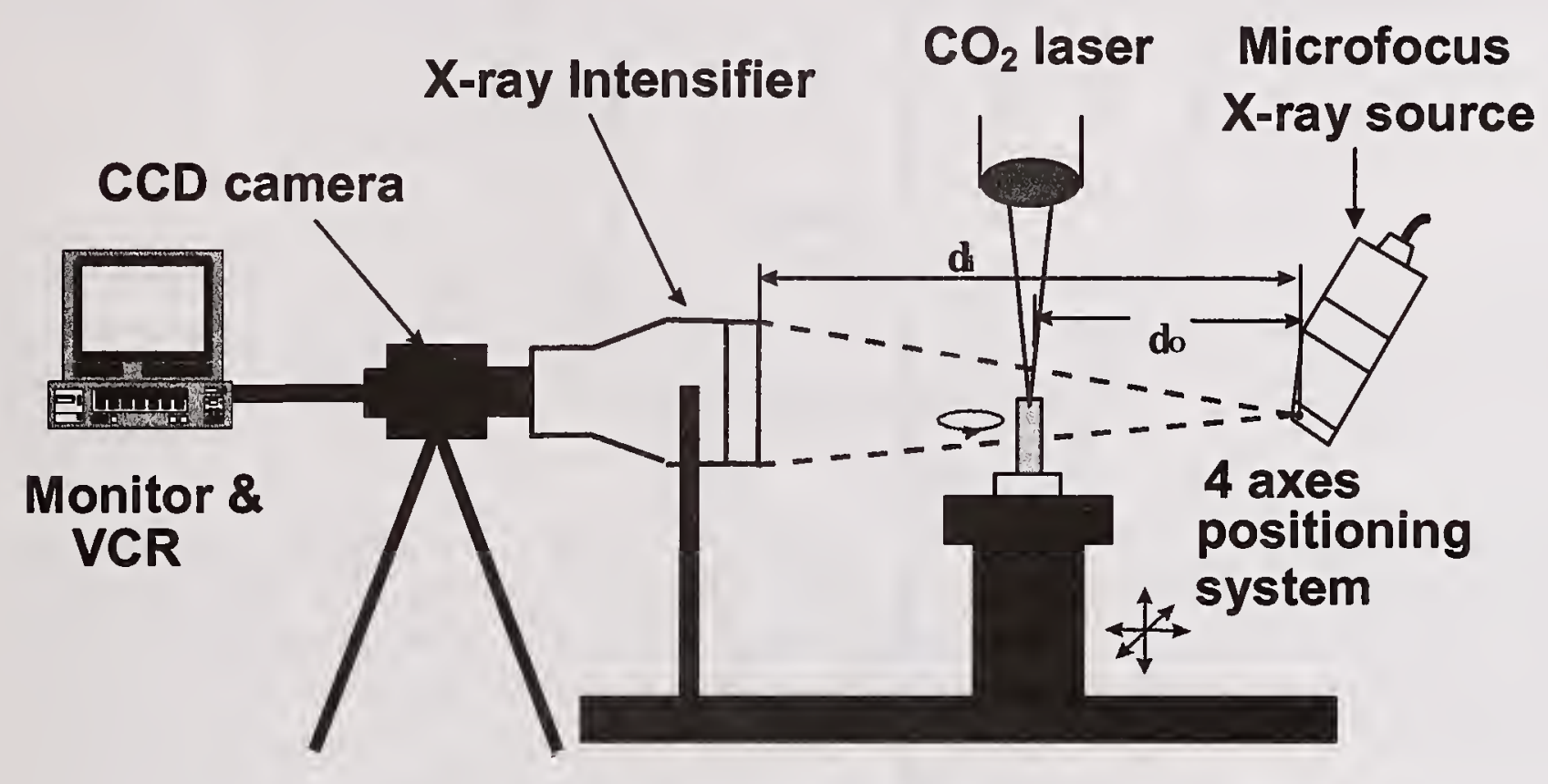

Figure1: Microradiographic setup for observation of keyhole behavior

A pre-welded sample was used to find the optimum positioning parameters to obtain the desired image of the keyhole before implementing the real time monitoring of laser welding keyholes. After the welded sample was set up in the optimum position the welded sample was replaced with the new sample and then the laser beam hit the edge of the plain carbon steel for about one second while the CCD camera took a picture every $0.22 \mathrm{~ms}$.

\section{Formulation for Numerical Simulation}

The simulation was comprised of a fluid/thermal simulation to calculate keyhole formation and a ray-tracing simulation to calculate propagation of the laser beam within the keyhole. The raytracing method of calculating beam propagation assumes that incident radiation consists of a number of rays, each having a specified direction and power. ${ }^{4}$ The absorption of laser power for a simplified conical shape is increasing compared to the flat surface due to multiple reflections within the keyhole as shown in Figure2. In this work, the laser beam incident on the work surface was assumed to consist of parallel rays, the power assigned to each calculated from a circularly symmetric Gaussian profile having specified $1 / \mathrm{e}^{2}$ radius. The ray tracing proceeded under the assumptions that the material surfaces were specular, that plume absorption was negligible and that the beam polarization was random. Consistent with the last assumption, the reflectivity of the iron surfaces was taken as the intermediate value between available "s" and " $p$ " reflectivity vs. angle curves. The number of reflections and the accumulated power density 
were calculated at each position on the keyhole wall, and the absorbed power density was given as the boundary condition to calculate the temperature of the keyhole.

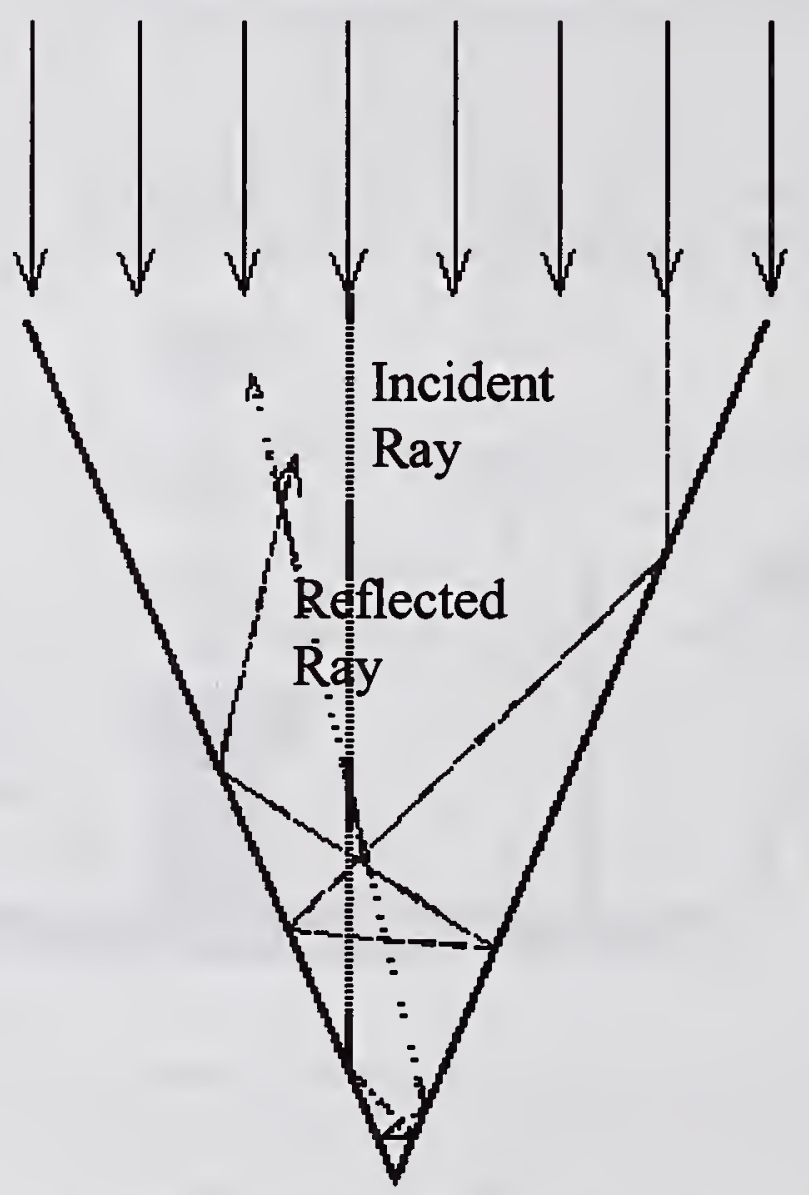

Figure2: Ray tracing in the keyhole cavity

The axisymmetric keyhole profile and flow velocity were calculated numerically using the Volume of Fluid (VOF) method. ${ }^{5}$ The governing equations for the VOF method consist of continuity and momentum and volume fraction equations

$$
\begin{aligned}
& \nabla \cdot \overline{\mathrm{v}}=0 \\
& \frac{\partial \overline{\mathrm{v}}}{\partial \mathrm{t}}+(\overline{\mathrm{v}} \cdot \nabla) \overline{\mathrm{v}}=-\frac{1}{\rho} \nabla \mathrm{P}+\nu \nabla^{2} \overline{\mathrm{v}}+\beta \overline{\mathrm{g}} \Delta \mathrm{T} \\
& \frac{\partial \mathrm{F}}{\partial \mathrm{t}}+(\overline{\mathrm{v}} \cdot \nabla) \mathrm{F}=0
\end{aligned}
$$

where $\bar{v}$ is the velocity vector, $P$ is pressure, $\rho$ is mass density, $v$ is viscosity, $\beta$ is thermal expansion coefficient, $\mathrm{g}$ is the gravity vector and $\mathrm{F}$ is volume fraction.

The temperature distribution was obtained by solving the energy equation

$$
\rho C_{p}\left(\frac{\partial T}{\partial t}+v \cdot \nabla T\right)=k \nabla^{2} T
$$


where $\mathrm{C}_{\mathrm{p}}$ represents the specific heat and $\mathrm{k}$ the thermal conductivity. Note that the cooling effect of vapor leaving the surface is neglected in this energy equation. The recoil pressure due to evaporation was taken as 0.54 times the pressure of vapor in equilibrium with the liquid surface (a common assumption that implies vapor flow velocity is at its maximum possible value). The equilibrium vapor pressure was calculated from the integral of the Clausius-Clapeyron equation ${ }^{6}$

$$
P_{r} \cong 0.54 P_{0} \exp \left(\Delta H_{V} \frac{T-T_{V}}{R T T_{V}}\right)
$$

where $\mathrm{P}_{0}$ denotes atmospheric pressure, $\mathrm{R}$ the universal gas constant, $\Delta \mathrm{H}_{\mathrm{v}}$ the latent heat of vaporization and $\mathrm{T}_{\mathrm{V}}$ evaporation temperature, respectively. When the temperature on the keyhole surface is given, the recoil pressure is calculated and imposed on the free surface as the boundary condition.

Since the VOF method was explained in details in other works ${ }^{7,8}$, the calculation procedure is briefly summarized as follows: (1) absorbed laser power density on the keyhole wall is estimated using the ray tracing method, (2) liquid and solid temperatures are calculated by solving the energy equation, (3) recoil pressure is obtained from the surface temperature and imposed on the free surface, (4) free surface profile and liquid flow velocities are calculated numerically using the VOF method, (5) time is incremented and steps (1)-(5) repeated.

\section{SUMMARY}

Figure 3 shows the real time x-ray images of the keyhole formation during $500 \mathrm{~W} \mathrm{CO}_{2}$ spot welding of plain carbon steel. These images show how fast the keyhole is fluctuating and how often the keyhole collapses during laser welding. The images in Figure 3 were selected to illustrate the keyhole dynamics.

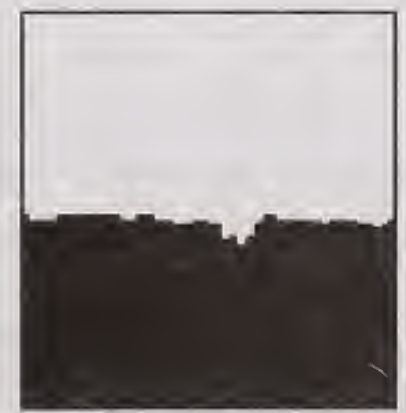

1) $1.75 \mathrm{~ms}$

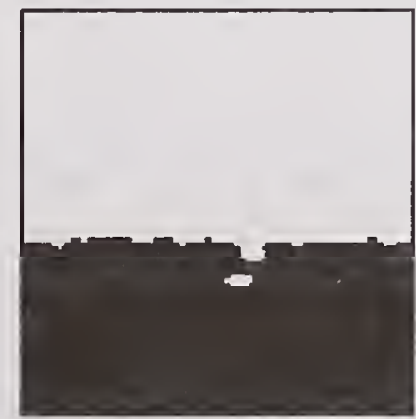

2) $(1.75+0.22) \mathrm{ms}$

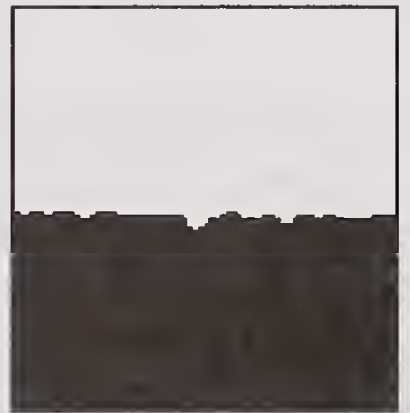

3) $(1.75+0.44) \mathrm{ms}$ 


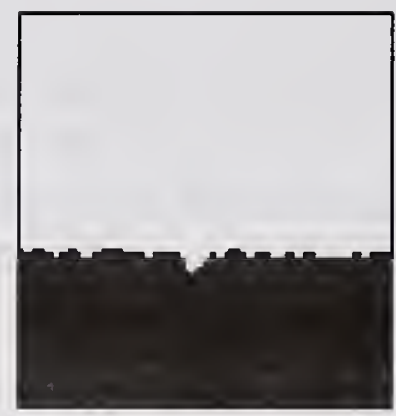

4) $(1.75+0.66) \mathrm{ms}$

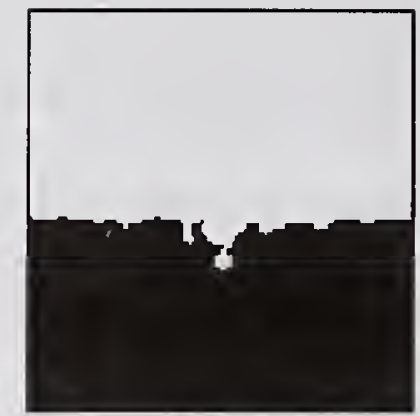

5) $(1.75+0.88) \mathrm{ms}$

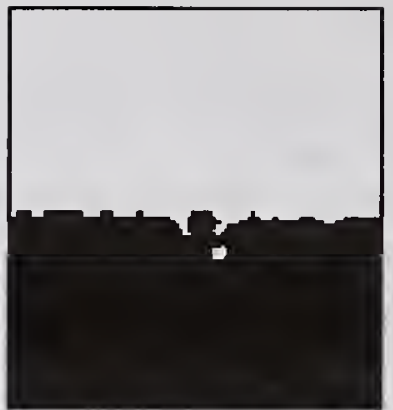

6) $(1.75+1.1) \mathrm{ms}$

Figure3: Keyhole behavior observed by X-ray image system

The first image was taken at $1.75 \mathrm{~ms}$ after the beam impinged on the material, and then others were taken every $0.22 \mathrm{~ms}$. This is the first keyhole formed during the weld. There is no pore at the bottom of the keyhole in the first image. The keyhole was collapsed in the second image, and a pore was trapped at the bottom of the keyhole after $0.22 \mathrm{~ms}$ since the complete keyhole formed. This was the first pore seen since the laser beam impinged on the edge of the sample. In the third image the pore suddenly disappeared because the continuous laser beam broke through the bridge and reopened the keyhole. The keyhole diameter increased and did not collapse in the fourth image. The keyhole was still in a stable state.

In the fifth image the protrusion appeared on the wall of the keyhole. Simulation results of shown later that the protrusion forms because of the collision the flow of the molten metals due to the recoil pressure acting upward and the surface tension acting downward. The protrusions on the keyhole walls were obviously growing toward the center of the keyhole and were almost connected each other. This is the beginning point of the keyhole collapse. Finally, the bridge was completely built and the pore was trapped at the bottom of the keyhole in the sixth image.

The complete keyhole was found $1.75 \mathrm{~ms}$ after the laser beam impinged on the plain carbon steel. After $1.97 \mathrm{~ms}$, the first keyhole collapse was observed and then the keyhole reopened by the continuous laser beam irradiation after $0.22 \mathrm{~ms}$. The period of the periodical collapse time is about $0.88 \mathrm{~ms}$.

The surface profile, flow velocities and temperature distributions corresponding to a $500 \mathrm{~W}$ laser power are shown in Figure 4. The recoil pressure is significantly higher and keyhole exists at a time of $10 \mathrm{~ms}$. Simulation results between 10 and $13 \mathrm{~ms}$ show molten metal oscillating upward and downward along the keyhole wall, a mode which contributes to the keyhole instability. Upward flow from the bottom of the keyhole, caused by the high recoil pressure at that location, delivers heat and molten material to the periphery. The surface profile at the periphery becomes a convex (e.g. at $13 \mathrm{~ms}$ ), increasing the inward pressure due to surface tension. This pressure induces a downward flow, opposite to that induced by recoil pressure. As the opposing flows collide at $13.2 \mathrm{~ms}$, an inward protrusion is formed near $\mathrm{z}=0.1 \mathrm{~mm}$. This protrusion becomes unstable, since the smaller keyhole radius at that location increases the inward radial component of surface tension pressure. Hence, the protruding liquid is accelerated inward and the keyhole collapsed shortly after $13.2 \mathrm{~ms}$. 
The keyhole profile from the simulation at $13.2 \mathrm{~ms}$ is similar to the keyhole shape from the $\mathrm{x}$-ray experiment at the fifth image in Figure 3 . Also, it takes $0.2 \mathrm{~ms}$ for the keyhole to collapse since the protrusion is generated in both cases, which are the x-ray videography experiment and the simulation.
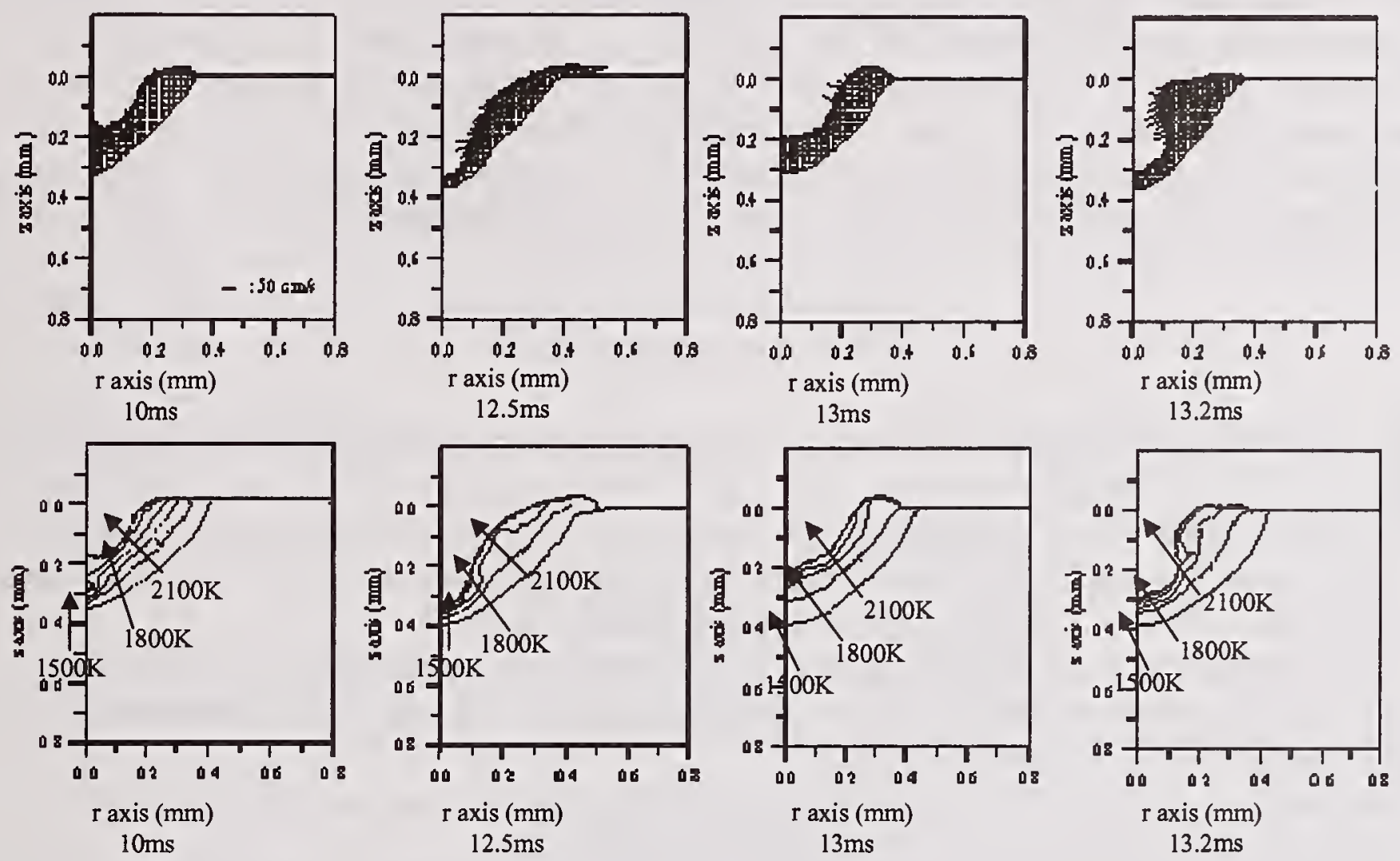

Figure 4: Surface profiles, temperature, and flow pattern for $500 \mathrm{~W}$ laser

That the cylindrical keyhole geometry is prone to instability of this type is not surprising. Conceptually, the dynamics of the keyhole configuration are related to that of a submerged gas jet in a liquid, first analyzed by Rayleigh. ${ }^{9}$ In that work, it was found that the cylindrical jet was unstable when the wavelength of a varicose perturbation was greater than its circumference. The maximum exponent, $\mathrm{q}$, of the rate of increase in the amplitude of a varicose perturbation $e^{q t}$ was calculated as

$$
q=\sqrt{0.696 \frac{\gamma}{\rho a^{3}}}
$$

where $\mathrm{a}$ is the radius of the jet, $\gamma$ is the surface tension, and $\rho$ is the density of the steel. For the simulated keyholes, this amounts to the time constant of about $1.8 \times 10^{-4} \mathrm{~s}$. This growth rate compares well to the time elapsed from protrusion formation to keyhole collapse in both the $500 \mathrm{~W}$ keyhole simulations and the direct $\mathrm{x}$-ray keyhole observation experiment of the $500 \mathrm{~W}$ laser weld. 


\section{CONCLUSION}

The direct observation of the keyhole dynamics by the $\mathrm{x}$-ray transmission imaging system showed the dynamic behavior of keyholes generated with a $500 \mathrm{~W} \mathrm{CO}$ laser beam. The keyhole formation was monitored and instability of the keyhole was observed by the analysis of images. Additionally the dynamic behavior of the keyhole was simulated and it was compared to the experimental results. Surface tension caused growth of protrusions on the keyhole wall. This caused instability of the keyhole and led to the collapse of the keyhole. In both cases the time for the complete collapse of keyhole when the protrusion appeared was about $0.2 \mathrm{~ms}$. This number shows reasonable correlation between the experiment and the simulation.

\section{REFERENCE}

1. Welding Handbook, Eighth Edition, Vol. 2. Miami: American Welding society, 1991:723725 .

2. Shigeki Fujinaga, Hiroo Takenaka, Toru Narikiyo, Seiji Katayama and Akira Matsunawa. Direct observation of keyhole behaviour during pulse modulated high-power Nd:YAG laser irradiation. J. Phys. D:Appl.Phys. 33 492-497.: November 1999.

3. Akira Matsunawa, Jong-Do Kim, Naoki Seto, Masami Mizutani and Seiji Katayama. Porosity Formation and Solidification Cracking in Laser Welding. Taiwan International Welding Conference.: September 1998.

4. Milewski J O and Barbe M B 1999 Modeling and analysis of laser melting within a narrow groove welding Welding J. 78 109-115s

5. Hirt C W and Nichols B D 1981 Volume of fluid (VOF) method for the dynamics of free boundaries $J$. of Computational Phys. 39 201-225

6. von Allmen M and Blatter M 1995 Laser-beam interactions with materials $2^{\text {nd }}$ ed (Springer)

7. Ko S H, Choi S K and Yoo C D 2001 Effects of surface depression on pool convection and geometry in stationary GTAW Welding J. 80 39-45s

8. Fan H G and Kovacevic R 1999 Keyhole formation and collapse in plasma arc welding $J$. Phys. D: Appl. Phys. 32 2902-2909

9. Rayleigh J W S 1892 "On the stability of cylindrical fluid surfaces", Phil. Mag. s.5 v.34 177180. 


\title{
FINITE ELEMENT MODELLING ON WELDING PROCESSES AND WELDING PHENOMENA
}

\author{
B. Palotas*
}

\begin{abstract}
The Finite Element Modelling is a wide applicable method in the field of welding as well. The paper shows application examples of modelling of resistance welding processes, modelling of cold pressure welding and some possibilities of FEM application.

The results of FEM can be used in the field of Computer Aided Process Planning applied in the field of welding processes.

The application of Finite Element Modelling helps to understand the welding processes, the welding phenomena and it is an effective tool of Welding Specialists and Researchers in our time.
\end{abstract}

\section{KEYWORDS}

Welding, Computer Aided Welding, Modelling of Welding, Finite Element Modelling

\section{INTRODUCTION}

The Welding Engineer is often faced with the task of developing a welding technology, after he has first selected the welding procedure in accordance with the prescribed characteristics and the geometrical requirements of the weld. Then it is necessary to calculate the course of the welding process, the criteria for optimisation being the weld form, the quality stability and the weldability of the material under pre-established physical, technical and economical conditions. The physical processes should be described by natural laws, such as the conservation, transmission and conversation of energy. The technical and economic conditions, on the other hand, such as time, output and cost can be influenced. They therefore form the basis for the optimisation problem. This complicated problem can be solved theoretically or empirically. Less and less time available for project planning tasks makes it increasingly necessary to avoid empirical test methods and to search for more effective methods of studying and developing welding technologies.

Automated preparation of the technological data increases the labour productivity of the engineer, but basically does not change the quality of the design work. This is due to the fact that the welding parameters are prescribed on the basis of empirical information.

\footnotetext{
* Associate Professor, Department of Materials Science and Engineering, Budapest University of Technology and Economics, Bertalan L. u. 7. Budapest, H-1111, Hungary, Tel: +36 (1) 463 1115, e-mail: palotasb@eik.bme.hu
} 
The quality of the design work can, however, be improved considerably using the theory of welding process in the form of certain mathematical models. These models should as far as possible contain complete information about the process to be calculated. It would be advantageous to create universal models for the form of the welds and the weld defects. The software for the mathematical model is only produced once and can be rendered more precise as knowledge of the process to be studied increases (Ref. 1.).

\section{MATHEMATICAL MODELLING OF WELDING PHENOMENA}

The welding phenomena should be written by differential equations given in the Table 1 . on the basis of (Ref. 2.). The general solution of these equations can be given by Gauss-Ostrogradsky's theorem:

$$
\oint_{\Sigma} q d S=\int_{V} \operatorname{div} q d V=\int_{V}\left(\frac{\partial q}{\partial x}+\frac{\partial q}{\partial y}+\frac{\partial q}{\partial z}\right) d V
$$

where $\mathrm{q}$ is a flow through a $\Sigma$ surface ( flux of q) when its source is written with its divergent (div) in $\mathrm{V}$ volume bordered by $\Sigma$ surface. The equation (1) can be used for analysis of phenomena occurring in solid, liquid, solid-liquid and gas agent during welding.

\begin{tabular}{|c|c|c|c|}
\hline Physical process & Process parameter & Coefficient & Flow \\
\hline Heat conduction & Temperature, $\mathrm{T}$ & Thermal conductivity, $\lambda$ & $\begin{array}{l}\text { Heat flowing } \\
q=-\lambda \text { grad } T \\
\text { (Fourier's law ) }\end{array}$ \\
\hline Diffusion & Concentration, $\mathrm{C}$ & $\begin{array}{l}\text { Coefficient of Diffusion, } \\
\text { D }\end{array}$ & \begin{tabular}{|l} 
Mass flow \\
$q=-D$ grad C \\
(Fick's law) \\
\end{tabular} \\
\hline Electrical field & Potential, U & Electrical conductivity, $\sigma$ & $\begin{array}{l}\text { Current density } \\
j=-\sigma \text { grad U } \\
\text { (Ohm's law ) }\end{array}$ \\
\hline Elastic deformation & Strain, $x$ & Young's modulus, $\mathrm{E}$ & $\begin{array}{l}\text { Stress } \\
\sigma=\text { E grad } \mathbf{x} \\
\text { (Hoock's law) }\end{array}$ \\
\hline $\begin{array}{l}\text { Viscous flow of } \\
\text { liquid }\end{array}$ & Flow velocity, $v$ & Coefficient of friction, $\mu$ & $\begin{array}{l}\text { Stress of friction } \\
\tau=-\mu \text { grad } v \\
\text { (Newton's law ) }\end{array}$ \\
\hline
\end{tabular}

Table 1. Base equations of welding phenomena

Unfortunately, the analytical solution of equations (Tab.1.) is very difficult and it requires a lot of neglect and we have to know all the processes occurring during welding. Of course all the processes are not known that occur during welding and negligence lead to information losses. 
Due to these facts, we have to use numerical mathematical methods to solve the above-mentioned equations (Ref. 3.).

One of these numerical methods is the Finite Element Method.

\section{FINITE ELEMENT MODELLING OF WELDING}

The processes occurring during welding are named coupled processes, because thermal, electrical, electro- magnetic, mechanical, metallurgical and surface processes are to be taken into consideration (Fig. 1.) (Ref. 4.). The heat source heats the material which heat causes thermal processes in the material with mechanical response and changes the microstructures.

The first publications were about FEM modelling of stress and strain distribution of welded joints and many articles can be found about this question for example (Ref. 4.), (Ref. 5.). At the time of first FEM modelling the software and computer possibility were limited, but in our time many FEM Software exist and they are applicable in the field of welding successfully (for example, ANSYS, SYSWELD, MARC).

In the following new results of FEM modelling will be shown, these acts of modelling were carried out at the Department of Materials Science and Engineering of Technical University of Budapest (new name is Budapest University of Technology and Economics ).

\section{MODELLING OF PRESSURE WELDING PROCESSES}

Pressure welding processes are generally fully mechanized processes, so the human factors do not effect in the case of these processes. It means that, the Computer Aided Process Planning (CAPP) is well applicable for these processes. CAPP requires probable equations based on physical laws. The FEM modelling can give the basis of CAPP.

The FEM modelling of pressure welding processes helps understanding physics of them as well. The simplest process is the cold pressure welding in the modelling.

\section{Cold Pressure Welding}

The cold pressure welding applies only force for jointing elements. When we use this welding process means we use a cold forming process. Cold forming processes are widely modelled by FEM. There are many software for FEM modelling of forming, for example MSC MARC AUTOFORGE 3.1.

We used this software for modelling of cold pressure but welding as well. The plastic deformation processes and the condition of formation of welding joint can also be investigated by this modelling.

The result of geometrical modelling of cold pressure welding of aluminium rods is given in the Fig. 2. The computer with a high speed can calculate the main welding parameters as pressure force and strain. This modelling should be become a theoretical basis of investigation of welded joint formation. If we can give a mathematical equation for conditions of welding joint formation then it will be a nice new technical result. We are working on this question nowadays. 
If we heat the materials during welding then the processes are more complicated, but we could model resistance welding processes by FEM.

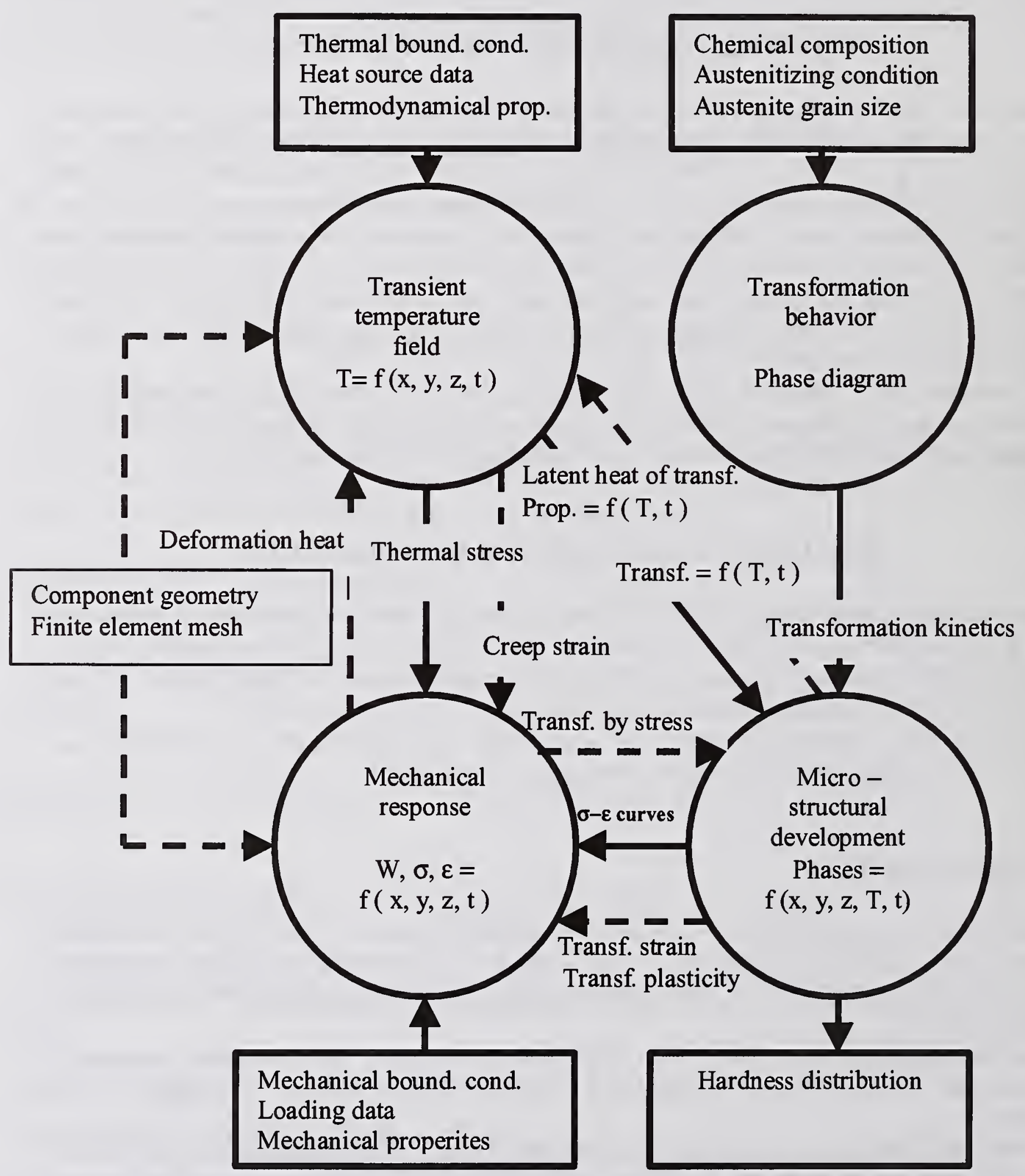

Figure 1: Coupled processes at welding analysed by FEM 


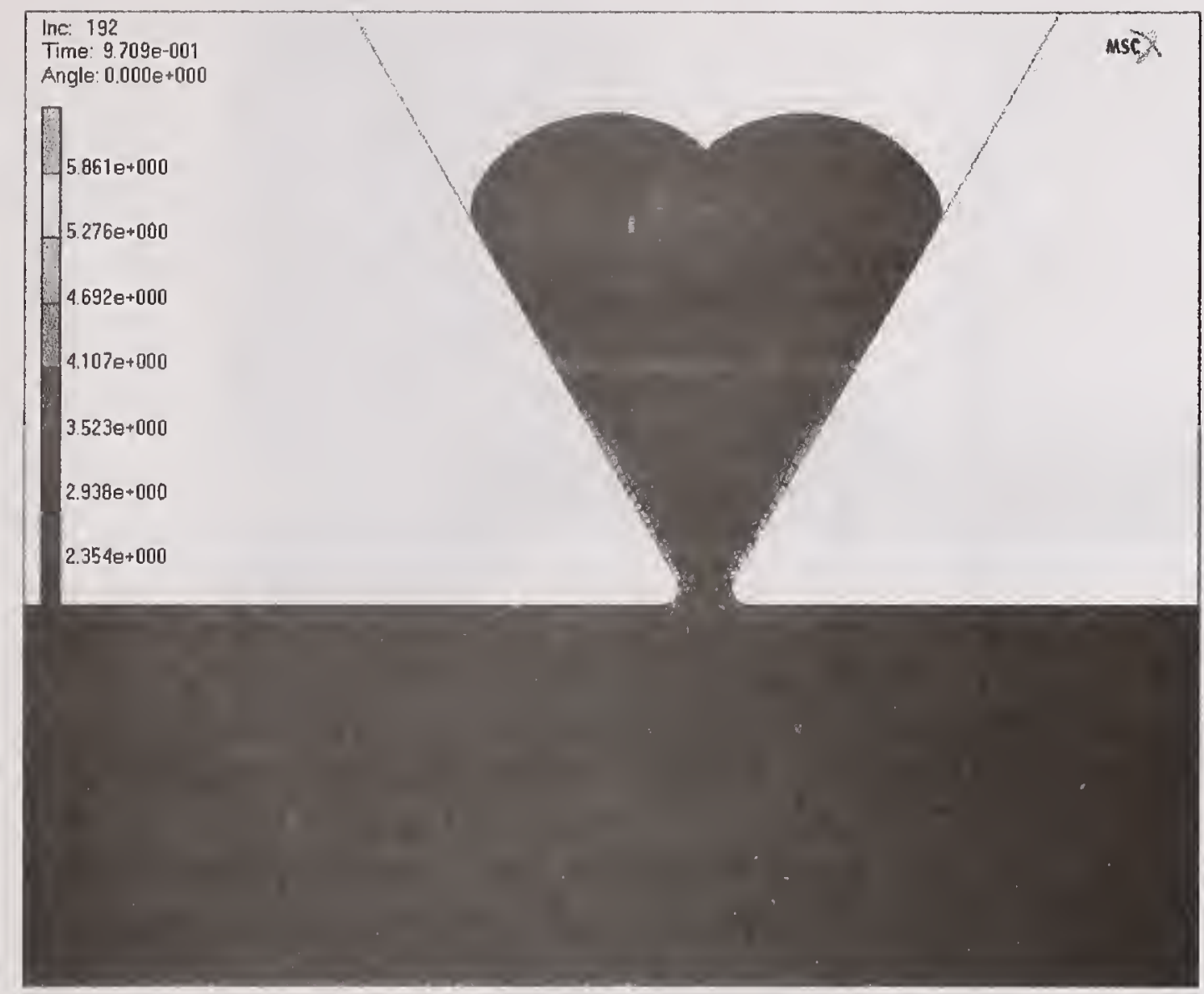

Figure 2. FEM model of cold pressure butt-welding

Another area of welding processes modelling is the FEM modelling of resistance welding processes at our department.

\section{Resistance Upset Welding}

The FEM modelling of resistance upset welding was carried out by MARC/AutoForge 2.2.0 software. The detailed conditions of modelling and results can be seen in Ref. 6 . The strain distribution is given in the Fig.3. and the pressure force requirement can be seen in the Fig.4. The results of modelling can be used for welding parameter planning. The practice proved that the results as pressure force and length change, were applicable, correct welding parameters.

The FEM modelling of resistance upset welding as pressure forming procedure is a new approach of welding parameter modelling and it has international interest.

\section{Resistance Spot Welding}

The Ref. 7. modelled the resistance spot welding, the result of temperature distribution in the moment of current turned off can be seen in the Fig. 5. The results of Ref. 7, are in accordance with international literature, the used software was the ANSYS 5.0.

The three-dimensional geometrical model can be seen in Fig. 6. 


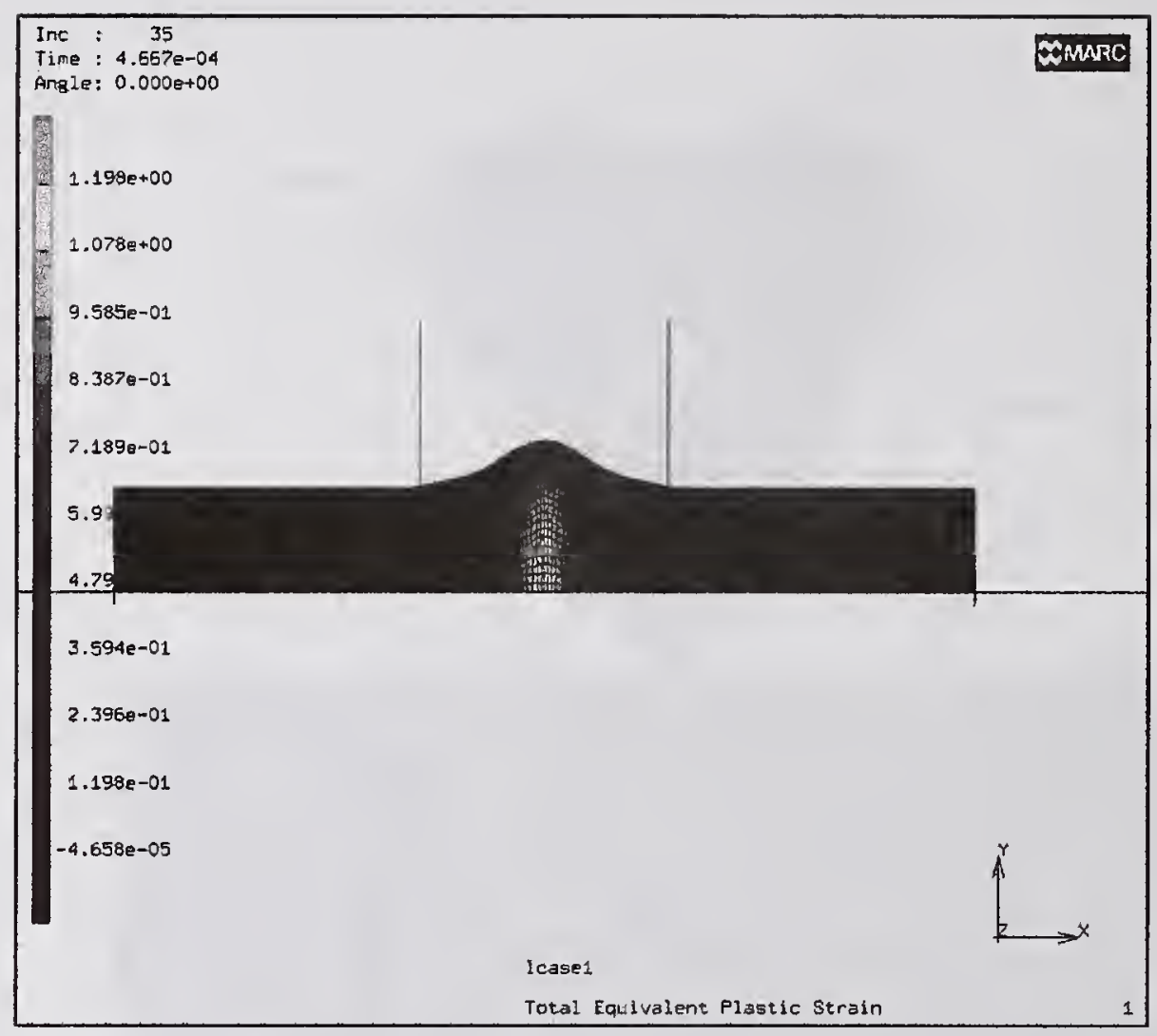

Figure 3: Strain distribution results at resistance upset welding

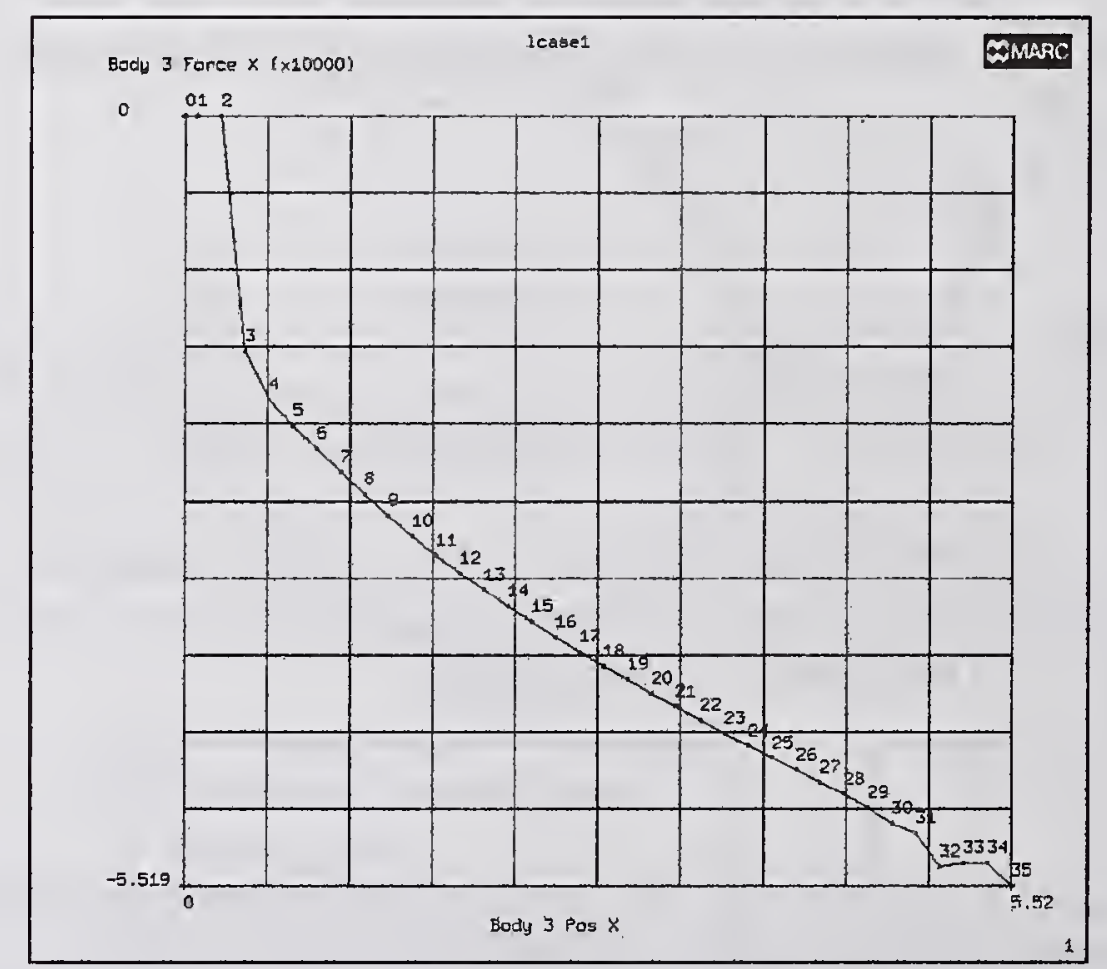

Figure 4: Pressure force requirement at resistance upset welding 

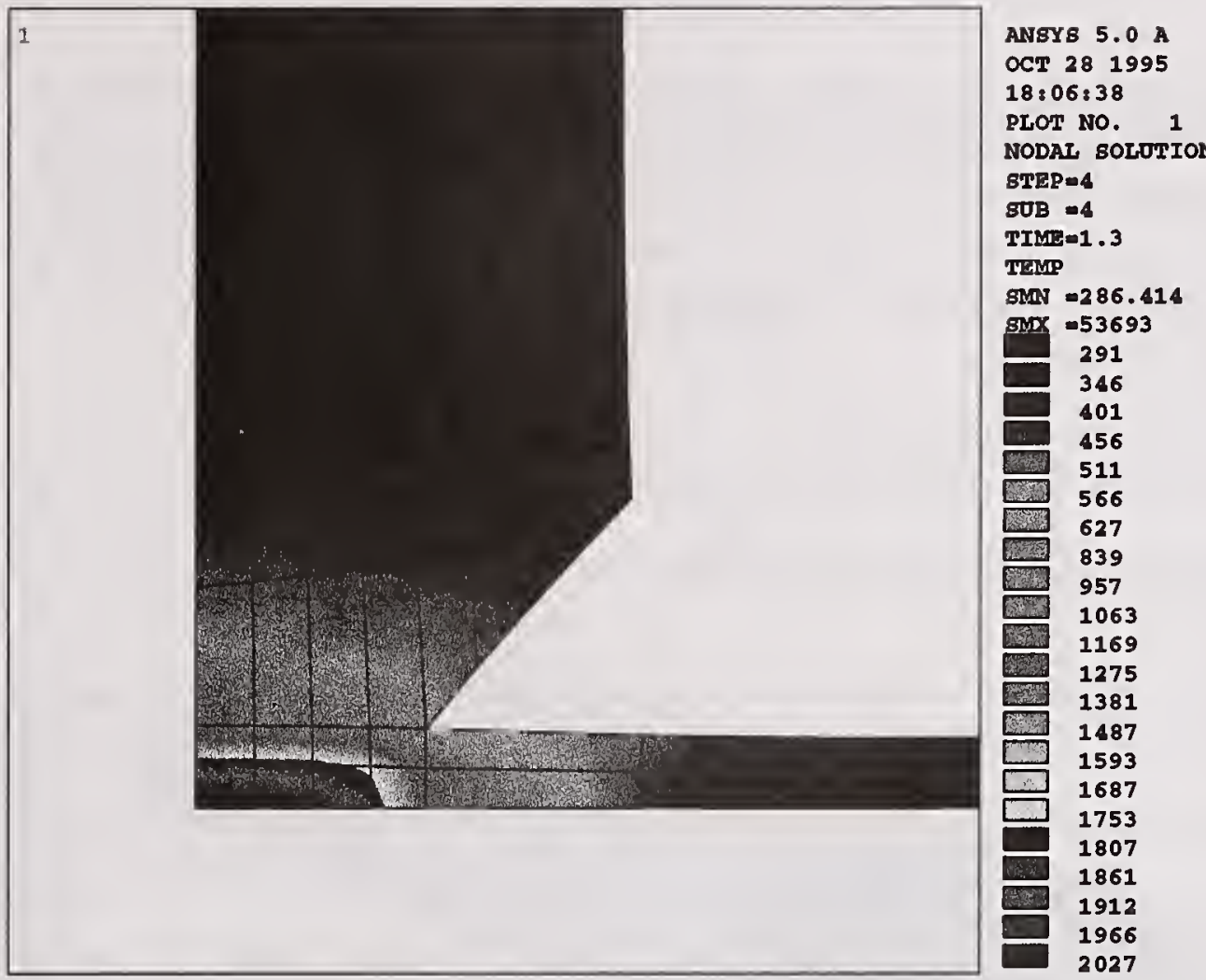

Figure 5: Temperature distribution of resistance spot welding (in the moment of current turned off)

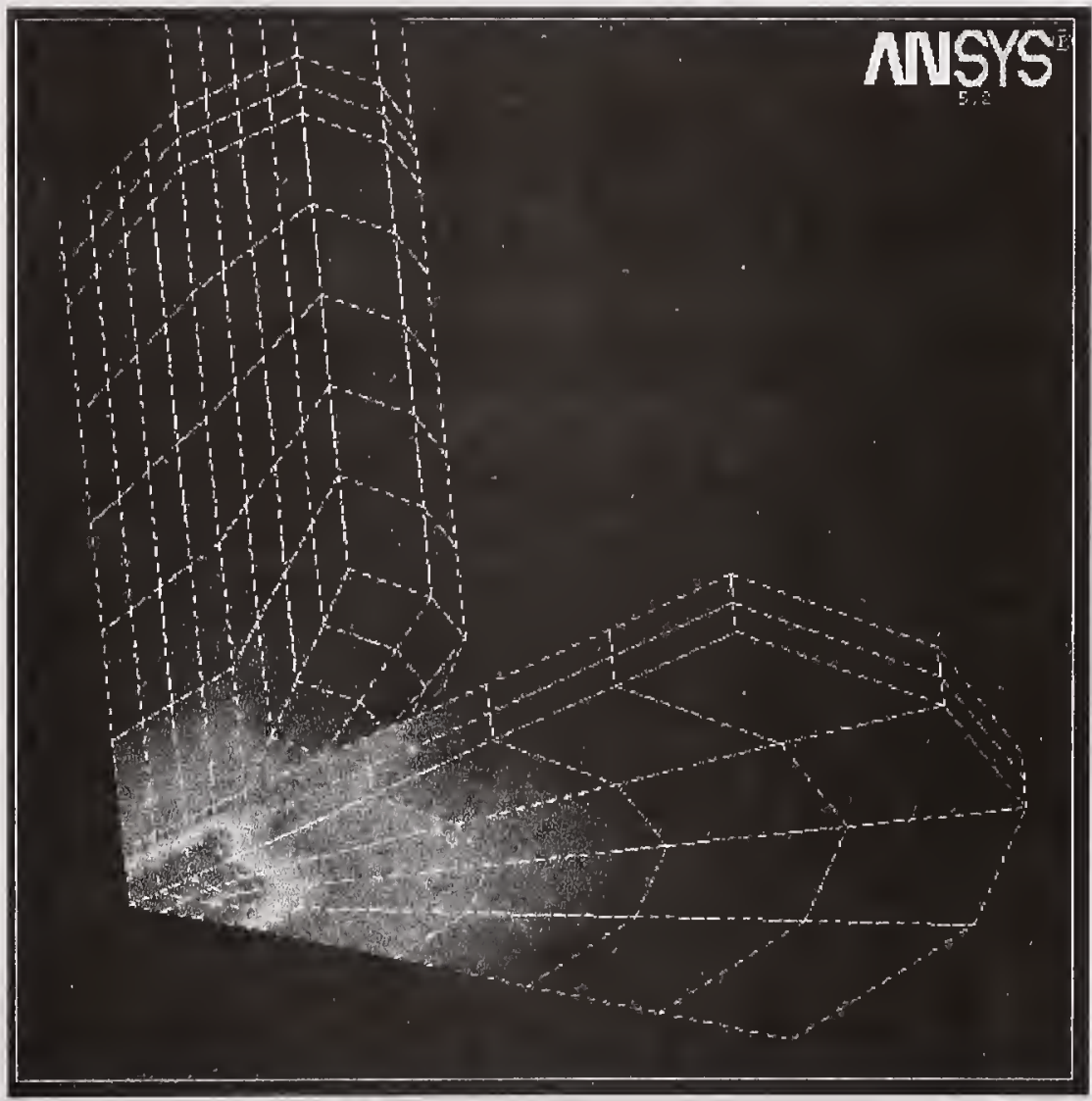

Figure 6: A three-dimensional geometrical model of resistance spot welding 


\section{MODELLING OF HEAT AFFECTED ZONE}

The new modelling area is the FEM modelling of heat-affected zone at our Department. Using the FEM modelling the critical points of heat-affected zone can be found.

Fig. 7. shows the result of temperature distribution in a given case for demonstration that, the FEM modelling can be applied for calculation of thermal cycles in the heat affected zones. From thermal cycle we can predict the structure changing in the heat-affected zones, which is the basis of mechanical properties prediction.

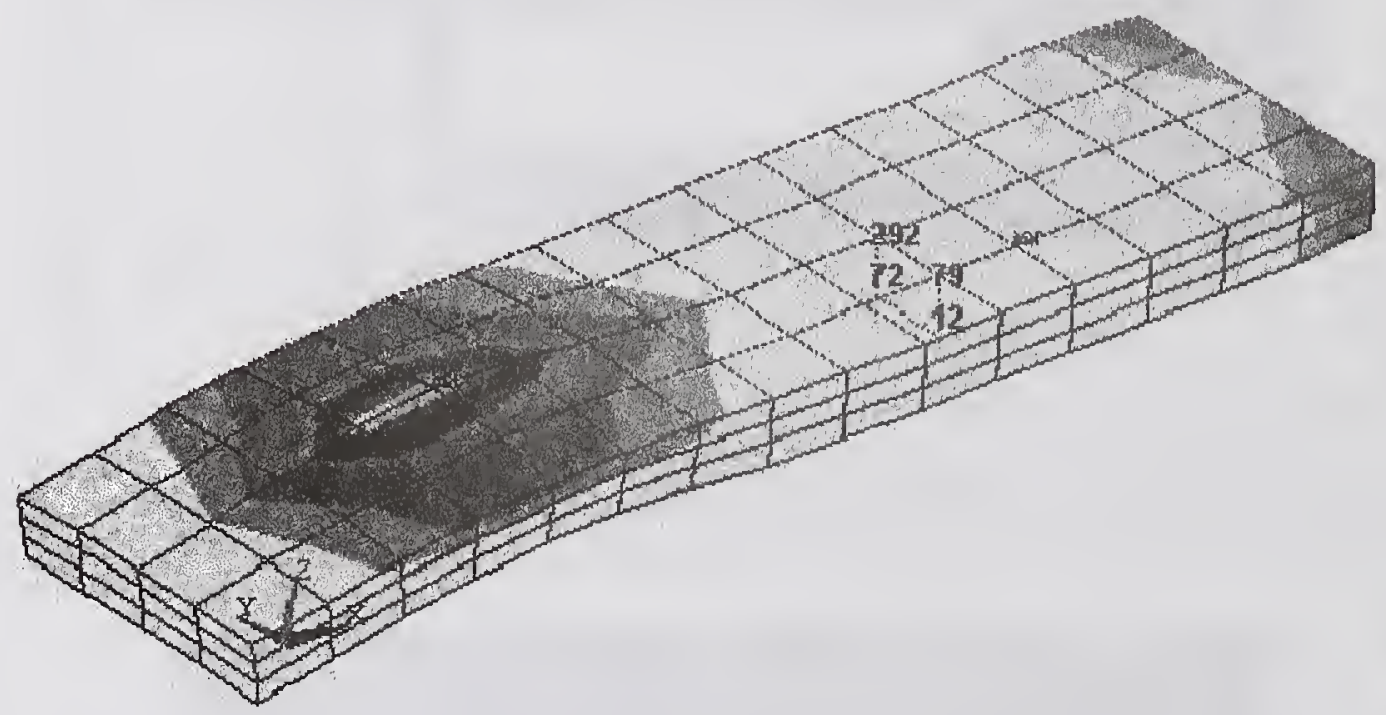

$$
\begin{aligned}
& \text { SEQV [AVG) } \\
& \text { DLX }=.045388 \\
& \text { SMN }=525.197 \\
& \sin =253620 \\
& \text { SIDXB }=492415 \\
& 525.197 \\
& 28647
\end{aligned}
$$

Figure 7: Heat affected zone modelling by FEM

\section{SUMMARY}

The Department of Materials Science and Engineering at Budapest University of Technology and Economics has experiences in the field of Finite Element Modelling of welding processes and welding phenomena. The paper shows some result of FEM modelling as cold pressure welding, resistance upset and spot welding and as heat affected zone modelling. The results of modelling can be used in the Computer Aided Process Planning of welding processes.

\section{CONCLUSION}

The Finite Element Modelling is a powerful method for modelling of processes occurring during welding as well. FEM modelling results of Materials Science and Engineering Department of BUTE proved that the pressure welding processes could be approached as cold or hot forming processes. The phenomena occurring in the heat-affected zone of welds can be also modelled by Finite Element Method, which we are working on presently. 


\section{ACKNOWLEGEMENT}

Students' work in the field of Finite Element Modelling is appreciated. Thanks a lot for work of Istvan Borhy, Dmitrij Malgin and Balazs Keskeny.

\section{REFERENCES}

1. W. Sudnik 1991. Research into fusion welding technologies based on physicalmathematical models. Schweissen und Schneiden: 43 (10): 588-s to 590-s.

2. W. A. Sudnik, W. A. Jerofejeev 1986. Computer aided welding process calculations: 100 Tula: Technical University of Tula.

3. B. Palotas, I. Borhy 1996. Mathematical modelling of welding phenomena. Welding Technology: 7 (2): 15 -s to 23 -s.

4. D. Radaj 1992. Heat Effects of Welding, Temperature Field, Residual Stress, Distortion: 348 Berlin Heidelberg: Spinger-Verlag.

5. Z. Dudas, N. Habib 1989. R \& D work. Computer Aided Welding: 261-s to 449-s Budapest: IEC, TUB.

6. B. Palotas, D. Malgin 1999. Finite Element Modelling of Resistance Upset Welding. Proc. $9^{\text {th }}$ International Conference on Computer Technology in Welding Eds. T. Siewert, and C. Pollock: 111-123. NIST Special Publication 949.

7. I. Borhy 1995. Modelling of resistance spot welding: Dt 3/1994 Budapest: TUB. (Supervisors: Gy. Krallics, B. Palotas). 

Session B4: Modeling IV 



\title{
THREE-DIMENSIONAL SIMULATION OF ROBOT PATH AND HEAT TRANSFER OF A TIG-WELDED PART WITH COMPLEX GEOMETRY
}

\author{
M. Ericsson*, P. Nylén ${ }^{* *}$, G. Bolmsjö ${ }^{* * *}$
}

\begin{abstract}
The applications of commercial software (OLP) packages for robot simulation, and programming, us interactive computer graphics, provide powerful tools for creating welding paths off-line. By the use of such software, problems of robot reach, accessibility, collision and timing can be eliminated during the planning stage. This paper describes how such software can be integrated with a numerical model that predicts temperature-time histories in the solid material. The objective of this integration is to develop a tool for the engineer where robot trajectories and process parameters can be optimized on parts with complex geometry. Such a tool would decrease the number of weld trials, increase productivity and reduce costs. Assumptions and principles behind the modeling techniques are presented together with experimental evaluation of the correlation between modeled and measured temperatures.
\end{abstract}

\section{INTRODUCTION}

The metallurgical structure of a metal, which determines its mechanical properties, is a function of its chemical composition, its initial structure and the thermal effects of the welding process. Theoretically, if both the thermal events and the response of the material to the thermal process is known, the resulting changes in microstructure and properties can be predicted. Several papers have been published concerning numerical modeling of thermal histories, residual stresses, and distortion (Ref. 1-7). Mainly two-dimensional studies have been performed. Three-dimensional studies are still restricted to simpler shapes such as plates and pipes.

The use of robots for arc welding started in the early 70's and is now extensively used in the MIG/MAG processes. Using robots for TIG (GTAW) welding is however still rare. One of the reasons is the increased demand for precise programming and control. Programming of welding robots is usually done manually by the jog teach method. Using this method the robot is off-line, the part stationary, and the robot arm jogged through the program under reduced power and at reduced speed, via a joystick. Generating a path by hand in this way can be time consuming. On a complex geometry, it is virtually impossible for a programmer to maintain constant gun velocity, distance from, and orientation to, the part. However, by using computer simulation this problem can be overcome. Using this method, the programming is moved away from the robot to a graphical computer system, often referred to as a "off-line programming" system (OLP). The technology in this area is well established and has been a research area (Ref. 8-11) for some ten years. Despite these extensive investigations, the two different simulation techniques (numerical process modeling and OLP) seems only to have been studied separately.

The need for a better simulation tool for arc welding was the starting point for a research program at the University Trollhättan/Uddevalla in collaboration with Volvo Aero Corporation. The

\footnotetext{
${ }^{*}$ University Trollhättan/Uddevalla Box 957 S-461 29 Trollhättan Sweden

${ }^{* *}$ Volvo Aero Corporation, S-461 81 Trollhättan, Sweden.

${ }^{* * *}$ Department of Production Engineering, Lund Institute of Technology, Box 118, S-221 00 Lund, Sweden
} 
objective of this program is to provide temperature -time histories and metallurgical- and mechanical-properties predictions on robot welded parts. The program is divided into four parts:

1. to off-line program parts with complex shapes,

2. to numerically predict the shape of the molten pool by the use of Computational Fluid Dynamics (CFD) techniques,

3. to numerically solve the energy equations in the solid material with sufficient accuracy that metallurgical predictions can be made, as well as to link the off-line programming model with this numerical model, and

4. to empirically establish relationships between temperature-time history and metallurgical and mechanical properties

This paper is concerned with parts 1 and 3 above; namely methods of programming robots offline and of predicting temperature-time histories on parts with complex shapes.

\section{PRINCIPLE OF OFF-LINE PROGRAMMING (OLP)}

Several commercial software packages for off-line programming of robots exist (CATIA, IGRIP, Robcad GRASP etc.). A brief description of the methodology using such systems is given below. A more detailed description is given in (Ref. 8). The methodology of OLP includes the following steps (Ref. 8):

1. modeling of the work cell,

2. modeling of the work-piece,

3. calibrating the work-cell,

4. adjusting and fine-tuning up and down loading of programs,

5. programming, and

6. test runs and macro programming enhancements

The first step to model the work cell concerns the construction of a geometric and a kinematic model of the robot, positioner etc. This demands access to design drawing of the cell together with measurements of critical dimensions in the cell. The workcell model is usually constructed directly in the OLP system. The IGRIP (Interactive Graphics Robot Instruction Program, Deneb Robotics) system was used in this study. In the second step a geometrical description (CAD data) of the part to be welded is generated either in a CAD/CAM software or in the off-line programming software (OLP). If this model is created in a CAD system the data is imported to the OLP software either using a neutral interface (for instance IGES) or a direct reader.

The accuracy of the modeled workcell is usually not high and the third step is therefor to make a calibration by measuring different points in the physical welding cell. This procedure might include several sub-steps depending on the complexity of the workcell. In this work a tool calibration and a calibration of the workpiece were performed. Tool calibration is performed to determine the tool center point and to determine the orientation of the weld torch. The procedure used in this study was to have a measuring arrow in a fixed position in the work cell and to move the robot to this position in different directions. The positions from the real robot cell were then uploaded to the OLP software and a "best fit" was performed by the system. The calibration of the workpiece was performed similarly by moving the robot to clearly identified positions on the workpiece. These positions were recorded and uploaded to the OLP software where the 
difference between model and measurements was calculated and an adjustment of the model using least squares fitting was done.

The motion of the robot is then programmed in steps four and five, either in a high level programming language (for instance GSL, which is the graphical simulation program in IGRIP) or in a specific robot language (such as RAPID, which is the program language for ABB robots). A robot trajectory is then defined by a set of coordinate frames specifying locations and gun orientation. After that, the motion may be simulated to check the results on the computer. High level languages are then translated and the program finally downloaded to the robot controller where in the final step, test runs are performed. Figure 1 shows a screen-capture during the simulation in the OLP system.

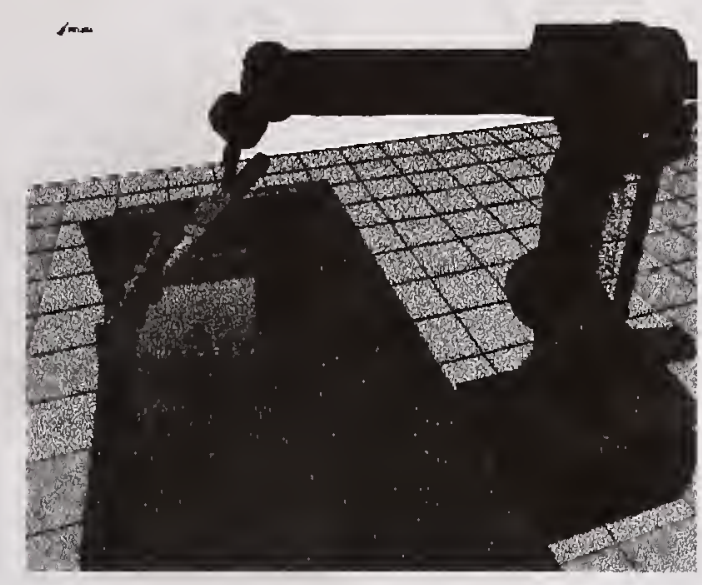

Figure1: OLP model.

\section{THE COMPUTATIONAL HEAT TRANSFER MODEL}

The primary aim of the numerical finite element model is to predict the temperature evolution outside the molten zone on a part with complex geometry. Here, the software ICEM CFD, HEXA, which is a commercial pre-processor for CFD and structural applications, was used to mesh the part. The commercial FEM program Marc from MSC Software was used in the heat transfer predictions. User subroutines had to be developed to simulate the moving heat source. A Gaussian surface distribution was used to model the heat source from the weld. This distribution was preferred to a volumetric one (Ref. 4) since it reduces the number of parameters (unknown variables) to be fit and because the plates to be welded were considered thin $(<1.5 \mathrm{~mm})$ The heat flux was expressed as (Ref. 4):

$$
\begin{aligned}
& q=q_{0} \cdot e^{-\alpha_{q} r^{2}} \\
& q_{0}=\frac{\eta E I \alpha_{q}}{\pi} \\
& q=\frac{\eta E I \alpha_{q}}{\pi} e^{-\alpha_{q} r^{2}}
\end{aligned}
$$

where $\mathrm{q}$ denotes the heat transferred to the workpiece, $\mathrm{E}$ the voltage, I the current, $\eta$ the efficiency factor, $\alpha_{q}$ the concentration factor, and $r$ the radial distance from the center of the heat 
source. The distribution was truncated in the radial direction, at a cut off limit of $5 \%$ of the maximal heat input, as proposed by D. Radaj (Ref. 4). Temperature dependent properties as well as phase change was included in the analysis. The thermal conductivity was increased by a factor 10 when the temperature reached the liquidus temperature to account for connective heat transfer in the melted zone (Ref. 4). This simplified model was used instead of more advanced CFD models to simulate the physics in the molten zone, since the latter methods are too computationally demanding. The part was divided into a non-uniform mesh with higher mesh densities close to the weld path (where the highest temperature gradients were assumed to occur), Figure 2. Eight-node brick elements were used. Six elements were defined in the thickness direction. Grid sensitivity trials were made by successively refining the mesh. The final number of nodes in the model was 181500 .

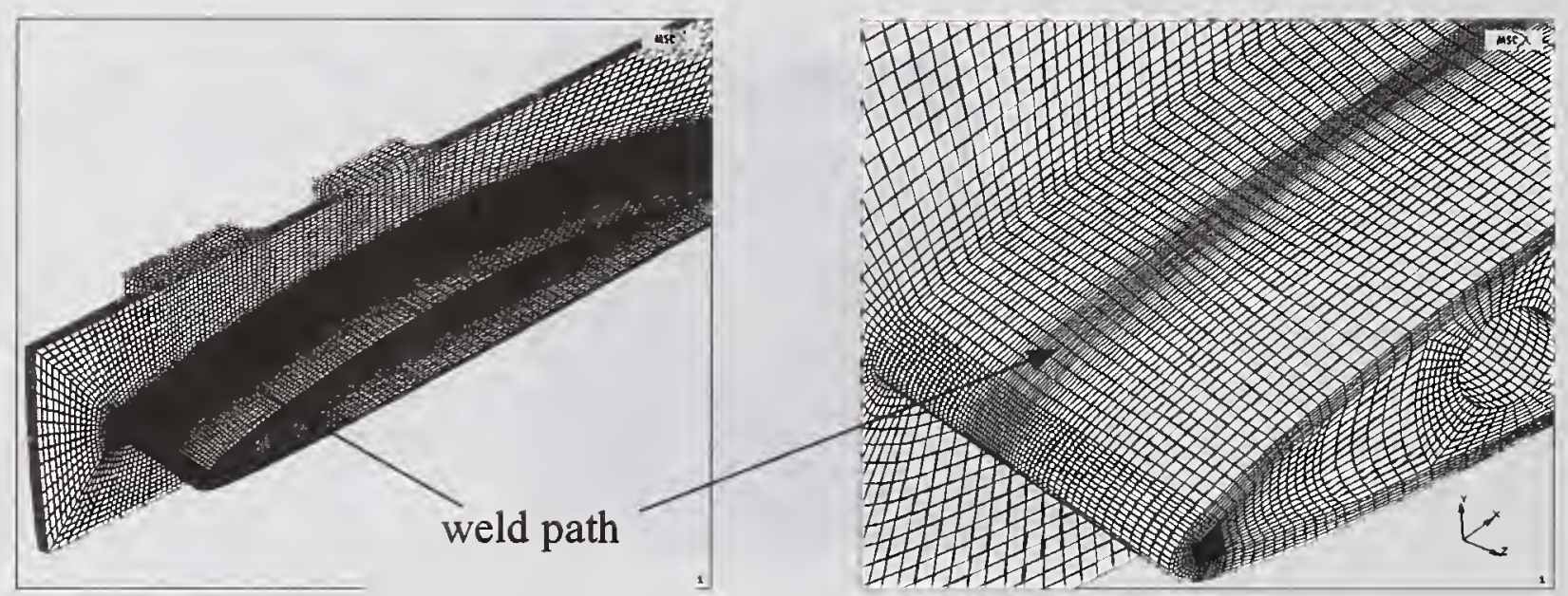

Figure 2: The non-uniform mesh. Note the higher densities along the weld path

The boundary conditions for the analysis are given in Table 1 . The model had to be restricted to a subsection of the part to be welded since otherwise to long computational times would have been required. To compensate for this simplification a "Metal-Metal" boundary condition was introduced which symbolizes pure heat conduction through these interfaces, Table 1.

Table 1: Boundary conditions in the heat transfer analysis

\begin{tabular}{|l|l|l|}
\hline Type of condition & Interface & Value \\
\hline Face film & Metal - Metal & $1000 \mathrm{E}-6$ \\
\hline Face film & Metal - Air & $20 \mathrm{E}-6$ \\
\hline Face film & Metal - Gas (Argon as root gas) & $200 \mathrm{E}-6$ \\
\hline Flux & Metal - Heat source & user subroutine ( q above) \\
\hline
\end{tabular}

The simulations were to time consuming to be run on a single workstation why parallel computing on a Linux cluster was used. By the use of the parallel computing option in Marc, the part is subdivided into several domains. For each domain, subsolutions are then calculated in parallel on different processor, and an iterative procedure assembles the subsolutions to the global solution. Ten $800 \mathrm{Mz}$ processors were used in the present study. 


\section{INTEGRATION OF THE HEAT TRANSFER AND OFF-LINE PROGRAMMING MODELS}

By the integration of the heat transfer model above with the off-line programming system IGRIP, a powerful, yet efficient tool for temperature prediction and optimization may be obtained. To that end, an interface translating the data; robot coordinates, welding speeds and currents between the two softwares was developed. This interface calculates a linear motion between each robot point, which controls the moving heat source in the finite element calculation. The two softwares (IGRIP and Marc) have to be installed on the same workstation since communication between different operating systems has not been considered. The overall architecture of the simulation system is given in Figure 3.

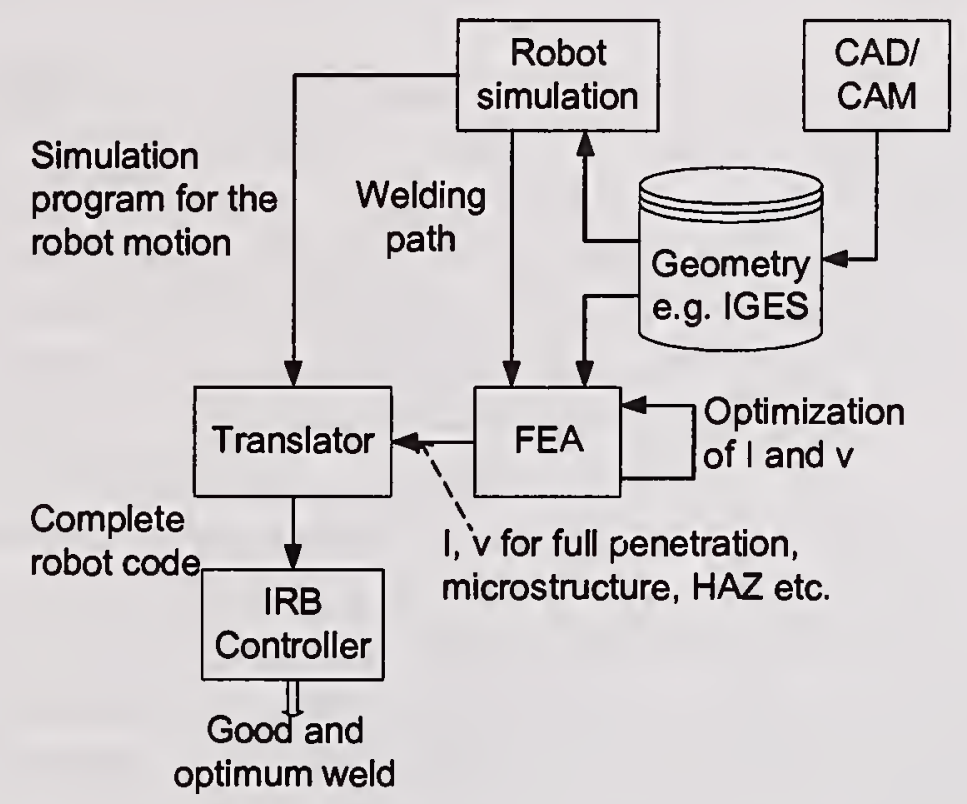

Figure 3: The overall architecture of the simulation system.

\section{EXPERIMENTAL}

TIG (GTAW) welding was performed using an in-house robotised welding cell. The torch used is from Binzel $A B$ and is linked to a six-axis robot from ABB, IRB1400. The power source is a TIG Commander $400 \mathrm{AC} / \mathrm{DC}$ from Migatronic AB. Throughout all experiments, thoriated tungsten electrodes were used. Welding experiments were performed on both plane plates and on a section of an aerospace part (in the following referred to as the production part), namely a turbine component from the V2500 engine provided from Volvo Aero Corporation. 1/13 was cut out of the real part, which originally consisted of an inner, an outer ring and 13 vanes, see Figure 4 . The reasons for the experiments on the plane plates were twofold: to calibrate the heat source parameters used in the simulations, and to determine the emissivity in the infrared temperature measurements. Both the plane plates and the production part were made of Greek Ascoloy with a thickness of $1.25 \mathrm{~mm}$. To avoid oxidation, argon was used as root gas in all weld trials. The types of welds performed were bead on plates, and no filler material was used. All plates were tacwelded together before performing experiments so that no gap or misalignments could be introduced. Two special fixtures were designed, one for the plane plates and one for the production part, Figure 4. 

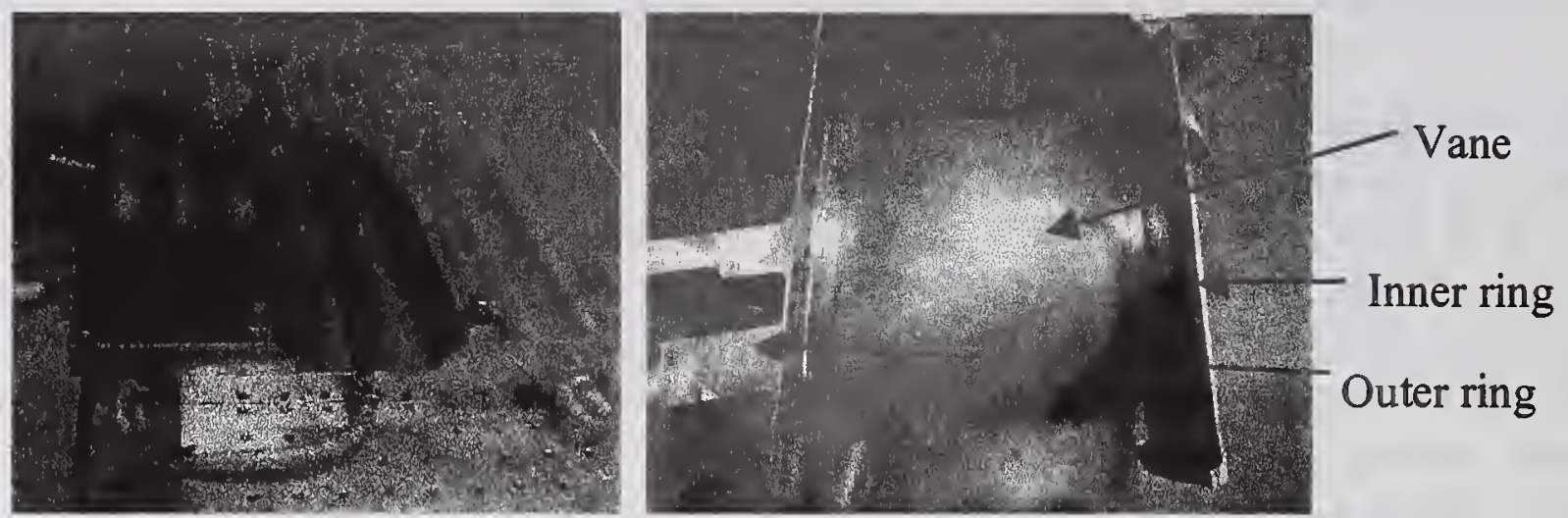

Figure 4: Fixture for the plan plates (left) and fixture with component (right).

To obtain temperature measurements, both thermocouples and high-resolution infrared (IR) emission measurements were used. Six thermocouples were positioned perpendicular to the welding direction. The first gauge was positioned as close to the melted zone as possible at a distance of $4 \mathrm{~mm}$ from the center of the weld. The second and third were positioned $0.5 \mathrm{~mm}$ radially from the previous one. The remaining gauges were positioned $1.0 \mathrm{~mm}$ radially from the preview one. The sampling frequency for all thermocouples was $270 \mathrm{~Hz}$. The IR-camera used is a VARIOSCAN High Resolution, from JENOPTIK, Laser, Optik, Systeme Gmbh, which works in the IR radiation spectrum of $8-12 \mu \mathrm{m}$. The camera was used both in a line scan mode with a scanning frequency of $270 \mathrm{~Hz}$ as well as in a full-frame mode with a frequency of $1 \mathrm{~Hz}$. The analysis of the IR measurements were made using the IRBIS Plus software provided by JENOPTIK. A comparison between the IR results with the thermocouple was made. Different techniques to soot the plane plates were evaluated to reduce emissivity -dependency in the IRmeasurements. Finally a method using an acetylene flame was selected. This technique was also used on the production part.

\section{RESULTS AND DISCUSSION}

The results of the robot programs made off-line showed a high accuracy and very little finetuning after calibrations had to be made. The method appears as a powerful tool, particularly in small batch production such as within the aerospace industry.

The computational time from the parallel calculation was $34 \mathrm{hrs}$ for the production part. The predicted fusion zone was $5.0 \mathrm{~mm}$ on the top-side and $4.8 \mathrm{~mm}$ at the root-side which agreed well with measured widths.

The predicted and IR-measured temperature histories in the point located $4 \mathrm{~mm}$ and $7 \mathrm{~mm}$ from the center of the weld are given in Figure 5. The IR-measurements were performed twice, corresponding to the captions IR1 and IR2 in the Figure 5. There is an excellent agreement between predictions and measurements for the $4.0 \mathrm{~mm}$ case both in peak temperature and in the cooling history. The agreement for the $7.0 \mathrm{~mm}$ case is not as good as for the $4 \mathrm{~mm}$ case but still good. An example of the comparison between the thermocouple and the IR-measurements are given in Figure 6. There is a very good agreement, which implies that that the technique to soot worked well. The reason why data is lacking in the IR-curve is that the camera-system collects data during a maximum time interval of 20 seconds. This data then have to be written to disk before a new sampling sequence can be gathered. A more extensive evaluation between thermocouple and IR measurements is planned. 

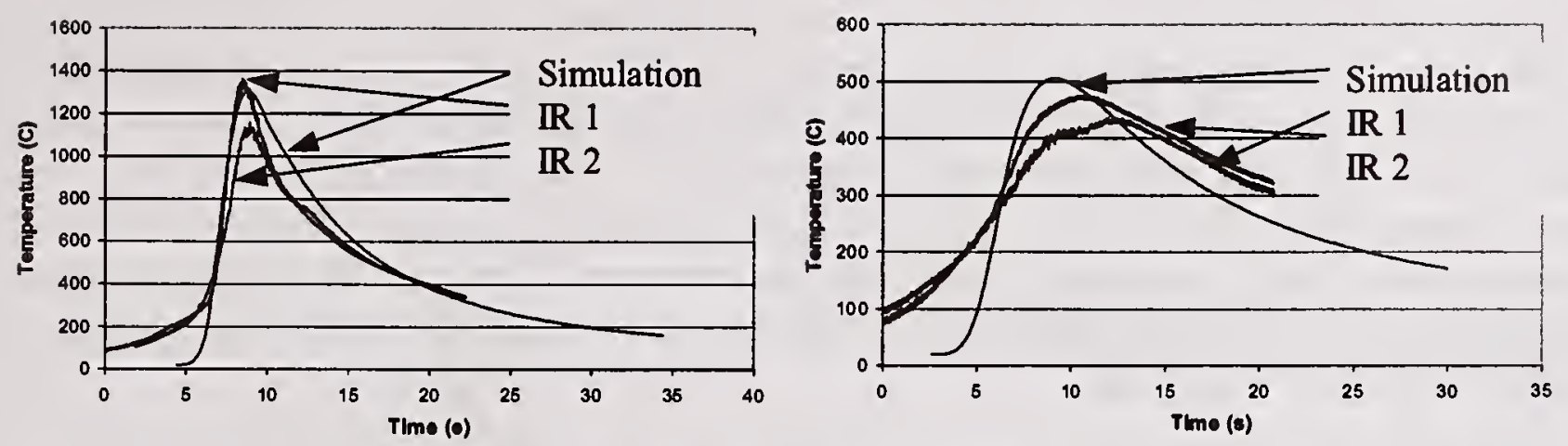

Figure 5: Predicted and measured temperature-time histories, $4 \mathrm{~mm}$ (left) and $7 \mathrm{~mm}$ (right) from the weld centerline.

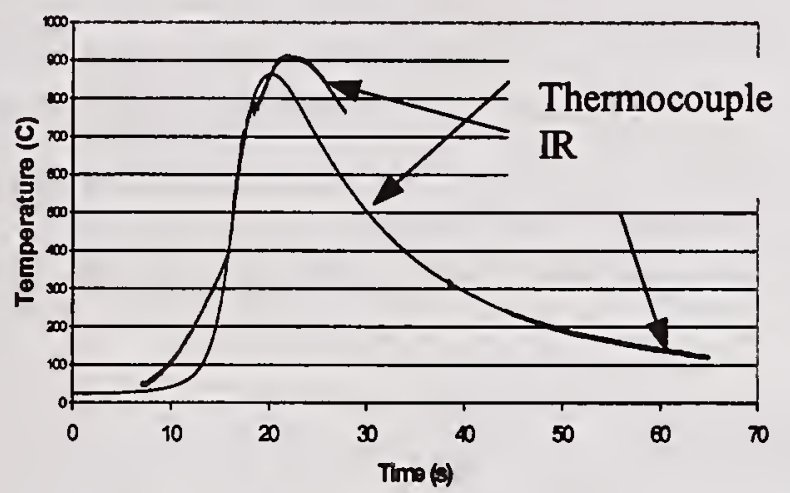

Figure 6: Comparison between thermocouple and IR measured temperatures.

The overall conclusion from the simulations is that the model predicts the thermal cycle very well. However, further research is needed until welded structures can be optimized without experiments. The temperature predictions are naturally dependent on the heat transfer coefficients (boundary conditions in Table 1). To determine these values, experiments are required since the heat flow can be convection dependent, specifically if the workpiece and fixturing are small. Also, the heat source parameters (in the expression for $q$ above) have to be calibrated by experiments. The on-going work at the laboratory at University Trollhättan/Uddevalla to numerically model the magnetohydrodynamics of the arc and to predict the shape of the molten pool by the use of Computational Fluid Dynamics (CFD) techniques seems as a promising tool to compute the heat source parameters without experiments. Such a model will establish a direct relationship between the welding current, speed, voltage and the shape of the molten zone, which can be used as a boundary condition in the temperature predictions in the solid region. Such model will also increase the knowledge of the stirring of the weld pool, the weld pool surface shape and the physics of the arc.

Several extensions of the modeling work described in this article are possible. The simulations can be extended to compute residual stresses, distortion and in the longer term to predict fracture strength and fatigue life of a structure. To extend the modeling work to include filler wire and pulsed current would also be valuable.

\section{SUMMARY AND CONCLUSIONS}

An engineering method and a simulation tool to define robot trajectories and to predict thermal histories on parts with complex geometries have been developed. The method was evaluated on a part with a complex shape where robot weld paths were defined off-line, and automatically 
downloaded to a FEM-model where transient temperatures were predicted. These predictions were compared with experimental measurements using both thermocouple and infrared emission measurements and good agreement was found. The described method provides a promising means to construct and optimize torch trajectories and process parameters off-line. Using this system, thermal histories can be predicted on complex shaped parts and thereby resulting changes in microstructure and mechanical properties be estimated. The models used may after futher development enable the optimization of welding processes, thus increasing productivity and reducing the need of weld trials.

\section{ACKNOWLEDGEMENT}

The authors wish to acknowledge the guidance in the temperature measurements of Per Henrikson (Volvo Aero Corporation), and the assistance in the laboratory by Xavier Guterbaum (University of Trollhättan/Uddevalla) and Börje Nordin (Volvo Aero Corporation). Appreciation is expressed to Peter Jonsson of Volvo Aero Corporation for providing samples for this research and to Anita Hansbo of University of Trollhättan/Uddevalla for linguistic revision. The work was funded by the Foundation for Knowledge and Competence Development and EC Structural Founds.

\section{REFERENCES}

1. Eagar T. W., Tsai, N. S. 1983. Temperature Fields Produced by Traveling Distributed Heat Sources. American Welding Society Journal 62(12) 346-s to 355-s.

2. Gu, M.; Goldak, J.; Hughes, E. 1993. Steady state thermal analysis of welds with filler metal addition. Canadian Metallurgical Quarterlv. 32 (1): 49-s to 55-s.

3. Kou, S.; Le Y. 1983. Three-dimensional heat flow and solidification during Autogenous GTA Welding of Aluminum Plates. Metallurgical Transactions A. 14A: 2245-s to 2253-s.

4. Radej, D. 1992 Heat Effects of Welding: Berlin: Springer Verlag.

5. Goldak, J.; McDill, M.; Oddy, A.; House, R.; Chi, M.; Bibby, M. 1987. Computational Heat Transfer for Weld Mechanics. Proc. of Int. Conf. on Trends in Welding Research, Advances in Welding Science and Technology. Eds S. A. David: 15-20. Metals Park ASM Int.

6. Jonsson, M.; Karlsson, L; Lindgren, L.E. 1985. Deformation and Stresses in Butt Welding of Large Plates with Special References to the Material Properties, J. of Eng. Mat. And Tech. 107: 265 -s to 270 -s.

7. Lindgren, L.E.; Karlsson, L. 1988. Deformation and Stresses in welding of Shell Structures. Int. J. for Numerical Methods in Eng. 25: 635-s to 655-s.

8. Bolmsjö, G.; Olsson, M.; Brink, K. 1997. Off- line programming of GMAW robotic systems - a case study. Int. J. for the Joining of Materials, 9 (3): 86-s to 93-s.

9. Buchal, R.O.; Cheras, D.B.; Sassani, F.; Duncan J.P. 1989. Simulated Off-Line Programming of Welding Robots. Int. J. of Robotics Research 8 (3): 31-s to 43-s.

10. Bolmsjö, G. 1999. Programming robot welding system using advanced simulation tools. Proc. of the International Conf. on the Joining of Materials JOM-9, 284-291. May 16-19, 1999, Helsingør, Denmark.

11. Walter S. 1994. Simulation and Calibration for Off-line Programming of Industrial Robots. Proc. of Computer Technology in Welding: Paper 54. Paris 15-16 June. 


\title{
DYNAMIC MODELING OF GTAW FOR RAPID MANUFACTURING
}

\author{
Z. Jandric, I. S. Kmecko, R. Kovacevic*
}

\begin{abstract}
A new parameter referred to as the geometrical factor was defined to reflect on the geometrical configuration around the molten pool, and an expression for heat conduction dissipation through the workpiece was established. The weld bead width, height, depth of penetration, and the weld bead cross-sectional area, were experimentally determined for different combinations of the welding currents, workpiece velocity, the filler metal flow rates, and for different geometrical configurations of the weald bead. The open-loop controller for the gas tungsten arc welding (GTAW) process based on the developed model was designed and experimentally tested by controlling the metal layer deposition process on top of a workpiece with complex geometrical features, such as conformal channels, edges, and corners.
\end{abstract}

\section{INTRODUCTION}

Rapid prototyping (RP) is the most common name given to a host of related technologies that are used to fabricate physical objects directly from CAD data sources. These methods are unique in that they add and bond material in layers to form objects. Such systems are also generally known as Freeform Fabrication (FFF), solid freeform fabrication (SFF) and Layered Manufacturing. The major advantages of the RP technologies are that:

- Objects can be formed with any geometric complexity or intricacy without the need for an elaborate machine setup or final assembly;

- Rapid prototyping systems reduce the construction of complex objects to a manageable, straightforward, and relatively fast process.

The materials used in rapid prototyping are limited and dependent on the production method chosen. Non-metal materials such as plastics, ceramics, waxes, and papers were almost exclusively used for many years. Parts made of these materials can not be used to test if the design of the parts meets the mechanical property requirements. Deposition by welding (DBW) enables the direct production of the metallic parts. The use of welding for creating free-standing shapes was first used in Germany in the 1960's (Ref. 1). Other work in this area has been undertaken by Babcock and Wilcox Co. who were working mainly on large components produced in an austenitic material. Also, work by Rolls Royce has centered on investigating the technique as a means of reducing the waste levels of expensive high performance alloys that can occur in conventional processing. Lately, work on $3 \mathrm{D}$ welding has also been in progress at the University of Nottingham, the United Kingdom and Southern Methodist University, Dallas, Texas.

Research Center for Advanced Manufacturing Southern Methodist University, 1500 International Pkwy. Suite \#100, Richardson, TX 75081

*Corresponding author - tel.: 214-768-4865, Email: kovacevi@engr.smu.edu 
Gas tungsten arc welding has many properties that makes it very attractive for the DBW such as: the wire feeding rate does not depend on the welding current; it is possible to completely stop the deposition process while keeping the arc on; the wire can be fed directly to the molten pool to let the surface tension to evenly spread the liquid metal; and the presence of the previously deposited bead will not affect the stability of the arc, etc. To make GTAW even more suitable for DBW, a constant heat input and cooling rate should be maintained during the deposition process in order to achieve a homogeneous crystallization and transformation structure throughout the whole workpiece mass, and to obtain the uniform geometry of weld beads across the deposited layer.

As mentioned, a major advantage of $\mathrm{RP}$ is the building of the $3 \mathrm{D}$ part with the complex geometrical features. Depositing layers above the complex geometrical features directly affect the heat transfer conditions, since the surrounding mass of the material is rapidly changed with the X-Y coordinates. Though, when the weld bead is deposited near the edges or above the channels, any excessive heat input will melt the underlying geometry together with a filler metal - the shape of the 3D part will be destroyed. It is not possible to successfully use the same heat input for depositing the weld bead over the changing volume of substrate. Clearly, heat input is strongly correlated to the geometry of the part (Ref. 2). In order to keep a uniform cooling rate, and subsequently, the uniform mechanical properties of the welded substrate, the heat input must be adjusted for each instance according to the mass of the surrounding material. A new parameter referred to as the geometrical factor $\left(\xi_{\mathrm{G}}\right)$ was designed to reflect on the geometrical configuration around the molten pool. Since the geometry of the built part is known, the geometrical factor can be calculated in advance and that represents the base for the open-loop controller.

To verify the correlation between the geometry of the part and the weld-bead width, height, depth of penetration for different combinations of the welding currents, welding speed, and the filler metal feeding speed, the number of experiments were designed and performed. An accurate dynamic model and an expression for the heat conduction dissipation through the workpiece were established. A number of researchers (Ref. 3-8) modeled the welding process without taking in consideration the geometry of the substrate. In this paper, an expression is presented that balances the mass and energy flow through the controlled volume set around the molten pool including the net heat input, filler metal feeding speed, welding speed, and geometrical factor. The open-loop controller based on this model is experimentally tested, by controlling the metal layer deposition process on top of the workpiece with complex geometry.

\section{EXPERIMENTAL SET-UP}

Figure 1 shows the experimental set-up. A torch is equipped with a step motor (1) in order to solve the problem of omnidirectionality, the filler metal has to be fed in front of the torch. The supply of the filler metal is provided by the step motor (2). The PC based data acquisition system controls the power source, wire-feeding speed, and orientation of the wire feed mechanism.

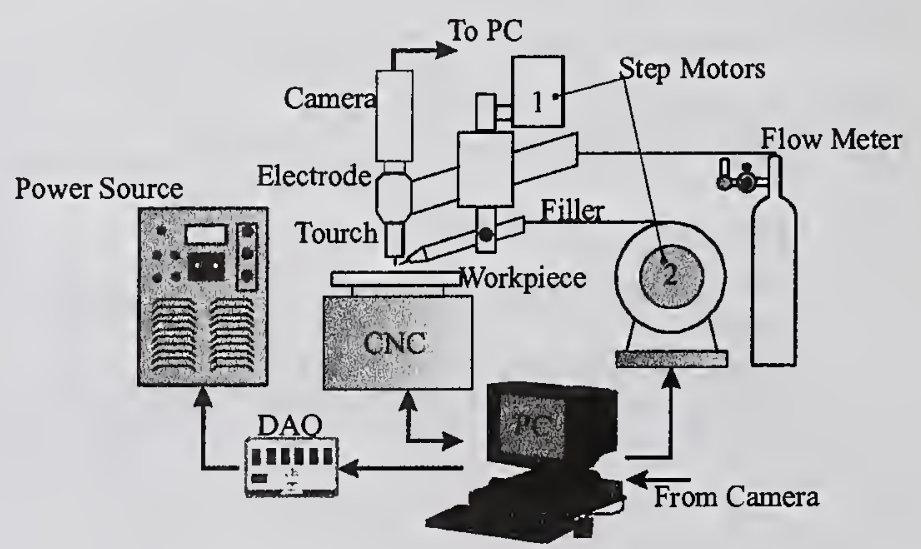

Fig. 1 Experimental Set-up 
Communication of the central controller and the CNC machine is established so that the movement of the $\mathrm{CNC}$ machine table and the orientation of the wire feeding mechanism are synchronized. A high resolution CCD color camera is integrated coaxially with the torch.

\section{EXPERIMENTAL PROCEDURE}

To determine the correlation between the weld-bead geometry and the welding current, the welding speed, and the filler metal feeding speed, for different geometrical configurations, a number of experiments are performed. Weld bead geometry is defined by the bead width $\left(w_{b}\right)$, the depth of penetration $\left(d_{w}\right)$, the height of buildup $\left(b_{b}\right)$, and by the cross-sectional area of the bead (A). Additionally, the cross-section area of the bead can be divided into two areas: the above-surface $\left(\mathrm{A}_{\mathrm{b}}\right)$ and below-surface $\left(\mathrm{A}_{\mathrm{w}}\right)$ areas, where the surface denotes the top plane of the workpiece before welding. Two sets of experiments are designed. The first set (Fig. 2) correlates the weld-bead geometry with the welding current and the filler metal feeding speed where the surrounding mass of the material varies between $12.5 \%$ and $100 \%$ of the maximum possible one. Experiment $I$ is done for nine combinations of the welding current $(80,120$ and $160 \mathrm{~A})$ and the filler metal feeding speed $(40,80$ and $120 \mathrm{~cm} / \mathrm{min})$. The second experiment (Fig. 3 ) is designed to correlate the weld-bead geometry with the welding speed, the welding current, and the filler metal feeding speed. Nine experiments are performed too (combinations of 100 , 120 and $160 \mathrm{~A} ; 40,80,120 \mathrm{~cm} / \mathrm{sec}$ ). In both cases during a single experiment, the filler metal feeding speed and the current are kept constant. While in the second experiment, the welding speed was increased.

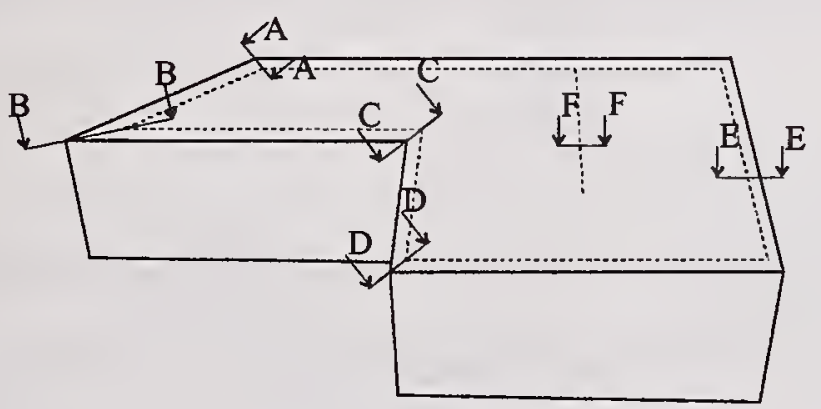

Fig. 2 Experiment I

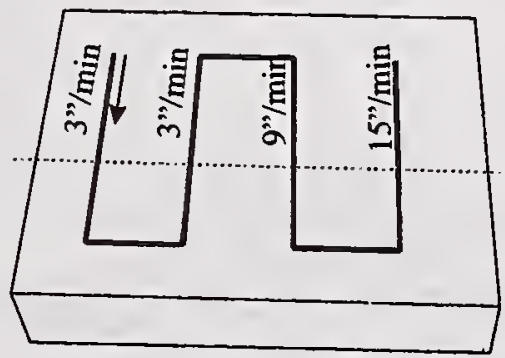

Fig. 3 Experiment II

\section{EXPERIMENTAL RESULTS}

After welding, each sample was cross-sectioned using an abrasive cut-off wheel, polished to a 1$\mu \mathrm{m}$ finish, and etched in a $2 \%-N i t a l$ solution. The workpiece from the first experiment is cut through the A-A, B-B, C-C, D-D, E-E and F-F cross-sections (Fig. 2). The cross-sections for the second experiment were done along the dotted line in Fig. 3. A few images of the cross-sections from the first experiment for the wire feeding speed of $40 \mathrm{~cm} / \mathrm{min}$ and for welding current of 80 , 120 and $160 \mathrm{~A}$, are shown in the Table 1. The individual cross-sectional areas of the melted substrate and deposited filler metal were then measured. Figures $4 a, 4 b$, and $4 c$ present the mean values of the bead widths, depths of the penetration and the height of bead build-ups as the function of the geometry. Experimental data are approximated with linear functions. It is interesting to note that the underlying geometry basically has no influence on the height of the build-up. A comparison of the maximum and minimum values from the Figs. $4 \mathrm{a}$ and $4 \mathrm{~b}$ show that the impact of the substrate geometry on the DBW can not be neglected. Further, some 
cross-sections of the second experiment are presented in the Table 5. Although the experimental data fluctuates, it is easy to conclude from the data presented in the Table 5 that the workpiece velocity has a great influence on the bead parameters. In the summary, experimental results showed that the 'geometrical factor' has to be included in the modeling of the rapid prototyping process based on the welding.

Table 1. Cross-sections of the bead for the different geometrical factors

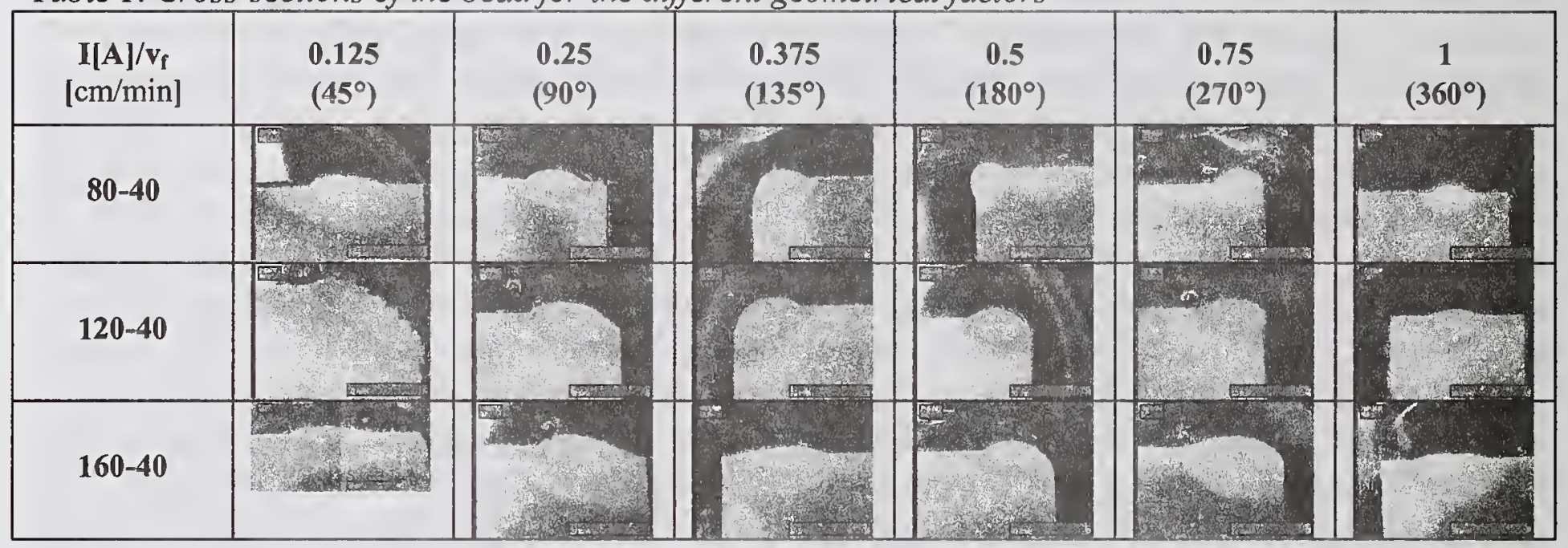

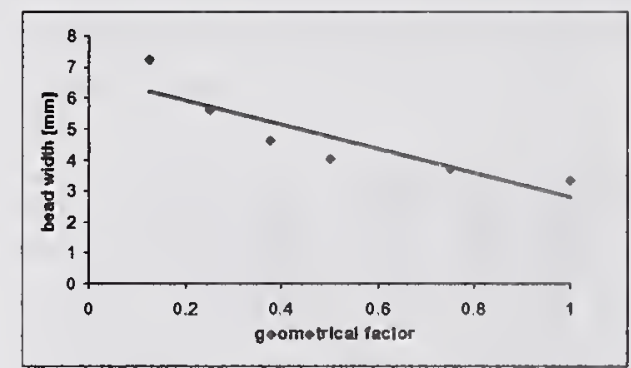

a) bead width

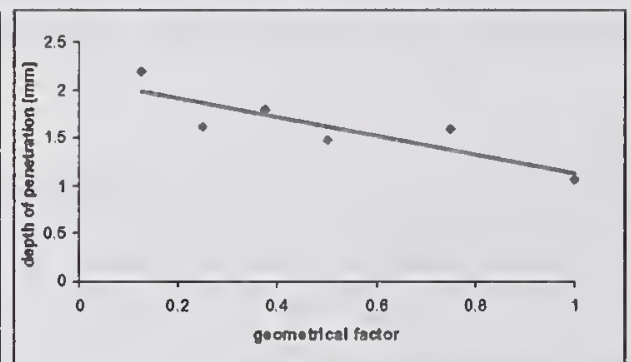

b) depth of penetration

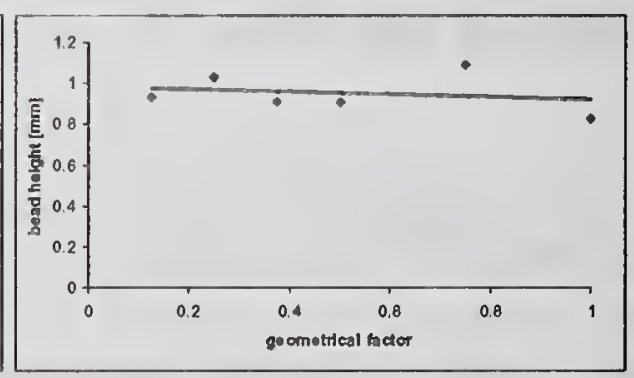

c) bead build-up

Diagrams $4 a, b$ and $c$-influence of the geometry on the bead

Table 5a. Cross-sections of the bead for the second experiment

\begin{tabular}{|c|c|c|c|c|}
\hline \multirow{2}{*}{$\begin{array}{c}\text { workpiece } \\
\text { velocity }\end{array}$} & \multicolumn{3}{|c|}{$\mathrm{I}[\mathrm{A}] / \mathrm{v}_{\mathrm{f}}[\mathrm{cm} / \mathrm{min}]$} \\
\hline & $100 / 40$ & $100 / 80$ & $100 / 120$ & \\
\hline \\
\cline { 2 - 5 }
\end{tabular}




\section{MATHEMATICAL MODEL}

An accurate dynamic model that provides guidance for sensing and corrective actions is needed for on-line adaptive control. The model provides a quantitative analysis of the thermal behavior of the welding process and aids in a fundamental understanding of the influence of the welding current, the filler metal flow rate, the workpiece velocity, and the geometrical factor. An expression that will combine the net heat input, the filler metal feeding speed, the traverse speed, and the heat conduction rate may be obtained by balancing the mass and energy flow through the control volume set around the molten pool as shown in Fig. 5.

Segment A-A of the controlled volume is positioned through the largest cross-sectional area of the liquid metal in the molten pool. By balancing the mass flow through the controlled volume, the maximum flow rate of the liquid metal

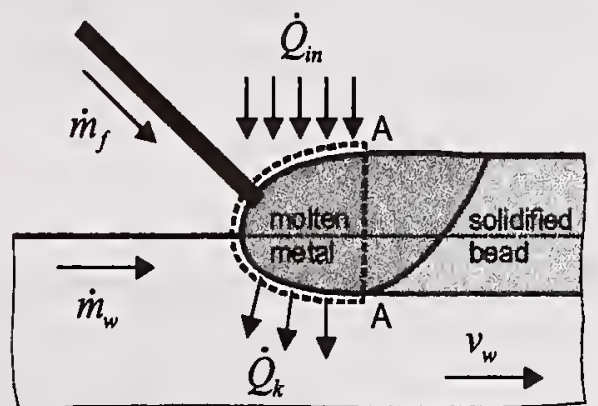

Fig. 5 Control volume may be calculated as the sum of the filler metal mass flow rate, $\dot{m}_{f}$, and the maximum molten substrate flow rate, $\dot{m}_{w}$. Then, the balance of energy at steady state is:

$$
\dot{m}_{f} h_{0}+\dot{m}_{w} h_{0}+\dot{Q}_{i n}=\left(\dot{m}_{f}+\dot{m}_{w}\right) h_{l i q}+\dot{Q}_{k}
$$

where the $h_{0}$ is the specific enthalpy of the filler metal, and the substrate metal entering the controlled volume, $h_{\text {liq }}$ is the specific enthalpy of the liquid metal leaving the control volume, $\dot{Q}_{i n}$ and $\dot{Q}_{k}$ is the net heat input and the conduction heat flow rate through the substrate. The balance (1) can be rewritten as:

$$
\dot{Q}_{i n}=\dot{Q}_{f}+\dot{Q}_{w}+\dot{Q}_{k}
$$

The net heat input $\dot{Q}_{\text {in }}$ is used for melting filler metal, $\dot{Q}_{f}$, for melting the substrate material, $\dot{Q}_{w}$, and for the heat dissipated by conduction, $\dot{Q}_{k}$. The heat used for heating and melting the filler metal is calculated as:

$$
\dot{Q}_{f}=\dot{m}_{f}\left(h_{l i q}-h_{0}\right)=\frac{d_{f}^{2} \pi}{4} v_{f}\left(\bar{h}_{l i q}-\bar{h}_{0}\right)
$$

where $d_{\mathrm{f}}$ is the filler-wire diameter, $\mathrm{v}_{\mathrm{f}}$ is the filler-wire velocity and $\bar{h}_{\text {liq }}-\bar{h}_{0}=12 \cdot 10^{9} \mathrm{~J} / \mathrm{m}^{3}$ for carbon steel. The heat-flow rate used to heat and melts the substrate material, $\dot{Q}_{w}$, is:

$$
\dot{Q}_{w}=\dot{m}_{w}\left(h_{l i q}-h_{0}\right)=\dot{v}_{w}\left(\bar{h}_{l i q}-\bar{h}_{0}\right)
$$

where $\dot{\nu}_{w}$ is the volumetric flow rate of the molten substrate metal. The heat conduction rate $\dot{Q}_{k}$ is calculated as (Ref. 10):

$$
\dot{Q}_{k}=2.217 \cdot \xi_{G} \cdot \frac{\alpha^{2}}{v_{w}}\left(\bar{h}_{l i q}-\bar{h}_{0}\right) \cdot\left(\frac{w_{b} \cdot v_{w}}{2 \alpha}\right)^{0.9666}
$$


where $\xi_{\mathrm{G}}$ is the geometrical factor, $\alpha$ is the temperature diffusivity, and $\mathrm{w}_{\mathrm{b}}$ is the bead width. The net heat input is used to calculate the welding current, I:

$$
I=\frac{1}{2 \cdot b_{1}}\left(\sqrt{b_{0}^{2}+\frac{4}{\eta_{\text {arc }}} b_{1} \dot{Q}_{\text {in }}}-b_{0}\right)
$$

where $b_{0}=7.952$ and $b_{1}=0.02117$ are non-dimensional experimentally obtained constants (Ref. 10 ), and $\eta_{\text {arc }}=0.48 \%$ is the arc efficiency for GTAW of mild steel.

\section{GEOMETRICAL MODEL}

The calculation of the influence of the geometry on the DBW is achieved in the four steps:

1. creating solid model

2. creating $3 \mathrm{D}$ matrix

3. calculation of a geometrical factor

4. generation of the 2D matrix for each layer to be deposited.

The first step is designing the 3D solid model (Fig. 6) by computer aided design (CAD). The 3D solid part should have all the details - same as a wanted part (Fig. 7), so that the model is as realistic as possible.

The second step creates the 3D matrix that consists only of the 'ones' and the 'zeros' (Fig. 8). 'Ones' mark the point where the material is supposed to be deposited, and 'zeros' mark the point where the material should be removed or does not exist. The $3 \mathrm{D}$ matrix is determined by its resolution defined as the number of the points ' $n$ ' within the unit length ' 1 '.

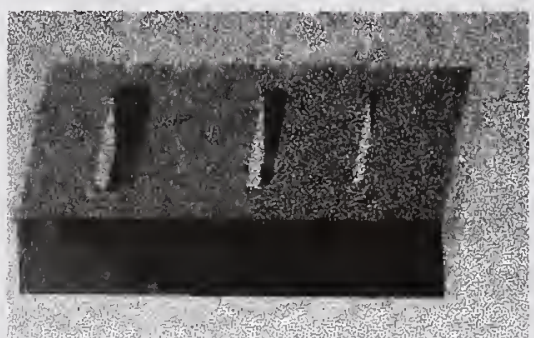

Fig. 6 3D Solid model

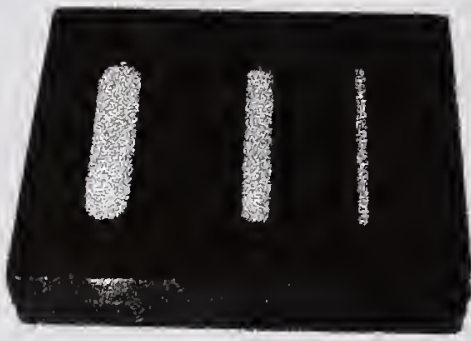

Fig. 7 Built 3D part

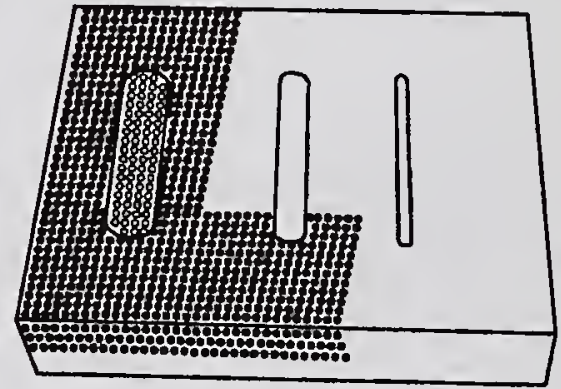

Fig. 8 Part as a matrix of 'ones' -filled circles and 'zeros' - empty circles

The third step is computationally most demanding. For each point in a 3D matrix geometrical factor, $\xi_{G}$, is calculated. The calculation of the geometrical factor is based on the fact that the heat affected zone, when it is welded on a semi-infinity solid is theoretically half of the sphere (Ref. 8). The size of the radius (R) of the half-sphere is experimentally obtained. The center of the sphere is the point for which the geometrical factor is to be calculated with the coordinates $\left.x_{c}, y_{c}, z_{c}\right)$. Then, all the points in the range $\left(x_{c} \pm R ; y_{c} \pm R ; z_{c}-R\right)$ are checked for their distance $(d)$ from the half-sphere center according to: 


$$
\begin{aligned}
& d=\sqrt{\left(x_{C}-x\right)^{2}+\left(y_{C}-y\right)^{2}+\left(z_{C}-z\right)^{2}} \\
& d \leq R
\end{aligned}
$$

where, $x, y$ and $z$ are the coordinates of an arbitrary point. All the points that satisfy $d \leq R$ are counted, where counter is denoted by ' $V_{\max }$ '. In addition, all points with value ' 1 ' that satisfy $\mathrm{d} \leq \mathrm{R}$ are counted with the counter denoted as ${ }^{\prime} V$. Now, the geometrical factor can be defined as:

$$
\xi_{G}=\frac{V}{V_{\max }}
$$

where, the geometrical factor is the ratio of the volumes, where ' $V_{\max }$ ' is constant and depends on the predetermined radius of the half-sphere, and ' $\mathrm{V}$ ' is the amount of the material that exists in the observed half-sphere. Here, the volume is digitized; and so, the model error is inherited. But it should be noticed that this is an off-line heat input planning model that allows us to increase the matrix resolution up to the virtually any value, if it is concluded that the model is not precise enough.

The fourth step extracts all geometrical factors from the 3D matrix with the same $z^{*}$ coordinate in a unique $2 \mathrm{D}$ matrix that will be used when the layer with a $z^{*}$ distance from base-plate is to be deposited. As an example a few diagrams with the calculated values of the geometrical factors along the directions A-A, B-B and C-C (from Fig. 9) are shown in the Figs. 10a, 10b and $10 \mathrm{c}$, respectively. These diagrams are obtained for
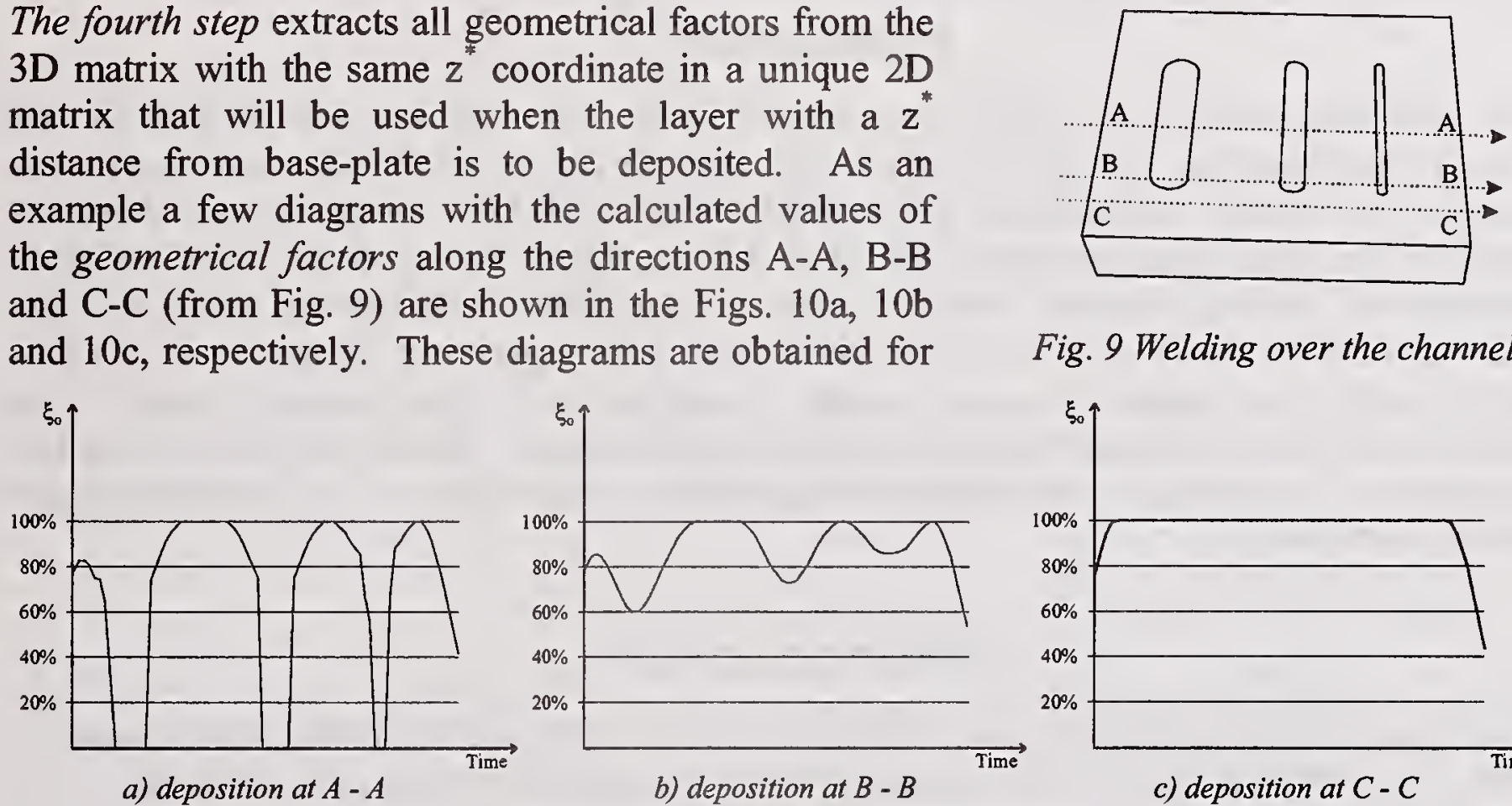

b) deposition at $B-B$

Fig. 9 Welding over the channels

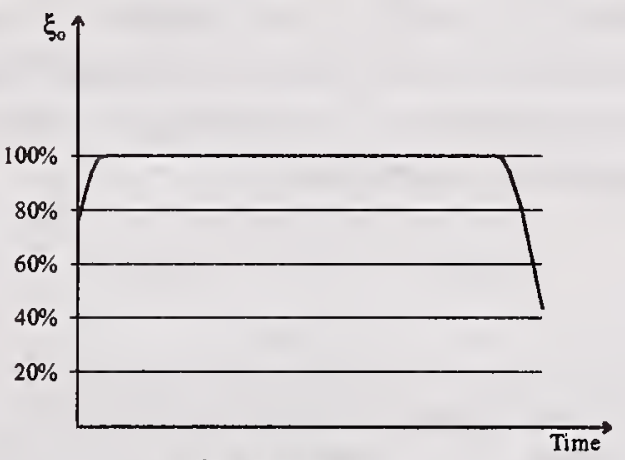

c) deposition at $C-C$

Fig. 10 Calculated geometrical factor

the top layer, where the bottom of the channels is sufficiently remote and doesn't have any influence on the geometrical factor. Results of the welding can be observed in the Fig. 11.

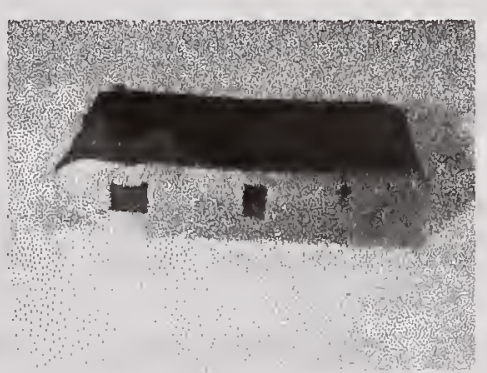

Fig. 11. Workpiece cross-section Since two separated controllers are involved, one of the most challenging problems is to synchronize the welding speed and update the heat-input (current). In order to coordinate the work of these two controllers, a new software based on the LabVIEW is developed. This software communicates through the PC's serial port with a CNC controller. The CNC controller sends the signal whenever the workpiece is about to change the direction. Instantaneously the software receives the signal (max. delay is $1 \mathrm{msec}$ ), reads the 
appropriate values from the 2D matrix, and calculates the analog output signal for the data acquisition board, according to the Eq. 7. Fast software is necessity since even very small delays can bring about the overheating and damage the part. In addition, this software controls the filler metal feeding speed, the motion of the torch, and monitors (record) the welding current and voltage by sensing through-the-arc. Recorded analog signals of the current and the voltage are shown in the Fig. 12. It should be noted that in the intervals where the calculated

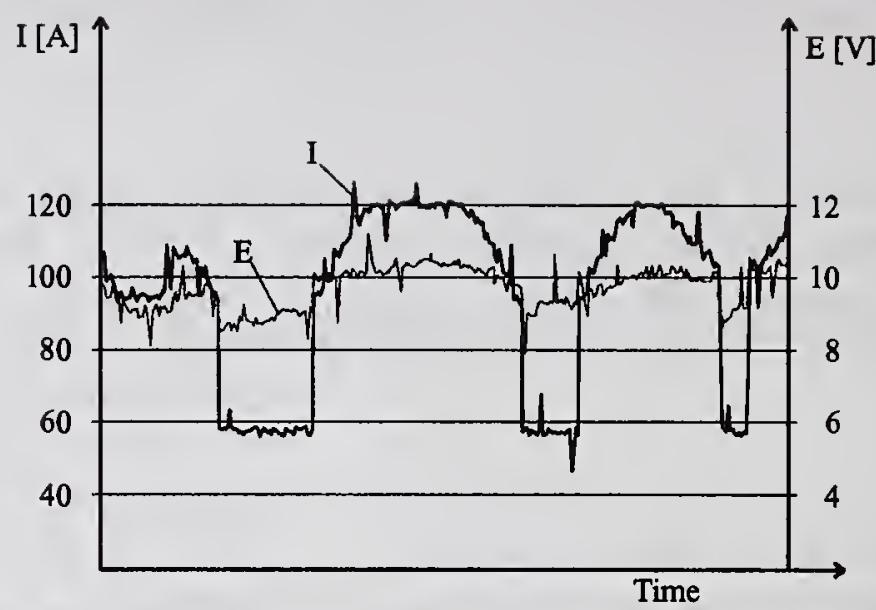

Fig. 12 Values of the welding current and voltage as a function of the geometry of the substrate geometrical factor has zero values, the real value of the current is not zero. That value presents the energy needed for melting the filler metal, and it is taken into the account through the expression for the heat conduction dissipation.

\section{CONCLUSION}

A new parameter referred to as the geometrical factor was introduced to reflect on the geometrical configuration around the molten pool, and an expression for the heat conduction dissipation through the workpiece is established. The weld bead width, height, depth of penetration, and weld bead cross-sectional area, are experimentally determined for different combinations of welding current, workpiece velocity, the filler metal feeding speed, and for different geometrical configurations of the weld bead. The open-loop controller for GTAW process, based on an accurate dynamic model is designed and experimentally tested. The controller shows very robust performance and fast response speed. Future work will include the development of a closed-loop controller that should enhance the versatility of the demonstrated open-loop controller.

\section{ACKNOWLEGEMENT}

This work was financially supported by THECB, Grant $\mathrm{N}_{0}$. 003613-0022001999, NSF, Grants $\mathrm{N}_{\mathrm{o}}$. DMI -9732848 and DMI - 9809198, and the U.S. Department of Education, Grant $\mathrm{N}_{\mathrm{o}}$. P200A-80806-98.

\section{REFERENCES}

1. Kovacevic, R., and Beardsley, H., (1998). Process Control of 3D Welding as a Droplet-Based Rapid Prototyping Technique. Proceedings of the $9^{\text {th }}$ Annual Solid Freeform Fabrication Symposium, August 10-12, Austin, TX, pp. 57-64.

2. Million, K., Datta, R., Zimmermann, H., (1986). Method of manufacture of a design piece by form creating deposition welding and a design piece manufactured by this method, European Patent Office, Publication number: $0172309 \mathrm{~A} 1$.

3. Christensen, N., Davies, V.L. and Gjermundsen, K. (1965). Distribution of Temperatures in Arc Welding. British Welding Journal. Vol. 7, pp.54-74.

4. Ghent, H.W., Roberts, D.W., Hemance, C.E., Kerr, H.W. and Strong, A.B. (1979). Arc Efficiencies in TIG Welds, in Arc Physics and Welding Pool Behaviour. The Welding Institute, Cambridge, pp. 17-23. 
5. Fuerschbach, P.W. and Knorovsky, G.A. (1991). A Study of Melting Efficiency in Plasma Arc and Gas Tungsten Arc Welding. Welding Journal (Miami, Fl.). Vol.70, No.11, pp.287s-297s.

6. Kannaty-Asibu, E. Jr. (1997). Milestone Developments in Welding and Joining Processes. Journal of Manufacturing Science and Engineering. Vol. 119, pp. 801-810.

7. Rykalin, N.N. (1957). Berechnung der Warmevorgange beim Schweisen. Verlag Technik, Berlin. pp. 68-69, pp. 100-105.

8. Rosental, D. (1946). The Theory of Moving Sources Of Heat And Its Application To Metal Treatments. Transactions of ASME. Vol. 68, No.11, pp.849-866.

9. Radaj, D. (1992). Heat Effects of Welding. Springer-Verlag, Berlin/Heidelberg/New York.

10. Kmecko, S.I., Jandric, Z., Kovacevic, R. (2001). Influence of Geometrical Factor on Heat Transfer Rate During GTAW RP. Accepted for the International Mechanical Congress, Nov. 11-16, 2001, New York, NY. 



\title{
THREE DIMENSIONAL TRANSIENT HEAT TRANSFER ANALYSIS FOR GTA WELDING PROCESS
}

\author{
Min Jou*
}

\begin{abstract}
It has been well recognized that the geometry of weld pool plays a fundamental role in determining the mechanical properties of weld joints. The major variables in GTA welding affeting on the formation of weld pool including welding current, voltage, welding speed, arc length, and electrode tip angle. Besides these variables, GTA welding is subject to numerous disturbances and uncertainties within and between welding cycles. These can arise from: workpiece thickness tolerances, fluctuations in welding current, voltage, welding speed, arc length, and root opening. Any or all of these complicate automation, reduce weld quality, and drive up production cost. For this reason, studying interaction and correlation of process variables, disturbances and uncertainties affecting on the formation of weld pool for GTA welding remains a major challenge and goal.
\end{abstract}

In this research, a series of experiment has been conducted to investigate the interaction and correlation of welding current, voltage, welding speed, and arc length affecting on the formation of weld pool. The arc length affecting on arc efficiency and heat distribution parameter are also examined and addressed in this paper.

In addition to experimental study, a three-dimensional finite element model has been developed to analyze transient heat flow and to predict the formation of weld pool. The correlation between the parameters including welding current, voltage, welding speed, arc length, open-loop response and the characteristic geometry of weld pool are established. The 3-D FEM can calculate not only the transient thermal histories but also the sizes of weld pool in single-pass arc welding. In order to obtain good weld quality, this model will determine the effect of arc length to the formation of weld. Furthermore, the effects of welding parameters on the Gaussian heat source parameters (arc efficiency and heat distribution parameter) are also studied. The experimental calibration and verification are carried out to verify the numerical model. Experimental data are consistent and in quantitative agreement with values from FEM simulations.

\section{KEYWORDS}

Weld pool size, Three-dimensional model, Transient heat transfer, Welding process variable, Process variation, Open-loop response, Arc length

\footnotetext{
* The author is in the Department of Industrial Education at the National Taiwan Normal University, 162, Section 1, Hoping East Road, Taipei City 106, Taiwan.
} 


\section{INTRODUCTION}

Gas tungsten arc welding is the most useful welding process used in cases which require higher weld strength joint such as aerospace, nuclear, large structure and automotive industries. The weld strength is affected by the geometry of weld and thermal characteristics. In order to create desired weld strength, the weld cross section geometry (i.e., such as weld bead width, weld depth, weld front width, and weld heat-affected zone) must be developed accurately (Figure $1)$.

The key geometric attribute of a major class of welds is the depth of penetration since it is a direct indicator of weld strength. However, direct measurement of this quantity is quite difficult since the depth is not directly visible. Several investigator [Refs.1-4] utilized reflection ultrasound methods to measure the size of weld pool, but this technique is not very accurate and unapplicable because of the problem of diffusion of the signal and the distortion caused by steep temperature gradients. The other methods have been studied to indirectly detect the pool shape based on the concept of pool oscillation [Refs.5-7], but it has been proved by Zacksenhouse and Hardt [Ref.8] that it is not practical to detect the pool shape in partial penetration using the concept of pool oscillation. The surface waves can only be correlated to bulk fluid oscillation for full penetration welds. In a recent report, an effort to study the pool oscillation on a stationary welding pool was made by Xiao and Ouden [Ref.9]. The case of partial penetration as well as full penetration was performed in this research but not in a practical welding situations which is a moving welding pool. Other Studies have been conducted to correlate the depth of penetration to variables which can be measured during welding including arc voltage sensing[Ref.10], x-ray sensing[Ref.11], video[Ref.12], acoustic sensing [Ref.13] and surface radiation techniques[Ref.14]. Every methods offers specific advantages, But each of these methods contain drawbacks such as signal disturbances from high temperatures, contact sensing devices, sensing performance corrupted by workpiece thickness or preheat changes, and gave no details on the actual pool shape. These drawbacks have limited their applicability in practical welding situations. Infrared sensing techniques have been studied as a possible sensor to detect weld penetration [Ref.15-17]. In order to develop a sensor based on infrared sensing that can be easily implemented in practical welding applications. The development of a mathematical relationship among different welding conditions (i.e., varied current, welding speed, material types, electrode tip angle, ...etc.), measurable signal, and size of weld pool becomes an important research goal.

The temperature history of the welded components has a significant influence on the residual stresses, distortion and hence the fatigue behavior of the welded structure. A large amount of research has been dedicated to the simulation of welding process in the past few decades. However, since the welding process causes complex mechanical, electric and metallurgical changes in weldments, the accurate solution of mathematical model, which is capable of addressing all the issues including material nonlinearity, phase transformation, magnetodynamic effects, fluid flow in weld pool, etc., is practically intangible. To solve the problem, the numerical methods, particularly the finite difference methods and the finite element methods, are widely used for the simulation of welding process and the resultant residual strain and stress fields. A quick review of literatures reveals that there are primarily two approaches in the simulation of welding process. One approach is to solve the decoupled heat transfer equation and Navier-Stokes equations so that the temperature distribution and the details of fluid flow in 
weld pool can be obtained. The typical examples following this approach can be found in Kovacevic, el al.[Ref 18] and Cao. et al, [Ref.19], where the effects of changing welding parameters on the characteristic geometry of weld pool is analyzed by using three dimensional finite difference method for transient analysis. More examples can be found, to name a few, in Zacharia, et.,[Ref.20,21] and Kim and $\mathrm{Na}$ [Ref.22]. Another approach for the simulation of the welding process is to solve the heat transfer equation directly with the consideration of temperature-dependent material properties and phase transformation. Due to the simplicity, yet the acceptable accuracy, of this approach, many numerical models have been developed based on it, especially when the resultant residual strain and stress is of the particular interest. The examples can be found in Tekriwal and Mazumder [Ref.23], Mahin, et al,[Ref.24], Brown and Song [Ref.25], Taljat, et al,[Ref.26] and Bonifaz [Ref.27]. Typically the heat flux input from arc is assumed to follow Gaussian function or double ellipsoidal model[Ref.27].

This research is focused on the critical single-pass bead-on-plate GTA welding process. Three dimensional finite element model for the simulation of transient heat transfer is adopted in our analysis in the sense that the simple 2-D model captures less information of the characteristic sizes of the weld pool than 3-D model does. The accuracy of the 2-D model has also been questioned by Brown and Song[Ref.25] and Bonifaz[Ref.27]. The aforementioned second approach is employed in our finite element model, and the effect of fluid flow in the weld pool is approximated by the temperature-dependent material properties. The distribution of the moving heat source generated by the arc is assumed to be 2-dimensional Gaussian function. The experimental calibration and verification are carried out to verify the numerical model. The correlation between the parameters of the GTA welding process, including torch speed, electric current and voltage, and the characteristic geometry of the weld pool are established. The major parameters which will affect the pool size are: arc length, current, speed, voltage, and vertex angle of electrode. However, the electrode is installed and cannot be changed during welding in automatic welding. So in this research we will not study the effects of electrode vertex angle.

\section{EXPERIMENTS}

A Hobart TIGWAVE ${ }^{\mathrm{TM}} 350$ AC/DC welding system (Figure 2) was employed for the gas tungsten arc welding in this study. The water-cooled torch was manipulated by a X-Y positional table. The electrode was made of EWTh-2 with $3.2 \mathrm{~mm}$ diameter and 120 (degrees) included tip angle. The electrode extension from the nozzle (with a diameter of $11 \mathrm{~mm}$ ) was $3 \mathrm{~mm}$. Shielding gas with a flow rate of 10 liters/min was industrial pure argon (99.99\%). Preparation of test coupons with dimensions of $130 \times 130 \mathrm{~mm}$ were made of SUS304 (Type 304) austenitic stainless plates $6 \mathrm{~mm}$ and $4 \mathrm{~mm}$ in thickness. Prior to welding, the coupons were sanded with \#200 sand paper and cleaned with acetone. But welds then carried out down the center of the plate. The samples were mounted on the flat surface of the plateform. During welding, the coupons were free of restraint. The joint setup was autogenous bead-on-plate welding. Welding parameters influence the quality of welded joints. Therefore, the determination of these parameters is an important step in this study. The criteria for selecting the optimum welding parameters include the external appearance of the weld, fusion geometry and penetration. To study the effects of welding parameters on the weld geometry, the tested coupons were sectioned perpendicularly to the welding direction for metallographic examinations. The sectioned samples 
were mounted, sanded using sand papers, and polished using $\mathrm{Al}_{2} \mathrm{O}_{3}$ ( 0.05 micron) colloidal solution. Samples were cleaned before etched with a solution with $10 \mathrm{~g} \mathrm{CuSO} 4+50 \mathrm{ml} \mathrm{HCl}+$ $50 \mathrm{ml}$ water. Samples were observed under an Olympus BX60M microscope to determine the weld profile.

In order to verify and calibrate the 3-D FEM model, experiments were performed to investigate the interaction and correlation of process variables and variations affecting on the formation of weld pool for GTA welding. The experimental results as shown in Figure 3, indicate that: (1). increasing the welding current increases the weld penetration; (2). increasing the welding speed decreases the weld penetration. The open-loop response is the key parameter for development of control system. Therefore, experiments were conducted to realize how the open-loop response will be affected by the controllable variables which is the welding current and welding speed. Figure 4 shows that increases input current magnitude speed up open-loop response. Increasing welding speed will slow down open-loop response as shown in Figure 5. Figure 6 indicates that welding speed has more effect on open-loop response than welding current.

Welding process anomalies occurs quite frequently when welding production sheet metal parts. The most significant effect of process anomalies is that it results from variation of arc length. The condition arises when the metal parts are not flat, when contours do not match dies/fixtures or one another precisely, when ripples or wrinkles are present, when tip of tungsten electrode is melt due to high temperature. In order to study the effect of variation of arc length on the formation of weld pool, welding on stepped down plates was performed (i.e., arc length increases from $2 \mathrm{~mm}$ to $4 \mathrm{~mm}$ ) as shown in Figure 7. Experimental data indicates that increasing the arc length decreases weld penetration under constant welding speed as shown in Figure 8. Figure 9 show that increases arc length decreases weld penetration under constant welding current. This indicates longer arc length will make the arc efficiency lower.

The arc length affecting on weld surface width is also examed. The results presented in Figure 10 and 11 show that the surface width slightly increases with increasing arc length. The longer arc length results in greater expansion of the arc. Therefore, the surface width increases with arc length increased. However, the surface width decreases with arc length increased when welding at lower current. The reason is that higher heat input (i.e.,higher welding current) provides sufficient heat for melting, and a wider arc distribution (i.e.,longer arc length) will result in a wider surface width. A very broad arc distribution (i.e.,longer arc length) at low heat input (i.e.,lower welding current) will not produce melting, thus results in decreasing surface width of weld.

\section{MODELING OF PROCESS DYNAMICS}

The objectives for modeling of process dynamics were: (1) to develop a 3-D FEM to calculate not only the transient thermal histories but also the sizes of weld pool in single-pass arc welding; (2) to determine the effect of arc length to the formation of weld; (3) to obtain the effect of welding parameters to the arc efficiency and heat distribution parameter; and (4) to identify 
the correlation among controllable variables (i.e., welding current and speed), arc efficiency and heat distribution parameter.

The weldment is made of SUS304 stainless steel. Temperature-dependent material properties are used in this study, which bring nonlinearity into the calculation. To account for the heat transfer due to the fluid flow in weld pool, the thermal conductivity is assumed to be enhanced linearly from 1 to 6 with temperature change from the solidus temperature (1673K) to $3100 \mathrm{~K}$ [Ref.24]. The liquid/solid phase transformation is approximated by taking into account the latent heat of fusion over melting range (between $1673 \mathrm{~K}$ and 1723K). The thermal properties used in our calculation are summarized in Table 1. The thickness of the weldment under investigation is $4 \mathrm{~mm}$ and $6 \mathrm{~mm}$. The length of the FE model is selected to be $36 \mathrm{~mm}$ and the width is $20 \mathrm{~mm}$. The symmetry condition along the arc path is assumed in our model. The fine mesh with $0.333 \mathrm{~mm}$ characteristic grid spacing is applied to the weld pool region, while the coarse mesh is used elsewhere in order to reduce the computation costs. The finite element mesh for the simulation model is depicted in Figure 12.

The overall input of heat flux is calculated as:

$$
Q_{0}=\eta E I
$$

where $\eta$ is arc efficiency factor, which accounts for the radiation and other losses from the arc to the environment, $E$ is voltage and $I$ is electric current. The radial heat flux distribution on the top surface of weldment is approximated to be a Gaussian function in the form of (as shown in Figure 13);

$$
q=\frac{Q_{0}}{2 \pi r_{0}^{2}} \exp \left(\frac{-r^{2}}{2 r_{0}^{2}}\right)
$$

where $r$ is the radial distance with respect to the origin at the center of torch; $r_{0}$ is the distribution parameter defined as the radial distance within which $95 \%$ of energy is transferred.

Convection and radiation boundary conditions are applied to the weldment to model the heat loss from the weldment surfaces. Particularly, the forced convection boundary condition is assumed on the entire top surface right below the arc. The natural convection boundary conditions are assumed on all the other surfaces. All the convection boundary conditions are modeled by Newton's law of cooling, i.e.

$$
q_{\text {conv }}=h\left(T-T_{\infty}\right)
$$

where $h$ is the convection coefficient which is assumed to be constant through the welding process; $T$ is the local temperature and $T_{\infty}$ is the ambient temperature. The radiation boundary condition is applied over the top surface following to the gray body radiation law:

$$
q_{\text {evap }}=\varepsilon\left(T^{4}-T_{\infty}^{4}\right)
$$




\section{INTRODUCTION}

Gas tungsten arc welding is the most useful welding process used in cases which require higher weld strength joint such as aerospace, nuclear, large structure and automotive industries. The weld strength is affected by the geometry of weld and thermal characteristics. In order to create desired weld strength, the weld cross section geometry (i.e., such as weld bead width, weld depth, weld front width, and weld heat-affected zone) must be developed accurately (Figure 1).

The key geometric attribute of a major class of welds is the depth of penetration since it is a direct indicator of weld strength. However, direct measurement of this quantity is quite difficult since the depth is not directly visible. Several investigator [Refs.1-4] utilized reflection ultrasound methods to measure the size of weld pool, but this technique is not very accurate and unapplicable because of the problem of diffusion of the signal and the distortion caused by steep temperature gradients. The other methods have been studied to indirectly detect the pool shape based on the concept of pool oscillation [Refs.5-7], but it has been proved by Zacksenhouse and Hardt [Ref.8] that it is not practical to detect the pool shape in partial penetration using the concept of pool oscillation. The surface waves can only be correlated to bulk fluid oscillation for full penetration welds. In a recent report, an effort to study the pool oscillation on a stationary welding pool was made by Xiao and Ouden [Ref.9]. The case of partial penetration as well as full penetration was performed in this research but not in a practical welding situations which is a moving welding pool. Other Studies have been conducted to correlate the depth of penetration to variables which can be measured during welding including arc voltage sensing[Ref.10], x-ray sensing[Ref.11], video[Ref.12], acoustic sensing [Ref.13] and surface radiation techniques[Ref.14]. Every methods offers specific advantages, But each of these methods contain drawbacks such as signal disturbances from high temperatures, contact sensing devices, sensing performance corrupted by workpiece thickness or preheat changes, and gave no details on the actual pool shape. These drawbacks have limited their applicability in practical welding situations. Infrared sensing techniques have been studied as a possible sensor to detect weld penetration [Ref.15-17]. In order to develop a sensor based on infrared sensing that can be easily implemented in practical welding applications. The development of a mathematical relationship among different welding conditions (i.e., varied current, welding speed, material types, electrode tip angle, ...etc.), measurable signal, and size of weld pool becomes an important research goal.

The temperature history of the welded components has a significant influence on the residual stresses, distortion and hence the fatigue behavior of the welded structure. A large amount of research has been dedicated to the simulation of welding process in the past few decades. However, since the welding process causes complex mechanical, electric and metallurgical changes in weldments, the accurate solution of mathematical model, which is capable of addressing all the issues including material nonlinearity, phase transformation, magnetodynamic effects, fluid flow in weld pool, etc., is practically intangible. To solve the problem, the numerical methods, particularly the finite difference methods and the finite element methods, are widely used for the simulation of welding process and the resultant residual strain and stress fields. A quick review of literatures reveals that there are primarily two approaches in the simulation of welding process. One approach is to solve the decoupled heat transfer equation and Navier-Stokes equations so that the temperature distribution and the details of fluid flow in 
weld pool can be obtained. The typical examples following this approach can be found in Kovacevic, el al.[Ref 18] and Cao. et al, [Ref.19], where the effects of changing welding parameters on the characteristic geometry of weld pool is analyzed by using three dimensional finite difference method for transient analysis. More examples can be found, to name a few, in Zacharia, et.,[Ref.20,21] and Kim and $\mathrm{Na}$ [Ref.22]. Another approach for the simulation of the welding process is to solve the heat transfer equation directly with the consideration of temperature-dependent material properties and phase transformation. Due to the simplicity, yet the acceptable accuracy, of this approach, many numerical models have been developed based on it, especially when the resultant residual strain and stress is of the particular interest. The examples can be found in Tekriwal and Mazumder [Ref.23], Mahin, et al,[Ref.24], Brown and Song [Ref.25], Taljat, et al,[Ref.26] and Bonifaz [Ref.27]. Typically the heat flux input from arc is assumed to follow Gaussian function or double ellipsoidal model[Ref.27].

This research is focused on the critical single-pass bead-on-plate GTA welding process. Three dimensional finite element model for the simulation of transient heat transfer is adopted in our analysis in the sense that the simple 2-D model captures less information of the characteristic sizes of the weld pool than 3-D model does. The accuracy of the 2-D model has also been questioned by Brown and Song[Ref.25] and Bonifaz[Ref.27]. The aforementioned second approach is employed in our finite element model, and the effect of fluid flow in the weld pool is approximated by the temperature-dependent material properties. The distribution of the moving heat source generated by the arc is assumed to be 2-dimensional Gaussian function. The experimental calibration and verification are carried out to verify the numerical model. The correlation between the parameters of the GTA welding process, including torch speed, electric current and voltage, and the characteristic geometry of the weld pool are established. The major parameters which will affect the pool size are: arc length, current, speed, voltage, and vertex angle of electrode. However, the electrode is installed and cannot be changed during welding in automatic welding. So in this research we will not study the effects of electrode vertex angle.

\section{EXPERIMENTS}

A Hobart TIGWAVE ${ }^{\mathrm{TM}} 350$ AC/DC welding system (Figure 2) was employed for the gas tungsten arc welding in this study. The water-cooled torch was manipulated by a X-Y positional table. The electrode was made of EWTh-2 with $3.2 \mathrm{~mm}$ diameter and 120 (degrees) included tip angle. The electrode extension from the nozzle (with a diameter of $11 \mathrm{~mm}$ ) was $3 \mathrm{~mm}$. Shielding gas with a flow rate of 10 liters/min was industrial pure argon (99.99\%). Preparation of test coupons with dimensions of $130 \times 130 \mathrm{~mm}$ were made of SUS304 (Type 304) austenitic stainless plates $6 \mathrm{~mm}$ and $4 \mathrm{~mm}$ in thickness. Prior to welding, the coupons were sanded with \#200 sand paper and cleaned with acetone. But welds then carried out down the center of the plate. The samples were mounted on the flat surface of the plateform. During welding, the coupons were free of restraint. The joint setup was autogenous bead-on-plate welding. Welding parameters influence the quality of welded joints. Therefore, the determination of these parameters is an important step in this study. The criteria for selecting the optimum welding parameters include the external appearance of the weld, fusion geometry and penetration. To study the effects of welding parameters on the weld geometry, the tested coupons were sectioned perpendicularly to the welding direction for metallographic examinations. The sectioned samples 
were mounted, sanded using sand papers, and polished using $\mathrm{Al}_{2} \mathrm{O}_{3}$ (0.05 micron) colloidal solution. Samples were cleaned before etched with a solution with $10 \mathrm{~g} \mathrm{CuSO} 4+50 \mathrm{ml} \mathrm{HCl}+$ $50 \mathrm{ml}$ water. Samples were observed under an Olympus BX60M microscope to determine the weld profile.

In order to verify and calibrate the 3-D FEM model, experiments were performed to investigate the interaction and correlation of process variables and variations affecting on the formation of weld pool for GTA welding. The experimental results as shown in Figure 3, indicate that: (1). increasing the welding current increases the weld penetration; (2). increasing the welding speed decreases the weld penetration. The open-loop response is the key parameter for development of control system. Therefore, experiments were conducted to realize how the open-loop response will be affected by the controllable variables which is the welding current and welding speed. Figure 4 shows that increases input current magnitude speed up open-loop response. Increasing welding speed will slow down open-loop response as shown in Figure 5. Figure 6 indicates that welding speed has more effect on open-loop response than welding current.

Welding process anomalies occurs quite frequently when welding production sheet metal parts. The most significant effect of process anomalies is that it results from variation of arc length. The condition arises when the metal parts are not flat, when contours do not match dies/fixtures or one another precisely, when ripples or wrinkles are present, when tip of tungsten electrode is melt due to high temperature. In order to study the effect of variation of arc length on the formation of weld pool, welding on stepped down plates was performed (i.e., arc length increases from $2 \mathrm{~mm}$ to $4 \mathrm{~mm}$ ) as shown in Figure 7. Experimental data indicates that increasing the arc length decreases weld penetration under constant welding speed as shown in Figure 8. Figure 9 show that increases arc length decreases weld penetration under constant welding current. This indicates longer arc length will make the arc efficiency lower.

The arc length affecting on weld surface width is also examed. The results presented in Figure 10 and 11 show that the surface width slightly increases with increasing arc length. The longer arc length results in greater expansion of the arc. Therefore, the surface width increases with arc length increased. However, the surface width decreases with arc length increased when welding at lower current. The reason is that higher heat input (i.e.,higher welding current) provides sufficient heat for melting, and a wider arc distribution (i.e.,longer arc length) will result in a wider surface width. A very broad arc distribution (i.e.,longer arc length) at low heat input (i.e.,lower welding current) will not produce melting, thus results in decreasing surface width of weld.

\section{MODELING OF PROCESS DYNAMICS}

The objectives for modeling of process dynamics were: (1) to develop a 3-D FEM to calculate not only the transient thermal histories but also the sizes of weld pool in single-pass arc welding; (2) to determine the effect of arc length to the formation of weld; (3) to obtain the effect of welding parameters to the arc efficiency and heat distribution parameter; and (4) to identify 
the correlation among controllable variables (i.e., welding current and speed), arc efficiency and heat distribution parameter.

The weldment is made of SUS304 stainless steel. Temperature-dependent material properties are used in this study, which bring nonlinearity into the calculation. To account for the heat transfer due to the fluid flow in weld pool, the thermal conductivity is assumed to be enhanced linearly from 1 to 6 with temperature change from the solidus temperature $(1673 \mathrm{~K})$ to $3100 \mathrm{~K}$ [Ref.24]. The liquid/solid phase transformation is approximated by taking into account the latent heat of fusion over melting range (between $1673 \mathrm{~K}$ and $1723 \mathrm{~K}$ ). The thermal properties used in our calculation are summarized in Table 1 . The thickness of the weldment under investigation is $4 \mathrm{~mm}$ and $6 \mathrm{~mm}$. The length of the FE model is selected to be $36 \mathrm{~mm}$ and the width is $20 \mathrm{~mm}$. The symmetry condition along the arc path is assumed in our model. The fine mesh with $0.333 \mathrm{~mm}$ characteristic grid spacing is applied to the weld pool region, while the coarse mesh is used elsewhere in order to reduce the computation costs. The finite element mesh for the simulation model is depicted in Figure 12.

The overall input of heat flux is calculated as:

$$
Q_{0}=\eta E I
$$

where $\eta$ is arc efficiency factor, which accounts for the radiation and other losses from the arc to the environment, $E$ is voltage and $I$ is electric current. The radial heat flux distribution on the top surface of weldment is approximated to be a Gaussian function in the form of (as shown in Figure 13);

$$
q=\frac{Q_{0}}{2 \pi r_{0}^{2}} \exp \left(\frac{-r^{2}}{2 r_{0}^{2}}\right)
$$

where $r$ is the radial distance with respect to the origin at the center of torch; $r_{0}$ is the distribution parameter defined as the radial distance within which $95 \%$ of energy is transferred.

Convection and radiation boundary conditions are applied to the weldment to model the heat loss from the weldment surfaces. Particularly, the forced convection boundary condition is assumed on the entire top surface right below the arc. The natural convection boundary conditions are assumed on all the other surfaces. All the convection boundary conditions are modeled by Newton's law of cooling, i.e.

$$
q_{\text {conv }}=h\left(T-T_{\infty}\right)
$$

where $h$ is the convection coefficient which is assumed to be constant through the welding process; $T$ is the local temperature and $T_{\infty}$ is the ambient temperature. The radiation boundary condition is applied over the top surface following to the gray body radiation law:

$$
q_{\text {evap }}=\varepsilon\left(T^{4}-T_{\infty}^{4}\right)
$$


where $\varepsilon$ is the surface emittance and $\sigma$ is Stephen-Boltzman constant. The evaporation of the welding pool is assumed to be negligible in this study.

\section{SIMULATION RESULTS AND EXPERIMENTAL VERIFICATION}

Figure 14 shows the transient temperature distribution of the weld created from the developed 3D FEM model under different welding conditions. The numerical simulations are carried out for two different workpiece thickness (i.e., $4 \mathrm{~mm}$ and $6 \mathrm{~mm}$ ) with various torch velocities. The weld penetration and surface width of different workpiece thickness can be predicted through the developed FEM model under various welding conditions as shown in Figure 15 to 18.

The heat distribution parameter $r_{0}$ has significant influences on both the shape and the size of weld pools. The numerical simulation reveals that the depth of weld pools is more sensitive to the changing of $r_{0}$ than the top-surface width and length. With the increase of $r_{0}$, the top surface of weld pools tends to evolving from approximately round shape to tear-drop shape. The curvature of the weld pool boundary in the depth decrease slightly faster at the front end than at the rear end when $r_{0}$ increases. The arc efficiency also has significant influences on the size weld pools since it directly relates to the amount of the net heat input from arc (i.e., $\eta^{*} E^{*} I$ ). Again, the depth of weld pools is more sensitive to the changing of the arc efficiency than the top-surface width and length. The shape of the top surface of weld pools changes slightly with the arc efficiency, while the shape in the depth is significantly affected. As for the effects of heat source parameter on the cooling rate, smaller $r_{0}$ and efficiency will lead to faster cooling process.

The dependence of heat distribution parameter, $r_{0}$, and arc efficiency on travel speed is shown in Figure 19 and Figure 20. The process exhibits a nearly constant value of heat distribution parameter with the exception of the higher current where a decrease is noted as increasing the welding speed. The small distribution parameter, which mean the heat source more concentrated on the surface under the torch, does not mean that the small pool size will be obtained. The depth of weld pool is very sensitive to the distribution parameter while surface width is relatively not. Figure 20 shows a nearly constant level of arc efficiency with the exception of the lower current where a decrease is noted.

Figure 21 and 22 show that the distribution parameter, $r_{0}$, increases rapidly with increasing arc length and increasing current. As the arc length is increased, the number of electron-ion collisions increases and the arc spreads from the sharply-tipped cathode to the flat anode. The increased electron-ion scattering of a longer arc results in greater expansion of the arc. However, distribution parameter slightly decreases with increasing arc length at lower welding current. This shows that lower current will not provide enough heat to melt the workpiece.

The arc length affecting on arc efficiency is also examed. The results presented in Figure 23 and 24 show that the arc efficiency decreases with increasing arc length. This indicates longer arc length will make the arc efficiency lower. This results are constanct with the experimental 
results as shown in Figure 8 and 9, where the weld penetration decreases as the arc length increasing.

\section{CONCLUSIONS}

1. The effects of variations in the welding process parameters on the heat distributions in gas tungsten arc welding have been reported. Arc length is the primary parameter govering the heat distributions. The results provide a link between the engineering parameters of current, welding speed to the scientific parameters which consist of the heat distribution parameter.

2. The relative insensitivity of the GTAW arc efficiency to travel speed has been reported.

3. Develop a meaningful between teoretical models and experimental observations.

4. The finite element solution was sensitive to heat distribution. The model results provided a straight forward approach to understanding the effects of heat distribution.

\section{ACKNOWLEDGEMENTS}

The work reported here was partially performed and funded under the support of the National Science Council of Taiwan under the Grant No. NSC 88-2212-E-239-004. The author would also like to thank National Center for High-performance Computing of Taiwan for furnishing computational systems which made this investigation possible.

\section{REFERENCES}

1. Lott, L.A., "Ultrasonic detection of molten/solid interfaces in weld pools," Material Evaluation, Vol.42, 1983, pp.337-341.

2. Hardt, D.E. and Katz, J.M., "Ultrasonic measurement of weld penetration," Welding Journal, Vol.63, No.9, 1984, pp.273s-281s.

3. Johnson, J.A., Carlson, N.M., and Lott, L.A., "Ultrasonic wave propagation in temperature gradients," Welding Journal, Vol.67, No.3, 1988, pp.147-157.

4. Carlson, N.M. and Johnson, J.A., "Ultrasonic sensing of weld pool penetration," Welding Journal, Vol.67, No.11, 1988, pp.239s-246s.

5. Renwick, R.J., and Richardson, R.W., "Experimental investigation of GTA weld pool oscillations," Welding Journal, Vol.62, No.2, 1983, pp.29s-35s.

6. Sorenson, C.D., "Digital signal processing as a diagnostic tool for gas tungsten arc welding," Ph.D Thesis, 1985, MIT Department of Material Science.

7. Tam, A.S., and Hardt, D.E., "Weld pool impedance for pool geometry measurement: stationary and non-stationary pools," ASME Journal of Dynamic Systems, Measurement, and Control, Vol.111, No.4, 1989, pp.545-553. 
8. Zacksenhouse, M., and Hardt, D.E., "Weld pool impedance identification for size measurement and control," ASME Journal of Dynamic Systems, Measurement, and Control, Vol.105, No.3, 1983, pp.179-184.

9. Xiao, Y.H., and den Ouden, G., "A study of GTA weld pool oscillation," Welding Journal, Vol.69, No.8, 1990, pp.293s-298s.

10. Gladkov, E.A., "Construction of parametric penetration depth regulators for welding with a non-consumable electrode," AVT, Svarka, No.4, 1976, pp.18-23.

11. Charbonnier, F.M., "Flash x-ray of welding, casting, and cast injury," Proceedings of the Flash Radiographic Symposium, American Society for Nondestructive Testing, September, 1976.

12. Richardson, R.W., Gutow, D.A., Anderson, R.A., and Farson, D.F., "Coaxial arc weld pool viewing for process monitoring and control," Welding Journal, Vol.63, No.3, 1984, pp.43-50.

13. Dickhaut, E., Eisenblatter, J., "Acoustic emission measurement during electron beam welding of nickel-base alloys," Journal of Engineering for Power, January, 1979, pp.47-52.

14. Nomura, H., Satoh, Y., Tohno, K., Satoh, Y., and Kurotori, M., "Arc light intensity controls current in SA welding system," Welding and Metal Fabrication, Vol.48, 1980, pp.457-463.

15. Khan, M.A., Madsen, N.H., Goodling, J.S., and Chin, B.A., "Infrared thermography as a control for the welding process," Optical Engineering, Vol.25, No.6, 1986, pp.799-805.

16. Lukens, W.E., and Morris, R.A., "Infrared temperature sensing of cooling rates for arc welding control," Welding Journal, Vol.61, No.1, 1982, pp.27-33.

17. Chen, W. and Chin, B.A., "Monitoring joint penetration using infrared sensing techniques," Welding Journal, Vol.69, No.4, 1990, pp.181s-185s.

18. Kovacevic, R., Cao, Z.N. and Zhang, Y.M., "Role of welding parameters in determining the geometrical appearance of weld pool," Journal of Engineering Materials and Technology, Vol. 118, 1996, pp.689-596.

19. Cao, Z.N., Zhang, Y.M. and Kovacevic, R., "Numerical Dynamic Analysis of moving GTA weld pool," Journal of Manufacturing Science and Engineering, Vol.120, No.1, 1998, pp.173-178.

20. Zacharia, T.,Eraslan, A.H. and Aidun, D.K.," Modeling of non-autogenous welding," Welding Journal, Vol.67, No.1, 1988, pp.18s-27s.

21. Zacharia, T.,Eraslan, A.H. and Aidun, D.K., "Modeling of autogenous welding," Welding Journal, Vol.67, No.3, 1988, pp.53s-62s.

22. Kim, J.W.and Na, S.J." "Study on the three-dimensional analysis of heat and fluid flow in gas metal arc welding using boundary-fitted coordinates," Journal of Engineering for Industry, Vol.116, No.1, 1994, pp.78-85. 
23. Tekriwal, P. and Mazumder, J., "Finite element analysis of three-dimensional transient heat transfer in GMA welding," Welding Journal, Vol.67, No.5, 1988, pp.150s-156s

24. Mahin, K.W., Winters, W., Holden, T.M., Hosbons, R.R. and MacEwen, S.R., "Prediction and measurement of residual plastic strain distributions in gas tungsten arc welding," Welding Journal, Vol.70, No.9, 1991, pp.245s-260s

25. Brown, S.B. and Song, H., "Implications of three-dimensional numerical simulations of welding of large structures," Welding Journal, Vol.71, No.2, 1992, pp.55s-62s

26. Taljat, B., Radhakrishnan, B. and Zacharia, T, "Numerical analysis of GTA welding process with emphasis on post-solidification phase transformation effects on residual stresses," Materials Science and Engineering, A246, 1998, pp.45-54

27. Bonifaz, E.A., "Finite element analysis of heat flow in single-pass arc welds," Welding Journal, Vol.79. No.5, 2000, pp.121s-125. 
Front Width

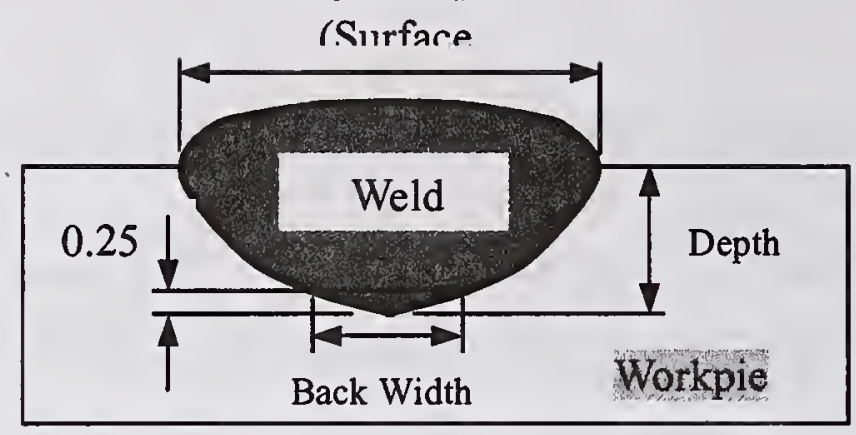

Figure 1. Geometry of weld cross section

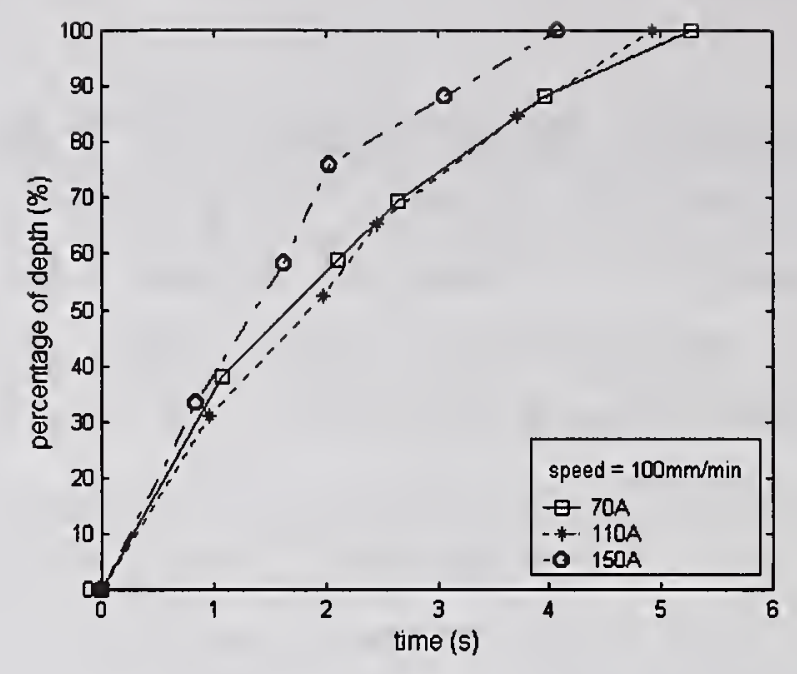

Figure 4 Effect of welding current on open-loop response

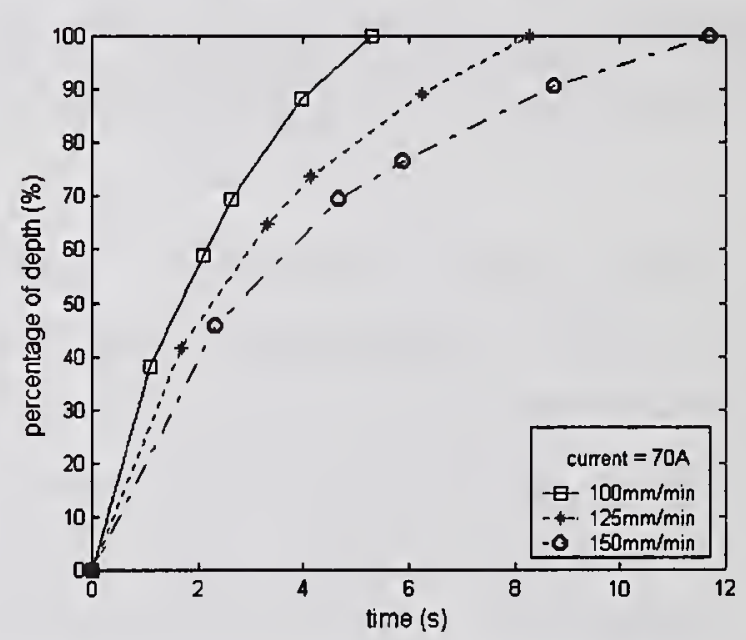

Figure 5 Effect of welding speed on open-loop response

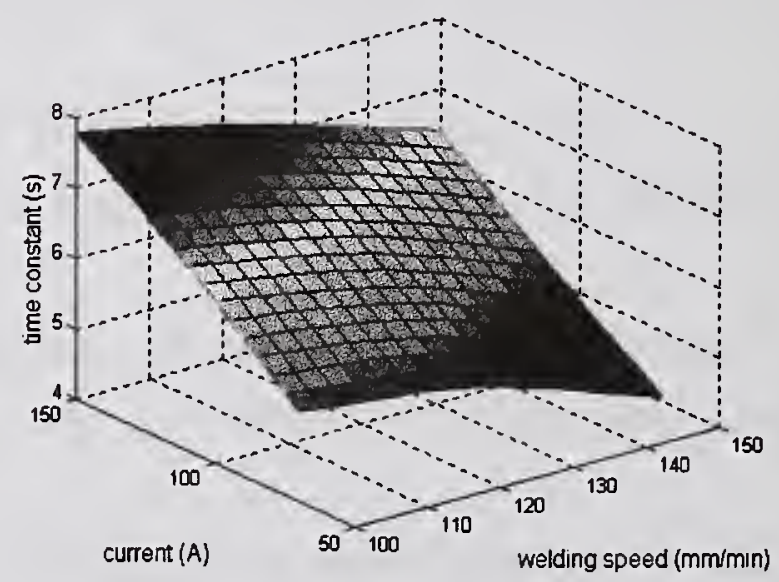

Figure 6 Correlation of welding current, speed, and open-loop response (arc length $=2 \mathrm{~mm}$, thickness $=6 \mathrm{~mm}$ ) 


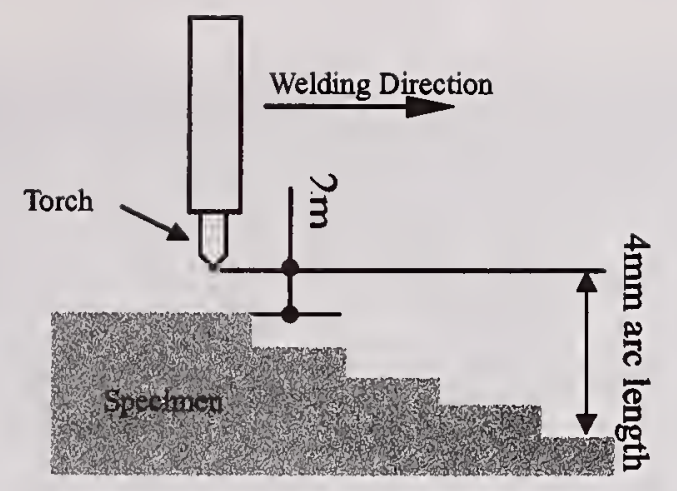

Figure 7 Welding on varying workpiece thickness

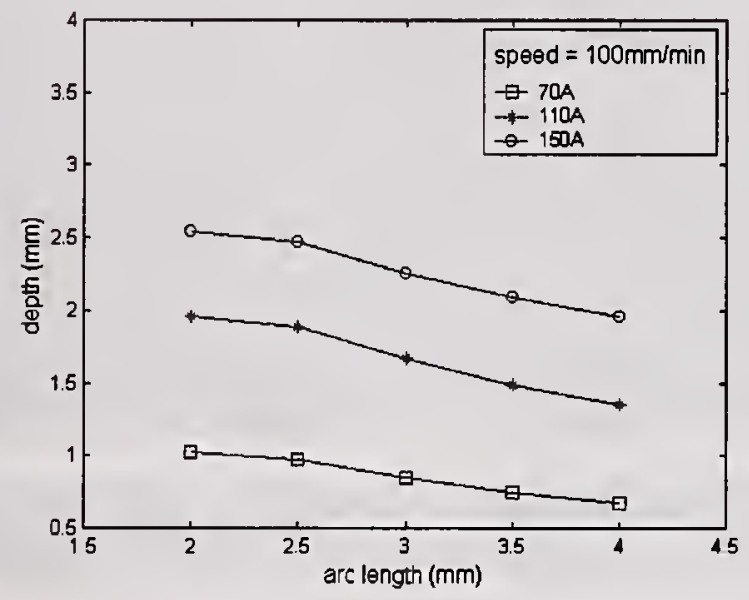

Figure $8 \mathrm{Effect}$ of arc length on GTA weld penetration under constant welding speed

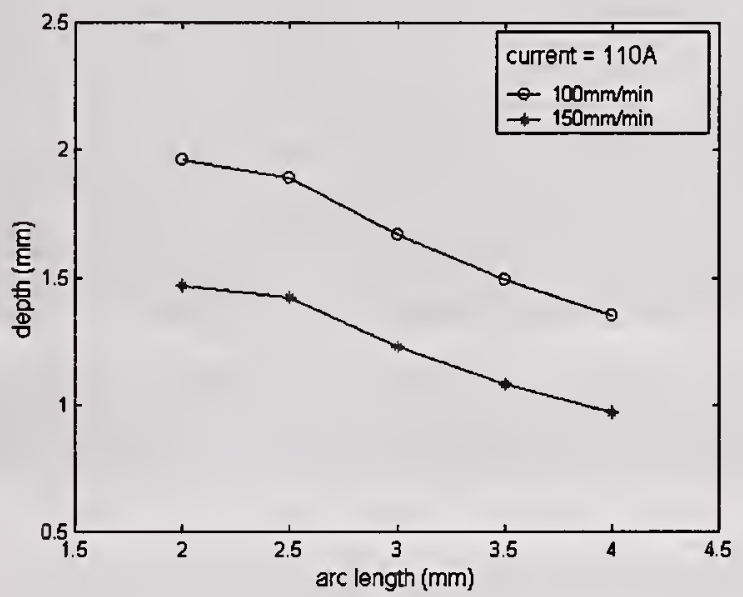

Figure 9 Effect of arc length on GTA weld penetration under constant welding current

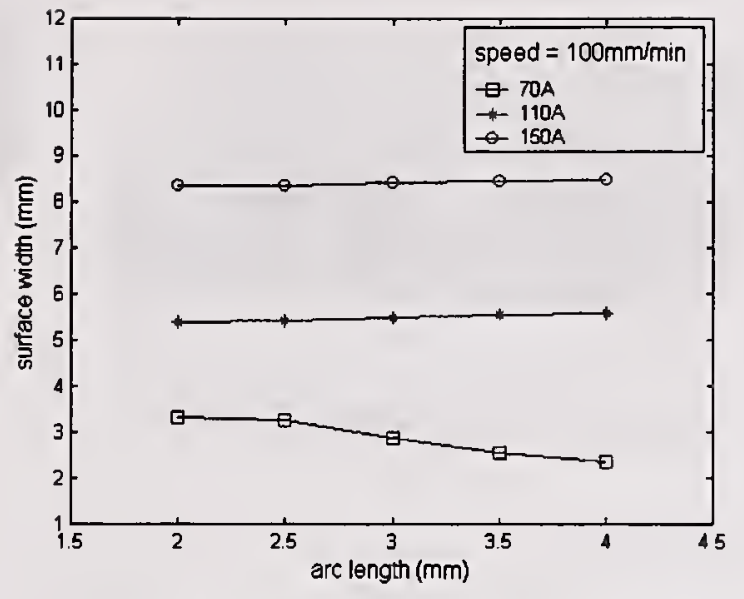

Figure 10 Effect of arc length on weld surface width under constant welding speed

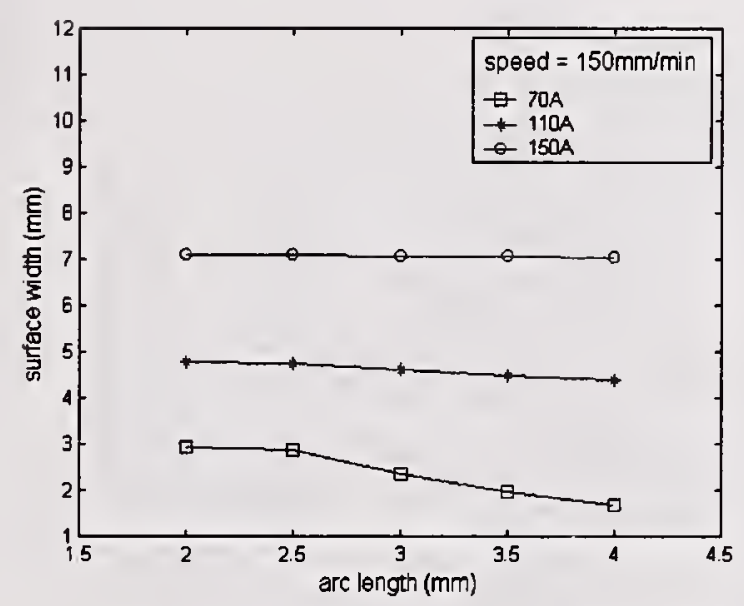

Figure 11 Effect of arc length on weld surface width under constant welding speed

Table 1: Thermal properties for SUS304 stainless steel

\begin{tabular}{|c|c|}
\hline $\begin{array}{l}\text { Thermal conductivity } \\
(\mathrm{W} / \mathrm{m}-\mathrm{K}) \\
\text { Solid }(T \leq 1673 \mathrm{~K}) \\
\text { Liquid }(\mathrm{K}) \\
\end{array}$ & $\begin{array}{l}8.116+1.618 \times 10^{-2} T \\
-171.1+0.1233 \mathrm{~T}\end{array}$ \\
\hline $\begin{array}{c}\text { Density }\left(\mathrm{kg} / \mathrm{m}^{3}\right) \\
\text { Solid }(<=1673 \mathrm{~K}) \\
\text { Liquid }(>1673 \mathrm{~K}) \\
\end{array}$ & $\begin{array}{l}8000 \\
7200 \\
\end{array}$ \\
\hline \begin{tabular}{|l|} 
Specific heat $(\mathrm{J} / \mathrm{kg}-\mathrm{K})$ \\
Solid $(T \leq 1673 \mathrm{~K})$ \\
Liquid $(\mathrm{T}>1723 \mathrm{~K})$ \\
Change linearly \\
between $1673 \mathrm{~K}$ and \\
$1723 \mathrm{~K}$ \\
\end{tabular} & $\begin{array}{l}465.4+0.1336 T \\
788\end{array}$ \\
\hline Latent heat of fusion $(\mathrm{J} / \mathrm{kg})$ & $2.5 \times 10^{5}$ \\
\hline $\begin{array}{l}\text { Convection coefficient }\left(\mathrm{W} / \mathrm{m}^{2}-\mathrm{K}\right) \\
\text { Natural convection } \\
\text { Forced convection }\end{array}$ & $\begin{array}{l}10 \\
40\end{array}$ \\
\hline Surface emissivity & 0.7 \\
\hline Ambient temperature (K) & 300 \\
\hline
\end{tabular}




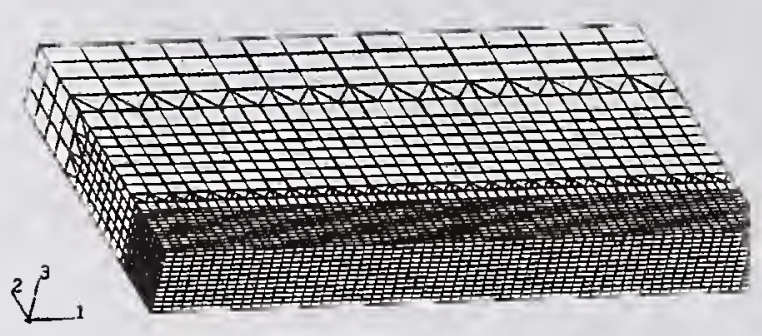

Figure 12 FE mesh of the model

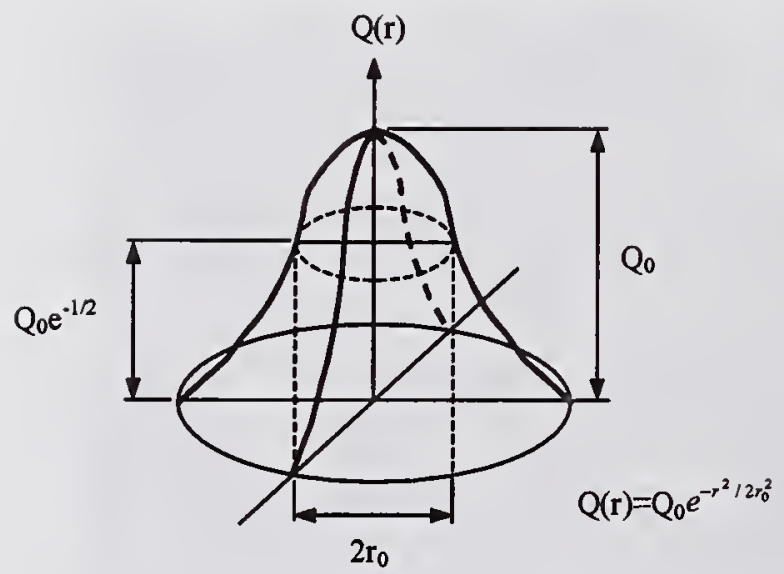

Figure 13 Gaussian distributed heat source
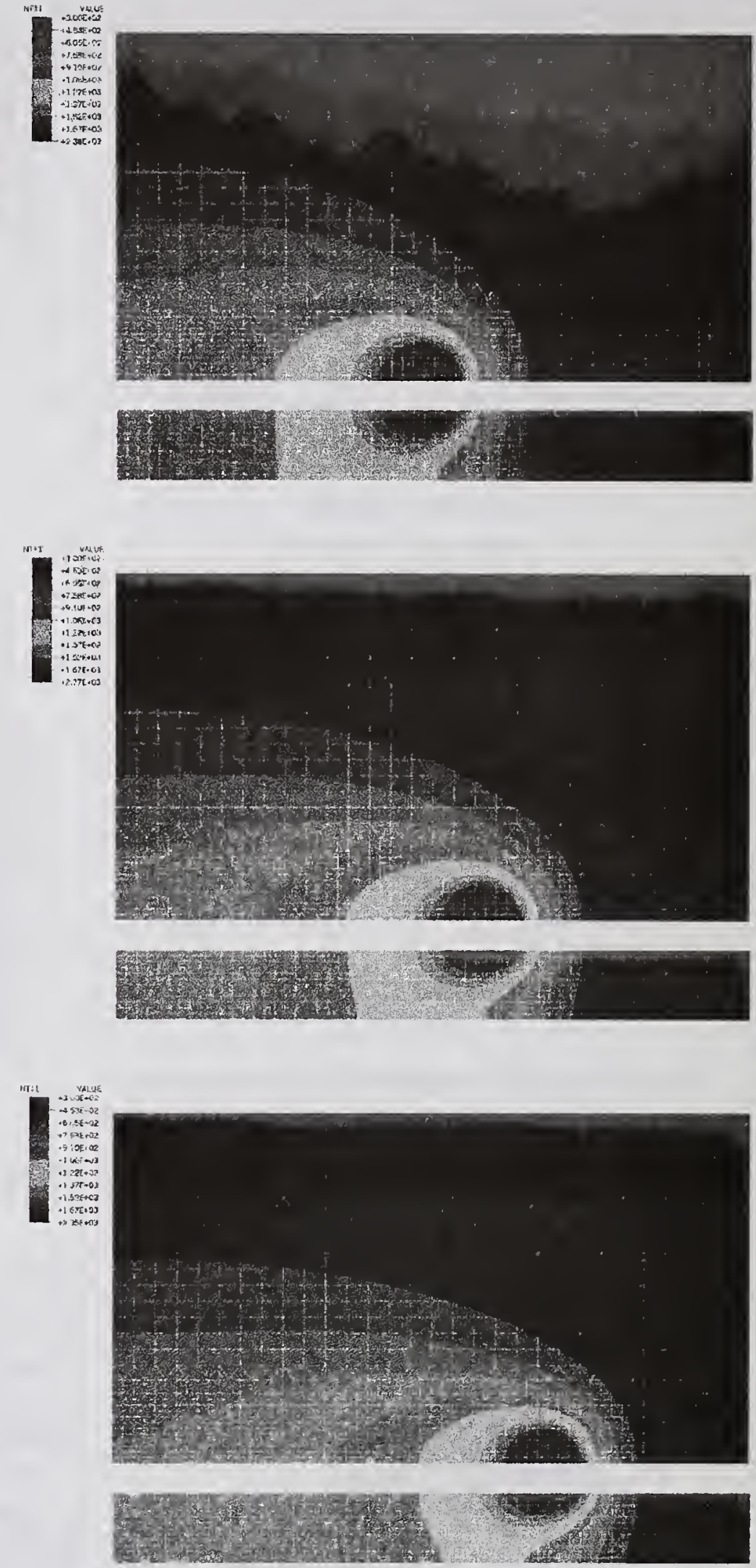

Figure 14 Temperature distributions at 9th second. (red areas stand for the weld pools $)(E=12.2 \mathrm{~V}, \mathrm{I}=90 \mathrm{~A}, \eta=0.50$, $r_{0}=1.05$ with speed 125,150 , $175 \mathrm{~mm} / \mathrm{min}$ respectively) 


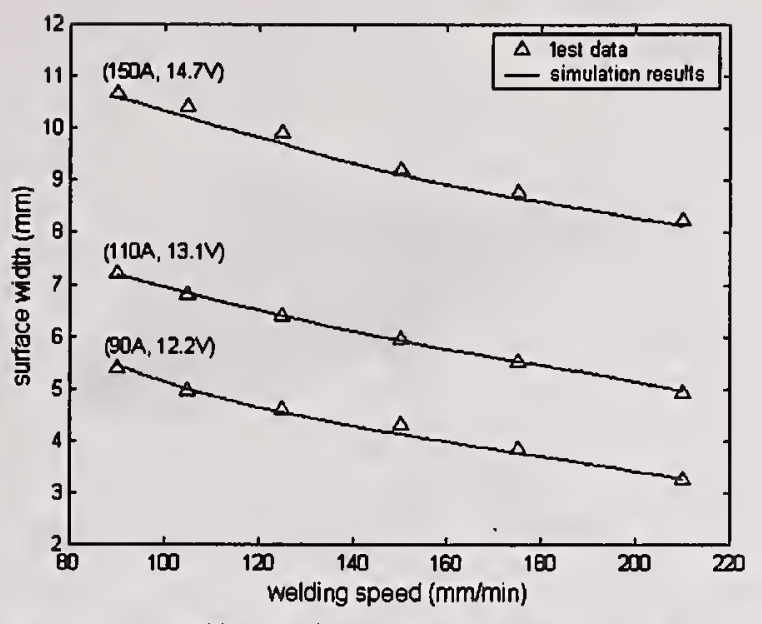

Figure 15 Effect of welding speed on the suface width of weld pool (arc length $=2 \mathrm{~mm}$, workpiece thickness $=4 \mathrm{~mm}$ )

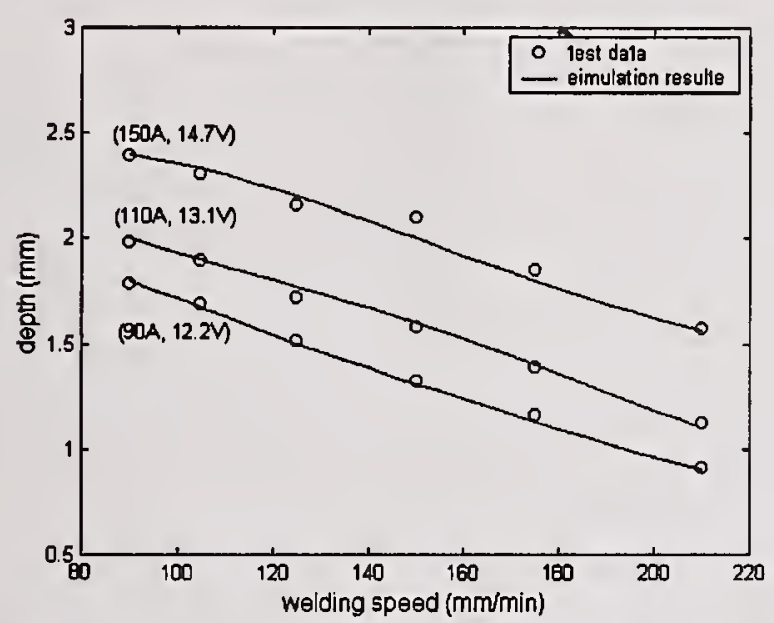

Figure 16 Effect of welding speed on the depth of weld pool (arc length $=2 \mathrm{~mm}$, workpiece thickness $=4 \mathrm{~mm}$ )

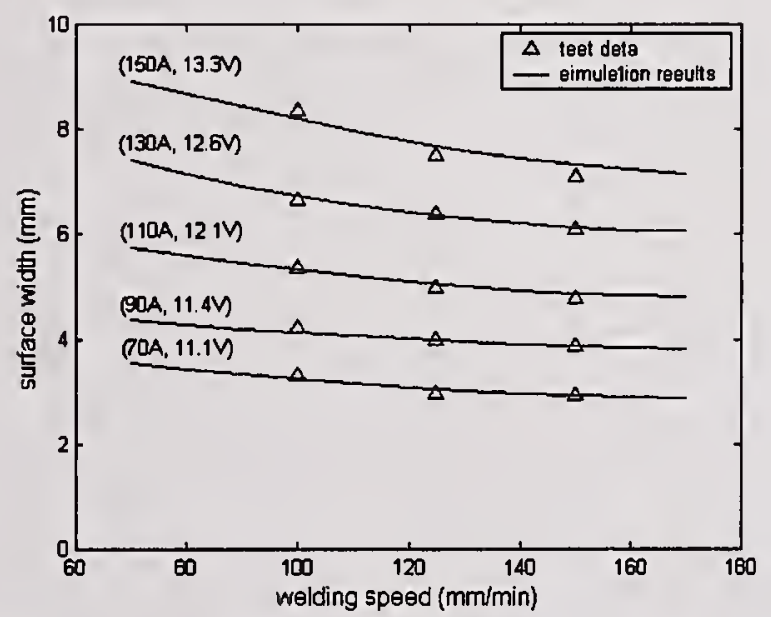

Figure 17 Effect of welding speed on the surface width of weld pool (arc length $=2 \mathrm{~mm}$, workpiece thickness $=6 \mathrm{~mm}$ )

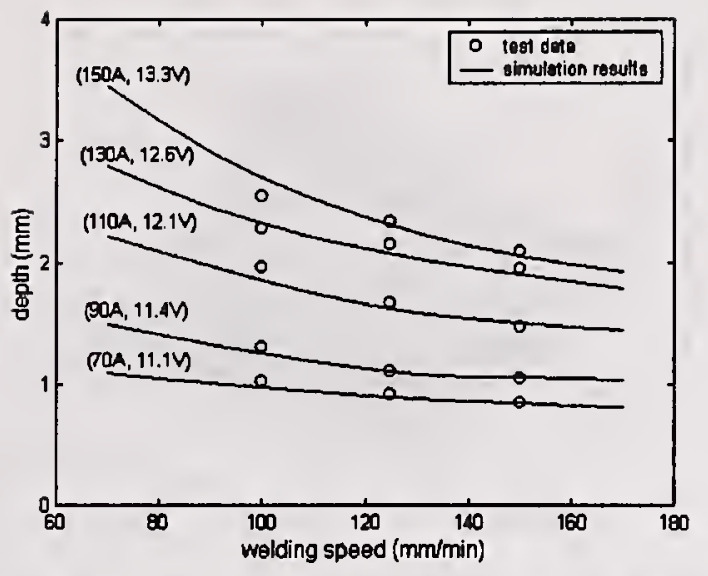

Figure 18 Effect of welding speed on the depth of weld pool (arc length $=2 \mathrm{~mm}$, workpiece thickness $=6 \mathrm{~mm}$ )

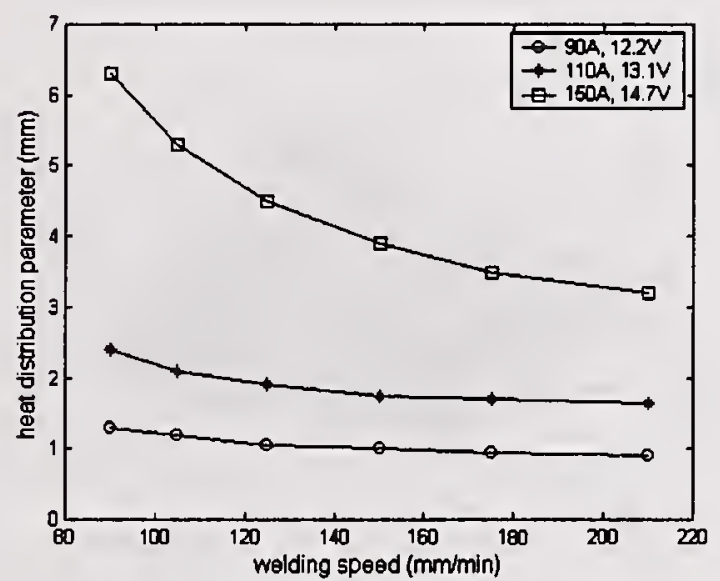

Figure 19 Effect of welding speed on the heat distribution parameter (arc length $=2 \mathrm{~mm}$, workpiece thickness $=4 \mathrm{~mm}$ )

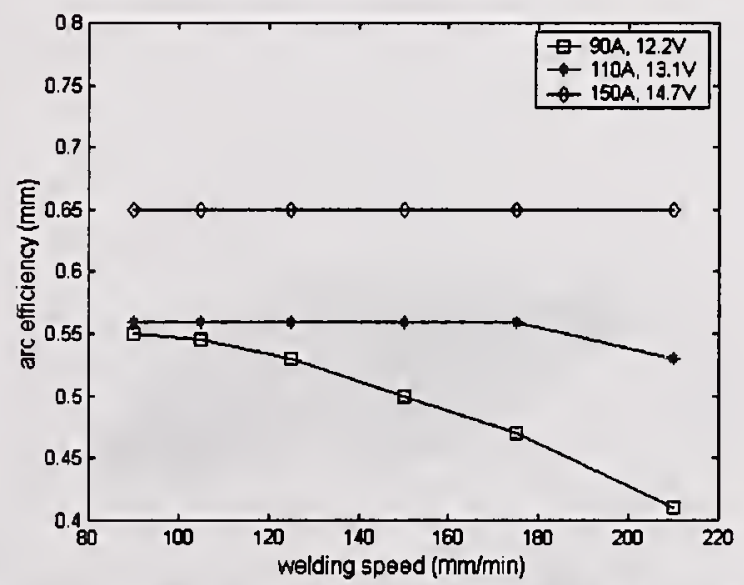

Figure 20 Effect of welding speed on arc efficiency (arc length $=2 \mathrm{~mm}$, workpiece thickness $=4 \mathrm{~mm}$ ) 


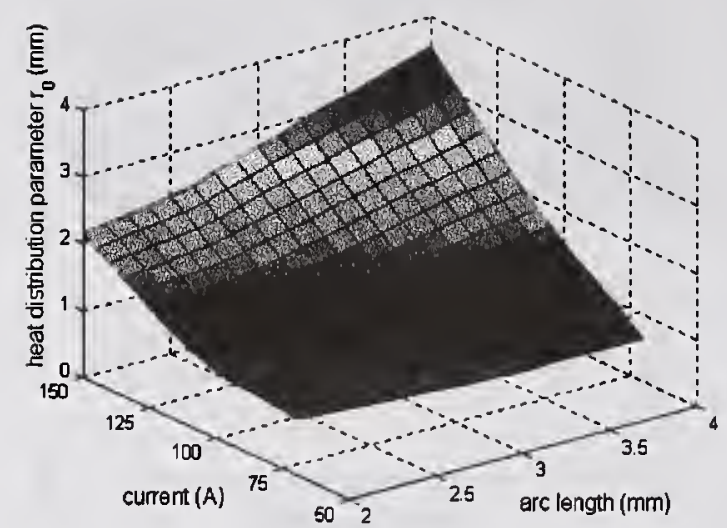

Figure 21 Correlation among heat distribution parameter, current and arc length under $1 \mathrm{nn} \mathrm{mm} / \mathrm{min}$ wxpldinn cneed

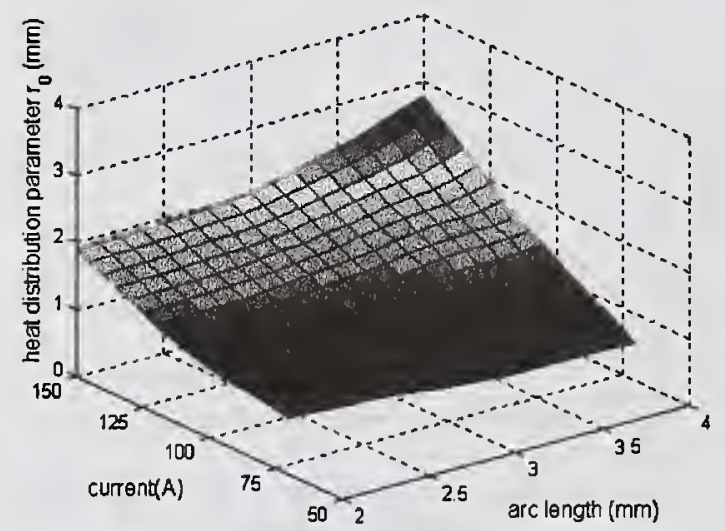

Figure 22 Correlation among heat distribution parameter, current and arc length under $150 \mathrm{~mm} / \mathrm{min}$ welding speed

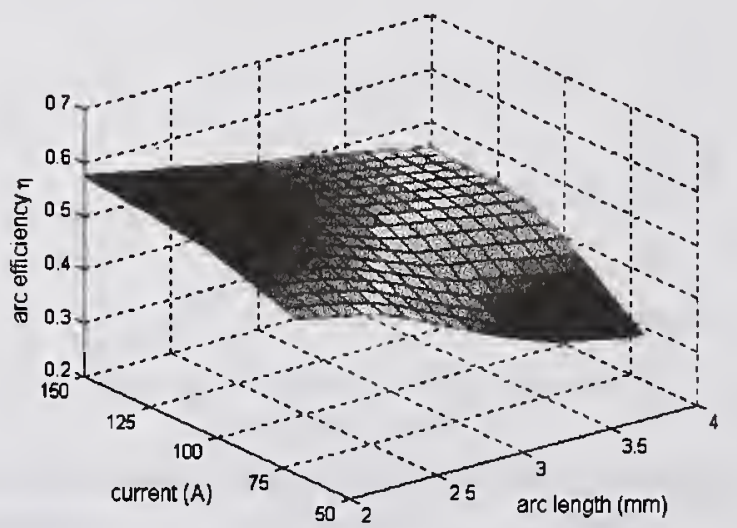

Figure 23 Correlation among arc efficiency, current and arc length under $100 \mathrm{~mm} / \mathrm{min}$ welding speed

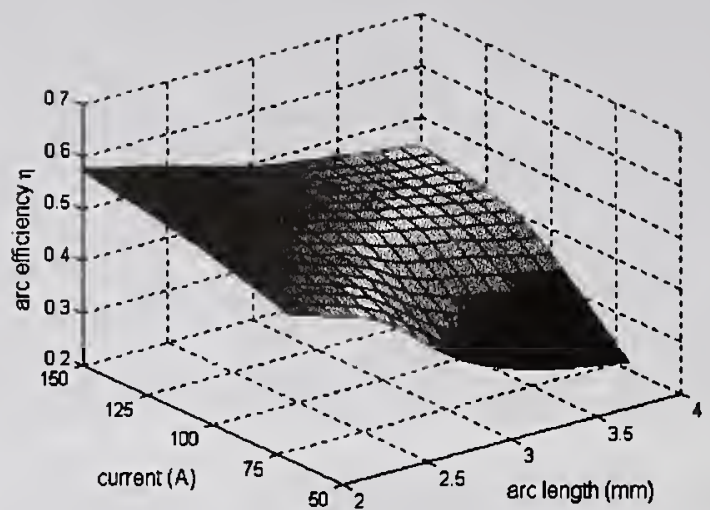

Figure 24 Correlation among arc efficiency, current and arc length under $150 \mathrm{~mm} / \mathrm{min}$ welding speed 




\section{NIST Technical Publications}

\section{Periodical}

Journal of Research of the National Institute of Standards and Technology-Reports NIST research and development in those disciplines of the physical and engineering sciences in which the Institute is active. These include physics, chemistry, engineering, mathematics, and computer sciences. Papers cover a broad range of subjects, with major emphasis on measurement methodology and the basic technology underlying standardization. Also included from time to time are survey articles on topics closely related to the Institute's technical and scientific programs. Issued six times a year.

\section{Nonperiodicals}

Monographs-Major contributions to the technical literature on various subjects related to the Institute's scientific and technical activities.

Handbooks-Recommended codes of engineering and industrial practice (including safety codes) developed in cooperation with interested industries, professional organizations, and regulatory bodies. Special Publications-Include proceedings of conferences sponsored by NIST, NIST annual reports, and other special publications appropriate to this grouping such as wall charts, pocket cards, and bibliographies. National Standard Reference Data Series-Provides quantitative data on the physical and chemical properties of materials, compiled from the world's literature and critically evaluated. Developed under a worldwide program coordinated by NIST under the authority of the National Standard Data Act (Public Law 90-396). NOTE: The Journal of Physical and Chemical Reference Data (JPCRD) is published bimonthly for NIST by the American Institute of Physics (AIP). Subscription orders and renewals are available from AIP, P.O. Box 503284, St. Louis, MO 63150-3284.

Building Science Series-Disseminates technical information developed at the Institute on building materials, components, systems, and whole structures. The series presents research results, test methods, and performance criteria related to the structural and environmental functions and the durability and safety characteristics of building elements and systems.

Technical Notes-Studies or reports which are complete in themselves but restrictive in their treatment of a subject. Analogous to monographs but not so comprehensive in scope or definitive in treatment of the subject area. Often serve as a vehicle for final reports of work performed at NIST under the sponsorship of other government agencies.

Voluntary Product Standards-Developed under procedures published by the Department of Commerce in Part 10, Title 15, of the Code of Federal Regulations. The standards establish nationally recognized requirements for products, and provide all concerned interests with a basis for common understanding of the characteristics of the products. NIST administers this program in support of the efforts of private-sector standardizing organizations.

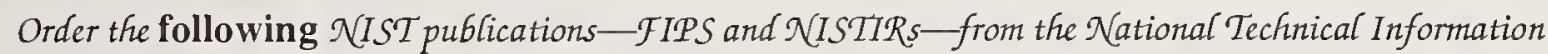

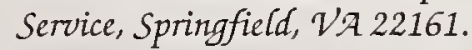

Federal Information Processing Standards Publications (FIPS PUB)_Publications in this series collectively constitute the Federal Information Processing Standards Register. The Register serves as the official source of information in the Federal Government regarding standards issued by NIST pursuant to the Federal Property and Administrative Services Act of 1949 as amended, Public Law 89-306 (79 Stat. 1127), and as implemented by Executive Order 11717 (38 FR 12315, dated May 11, 1973) and Part 6 of Title 15 CFR (Code of Federal Regulations).

NIST Interagency or Internal Reports (NISTIR)-The series includes interim or final reports on work performed by NIST for outside sponsors (both government and nongovernment). In general, initial distribution is handled by the sponsor; public distribution is handled by sales through the National Technical Information Service, Springfield, VA 22161, in hard copy, electronic media, or microfiche form. NISTIR's may also report results of NIST projects of transitory or limited interest, including those that will be published subsequently in more comprehensive form. 


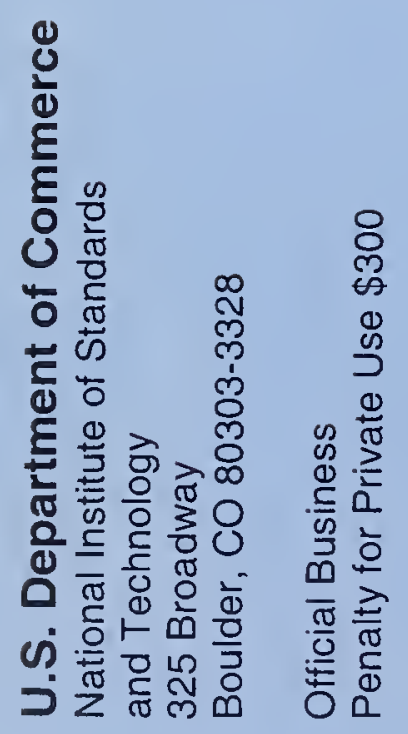

